Keywords: SB6, Frit 418, variability, durability

Retention: Permanent

\title{
Sludge Batch 6 Variability Study with Frit 418
}

\author{
F.C. Johnson
}

T.B. Edwards

November 2010

Savannah River National Laboratory Savannah River Nuclear Solutions Aiken, SC 29808

Prepared for the U.S. Department of Energy under contract number DE-AC09-08SR22470.

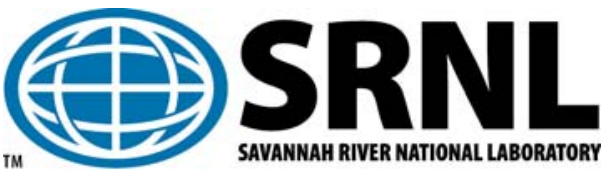


SRNL-STI-2010-00242

Revision 0

\section{DISCLAIMER}

This work was prepared under an agreement with and funded by the U.S. Government. Neither the U.S. Government or its employees, nor any of its contractors, subcontractors or their employees, makes any express or implied:

1. warranty or assumes any legal liability for the accuracy, completeness, or for the use or results of such use of any information, product, or process disclosed; or

2. representation that such use or results of such use would not infringe privately owned rights; or

3. endorsement or recommendation of any specifically identified commercial product, process, or service.

Any views and opinions of authors expressed in this work do not necessarily state or reflect those of the United States Government, or its contractors, or subcontractors.

\section{Printed in the United States of America \\ Prepared for \\ U.S. Department of Energy}




\section{REVIEWS AND APPROVALS}

\section{AUTHORS:}

F.C. Johnson, Process Technology Programs

Date

T.B. Edwards, Applied Computational Engineering and Statistics

Date

TECHNICAL REVIEW:

D.K. Peeler, Process Technology Programs

Date

APPROVAL:

C.C. Herman, Manager

Date

Process Technology Programs

S.L. Marra, Manager

Date

Environmental \& Chemical Process Technology Research Programs

J.E. Occhipinti, Manager

Date

Waste Solidification Engineering 
SRNL-STI-2010-00242

Revision 0

\section{EXECUTIVE SUMMARY}

The Defense Waste Processing Facility (DWPF) initiated processing Sludge Batch 6 (SB6) in the summer of 2010. In support of processing, the Savannah River National Laboratory (SRNL) provided a recommendation to utilize Frit 418 to process SB6. This recommendation was based on assessments of the compositional projections for SB6 available at the time from the Liquid Waste Organization (LWO) and SRNL (using a model-based approach). To support qualification of SB6, SRNL executed a variability study to assess the applicability of the current durability models for SB6. The durability models were assessed over the expected Frit 418-SB6 composition range.

Seventeen glasses were selected for the variability study based on the sludge projections used in the frit recommendation. Five of the glasses are based on the centroid of the compositional region, spanning a waste loading (WL) range of 32 to $40 \%$. The remaining twelve glasses are extreme vertices (EVs) of the sludge region of interest for SB6 combined with Frit 418 and are all at $36 \% \mathrm{WL}$. These glasses were fabricated and characterized using chemical composition analysis, X-ray diffraction (XRD) and the Product Consistency Test (PCT).

After initiating the SB6 variability study, the measured composition of the SB6 Tank 51 qualification glass produced at the SRNL Shielded Cells Facility indicated that thorium was present in the glass at an appreciable concentration $(1.03 \mathrm{wt} \%)$, which made it a reportable element for SB6. This concentration of $\mathrm{ThO}_{2}$ resulted in a second phase of experimental studies. Five glasses were formulated that were based on the centroid of the new sludge compositional region combined with Frit 418, spanning a WL range of 32 to $40 \%$. These glasses were fabricated and characterized using chemical composition analysis and the PCT.

Based on the measured PCT response, all of the glasses (with and without thorium) were acceptable with respect to the Environmental Assessment (EA) reference glass regardless of thermal history. All of the normalized boron releases were less than $1 \mathrm{~g} / \mathrm{L}$. While all of the targeted glass compositions were predictable with respect to the Product Composition Control System (PCCS) models for durability, a small number of the measured glass compositions were located outside of the lower prediction limit indicating poorer durability than what was actually measured. These unpredictable glasses were in the same lithium metaborate (LM) preparation block during the chemical analyses, which resulted in measured compositions that were not representative of the target compositions. A review of the data did not indicate a clear cause for the problem. Re-digestion and re-measurement of three glasses from this preparation block yielded glass compositions closer to the target values and predicted PCT responses within the PCCS model uncertainty. Therefore, it is believed that the glasses were correctly fabricated and the targeted compositions are closer representations of the true compositions.

Per the requirements of the DWPF Glass Product Control Program, the PCCS durability models have been shown to be applicable for the SB6/Frit 418 glass system. PCT results from the glasses fabricated as part of the variability study were shown to be predictable and/or acceptable with respect to the DWPF PCCS models. In addition, the inclusion of $\mathrm{ThO}_{2}$ was shown to have minimal impact on the acceptability and predictability of the variability study glasses. 


\section{TABLE OF CONTENTS}

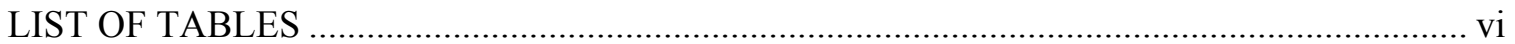

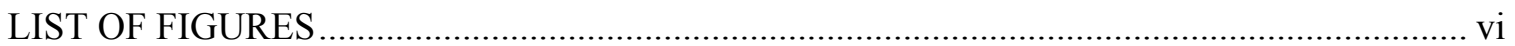

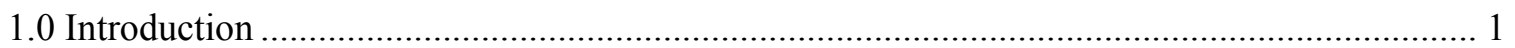

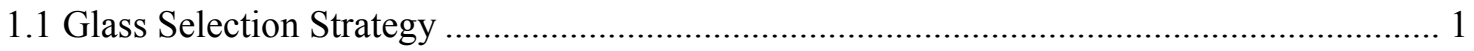

1.1.1 Non-thorium Variability Study Glasses ................................................................... 1

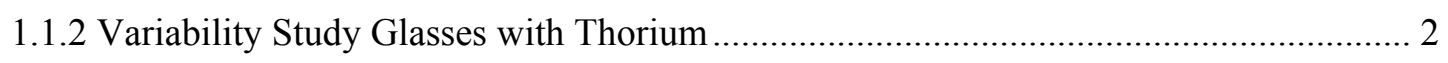

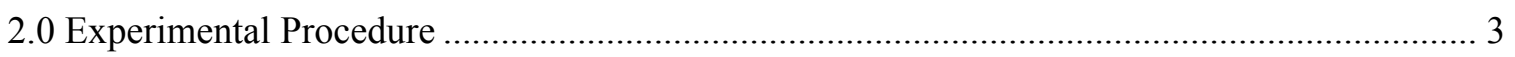

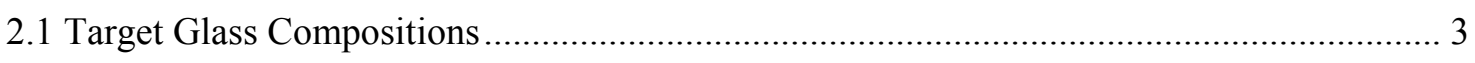

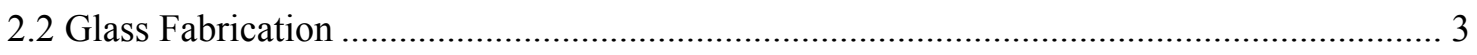

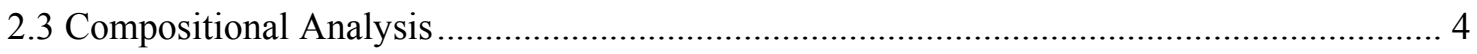

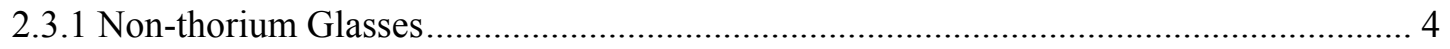

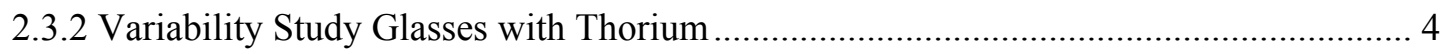

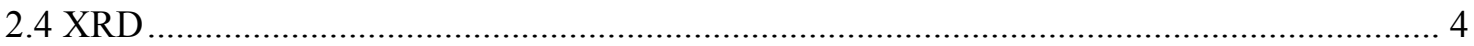

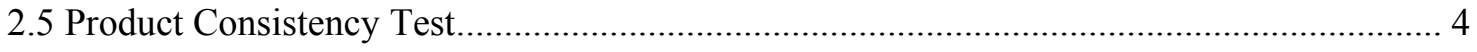

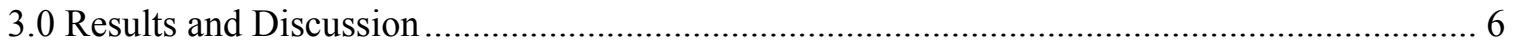

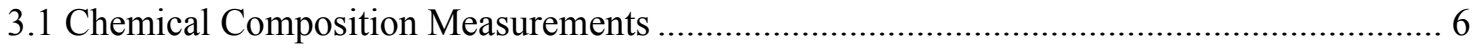

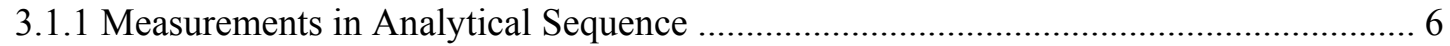

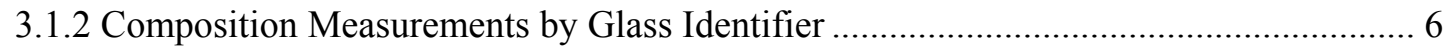

3.1.3 Statistical Evaluations of the Results from the Standard Glasses ................................... 7

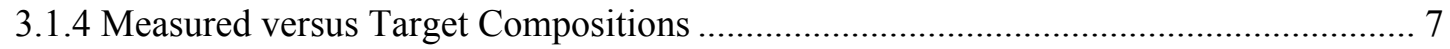

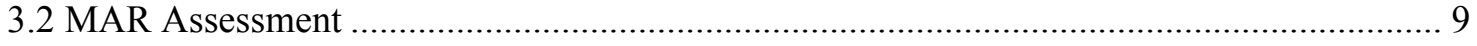

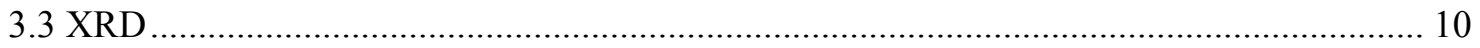

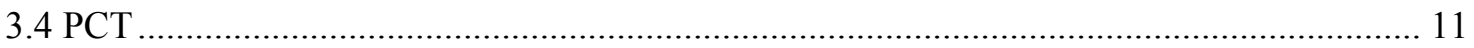

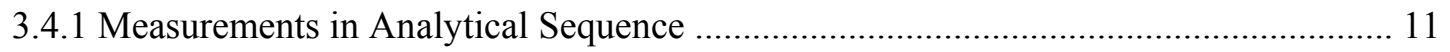

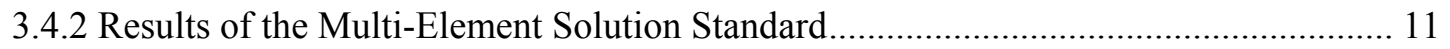

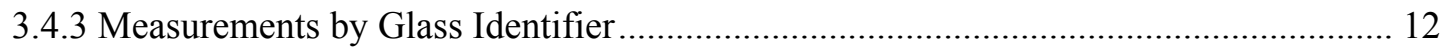

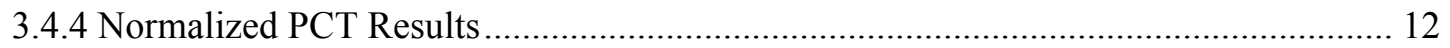

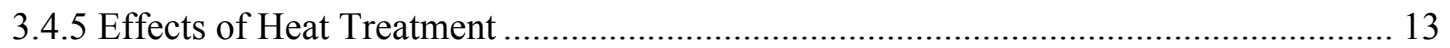

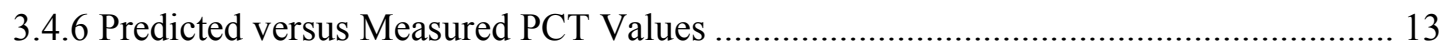

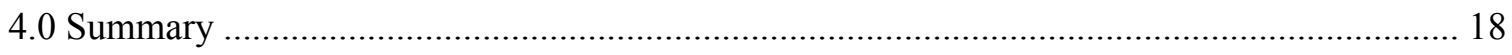

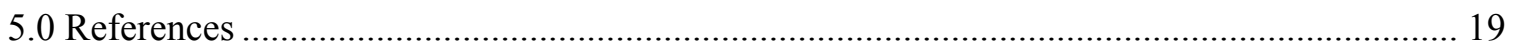




\section{LIST OF TABLES}

Table 1-1. SB6 Sludge Composition Region........................................................................... 2

Table 1-2 Centroid of the SB6 Composition Region Used for Variation Stage Assessments ....... 3

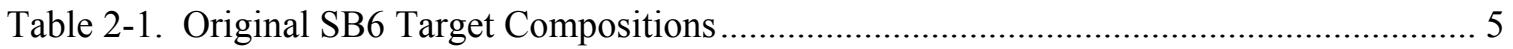

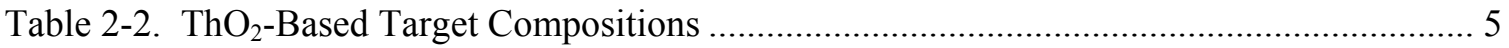

Table 3-1. Re-measured Values of SB6VS-02, -04 and -15 ............................................................ 8

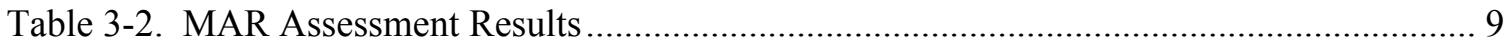

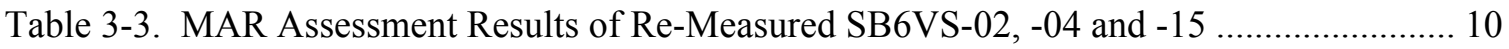

Table 3-4. Results from Samples of the Multi-Element Solution Standard ................................. 11

Table 3-5. Normalized PCT Results of the Non-Thorium Glasses ............................................ 14

Table 3-6. Normalized PCT Results of the Thorium Glasses .................................................... 15

Table 3-7. Normalized PCT Results of the Re-Measured Glasses.............................................. 15

\section{LIST OF FIGURES}

Figure 3-1. Representative XRD pattern from a quenched (a) and ccc (b) sample..................... 10

Figure 3-2. del Gp $\left(\Delta \mathrm{G}_{\mathrm{p}}\right)$ predictions versus the common logarithm of the normalized leachate $(\log$ NL[.]) for B. The PCT responses are normalized to the measured compositions of the

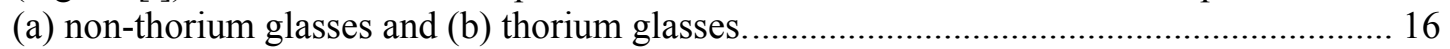

Figure 3-3. del $\mathrm{Gp}\left(\Delta \mathrm{G}_{\mathrm{p}}\right)$ predictions versus the common logarithm of the normalized leachate ( $\log$ NL[.]) for B for SB6VS-02, -04 and -15 . The PCT responses are normalized to the measured compositions of the (a) re-measured glasses and (b) original glasses. 17 


\section{LIST OF ABBREVIATIONS}

$\begin{array}{ll}\text { AD } & \text { Analytical Development } \\ \text { ARM } & \text { Approved Reference Material } \\ \text { ARP } & \text { Actinide Removal Process } \\ \text { DWPF } & \text { Defense Waste Processing Facility } \\ \text { EA } & \text { Environmental Assessment } \\ \text { EV } & \text { Extreme Vertices } \\ \text { ICP-AES } & \text { Inductively Coupled Plasma-Atomic Emission Spectroscopy } \\ \text { LM } & \text { Lithium Metaborate } \\ \text { LWO } & \text { Liquid Waste Organization } \\ \text { MA } & \text { Mixed Acid } \\ \text { PCT } & \text { Product Consistency Test } \\ \text { PF } & \text { Peroxide Fusion } \\ \text { PSAL } & \text { Process Science and Analytical Laboratory } \\ \text { SB6 } & \text { Sludge Batch 6 } \\ \text { SRNL } & \text { Savannah River National Laboratory } \\ \text { SRNL } & \text { Savannah River National Laboratory } \\ \text { TT\&QAP } & \text { Task Technical and Quality Assurance Plan } \\ \text { TTR } & \text { Technical Task Request } \\ \text { XRD } & \text { X-ray Diffraction }\end{array}$




\subsection{Introduction}

The Defense Waste Processing Facility (DWPF) initiated processing Sludge Batch 6 (SB6) in the summer of 2010. In support of the upcoming processing, the Savannah River National Laboratory (SRNL) assessed the applicability of using Frit 418 to process SB6. ${ }^{1}$ This assessment was based on the compositional projections for SB6 available at the time from the Liquid Waste Organization (LWO) and SRNL (using a model-based approach).

To support qualification of SB6 and the Glass Product Control Program, SRNL executed a variability study to assess the applicability of the current durability models for SB6. The durability models were assessed over the expected Frit 418-SB6 composition range. The experimental portion of the original variability study was initiated by a DWPF Technical Task Request $^{2}$ (TTR) and was performed under a SRNL Task Technical and Quality Assurance Plan ${ }^{3}$ (TT\&QAP).

After initiating the SB6 variability study, the measured composition of the SB6 Tank 51 qualification glass produced at the SRNL Shielded Cells Facility indicated that thorium was present in the glass at an appreciable concentration $(1.03 \mathrm{wt} \%)$, which made it a reportable element for SB6. ${ }^{4}$ This concentration of $\mathrm{ThO}_{2}$ resulted in a second phase of experimental studies that were initiated by an addendum to the original TTR. ${ }^{\mathrm{a}}$

\subsection{Glass Selection Strategy}

For a complete discussion of the sludge projections and assessments leading up to the frit selection see SRNL-L3100-2010-00043 and SRNL-STI-2010-00137. ${ }^{1,5}$ Only a brief summary will be provided in this section.

\subsubsection{Non-thorium Variability Study Glasses}

SRNL generated projections using updated compositional data from the Tank 51 SB6 qualification sample ${ }^{6}$ and a washing spreadsheet ${ }^{b}$ provided by LWO. These projections included several scenarios for the SB6 blend as well as the impact of additions of material from the Actinide Removal Process (ARP). The bounding intervals for each of the sludge components developed for the frit recommendation are repeated in Table 1-1. Note that "Others" includes $\mathrm{BaO}, \mathrm{CdO}, \mathrm{Ce}_{2} \mathrm{O}_{3}, \mathrm{Cr}_{2} \mathrm{O}_{3}, \mathrm{CuO}, \mathrm{Gd}_{2} \mathrm{O}_{3}, \mathrm{~K}_{2} \mathrm{O}, \mathrm{La}_{2} \mathrm{O}_{3}, \mathrm{MoO}_{3} \mathrm{P}_{2} \mathrm{O}_{5}, \mathrm{PbO}, \mathrm{SrO}, \mathrm{ZnO}$ and $\mathrm{ZrO}_{2}$.

Seventeen glasses were selected for the variability study based on the sludge projections used in the frit selection. ${ }^{1}$ Five of the glasses were based on the centroid (average) of the compositional space combined with Frit 418, spanning a waste loading (WL) range of 32 to $40 \%$ (2 wt $\%$ increments). The remaining twelve glasses were extreme vertices (EVs) of the sludge region of interest for SB6 combined with Frit 418 and were all at 36\% WL. ${ }^{7, c}$

These glasses were fabricated and characterized using chemical composition analysis, X-ray diffraction (XRD) and the Product Consistency Test (PCT).

\footnotetext{
${ }^{\text {a }}$ Email communication from J. Bricker on 5/7/2010.

b Excel workbook "SB6_011310.xls." Sent by Jeff Gillam via email on 1/19/2010.

${ }^{c}$ The twelve sludge EVs were selected using the D-optimality routine available in the Custom Design platform in $\mathrm{JMP}^{\mathrm{TM}}$ to support investigation of linear affects of the sludge components.
} 
SRNL-STI-2010-00242

Revision 0

Table 1-1. SB6 Sludge Composition Region (wt $\%$ calcined basis)

\begin{tabular}{|c|c|c|}
\hline \multirow{2}{*}{ Oxide } & Maximum & Minimum \\
\hline & \multicolumn{2}{|c|}{$(\mathrm{wt} \%)$} \\
\hline $\mathrm{Al}_{2} \mathrm{O}_{3}$ & 23.49 & 29.25 \\
\hline $\mathrm{CaO}$ & 0.78 & 2.03 \\
\hline $\mathrm{Fe}_{2} \mathrm{O}_{3}$ & 21.69 & 26.82 \\
\hline $\mathrm{MgO}$ & 0.17 & 1.38 \\
\hline $\mathrm{MnO}$ & 6.25 & 7.58 \\
\hline $\mathrm{Na}_{2} \mathrm{O}$ & 23.92 & 29.55 \\
\hline $\mathrm{NiO}$ & 2.39 & 3.51 \\
\hline $\mathrm{SiO}_{2}$ & 1.59 & 2.74 \\
\hline $\mathrm{SO}_{4}$ & 1.06 & 1.73 \\
\hline $\mathrm{TiO}_{2}$ & 0 & 1.84 \\
\hline $\mathrm{U}_{3} \mathrm{O}_{8}$ & 3.95 & 5.65 \\
\hline Others & 0.99 & 2.11 \\
\hline
\end{tabular}

\subsubsection{Variability Study Glasses with Thorium}

Peeler and Edwards conducted a paper study, which determined the impact of $\mathrm{ThO}_{2} \mathrm{PCCS}$ predictions for durability, viscosity and liquidus temperature. ${ }^{8}$ Based on the results, it was recommended that DWPF could process the SB6-Frit 418 system with $\mathrm{ThO}_{2}$ concentrations up to $1.8 \mathrm{wt} \%$. In order to confirm the affect of $\mathrm{TiO}_{2}$ concentrations on durability, five additional glasses were generated using updated SB6 projections based on a thorium projection provided by Savannah River Remediation (SRR). ${ }^{\mathrm{d}}$ These projection included several scenarios for the SB6 blend as well as the impact of additions of material from ARP. ${ }^{9}$ EVs were developed with these projections, which resulted in a centroid composition. Five glasses were formulated that were based on the centroid of the compositional region (Table 1-2) combined with Frit 418, spanning a WL range of 32 to $40 \%$ ( $2 \mathrm{wt} \%$ increments). The "Others" are shown individually in this table.

These glasses were fabricated and characterized using chemical composition analysis and the PCT.

\footnotetext{
${ }^{d}$ Thorium oxide concentration provided via email by H. H. Elder on May 6, 2010.
} 
SRNL-STI-2010-00242

Revision 0

Table 1-2 Centroid of the SB6 Composition Region Used for Variation Stage Assessments

\begin{tabular}{|c|c|}
\hline Sludge Oxide & Concentration (wt\%) \\
\hline $\mathrm{Al}_{2} \mathrm{O}_{3}$ & 25.36 \\
\hline $\mathrm{BaO}$ & 0.13 \\
\hline $\mathrm{CaO}$ & 1.37 \\
\hline $\mathrm{Ce}_{2} \mathrm{O}_{3}$ & 0.20 \\
\hline $\mathrm{Cr}_{2} \mathrm{O}_{3}$ & 0.12 \\
\hline $\mathrm{CuO}$ & 0.10 \\
\hline $\mathrm{Fe}_{2} \mathrm{O}_{3}$ & 23.46 \\
\hline $\mathrm{K}_{2} \mathrm{O}$ & 0.08 \\
\hline $\mathrm{La}_{2} \mathrm{O}_{3}$ & 0.10 \\
\hline $\mathrm{MgO}_{3}$ & 0.76 \\
\hline $\mathrm{MnO}$ & 6.68 \\
\hline $\mathrm{Na}_{2} \mathrm{O}$ & 25.79 \\
\hline $\mathrm{NiO}_{2}$ & 2.86 \\
\hline $\mathrm{P}_{2} \mathrm{O}_{5}$ & 0.43 \\
\hline $\mathrm{PbO}_{3}$ & 0.03 \\
\hline $\mathrm{SO}_{4}{ }^{2-}$ & 1.35 \\
\hline $\mathrm{SiO}_{2}$ & 2.10 \\
\hline $\mathrm{ThO}_{2}$ & 3.16 \\
\hline $\mathrm{TiO}_{2}$ & 0.92 \\
\hline $\mathrm{U}_{3} \mathrm{O}_{8}$ & 4.68 \\
\hline $\mathrm{ZnO}_{2 \mathrm{ZrO}_{2}}$ & 0.07 \\
\hline & 0.23 \\
\hline
\end{tabular}

\subsection{Experimental Procedure}

\subsection{Target Glass Compositions}

Target glass compositions of the original seventeen variability study glasses are shown in Table 2-1. The first twelve glasses (SB6VS-01 through SB6VS-12) are based on the EVs, while the last five (SB6VS-13 through SB6VS-17) are based on the centroid of the compositional region.

Target glass compositions of the five $\mathrm{ThO}_{2}$-based variability study glasses are shown in Table 2-2 .

\subsection{Glass Fabrication}

Each variability study glass was prepared from the proper proportions of reagent-grade metal oxides, carbonates, $\mathrm{H}_{3} \mathrm{BO}_{3}$, and salts in $150 \mathrm{~g}$ batches. ${ }^{10}$ The raw materials were thoroughly mixed and placed into a platinum alloy, $250 \mathrm{ml}$ crucible. Batched materials were placed into a high-temperature furnace at the target melt temperature of $1150^{\circ} \mathrm{C}$. The crucible was removed from the furnace after an isothermal hold at $1150^{\circ} \mathrm{C}$ for 1 hour. The molten glass was quenched by pouring the liquid onto a clean, stainless steel plate. The glass pour patty was used as a sampling stock for the various property measurements (i.e., chemical composition, durability testing and XRD). 
Approximately $25 \mathrm{~g}$ of each glass was heat-treated to simulate cooling along the centerline of a DWPF-type canister to gauge the effects of thermal history on the product performance. ${ }^{11}$ This cooling schedule is referred to as the centerline canister cooling (ccc) curve.

\subsection{Compositional Analysis}

\subsubsection{Non-thorium Glasses}

Two dissolution methods were utilized by the Process Science Analytical Laboratory (PSAL) to allow measurement of the compositions: lithium metaborate fusion (LM) and sodium peroxide fusion (PF). For each study glass, measurements were obtained from samples prepared in duplicate by each of these dissolution methods. A representative sample from each glass was submitted to Analytical Development (AD). ${ }^{12}$ All of the prepared samples were analyzed (twice for each element of interest) by Inductively Coupled Plasma-Atomic Emission Spectroscopy (ICP-AES) with the instrumentation being re-calibrated between the duplicate analyses. The analytical plan was developed in such a way as to provide the opportunity to evaluate potential sources of bias and error. Glass standards were also intermittently measured to assess the performance of the ICP-AES instrument over the course of these analyses.

\subsubsection{Variability Study Glasses with Thorium}

Representative samples of each glass were submitted to AD for ICP-AES. ${ }^{13}$ Two dissolution methods were utilized by AD, which include: PF and mixed acid (MA).

\subsection{XRD}

Representative samples of quenched and ccc glasses were submitted to AD for XRD analysis. ${ }^{\mathrm{e}}$ Samples were analyzed under conditions providing a detection limit of approximately $0.5 \mathrm{vol} \%$, i.e. no crystals can be detected if the amount in the sample is less than $\sim 0.5 \mathrm{vol} \%$.

\subsection{Product Consistency Test}

The PCT was performed in triplicate on each quenched and ccc glass to assess chemical durability using Method A of the procedure. ${ }^{14}$ Also included in the experimental test matrix was the Environmental Assessment (EA) glass, the Approved Reference Material (ARM) glass, and blanks from the sample cleaning batch. Samples were ground, washed, and prepared according to the standard procedure. The resulting solutions were sampled (filtered and acidified) and analyzed by AD under the auspices of three analytical plans. ${ }^{15-17}$ Samples of a multi-element, standard solution were also included in the analytical plan (as a check on the accuracy of the ICPAES instrument). Normalized release rates were calculated based on target and measured compositional views using the average of the leachate concentrations.

${ }^{\mathrm{e}}$ SB6VS-01 through SB6VS-17 only. 
Table 2-1. Original SB6 Target Compositions

\begin{tabular}{|c|c|c|c|c|c|c|c|c|c|c|c|c|c|c|c|c|c|c|c|c|c|c|c|c|}
\hline Glass ID & L (\%) & $\mathrm{Al}_{2} \mathbf{O}_{3}$ & $\mathrm{~B}_{2} \mathbf{O}_{3}$ & $\mathrm{BaO}$ & $\mathrm{CaO}$ & $\mathrm{Ce}_{2} \mathrm{O}_{3}$ & $\mathrm{Cr}_{2} \mathrm{O}_{3}$ & $\mathrm{CuO}$ & $\mathrm{Fe}_{2} \mathrm{O}_{3}$ & $\mathrm{~K}_{2} \mathrm{O}$ & $\mathrm{La}_{2} \mathrm{O}_{3}$ & $\mathrm{Li}_{2} \mathbf{O}$ & MgO & MnO & $\mathrm{Na}_{2} \mathrm{O}$ & $\mathrm{NiO}$ & $\mathbf{P}_{2} \mathbf{O}_{5}$ & PbO & $\mathrm{SO}_{4}$ & $\mathrm{SiO}_{2}$ & $\mathrm{TiO}_{2}$ & $\mathbf{U}_{3} \mathbf{O}_{8}$ & $\mathrm{ZnO}$ & $\mathrm{ZrO}_{2}$ \\
\hline SB6VS-01 & 36 & 10.03 & 5.12 & .05 & 0.28 & 0.08 & & 0.04 & 9.66 & 0.03 & 0.04 & 5.12 & 0.50 & 2.25 & 13.73 & 0.86 & 0.17 & 0.01 & 0.62 & 49.21 & 0.00 & 2.03 & 0.03 & 0.09 \\
\hline SB6V: & 36 & .79 & 5.12 & 0.05 & 0.73 & 08 & 05 & 0.04 & 9.66 & 0.03 & 0.04 & 5.12 & 0.06 & 2.25 & 13.73 & 0.86 & 0.17 & 0.01 & 0.38 & 49.63 & 0.66 & 1.42 & 0.03 & 0.09 \\
\hline SB6VS-03 & 36 & 10.35 & 5.12 & 0.05 & 0.28 & 0.08 & & 0.04 & 7.81 & 0.03 & 0.04 & 5.12 & 0.06 & 2.73 & 13.73 & 1.26 & 0.17 & 0.01 & 0.62 & 49.63 & 0.66 & 2.03 & 0.03 & 0.09 \\
\hline SB6V & & 9.76 & 12 & 0.02 & 0.73 & 03 & 02 & 0.01 & 9.66 & 0.01 & 0.01 & 5.12 & 0.06 & 2.73 & 13.73 & 1.26 & 0.06 & 0.00 & 0.38 & 49.21 & 0.00 & 2.03 & 0.01 & 0.03 \\
\hline SB6VS-05 & 36 & 10.53 & 5.12 & 0.05 & 0.28 & 0.08 & 0.05 & 0.04 & 7.93 & 0.03 & 0.04 & 5.12 & 0.06 & 2.73 & 15.76 & 0.86 & 0.17 & 0.01 & 0.38 & 49.21 & 0.00 & 1.42 & 0.03 & 0.09 \\
\hline SB6V: & & 9 & 5.12 & 0.02 & 0.73 & 0.03 & 02 & 0.01 & 7.81 & 0.01 & 0.01 & 5.12 & 0.06 & 2.25 & 15.76 & 0.86 & 0.06 & 0.00 & 0.62 & 49.63 & 0.00 & 2.03 & 0.01 & 0.03 \\
\hline SB6VS-07 & 36 & 10.53 & 5.12 & 0.02 & 0.28 & 0.03 & 0.02 & 0.01 & 7.81 & 0.01 & 0.01 & 5.12 & 0.50 & 2.25 & 15.50 & 1.26 & 0.06 & 0.00 & 0.38 & 49.63 & 0.00 & 1.42 & 0.01 & 0.03 \\
\hline SB6VS-08 & 36 & 8.46 & 5.12 & 0.02 & 0.28 & 0.03 & 02 & 0.01 & 9.66 & 0.01 & 0.01 & 5.12 & 0.06 & 2.25 & 15.68 & 1.26 & 0.06 & 0.00 & 0.62 & 49.21 & 0.66 & 1.42 & 0.01 & 0.03 \\
\hline SB6VS-09 & 36 & 8.46 & 5.12 & 0.05 & 0.73 & 0.08 & 0.05 & 0.04 & 9.66 & 0.03 & 0.04 & 5.12 & 0.50 & 2.73 & 14.16 & 1.26 & 0.17 & 0.01 & 0.62 & 49.63 & 0.00 & 1.42 & 0.03 & 0.09 \\
\hline SB6VS-10 & 36 & 8.46 & 5.12 & 0.02 & 0.28 & 0.03 & 0.02 & 0.01 & 9.66 & 0.01 & 0.01 & 5.12 & 0.50 & 2.73 & 14.38 & 0.86 & 0.06 & 0.00 & 0.38 & 49.63 & 0.66 & 2.03 & 0.01 & 0.03 \\
\hline SB6VS-11 & 36 & 10.53 & 5.12 & 0.02 & 0.73 & 0.03 & 02 & 0.01 & 7.81 & 0.01 & 0.01 & 5.12 & 0.50 & 2.73 & 14.49 & 0.86 & 0.06 & 0.00 & 0.62 & 49.21 & 0.66 & 1.42 & 0.01 & 0.03 \\
\hline SB6VS-12 & 36 & 8.57 & 5.12 & 0.05 & 0.73 & 0.08 & 0.05 & 0.04 & 7.81 & 0.03 & 0.04 & 5.12 & 0.50 & 2.25 & 15.76 & 1.26 & 0.17 & 0.01 & 0.38 & 49.21 & 0.66 & 2.03 & 0.03 & 0.09 \\
\hline SB6VS-13 & 32 & 8.47 & 5.44 & 0.03 & 0.45 & 0.05 & 0.03 & 0.02 & 7.79 & 0.02 & 0.02 & 5.44 & 0.25 & 2.21 & 14.02 & 0.94 & 0.10 & 0.01 & 0.45 & 52.37 & 0.29 & 1.53 & 0.02 & 0.05 \\
\hline SB6VS-14 & 34 & 9.00 & 5.28 & 0.03 & 0.48 & 0.05 & 0.03 & 0.03 & 8.28 & 0.02 & 0.03 & 5.28 & 0.26 & 2.35 & 14.39 & 1.00 & 0.11 & 0.01 & 0.48 & 50.90 & 0.31 & 1.63 & 0.02 & 0.06 \\
\hline SB6VS-15 & 36 & 9.53 & 5.12 & 0.03 & 0.50 & 0.05 & 0.03 & 0.03 & 8.76 & 0.02 & 0.03 & 5.12 & 0.28 & 2.49 & 14.77 & 1.06 & 0.11 & 0.01 & 0.50 & 49.42 & 0.33 & 1.72 & 0.02 & 0.06 \\
\hline SB6VS-16 & 38 & 10.06 & 4.96 & 0.04 & 0.53 & 0.06 & 0.03 & 0.03 & 9.25 & 0.02 & 0.03 & 4.96 & 0.29 & 2.63 & 15.14 & 1.12 & 0.12 & 0.01 & 0.53 & 47.94 & 0.35 & 1.82 & 0.02 & 0.06 \\
\hline SB6VS-17 & 40 & 10.59 & 4.80 & 0.04 & 0.56 & 0.06 & 0.03 & 0.03 & 9.74 & 0.02 & 0.03 & 4.80 & 0.31 & 2.77 & 15.52 & 1.18 & 0.13 & 0.01 & 0.56 & 46.46 & 0.36 & 1.91 & 0.02 & 0.07 \\
\hline
\end{tabular}

Table 2-2. $\mathrm{ThO}_{2}$-Based Target Compositions

\begin{tabular}{|c|c|c|c|c|c|c|c|c|c|c|c|c|c|c|c|c|c|c|c|c|c|c|c|c|c|}
\hline Glass ID & WL (\%) & $\mathrm{Al}_{2} \mathrm{O}_{3}$ & $\mathrm{~B}_{2} \mathrm{O}_{3}$ & $\mathrm{BaO}$ & $\mathrm{CaO}$ & $\mathrm{Ce}_{2} \mathrm{O}_{3}$ & $\mathrm{Cr}_{2} \mathrm{O}_{3}$ & $\mathrm{CuO}$ & $\mathrm{Fe}_{2} \mathrm{O}_{3}$ & $\mathrm{~K}_{2} \mathrm{O}$ & $\mathrm{La}_{2} \mathrm{O}_{3}$ & $\mathrm{Li}_{2} \mathrm{O}$ & $\mathrm{MgO}$ & $\mathrm{MnO}$ & $\mathrm{Na}_{2} \mathrm{O}$ & $\mathrm{NiO}$ & $\mathrm{P}_{2} \mathrm{O}_{5}$ & $\mathrm{PbO}$ & $\mathrm{SO}_{4}$ & $\mathrm{SiO}_{2}$ & $\mathrm{ThO}_{2}$ & $\mathrm{TiO}_{2}$ & $\mathrm{U}_{3} \mathrm{O}_{8}$ & $\mathrm{ZnO}$ & $\mathrm{ZrO}_{2}$ \\
\hline SB6VS-18 & 32 & 8.12 & 5.44 & 0.04 & 0.44 & 0.06 & 0.04 & 0.03 & 7.51 & 0.03 & 0.03 & 5.44 & 0.24 & 2.14 & 13.69 & 0.92 & 0.14 & 0.01 & 0.43 & 52.35 & 1.01 & 0.29 & 1.50 & 0.02 & 0.07 \\
\hline SB6VS-19 & 34 & 8.62 & 5.28 & 0.04 & 0.47 & 0.07 & 0.04 & 0.04 & 7.98 & 0.03 & 0.04 & 5.28 & 0.26 & 2.27 & 14.05 & 0.97 & 0.15 & 0.01 & 0.46 & 50.88 & 1.07 & 0.31 & 1.59 & 0.02 & 0.08 \\
\hline SB6VS-20 & 36 & 9.13 & 5.12 & 0.05 & 0.49 & 0.07 & 0.04 & 0.04 & 8.45 & 0.03 & 0.04 & 5.12 & 0.27 & 2.40 & 14.40 & 1.03 & 0.16 & 0.01 & 0.49 & 49.40 & 1.14 & 0.33 & 1.68 & 0.02 & 0.08 \\
\hline SB6VS-21 & 38 & 9.64 & 4.96 & 0.05 & 0.52 & 0.08 & 0.04 & 0.04 & 8.92 & 0.03 & 0.04 & 4.96 & 0.29 & 2.54 & 14.76 & 1.09 & 0.16 & 0.01 & 0.51 & 47.92 & 1.20 & 0.35 & 1.78 & 0.03 & 0.09 \\
\hline SB6VS-22 & 40 & 10.14 & 4.80 & 0.05 & 0.55 & 0.08 & 0.05 & 0.04 & 9.39 & 0.03 & 0.04 & 4.80 & 0.31 & 2.67 & 15.12 & 1.14 & 0.17 & 0.01 & 0.54 & 46.44 & 1.26 & 0.37 & 1.87 & 0.03 & 0.09 \\
\hline
\end{tabular}




\subsection{Results and Discussion}

\subsection{Chemical Composition Measurements}

Tables A1 and A2 in Appendix A provide the elemental concentration measurements from the non-thorium study glasses that were prepared using LM and Table A3 in Appendix A provides the measurements from these glasses using PF. Tables $\mathrm{C} 1$ and $\mathrm{C} 2$ in Appendix $\mathrm{C}$ provide the elemental concentration measurements from the thorium study glasses that were prepared using MA with a $10 \mathrm{X}$ and $2 \mathrm{X}$ dilution, respectively. Tables $\mathrm{C} 3$ and $\mathrm{C} 4$ in Appendix $\mathrm{C}$ provide the measurements from these glasses prepared using PF. Measurements for the Batch 1 and uranium standard $\left(\mathrm{U}_{\text {std }}\right)$ glass are also provided in the tables of Appendix A while measurements of ARG-1 and $\mathrm{U}_{\text {std }}$ are provided in the tables of Appendix C.

Elemental concentrations were converted to oxide concentrations by multiplying the values for each element by the gravimetric factor for the corresponding oxide. During this process, an elemental concentration that was determined to be below the detection limit of the analytical procedures used was reduced to half of that detection limit as the oxide concentration was determined. ${ }^{18}$

\subsubsection{Measurements in Analytical Sequence}

Exhibit A1 in Appendix A provides plots in analytical sequence of the sample measurements generated by $\mathrm{AD}$ for each oxide by preparation method (i.e., LM and PF) for the non-thorium glass study. Exhibit $\mathrm{C} 1$ in Appendix $\mathrm{C}$ provides similar plots for the MA and PF measurements of the thorium glasses. These plots include all of the measurement data, and in addition to analytical sequence of the measurements, the laboratory identifiers of each sample are shown.

\subsubsection{Composition Measurements by Glass Identifier}

Exhibit A2 in Appendix A provides plots of the oxide concentration measurements by Glass ID/Lab ID (including Batch 1 and $U_{\text {std }}$ ) for the non-thorium glasses. Similar plots for the thorium glasses are provided in Exhibit C2 of Appendix C, which include measurements of ARG-1 and $\mathrm{U}_{\text {std. }}$ For both Exhibit A2 and Exhibit C2, the measurements are arranged by the target concentrations of the study glasses. The plots in both exhibits demonstrate the individual measurements across the duplicates of each preparation method and the two ICP-AES calibrations for each study glass. Exhibit $\mathrm{C} 2$ provides a comparison of the MA and PF measurements for the thorium glasses. While there appears to be good repeatability among the measurements for each of the oxides for most of the glasses, there are some issues that should be noted.

The following observations are made regarding Exhibit A2. The $\mathrm{Al}_{2} \mathrm{O}_{3}$ measurements for one of SB6VS-13 are significantly higher than intended for that glass. There also appears to be a preparation issue for this oxide for this glass. Preparation issues are also indicated for other results; these include: $\mathrm{MnO}, \mathrm{Na}_{2} \mathrm{O}, \mathrm{NiO}$, and $\mathrm{U}_{3} \mathrm{O}_{8}$ for SB6VS-14 and several glasses showing higher than targeted $\mathrm{Na}_{2} \mathrm{O}$ measurements for both preparations. In general, the $\mathrm{SiO}_{2}$ values of all of the glasses are low compared to the target values. Scatter in the data is also observed for $\mathrm{B}_{2} \mathrm{O}_{3}$, $\mathrm{CaO}, \mathrm{Li}_{2} \mathrm{O}, \mathrm{MnO}$ and $\mathrm{U}_{3} \mathrm{O}_{8}$. A more thorough discussion of these analytical issues is provided in the following sections.

Based on a review of Exhibit C2, a decision was made by the authors to use the PF measurements 
rather than the MA measurements for all of the oxides for which measurements from both preparations are available. For example, the MA values of $\mathrm{ThO}_{2}$ for SB6VS-18 through 22 are consistently low when compared to both the PF and target values. Thus, the MA measurements for $\mathrm{CaO}, \mathrm{Na}_{2} \mathrm{O}$ and $\mathrm{ZrO}_{2}$ will be used to represent the measured compositions of the thorium glasses, while the PF measurements will be used for the remaining oxides. ${ }^{f}$

\subsubsection{Statistical Evaluations of the Results from the Standard Glasses}

Exhibit A3 in Appendix A provides statistical analyses of the Batch 1 and $\mathrm{U}_{\text {std }}$ results from the non-thorium glass study by calibration block for each oxide of interest for both the LM and PF preparation methods. The results include analysis of variance (ANOVA) investigations, which determine statistically significant differences among the means of these groups for each of the oxides for each of the standards. The following components indicate a significant ICP-AES calibration effect on the block averages at the 5\% significance level:

- $\quad$ Batch 1: $\mathrm{BaO}, \mathrm{CaO}, \mathrm{Cr}_{2} \mathrm{O}_{3}, \mathrm{CuO}$, and $\mathrm{TiO}_{2}$

- $\mathrm{U}_{\text {std }}: \mathrm{Al}_{2} \mathrm{O}_{3}, \mathrm{~B}_{2} \mathrm{O}_{3}, \mathrm{CaO}, \mathrm{Fe}_{2} \mathrm{O}_{3}, \mathrm{MgO}, \mathrm{Na}_{2} \mathrm{O}, \mathrm{TiO}_{2}$, and $\mathrm{U}_{3} \mathrm{O}_{8}$

Exhibit C3 in Appendix C provides statistical analyses of the ARG-1 and $\mathrm{U}_{\text {std }}$ results from the thorium glass study by calibration block and ANOVA investigations for each oxide of interest from the PF and MA preparation methods. The following components indicate a significant ICPAES calibration effect on the block averages at the 5\% significance level:

$$
\text { - } \mathrm{U}_{\text {std }}: \mathrm{Cr}_{2} \mathrm{O}_{3}(\mathrm{PF})
$$

Reference values for the oxide concentrations of the standards are given in the header for each set of measurements in Exhibits A3 and C3.

\subsubsection{Measured versus Target Compositions}

Table A4 in Appendix A provides a summary of the average compositions of the non-thorium glasses as well as the target compositions and some associated differences and relative differences. Exhibit A4 in Appendix A provides plots showing results for each glass for each oxide to help highlight the comparisons among the measured and targeted values for the nonthorium glasses. Table C5 and Exhibit C4 in Appendix C provide similar information for the results of the thorium glass study. In general, the measured values are consistent with the target oxide content in each of the study glasses. Some exceptions include: (i) high measured values of $\mathrm{CaO}, \mathrm{Na}_{2} \mathrm{O}, \mathrm{MnO}, \mathrm{NiO}$ and $\mathrm{SO}_{4}$ in some of the non-thorium glasses, (ii) low measured measured values of $\mathrm{Fe}_{2} \mathrm{O}_{3}, \mathrm{Li}_{2} \mathrm{O}$ and $\mathrm{SiO}_{2}$ in some of the non-thorium glasses, (iii) low measured values of $\mathrm{ThO}_{2}$ in SB6VS-18 through 22, and (iv) high measured values of $\mathrm{SiO}_{2}$ and $\mathrm{SO}_{4}$ in the thorium glasses. Although these issues exist, the measured sums of oxides for all of the study glasses are still within the interval of 95 to $105 \mathrm{wt} \%$. Despite the inconsistencies of some of the components, the compositions of the thorium glasses are thought to be accurate representations of the target compositions.

After thoroughly studying the data from the non-thorium glasses, it was hypothesized that the high $\mathrm{Na}_{2} \mathrm{O}, \mathrm{MnO}$ and $\mathrm{NiO}$ concentrations were likely due to preparation issues, specific to block

\footnotetext{
${ }^{\mathrm{f}}$ Calcium $(\mathrm{Ca})$ and sodium $(\mathrm{Na})$ are contaminants in the fusion reagents and a significant amount of the $\mathrm{Zr}$ crucible is also dissolved during fusion digestion.
} 
2 of the LM dissolutions. SB6VS-02, SB6VS-04 and SB6VS-15 were re-digested and remeasured by $\mathrm{AD}$. In general, the re-measured compositions are consistent with the target compositions, as shown in Table 3-1, which confirms the presence of an analytical preparation issue. All of the sums of oxides are within 95 and $105 \mathrm{wt} \%$. Based on these results, it should be recognized that the rest of the glass compositions from preparation block 2 are not representative of the target compositions and should not be included in the interpretation of the overall results of this study. The remainder of the glasses from preparation block 2 include: SB6VS-01, $-03,-05$ and -10 .

Due to the low measured values of $\mathrm{ThO}_{2}$, SB6VS-19 and SB6VS-22 were re-digested and remeasured. The re-measured results of these two glasses indicate that the measured values are consistent with the target values. At the present time the reason for the low $\mathrm{ThO}_{2}$ recoveries in the first set of measurements remains unclear. A more thorough discussion of the $\mathrm{ThO}_{2}$ analysis and results is provided in SRNL-L4000-2010-00052. ${ }^{19}$

Table 3-1. Re-measured Values of SB6VS-02, -04 and -15

\begin{tabular}{|c|c|c|c|c|c|c|}
\hline Glass ID & \multicolumn{2}{|c|}{ SB6VS-02 } & \multicolumn{2}{c|}{ SB6VS-04 } & \multicolumn{2}{c|}{ SB6VS-15 } \\
\hline Comp View & target & measured & target & measured & target & measured \\
\hline $\mathrm{Al}_{2} \mathrm{O}_{3}$ & 9.789 & 10.543 & 9.761 & 10.525 & 9.531 & 10.109 \\
\hline $\mathrm{B}_{2} \mathrm{O}_{3}$ & 5.12 & 5.023 & 5.12 & 5.055 & 5.12 & 5.023 \\
\hline $\mathrm{BaO}$ & 0.052 & 0.055 & 0.017 & 0.02 & 0.034 & 0.033 \\
\hline $\mathrm{CaO}$ & 0.729 & 0.775 & 0.729 & 0.708 & 0.504 & 0.498 \\
\hline $\mathrm{Ce}_{2} \mathrm{O}_{3}$ & 0.08 & 0.078 & 0.027 & 0.038 & 0.053 & 0.038 \\
\hline $\mathrm{Cr}_{2} \mathrm{O}_{3}$ & 0.046 & 0.052 & 0.016 & 0.037 & 0.031 & 0.044 \\
\hline $\mathrm{CuO}$ & 0.041 & 0.036 & 0.014 & 0.009 & 0.028 & 0.039 \\
\hline $\mathrm{Fe}_{2} \mathrm{O}_{3}$ & 9.656 & 9.25 & 9.656 & 8.55 & 8.763 & 8.421 \\
\hline $\mathrm{K}_{2} \mathrm{O}$ & 0.033 & 0.119 & 0.011 & 0.119 & 0.022 & 0.119 \\
\hline $\mathrm{La}_{2} \mathrm{O}_{3}$ & 0.041 & 0.031 & 0.014 & 0.006 & 0.027 & 0.018 \\
\hline $\mathrm{Li}_{2} \mathrm{O}$ & 5.12 & 4.952 & 5.12 & 4.952 & 5.12 & 4.909 \\
\hline $\mathrm{MgO} \mathrm{O}$ & 0.062 & 0.069 & 0.062 & 0.066 & 0.278 & 0.274 \\
\hline $\mathrm{MnO}_{\mathrm{Na}} \mathrm{O}$ & 2.249 & 2.208 & 2.729 & 2.699 & 2.489 & 2.453 \\
\hline $\mathrm{NiO}_{2}$ & 13.73 & 14.289 & 13.73 & 13.224 & 14.768 & 13.884 \\
\hline $\mathrm{P}_{2} \mathrm{O}_{5}$ & 0.861 & 0.827 & 1.264 & 1.185 & 1.06 & 1.007 \\
\hline $\mathrm{PbO}^{2}$ & 0.011 & 0.38 & 0.058 & 0.38 & 0.114 & 0.38 \\
\hline $\mathrm{SO}_{4}$ & 0.383 & 1.107 & 0.383 & 1.107 & 0.5034 & 1.1055 \\
\hline $\mathrm{SiO}_{2}$ & 49.627 & 52.627 & 49.212 & 51.985 & 49.418 & 51.985 \\
\hline $\mathrm{TiO}_{2}$ & 0.661 & 0.647 & 0 & 0.006 & 0.328 & 0.319 \\
\hline $\mathrm{U}_{3} \mathrm{O}_{8}$ & 1.422 & 1.226 & 2.034 & 1.722 & 1.723 & 1.474 \\
\hline $\mathrm{ZnO}_{\mathrm{ZrO}_{2}}$ & 0.027 & 0.054 & 0.009 & 0.009 & 0.018 & 0.03 \\
\hline $\mathrm{Sum}$ & 100.00 & 104.53 & 100.00 & 102.53 & 100.00 & 102.31 \\
\hline
\end{tabular}




\section{2 $\underline{\text { MAR Assessment }}$}

MAR assessment results for both sets of glasses are provided in Table 3-2. The columns in the table list the glass identifier with compositional view, nepheline value, $\mathrm{TiO}_{2}$ content $(\mathrm{wt} \%)$ and the overall MAR assessment and the predicted values for: $\Delta \mathrm{G}_{\mathrm{p}}$ value for boron (B Del $\mathrm{G}_{\mathrm{p}}$ value), normalized leachate for boron in grams/Liter (NL $[\mathrm{B}(\mathrm{g} / \mathrm{L})])$, liquidus temperature in degrees Celsius $\left(\mathrm{T}_{\mathrm{L}}\left({ }^{\circ} \mathrm{C}\right)\right)$ and viscosity in Poise (Visc $\left.(\mathrm{P})\right)$. No issues are present in any of the target compositions; however, a few issues appear for the measured compositions, which include: Del $\mathrm{G}_{\mathrm{P}}$ and nepheline. Based on the data analyses in the previous section, these issues should not be a concern as the measured compositions used to calculate the MAR status were not representative of the target compositions or the actual glasses. The MAR assessment results of the three remeasured glasses are shown in

Table 3-3. No issues are present in any of these glasses based on model predictions.

Table 3-2. MAR Assessment Results

\begin{tabular}{|c|c|c|c|c|c|c|c|c|}
\hline Glass ID & \begin{tabular}{|c|} 
Compositional View \\
\end{tabular} & B Del Gp Value & $\mathrm{NL}[\mathrm{B}(\mathrm{g} / \mathrm{L})]$ & TL Pred (oC) & Visc Pred (P) & $\mathrm{TiO}_{2} \mathrm{wt}^{2} \%$ & Neph Val & MAR Status \\
\hline SB6VS-01 & \multirow{17}{*}{ target } & $\begin{array}{l}-9.79 \\
\end{array}$ & 0.75 & 903 & 54 & 0.00 & 0.67 & \\
\hline SB6VS-02 & & -9.74 & 0.73 & 893 & 55 & 0.66 & 0.68 & \\
\hline SB6VS-03 & & -9.77 & 0.74 & 894 & 64 & 0.66 & 0.67 & \\
\hline SB6VS-04 & & -10.05 & 0.83 & 907 & 53 & 0.00 & 0.68 & \\
\hline SB6VS-05 & & -11.58 & 1.57 & 832 & 46 & 0.00 & 0.65 & \\
\hline SB6VS-06 & & -11.74 & 1.68 & 810 & 45 & 0.00 & 0.66 & \\
\hline SB6VS-07 & & -11.20 & 1.34 & 864 & 50 & 0.00 & 0.66 & \\
\hline SB6VS-08 & & -11.77 & 1.70 & 864 & 35 & 0.66 & 0.67 & \\
\hline SB6VS-09 & & -10.97 & 1.22 & 903 & 45 & 0.00 & 0.69 & \\
\hline SB6VS-10 & & -10.88 & 1.18 & 866 & 44 & 0.66 & 0.69 & \\
\hline SB6VS-11 & & -10.50 & 1.00 & 857 & 57 & 0.66 & 0.66 & \\
\hline SB6VS-12 & & -12.21 & 2.04 & 850 & 39 & 0.66 & 0.67 & \\
\hline SB6VS-13 & & -10.76 & 1.12 & 827 & 56 & 0.29 & 0.70 & \\
\hline SB6VS-14 & & -10.85 & 1.16 & 848 & 52 & 0.31 & 0.69 & \\
\hline SB6VS-15 & & -10.93 & 1.20 & 869 & 47 & 0.33 & 0.67 & \\
\hline SB6VS-16 & & -11.02 & 1.25 & 888 & 43 & 0.35 & 0.66 & \\
\hline SB6VS-17 & & -11.11 & 1.29 & 906 & 39 & 0.37 & 0.64 & \\
\hline SB6VS-01 & \multirow{17}{*}{ measured } & -12.10 & 1.96 & 866 & 41 & 0.01 & 0.64 & \\
\hline SB6VS-02 & & -12.28 & 2.11 & 852 & 38 & 0.75 & 0.64 & \\
\hline SB6VS-03 & & -12.48 & 2.29 & 849 & 46 & 0.78 & 0.64 & \\
\hline SB6VS-04 & & -12.56 & 2.37 & 844 & 36 & 0.01 & 0.64 & \\
\hline SB6VS-05 & & -14.68 & 5.74 & 794 & 28 & 0.01 & 0.61 & Del Gp Neph \\
\hline SB6VS-06 & & -11.60 & 1.59 & 812 & 48 & 0.01 & 0.65 & \\
\hline SB6VS-07 & & -11.55 & 1.55 & 852 & 49 & 0.01 & 0.64 & \\
\hline SB6VS-08 & & -12.76 & 2.58 & 838 & 27 & 0.71 & 0.65 & \\
\hline SB6VS-09 & & -11.62 & 1.60 & 887 & 42 & 0.01 & 0.67 & \\
\hline SB6VS-10 & & -13.34 & 3.28 & 832 & 29 & 0.82 & 0.65 & \\
\hline SB6VS-11 & & -11.55 & 1.55 & 820 & 52 & 0.71 & 0.64 & \\
\hline SB6VS-12 & & -12.53 & 2.34 & 838 & 36 & 0.69 & 0.66 & \\
\hline SB6VS-13 & & -10.98 & 1.22 & 848 & 55 & 0.33 & 0.65 & \\
\hline SB6VS-14 & & -10.32 & 0.93 & 841 & 58 & 0.32 & 0.68 & \\
\hline SB6VS-15 & & -13.52 & 3.53 & 831 & 31 & 0.41 & 0.63 & Del Gp \\
\hline SB6VS-16 & & -11.43 & 1.48 & 879 & 45 & 0.38 & 0.65 & \\
\hline SB6VS-17 & & -11.66 & 1.62 & 890 & 37 & 0.40 & 0.62 & Neph \\
\hline SB6VS18 & \multirow{5}{*}{ target } & -10.55 & 1.02 & 822 & 58 & 0.29 & 0.71 & \\
\hline SB6VS19 & & -10.63 & 1.06 & 844 & 54 & 0.31 & 0.69 & \\
\hline SB6VS20 & & -10.70 & 1.09 & 865 & 49 & 0.33 & 0.68 & \\
\hline SB6VS21 & & -10.78 & 1.12 & 885 & 45 & 0.35 & 0.66 & \\
\hline SB6VS22 & & -10.85 & 1.16 & 904 & 41 & 0.37 & 0.65 & \\
\hline SB6VS18 & \multirow{5}{*}{ measured } & -9.61 & 0.69 & 830 & 79 & 0.30 & 0.72 & \\
\hline SB6VS19 & & -10.37 & 0.95 & 839 & 66 & 0.32 & 0.70 & \\
\hline SB6VS20 & & -9.42 & 0.64 & 878 & 71 & 0.33 & 0.69 & \\
\hline SB6VS21 & & -10.70 & 1.09 & 874 & 54 & 0.35 & 0.67 & \\
\hline SB6VS22 & & -9.23 & 0.59 & 922 & 65 & 0.37 & 0.67 & \\
\hline
\end{tabular}


SRNL-STI-2010-00242

Revision 0

Table 3-3. MAR Assessment Results of Re-Measured SB6VS-02, -04 and -15

\begin{tabular}{|c|c|c|c|c|c|c|c|c|}
\hline Glass ID & Compositional View & B Del Gp Value & NL[B (g/L)] & TL Pred (oC) & \begin{tabular}{|l|} 
Visc Pred (P) \\
\end{tabular} & $\mathrm{TiO}_{2}$ wt\% & Neph Val & MAR Status \\
\hline \multirow{2}{*}{ SB6VS-02 } & target & -9.74 & 0.73 & 893 & 55 & 0.661 & 0.68 & \\
\hline & measured & -9.72 & 0.72 & 882 & 72 & 0.647 & 0.68 & \\
\hline \multirow{2}{*}{ SB6VS-04 } & target & -10.05 & 0.83 & 907 & 53 & 0 & 0.68 & \\
\hline & measured & -9.22 & 0.59 & 902 & 83 & 0.006 & 0.69 & \\
\hline \multirow{2}{*}{ SB6VS-15 } & target & -10.93 & 1.20 & 869 & 47 & 0.328 & 0.67 & \\
\hline & measured & -9.74 & 0.73 & 883 & 74 & 0.319 & 0.68 & \\
\hline
\end{tabular}

\section{$3.3 \underline{\mathrm{XRD}}$}

Each of the quenched and ccc glasses were amorphous (within the detection limit of the instrument $[0.5 \mathrm{vol} \%])$. Representative XRD scans from a quenched and ccc glass are shown in Figure $3-1 .{ }^{\mathrm{g}}$

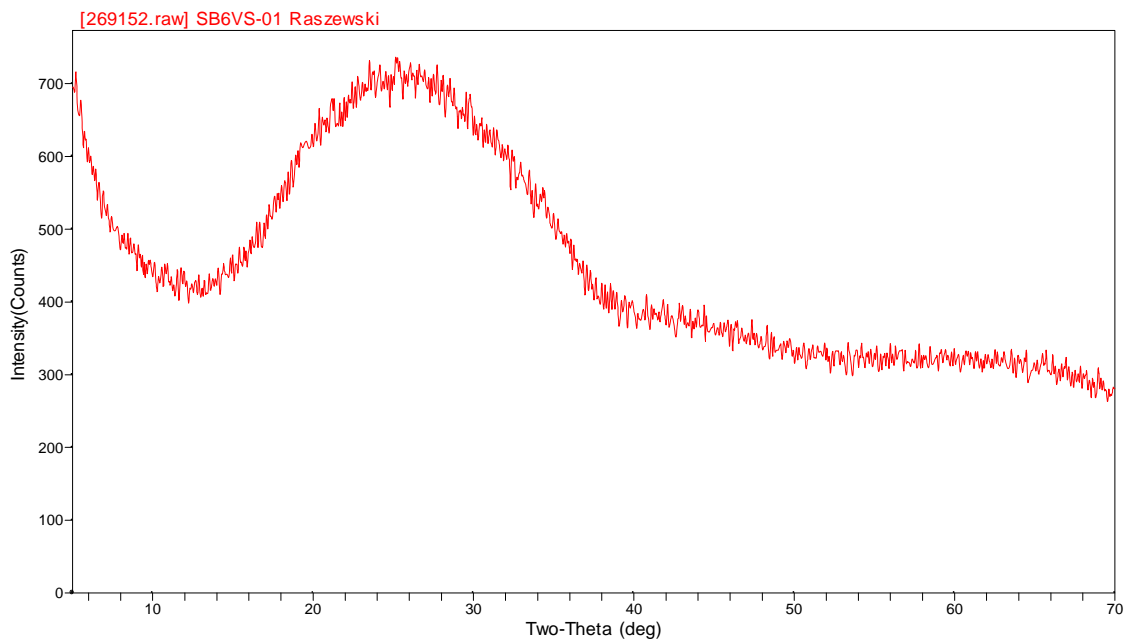

(a)

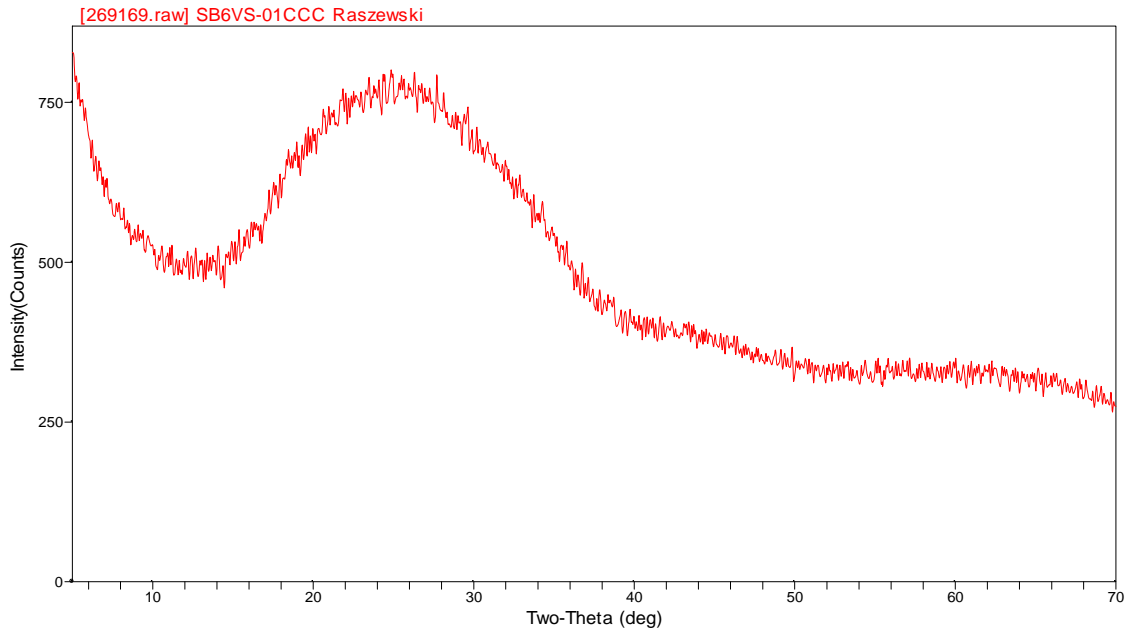

(b)

Figure 3-1. Representative XRD pattern from a quenched (a) and ccc (b) sample.

g The remainder of the XRD patterns (quenched and ccc) can be viewed in the laboratory notebook for the SB6 variability study on pages 25 through 29 (WSRC-NB-2003-00041). XRD spectra of the thorium glasses were not measured as no issues with durability were observed. 


\section{$3.4 \underline{\mathrm{PCT}}$}

Table B1 in Appendix B and Table D1 in Appendix D provide the elemental leachate concentration measurements for the solution samples generated by the PCTs for the non-thorium study glasses and the thorium-based study glasses, respectively. Any measurement below the detection limit of the analytical procedure (indicated by a " $<$ ") was replaced by $1 / 2$ of the detection limit in subsequent analyses. ${ }^{18}$ In addition to adjustments for detection limits, the values were adjusted for the dilution factors: study glasses, blanks and the ARM glass were multiplied by 1.6667 to determine the values in parts per million (ppm) and the values for EA were multiplied by 16.6667. Table B2 in Appendix B provides the resulting values. Over the course of the 7-day test, there were no water loss issues.

\subsubsection{Measurements in Analytical Sequence}

Exhibit B1 in Appendix B provides plots of the common logarithms of the leachate (ppm) concentrations in analytical sequence for all of the data from all two sets of PCTs for the nonthorium study glasses. Exhibit D1 in Appendix D provides similar plots for the PCTs for the thorium glasses. No issues are observed in these plots.

\subsubsection{Results of the Multi-Element Solution Standard}

Exhibit B2 in Appendix B provides analyses of measurements of the multi-element solution standard by analytical set/ICP calibration block, and ANOVA investigations for each element of interest. A statistically significant difference (at a 5\% level) among the averages of these measurements is indicated for B and Na. Exhibit D2 in Appendix D provides a similar analysis for the thorium glass study. No statistically significant differences are shown (at a 5\% level) among the averages of these measurements for any of the elements of interest.

Table 3-4 summarizes the average measurements and the reference values for the 4 elements of interest for both sets of PCTs. The results indicate consistent and accurate measurements throughout the measurement process.

Table 3-4. Results from Samples of the Multi-Element Solution Standard

\begin{tabular}{|c|c|c|c|c|c|}
\hline & \multirow{2}{*}{$\begin{array}{l}\text { Analytical } \\
\text { Set/Block }\end{array}$} & Avg B & $\operatorname{Avg~Li}$ & Avg Na & Avg Si \\
\hline & & \multicolumn{4}{|c|}{ (ppm) } \\
\hline \multirow{6}{*}{$\begin{array}{c}\text { Non- } \\
\text { Thorium } \\
\text { Glasses }\end{array}$} & $1 / 1$ & 20 & 9.9 & 82.5 & 50.9 \\
\hline & $1 / 2$ & 20.1 & 10 & 82.3 & 50.9 \\
\hline & $1 / 3$ & 20 & 10 & 82.5 & 50.2 \\
\hline & $2 / 1$ & 20.1 & 10.1 & 82.5 & 50.8 \\
\hline & $2 / 2$ & 19.8 & 10 & 81.8 & 50.4 \\
\hline & $2 / 3$ & 19.9 & 10 & 80.9 & 50.5 \\
\hline \multirow{6}{*}{$\begin{array}{l}\text { Thorium } \\
\text { Glasses }\end{array}$} & 1 & 19.8 & 9.8 & 82.3 & 52 \\
\hline & 2 & 19.8 & 9.8 & 82.4 & 51.8 \\
\hline & 3 & 19.9 & 9.9 & 82.2 & 52 \\
\hline & Grand Average & 19.9 & 9.9 & 82.2 & 51.1 \\
\hline & Reference Value & 20 & 10 & 81 & 50 \\
\hline & $\%$ difference & $-0.48 \%$ & $-0.66 \%$ & $1.43 \%$ & $2.13 \%$ \\
\hline
\end{tabular}




\subsubsection{Measurements by Glass Identifier}

Exhibit B3 in Appendix B provides plots of the leachate concentrations for both the quenched and ccc version of each of the non-thorium study glasses, as well as the standards (EA, ARM ${ }^{\mathrm{h}}$, multielement solution standard and blanks). ${ }^{20}$ Two units of measure are used in these plots: ppm and the common logarithms of the ppm values. The common logarithm plots allow for the assessment of the repeatability of the measurements and any differences between the quenched and cec version of a given glass. A similar set of plots is provided in Exhibit D3 of Appendix D for the thorium study glasses.

For some of the glasses, scatter in the triplicate values of some analytes is observed; however, these results do not affect the outcome of this study.

\subsubsection{Normalized PCT Results}

For all of the PCT results, the PCT leachate concentrations were normalized using the target and measured cation compositions $(\mathrm{wt} \%)$ in the glass to obtain a grams-per-liter $(\mathrm{g} / \mathrm{L})$ leachate concentration.

As is the usual convention, the common logarithm of the normalized PCT (normalized leachate, NL) for each element of interest was determined and used for comparison. To accomplish this computation, one must:

1. Determine the common logarithm of the elemental leachate concentration (ppm) for each of the triplicates and each of the elements of interest (these values are provided in Table B1 of Appendix B for the non-throium set of glasses and in Table D1 of Appendix D for the thorium study glasses).

2. Average the common logarithms over the triplicates for each element of interest.

\section{Normalizing Using Measured Composition}

3. Subtract a quantity equal to 1 plus the common logarithm of the average cation measured concentration (expressed as a weight percent of the glass) from the average computed in step 2 .

Or Normalizing Using Target Composition

3. Subtract a quantity equal to 1 plus the common logarithm of the target cation concentration (expressed as a weight percent of the glass) from the average computed in step 2.

Exhibit B4 in Appendix B provides scatter plots that contain normalized released rates for both the quenched and ccc version of the non-thorium study glasses based on the target and measured compositions. These plots offer an opportunity to investigate the consistency in the leaching across the elements for the glasses of this study. Consistency in the leaching across the elements is typically demonstrated by a high degree of linear correlation among the values for pairs of these elements. The smallest correlation in this plot is for $\mathrm{Na}$ and $\mathrm{Si}$, with a value of $\sim 95.4 \%$, which demonstrates the consistency of the results among the 4 analytes. A similar set of results

\footnotetext{
${ }^{\mathrm{h}}$ The concentrations of each element of interest for ARM are within the controls limits in THERMO ${ }^{\mathrm{TM}}$.
} 
for the thorium glasses is provided in Exhibit D4 of Appendix D. The smallest correlation for these results is for $\mathrm{B}$ and $\mathrm{Si}$, with a value of $98.5 \%$.

Table 3-5 and Table 3-6 summarize the normalized PCTs for all of the glasses of this study, which are listed by heat treatment and compositional view for each glass. The PCTs for all of the glasses are acceptable based upon comparisons to the EA results.

\subsubsection{Effects of Heat Treatment}

Exhibit B5 in Appendix B provides plots of the normalized PCT responses between the two heat treatments for the non-thorium set of study glasses and Exhibit D5 of Appendix D providing similar plots for the thorium study glasses. These plots provide a basis for judging the practical impact of differences in the PCT response due to the heat treatment of the glass. In general, the ccc versions are relatively consistent with the quenched versions (within experimental error). The boron releases of each compositional view are well below EA and are acceptable.

\subsubsection{Predicted versus Measured PCT Values}

Exhibits B6 and B7 in Appendix B provide plots of the DWPF models for B, Li, Na, and Si that relate the logarithm of the normalized PCT (for each element of interest) to a linear function of a free energy of hydration term $\left(\Delta \mathrm{G}_{\mathrm{p}}\right.$, kcal/100g glass) derived for the target and measured compositional views, respectively, for the non-thorium study glasses. The results for both heat treatments are shown on each plot. Prediction limits at a 95\% confidence level for an individual PCT result are also plotted along with the linear fit. The EA and ARM results are also indicated on these plots. A similar set of plots for the thorium study glasses is provided in Exhibits D6 and D7 of Appendix D. Figure 3-2 provides a closer look at the boron PCT responses from both sets of glasses.

Note that the PCT results for the measured compositions of the non-thorium glasses show some that are not predictable by the DWPF durability models. Table B2 in Appendix B highlights those glasses that are unpredictable. As stated in Section 3.1.4, it was determined that there were issues with analyses of block 2 of the LM dissolutions and that the target compositions are more representative of the actual glass compositions. In general, the unpredictable glasses highlighted in Table B2 correspond to those glasses of preparation block 2. PCT results of the re-digested and re-measured glasses from preparation block 2 (SB6VS-02, -04 and -15) are shown in Table 3-7. Figure 3-3 contains the boron PCT responses of the re-measured glasses. A plot of the original data is shown for comparison. Using the re-measured compositions, the glasses are both acceptable with respect to EA and predictable. 
Table 3-5. Normalized PCT Results of the Non-Thorium Glasses

\begin{tabular}{|c|c|c|c|c|c|c|c|c|c|c|c|}
\hline Set & Glass ID & $\begin{array}{c}\text { Heat } \\
\text { Treatment }\end{array}$ & Comp View & $\begin{array}{c}\log \mathrm{NL} \\
{[\mathrm{B}(\mathrm{g} / \mathrm{L})]}\end{array}$ & $\begin{array}{c}\log \mathrm{NL} \\
{[\mathrm{Li}(\mathrm{g} / \mathrm{L})]}\end{array}$ & \begin{tabular}{|c|}
$\log \mathrm{NL}$ \\
{$[\mathrm{Na}(\mathrm{g} / \mathrm{L})]$}
\end{tabular} & \begin{tabular}{|c|}
$\log \mathrm{NL}$ \\
{$[\mathrm{Si}(\mathrm{g} / \mathrm{L})]$}
\end{tabular} & $\begin{array}{c}\text { NL } \\
B(g / L)\end{array}$ & $\begin{array}{c}\mathrm{NL} \\
\mathrm{Li}(\mathrm{g} / \mathrm{L})\end{array}$ & $\begin{array}{c}\mathrm{NL} \\
\mathrm{Na}(\mathrm{g} / \mathrm{L})\end{array}$ & $\begin{array}{c}\mathrm{NL} \\
\mathrm{Si}(\mathrm{g} / \mathrm{L})\end{array}$ \\
\hline 1 & \multirow{2}{*}{ ARM } & \multirow{4}{*}{ ref } & \multirow{4}{*}{ reference } & -0.34 & -0.264 & -0.325 & -0.572 & 0.46 & 0.54 & 0.47 & 0.27 \\
\hline 2 & & & & -0.309 & -0.226 & -0.288 & -0.541 & 0.49 & 0.59 & 0.52 & 0.29 \\
\hline 1 & \multirow{2}{*}{ EA } & & & 1.212 & 0.959 & 1.107 & 0.587 & 16.3 & 9.1 & 12.8 & 3.86 \\
\hline 2 & & & & 1.048 & 0.839 & 0.958 & 0.488 & 11.16 & 6.91 & 9.09 & 3.08 \\
\hline & \multirow{4}{*}{ SB6VS-01 } & \multirow{2}{*}{$\mathrm{ccc}$} & measured & -0.206 & -0.132 & -0.222 & -0.291 & 0.62 & 0.74 & 0.6 & 0.51 \\
\hline & & & target & -0.223 & -0.153 & -0.145 & -0.297 & 0.6 & 0.7 & 0.72 & 0.5 \\
\hline & & \multirow{2}{*}{ quenched } & measured & -0.168 & -0.111 & -0.174 & -0.268 & 0.68 & 0.77 & 0.67 & 0.54 \\
\hline & & & target & -0.185 & -0.132 & -0.097 & -0.274 & 0.65 & 0.74 & 0.8 & 0.53 \\
\hline & & & measured & -0.216 & -0.133 & -0.234 & -0.294 & 0.61 & 0.74 & 0.58 & 0.51 \\
\hline & SB6VS_0? & $\mathrm{ccc}$ & target & -0.246 & -0.156 & -0.148 & -0.305 & 0.57 & 0.7 & 0.71 & 0.5 \\
\hline & SBOVS-0L & aunched & measured & -0.177 & -0.109 & -0.181 & -0.267 & 0.66 & 0.78 & 0.66 & 0.54 \\
\hline & & quencned & target & -0.208 & -0.132 & -0.096 & -0.278 & 0.62 & 0.74 & 0.8 & 0.53 \\
\hline & & & measured & -0.232 & -0.152 & -0.246 & -0.307 & 0.59 & 0.71 & 0.57 & 0.49 \\
\hline & $\mathrm{SB}_{6 \mathrm{~V}} \mathrm{~S}_{03}$ & $\operatorname{ccc}$ & target & -0.253 & -0.173 & -0.155 & -0.314 & 0.56 & 0.67 & 0.7 & 0.49 \\
\hline & SB6VS-03 & & measured & -0.228 & -0.155 & -0.22 & -0.301 & 0.59 & 0.7 & 0.6 & 0.5 \\
\hline & & quenched & target & -0.25 & -0.176 & -0.129 & -0.308 & 0.56 & 0.67 & 0.74 & 0.49 \\
\hline & & $c c c$ & measured & -0.176 & -0.103 & -0.21 & -0.268 & 0.67 & 0.79 & 0.62 & 0.54 \\
\hline & SB6VS-04 & $\mathrm{ccc}$ & target & -0.22 & -0.139 & -0.129 & -0.293 & 0.6 & 0.73 & 0.74 & 0.51 \\
\hline & SBOV S-04 & auenched & measured & -0.175 & -0.123 & -0.187 & -0.265 & 0.67 & 0.75 & 0.65 & 0.54 \\
\hline & & quenched & target & -0.22 & -0.159 & -0.106 & -0.29 & 0.6 & 0.69 & 0.78 & 0.51 \\
\hline & & & measured & -0.164 & -0.093 & -0.143 & -0.243 & 0.69 & 0.81 & 0.72 & 0.57 \\
\hline 1 & $\mathrm{SB}_{6 \mathrm{~V}}-05$ & $\operatorname{ccc}$ & target & -0.194 & -0.117 & -0.053 & -0.255 & 0.64 & 0.76 & 0.88 & 0.56 \\
\hline 1 & SBOVS-US & oupnched & measured & -0.175 & -0.131 & -0.116 & -0.255 & 0.67 & 0.74 & 0.77 & 0.56 \\
\hline & & quencried & target & -0.205 & -0.154 & -0.026 & -0.267 & 0.62 & 0.7 & 0.94 & 0.54 \\
\hline & & & measured & -0.166 & -0.076 & -0.025 & -0.24 & 0.68 & 0.84 & 0.94 & 0.58 \\
\hline & SB6YS-06 & $\operatorname{ccc}$ & target & -0.178 & -0.095 & -0.023 & -0.246 & 0.66 & 0.8 & 0.95 & 0.57 \\
\hline & SBOV S-00 & guenched & measured & -0.149 & -0.076 & 0.022 & -0.225 & 0.71 & 0.84 & 1.05 & 0.6 \\
\hline & & quencned & target & -0.162 & -0.095 & 0.025 & -0.231 & 0.69 & 0.8 & 1.06 & 0.59 \\
\hline & & $\mathrm{ccc}$ & measured & -0.149 & -0.066 & -0.061 & -0.239 & 0.71 & 0.86 & 0.87 & 0.58 \\
\hline & SR6VS 07 & $\operatorname{ccc}$ & target & -0.171 & -0.099 & -0.043 & -0.252 & 0.67 & 0.8 & 0.91 & 0.56 \\
\hline & SDOV S-O & guenched & measured & -0.145 & -0.094 & -0.032 & -0.233 & 0.72 & 0.81 & 0.93 & 0.58 \\
\hline & & quenched & target & -0.167 & -0.126 & -0.013 & -0.246 & 0.68 & 0.75 & 0.97 & 0.57 \\
\hline & & & measured & -0.088 & -0.031 & -0.038 & -0.186 & 0.82 & 0.93 & 0.92 & 0.65 \\
\hline & SB6VS-08 & $\operatorname{ccc}$ & target & -0.136 & -0.072 & -0.008 & -0.218 & 0.73 & 0.85 & 0.98 & 0.61 \\
\hline & SBOV S-U8 & guenched & measured & -0.071 & -0.036 & 0.008 & -0.18 & 0.85 & 0.92 & 1.02 & 0.66 \\
\hline & & quencned & target & -0.119 & -0.078 & 0.038 & -0.211 & 0.76 & 0.84 & 1.09 & 0.62 \\
\hline & & $\operatorname{ccc}$ & measured & -0.152 & -0.099 & -0.127 & -0.285 & 0.7 & 0.8 & 0.75 & 0.52 \\
\hline & SB6VS-09 & $\mathrm{ccc}$ & target & -0.176 & -0.131 & -0.101 & -0.299 & 0.67 & 0.74 & 0.79 & 0.5 \\
\hline & SBOV S-09 & gunched & measured & -0.115 & -0.067 & -0.064 & -0.248 & 0.77 & 0.86 & 0.86 & 0.56 \\
\hline & & quencned & target & -0.138 & -0.099 & -0.038 & -0.263 & 0.73 & 0.8 & 0.92 & 0.55 \\
\hline & & ccc & measured & -0.109 & -0.048 & -0.135 & -0.197 & 0.78 & 0.9 & 0.73 & 0.64 \\
\hline & SB6VS-10 & $\operatorname{cec}$ & target & -0.141 & -0.076 & -0.056 & -0.212 & 0.72 & 0.84 & 0.88 & 0.61 \\
\hline & SBovs-10 & gunched & measured & -0.057 & -0.028 & -0.086 & -0.176 & 0.88 & 0.94 & 0.82 & 0.67 \\
\hline & & quenched & target & -0.089 & -0.056 & -0.007 & -0.191 & 0.81 & 0.88 & 0.98 & 0.64 \\
\hline & & ccc & measured & -0.176 & -0.099 & -0.135 & -0.268 & 0.67 & 0.8 & 0.73 & 0.54 \\
\hline & SB6VS-11 & $\operatorname{cec}$ & target & -0.208 & -0.131 & -0.1 & -0.283 & 0.62 & 0.74 & 0.8 & 0.52 \\
\hline & SBOV & auenched & measured & -0.147 & -0.076 & -0.079 & -0.237 & 0.71 & 0.84 & 0.83 & 0.58 \\
\hline & & quenched & target & -0.179 & -0.108 & -0.043 & -0.251 & 0.66 & 0.78 & 0.91 & 0.56 \\
\hline & & $c c c$ & measured & -0.081 & -0.018 & -0.008 & -0.199 & 0.83 & 0.96 & 0.98 & 0.63 \\
\hline & SR6VS-12 & $\operatorname{ccc}$ & target & -0.12 & -0.053 & 0.005 & -0.22 & 0.76 & 0.89 & 1.01 & 0.6 \\
\hline & SB6V S-12 & guenched & measured & -0.055 & -0.005 & 0.048 & -0.178 & 0.88 & 0.99 & 1.12 & 0.66 \\
\hline & & quenched & target & -0.093 & -0.04 & 0.061 & -0.199 & 0.81 & 0.91 & 1.15 & 0.63 \\
\hline & & ccc & measured & -0.176 & -0.08 & -0.165 & -0.244 & 0.67 & 0.83 & 0.68 & 0.57 \\
\hline & SR6VS 13 & $\operatorname{ccc}$ & target & -0.222 & -0.134 & -0.12 & -0.274 & 0.6 & 0.73 & 0.76 & 0.53 \\
\hline & SB6vs-13 & guenched & measured & -0.163 & -0.066 & -0.125 & -0.246 & 0.69 & 0.86 & 0.75 & 0.57 \\
\hline 2 & & quenched & target & -0.21 & -0.12 & -0.08 & -0.276 & 0.62 & 0.76 & 0.83 & 0.53 \\
\hline 2 & & ccc & measured & -0.123 & -0.038 & -0.03 & -0.2 & 0.75 & 0.92 & 0.93 & 0.63 \\
\hline & SB6VS-14 & $\operatorname{ccc}$ & target & -0.157 & -0.067 & -0.042 & -0.217 & 0.7 & 0.86 & 0.91 & 0.61 \\
\hline & SBOVS-14 & ourenched & measured & -0.157 & -0.087 & -0.044 & -0.23 & 0.7 & 0.82 & 0.9 & 0.59 \\
\hline & & quencied & target & -0.19 & -0.116 & -0.055 & -0.247 & 0.65 & 0.77 & 0.88 & 0.57 \\
\hline & & $\operatorname{ccc}$ & measured & -0.106 & -0.029 & -0.106 & -0.189 & 0.78 & 0.94 & 0.78 & 0.65 \\
\hline & SB6YS-15 & $\mathrm{ccc}$ & target & -0.135 & -0.066 & -0.026 & -0.207 & 0.73 & 0.86 & 0.94 & 0.62 \\
\hline & SB6VS-15 & ayenched & measured & -0.11 & -0.041 & -0.086 & -0.194 & 0.78 & 0.91 & 0.82 & 0.64 \\
\hline & & quencined & target & -0.14 & -0.078 & -0.006 & -0.212 & 0.72 & 0.84 & 0.99 & 0.61 \\
\hline & & & measured & -0.117 & -0.064 & -0.048 & -0.214 & 0.76 & 0.86 & 0.89 & 0.61 \\
\hline & SB6VS-16 & $\operatorname{ccc}$ & target & -0.133 & -0.08 & -0.028 & -0.213 & 0.74 & 0.83 & 0.94 & 0.61 \\
\hline & & guenched & measured & -0.108 & -0.056 & -0.006 & -0.211 & 0.78 & 0.88 & 0.99 & 0.62 \\
\hline & & quencned & target & -0.124 & -0.072 & 0.014 & -0.21 & 0.75 & 0.85 & 1.03 & 0.62 \\
\hline & & $\mathrm{ccc}$ & measured & -0.093 & -0.066 & -0.049 & -0.215 & 0.81 & 0.86 & 0.89 & 0.61 \\
\hline & SB6VS-17 & $\mathrm{ccc}$ & target & -0.128 & -0.093 & -0.027 & -0.229 & 0.75 & 0.81 & 0.94 & 0.59 \\
\hline & SDOV & guenched & measured & -0.073 & -0.057 & 0.004 & -0.194 & 0.85 & 0.88 & 1.01 & 0.64 \\
\hline & & quenched & target & -0.108 & -0.084 & 0.026 & -0.208 & 0.78 & 0.82 & 1.06 & 0.62 \\
\hline
\end{tabular}


Table 3-6. Normalized PCT Results of the Thorium Glasses

\begin{tabular}{|c|c|c|c|c|c|c|c|c|c|c|c|}
\hline Set & Glass ID & $\begin{array}{c}\text { Heat } \\
\text { Treatment }\end{array}$ & Comp View & $\begin{array}{c}\log N L \\
{[B(g / L)]}\end{array}$ & $\begin{array}{c}\log N \mathrm{~L} \\
{[\mathrm{Li}(\mathrm{g} / \mathrm{L})]}\end{array}$ & \begin{tabular}{|c|}
$\log \mathrm{NL}$ \\
{$[\mathrm{Na}(\mathrm{g} / \mathrm{L})]$}
\end{tabular} & $\begin{array}{c}\log \mathrm{NL} \\
{[\mathrm{Si}(\mathrm{g} / \mathrm{L})]}\end{array}$ & $\begin{array}{c}\text { NL } \\
\text { B (g/L) }\end{array}$ & $\begin{array}{c}\text { NL } \\
\mathrm{Li}(\mathrm{g} / \mathrm{L})\end{array}$ & $\begin{array}{c}\mathrm{NL} \\
\mathrm{Na}(\mathrm{g} / \mathrm{L})\end{array}$ & $\begin{array}{c}\mathrm{NL} \\
\mathrm{Si}(\mathrm{g} / \mathrm{L})\end{array}$ \\
\hline \multirow{22}{*}{3} & ARM & \multirow{2}{*}{ ref } & \multirow{2}{*}{ reference } & -0.304 & -0.231 & -0.287 & -0.536 & 0.5 & 0.59 & 0.52 & 0.29 \\
\hline & EA & & & 1.193 & 0.945 & 1.089 & 0.594 & 15.59 & 8.81 & 12.28 & 3.92 \\
\hline & \multirow{4}{*}{ SB6VS-18 } & \multirow{2}{*}{$\mathrm{ccc}$} & measured & -0.146 & -0.073 & -0.059 & -0.288 & 0.72 & 0.85 & 0.87 & 0.51 \\
\hline & & & target & -0.161 & -0.084 & -0.085 & -0.274 & 0.69 & 0.82 & 0.82 & 0.53 \\
\hline & & \multirow{2}{*}{ quenched } & measured & -0.165 & -0.093 & -0.042 & -0.298 & 0.68 & 0.81 & 0.91 & 0.5 \\
\hline & & & target & -0.18 & -0.104 & -0.068 & -0.284 & 0.66 & 0.79 & 0.85 & 0.52 \\
\hline & \multirow{4}{*}{ SB6VS-19 } & \multirow{2}{*}{$\mathrm{ccc}$} & measured & -0.165 & -0.096 & -0.09 & -0.31 & 0.68 & 0.8 & 0.81 & 0.49 \\
\hline & & & target & -0.182 & -0.106 & -0.092 & -0.294 & 0.66 & 0.78 & 0.81 & 0.51 \\
\hline & & \multirow{2}{*}{ quenched } & measured & -0.155 & -0.094 & -0.052 & -0.302 & 0.7 & 0.8 & 0.89 & 0.5 \\
\hline & & & target & -0.171 & -0.105 & -0.054 & -0.287 & 0.67 & 0.79 & 0.88 & 0.52 \\
\hline & \multirow{4}{*}{ SB6VS-20 } & \multirow{2}{*}{$\mathrm{ccc}$} & measured & -0.157 & -0.09 & -0.038 & -0.299 & 0.7 & 0.81 & 0.92 & 0.5 \\
\hline & & & target & -0.174 & -0.099 & -0.082 & -0.287 & 0.67 & 0.8 & 0.83 & 0.52 \\
\hline & & \multirow{2}{*}{ quenched } & measured & -0.156 & -0.1 & -0.003 & -0.298 & 0.7 & 0.79 & 0.99 & 0.5 \\
\hline & & & target & -0.173 & -0.109 & -0.047 & -0.286 & 0.67 & 0.78 & 0.9 & 0.52 \\
\hline & \multirow{4}{*}{ SB6VS-21 } & \multirow{2}{*}{$\mathrm{ccc}$} & measured & -0.146 & -0.095 & -0.073 & -0.3 & 0.72 & 0.8 & 0.85 & 0.5 \\
\hline & & & target & -0.164 & -0.105 & -0.07 & -0.285 & 0.68 & 0.79 & 0.85 & 0.52 \\
\hline & & \multirow{2}{*}{ quenched } & measured & -0.159 & -0.128 & -0.057 & -0.314 & 0.69 & 0.74 & 0.88 & 0.48 \\
\hline & & & target & -0.178 & -0.138 & -0.054 & -0.3 & 0.66 & 0.73 & 0.88 & 0.5 \\
\hline & \multirow{4}{*}{ SB6VS-22 } & \multirow{2}{*}{$\mathrm{ccc}$} & measured & -0.143 & -0.1 & -0.019 & -0.301 & 0.72 & 0.79 & 0.96 & 0.5 \\
\hline & & & target & -0.16 & -0.108 & -0.071 & -0.287 & 0.69 & 0.78 & 0.85 & 0.52 \\
\hline & & \multirow{2}{*}{ quenched } & measured & -0.142 & -0.129 & 0.021 & -0.305 & 0.72 & 0.74 & 1.05 & 0.5 \\
\hline & & & target & -0.159 & -0.137 & -0.032 & -0.291 & 0.69 & 0.73 & 0.93 & 0.51 \\
\hline
\end{tabular}

Table 3-7. Normalized PCT Results of the Re-Measured Glasses

\begin{tabular}{|c|c|c|c|c|c|c|c|c|c|c|}
\hline Glass ID & \begin{tabular}{|c|} 
Heat \\
Treatment
\end{tabular} & Comp View & $\begin{array}{c}\log N \mathrm{~L} \\
{[\mathrm{~B}(\mathrm{~g} / \mathrm{L})]}\end{array}$ & $\begin{array}{c}\log \mathbf{N L} \\
{[\mathrm{Li}(\mathrm{g} / \mathrm{L})]}\end{array}$ & $\begin{array}{c}\log \mathrm{NL} \\
{[\mathrm{Na}(\mathrm{g} / \mathrm{L})]}\end{array}$ & $\begin{array}{c}\log \mathrm{NL} \\
{[\mathrm{Si}(\mathrm{g} / \mathrm{L})]}\end{array}$ & $\begin{array}{c}\text { NL } \\
\text { B (g/L) }\end{array}$ & $\begin{array}{c}\text { NL } \\
\mathrm{Li}(\mathrm{g} / \mathrm{L})\end{array}$ & $\begin{array}{c}\mathrm{NL} \\
\mathrm{Na}(\mathrm{g} / \mathrm{L})\end{array}$ & $\begin{array}{c}\text { NL } \\
\mathrm{Si}(\mathrm{g} / \mathrm{L})\end{array}$ \\
\hline \multirow{4}{*}{ SB6VS-02 } & \multirow{2}{*}{$\mathrm{ccc}$} & measured & -0.238 & -0.142 & -0.166 & -0.331 & 0.58 & 0.72 & 0.68 & 0.47 \\
\hline & & target & -0.246 & -0.156 & -0.148 & -0.305 & 0.57 & 0.70 & 0.71 & 0.50 \\
\hline & \multirow{2}{*}{ quenched } & measured & -0.199 & -0.117 & -0.113 & -0.304 & 0.63 & 0.76 & 0.77 & 0.50 \\
\hline & & target & -0.208 & -0.132 & -0.096 & -0.278 & 0.62 & 0.74 & 0.80 & 0.53 \\
\hline \multirow{4}{*}{ SB6VS-04 } & \multirow{2}{*}{$\mathrm{ccc}$} & measured & -0.215 & -0.124 & -0.113 & -0.317 & 0.61 & 0.75 & 0.77 & 0.48 \\
\hline & & target & -0.22 & -0.139 & -0.129 & -0.293 & 0.60 & 0.73 & 0.74 & 0.51 \\
\hline & \multirow{2}{*}{ quenched } & measured & -0.214 & -0.144 & -0.09 & -0.314 & 0.61 & 0.72 & 0.81 & 0.49 \\
\hline & & target & -0.22 & -0.159 & -0.106 & -0.29 & 0.60 & 0.69 & 0.78 & 0.51 \\
\hline \multirow{4}{*}{ SB6VS-15 } & \multirow{2}{*}{$\mathrm{ccc}$} & measured & -0.127 & -0.047 & 0 & -0.229 & 0.75 & 0.90 & 1.00 & 0.59 \\
\hline & & target & -0.135 & -0.066 & -0.026 & -0.207 & 0.73 & 0.86 & 0.94 & 0.62 \\
\hline & \multirow{2}{*}{ quenched } & measured & -0.131 & -0.059 & 0.021 & -0.234 & 0.74 & 0.87 & 1.05 & 0.58 \\
\hline & & target & -0.14 & -0.078 & -0.006 & -0.212 & 0.72 & 0.84 & 0.99 & 0.61 \\
\hline
\end{tabular}


SRNL-STI-2010-00242

Revision 0

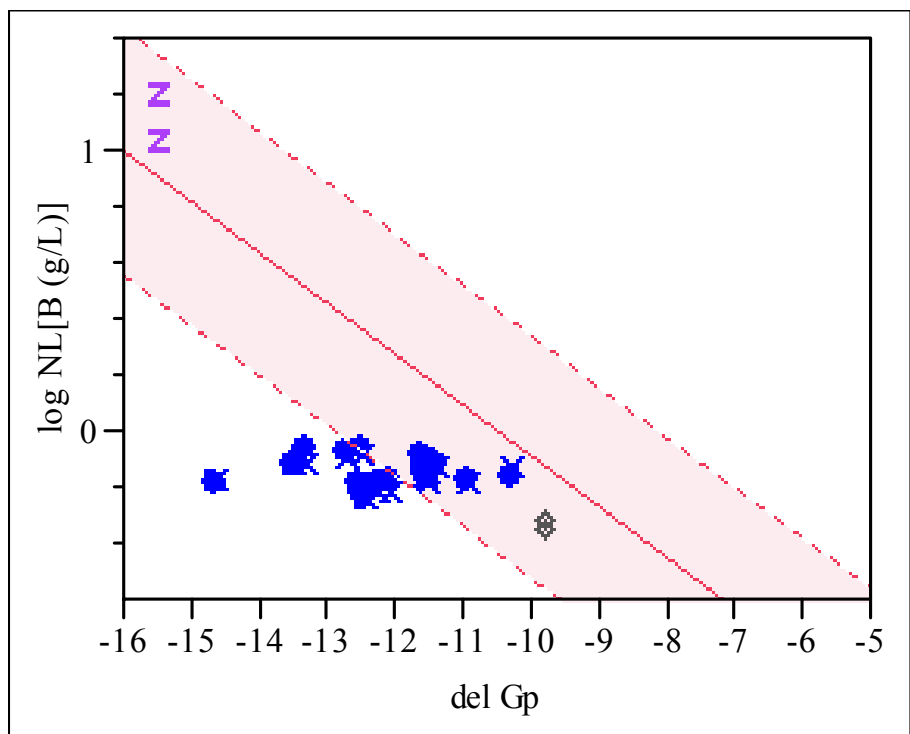

Legend

\begin{tabular}{|c|c|}
\hline Symbol & $\begin{array}{c}\text { Standard/ } \\
\text { Comp View-Heat Treatment }\end{array}$ \\
\hline$Z$ & EA \\
\hline$\diamond$ & ARM \\
\hline$\times$ & Measured-ccc \\
\hline$\bullet$ & Measured-quenched \\
\hline
\end{tabular}

(a) Non-thorium Glasses

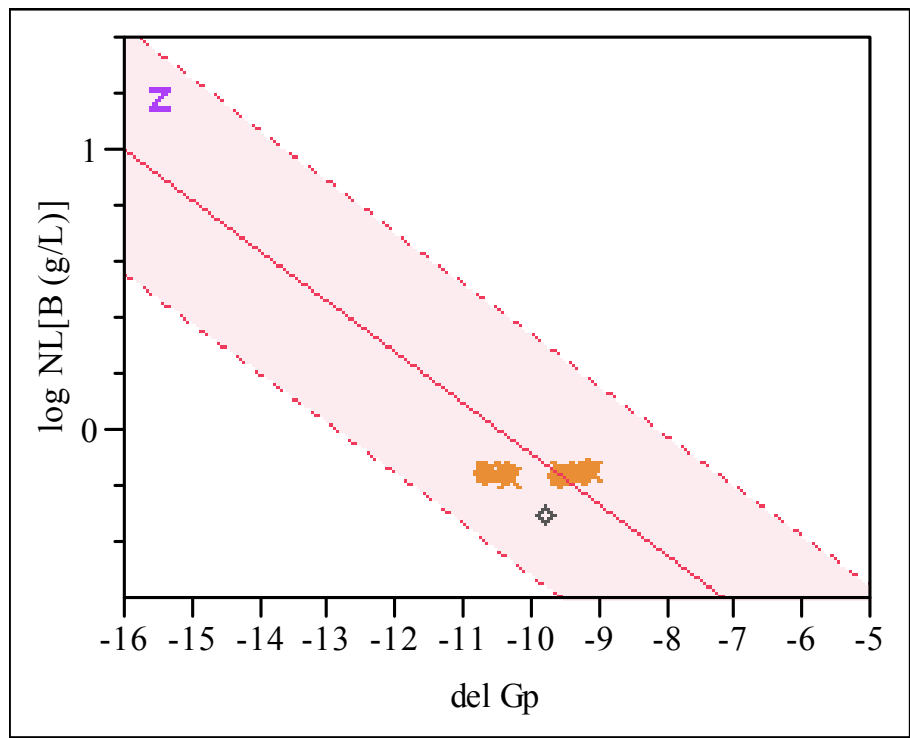

Legend

\begin{tabular}{|c|c|}
\hline Symbol & $\begin{array}{c}\text { Standard/ } \\
\text { Comp View-Heat Treatment }\end{array}$ \\
\hline$z$ & EA \\
\hline$\diamond$ & ARM \\
\hline$\times$ & Measured-ccc \\
\hline$\bullet$ & Measured-quenched \\
\hline
\end{tabular}

(b) Thorium Glasses

Figure 3-2. del $\mathrm{Gp}\left(\Delta \mathrm{G}_{\mathrm{p}}\right)$ predictions versus the common logarithm of the normalized leachate $(\log \mathrm{NL}[]$.$) for B. The PCT responses are normalized to the measured compositions of the (a)$ non-thorium glasses and (b) thorium glasses. 
SRNL-STI-2010-00242

Revision 0

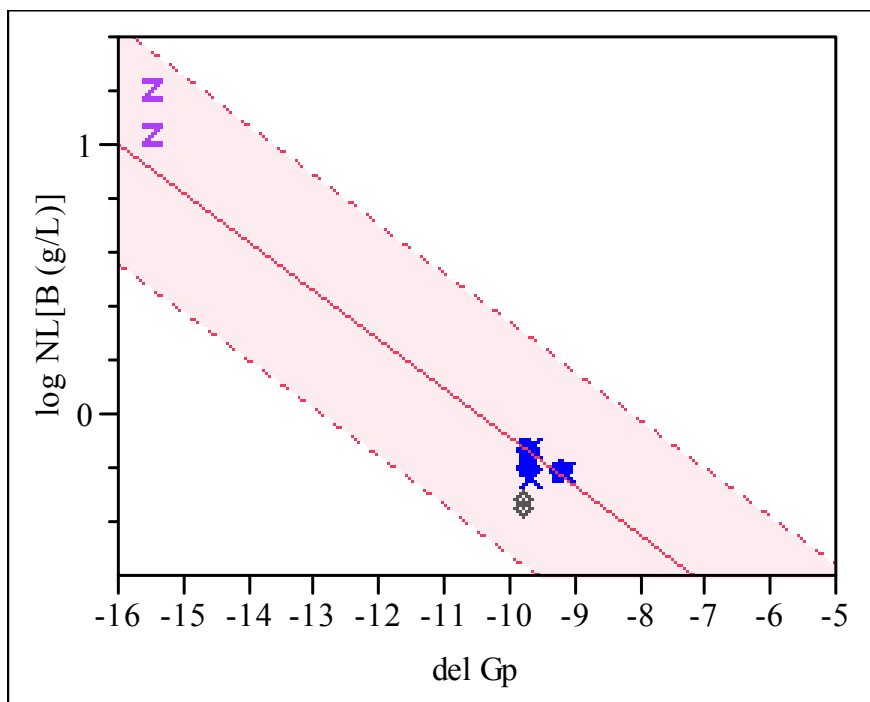

Legend

\begin{tabular}{|c|c|}
\hline Symbol & $\begin{array}{c}\text { Standard/ } \\
\text { Comp View-Heat Treatment }\end{array}$ \\
\hline$z$ & EA \\
\hline$\diamond$ & ARM \\
\hline$\times$ & Measured-ccc \\
\hline$\bullet$ & Measured-quenched \\
\hline
\end{tabular}

(a) Re-measured Non-Thorium Glasses

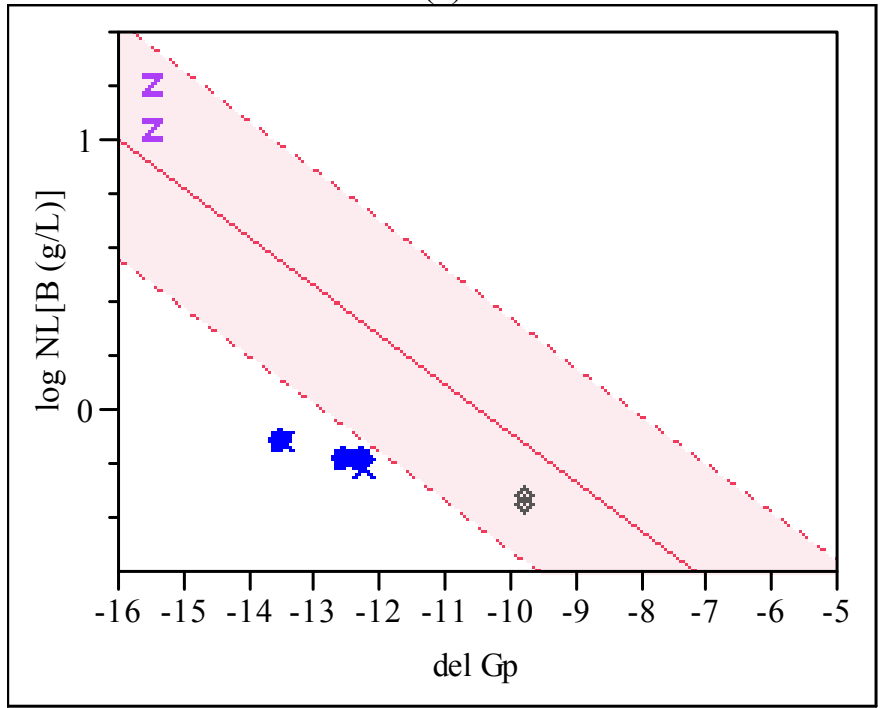

Legend

\begin{tabular}{|c|c|}
\multicolumn{2}{c}{ Legend } \\
\hline Symbol & $\begin{array}{c}\text { Standard/ } \\
\text { Comp View-Heat Treatment }\end{array}$ \\
\hline$z$ & EA \\
\hline$\triangleright$ & ARM \\
\hline$\times$ & Measured-ccc \\
\hline$\bullet$ & Measured-quenched \\
\hline
\end{tabular}

(b) Original Non-Thorium Glasses

Figure 3-3. del $\mathrm{Gp}\left(\Delta \mathrm{G}_{\mathrm{p}}\right)$ predictions versus the common logarithm of the normalized leachate $(\log \mathrm{NL}[]$.$) for B for SB6VS-02, -04$ and -15. The PCT responses are normalized to the measured compositions of the (a) re-measured glasses and (b) original glasses. 


\subsection{Summary}

To comply with the DWPF Glass Product Control Program, a total of twenty-two glasses were fabricated to assess the applicability of the current DWPF PCCS durability models for the SB6/Frit 418 system. Five of the twenty-two glasses contained thorium oxide, which were fabricated after it was discovered that thorium was a reportable element in the SRNL qualification glass. Based on the measured PCT response, all of the glasses (with and without thorium) are acceptable with respect to the EA reference glass regardless of thermal history. While all of the targeted glass compositions were predictable with respect to the PCCS models for durability, a small number of the measured glass compositions were located outside of the lower prediction limit indicating poorer durability than what was actually measured. These unpredictable glasses were in the same LM preparation block during the chemical analyses, which resulted in measured compositions that were not representative of the target compositions. A review of the data did not indicate a clear cause for the problem. Re-digestion and re-measurement of three glasses from this preparation block yielded glass compositions closer to the targets and predicted PCT responses within the PCCS model uncertainty. Therefore, it is believed that the glasses were correctly fabricated and the targeted compositions are closer representations of the true compositions.

Per the requirements of the DWPF Glass Product Control Program, the PCCS durability models have been shown to be applicable to the SB6/Frit 418 glass system. PCT results from the glasses fabricated as part of the variability study were shown to be predictable and/or acceptable with respect to the DWPF PCCS models. In addition, the inclusion of $\mathrm{ThO}_{2}$ was shown to have minimal impact on the acceptability and predictability of the variability study glasses. 


\subsection{References}

1. K.M. Fox, "Glass Frit Composition for Sludge Batch 6 Vitrification at the Defense Waste Processing Facility," Savannah River National Laboratory, Aiken, SC, SRNL-L31002010-00043, 2010.

2. J.M. Bricker, "Sludge Batch 6 Glass Variability Study," Savannah River Remediation, Aiken, SC, HLW-DWPF-TTR-2009-0012, 2009.

3. F.C. Raszewski, "Sludge Batch 6 Glass Variability Studies," Savannah River National Laboratory, Aiken, SC, SRNL-STI-2009-00156, 2009.

4. J.M. Pareizs, B.R. Pickenheim, C.J. Bannochie, A.L. Billings, N.E. Bibler, and D.R. Click, "Tank 51 SB6 Qualification SME Cycle Observations and Key Results," Savannah River National Laboratory, Aiken, SC, SRNL-L3100-2010-00085, 2010.

5. K.M. Fox, T.B. Edwards, and J.R. Zamecnik, "Frit Development for Sludge Batch 6," Savannah River National Laboratory, Aiken, SC, SRNL-STI-2010-00137, 2010.

6. C.J. Bannochie, J.M. Pareizs, and D.R. Click, "Tank 51 SB6 Qualification SRAT Receipt Characterization," Savannah River National Laboratory, Aiken, SC, SRNL-L3100-201000027, 2010.

7. JMP ${ }^{\mathrm{TM}}$ Version 7.0.2, SAS Institute Inc., Cary, NC, 2008.

8. D.K. Peeler and T.B. Edwards, "Impact of Thorium on PCCS Predictions with the Frit 418 - SB6 System " Savannah River National Laboratory, Aiken, SC, SRNL-L31002010-00099, 2010.

9. K.M. Fox and T.B. Edwards, "Impact of Thorium on Projected Operating Windows for Sludge Batch 6 with Frit 418," Aiken, SC, SRNL-L3100-2010-00094, 2010.

10. F.C. Raszewski and M.E. Stone, "Experimental Testing to Support Additional SB4 Tank 40 Decants (TT\&QAP)," Savannah River National Laboratory, Aiken, SC, WSRC-STI2008-00254, Rev. 0, 2008.

11. S.L. Marra and C.M. Jantzen, "Characterization of Projected DWPF Glass Heat Treated to Simulate Canister Centerline Cooling," Westinghouse Savannah River Company, Aiken, SC, WSRC-TR-92-142, Rev. 1, 1993.

12. T.B. Edwards, "An Analytical Plan for Measuring the Chemical Compositions of the SB6 Variability Study Glasses," Savannah River National Laboratory, Aiken, SC, SRNLL5200-2010-00006, 2010.

13. T.B. Edwards, "An Analytical Plan for Measuring the Chemical Compositions of the Supplemental SB6 Variability Study Glasses with Thorium," Aiken, SC, SRNL-L52002010-00022, 2010.

14. "Standard Test Methods for Determining Chemical Durability of Nuclear, Hazardous, and Mixed Waste Glasses and Multiphase Glass Ceramics: The Product Consistency Test (Pct),” ASTM International, West Conshohocken, PA, ASTM C 1285-02, 2002. 
15. T.B. Edwards, "An Analytical Plan for Measuring the PCTs for the First Set of SB6 VS Glasses," Savannah River National Laboratory, Aiken, SC, SRNL-L5200-2010-00007, 2010 .

16. T.B. Edwards, "An Analytical Plan for Measuring the PCTs for the Second Set of SB6 VS Glasses," Savannah River National Laboratory, Aiken, SC, SRNL-L5200-201000009, 2010.

17. T.B. Edwards, "An Analytical Plan for Measuring the PCTs for the Supplemental SB6 VS Glasses with $\mathrm{ThO}_{2}$," Aiken, SC, SRNL-L5200-2010-00019, 2010.

18. "Statistical Analysis of Ground-Water Monitoring Data at RCRA Facilities," United States Environmental Protection Agency, Washington, D.C., 530-SW-89-026, 1989.

19. D.R. Click, M.A. Jones, F.C. Johnson, and T.B. Edwards, "Thorium Measurements in the Sludge Batch 6 Variability Study Glasses " Savannah River National Laboratory, Aiken, SC, SRNL-L4000-2010-00052, 2010.

20. C.M. Jantzen, J.B. Pickett, K.G. Brown, T.B. Edwards, and D.C. Beam, "Process/Product Models for the Defense Waste Processing Facility (DWPF): Part I. Predicting Glass Durability from Composition Using a Thermodynamic Hydration Energy Reaction Model (Thermo)," Westinghouse Savannah River Company, Aiken, SC, WSRC-TR-93672, Rev. 1, 1995. 


\section{Appendix A:}

\section{Tables and Exhibits Supporting the Analysis of the Chemical Composition Measurements of the Non-Thorium SB6 VS Glasses}


SRNL-STI-2010-00242

Revision 0

This page intentionally left blank. 
Table A1. Measured Elemental Concentrations (wt\%) for the Study Glasses Prepared Using Lithium Metaborate (part 1)

\begin{tabular}{|c|c|c|c|c|c|c|c|c|c|c|c|c|c|c|}
\hline Glass ID & Block & $\begin{array}{c}\text { Sub- } \\
\text { Block }\end{array}$ & Seq. & Lab ID & $\begin{array}{c}\text { Ba } \\
(w t \%)\end{array}$ & $\begin{array}{c}\mathrm{Ca} \\
(\mathrm{wt} \%)\end{array}$ & $\begin{array}{c}\mathrm{Cd} \\
(\mathrm{wt} \%)\end{array}$ & $\begin{array}{c}\mathrm{Ce} \\
(w t \%)\end{array}$ & $\begin{array}{c}\mathrm{Cr} \\
(\mathbf{w t} \%)\end{array}$ & $\begin{array}{c}\mathrm{Cu} \\
(\mathrm{wt} \%)\end{array}$ & $\begin{array}{c}K \\
(w t \%)\end{array}$ & $\begin{array}{c}\text { La } \\
(w t \%)\end{array}$ & $\begin{array}{c}\text { Mg } \\
(\mathbf{w t} \%)\end{array}$ & $\begin{array}{c}\text { Mn } \\
(w t \%)\end{array}$ \\
\hline Batch 1 & 1 & 1 & 1 & BCHLM111 & 0.126 & 0.850 & $<0.001$ & $<0.012$ & 0.070 & 0.287 & 2.83 & $<0.005$ & 0.793 & 1.31 \\
\hline Ustd & 1 & 1 & 2 & UstdLM111 & $<0.003$ & 0.966 & $<0.001$ & $<0.012$ & 0.171 & $<0.003$ & 2.59 & $<0.005$ & 0.713 & 2.14 \\
\hline SB6VS-11 & 1 & 1 & 3 & K03LM21 & 0.019 & 0.592 & $<0.001$ & $<0.012$ & 0.017 & 0.011 & 0.05 & 0.011 & 0.316 & 2.23 \\
\hline SB6VS-07 & 1 & 1 & 4 & K04LM11 & 0.017 & 0.246 & $<0.001$ & $<0.012$ & 0.016 & 0.010 & 0.05 & 0.008 & 0.301 & 1.77 \\
\hline SB6VS-17 & 1 & 1 & 5 & K08LM11 & 0.035 & 0.426 & $<0.001$ & 0.019 & 0.027 & 0.020 & 0.06 & 0.021 & 0.182 & 2.10 \\
\hline SB6VS-06 & 1 & 1 & 6 & K09LM21 & 0.017 & 0.556 & $<0.001$ & $<0.012$ & 0.015 & 0.008 & 0.03 & 0.008 & 0.041 & 1.74 \\
\hline SB6VS-09 & 1 & 1 & 7 & K01LM21 & 0.049 & 0.576 & $<0.001$ & 0.042 & 0.034 & 0.030 & 0.06 & 0.030 & 0.325 & 2.17 \\
\hline SB6VS-12 & 1 & 1 & 8 & K11LM11 & 0.048 & 0.564 & $<0.001$ & 0.039 & 0.033 & 0.028 & 0.08 & 0.031 & 0.304 & 1.77 \\
\hline SB6VS-16 & 1 & 1 & 9 & K17LM21 & 0.034 & 0.434 & $<0.001$ & 0.021 & 0.029 & 0.022 & 0.08 & 0.021 & 0.181 & 2.08 \\
\hline SB6VS-12 & 1 & 1 & 10 & K11LM21 & 0.047 & 0.551 & $<0.001$ & 0.037 & 0.033 & 0.029 & 0.07 & 0.030 & 0.297 & 1.74 \\
\hline SB6VS-08 & 1 & 1 & 11 & K06LM21 & 0.018 & 0.230 & $<0.001$ & $<0.012$ & 0.016 & 0.008 & 0.04 & 0.009 & 0.042 & 1.79 \\
\hline Batch 1 & 1 & 1 & 12 & BCHLM121 & 0.126 & 0.850 & $<0.001$ & $<0.012$ & 0.071 & 0.285 & 2.81 & $<0.005$ & 0.792 & 1.31 \\
\hline Ustd & 1 & 1 & 13 & UstdLM121 & $<0.003$ & 0.965 & $<0.001$ & $<0.012$ & 0.172 & $<0.003$ & 2.53 & $<0.005$ & 0.713 & 2.16 \\
\hline SB6VS-09 & 1 & 1 & 14 & K01LM11 & 0.050 & 0.587 & $<0.001$ & 0.043 & 0.035 & 0.031 & 0.07 & 0.030 & 0.329 & 2.21 \\
\hline SB6VS-16 & 1 & 1 & 15 & K17LM11 & 0.034 & 0.426 & $<0.001$ & 0.016 & 0.029 & 0.021 & 0.05 & 0.021 & 0.181 & 2.11 \\
\hline SB6VS-17 & 1 & 1 & 16 & K08LM21 & 0.038 & 0.476 & $<0.001$ & 0.025 & 0.029 & 0.022 & 0.05 & 0.023 & 0.200 & 2.26 \\
\hline SB6VS-06 & 1 & 1 & 17 & K09LM11 & 0.016 & 0.551 & $<0.001$ & $<0.012$ & 0.017 & 0.009 & 0.04 & 0.007 & 0.042 & 1.74 \\
\hline SB6VS-07 & 1 & 1 & 18 & K04LM21 & 0.017 & 0.230 & $<0.001$ & $<0.012$ & 0.013 & 0.009 & 0.03 & 0.008 & 0.303 & 1.75 \\
\hline SB6VS-08 & 1 & 1 & 19 & K06LM11 & 0.019 & 0.244 & $<0.001$ & $<0.012$ & 0.018 & 0.009 & 0.04 & 0.009 & 0.045 & 1.85 \\
\hline SB6VS-13 & 1 & 1 & 20 & K05LM21 & 0.031 & 0.415 & $<0.001$ & 0.017 & 0.024 & 0.019 & 0.05 & 0.018 & 0.164 & 1.83 \\
\hline SB6VS-13 & 1 & 1 & 21 & K05LM11 & 0.029 & 0.362 & $<0.001$ & 0.016 & 0.025 & 0.018 & 0.08 & 0.017 & 0.162 & 1.75 \\
\hline SB6VS-11 & 1 & 1 & 22 & K03LM11 & 0.018 & 0.598 & $<0.001$ & $<0.012$ & 0.015 & 0.010 & 0.05 & 0.010 & 0.306 & 2.14 \\
\hline Batch 1 & 1 & 1 & 23 & BCHLM131 & 0.127 & 0.864 & $<0.001$ & $<0.012$ & 0.071 & 0.286 & 2.51 & $<0.005$ & 0.808 & 1.32 \\
\hline Ustd & 1 & 1 & 24 & UstdLM131 & $<0.003$ & 0.975 & $<0.001$ & $<0.012$ & 0.171 & $<0.003$ & 2.41 & $<0.005$ & 0.725 & 2.15 \\
\hline Batch 1 & 1 & 2 & 1 & BCHLM112 & 0.126 & 0.843 & $<0.001$ & $<0.012$ & 0.071 & 0.289 & 2.57 & $<0.005$ & 0.798 & 1.31 \\
\hline Ustd & 1 & 2 & 2 & UstdLM112 & $<0.003$ & 0.954 & $<0.001$ & $<0.012$ & 0.172 & $<0.003$ & 2.58 & $<0.005$ & 0.716 & 2.15 \\
\hline SB6VS-09 & 1 & 2 & 3 & K01LM22 & 0.049 & 0.568 & $<0.001$ & 0.042 & 0.035 & 0.031 & 0.065 & 0.030 & 0.325 & 2.18 \\
\hline SB6VS-11 & 1 & 2 & 4 & K03LM22 & 0.019 & 0.583 & $<0.001$ & $<0.012$ & 0.017 & 0.011 & 0.048 & 0.011 & 0.316 & 2.23 \\
\hline SB6VS-08 & 1 & 2 & 5 & K06LM12 & 0.019 & 0.236 & $<0.001$ & $<0.012$ & 0.018 & 0.009 & 0.044 & 0.009 & 0.044 & 1.85 \\
\hline SB6VS-13 & 1 & 2 & 6 & K05LM12 & 0.029 & 0.351 & $<0.001$ & 0.016 & 0.026 & 0.018 & 0.083 & 0.018 & 0.159 & 1.75 \\
\hline SB6VS-07 & 1 & 2 & 7 & K04LM22 & 0.017 & 0.221 & $<0.001$ & $<0.012$ & 0.013 & 0.009 & 0.031 & 0.008 & 0.296 & 1.75 \\
\hline SB6VS-17 & 1 & 2 & 8 & K08LM12 & 0.035 & 0.418 & $<0.001$ & 0.021 & 0.027 & 0.020 & 0.060 & 0.021 & 0.180 & 2.10 \\
\hline SB6VS-12 & 1 & 2 & 9 & K11LM22 & 0.047 & 0.541 & $<0.001$ & 0.037 & 0.033 & 0.029 & 0.075 & 0.031 & 0.294 & 1.74 \\
\hline SB6VS-08 & 1 & 2 & 10 & K06LM22 & 0.018 & 0.224 & $<0.001$ & $<0.012$ & 0.016 & 0.009 & 0.043 & 0.009 & 0.042 & 1.79 \\
\hline SB6VS-17 & 1 & 2 & 11 & K08LM22 & 0.038 & 0.462 & $<0.001$ & 0.025 & 0.030 & 0.022 & 0.055 & 0.023 & 0.194 & 2.27 \\
\hline Batch 1 & 1 & 2 & $\frac{1}{12}$ & BCHLM122 & 0.127 & 0.840 & $<0.001$ & $<0.012$ & 0.071 & 0.289 & 2.70 & $<0.005$ & 0.787 & 1.32 \\
\hline Ustd & 1 & 2 & 13 & UstdLM122 & $<0.003$ & 0.951 & $<0.001$ & $<0.012$ & 0.173 & $<0.003$ & 2.51 & $<0.005$ & 0.706 & 2.16 \\
\hline SB6VS-12 & 1 & 2 & 14 & K11LM12 & 0.049 & 0.554 & $<0.001$ & 0.040 & 0.034 & 0.028 & 0.079 & 0.031 & 0.300 & 1.78 \\
\hline SB6VS-06 & 1 & 2 & 15 & K09LM12 & 0.016 & 0.529 & $<0.001$ & $<0.012$ & 0.018 & 0.009 & 0.038 & 0.008 & 0.038 & 1.74 \\
\hline SB6VS-09 & 1 & 2 & 16 & K01LM12 & 0.050 & 0.571 & $<0.001$ & 0.043 & 0.035 & 0.031 & 0.065 & 0.030 & 0.322 & 2.22 \\
\hline SB6VS-16 & 1 & 2 & 17 & K17LM22 & 0.034 & 0.425 & $<0.001$ & 0.021 & 0.029 & 0.022 & 0.077 & 0.021 & 0.178 & 2.09 \\
\hline SB6VS-07 & 1 & 2 & 18 & K04LM12 & 0.017 & 0.239 & $<0.001$ & $<0.012$ & 0.016 & 0.010 & 0.050 & 0.008 & 0.294 & 1.78 \\
\hline SB6VS-13 & 1 & 2 & 19 & K05LM22 & 0.031 & 0.400 & $<0.001$ & 0.017 & 0.024 & 0.019 & 0.054 & 0.018 & 0.157 & 1.84 \\
\hline SB6VS-11 & 1 & 2 & 20 & K03LM12 & 0.018 & 0.579 & $<0.001$ & $<0.012$ & 0.015 & 0.010 & 0.051 & 0.010 & 0.295 & 2.14 \\
\hline SB6VS-06 & 1 & 2 & 21 & K09LM22 & 0.017 & 0.544 & $<0.001$ & $<0.012$ & 0.016 & 0.008 & 0.033 & 0.008 & 0.039 & 1.76 \\
\hline SB6VS-16 & 1 & 2 & 22 & K17LM12 & 0.034 & 0.407 & $<0.001$ & 0.018 & 0.030 & 0.021 & 0.053 & 0.020 & 0.173 & 2.12 \\
\hline Batch 1 & 1 & 2 & 23 & BCHLM132 & 0.127 & 0.837 & $<0.001$ & $<0.012$ & 0.072 & 0.287 & 2.74 & $<0.005$ & 0.778 & 1.32 \\
\hline Ustd & 1 & 2 & 24 & UstdLM132 & $<0.003$ & 0.947 & $<0.001$ & $<0.012$ & 0.173 & $<0.003$ & 2.56 & $<0.005$ & 0.699 & 2.16 \\
\hline Batch 1 & 2 & 1 & 1 & BCHLM211 & 0.128 & 0.845 & $<0.001$ & $<0.012$ & 0.072 & 0.284 & 2.69 & $<0.005$ & 0.789 & 1.31 \\
\hline Ustd & 2 & 1 & 2 & UstdLM211 & $<0.003$ & 0.963 & $<0.001$ & $<0.012$ & 0.174 & $<0.003$ & 2.58 & $<0.005$ & 0.715 & 2.16 \\
\hline SB6VS-15 & 2 & 1 & 3 & K15LM11 & 0.037 & 0.455 & $<0.001$ & 0.022 & 0.032 & 0.023 & 0.049 & 0.023 & 0.195 & 2.28 \\
\hline SB6VS-05 & 2 & 1 & 4 & K12LM21 & 0.055 & 0.256 & $<0.001$ & 0.045 & 0.039 & 0.035 & 0.077 & 0.035 & 0.047 & 2.48 \\
\hline SB6VS-01 & 2 & 1 & 5 & K14LM21 & 0.057 & 0.264 & $<0.001$ & 0.041 & 0.036 & 0.032 & 0.072 & 0.032 & 0.350 & 2.08 \\
\hline SB6VS-03 & 2 & 1 & 6 & K13LM11 & 0.051 & 0.260 & $<0.001$ & 0.042 & 0.038 & 0.033 & 0.072 & 0.034 & 0.047 & 2.48 \\
\hline SB6VS-10 & 2 & 1 & 7 & K16LM21 & 0.021 & 0.258 & $<0.001$ & $<0.012$ & 0.019 & 0.007 & 0.035 & 0.010 & 0.346 & 2.51 \\
\hline SB6VS-01 & 2 & 1 & 8 & K14LM11 & 0.056 & 0.254 & $<0.001$ & 0.038 & 0.049 & 0.034 & 0.077 & 0.032 & 0.345 & 2.06 \\
\hline SB6VS-14 & 2 & 1 & 9 & K02LM11 & 0.029 & 0.345 & $<0.001$ & 0.016 & 0.025 & 0.017 & 0.034 & 0.017 & 0.147 & 1.74 \\
\hline SB6VS-14 & 2 & 1 & 10 & K02LM21 & 0.036 & 0.424 & $<0.001$ & 0.014 & 0.031 & 0.023 & 0.050 & 0.021 & 0.183 & 2.11 \\
\hline Batch 1 & 2 & 1 & 11 & BCHLM221 & 0.130 & 0.847 & $<0.001$ & $<0.012$ & 0.073 & 0.282 & 2.63 & $<0.005$ & 0.796 & 1.33 \\
\hline Ustd & 2 & 1 & 12 & UstdLM221 & $<0.003$ & 0.959 & $<0.001$ & $<0.012$ & 0.176 & $<0.003$ & 2.60 & $<0.005$ & 0.716 & 2.19 \\
\hline SB6VS-02 & 2 & 1 & 13 & K07LM11 & 0.058 & 0.649 & $<0.001$ & 0.041 & 0.039 & 0.036 & 0.080 & 0.034 & 0.048 & 2.07 \\
\hline SB6VS-03 & 2 & 1 & 14 & K13LM21 & 0.053 & 0.263 & $<0.001$ & 0.042 & 0.039 & 0.034 & 0.068 & 0.034 & 0.048 & 2.56 \\
\hline SB6VS-05 & 2 & 1 & 15 & K12LM11 & 0.057 & 0.261 & $<0.001$ & 0.043 & 0.039 & 0.035 & 0.073 & 0.036 & 0.047 & 2.54 \\
\hline SB6VS-15 & 2 & $\frac{1}{1}$ & 16 & K15LM21 & 0.037 & 0.443 & $<0.001$ & 0.019 & 0.031 & 0.021 & 0.046 & 0.022 & 0.192 & 2.24 \\
\hline SB6VS-10 & 2 & 1 & 17 & K16LM11 & 0.020 & 0.247 & $<0.001$ & $<0.012$ & 0.017 & 0.006 & 0.030 & 0.010 & 0.339 & 2.44 \\
\hline SB6VS-04 & 2 & 1 & 18 & K10LM11 & 0.020 & 0.632 & $<0.001$ & $<0.012$ & 0.017 & 0.011 & 0.033 & 0.010 & 0.047 & 2.36 \\
\hline SB6VS-02 & 2 & 1 & 19 & K07LM21 & 0.058 & 0.641 & $<0.001$ & 0.043 & 0.037 & 0.033 & 0.072 & 0.034 & 0.048 & 2.03 \\
\hline SB6VS-04 & 2 & 1 & 20 & K10LM21 & 0.020 & 0.618 & $<0.001$ & $<0.012$ & 0.017 & 0.011 & 0.034 & 0.010 & 0.045 & 2.38 \\
\hline Batch 1 & 2 & 1 & 21 & BCHLM231 & 0.132 & 0.846 & $<0.001$ & $<0.012$ & 0.074 & 0.279 & 2.67 & $<0.005$ & 0.800 & 1.34 \\
\hline
\end{tabular}


SRNL-STI-2010-00242

Revision 0

Table A1. Measured Elemental Concentrations (wt\%) for the Study Glasses Prepared Using Lithium Metaborate (part 1)

\begin{tabular}{|c|c|c|c|c|c|c|c|c|c|c|c|c|c|c|}
\hline Glass ID & Block & $\begin{array}{c}\text { Sub- } \\
\text { Block }\end{array}$ & Seq. & Lab ID & $\begin{array}{c}\text { Ba } \\
(w t \%)\end{array}$ & $\begin{array}{c}\mathrm{Ca} \\
(\mathrm{wt} \%)\end{array}$ & $\begin{array}{c}\mathrm{Cd} \\
(\mathrm{wt} \%)\end{array}$ & $\begin{array}{c}\mathrm{Ce} \\
(\mathrm{wt} \%)\end{array}$ & $\begin{array}{c}\mathrm{Cr} \\
(\mathrm{wt} \%)\end{array}$ & $\begin{array}{c}\mathrm{Cu} \\
(\mathrm{wt} \%)\end{array}$ & $\begin{array}{c}K \\
(w t \%)\end{array}$ & $\begin{array}{c}\text { La } \\
(w t \%)\end{array}$ & $\begin{array}{c}\mathrm{Mg} \\
(\mathrm{wt} \%)\end{array}$ & $\begin{array}{c}\text { Mn } \\
(\mathbf{w t} \%)\end{array}$ \\
\hline Ustd & 2 & 1 & 22 & UstdLM231 & $<0.003$ & 0.957 & $<0.001$ & $<0.012$ & 0.180 & $<0.003$ & 2.42 & $<0.005$ & 0.721 & 2.21 \\
\hline Batch 1 & 2 & 2 & 1 & BCHLM212 & 0.127 & 0.844 & $<0.001$ & $<0.012$ & 0.071 & 0.286 & 2.52 & $<0.005$ & 0.798 & 1.30 \\
\hline Ustd & 2 & 2 & 2 & UstdLM212 & $<0.003$ & 0.960 & $<0.001$ & $<0.012$ & 0.172 & $<0.003$ & 2.47 & $<0.005$ & 0.725 & 2.15 \\
\hline SB6VS-01 & 2 & 2 & 3 & K14LM12 & 0.055 & 0.255 & $<0.001$ & 0.036 & 0.048 & 0.034 & 0.076 & 0.032 & 0.351 & 2.03 \\
\hline SB6VS-14 & 2 & 2 & 4 & K02LM12 & 0.028 & 0.345 & $<0.001$ & 0.013 & 0.024 & 0.017 & 0.034 & 0.018 & 0.151 & 1.70 \\
\hline SB6VS-02 & 2 & 2 & 5 & K07LM12 & 0.056 & 0.648 & $<0.001$ & 0.039 & 0.039 & 0.037 & 0.078 & 0.035 & 0.050 & 2.05 \\
\hline SB6VS-05 & 2 & 2 & 6 & K12LM12 & 0.056 & 0.261 & $<0.001$ & 0.043 & 0.038 & 0.036 & 0.071 & 0.036 & 0.049 & 2.51 \\
\hline SB6VS-14 & 2 & 2 & 7 & K02LM22 & 0.035 & 0.421 & $<0.001$ & $<0.012$ & 0.031 & 0.023 & 0.049 & 0.021 & 0.185 & 2.06 \\
\hline SB6VS-15 & 2 & 2 & 8 & K15LM12 & 0.037 & 0.455 & $<0.001$ & 0.016 & 0.032 & 0.023 & 0.048 & 0.023 & 0.199 & 2.28 \\
\hline SB6VS-03 & 2 & 2 & 9 & K13LM12 & 0.051 & 0.260 & $<0.001$ & 0.037 & 0.038 & 0.033 & 0.070 & 0.034 & 0.049 & 2.48 \\
\hline SB6VS-15 & 2 & 2 & 10 & K15LM22 & 0.036 & 0.449 & $<0.001$ & 0.016 & 0.031 & 0.021 & 0.045 & 0.022 & 0.197 & 2.21 \\
\hline Batch 1 & 2 & 2 & 11 & BCHLM222 & 0.128 & 0.846 & $<0.001$ & $<0.012$ & 0.072 & 0.286 & 2.57 & $<0.005$ & 0.812 & 1.31 \\
\hline Ustd & 2 & 2 & 12 & UstdLM222 & $<0.003$ & 0.959 & $<0.001$ & $<0.012$ & 0.176 & $<0.003$ & 2.41 & $<0.005$ & 0.731 & 2.19 \\
\hline SB6VS-02 & 2 & 2 & 13 & K07LM22 & 0.056 & 0.640 & $<0.001$ & 0.042 & 0.036 & 0.034 & 0.072 & 0.034 & 0.049 & 1.98 \\
\hline SB6VS-10 & 2 & 2 & 14 & K16LM12 & 0.020 & 0.248 & $<0.001$ & $<0.012$ & 0.017 & 0.006 & 0.029 & 0.010 & 0.345 & 2.40 \\
\hline SB6VS-10 & 2 & 2 & 15 & K16LM22 & 0.020 & 0.256 & $<0.001$ & $<0.012$ & 0.019 & 0.007 & 0.034 & 0.010 & 0.353 & 2.51 \\
\hline SB6VS-03 & 2 & 2 & 16 & K13LM22 & 0.053 & 0.263 & $<0.001$ & 0.039 & 0.038 & 0.034 & 0.068 & 0.035 & 0.050 & 2.54 \\
\hline SB6VS-04 & 2 & 2 & 17 & K10LM12 & 0.020 & 0.631 & $<0.001$ & $<0.012$ & 0.016 & 0.012 & 0.033 & 0.011 & 0.049 & 2.34 \\
\hline SB6VS-05 & 2 & 2 & 18 & K12LM22 & 0.055 & 0.254 & $<0.001$ & 0.036 & 0.039 & 0.035 & 0.074 & 0.035 & 0.049 & 2.48 \\
\hline SB6VS-01 & 2 & 2 & 19 & K14LM22 & 0.057 & 0.247 & $<0.001$ & 0.032 & 0.036 & 0.033 & 0.070 & 0.033 & 0.336 & 2.08 \\
\hline SB6VS-04 & 2 & 2 & 20 & K10LM22 & 0.019 & 0.618 & $<0.001$ & $<0.012$ & 0.017 & 0.011 & 0.034 & 0.010 & 0.047 & 2.36 \\
\hline Batch 1 & 2 & 2 & 21 & BCHLM232 & 0.129 & 0.844 & $<0.001$ & $<0.012$ & 0.073 & 0.285 & 2.57 & $<0.005$ & 0.818 & 1.33 \\
\hline Ustd & 2 & 2 & 22 & UstdLM232 & $<0.003$ & 0.958 & $<0.001$ & $<0.012$ & 0.177 & $<0.003$ & 2.45 & $<0.005$ & 0.738 & 2.20 \\
\hline
\end{tabular}


Table A2. Measured Elemental Concentrations (wt\%) for the Study Glasses Prepared Using Lithium Metaborate (part 2)

\begin{tabular}{|c|c|c|c|c|c|c|c|c|c|c|c|c|c|}
\hline Glass ID & Block & Sub-Block & Seq & Lab ID & $\begin{array}{c}\mathrm{Na} \\
(\mathbf{w t} \%)\end{array}$ & $\begin{array}{c}\mathrm{Ni} \\
(w t \%)\end{array}$ & $\begin{array}{c}P \\
(w t \%)\end{array}$ & $\begin{array}{c}\mathrm{Pb} \\
(\mathbf{w t} \%)\end{array}$ & $\begin{array}{c}S \\
(w t \%)\end{array}$ & $\begin{array}{c}\mathbf{T i} \\
(\mathbf{w t} \%)\end{array}$ & $\begin{array}{c}\mathrm{U} \\
(w t \%)\end{array}$ & $\begin{array}{c}\mathrm{Zn} \\
(\mathbf{w t} \%)\end{array}$ & $\begin{array}{c}\mathrm{Zr} \\
(\mathbf{w t} \%)\end{array}$ \\
\hline Batch 1 & 1 & 1 & 1 & BCHLM111 & 6.78 & 0.586 & $<0.025$ & $<0.005$ & $<0.110$ & 0.393 & $<0.500$ & $<0.005$ & 0.062 \\
\hline Ustd & 1 & 1 & 2 & \begin{tabular}{|l|} 
UstdLM111 \\
\end{tabular} & 9.09 & 0.858 & $<0.025$ & $<0.005$ & $<0.110$ & 0.599 & 2.09 & $<0.005$ & $<0.002$ \\
\hline SB6VS-11 & 1 & 1 & 3 & K03LM21 & 12.00 & 0.724 & 0.030 & $<0.005$ & 0.282 & 0.436 & 1.36 & 0.010 & 0.025 \\
\hline SB6VS-07 & 1 & 1 & 4 & K04LM11 & 12.20 & 0.960 & 0.030 & $<0.005$ & 0.192 & 0.007 & 1.21 & 0.012 & 0.022 \\
\hline SB6VS-17 & 1 & 1 & 5 & K08LM11 & 11.70 & 0.899 & 0.060 & $<0.005$ & 0.243 & 0.230 & 1.65 & 0.018 & 0.046 \\
\hline SB6VS-06 & 1 & 1 & 6 & K09LM21 & 12.00 & 0.669 & 0.035 & $<0.005$ & 0.241 & 0.006 & 1.77 & 0.008 & 0.023 \\
\hline SB6VS-09 & 1 & 1 & 7 & K01LM21 & 11.20 & 1.010 & 0.076 & 0.011 & 0.270 & 0.006 & 1.27 & 0.022 & 0.066 \\
\hline SB6VS-12 & 1 & 1 & 8 & K11LM11 & 12.30 & 0.990 & 0.079 & $<0.005$ & 0.194 & 0.418 & 1.73 & 0.023 & 0.068 \\
\hline SB6VS-16 & 1 & 1 & 9 & K17LM21 & 12.10 & 0.898 & 0.056 & 0.005 & 0.264 & 0.229 & 1.59 & 0.020 & 0.047 \\
\hline SB6VS-12 & 1 & 1 & 10 & K11LM21 & 12.10 & 0.976 & 0.080 & $<0.005$ & 0.185 & 0.409 & 1.73 & 0.023 & 0.066 \\
\hline SB6VS-08 & 1 & 1 & 11 & K06LM21 & 12.30 & 0.993 & 0.032 & $<0.005$ & 0.266 & 0.411 & 1.23 & 0.009 & 0.024 \\
\hline Batch 1 & 1 & 1 & 12 & BCHLM121 & 7.04 & 0.596 & $<0.025$ & $<0.005$ & $<0.110$ & 0.392 & $<0.500$ & $<0.005$ & 0.063 \\
\hline Ustd & 1 & 1 & 13 & \begin{tabular}{|l|} 
UstdLM121 \\
\end{tabular} & 9.20 & 0.870 & $<0.025$ & $<0.005$ & $<0.110$ & 0.597 & 2.05 & $<0.005$ & $<0.002$ \\
\hline SB6VS-09 & 1 & 1 & 14 & K01LM11 & 11.40 & 1.030 & 0.073 & 0.010 & 0.281 & 0.006 & 1.28 & 0.023 & 0.067 \\
\hline SB6VS-16 & 1 & 1 & 15 & K17LM11 & 11.90 & 0.904 & 0.065 & 0.008 & 0.267 & 0.226 & 1.66 & 0.018 & 0.045 \\
\hline SB6VS-17 & 1 & 1 & 16 & K08LM21 & 12.80 & 0.971 & 0.064 & $<0.005$ & 0.238 & 0.246 & 1.73 & 0.019 & 0.050 \\
\hline SB6VS-06 & 1 & 1 & 17 & K09LM11 & 12.00 & 0.670 & 0.039 & 0.007 & 0.280 & 0.006 & 1.69 & 0.008 & 0.022 \\
\hline SB6VS-07 & 1 & 1 & 18 & K04LM21 & 12.20 & 0.957 & 0.031 & $<0.005$ & 0.176 & 0.006 & 1.24 & 0.010 & 0.022 \\
\hline SB6VS-08 & 1 & 1 & 19 & K06LM11 & 13.00 & 1.030 & 0.033 & $<0.005$ & 0.259 & 0.425 & 1.25 & 0.009 & 0.024 \\
\hline SB6VS-13 & 1 & 1 & 20 & K05LM21 & 11.80 & 0.792 & 0.056 & $<0.005$ & 0.222 & 0.199 & 1.37 & 0.020 & 0.041 \\
\hline SB6VS-13 & 1 & 1 & 21 & K05LM11 & 11.90 & 0.758 & 0.051 & $<0.005$ & 0.209 & 0.193 & 1.32 & 0.015 & 0.040 \\
\hline SB6VS-11 & 1 & 1 & 22 & K03LM11 & 11.80 & 0.698 & 0.034 & $<0.005$ & 0.273 & 0.413 & 1.19 & 0.009 & 0.024 \\
\hline Batch 1 & 1 & 1 & 23 & BCHLM131 & 7.16 & 0.600 & $<0.025$ & $<0.005$ & $<0.110$ & 0.393 & $<0.500$ & 0.005 & 0.063 \\
\hline Ustd & 1 & 1 & 24 & UstdLM131 & 9.43 & 0.867 & $<0.025$ & $<0.005$ & $<0.110$ & 0.593 & 2.06 & $<0.005$ & $<0.002$ \\
\hline Batch 1 & 1 & 2 & 1 & BCHLM112 & 6.71 & 0.581 & $<0.025$ & $<0.005$ & $<0.110$ & 0.398 & $<0.500$ & $<0.005$ & 0.063 \\
\hline Ustd & 1 & 2 & 2 & \begin{tabular}{|l|} 
UstdLM112 \\
\end{tabular} & 8.95 & 0.851 & $<0.025$ & $<0.005$ & $<0.110$ & 0.606 & 2.02 & $<0.005$ & $<0.002$ \\
\hline SB6VS-09 & 1 & 2 & 3 & K01LM22 & 11.00 & 0.994 & 0.076 & 0.009 & 0.275 & 0.006 & 1.23 & 0.023 & 0.066 \\
\hline SB6VS-11 & 1 & 2 & 4 & K03LM22 & 11.90 & 0.720 & 0.029 & $<0.005$ & 0.293 & 0.441 & 1.26 & 0.011 & 0.024 \\
\hline SB6VS-08 & 1 & 2 & 5 & K06LM12 & 12.50 & 1.010 & 0.034 & $<0.005$ & 0.289 & 0.435 & 1.24 & 0.010 & 0.024 \\
\hline SB6VS-13 & 1 & 2 & 6 & K05LM12 & 11.50 & 0.743 & 0.052 & $<0.005$ & 0.206 & 0.198 & 1.27 & 0.016 & 0.040 \\
\hline SB6VS-07 & 1 & 2 & 7 & K04LM22 & 11.80 & 0.942 & 0.029 & $<0.005$ & 0.185 & 0.006 & 1.19 & 0.011 & 0.022 \\
\hline SB6VS-17 & 1 & 2 & 8 & K08LM12 & 11.50 & 0.891 & 0.065 & $<0.005$ & 0.254 & 0.233 & 1.54 & 0.019 & 0.046 \\
\hline SB6VS-12 & 1 & 2 & 9 & K11LM22 & 11.90 & 0.960 & 0.083 & $<0.005$ & 0.200 & 0.416 & 1.71 & 0.024 & 0.066 \\
\hline SB6VS-08 & 1 & 2 & 10 & K06LM22 & 12.10 & 0.981 & 0.031 & $<0.005$ & 0.275 & 0.419 & 1.20 & 0.010 & 0.024 \\
\hline SB6VS-17 & 1 & 2 & 11 & K08LM22 & 12.40 & 0.962 & 0.065 & $<0.005$ & 0.263 & 0.252 & 1.70 & 0.020 & 0.050 \\
\hline Batch 1 & 1 & 2 & 12 & \begin{tabular}{|l|} 
BCHLM122 \\
\end{tabular} & 6.81 & 0.589 & $<0.025$ & $<0.005$ & $<0.110$ & 0.399 & $<0.500$ & $<0.005$ & 0.063 \\
\hline Ustd & 1 & 2 & 13 & \begin{tabular}{|l|} 
UstdLM122 \\
\end{tabular} & 9.01 & 0.861 & $<0.025$ & $<0.005$ & $<0.110$ & 0.606 & 2.03 & $<0.005$ & $<0.002$ \\
\hline SB6VS-12 & 1 & 2 & 14 & K11LM12 & 11.90 & 0.989 & 0.086 & $<0.005$ & 0.201 & 0.422 & 1.73 & 0.024 & 0.068 \\
\hline SB6VS-06 & 1 & 2 & 15 & K09LM12 & 11.40 & 0.664 & 0.040 & 0.007 & 0.259 & 0.006 & 1.69 & 0.009 & 0.022 \\
\hline SB6VS-09 & 1 & 2 & 16 & K01LM12 & 11.00 & 1.020 & 0.078 & 0.011 & 0.288 & 0.006 & 1.26 & 0.024 & 0.068 \\
\hline SB6VS-16 & 1 & 2 & 17 & K17LM22 & 11.70 & 0.895 & 0.068 & 0.005 & 0.270 & 0.232 & 1.62 & 0.021 & 0.047 \\
\hline SB6VS-07 & 1 & 2 & 18 & K04LM12 & 11.80 & 0.965 & 0.032 & $<0.005$ & 0.191 & 0.007 & 1.17 & 0.012 & 0.022 \\
\hline SB6VS-13 & 1 & 2 & 19 & K05LM22 & 11.00 & 0.787 & 0.056 & $<0.005$ & 0.225 & 0.203 & 1.36 & 0.022 & 0.041 \\
\hline SB6VS-11 & 1 & 2 & 20 & K03LM12 & 11.00 & 0.691 & 0.035 & $<0.005$ & 0.276 & 0.420 & 1.23 & 0.009 & 0.024 \\
\hline SB6VS-06 & 1 & 2 & 21 & K09LM22 & 11.60 & 0.678 & 0.039 & 0.005 & 0.268 & 0.006 & 1.68 & 0.008 & 0.023 \\
\hline SB6VS-16 & 1 & 2 & 22 & K17LM12 & 11.40 & 0.902 & 0.066 & 0.008 & 0.265 & 0.229 & 1.69 & 0.020 & 0.045 \\
\hline Batch 1 & 1 & 2 & 23 & BCHLM132 & 6.65 & 0.598 & $<0.025$ & $<0.005$ & $<0.110$ & 0.397 & $<0.500$ & $<0.005$ & 0.063 \\
\hline Ustd & 1 & 2 & 24 & \begin{tabular}{|l|} 
UstdLM132 \\
\end{tabular} & 8.81 & 0.869 & $<0.025$ & $<0.005$ & $<0.110$ & 0.599 & 2.01 & $<0.005$ & $<0.002$ \\
\hline Batch 1 & 2 & 1 & 1 & BCHLM211 & 6.80 & 0.580 & $<0.025$ & $<0.005$ & $<0.110$ & 0.397 & $<0.500$ & $<0.005$ & 0.062 \\
\hline Ustd & 2 & 1 & 2 & UstdLM211 & 9.11 & 0.853 & $<0.025$ & $<0.005$ & $<0.110$ & 0.603 & 2.06 & $<0.005$ & $<0.002$ \\
\hline SB6VS-15 & 2 & 1 & 3 & \begin{tabular}{|l|} 
K15LM11 \\
\end{tabular} & 13.30 & 0.958 & 0.054 & $<0.005$ & 0.251 & 0.248 & 1.71 & 0.021 & 0.053 \\
\hline SB6VS-05 & 2 & 1 & 4 & K12LM21 & 14.10 & 0.784 & 0.082 & 0.013 & 0.217 & 0.007 & 1.34 & 0.028 & 0.077 \\
\hline SB6VS-01 & 2 & 1 & 5 & K14LM21 & 12.40 & 0.791 & 0.093 & 0.011 & 0.315 & 0.007 & 2.03 & 0.030 & 0.079 \\
\hline SB6VS-03 & 2 & 1 & 6 & K13LM11 & 12.30 & 1.140 & 0.090 & 0.005 & 0.261 & 0.464 & 2.01 & 0.029 & 0.080 \\
\hline SB6VS-10 & 2 & 1 & 7 & K16LM21 & 12.80 & 0.794 & 0.040 & 0.005 & 0.150 & 0.497 & 2.00 & 0.012 & 0.026 \\
\hline SB6VS-01 & 2 & 1 & 8 & K14LM11 & 12.20 & 0.795 & 0.093 & 0.013 & 0.318 & 0.007 & 1.92 & 0.032 & 0.076 \\
\hline SB6VS-14 & 2 & 1 & 9 & K02LM11 & 10.30 & 0.719 & 0.049 & $<0.005$ & 0.204 & 0.191 & 1.32 & 0.015 & 0.040 \\
\hline SB6VS-14 & 2 & 1 & 10 & K02LM21 & 12.90 & 0.889 & 0.057 & $<0.005$ & 0.250 & 0.227 & 1.55 & 0.018 & 0.050 \\
\hline Batch 1 & 2 & 1 & 11 & BCHLM221 & 6.87 & 0.598 & $<0.025$ & $<0.005$ & $<0.110$ & 0.398 & $<0.500$ & $<0.005$ & 0.063 \\
\hline Ustd & 2 & 1 & 12 & UstdLM221 & 9.16 & 0.867 & $<0.025$ & $<0.005$ & $<0.110$ & 0.602 & 2.06 & $<0.005$ & $<0.002$ \\
\hline SB6VS-02 & 2 & 1 & 13 & K07LM11 & 12.30 & 0.786 & 0.087 & 0.010 & 0.198 & 0.455 & 1.49 & 0.029 & 0.077 \\
\hline SB6VS-03 & 2 & 1 & 14 & K13LM21 & 12.60 & 1.180 & 0.099 & 0.005 & 0.273 & 0.472 & 2.05 & 0.030 & 0.082 \\
\hline SB6VS-05 & 2 & 1 & 15 & K12LM11 & 14.4 & 0.809 & 0.087 & 0.014 & 0.214 & 0.007 & 1.43 & 0.029 & 0.078 \\
\hline SB6VS-15 & 2 & 1 & 16 & K15LM21 & 12.9 & 0.940 & 0.049 & 0.006 & 0.236 & 0.239 & 1.63 & 0.021 & 0.051 \\
\hline SB6VS-10 & 2 & 1 & 17 & K16LM11 & 12.50 & 0.772 & 0.041 & $<0.005$ & 0.196 & 0.478 & 1.94 & 0.011 & 0.026 \\
\hline SB6VS-04 & 2 & 1 & 18 & K10LM11 & 12.30 & 1.080 & 0.035 & 0.009 & 0.213 & 0.007 & 2.02 & 0.012 & 0.028 \\
\hline SB6VS-02 & 2 & 1 & 19 & K07LM21 & 12.30 & 0.778 & 0.088 & 0.010 & 0.194 & 0.438 & 1.39 & 0.030 & 0.078 \\
\hline SB6VS-04 & 2 & 1 & 20 & K10LM21 & 12.00 & 1.090 & 0.039 & 0.010 & 0.210 & 0.007 & 1.97 & 0.012 & 0.026 \\
\hline Batch 1 & 2 & 1 & 21 & BCHLM231 & 6.89 & 0.611 & $<0.025$ & $<0.005$ & $<0.110$ & 0.397 & $<0.500$ & $<0.005$ & 0.062 \\
\hline
\end{tabular}


SRNL-STI-2010-00242

Revision 0

Table A2. Measured Elemental Concentrations (wt\%) for the Study Glasses Prepared Using Lithium Metaborate (part 2)

\begin{tabular}{|c|c|c|c|c|c|c|c|c|c|c|c|c|c|}
\hline Glass ID & Block & Sub-Block & Seq & Lab ID & $\begin{array}{c}\mathrm{Na} \\
(\mathrm{wt} \%)\end{array}$ & $\begin{array}{c}\mathrm{Ni} \\
(\mathrm{wt} \%)\end{array}$ & $\begin{array}{c}\mathbf{P} \\
(w t \%)\end{array}$ & $\begin{array}{c}\mathrm{Pb} \\
(\mathbf{w t} \%)\end{array}$ & $\begin{array}{c}S \\
(w t \%)\end{array}$ & $\begin{array}{c}\mathbf{T i} \\
(w t \%)\end{array}$ & $\begin{array}{c}\mathrm{U} \\
(w t \%)\end{array}$ & $\begin{array}{c}\mathrm{Zn} \\
(\mathbf{w t} \%)\end{array}$ & $\begin{array}{c}\mathrm{Zr} \\
(\mathbf{w t} \%)\end{array}$ \\
\hline Ustd & 2 & 1 & 22 & UstdLM231 & 9.18 & 0.888 & $<0.025$ & $<0.005$ & $<0.110$ & 0.598 & 2.07 & $<0.005$ & $<0.002$ \\
\hline Batch 1 & 2 & 2 & 1 & BCHLM212 & 6.87 & 0.582 & $<0.025$ & $<0.005$ & $<0.110$ & 0.399 & $<0.500$ & $<0.005$ & 0.063 \\
\hline Ustd & 2 & 2 & 2 & UstdLM212 & 9.27 & 0.842 & $<0.025$ & $<0.005$ & $<0.110$ & 0.606 & 1.98 & $<0.005$ & $<0.002$ \\
\hline SB6VS-01 & 2 & 2 & 3 & K14LM12 & 12.30 & 0.779 & 0.093 & 0.010 & 0.304 & 0.007 & 1.88 & 0.031 & 0.076 \\
\hline SB6VS-14 & 2 & 2 & 4 & K02LM12 & 10.50 & 0.703 & 0.050 & $<0.005$ & 0.187 & 0.192 & 1.29 & 0.014 & 0.040 \\
\hline SB6VS-02 & 2 & 2 & 5 & K07LM12 & 12.60 & 0.777 & 0.093 & 0.009 & 0.203 & 0.459 & 1.35 & 0.028 & 0.077 \\
\hline SB6VS-05 & 2 & 2 & 6 & K12LM12 & 14.6 & 0.799 & 0.090 & 0.014 & 0.236 & 0.007 & 1.42 & 0.028 & 0.079 \\
\hline SB6VS-14 & 2 & 2 & 7 & K02LM22 & 13.00 & 0.872 & 0.059 & $<0.005$ & 0.236 & 0.227 & 1.55 & 0.018 & 0.049 \\
\hline SB6VS-15 & 2 & 2 & 8 & K15LM12 & 13.4 & 0.964 & 0.062 & $<0.005$ & 0.259 & 0.250 & 1.68 & 0.021 & 0.053 \\
\hline SB6VS-03 & 2 & 2 & 9 & K13LM12 & 12.50 & 1.140 & 0.105 & 0.005 & 0.273 & 0.466 & 1.92 & 0.029 & 0.081 \\
\hline SB6VS-15 & 2 & 2 & 10 & K15LM22 & 13.1 & 0.932 & 0.064 & $<0.005$ & 0.237 & 0.243 & 1.63 & 0.020 & 0.051 \\
\hline Batch 1 & 2 & 2 & 11 & BCHLM222 & 7.04 & 0.593 & $<0.025$ & $<0.005$ & $<0.110$ & 0.401 & $<0.500$ & $<0.005$ & 0.063 \\
\hline Ustd & 2 & 2 & 12 & UstdLM222 & 9.33 & 0.871 & $<0.025$ & $<0.005$ & $<0.110$ & 0.612 & 2.01 & $<0.005$ & $<0.002$ \\
\hline SB6VS-02 & 2 & 2 & 13 & K07LM22 & 12.40 & 0.757 & 0.092 & 0.009 & 0.197 & 0.442 & 1.37 & 0.028 & 0.078 \\
\hline SB6VS-10 & 2 & 2 & 14 & K16LM12 & 12.80 & 0.763 & 0.038 & $<0.005$ & 0.165 & 0.482 & 1.90 & 0.011 & 0.026 \\
\hline SB6VS-10 & 2 & 2 & 15 & K16LM22 & 13.10 & 0.804 & 0.046 & 0.005 & 0.202 & 0.501 & 1.93 & 0.012 & 0.026 \\
\hline SB6VS-03 & 2 & 2 & 16 & K13LM22 & 12.80 & 1.170 & 0.100 & 0.006 & 0.277 & 0.476 & 2.01 & 0.030 & 0.082 \\
\hline SB6VS-04 & 2 & 2 & 17 & K10LM12 & 12.60 & 1.080 & 0.044 & 0.009 & 0.230 & 0.007 & 1.97 & 0.012 & 0.028 \\
\hline SB6VS-05 & 2 & 2 & 18 & K12LM22 & 14.40 & 0.799 & 0.095 & 0.011 & 0.225 & 0.007 & 1.40 & 0.029 & 0.077 \\
\hline SB6VS-01 & 2 & 2 & 19 & K14LM22 & 11.80 & 0.805 & 0.106 & 0.012 & 0.324 & 0.007 & 1.88 & 0.030 & 0.078 \\
\hline SB6VS-04 & 2 & 2 & 20 & K10LM22 & 12.20 & 1.070 & 0.040 & 0.009 & 0.235 & 0.007 & 1.92 & 0.012 & 0.026 \\
\hline Batch 1 & 2 & 2 & 21 & BCHLM232 & 7.04 & 0.603 & $<0.025$ & $<0.005$ & $<0.110$ & 0.403 & $<0.500$ & $<0.005$ & 0.063 \\
\hline Ustd & 2 & 2 & 22 & UstdLM232 & 9.40 & 0.879 & $<0.025$ & $<0.005$ & $<0.110$ & 0.611 & 2.02 & $<0.005$ & $<0.002$ \\
\hline
\end{tabular}


Table A3. Measured Elemental Concentrations (wt\%) for the Study Glasses Prepared Using Peroxide Fusion

\begin{tabular}{|c|c|c|c|c|c|c|c|c|c|}
\hline Glass ID & Block & Sub-Block & Sequence & Lab ID & Al (wt\%) & B (wt\%) & $\mathrm{Fe}(\mathrm{wt} \%)$ & Li (wt\%) & $\mathrm{Si}(w \mathrm{t} \%)$ \\
\hline Batch 1 & 1 & 1 & 1 & BCHPF111 & 2.63 & 2.34 & 8.99 & 2.03 & 23.5 \\
\hline Ustd & 1 & 1 & 2 & UstdPF111 & 2.19 & 2.76 & 9.24 & 1.39 & 21.2 \\
\hline SB6VS-02 & 1 & 1 & 3 & K07PF21 & 5.44 & 1.51 & 6.20 & 2.30 & 23.0 \\
\hline SB6VS-03 & 1 & 1 & 4 & K13PF21 & 5.76 & 1.51 & 4.58 & 2.29 & 22.8 \\
\hline SB6VS-10 & 1 & 1 & 5 & K16PF21 & 4.61 & 1.47 & 6.30 & 2.27 & 22.6 \\
\hline SB6VS-02 & 1 & 1 & 6 & K07PF11 & 5.29 & 1.43 & 6.00 & 2.24 & 22.1 \\
\hline SB6VS-14 & 1 & 1 & 7 & K02PF11 & 4.84 & 1.49 & 5.15 & 2.31 & 22.6 \\
\hline SB6VS-04 & 1 & 1 & 8 & K10PF11 & 5.23 & 1.42 & 5.36 & 2.21 & 21.6 \\
\hline SB6VS-17 & 1 & 1 & 9 & K08PF11 & 5.65 & 1.35 & 6.15 & 2.07 & 20.8 \\
\hline SB6VS-12 & 1 & 1 & 10 & K11PF11 & 4.49 & 1.44 & 5.04 & 2.22 & 21.9 \\
\hline SB6VS-03 & 1 & 1 & 11 & K13PF11 & 5.74 & 1.48 & 4.57 & 2.28 & 22.7 \\
\hline Batch 1 & 1 & 1 & 12 & BCHPF121 & 2.63 & 2.29 & 8.96 & 2.04 & 23.5 \\
\hline Ustd & 1 & 1 & 13 & UstdPF121 & 2.20 & 2.72 & 9.22 & 1.39 & 21.2 \\
\hline SB6VS-08 & 1 & 1 & 14 & K06PF11 & 4.35 & 1.41 & 5.95 & 2.20 & 21.2 \\
\hline SB6VS-08 & 1 & 1 & 15 & K06PF21 & 4.40 & 1.41 & 6.00 & 2.19 & 21.4 \\
\hline SB6VS-14 & 1 & 1 & 16 & K02PF21 & 4.82 & 1.50 & 5.18 & 2.34 & 22.9 \\
\hline SB6VS-05 & 1 & 1 & 17 & K12PF11 & 5.62 & 1.43 & 5.05 & 2.23 & 21.9 \\
\hline SB6VS-12 & 1 & 1 & 18 & K11PF21 & 4.49 & 1.42 & 4.97 & 2.21 & 21.8 \\
\hline SB6VS-10 & 1 & 1 & 19 & K16PF11 & 4.47 & 1.43 & 6.22 & 2.24 & 22.1 \\
\hline SB6VS-04 & 1 & 1 & 20 & K10PF21 & 5.22 & 1.42 & 5.37 & 2.22 & 21.7 \\
\hline SB6VS-17 & 1 & 1 & 21 & K08PF21 & 5.78 & 1.37 & 6.26 & 2.15 & 21.2 \\
\hline SB6VS-05 & 1 & 1 & 22 & K12PF21 & 5.85 & 1.50 & 5.27 & 2.34 & 22.8 \\
\hline Batch 1 & 1 & 1 & 23 & BCHPF131 & 2.62 & 2.31 & 8.97 & 2.06 & 23.6 \\
\hline Ustd & 1 & 1 & 24 & UstdPF131 & 2.20 & 2.72 & 9.23 & 1.41 & 21.2 \\
\hline Batch 1 & 1 & 2 & 1 & BCHPF112 & 2.62 & 2.37 & 8.97 & 2.03 & 23.5 \\
\hline Ustd & 1 & 2 & 2 & UstdPF112 & 2.18 & 2.77 & 9.26 & 1.37 & 21.2 \\
\hline SB6VS-04 & 1 & 2 & 3 & K10PF22 & 5.23 & 1.45 & 5.40 & 2.16 & 21.8 \\
\hline SB6VS-03 & 1 & 2 & 4 & K13PF12 & 5.73 & 1.53 & 4.58 & 2.25 & 22.8 \\
\hline SB6VS-17 & 1 & 2 & 5 & K08PF12 & 5.68 & 1.38 & 6.20 & 2.07 & 20.9 \\
\hline SB6VS-04 & 1 & 2 & 6 & K10PF12 & 5.24 & 1.45 & 5.40 & 2.17 & 21.7 \\
\hline SB6VS-02 & 1 & 2 & 7 & K07PF12 & 5.27 & 1.47 & 6.06 & 2.20 & 22.3 \\
\hline SB6VS-08 & 1 & 2 & 8 & K06PF22 & 4.40 & 1.44 & 6.05 & 2.13 & 21.6 \\
\hline SB6VS-08 & 1 & 2 & 9 & K06PF12 & 4.34 & 1.44 & 5.99 & 2.13 & 21.4 \\
\hline SB6VS-02 & 1 & 2 & 10 & K07PF22 & 5.44 & 1.52 & 6.24 & 2.28 & 23.1 \\
\hline SB6VS-17 & 1 & 2 & 11 & K08PF22 & 5.76 & 1.40 & 6.28 & 2.09 & 21.2 \\
\hline Batch 1 & 1 & 2 & 12 & BCHPF122 & 2.62 & 2.35 & 9.00 & 2.03 & 23.6 \\
\hline Ustd & 1 & 2 & 13 & UstdPF122 & 2.16 & 2.80 & 9.28 & 1.37 & 21.2 \\
\hline SB6VS-05 & 1 & 2 & 14 & K12PF22 & 5.85 & 1.53 & 5.30 & 2.28 & 22.8 \\
\hline SB6VS-14 & 1 & 2 & 15 & K02PF22 & 4.85 & 1.55 & 5.24 & 2.28 & 23.1 \\
\hline SB6VS-14 & 1 & 2 & 16 & K02PF12 & 4.80 & 1.53 & 5.18 & 2.24 & 22.9 \\
\hline SB6VS-10 & 1 & 2 & 17 & K16PF12 & 4.51 & 1.48 & 6.27 & 2.19 & 22.3 \\
\hline SB6VS-03 & 1 & 2 & 18 & K13PF22 & 5.74 & 1.53 & 4.62 & 2.24 & 23.0 \\
\hline SB6VS-10 & 1 & 2 & 19 & K16PF22 & 4.57 & 1.53 & 6.37 & 2.20 & 22.7 \\
\hline SB6VS-05 & 1 & 2 & 20 & K12PF12 & 5.58 & 1.47 & 5.13 & 2.16 & 22.1 \\
\hline SB6VS-12 & 1 & 2 & 21 & K11PF12 & 4.54 & 1.50 & 5.09 & 2.20 & 22.2 \\
\hline SB6VS-12 & 1 & 2 & 22 & K11PF22 & 4.50 & 1.46 & 5.00 & 2.14 & 21.8 \\
\hline Batch 1 & 1 & 2 & 23 & BCHPF132 & 2.59 & 2.36 & 9.02 & 2.01 & 23.7 \\
\hline Ustd & 1 & 2 & 24 & UstdPF132 & 2.18 & 2.80 & 9.32 & 1.39 & 21.3 \\
\hline Batch 1 & 2 & 1 & 1 & BCHPF211 & 2.61 & 2.34 & 8.94 & 2.01 & 23.3 \\
\hline Ustd & 2 & 1 & 2 & UstdPF211 & 2.15 & 2.76 & 9.20 & 1.37 & 21.2 \\
\hline SB6VS-15 & 2 & 1 & 3 & K15PF11 & 4.98 & 1.45 & 5.43 & 2.17 & 21.7 \\
\hline SB6VS-11 & 2 & 1 & 4 & K03PF11 & 5.61 & 1.48 & 4.32 & 2.22 & 22.3 \\
\hline SB6VS-06 & 2 & 1 & 5 & K09PF21 & 5.47 & 1.58 & 5.35 & 2.34 & 23.4 \\
\hline SB6VS-16 & 2 & 1 & 6 & K17PF11 & 5.56 & 1.48 & 6.06 & 2.21 & 22.5 \\
\hline SB6VS-01 & 2 & 1 & 7 & K14PF11 & 5.48 & 1.52 & 5.99 & 2.26 & 22.4 \\
\hline SB6VS-13 & 2 & 1 & 8 & K05PF11 & 5.60 & 1.48 & 5.03 & 2.19 & 22.2 \\
\hline SB6VS-06 & 2 & 1 & 9 & K09PF11 & 5.26 & 1.48 & 5.11 & 2.24 & 22.4 \\
\hline SB6VS-09 & 2 & 1 & 10 & K01PF21 & 4.58 & 1.50 & 6.29 & 2.24 & 22.5 \\
\hline Batch 1 & 2 & 1 & 11 & BCHPF221 & 2.63 & 2.37 & 9.06 & 2.03 & 23.6 \\
\hline Ustd & 2 & 1 & 12 & UstdPF221 & 2.16 & 2.77 & 9.23 & 1.37 & 21.2 \\
\hline SB6VS-13 & 2 & 1 & 13 & K05PF21 & 5.95 & 1.59 & 5.35 & 2.33 & 23.4 \\
\hline SB6VS-01 & 2 & 1 & 14 & K14PF21 & 5.56 & 1.54 & 6.09 & 2.31 & 23.0 \\
\hline SB6VS-11 & 2 & 1 & 15 & K03PF21 & 5.64 & 1.49 & 4.34 & 2.22 & 22.3 \\
\hline SB6VS-15 & 2 & 1 & 16 & K15PF21 & 5.14 & 1.52 & 5.63 & 2.24 & 22.6 \\
\hline SB6VS-16 & 2 & 1 & 17 & K17PF21 & 5.60 & 1.51 & 6.11 & 2.24 & 22.5 \\
\hline SB6VS-09 & 2 & 1 & 18 & K01PF11 & 4.50 & 1.49 & 6.21 & 2.21 & 22.5 \\
\hline SB6VS-07 & 2 & 1 & 19 & K04PF21 & 5.67 & 1.50 & 5.10 & 2.22 & 22.5 \\
\hline SB6VS-07 & 2 & 1 & 20 & K04PF11 & 5.72 & 1.52 & 5.16 & 2.23 & 22.7 \\
\hline Batch 1 & 2 & 1 & 21 & BCHPF231 & 2.63 & 2.38 & 9.06 & 2.02 & 23.7 \\
\hline
\end{tabular}


SRNL-STI-2010-00242

Revision 0

Table A3. Measured Elemental Concentrations (wt\%) for the Study Glasses Prepared Using Peroxide Fusion

\begin{tabular}{|c|c|c|c|c|c|c|c|c|c|}
\hline Glass ID & Block & Sub-Block & Sequence & Lab ID & Al (wt\%) & B (wt\%) & $\mathrm{Fe}(\mathrm{wt} \%)$ & $\mathrm{Li}(\mathrm{wt} \%)$ & $\mathrm{Si}\left(w \mathrm{w}^{\circ} \%\right)$ \\
\hline Ustd & 2 & 1 & 22 & UstdPF231 & 2.15 & 2.78 & 9.24 & 1.37 & 21.3 \\
\hline Batch 1 & 2 & 2 & 1 & BCHPF212 & 2.60 & 2.32 & 8.96 & 2.02 & 23.7 \\
\hline Ustd & 2 & 2 & 2 & UstdPF212 & 2.12 & 2.75 & 9.21 & 1.39 & 21.4 \\
\hline SB6VS-06 & 2 & 2 & 3 & K09PF22 & 5.49 & 1.62 & 5.30 & 2.36 & 23.5 \\
\hline SB6VS-09 & 2 & 2 & 4 & K01PF22 & 4.52 & 1.53 & 6.19 & 2.24 & 22.6 \\
\hline SB6VS-07 & 2 & 2 & 5 & K04PF22 & 5.64 & 1.50 & 5.05 & 2.20 & 22.5 \\
\hline SB6VS-01 & 2 & 2 & 6 & K14PF22 & 5.52 & 1.53 & 6.03 & 2.29 & 23.0 \\
\hline SB6VS-15 & 2 & 2 & 7 & K15PF12 & 4.96 & 1.43 & 5.41 & 2.14 & 21.8 \\
\hline SB6VS-11 & 2 & 2 & 8 & K03PF12 & 5.58 & 1.44 & 4.28 & 2.20 & 22.2 \\
\hline SB6VS-13 & 2 & 2 & 9 & K05PF22 & 5.93 & 1.52 & 5.32 & 2.29 & 23.6 \\
\hline SB6VS-16 & 2 & 2 & 10 & K17PF22 & 5.58 & 1.44 & 6.06 & 2.22 & 22.5 \\
\hline Batch 1 & 2 & 2 & 11 & BCHPF222 & 2.60 & 2.29 & 8.99 & 2.05 & 23.7 \\
\hline Ustd & 2 & 2 & 12 & UstdPF222 & 2.11 & 2.68 & 9.15 & 1.34 & 21.1 \\
\hline SB6VS-11 & 2 & 2 & 13 & K03PF22 & 5.61 & 1.50 & 4.32 & 2.21 & 22.2 \\
\hline SB6VS-16 & 2 & 2 & 14 & K17PF12 & 5.53 & 1.51 & 6.01 & 2.20 & 22.3 \\
\hline SB6VS-01 & 2 & 2 & 15 & K14PF12 & 5.44 & 1.53 & 5.95 & 2.21 & 22.3 \\
\hline SB6VS-15 & 2 & 2 & 16 & K15PF22 & 5.15 & 1.54 & 5.61 & 2.19 & 22.5 \\
\hline SB6VS-09 & 2 & 2 & 17 & K01PF12 & 4.51 & 1.51 & 6.18 & 2.16 & 22.2 \\
\hline SB6VS-13 & 2 & 2 & 18 & K05PF12 & 5.58 & 1.48 & 5.02 & 2.12 & 22.1 \\
\hline SB6VS-06 & 2 & 2 & 19 & K09PF12 & 5.24 & 1.50 & 5.10 & 2.16 & 22.3 \\
\hline SB6VS-07 & 2 & 2 & 20 & K04PF12 & 5.72 & 1.52 & 5.13 & 2.18 & 22.5 \\
\hline Batch 1 & 2 & 2 & 21 & BCHPF232 & 2.61 & 2.36 & 9.02 & 1.98 & 23.5 \\
\hline Ustd & 2 & 2 & 22 & UstdPF232 & 2.13 & 2.75 & 9.19 & 1.32 & 21.1 \\
\hline
\end{tabular}


SRNL-STI-2010-00242

Revision 0

Table A4. Average Measured Chemical Compositions Versus Targeted Compositions

\begin{tabular}{|c|c|c|c|c|c|}
\hline & & Measured & Targeted & Difference of & $\%$ Difference of \\
\hline Glass ID & Oxide & $(w t \%)$ & $(w t \%)$ & Measured vs Targeted (wt\%) & Measured vs Targeted \\
\hline Batch 1 & $\mathrm{Al}_{2} \mathrm{O}_{3}$ & 4.9426 & 4.8770 & 0.0656 & $1.3 \%$ \\
\hline Batch 1 & $\mathrm{~B}_{2} \mathrm{O}_{3}$ & 7.5346 & 7.7770 & -0.2424 & $-3.1 \%$ \\
\hline Batch 1 & $\mathrm{BaO}$ & 0.1426 & 0.1510 & -0.0084 & $-5.5 \%$ \\
\hline Batch 1 & $\mathrm{CaO}$ & 1.1842 & 1.2200 & -0.0358 & $-2.9 \%$ \\
\hline Batch 1 & $\mathrm{Ce}_{2} \mathrm{O}_{3}$ & 0.0070 & 0.0000 & 0.0070 & \\
\hline Batch 1 & $\mathrm{Cr}_{2} \mathrm{O}_{3}$ & 0.1049 & 0.1070 & -0.0021 & $-2.0 \%$ \\
\hline Batch 1 & $\mathrm{CuO}$ & 0.3573 & 0.3990 & -0.0417 & $-10.5 \%$ \\
\hline Batch 1 & $\mathrm{Fe}_{2} \mathrm{O}_{3}$ & 12.8602 & 12.8390 & 0.0212 & $0.2 \%$ \\
\hline Batch 1 & $\mathrm{~K}_{2} \mathrm{O}$ & 3.1932 & 3.3270 & -0.1338 & $-4.0 \%$ \\
\hline Batch 1 & $\mathrm{La}_{2} \mathrm{O}_{3}$ & 0.0029 & 0.0000 & 0.0029 & \\
\hline Batch 1 & $\mathrm{Li}_{2} \mathrm{O}$ & 4.3614 & 4.4290 & -0.0676 & $-1.5 \%$ \\
\hline Batch 1 & $\mathrm{MgO}$ & 1.3224 & 1.4190 & -0.0966 & $-6.8 \%$ \\
\hline Batch 1 & $\mathrm{MnO}$ & 1.7012 & 1.7260 & -0.0248 & $-1.4 \%$ \\
\hline Batch 1 & $\mathrm{Na}_{2} \mathrm{O}$ & 9.2855 & 9.0030 & 0.2825 & $3.1 \%$ \\
\hline Batch 1 & $\mathrm{NiO}$ & 0.7547 & 0.7510 & 0.0037 & $0.5 \%$ \\
\hline Batch 1 & $\mathrm{P}_{2} \mathrm{O}_{5}$ & 0.0286 & 0.0000 & 0.0286 & \\
\hline Batch 1 & $\mathrm{PbO}$ & 0.0027 & 0.0000 & 0.0027 & \\
\hline Batch 1 & $\mathrm{SiO}_{2}$ & 50.4340 & 50.2200 & 0.2140 & $0.4 \%$ \\
\hline Batch 1 & $\mathrm{SO}_{4}$ & 0.1648 & 0.0000 & 0.1648 & \\
\hline Batch 1 & $\mathrm{TiO}_{2}$ & 0.6626 & 0.6770 & -0.0144 & $-2.1 \%$ \\
\hline Batch 1 & $\mathrm{U}_{3} \mathrm{O}_{8}$ & 0.2948 & 0.0000 & 0.2948 & \\
\hline Batch 1 & $\mathrm{ZnO}$ & 0.0034 & 0.0000 & 0.0034 & \\
\hline Batch 1 & $\mathrm{ZrO}_{2}$ & 0.0848 & 0.0980 & -0.0132 & $-13.5 \%$ \\
\hline Batch 1 & Sum & 99.4308 & 99.0200 & 0.4108 & $0.4 \%$ \\
\hline SB6VS-01 & $\mathrm{Al}_{2} \mathrm{O}_{3}$ & 10.3923 & 10.0274 & 0.3648 & $3.6 \%$ \\
\hline SB6VS-01 & $\mathrm{B}_{2} \mathrm{O}_{3}$ & 4.9264 & 5.1200 & -0.1936 & $-3.8 \%$ \\
\hline SB6VS-01 & $\mathrm{BaO}$ & 0.0628 & 0.0515 & 0.0113 & $21.9 \%$ \\
\hline SB6VS-01 & $\mathrm{CaO}$ & 0.3568 & 0.2794 & 0.0774 & $27.7 \%$ \\
\hline SB6VS-01 & $\mathrm{Ce}_{2} \mathrm{O}_{3}$ & 0.0430 & 0.0802 & -0.0372 & $-46.3 \%$ \\
\hline SB6VS-01 & $\mathrm{Cr}_{2} \mathrm{O}_{3}$ & 0.0618 & 0.0463 & 0.0155 & $33.4 \%$ \\
\hline SB6VS-01 & $\mathrm{CuO}$ & 0.0416 & 0.0414 & 0.0002 & $0.5 \%$ \\
\hline SB6VS-01 & $\mathrm{Fe}_{2} \mathrm{O}_{3}$ & 8.5996 & 9.6556 & -1.0559 & $-10.9 \%$ \\
\hline SB6VS-01 & $\mathrm{K}_{2} \mathrm{O}$ & 0.0888 & 0.0332 & 0.0556 & $167.3 \%$ \\
\hline SB6VS-01 & $\mathrm{La}_{2} \mathrm{O}_{3}$ & 0.0378 & 0.0410 & -0.0031 & $-7.7 \%$ \\
\hline SB6VS-01 & $\mathrm{Li}_{2} \mathrm{O}$ & 4.8817 & 5.1200 & -0.2383 & $-4.7 \%$ \\
\hline SB6VS-01 & $\mathrm{MgO}$ & 0.5729 & 0.4964 & 0.0765 & $15.4 \%$ \\
\hline SB6VS-01 & $\mathrm{MnO}$ & 2.6631 & 2.2489 & 0.4142 & $18.4 \%$ \\
\hline SB6VS-01 & $\mathrm{Na}_{2} \mathrm{O}$ & 16.4119 & 13.7301 & 2.6818 & $19.5 \%$ \\
\hline SB6VS-01 & $\mathrm{NiO}$ & 1.0085 & 0.8608 & 0.1477 & $17.2 \%$ \\
\hline SB6VS-01 & $\mathrm{P}_{2} \mathrm{O}_{5}$ & 0.2205 & 0.1712 & 0.0494 & $28.8 \%$ \\
\hline SB6VS-01 & $\mathrm{PbO}$ & 0.0124 & 0.0110 & 0.0014 & $13.1 \%$ \\
\hline SB6VS-01 & $\mathrm{SiO}_{2}$ & 48.5086 & 49.2120 & -0.7034 & $-1.4 \%$ \\
\hline SB6VS-01 & $\mathrm{SO}_{4}$ & 0.9445 & 0.6228 & 0.3217 & $51.6 \%$ \\
\hline SB6VS-01 & $\mathrm{TiO}_{2}$ & 0.0117 & 0.0000 & 0.0117 & \\
\hline SB6VS-01 & $\mathrm{U}_{3} \mathrm{O}_{8}$ & 2.2729 & 2.0340 & 0.2389 & $11.7 \%$ \\
\hline
\end{tabular}


SRNL-STI-2010-00242

Revision 0

Table A4. Average Measured Chemical Compositions Versus Targeted Compositions

\begin{tabular}{|c|c|c|c|c|c|}
\hline & & Measured & Targeted & Difference of & $\%$ Difference of \\
\hline Glass ID & Oxide & $(w t \%)$ & $(w t \%)$ & Measured vs Targeted (wt\%) & Measured vs Targeted \\
\hline SB6VS-01 & $\mathrm{ZnO}$ & 0.0383 & 0.0270 & 0.0113 & $41.8 \%$ \\
\hline SB6VS-01 & $\mathrm{ZrO}_{2}$ & 0.1043 & 0.0898 & 0.0146 & $16.2 \%$ \\
\hline SB6VS-01 & Sum & 102.2629 & 100.0000 & 2.2629 & $2.3 \%$ \\
\hline SB6VS-02 & $\mathrm{Al}_{2} \mathrm{O}_{3}$ & 10.1277 & 9.7888 & 0.3390 & $3.5 \%$ \\
\hline SB6VS-02 & $\mathrm{B}_{2} \mathrm{O}_{3}$ & 4.7735 & 5.1200 & -0.3465 & $-6.8 \%$ \\
\hline SB6VS-02 & $\mathrm{BaO}$ & 0.0636 & 0.0515 & 0.0121 & $23.5 \%$ \\
\hline SB6VS-02 & $\mathrm{CaO}$ & 0.9018 & 0.7294 & 0.1724 & $23.6 \%$ \\
\hline SB6VS-02 & $\mathrm{Ce}_{2} \mathrm{O}_{3}$ & 0.0483 & 0.0802 & -0.0319 & $-39.8 \%$ \\
\hline SB6VS-02 & $\mathrm{Cr}_{2} \mathrm{O}_{3}$ & 0.0552 & 0.0463 & 0.0089 & $19.2 \%$ \\
\hline SB6VS-02 & $\mathrm{CuO}$ & 0.0438 & 0.0414 & 0.0024 & $5.8 \%$ \\
\hline SB6VS-02 & $\mathrm{Fe}_{2} \mathrm{O}_{3}$ & 8.7569 & 9.6556 & $\begin{array}{l}-0.8986 \\
\end{array}$ & $-9.3 \%$ \\
\hline SB6VS-02 & $\mathrm{K}_{2} \mathrm{O}$ & 0.0909 & 0.0332 & 0.0577 & $173.6 \%$ \\
\hline SB6VS-02 & $\mathrm{La}_{2} \mathrm{O}_{3}$ & 0.0402 & 0.0410 & -0.0008 & $-1.9 \%$ \\
\hline SB6VS-02 & $\mathrm{Li}_{2} \mathrm{O}$ & 4.8548 & 5.1200 & -0.2652 & $-5.2 \%$ \\
\hline SB6VS-02 & $\mathrm{MgO}$ & 0.0808 & 0.0616 & 0.0193 & $31.3 \%$ \\
\hline SB6VS-02 & $\mathrm{MnO}$ & 2.6244 & 2.2489 & 0.3754 & $16.7 \%$ \\
\hline SB6VS-02 & $\mathrm{Na}_{2} \mathrm{O}$ & 16.7152 & 13.7301 & 2.9851 & $21.7 \%$ \\
\hline SB6VS-02 & $\mathrm{NiO}$ & 0.9856 & 0.8608 & 0.1248 & $14.5 \%$ \\
\hline SB6VS-02 & $\mathrm{P}_{2} \mathrm{O}_{5}$ & 0.2062 & 0.1712 & 0.0350 & $20.5 \%$ \\
\hline SB6VS-02 & $\mathrm{PbO}$ & 0.0102 & 0.0110 & -0.0007 & $-6.6 \%$ \\
\hline SB6VS-02 & $\mathrm{SiO}_{2}$ & 48.4017 & 49.6271 & -1.2255 & $-2.5 \%$ \\
\hline SB6VS-02 & $\mathrm{SO}_{4}$ & 0.5932 & 0.3830 & 0.2101 & $54.9 \%$ \\
\hline SB6VS-02 & $\mathrm{TiO}_{2}$ & 0.7481 & 0.6606 & 0.0875 & $13.2 \%$ \\
\hline SB6VS-02 & $\mathrm{U}_{3} \mathrm{O}_{8}$ & 1.6509 & 1.4216 & 0.2292 & $16.1 \%$ \\
\hline SB6VS-02 & $\mathrm{ZnO}$ & 0.0358 & 0.0270 & 0.0088 & $32.6 \%$ \\
\hline SB6VS-02 & $\mathrm{ZrO}_{2}$ & 0.1047 & 0.0898 & 0.0149 & $16.6 \%$ \\
\hline SB6VS-02 & Sum & 101.9141 & 100.0000 & 1.9141 & $1.9 \%$ \\
\hline SB6VS-03 & $\mathrm{Al}_{2} \mathrm{O}_{3}$ & 10.8505 & 10.3511 & 0.4994 & $4.8 \%$ \\
\hline SB6VS-03 & $\mathrm{B}_{2} \mathrm{O}_{3}$ & 4.8701 & 5.1200 & $\begin{array}{l}-0.2499 \\
\end{array}$ & $-4.9 \%$ \\
\hline SB6VS-03 & $\mathrm{BaO}$ & 0.0581 & 0.0515 & 0.0065 & $12.7 \%$ \\
\hline SB6VS-03 & $\mathrm{CaO}$ & 0.3659 & 0.2794 & 0.0865 & $31.0 \%$ \\
\hline SB6VS-03 & $\mathrm{Ce}_{2} \mathrm{O}_{3}$ & 0.0469 & 0.0802 & -0.0334 & $-41.6 \%$ \\
\hline SB6VS-03 & $\mathrm{Cr}_{2} \mathrm{O}_{3}$ & 0.0559 & 0.0463 & 0.0096 & $20.8 \%$ \\
\hline SB6VS-03 & $\mathrm{CuO}$ & 0.0419 & 0.0414 & 0.0005 & $1.2 \%$ \\
\hline SB6VS-03 & $\mathrm{Fe}_{2} \mathrm{O}_{3}$ & 6.5587 & 7.8077 & -1.2489 & $-16.0 \%$ \\
\hline SB6VS-03 & $\mathrm{K}_{2} \mathrm{O}$ & 0.0837 & 0.0332 & 0.0505 & $151.9 \%$ \\
\hline SB6VS-03 & $\mathrm{La}_{2} \mathrm{O}_{3}$ & 0.0402 & 0.0410 & -0.0008 & $-1.9 \%$ \\
\hline SB6VS-03 & $\mathrm{Li}_{2} \mathrm{O}$ & 4.8763 & 5.1200 & -0.2437 & $-4.8 \%$ \\
\hline SB6VS-03 & $\mathrm{MgO}$ & 0.0804 & 0.0616 & 0.0189 & $30.6 \%$ \\
\hline SB6VS-03 & $\mathrm{MnO}$ & 3.2474 & 2.7288 & 0.5186 & $19.0 \%$ \\
\hline SB6VS-03 & $\mathrm{Na}_{2} \mathrm{O}$ & 16.9174 & 13.7301 & 3.1873 & $23.2 \%$ \\
\hline SB6VS-03 & $\mathrm{NiO}$ & 1.4729 & 1.2643 & 0.2086 & $16.5 \%$ \\
\hline SB6VS-03 & $\mathrm{P}_{2} \mathrm{O}_{5}$ & 0.2257 & 0.1712 & 0.0545 & $31.8 \%$ \\
\hline SB6VS-03 & $\mathrm{PbO}$ & 0.0057 & 0.0110 & -0.0053 & $-48.4 \%$ \\
\hline SB6VS-03 & $\mathrm{SiO}_{2}$ & 48.8295 & 49.6271 & -0.7976 & $-1.6 \%$ \\
\hline
\end{tabular}


SRNL-STI-2010-00242

Revision 0

Table A4. Average Measured Chemical Compositions Versus Targeted Compositions

\begin{tabular}{|c|c|c|c|c|c|}
\hline & & Measured & Targeted & Difference of & $\%$ Difference of \\
\hline Glass ID & Oxide & $(w t \%)$ & $(w t \%)$ & Measured vs Targeted (wt\%) & Measured vs Targeted \\
\hline SB6VS-03 & $\mathrm{SO}_{4}$ & 0.8119 & 0.6228 & 0.1891 & $30.4 \%$ \\
\hline SB6VS-03 & $\mathrm{TiO}_{2}$ & 0.7831 & 0.6606 & 0.1225 & $18.5 \%$ \\
\hline SB6VS-03 & $\mathrm{U}_{3} \mathrm{O}_{8}$ & 2.3555 & 2.0340 & 0.3215 & $15.8 \%$ \\
\hline SB6VS-03 & $\mathrm{ZnO}$ & 0.0367 & 0.0270 & 0.0097 & $36.0 \%$ \\
\hline SB6VS-03 & $\mathrm{ZrO}_{2}$ & 0.1098 & 0.0898 & 0.0200 & $22.2 \%$ \\
\hline SB6VS-03 & Sum & 102.7247 & 100.0000 & 2.7247 & $2.7 \%$ \\
\hline SB6VS-04 & $\mathrm{Al}_{2} \mathrm{O}_{3}$ & 9.8821 & 9.7614 & 0.1207 & $1.2 \%$ \\
\hline SB6VS-04 & $\mathrm{B}_{2} \mathrm{O}_{3}$ & 4.6206 & 5.1200 & -0.4994 & $-9.8 \%$ \\
\hline SB6VS-04 & $\mathrm{BaO}$ & 0.0221 & 0.0174 & 0.0047 & $26.9 \%$ \\
\hline SB6VS-04 & $\mathrm{CaO}$ & 0.8742 & 0.7294 & 0.1448 & $19.9 \%$ \\
\hline SB6VS-04 & $\mathrm{Ce}_{2} \mathrm{O}_{3}$ & 0.0070 & 0.0270 & -0.0200 & $-74.0 \%$ \\
\hline SB6VS-04 & $\mathrm{Cr}_{2} \mathrm{O}_{3}$ & 0.0245 & 0.0156 & 0.0089 & $56.8 \%$ \\
\hline SB6VS-04 & $\mathrm{CuO}$ & 0.0141 & 0.0140 & 0.0001 & $0.8 \%$ \\
\hline SB6VS-04 & $\mathrm{Fe}_{2} \mathrm{O}_{3}$ & 7.6954 & 9.6556 & -1.9602 & $-20.3 \%$ \\
\hline SB6VS-04 & $\mathrm{K}_{2} \mathrm{O}$ & 0.0404 & 0.0112 & 0.0291 & $260.0 \%$ \\
\hline SB6VS-04 & $\mathrm{La}_{2} \mathrm{O}_{3}$ & 0.0120 & 0.0138 & -0.0018 & $-13.0 \%$ \\
\hline SB6VS-04 & $\mathrm{Li}_{2} \mathrm{O}$ & 4.7149 & 5.1200 & -0.4051 & $-7.9 \%$ \\
\hline SB6VS-04 & $\mathrm{MgO}$ & 0.0779 & 0.0616 & 0.0164 & $26.6 \%$ \\
\hline SB6VS-04 & $\mathrm{MnO}$ & 3.0472 & 2.7288 & 0.3184 & $11.7 \%$ \\
\hline SB6VS-04 & $\mathrm{Na}_{2} \mathrm{O}$ & 16.5467 & 13.7301 & 2.8166 & $20.5 \%$ \\
\hline SB6VS-04 & $\mathrm{NiO}$ & 1.3743 & 1.2643 & 0.1100 & $8.7 \%$ \\
\hline SB6VS-04 & $\mathrm{P}_{2} \mathrm{O}_{5}$ & 0.0905 & 0.0577 & 0.0328 & $56.8 \%$ \\
\hline SB6VS-04 & $\mathrm{PbO}$ & 0.0100 & 0.0037 & 0.0063 & $169.8 \%$ \\
\hline SB6VS-04 & $\mathrm{SiO}_{2}$ & 46.4228 & 49.2120 & -2.7892 & $-5.7 \%$ \\
\hline SB6VS-04 & $\mathrm{SO}_{4}$ & 0.6651 & 0.3830 & 0.2820 & $73.6 \%$ \\
\hline SB6VS-04 & $\mathrm{TiO}_{2}$ & 0.0117 & 0.0000 & 0.0117 & \\
\hline SB6VS-04 & $\mathrm{U}_{3} \mathrm{O}_{8}$ & 2.3230 & 2.0340 & 0.2890 & $14.2 \%$ \\
\hline SB6VS-04 & $\mathrm{ZnO}$ & 0.0149 & 0.0091 & 0.0058 & $64.1 \%$ \\
\hline SB6VS-04 & $\mathrm{ZrO}_{2}$ & 0.0365 & 0.0303 & 0.0062 & $20.5 \%$ \\
\hline SB6VS-04 & Sum & 98.5282 & 100.0000 & -1.4718 & $-1.5 \%$ \\
\hline SB6VS-05 & $\mathrm{Al}_{2} \mathrm{O}_{3}$ & 10.8174 & 10.5307 & 0.2867 & $2.7 \%$ \\
\hline SB6VS-05 & $\mathrm{B}_{2} \mathrm{O}_{3}$ & 4.7735 & 5.1200 & -0.3465 & $-6.8 \%$ \\
\hline SB6VS-05 & $\mathrm{BaO}$ & 0.0622 & 0.0515 & 0.0107 & $20.8 \%$ \\
\hline SB6VS-05 & $\mathrm{CaO}$ & 0.3610 & 0.2794 & 0.0816 & $29.2 \%$ \\
\hline SB6VS-05 & $\mathrm{Ce}_{2} \mathrm{O}_{3}$ & 0.0489 & 0.0802 & -0.0313 & $-39.0 \%$ \\
\hline SB6VS-05 & $\mathrm{Cr}_{2} \mathrm{O}_{3}$ & 0.0566 & 0.0463 & 0.0103 & $22.3 \%$ \\
\hline SB6VS-05 & $\mathrm{CuO}$ & 0.0441 & 0.0414 & 0.0027 & $6.5 \%$ \\
\hline SB6VS-05 & $\mathrm{Fe}_{2} \mathrm{O}_{3}$ & 7.4166 & 7.9319 & -0.5153 & $-6.5 \%$ \\
\hline SB6VS-05 & $\mathrm{K}_{2} \mathrm{O}$ & 0.0888 & 0.0332 & 0.0556 & $167.3 \%$ \\
\hline SB6VS-05 & $\mathrm{La}_{2} \mathrm{O}_{3}$ & 0.0416 & 0.0410 & 0.0007 & $1.6 \%$ \\
\hline SB6VS-05 & $\mathrm{Li}_{2} \mathrm{O}$ & 4.8494 & 5.1200 & -0.2706 & $-5.3 \%$ \\
\hline SB6VS-05 & $\mathrm{MgO}$ & 0.0796 & 0.0616 & 0.0180 & $29.3 \%$ \\
\hline SB6VS-05 & $\mathrm{MnO}$ & 3.2312 & 2.7288 & 0.5024 & $18.4 \%$ \\
\hline SB6VS-05 & $\mathrm{Na}_{2} \mathrm{O}$ & 19.3775 & 15.7576 & 3.6199 & $23.0 \%$ \\
\hline SB6VS-05 & $\mathrm{NiO}$ & 1.0151 & 0.8608 & 0.1544 & $17.9 \%$ \\
\hline
\end{tabular}


SRNL-STI-2010-00242

Revision 0

Table A4. Average Measured Chemical Compositions Versus Targeted Compositions

\begin{tabular}{|c|c|c|c|c|c|}
\hline & & Measured & Targeted & Difference of & $\%$ Difference of \\
\hline Glass ID & Oxide & $(w t \%)$ & $(w t \%)$ & Measured vs Targeted (wt\%) & Measured vs Targeted \\
\hline SB6VS-05 & $\mathrm{P}_{2} \mathrm{O}_{5}$ & 0.2028 & 0.1712 & 0.0316 & $18.5 \%$ \\
\hline SB6VS-05 & $\mathrm{PbO}$ & 0.0140 & 0.0110 & 0.0031 & $27.9 \%$ \\
\hline SB6VS-05 & $\mathrm{SiO}_{2}$ & 47.9203 & 49.2120 & -1.2917 & $-2.6 \%$ \\
\hline SB6VS-05 & $\mathrm{SO}_{4}$ & 0.6681 & 0.3830 & 0.2850 & $74.4 \%$ \\
\hline SB6VS-05 & $\mathrm{TiO}_{2}$ & 0.0117 & 0.0000 & 0.0117 & \\
\hline SB6VS-05 & $\mathrm{U}_{3} \mathrm{O}_{8}$ & 1.6479 & 1.4216 & 0.2263 & $15.9 \%$ \\
\hline SB6VS-05 & $\mathrm{ZnO}$ & 0.0355 & 0.0270 & 0.0085 & $31.4 \%$ \\
\hline SB6VS-05 & $\mathrm{ZrO}_{2}$ & 0.1050 & 0.0898 & 0.0152 & $17.0 \%$ \\
\hline SB6VS-05 & Sum & 102.8696 & 100.0000 & 2.8696 & $2.9 \%$ \\
\hline SB6VS-06 & $\mathrm{Al}_{2} \mathrm{O}_{3}$ & 10.1372 & 9.8104 & 0.3268 & $3.3 \%$ \\
\hline SB6VS-06 & $\mathrm{B}_{2} \mathrm{O}_{3}$ & 4.9747 & 5.1200 & -0.1453 & $-2.8 \%$ \\
\hline SB6VS-06 & $\mathrm{BaO}$ & 0.0184 & 0.0174 & 0.0011 & $6.1 \%$ \\
\hline SB6VS-06 & $\mathrm{CaO}$ & 0.7626 & 0.7294 & 0.0332 & $4.6 \%$ \\
\hline SB6VS-06 & $\mathrm{Ce}_{2} \mathrm{O}_{3}$ & 0.0070 & 0.0270 & -0.0200 & $-74.0 \%$ \\
\hline SB6VS-06 & $\mathrm{Cr}_{2} \mathrm{O}_{3}$ & 0.0241 & 0.0156 & 0.0085 & $54.5 \%$ \\
\hline SB6VS-06 & $\mathrm{CuO}$ & 0.0106 & 0.0140 & -0.0033 & $-23.8 \%$ \\
\hline SB6VS-06 & $\mathrm{Fe}_{2} \mathrm{O}_{3}$ & 7.4559 & 7.8077 & -0.3518 & $-4.5 \%$ \\
\hline SB6VS-06 & $\mathrm{K}_{2} \mathrm{O}$ & 0.0425 & 0.0112 & 0.0313 & $278.8 \%$ \\
\hline SB6VS-06 & $\mathrm{La}_{2} \mathrm{O}_{3}$ & 0.0091 & 0.0138 & -0.0047 & $-34.2 \%$ \\
\hline SB6VS-06 & $\mathrm{Li}_{2} \mathrm{O}$ & 4.8978 & 5.1200 & -0.2222 & $-4.3 \%$ \\
\hline SB6VS-06 & $\mathrm{MgO}$ & 0.0663 & 0.0616 & 0.0048 & $7.8 \%$ \\
\hline SB6VS-06 & $\mathrm{MnO}$ & 2.2531 & 2.2489 & 0.0042 & $0.2 \%$ \\
\hline SB6VS-06 & $\mathrm{Na}_{2} \mathrm{O}$ & 15.8390 & 15.7576 & 0.0814 & $0.5 \%$ \\
\hline SB6VS-06 & $\mathrm{NiO}$ & 0.8529 & 0.8608 & -0.0079 & $-0.9 \%$ \\
\hline SB6VS-06 & $\mathrm{P}_{2} \mathrm{O}_{5}$ & 0.0876 & 0.0577 & 0.0299 & $51.8 \%$ \\
\hline SB6VS-06 & $\mathrm{PbO}$ & 0.0058 & 0.0037 & 0.0021 & $56.8 \%$ \\
\hline SB6VS-06 & $\mathrm{SiO}_{2}$ & 48.9900 & 49.6271 & -0.6371 & $-1.3 \%$ \\
\hline SB6VS-06 & $\mathrm{SO}_{4}$ & 0.7849 & 0.6228 & 0.1621 & $26.0 \%$ \\
\hline SB6VS-06 & $\mathrm{TiO}_{2}$ & 0.0100 & 0.0000 & 0.0100 & \\
\hline SB6VS-06 & $\mathrm{U}_{3} \mathrm{O}_{8}$ & 2.0135 & 2.0340 & -0.0205 & $-1.0 \%$ \\
\hline SB6VS-06 & $\mathrm{ZnO}$ & 0.0103 & 0.0091 & 0.0012 & $12.8 \%$ \\
\hline SB6VS-06 & $\mathrm{ZrO}_{2}$ & 0.0304 & 0.0303 & 0.0001 & $0.4 \%$ \\
\hline SB6VS-06 & Sum & 99.2844 & 100.0000 & -0.7156 & $-0.7 \%$ \\
\hline SB6VS-07 & $\mathrm{Al}_{2} \mathrm{O}_{3}$ & 10.7465 & 10.5307 & 0.2158 & $2.0 \%$ \\
\hline SB6VS-07 & $\mathrm{B}_{2} \mathrm{O}_{3}$ & 4.8620 & 5.1200 & -0.2580 & $-5.0 \%$ \\
\hline SB6VS-07 & $\mathrm{BaO}$ & 0.0190 & 0.0174 & 0.0016 & $9.3 \%$ \\
\hline SB6VS-07 & $\mathrm{CaO}$ & 0.3274 & 0.2794 & 0.0481 & $17.2 \%$ \\
\hline SB6VS-07 & $\mathrm{Ce}_{2} \mathrm{O}_{3}$ & 0.0070 & 0.0270 & -0.0200 & $-74.0 \%$ \\
\hline SB6VS-07 & $\mathrm{Cr}_{2} \mathrm{O}_{3}$ & 0.0212 & 0.0156 & 0.0056 & $35.8 \%$ \\
\hline SB6VS-07 & $\mathrm{CuO}$ & 0.0119 & 0.0140 & -0.0021 & $-14.9 \%$ \\
\hline SB6VS-07 & $\mathrm{Fe}_{2} \mathrm{O}_{3}$ & 7.3058 & 7.8077 & -0.5019 & $-6.4 \%$ \\
\hline SB6VS-07 & $\mathrm{K}_{2} \mathrm{O}$ & 0.0485 & 0.0112 & 0.0373 & $332.6 \%$ \\
\hline SB6VS-07 & $\mathrm{La}_{2} \mathrm{O}_{3}$ & 0.0094 & 0.0138 & -0.0044 & $-32.1 \%$ \\
\hline SB6VS-07 & $\mathrm{Li}_{2} \mathrm{O}$ & 4.7525 & 5.1200 & -0.3675 & $-7.2 \%$ \\
\hline SB6VS-07 & $\mathrm{MgO}$ & 0.4950 & 0.4964 & -0.0014 & $-0.3 \%$ \\
\hline
\end{tabular}


SRNL-STI-2010-00242

Revision 0

Table A4. Average Measured Chemical Compositions Versus Targeted Compositions

\begin{tabular}{|c|c|c|c|c|c|}
\hline & & Measured & Targeted & Difference of & $\%$ Difference of \\
\hline Glass ID & Oxide & $(w t \%)$ & $(w t \%)$ & Measured vs Targeted (wt\%) & Measured vs Targeted \\
\hline SB6VS-07 & $\mathrm{MnO}$ & 2.2757 & 2.2489 & 0.0268 & $1.2 \%$ \\
\hline SB6VS-07 & $\mathrm{Na}_{2} \mathrm{O}$ & 16.1760 & 15.5010 & 0.6750 & $4.4 \%$ \\
\hline SB6VS-07 & $\mathrm{NiO}$ & 1.2165 & 1.2643 & -0.0478 & $-3.8 \%$ \\
\hline SB6VS-07 & $\mathrm{P}_{2} \mathrm{O}_{5}$ & 0.0699 & 0.0577 & 0.0122 & $21.1 \%$ \\
\hline SB6VS-07 & $\mathrm{PbO}$ & 0.0027 & 0.0037 & -0.0010 & $-27.1 \%$ \\
\hline SB6VS-07 & $\mathrm{SiO}_{2}$ & 48.2412 & 49.6271 & -1.3859 & $-2.8 \%$ \\
\hline SB6VS-07 & $\mathrm{SO}_{4}$ & 0.5572 & 0.3830 & 0.1742 & $45.5 \%$ \\
\hline SB6VS-07 & $\mathrm{TiO}_{2}$ & 0.0108 & 0.0000 & 0.0108 & \\
\hline SB6VS-07 & $\mathrm{U}_{3} \mathrm{O}_{8}$ & 1.4180 & 1.4216 & -0.0037 & $-0.3 \%$ \\
\hline SB6VS-07 & $\mathrm{ZnO}$ & 0.0140 & 0.0091 & 0.0049 & $53.8 \%$ \\
\hline SB6VS-07 & $\mathrm{ZrO}_{2}$ & 0.0297 & 0.0303 & -0.0006 & $-1.8 \%$ \\
\hline SB6VS-07 & Sum & 98.6187 & 100.0000 & -1.3813 & $-1.4 \%$ \\
\hline SB6VS-08 & $\mathrm{Al}_{2} \mathrm{O}_{3}$ & 8.2618 & 8.4575 & -0.1956 & $-2.3 \%$ \\
\hline SB6VS-08 & $\mathrm{B}_{2} \mathrm{O}_{3}$ & 4.5884 & 5.1200 & -0.5316 & $-10.4 \%$ \\
\hline SB6VS-08 & $\mathrm{BaO}$ & 0.0207 & 0.0174 & 0.0033 & $18.9 \%$ \\
\hline SB6VS-08 & $\mathrm{CaO}$ & 0.3267 & 0.2794 & 0.0474 & $17.0 \%$ \\
\hline SB6VS-08 & $\mathrm{Ce}_{2} \mathrm{O}_{3}$ & 0.0070 & 0.0270 & -0.0200 & $-74.0 \%$ \\
\hline SB6VS-08 & $\mathrm{Cr}_{2} \mathrm{O}_{3}$ & 0.0248 & 0.0156 & 0.0092 & $59.2 \%$ \\
\hline SB6VS-08 & $\mathrm{CuO}$ & 0.0110 & 0.0140 & -0.0030 & $-21.6 \%$ \\
\hline SB6VS-08 & $\mathrm{Fe}_{2} \mathrm{O}_{3}$ & 8.5746 & 9.6556 & -1.0809 & $-11.2 \%$ \\
\hline SB6VS-08 & $\mathrm{K}_{2} \mathrm{O}$ & 0.0503 & 0.0112 & 0.0391 & $348.7 \%$ \\
\hline SB6VS-08 & $\mathrm{La}_{2} \mathrm{O}_{3}$ & 0.0106 & 0.0138 & -0.0033 & $-23.6 \%$ \\
\hline SB6VS-08 & $\mathrm{Li}_{2} \mathrm{O}$ & 4.6556 & 5.1200 & -0.4644 & $-9.1 \%$ \\
\hline SB6VS-08 & $\mathrm{MgO}$ & 0.0717 & 0.0616 & 0.0102 & $16.5 \%$ \\
\hline SB6VS-08 & $\mathrm{MnO}$ & 2.3500 & 2.2489 & 0.1011 & $4.5 \%$ \\
\hline SB6VS-08 & $\mathrm{Na}_{2} \mathrm{O}$ & 16.8163 & 15.6759 & 1.1404 & $7.3 \%$ \\
\hline SB6VS-08 & $\mathrm{NiO}$ & 1.2770 & 1.2643 & 0.0126 & $1.0 \%$ \\
\hline SB6VS-08 & $\mathrm{P}_{2} \mathrm{O}_{5}$ & 0.0745 & 0.0577 & 0.0167 & $29.0 \%$ \\
\hline SB6VS-08 & $\mathrm{PbO}$ & 0.0027 & 0.0037 & -0.0010 & $-27.1 \%$ \\
\hline SB6VS-08 & $\mathrm{SiO}_{2}$ & 45.7810 & 49.2120 & -3.4310 & $-7.0 \%$ \\
\hline SB6VS-08 & $\mathrm{SO}_{4}$ & 0.8156 & 0.6228 & 0.1928 & $31.0 \%$ \\
\hline SB6VS-08 & $\mathrm{TiO}_{2}$ & 0.7047 & 0.6606 & 0.0441 & $6.7 \%$ \\
\hline SB6VS-08 & $\mathrm{U}_{3} \mathrm{O}_{8}$ & 1.4504 & 1.4216 & 0.0288 & $2.0 \%$ \\
\hline SB6VS-08 & $\mathrm{ZnO}$ & 0.0118 & 0.0091 & 0.0027 & $29.9 \%$ \\
\hline SB6VS-08 & $\mathrm{ZrO}_{2}$ & 0.0324 & 0.0303 & 0.0021 & $7.1 \%$ \\
\hline SB6VS-08 & Sum & 95.9203 & 100.0000 & -4.0797 & $-4.1 \%$ \\
\hline SB6VS-09 & $\mathrm{Al}_{2} \mathrm{O}_{3}$ & 8.5547 & 8.4575 & 0.0972 & $1.1 \%$ \\
\hline SB6VS-09 & $\mathrm{B}_{2} \mathrm{O}_{3}$ & 4.8540 & 5.1200 & -0.2660 & $-5.2 \%$ \\
\hline SB6VS-09 & $\mathrm{BaO}$ & 0.0553 & 0.0515 & 0.0038 & $7.3 \%$ \\
\hline SB6VS-09 & $\mathrm{CaO}$ & 0.8052 & 0.7294 & 0.0759 & $10.4 \%$ \\
\hline SB6VS-09 & $\mathrm{Ce}_{2} \mathrm{O}_{3}$ & 0.0498 & 0.0802 & -0.0304 & $-37.9 \%$ \\
\hline SB6VS-09 & $\mathrm{Cr}_{2} \mathrm{O}_{3}$ & 0.0508 & 0.0463 & 0.0045 & $9.7 \%$ \\
\hline SB6VS-09 & $\mathrm{CuO}$ & 0.0385 & 0.0414 & -0.0029 & $-7.1 \%$ \\
\hline SB6VS-09 & $\mathrm{Fe}_{2} \mathrm{O}_{3}$ & 8.8892 & 9.6556 & -0.7664 & $-7.9 \%$ \\
\hline SB6VS-09 & $\mathrm{K}_{2} \mathrm{O}$ & 0.0783 & 0.0332 & 0.0451 & $135.5 \%$ \\
\hline
\end{tabular}


SRNL-STI-2010-00242

Revision 0

Table A4. Average Measured Chemical Compositions Versus Targeted Compositions

\begin{tabular}{|c|c|c|c|c|c|}
\hline & & Measured & Targeted & Difference of & $\%$ Difference of \\
\hline Glass ID & Oxide & $(w t \%)$ & $(w t \%)$ & Measured vs Targeted (wt\%) & Measured vs Targeted \\
\hline SB6VS-09 & $\mathrm{La}_{2} \mathrm{O}_{3}$ & 0.0352 & 0.0410 & -0.0058 & $-14.1 \%$ \\
\hline SB6VS-09 & $\mathrm{Li}_{2} \mathrm{O}$ & 4.7633 & 5.1200 & -0.3567 & $-7.0 \%$ \\
\hline SB6VS-09 & $\mathrm{MgO}$ & 0.5394 & 0.4964 & 0.0429 & $8.6 \%$ \\
\hline SB6VS-09 & $\mathrm{MnO}$ & 2.8342 & 2.7288 & 0.1054 & $3.9 \%$ \\
\hline SB6VS-09 & $\mathrm{Na}_{2} \mathrm{O}$ & 15.0302 & 14.1639 & 0.8663 & $6.1 \%$ \\
\hline SB6VS-09 & $\mathrm{NiO}$ & 1.2897 & 1.2643 & 0.0254 & $2.0 \%$ \\
\hline SB6VS-09 & $\mathrm{P}_{2} \mathrm{O}_{5}$ & 0.1736 & 0.1712 & 0.0024 & $1.4 \%$ \\
\hline SB6VS-09 & $\mathrm{PbO}$ & 0.0110 & 0.0110 & 0.0001 & $0.8 \%$ \\
\hline SB6VS-09 & $\mathrm{SiO}_{2}$ & 48.0273 & 49.6271 & -1.5998 & $-3.2 \%$ \\
\hline SB6VS-09 & $\mathrm{SO}_{4}$ & 0.8344 & 0.6228 & 0.2116 & $34.0 \%$ \\
\hline SB6VS-09 & $\mathrm{TiO}_{2}$ & 0.0100 & 0.0000 & 0.0100 & \\
\hline SB6VS-09 & $\mathrm{U}_{3} \mathrm{O}_{8}$ & 1.4858 & 1.4216 & 0.0642 & $4.5 \%$ \\
\hline SB6VS-09 & $\mathrm{ZnO}$ & 0.0286 & 0.0270 & 0.0016 & $6.1 \%$ \\
\hline SB6VS-09 & $\mathrm{ZrO}_{2}$ & 0.0902 & 0.0898 & 0.0004 & $0.4 \%$ \\
\hline SB6VS-09 & Sum & 98.5291 & 100.0000 & -1.4709 & $-1.5 \%$ \\
\hline SB6VS-10 & $\mathrm{Al}_{2} \mathrm{O}_{3}$ & 8.5783 & 8.4575 & 0.1208 & $1.4 \%$ \\
\hline SB6VS-10 & $\mathrm{B}_{2} \mathrm{O}_{3}$ & 4.7574 & 5.1200 & -0.3626 & $-7.1 \%$ \\
\hline SB6VS-10 & $\mathrm{BaO}$ & 0.0226 & 0.0174 & 0.0052 & $30.2 \%$ \\
\hline SB6VS-10 & $\mathrm{CaO}$ & 0.3529 & 0.2794 & 0.0736 & $26.3 \%$ \\
\hline SB6VS-10 & $\mathrm{Ce}_{2} \mathrm{O}_{3}$ & 0.0070 & 0.0270 & -0.0200 & $-74.0 \%$ \\
\hline SB6VS-10 & $\mathrm{Cr}_{2} \mathrm{O}_{3}$ & 0.0263 & 0.0156 & 0.0107 & $68.5 \%$ \\
\hline SB6VS-10 & $\mathrm{CuO}$ & 0.0081 & 0.0140 & -0.0058 & $-41.7 \%$ \\
\hline SB6VS-10 & $\mathrm{Fe}_{2} \mathrm{O}_{3}$ & 8.9928 & 9.6556 & -0.6627 & $-6.9 \%$ \\
\hline SB6VS-10 & $\mathrm{K}_{2} \mathrm{O}$ & 0.0385 & 0.0112 & 0.0273 & $243.9 \%$ \\
\hline SB6VS-10 & $\mathrm{La}_{2} \mathrm{O}_{3}$ & 0.0117 & 0.0138 & -0.0021 & $-15.1 \%$ \\
\hline SB6VS-10 & $\mathrm{Li}_{2} \mathrm{O}$ & 4.7902 & 5.1200 & -0.3298 & $-6.4 \%$ \\
\hline SB6VS-10 & $\mathrm{MgO}$ & 0.5734 & 0.4964 & 0.0769 & $15.5 \%$ \\
\hline SB6VS-10 & $\mathrm{MnO}$ & 3.1828 & 2.7288 & 0.4540 & $16.6 \%$ \\
\hline SB6VS-10 & $\mathrm{Na}_{2} \mathrm{O}$ & 17.2544 & 14.3770 & 2.8774 & $20.0 \%$ \\
\hline SB6VS-10 & $\mathrm{NiO}$ & 0.9967 & 0.8608 & 0.1359 & $15.8 \%$ \\
\hline SB6VS-10 & $\mathrm{P}_{2} \mathrm{O}_{5}$ & 0.0945 & 0.0577 & 0.0368 & $63.8 \%$ \\
\hline SB6VS-10 & $\mathrm{PbO}$ & 0.0040 & 0.0037 & 0.0003 & $9.4 \%$ \\
\hline SB6VS-10 & $\mathrm{SiO}_{2}$ & 47.9738 & 49.6271 & -1.6533 & $-3.3 \%$ \\
\hline SB6VS-10 & $\mathrm{SO}_{4}$ & 0.5340 & 0.3830 & 0.1510 & $39.4 \%$ \\
\hline SB6VS-10 & $\mathrm{TiO}_{2}$ & 0.8165 & 0.6606 & 0.1559 & $23.6 \%$ \\
\hline SB6VS-10 & $\mathrm{U}_{3} \mathrm{O}_{8}$ & 2.2906 & 2.0340 & 0.2566 & $12.6 \%$ \\
\hline SB6VS-10 & $\mathrm{ZnO}$ & 0.0143 & 0.0091 & 0.0052 & $57.3 \%$ \\
\hline SB6VS-10 & $\mathrm{ZrO}_{2}$ & 0.0351 & 0.0303 & 0.0048 & $16.0 \%$ \\
\hline SB6VS-10 & Sum & 101.3568 & 100.0000 & 1.3568 & $1.4 \%$ \\
\hline SB6VS-11 & $\mathrm{Al}_{2} \mathrm{O}_{3}$ & 10.6001 & 10.5307 & 0.0694 & $0.7 \%$ \\
\hline SB6VS-11 & $\mathrm{B}_{2} \mathrm{O}_{3}$ & 4.7574 & 5.1200 & -0.3626 & $-7.1 \%$ \\
\hline SB6VS-11 & $\mathrm{BaO}$ & 0.0207 & 0.0174 & 0.0033 & $18.9 \%$ \\
\hline SB6VS-11 & $\mathrm{CaO}$ & 0.8227 & 0.7294 & 0.0934 & $12.8 \%$ \\
\hline SB6VS-11 & $\mathrm{Ce}_{2} \mathrm{O}_{3}$ & 0.0070 & 0.0270 & -0.0200 & $-74.0 \%$ \\
\hline SB6VS-11 & $\mathrm{Cr}_{2} \mathrm{O}_{3}$ & 0.0234 & 0.0156 & 0.0078 & $49.8 \%$ \\
\hline
\end{tabular}


SRNL-STI-2010-00242

Revision 0

Table A4. Average Measured Chemical Compositions Versus Targeted Compositions

\begin{tabular}{|c|c|c|c|c|c|}
\hline & & Measured & Targeted & Difference of & $\%$ Difference of \\
\hline Glass ID & Oxide & $(w t \%)$ & $(w t \%)$ & Measured vs Targeted (wt\%) & Measured vs Targeted \\
\hline SB6VS-11 & $\mathrm{CuO}$ & 0.0131 & 0.0140 & -0.0008 & $-5.9 \%$ \\
\hline SB6VS-11 & $\mathrm{Fe}_{2} \mathrm{O}_{3}$ & 6.1692 & 7.8077 & -1.6385 & $-21.0 \%$ \\
\hline SB6VS-11 & $\mathrm{K}_{2} \mathrm{O}$ & 0.0599 & 0.0112 & 0.0487 & $434.7 \%$ \\
\hline SB6VS-11 & $\mathrm{La}_{2} \mathrm{O}_{3}$ & 0.0123 & 0.0138 & -0.0015 & $-10.8 \%$ \\
\hline SB6VS-11 & $\mathrm{Li}_{2} \mathrm{O}$ & 4.7633 & 5.1200 & -0.3567 & $-7.0 \%$ \\
\hline SB6VS-11 & $\mathrm{MgO}$ & 0.5112 & 0.4964 & 0.0147 & $3.0 \%$ \\
\hline SB6VS-11 & $\mathrm{MnO}$ & 2.8213 & 2.7288 & 0.0925 & $3.4 \%$ \\
\hline SB6VS-11 & $\mathrm{Na}_{2} \mathrm{O}$ & 15.7379 & 14.4894 & 1.2485 & $8.6 \%$ \\
\hline SB6VS-11 & $\mathrm{NiO}$ & 0.9012 & 0.8608 & 0.0405 & $4.7 \%$ \\
\hline SB6VS-11 & $\mathrm{P}_{2} \mathrm{O}_{5}$ & 0.0733 & 0.0577 & 0.0156 & $27.0 \%$ \\
\hline SB6VS-11 & $\mathrm{PbO}$ & 0.0027 & 0.0037 & -0.0010 & $-27.1 \%$ \\
\hline SB6VS-11 & $\mathrm{SiO}_{2}$ & 47.5994 & 49.2120 & -1.6126 & $-3.3 \%$ \\
\hline SB6VS-11 & $\mathrm{SO}_{4}$ & 0.8418 & 0.6228 & 0.2190 & $35.2 \%$ \\
\hline SB6VS-11 & $\mathrm{TiO}_{2}$ & 0.7131 & 0.6606 & 0.0525 & $7.9 \%$ \\
\hline SB6VS-11 & $\mathrm{U}_{3} \mathrm{O}_{8}$ & 1.4858 & 1.4216 & 0.0642 & $4.5 \%$ \\
\hline SB6VS-11 & $\mathrm{ZnO}$ & 0.0121 & 0.0091 & 0.0030 & $33.3 \%$ \\
\hline SB6VS-11 & $\mathrm{ZrO}_{2}$ & 0.0328 & 0.0303 & 0.0025 & $8.2 \%$ \\
\hline SB6VS-11 & Sum & 97.9823 & 100.0000 & -2.0177 & $-2.0 \%$ \\
\hline SB6VS-12 & $\mathrm{Al}_{2} \mathrm{O}_{3}$ & 8.5122 & 8.5734 & -0.0612 & $-0.7 \%$ \\
\hline SB6VS-12 & $\mathrm{B}_{2} \mathrm{O}_{3}$ & 4.6850 & 5.1200 & -0.4350 & $-8.5 \%$ \\
\hline SB6VS-12 & $\mathrm{BaO}$ & 0.0533 & 0.0515 & 0.0018 & $3.5 \%$ \\
\hline SB6VS-12 & $\mathrm{CaO}$ & 0.7731 & 0.7294 & 0.0437 & $6.0 \%$ \\
\hline SB6VS-12 & $\mathrm{Ce}_{2} \mathrm{O}_{3}$ & 0.0448 & 0.0802 & -0.0354 & $-44.1 \%$ \\
\hline SB6VS-12 & $\mathrm{Cr}_{2} \mathrm{O}_{3}$ & 0.0486 & 0.0463 & 0.0023 & $5.0 \%$ \\
\hline SB6VS-12 & $\mathrm{CuO}$ & 0.0357 & 0.0414 & -0.0057 & $-13.9 \%$ \\
\hline SB6VS-12 & $\mathrm{Fe}_{2} \mathrm{O}_{3}$ & 7.1842 & 7.8077 & -0.6234 & $-8.0 \%$ \\
\hline SB6VS-12 & $\mathrm{K}_{2} \mathrm{O}$ & 0.0915 & 0.0332 & 0.0583 & $175.4 \%$ \\
\hline SB6VS-12 & $\mathrm{La}_{2} \mathrm{O}_{3}$ & 0.0361 & 0.0410 & -0.0049 & $-12.0 \%$ \\
\hline SB6VS-12 & $\mathrm{Li}_{2} \mathrm{O}$ & 4.7202 & 5.1200 & $\begin{array}{l}-0.3998 \\
\end{array}$ & $-7.8 \%$ \\
\hline SB6VS-12 & $\mathrm{MgO}$ & 0.4954 & 0.4964 & -0.0010 & $-0.2 \%$ \\
\hline SB6VS-12 & $\mathrm{MnO}$ & 2.2693 & 2.2489 & 0.0204 & $0.9 \%$ \\
\hline SB6VS-12 & $\mathrm{Na}_{2} \mathrm{O}$ & 16.2434 & 15.7576 & 0.4858 & $3.1 \%$ \\
\hline SB6VS-12 & $\mathrm{NiO}$ & 1.2455 & 1.2643 & -0.0189 & $-1.5 \%$ \\
\hline SB6VS-12 & $\mathrm{P}_{2} \mathrm{O}_{5}$ & 0.1879 & 0.1712 & 0.0167 & $9.8 \%$ \\
\hline SB6VS-12 & $\mathrm{PbO}$ & 0.0027 & 0.0110 & $\begin{array}{l}-0.0083 \\
\end{array}$ & $-75.4 \%$ \\
\hline SB6VS-12 & $\mathrm{SiO}_{2}$ & 46.9042 & 49.2120 & -2.3079 & $-4.7 \%$ \\
\hline SB6VS-12 & $\mathrm{SO}_{4}$ & 0.5842 & 0.3830 & 0.2012 & $52.5 \%$ \\
\hline SB6VS-12 & $\mathrm{TiO}_{2}$ & 0.6943 & 0.6606 & 0.0337 & $5.1 \%$ \\
\hline SB6VS-12 & $\mathrm{U}_{3} \mathrm{O}_{8}$ & 2.0341 & 2.0340 & 0.0001 & $0.0 \%$ \\
\hline SB6VS-12 & $\mathrm{ZnO}$ & 0.0293 & 0.0270 & 0.0023 & $8.4 \%$ \\
\hline SB6VS-12 & $\mathrm{ZrO}_{2}$ & 0.0905 & 0.0898 & 0.0007 & $0.8 \%$ \\
\hline SB6VS-12 & Sum & 96.9659 & 100.0000 & -3.0341 & $-3.0 \%$ \\
\hline SB6VS-13 & $\mathrm{Al}_{2} \mathrm{O}_{3}$ & 10.8930 & 8.4717 & 2.4213 & $28.6 \%$ \\
\hline SB6VS-13 & $\mathrm{B}_{2} \mathrm{O}_{3}$ & 4.8862 & 5.4400 & -0.5538 & $-10.2 \%$ \\
\hline SB6VS-13 & $\mathrm{BaO}$ & 0.0335 & 0.0304 & 0.0031 & $10.0 \%$ \\
\hline
\end{tabular}


SRNL-STI-2010-00242

Revision 0

Table A4. Average Measured Chemical Compositions Versus Targeted Compositions

\begin{tabular}{|c|c|c|c|c|c|}
\hline & & Measured & Targeted & Difference of & $\%$ Difference of \\
\hline Glass ID & Oxide & $(w t \%)$ & $(w t \%)$ & Measured vs Targeted (wt\%) & Measured vs Targeted \\
\hline SB6VS-13 & $\mathrm{CaO}$ & 0.5345 & 0.4481 & 0.0864 & $19.3 \%$ \\
\hline SB6VS-13 & $\mathrm{Ce}_{2} \mathrm{O}_{3}$ & 0.0193 & 0.0474 & -0.0281 & $-59.2 \%$ \\
\hline SB6VS-13 & $\mathrm{Cr}_{2} \mathrm{O}_{3}$ & 0.0362 & 0.0274 & 0.0088 & $32.3 \%$ \\
\hline SB6VS-13 & $\mathrm{CuO}$ & 0.0232 & 0.0245 & -0.0013 & $-5.4 \%$ \\
\hline SB6VS-13 & $\mathrm{Fe}_{2} \mathrm{O}_{3}$ & 7.4058 & 7.7897 & -0.3839 & $-4.9 \%$ \\
\hline SB6VS-13 & $\mathrm{K}_{2} \mathrm{O}$ & 0.0804 & 0.0196 & 0.0608 & $309.4 \%$ \\
\hline SB6VS-13 & $\mathrm{La}_{2} \mathrm{O}_{3}$ & 0.0208 & 0.0242 & -0.0034 & $-14.0 \%$ \\
\hline SB6VS-13 & $\mathrm{Li}_{2} \mathrm{O}$ & 4.8063 & 5.4400 & -0.6337 & $-11.6 \%$ \\
\hline SB6VS-13 & $\mathrm{MgO}$ & 0.2662 & 0.2470 & 0.0192 & $7.8 \%$ \\
\hline SB6VS-13 & $\mathrm{MnO}$ & 2.3145 & 2.2125 & 0.1020 & $4.6 \%$ \\
\hline SB6VS-13 & $\mathrm{Na}_{2} \mathrm{O}$ & 15.5694 & 14.0158 & 1.5536 & $11.1 \%$ \\
\hline SB6VS-13 & $\mathrm{NiO}$ & 0.9798 & 0.9424 & 0.0375 & $4.0 \%$ \\
\hline SB6VS-13 & $\mathrm{P}_{2} \mathrm{O}_{5}$ & 0.1232 & 0.1011 & 0.0220 & $21.8 \%$ \\
\hline SB6VS-13 & $\mathrm{PbO}$ & 0.0027 & 0.0065 & -0.0038 & $-58.4 \%$ \\
\hline SB6VS-13 & $\mathrm{SiO}_{2}$ & 48.8295 & 52.3718 & -3.5423 & $-6.8 \%$ \\
\hline SB6VS-13 & $\mathrm{SO}_{4}$ & 0.6456 & 0.4475 & 0.1981 & $44.3 \%$ \\
\hline SB6VS-13 & $\mathrm{TiO}_{2}$ & 0.3307 & 0.2918 & 0.0389 & $13.3 \%$ \\
\hline SB6VS-13 & $\mathrm{U}_{3} \mathrm{O}_{8}$ & 1.5683 & 1.5316 & 0.0367 & $2.4 \%$ \\
\hline SB6VS-13 & $\mathrm{ZnO}$ & 0.0227 & 0.0160 & 0.0068 & $42.4 \%$ \\
\hline SB6VS-13 & $\mathrm{ZrO}_{2}$ & 0.0547 & 0.0530 & 0.0017 & $3.1 \%$ \\
\hline SB6VS-13 & Sum & 99.4471 & 100.0000 & -0.5529 & $-0.6 \%$ \\
\hline SB6VS-14 & $\mathrm{Al}_{2} \mathrm{O}_{3}$ & 9.1216 & 9.0012 & 0.1204 & $1.3 \%$ \\
\hline SB6VS-14 & $\mathrm{B}_{2} \mathrm{O}_{3}$ & 4.8862 & 5.2800 & -0.3938 & $-7.5 \%$ \\
\hline SB6VS-14 & $\mathrm{BaO}$ & 0.0318 & 0.0323 & -0.0005 & $-1.6 \%$ \\
\hline SB6VS-14 & $\mathrm{CaO}$ & 0.4827 & 0.4761 & 0.0067 & $1.4 \%$ \\
\hline SB6VS-14 & $\mathrm{Ce}_{2} \mathrm{O}_{3}$ & 0.0170 & 0.0504 & -0.0334 & $-66.3 \%$ \\
\hline SB6VS-14 & $\mathrm{Cr}_{2} \mathrm{O}_{3}$ & 0.0358 & 0.0291 & 0.0067 & $23.2 \%$ \\
\hline SB6VS-14 & $\mathrm{CuO}$ & 0.0213 & 0.0260 & -0.0047 & $-18.2 \%$ \\
\hline SB6VS-14 & $\mathrm{Fe}_{2} \mathrm{O}_{3}$ & 7.4166 & 8.2766 & -0.8600 & $-10.4 \%$ \\
\hline SB6VS-14 & $\mathrm{K}_{2} \mathrm{O}$ & 0.0410 & 0.0209 & 0.0201 & $96.3 \%$ \\
\hline SB6VS-14 & $\mathrm{La}_{2} \mathrm{O}_{3}$ & 0.0205 & 0.0257 & -0.0052 & $-20.2 \%$ \\
\hline SB6VS-14 & $\mathrm{Li}_{2} \mathrm{O}$ & 4.9355 & 5.2800 & -0.3445 & $-6.5 \%$ \\
\hline SB6VS-14 & $\mathrm{MgO}$ & 0.2471 & 0.2624 & -0.0154 & $-5.8 \%$ \\
\hline SB6VS-14 & $\mathrm{MnO}$ & 2.2209 & 2.3508 & -0.1299 & $-5.5 \%$ \\
\hline SB6VS-14 & $\mathrm{Na}_{2} \mathrm{O}$ & 14.0192 & 14.3918 & -0.3726 & $-2.6 \%$ \\
\hline SB6VS-14 & $\mathrm{NiO}$ & 0.9047 & 1.0013 & -0.0965 & $-9.6 \%$ \\
\hline SB6VS-14 & $\mathrm{P}_{2} \mathrm{O}_{5}$ & 0.1134 & 0.1075 & 0.0060 & $5.5 \%$ \\
\hline SB6VS-14 & $\mathrm{PbO}$ & 0.0027 & 0.0069 & -0.0042 & $-60.8 \%$ \\
\hline SB6VS-14 & $\mathrm{SiO}_{2}$ & 48.9365 & 50.8951 & -1.9586 & $-3.8 \%$ \\
\hline SB6VS-14 & $\mathrm{SO}_{4}$ & 0.5857 & 0.4754 & 0.1103 & $23.2 \%$ \\
\hline SB6VS-14 & $\mathrm{TiO}_{2}$ & 0.3194 & 0.3101 & 0.0094 & $3.0 \%$ \\
\hline SB6VS-14 & $\mathrm{U}_{3} \mathrm{O}_{8}$ & 1.5389 & 1.6274 & -0.0885 & $-5.4 \%$ \\
\hline SB6VS-14 & $\mathrm{ZnO}$ & 0.0180 & 0.0169 & 0.0011 & $6.5 \%$ \\
\hline SB6VS-14 & $\mathrm{ZrO}_{2}$ & 0.0540 & 0.0564 & -0.0023 & $-4.1 \%$ \\
\hline SB6VS-14 & Sum & 95.9711 & 100.0000 & -4.0289 & $-4.0 \%$ \\
\hline
\end{tabular}


SRNL-STI-2010-00242

Revision 0

Table A4. Average Measured Chemical Compositions Versus Targeted Compositions

\begin{tabular}{|c|c|c|c|c|c|}
\hline & & Measured & Targeted & Difference of & $\%$ Difference of \\
\hline Glass ID & Oxide & $(w t \%)$ & $(w t \%)$ & Measured vs Targeted (wt\%) & Measured vs Targeted \\
\hline SB6VS-15 & $\mathrm{Al}_{2} \mathrm{O}_{3}$ & 9.5561 & 9.5307 & 0.0255 & $0.3 \%$ \\
\hline SB6VS-15 & $\mathrm{B}_{2} \mathrm{O}_{3}$ & 4.7816 & 5.1200 & -0.3384 & $-6.6 \%$ \\
\hline SB6VS-15 & $\mathrm{BaO}$ & 0.0410 & 0.0342 & 0.0068 & $19.8 \%$ \\
\hline SB6VS-15 & $\mathrm{CaO}$ & 0.6303 & 0.5041 & 0.1263 & $25.1 \%$ \\
\hline SB6VS-15 & $\mathrm{Ce}_{2} \mathrm{O}_{3}$ & 0.0214 & 0.0533 & -0.0319 & $-59.9 \%$ \\
\hline SB6VS-15 & $\mathrm{Cr}_{2} \mathrm{O}_{3}$ & 0.0460 & 0.0308 & 0.0153 & $49.6 \%$ \\
\hline SB6VS-15 & $\mathrm{CuO}$ & 0.0275 & 0.0275 & 0.0000 & $0.0 \%$ \\
\hline SB6VS-15 & $\mathrm{Fe}_{2} \mathrm{O}_{3}$ & 7.8919 & 8.7634 & -0.8715 & $-9.9 \%$ \\
\hline SB6VS-15 & $\mathrm{K}_{2} \mathrm{O}$ & 0.0566 & 0.0221 & 0.0345 & $156.2 \%$ \\
\hline SB6VS-15 & $\mathrm{La}_{2} \mathrm{O}_{3}$ & 0.0264 & 0.0272 & -0.0008 & $-3.1 \%$ \\
\hline SB6VS-15 & $\mathrm{Li}_{2} \mathrm{O}$ & 4.7041 & 5.1200 & -0.4159 & $-8.1 \%$ \\
\hline SB6VS-15 & $\mathrm{MgO}$ & 0.3246 & 0.2779 & 0.0467 & $16.8 \%$ \\
\hline SB6VS-15 & $\mathrm{MnO}$ & 2.9084 & 2.4890 & 0.4194 & $16.8 \%$ \\
\hline SB6VS-15 & $\mathrm{Na}_{2} \mathrm{O}$ & 17.7599 & 14.7678 & 2.9921 & $20.3 \%$ \\
\hline SB6VS-15 & $\mathrm{NiO}$ & 1.2070 & 1.0602 & 0.1468 & $13.8 \%$ \\
\hline SB6VS-15 & $\mathrm{P}_{2} \mathrm{O}_{5}$ & 0.1312 & 0.1138 & 0.0174 & $15.3 \%$ \\
\hline SB6VS-15 & $\mathrm{PbO}$ & 0.0036 & 0.0073 & -0.0036 & $-50.1 \%$ \\
\hline SB6VS-15 & $\mathrm{SiO}_{2}$ & 47.3855 & 49.4183 & -2.0328 & $-4.1 \%$ \\
\hline SB6VS-15 & $\mathrm{SO}_{4}$ & 0.7362 & 0.5034 & 0.2328 & $46.3 \%$ \\
\hline SB6VS-15 & $\mathrm{TiO}_{2}$ & 0.4087 & 0.3283 & 0.0804 & $24.5 \%$ \\
\hline SB6VS-15 & $\mathrm{U}_{3} \mathrm{O}_{8}$ & 1.9604 & 1.7231 & 0.2373 & $13.8 \%$ \\
\hline SB6VS-15 & $\mathrm{ZnO}$ & 0.0258 & 0.0179 & 0.0079 & $43.9 \%$ \\
\hline SB6VS-15 & $\mathrm{ZrO}_{2}$ & 0.0702 & 0.0597 & 0.0106 & $17.7 \%$ \\
\hline SB6VS-15 & Sum & 100.7052 & 100.0000 & 0.7052 & $0.7 \%$ \\
\hline SB6VS-16 & $\mathrm{Al}_{2} \mathrm{O}_{3}$ & 10.5198 & 10.0601 & 0.4596 & $4.6 \%$ \\
\hline SB6VS-16 & $\mathrm{B}_{2} \mathrm{O}_{3}$ & 4.7816 & 4.9600 & -0.1784 & $-3.6 \%$ \\
\hline SB6VS-16 & $\mathrm{BaO}$ & 0.0380 & 0.0361 & 0.0018 & $5.0 \%$ \\
\hline SB6VS-16 & $\mathrm{CaO}$ & 0.5919 & 0.5321 & 0.0598 & $11.2 \%$ \\
\hline SB6VS-16 & $\mathrm{Ce}_{2} \mathrm{O}_{3}$ & 0.0223 & 0.0563 & -0.0340 & $-60.5 \%$ \\
\hline SB6VS-16 & $\mathrm{Cr}_{2} \mathrm{O}_{3}$ & 0.0428 & 0.0325 & 0.0103 & $31.6 \%$ \\
\hline SB6VS-16 & $\mathrm{CuO}$ & 0.0269 & 0.0291 & -0.0021 & $-7.4 \%$ \\
\hline SB6VS-16 & $\mathrm{Fe}_{2} \mathrm{O}_{3}$ & 8.6640 & 9.2503 & -0.5863 & $-6.3 \%$ \\
\hline SB6VS-16 & $\mathrm{K}_{2} \mathrm{O}$ & 0.0783 & 0.0233 & 0.0550 & $235.7 \%$ \\
\hline SB6VS-16 & $\mathrm{La}_{2} \mathrm{O}_{3}$ & 0.0243 & 0.0287 & -0.0044 & $-15.3 \%$ \\
\hline SB6VS-16 & $\mathrm{Li}_{2} \mathrm{O}$ & 4.7741 & 4.9600 & -0.1859 & $-3.7 \%$ \\
\hline SB6VS-16 & $\mathrm{MgO}$ & 0.2956 & 0.2933 & 0.0023 & $0.8 \%$ \\
\hline SB6VS-16 & $\mathrm{MnO}$ & 2.7115 & 2.6273 & 0.0842 & $3.2 \%$ \\
\hline SB6VS-16 & $\mathrm{Na}_{2} \mathrm{O}$ & 15.8727 & 15.1438 & 0.7289 & $4.8 \%$ \\
\hline SB6VS-16 & $\mathrm{NiO}$ & 1.1449 & 1.1190 & 0.0259 & $2.3 \%$ \\
\hline SB6VS-16 & $\mathrm{P}_{2} \mathrm{O}_{5}$ & 0.1461 & 0.1201 & 0.0260 & $21.6 \%$ \\
\hline SB6VS-16 & $\mathrm{PbO}$ & 0.0070 & 0.0077 & -0.0007 & $-8.9 \%$ \\
\hline SB6VS-16 & $\mathrm{SiO}_{2}$ & 48.0273 & 47.9416 & 0.0857 & $0.2 \%$ \\
\hline SB6VS-16 & $\mathrm{SO}_{4}$ & 0.7984 & 0.5314 & 0.2670 & $50.3 \%$ \\
\hline SB6VS-16 & $\mathrm{TiO}_{2}$ & 0.3820 & 0.3465 & 0.0354 & $10.2 \%$ \\
\hline SB6VS-16 & $\mathrm{U}_{3} \mathrm{O}_{8}$ & 1.9339 & 1.8188 & 0.1151 & $6.3 \%$ \\
\hline
\end{tabular}


SRNL-STI-2010-00242

Revision 0

Table A4. Average Measured Chemical Compositions Versus Targeted Compositions

\begin{tabular}{|c|c|c|c|c|c|}
\hline & & Measured & Targeted & Difference of & $\%$ Difference of \\
\hline Glass ID & Oxide & $(w t \%)$ & $(w t \%)$ & Measured vs Targeted (wt\%) & Measured vs Targeted \\
\hline SB6VS-16 & $\mathrm{ZnO}$ & 0.0246 & 0.0189 & 0.0056 & $29.8 \%$ \\
\hline SB6VS-16 & $\mathrm{ZrO}_{2}$ & 0.0621 & 0.0630 & -0.0009 & $-1.4 \%$ \\
\hline SB6VS-16 & Sum & 100.9704 & 100.0000 & 0.9704 & $1.0 \%$ \\
\hline SB6VS-17 & $\mathrm{Al}_{2} \mathrm{O}_{3}$ & 10.8032 & 10.5896 & 0.2136 & $2.0 \%$ \\
\hline SB6VS-17 & $\mathrm{B}_{2} \mathrm{O}_{3}$ & 4.4274 & 4.8000 & -0.3726 & $-7.8 \%$ \\
\hline SB6VS-17 & $\mathrm{BaO}$ & 0.0408 & 0.0380 & 0.0027 & $7.1 \%$ \\
\hline SB6VS-17 & $\mathrm{CaO}$ & 0.6233 & 0.5601 & 0.0633 & $11.3 \%$ \\
\hline SB6VS-17 & $\mathrm{Ce}_{2} \mathrm{O}_{3}$ & 0.0264 & 0.0592 & -0.0329 & $-55.5 \%$ \\
\hline SB6VS-17 & $\mathrm{Cr}_{2} \mathrm{O}_{3}$ & 0.0413 & 0.0342 & 0.0071 & $20.8 \%$ \\
\hline SB6VS-17 & $\mathrm{CuO}$ & 0.0263 & 0.0306 & -0.0043 & $-14.1 \%$ \\
\hline SB6VS-17 & $\mathrm{Fe}_{2} \mathrm{O}_{3}$ & 8.8963 & 9.7371 & -0.8408 & $-8.6 \%$ \\
\hline SB6VS-17 & $\mathrm{K}_{2} \mathrm{O}$ & 0.0678 & 0.0246 & 0.0432 & $176.0 \%$ \\
\hline SB6VS-17 & $\mathrm{La}_{2} \mathrm{O}_{3}$ & 0.0258 & 0.0303 & -0.0045 & $-14.7 \%$ \\
\hline SB6VS-17 & $\mathrm{Li}_{2} \mathrm{O}$ & 4.5103 & 4.8000 & -0.2897 & $-6.0 \%$ \\
\hline SB6VS-17 & $\mathrm{MgO}$ & 0.3134 & 0.3087 & 0.0047 & $1.5 \%$ \\
\hline SB6VS-17 & $\mathrm{MnO}$ & 2.8180 & 2.7656 & 0.0524 & $1.9 \%$ \\
\hline SB6VS-17 & $\mathrm{Na}_{2} \mathrm{O}$ & 16.3108 & 15.5198 & 0.7910 & $5.1 \%$ \\
\hline SB6VS-17 & $\mathrm{NiO}$ & 1.1844 & 1.1779 & 0.0064 & $0.5 \%$ \\
\hline SB6VS-17 & $\mathrm{P}_{2} \mathrm{O}_{5}$ & 0.1455 & 0.1264 & 0.0191 & $15.1 \%$ \\
\hline SB6VS-17 & $\mathrm{PbO}$ & 0.0027 & 0.0081 & -0.0054 & $-66.7 \%$ \\
\hline SB6VS-17 & $\mathrm{SiO}_{2}$ & 44.9788 & 46.4648 & -1.4860 & $-3.2 \%$ \\
\hline SB6VS-17 & $\mathrm{SO}_{4}$ & 0.7475 & 0.5593 & 0.1881 & $33.6 \%$ \\
\hline SB6VS-17 & $\mathrm{TiO}_{2}$ & 0.4007 & 0.3648 & 0.0360 & $9.9 \%$ \\
\hline SB6VS-17 & $\mathrm{U}_{3} \mathrm{O}_{8}$ & 1.9516 & 1.9145 & 0.0370 & $1.9 \%$ \\
\hline SB6VS-17 & $\mathrm{ZnO}$ & 0.0237 & 0.0199 & 0.0037 & $18.6 \%$ \\
\hline SB6VS-17 & $\mathrm{ZrO}_{2}$ & 0.0648 & 0.0663 & -0.0015 & $-2.2 \%$ \\
\hline SB6VS-17 & Sum & 98.4313 & 100.0000 & -1.5687 & $-1.6 \%$ \\
\hline Ustd & $\mathrm{Al}_{2} \mathrm{O}_{3}$ & 4.0829 & 4.1000 & -0.0171 & $-0.4 \%$ \\
\hline Ustd & $\mathrm{B}_{2} \mathrm{O}_{3}$ & 8.8708 & 9.2090 & -0.3382 & $-3.7 \%$ \\
\hline Ustd & $\mathrm{BaO}$ & 0.0017 & 0.0000 & 0.0017 & \\
\hline Ustd & $\mathrm{CaO}$ & 1.3425 & 1.3010 & 0.0415 & $3.2 \%$ \\
\hline Ustd & $\mathrm{Ce}_{2} \mathrm{O}_{3}$ & 0.0070 & 0.0000 & 0.0070 & \\
\hline Ustd & $\mathrm{Cr}_{2} \mathrm{O}_{3}$ & 0.2542 & 0.0000 & 0.2542 & \\
\hline Ustd & $\mathrm{CuO}$ & 0.0019 & 0.0000 & 0.0019 & \\
\hline Ustd & $\mathrm{Fe}_{2} \mathrm{O}_{3}$ & 13.1973 & 13.1960 & 0.0013 & $0.0 \%$ \\
\hline Ustd & $\mathrm{K}_{2} \mathrm{O}$ & 3.0225 & 2.9990 & 0.0235 & $0.8 \%$ \\
\hline Ustd & $\mathrm{La}_{2} \mathrm{O}_{3}$ & 0.0029 & 0.0000 & 0.0029 & \\
\hline Ustd & $\mathrm{Li}_{2} \mathrm{O}$ & 2.9566 & 3.0570 & -0.1004 & $-3.3 \%$ \\
\hline Ustd & $\mathrm{MgO}$ & 1.1909 & 1.2100 & -0.0191 & $-1.6 \%$ \\
\hline Ustd & $\mathrm{MnO}$ & 2.7998 & 2.8920 & -0.0922 & $-3.2 \%$ \\
\hline Ustd & $\mathrm{Na}_{2} \mathrm{O}$ & 12.3499 & 11.7950 & 0.5549 & $4.7 \%$ \\
\hline Ustd & $\mathrm{NiO}$ & 1.1003 & 1.1200 & -0.0197 & $-1.8 \%$ \\
\hline Ustd & $\mathrm{P}_{2} \mathrm{O}_{5}$ & 0.0286 & 0.0000 & 0.0286 & \\
\hline Ustd & $\mathrm{PbO}$ & 0.0027 & 0.0000 & 0.0027 & \\
\hline Ustd & $\mathrm{SiO}_{2}$ & 45.3888 & 45.3530 & 0.0358 & $0.1 \%$ \\
\hline
\end{tabular}


SRNL-STI-2010-00242

Revision 0

Table A4. Average Measured Chemical Compositions Versus Targeted Compositions

\begin{tabular}{|c|c|c|c|c|c|}
\hline & & Measured & Targeted & Difference of & \% Difference of \\
\hline Glass ID & Oxide & $\mathbf{( w t \% )}$ & $\mathbf{( w t \% )}$ & Measured vs Targeted (wt\%) & Measured vs Targeted \\
\hline Ustd & $\mathrm{SO}_{4}$ & 0.1648 & 0.0000 & 0.1648 & $-4.2 \%$ \\
\hline Ustd & $\mathrm{TiO}_{2}$ & 1.0052 & 1.0490 & -0.0438 & $-0.1 \%$ \\
\hline Ustd & $\mathrm{U}_{3} \mathrm{O}_{8}$ & 2.4036 & 2.4060 & -0.0024 & \\
\hline Ustd & $\mathrm{ZnO}$ & 0.0031 & 0.0000 & 0.0031 & $0.5 \%$ \\
\hline Ustd & $\mathrm{ZrO}_{2}$ & 0.0014 & 0.0000 & 0.0014 & 0.4932 \\
\hline Ustd & $\mathrm{Sum}$ & 100.1802 & 99.6870 & & \\
\hline
\end{tabular}


Exhibit A1. Measurements of Initial Glasses in Analytical Sequence for Samples by Prep Method by Oxide

LM Prep Method - Variability Chart for BaO (wt\%)

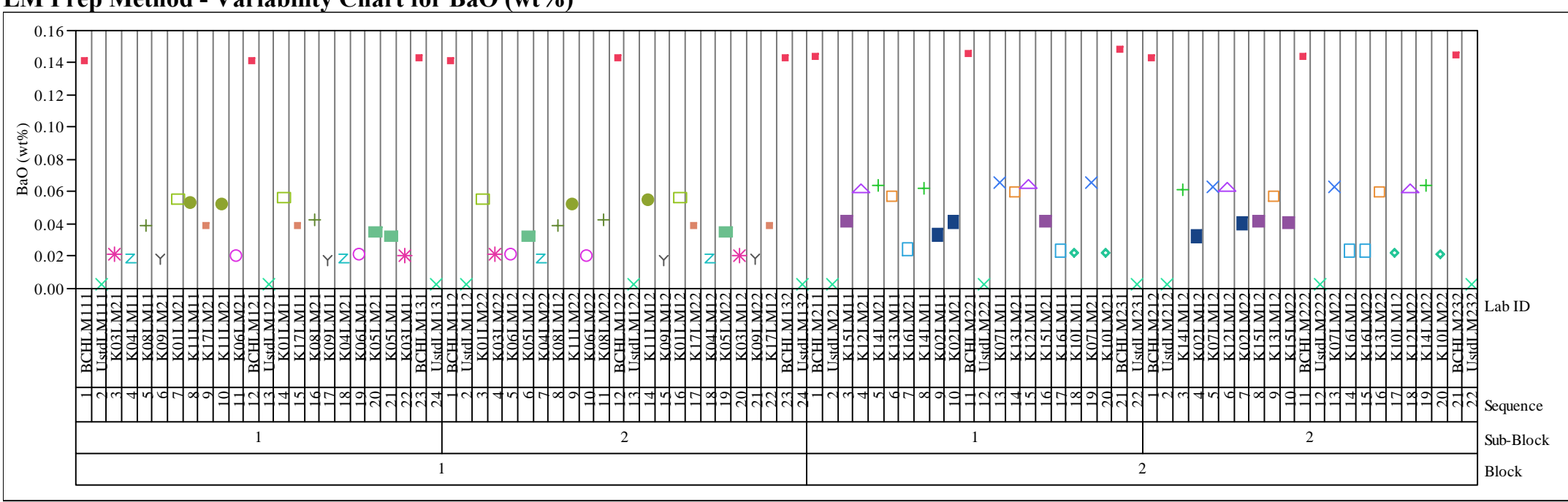

\section{LM Prep Method - Variability Chart for $\mathrm{CaO}(\mathrm{wt} \%)$}

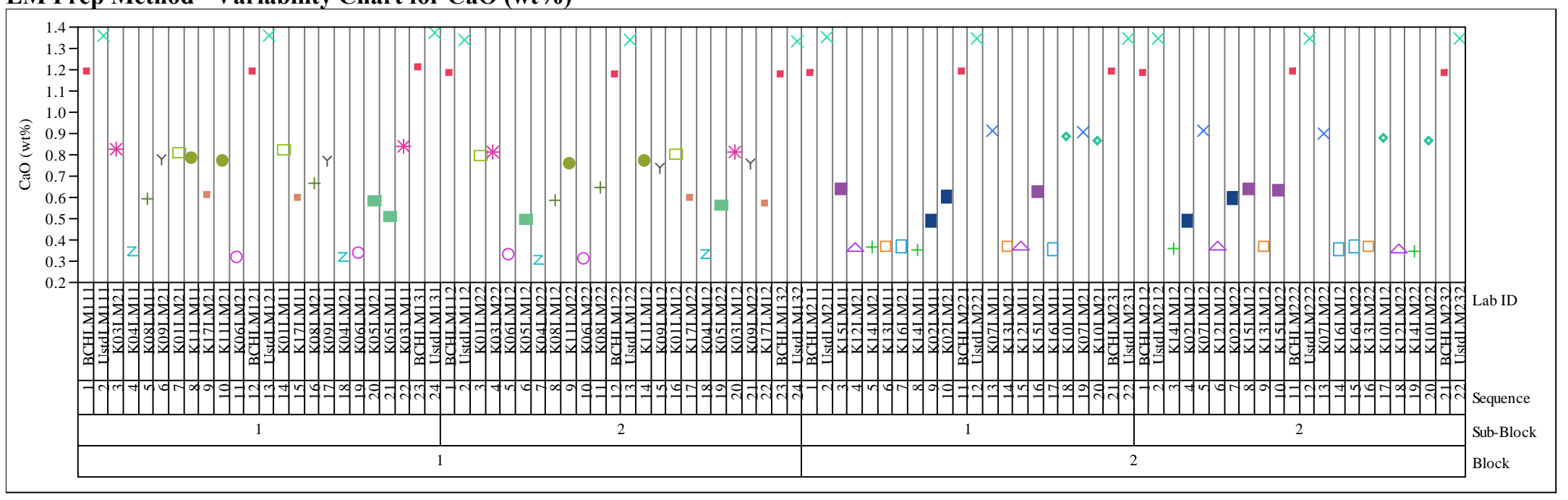


Exhibit A1. Measurements of Initial Glasses in Analytical Sequence for Samples by Prep Method by Oxide

LM Prep Method - Variability Chart for CdO (wt\%)

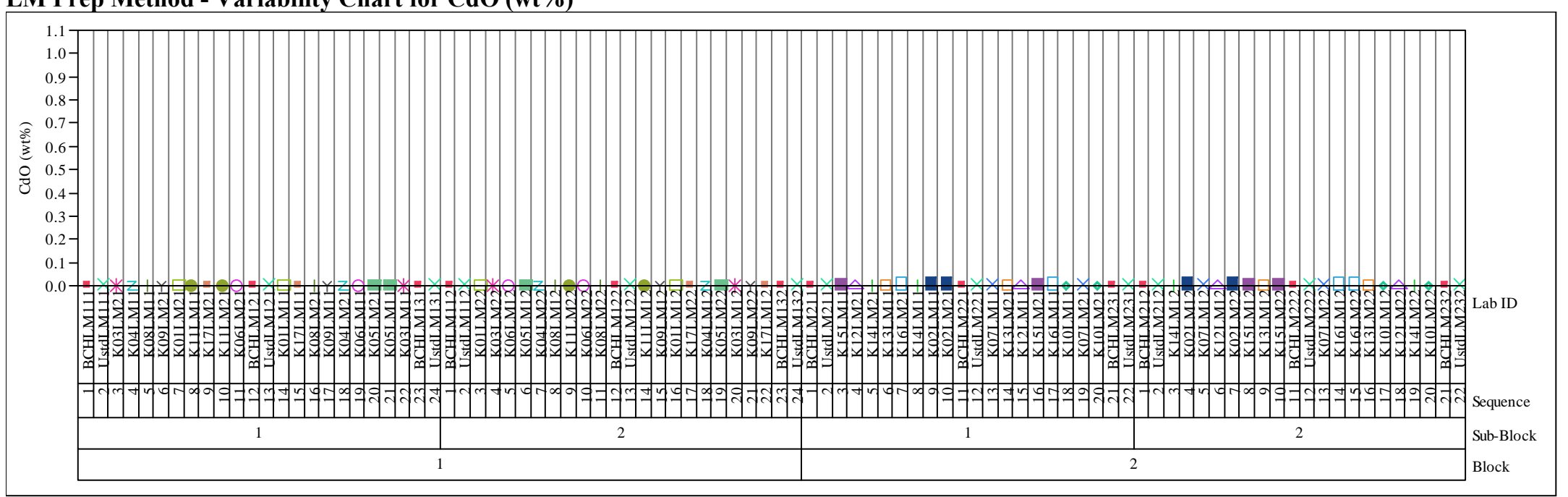

LM Prep Method - Variability Chart for Ce2O3 (wt\%)

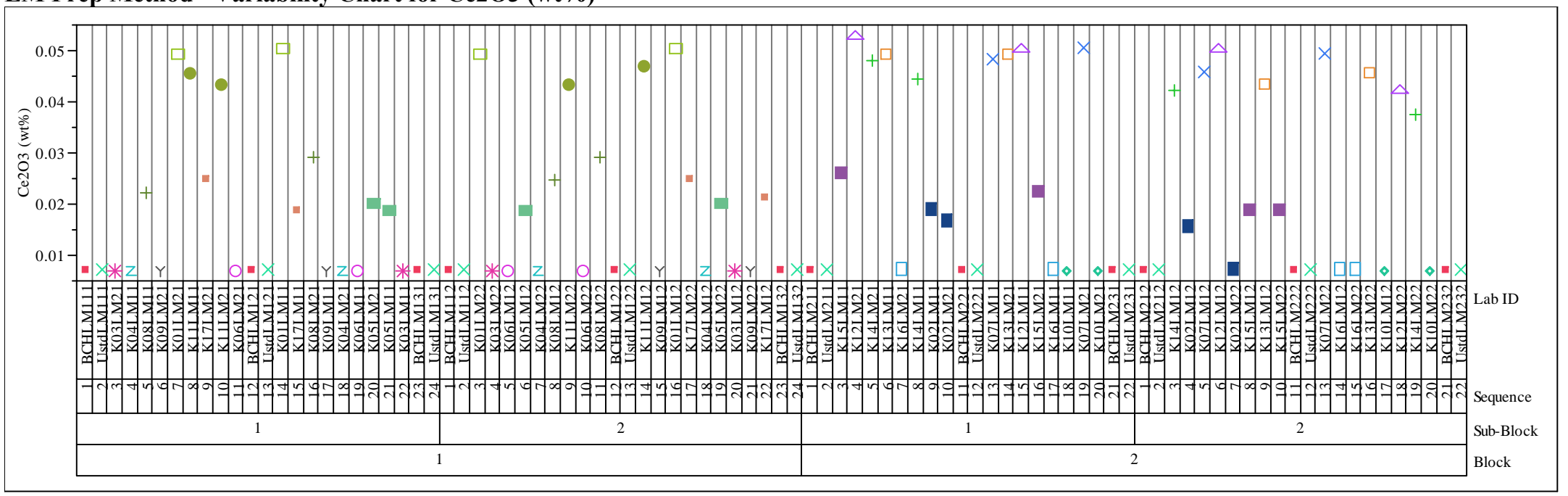


Exhibit A1. Measurements of Initial Glasses in Analytical Sequence for Samples by Prep Method by Oxide

LM Prep Method - Variability Chart for Cr2O3 (wt\%)

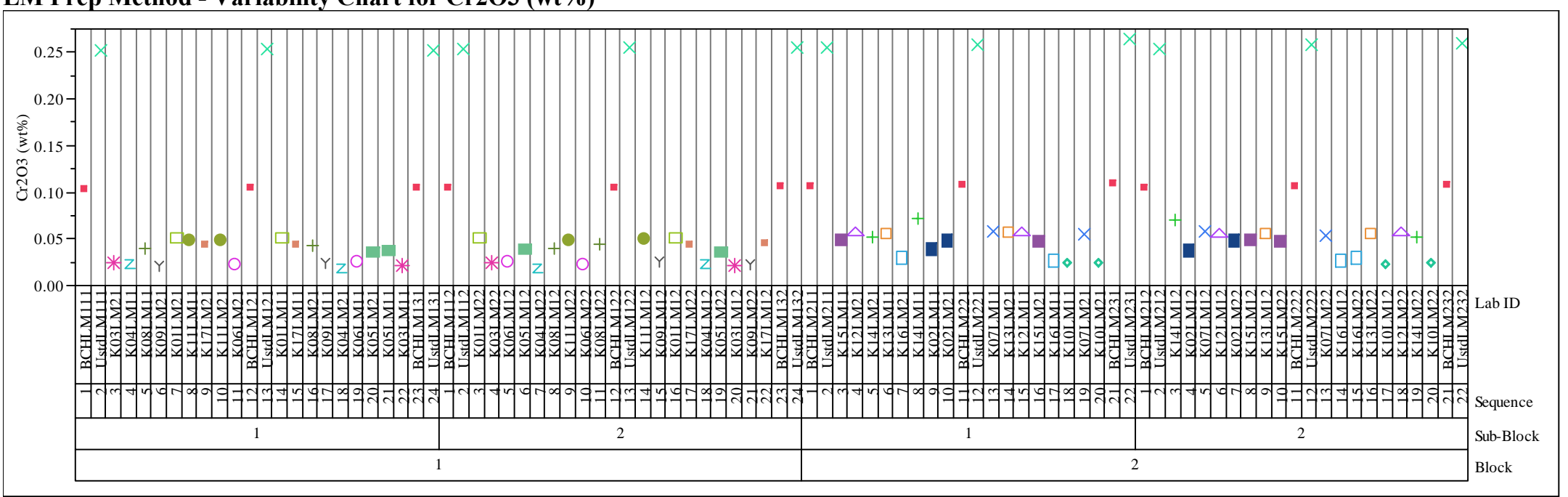

LM Prep Method - Variability Chart for $\mathrm{CuO}(\mathrm{wt} \%)$

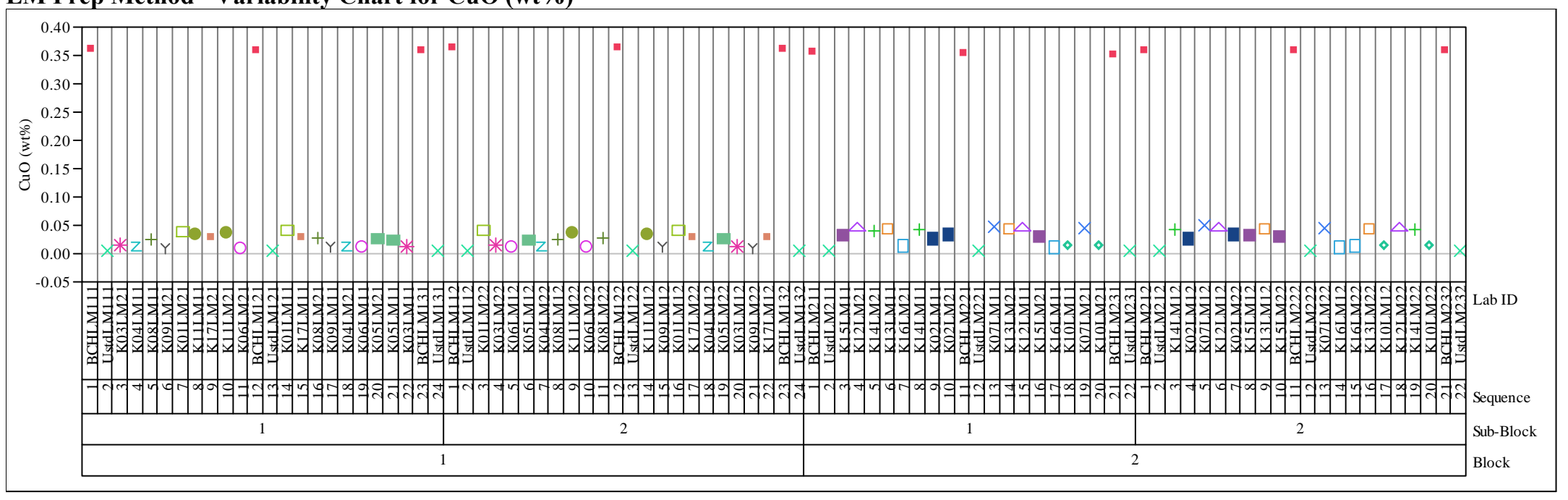




\section{Exhibit A1. Measurements of Initial Glasses in Analytical Sequence for Samples by Prep Method by Oxide}

\section{LM Prep Method - Variability Chart for K2O (wt\%)}

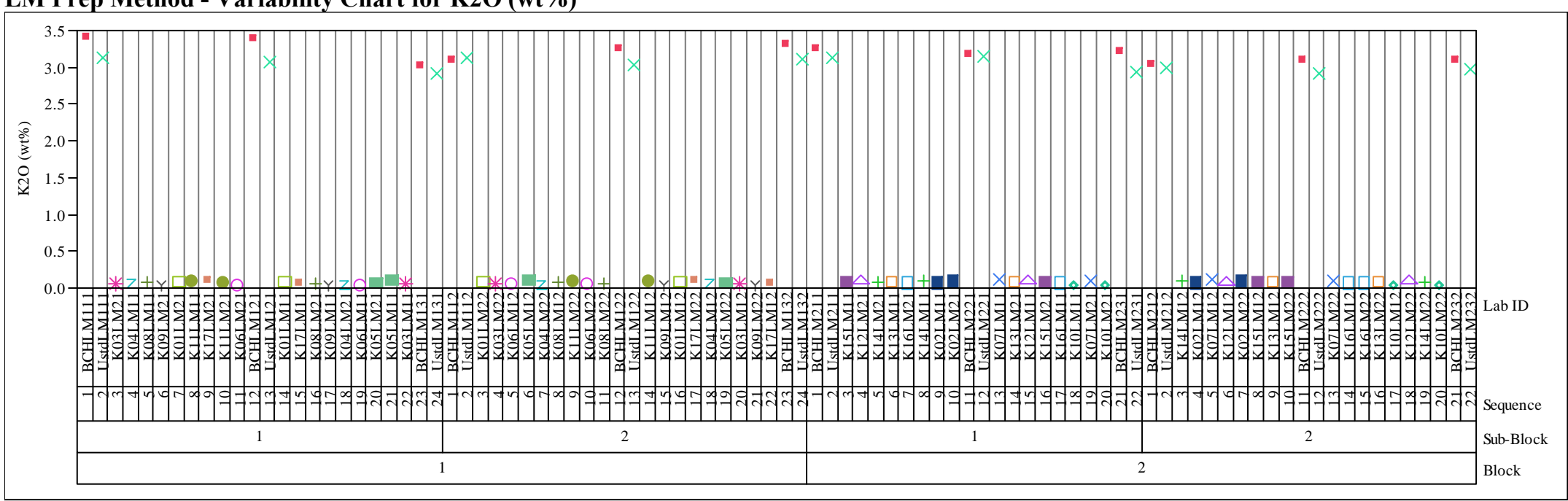

\section{LM Prep Method - Variability Chart for La2O3 (wt\%)}

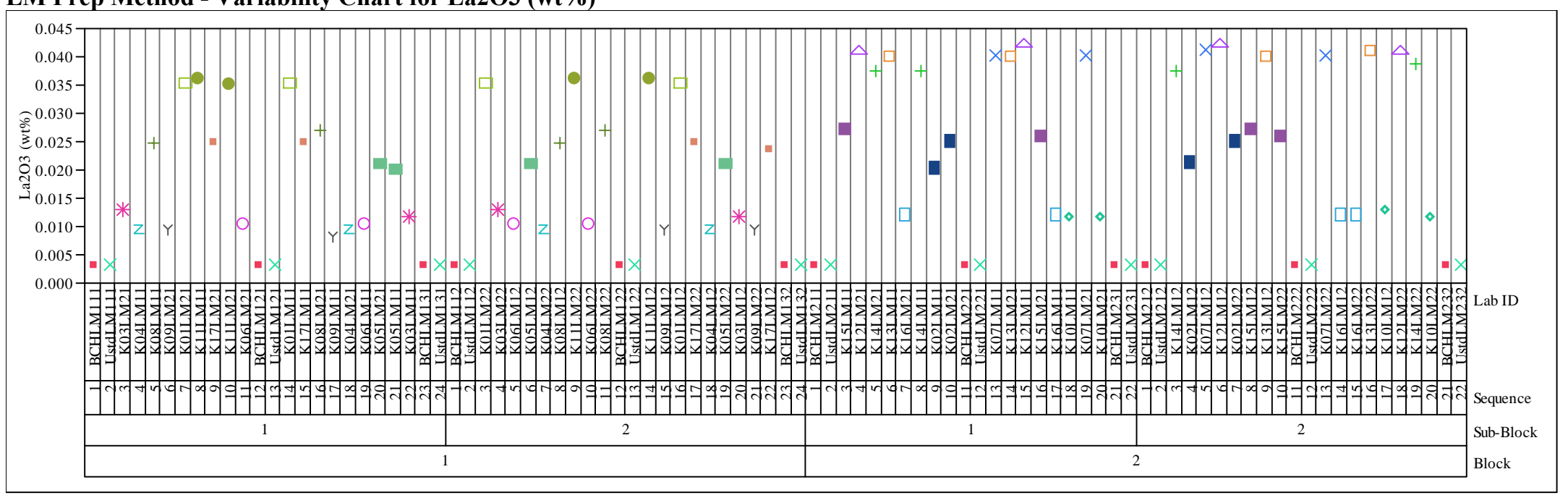


Exhibit A1. Measurements of Initial Glasses in Analytical Sequence for Samples by Prep Method by Oxide

LM Prep Method - Variability Chart for MgO (wt\%)

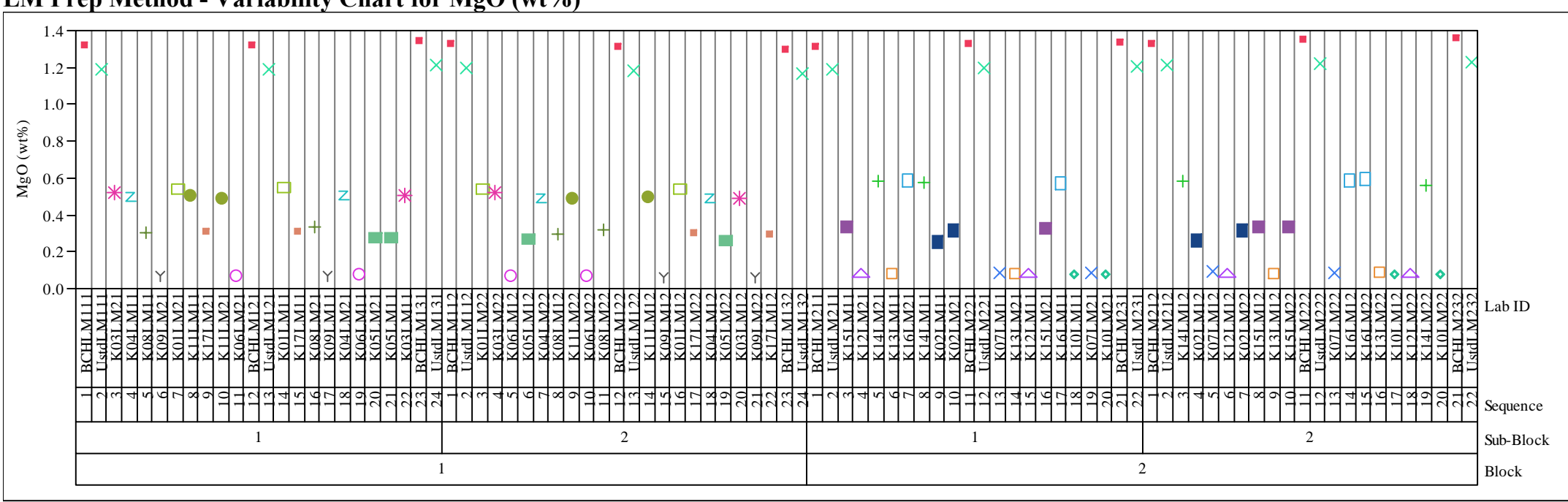

LM Prep Method - Variability Chart for MnO (wt\%)

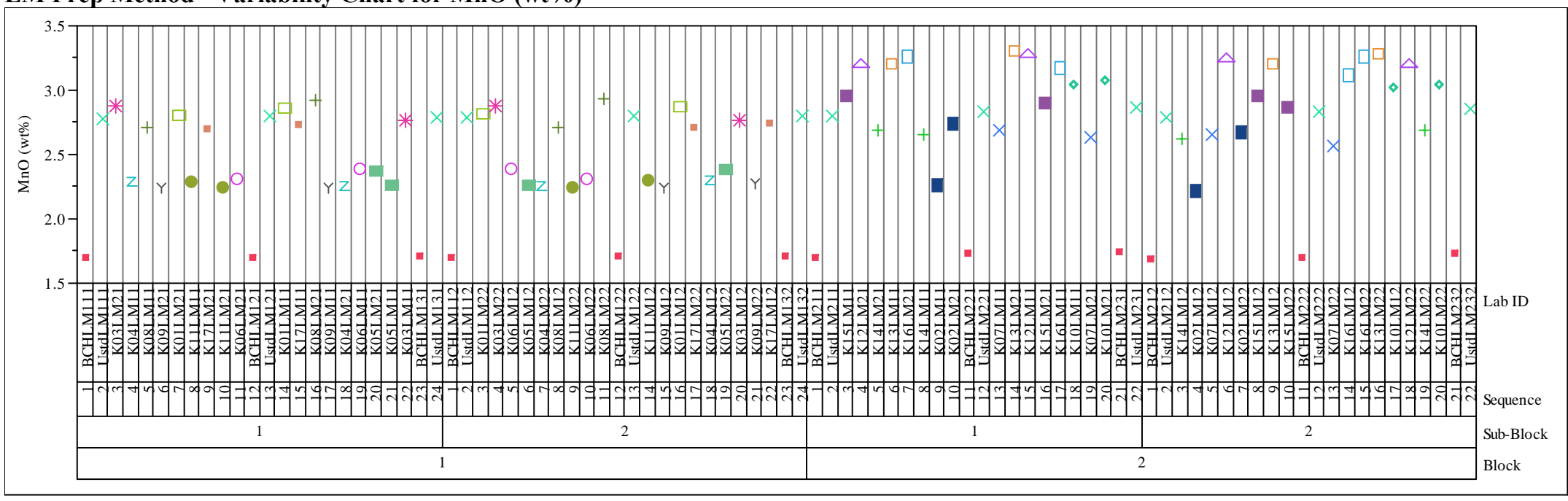




\section{Exhibit A1. Measurements of Initial Glasses in Analytical Sequence for Samples by Prep Method by Oxide}

\section{LM Prep Method - Variability Chart for Na2O (wt\%)}

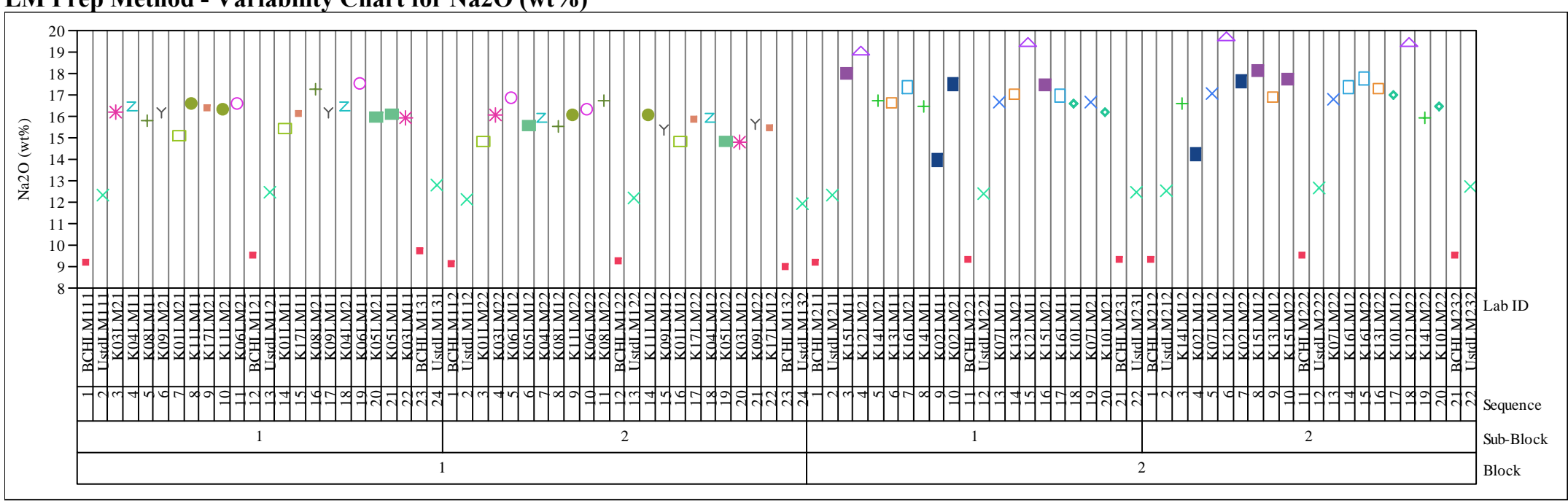

\section{LM Prep Method - Variability Chart for NiO (wt\%)}

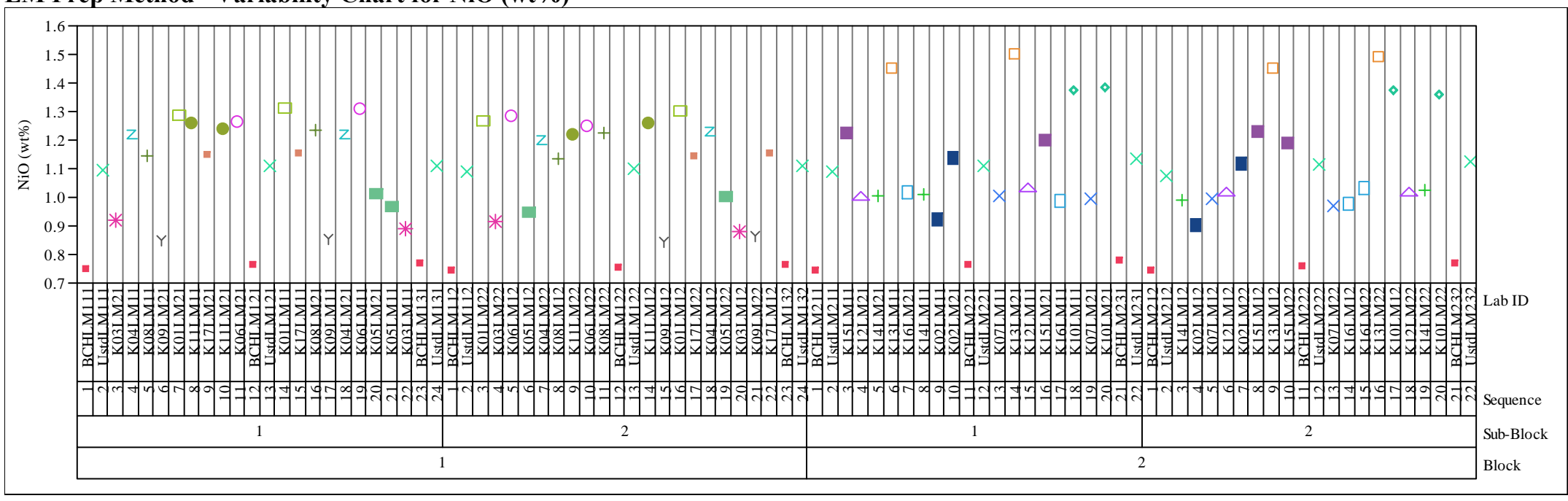




\section{Exhibit A1. Measurements of Initial Glasses in Analytical Sequence for Samples by Prep Method by Oxide}

\section{LM Prep Method - Variability Chart for P2O5 (wt\%)}

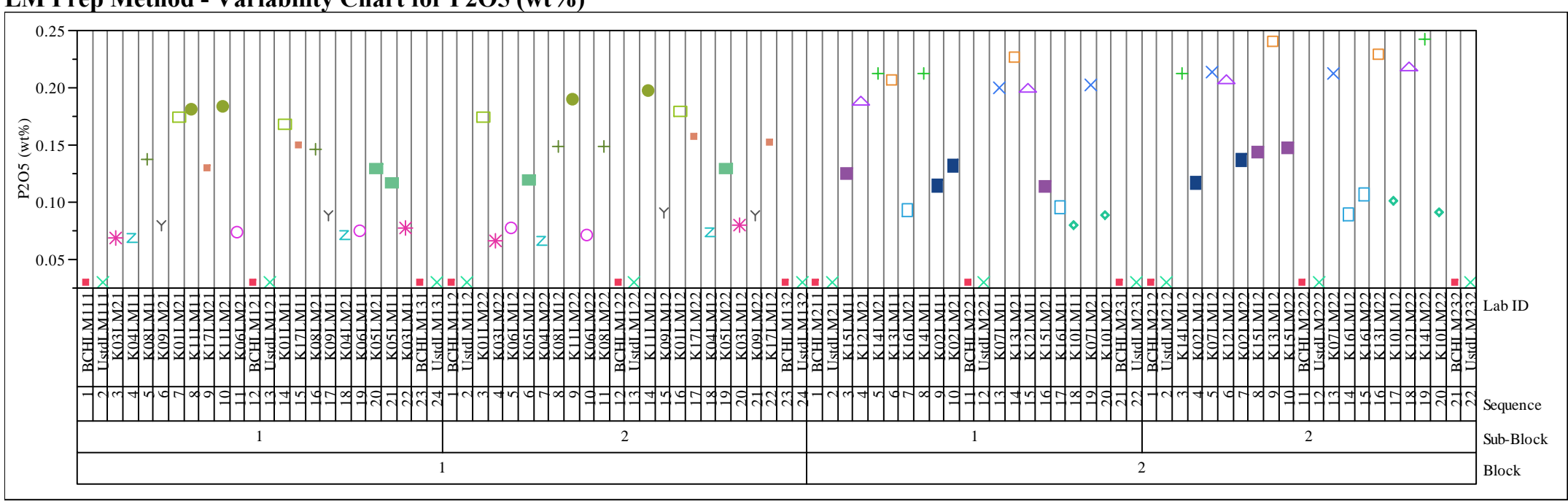

\section{LM Prep Method - Variability Chart for PbO (wt\%)}

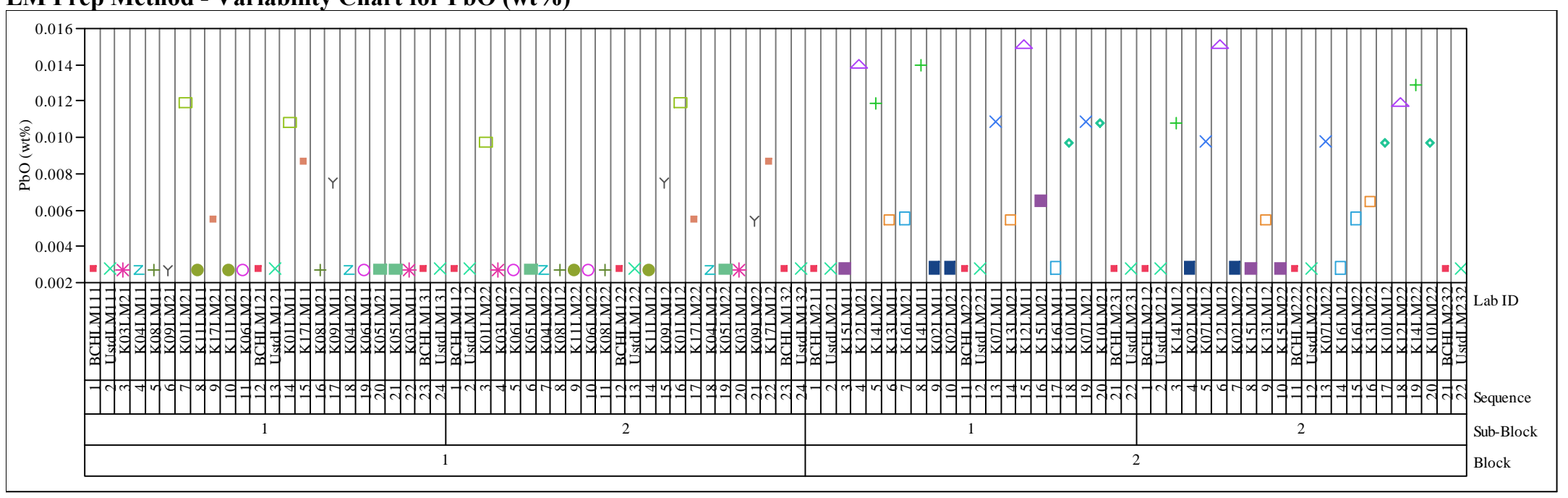




\section{Exhibit A1. Measurements of Initial Glasses in Analytical Sequence for Samples by Prep Method by Oxide}

\section{LM Prep Method - Variability Chart for SO4 (wt\%)}

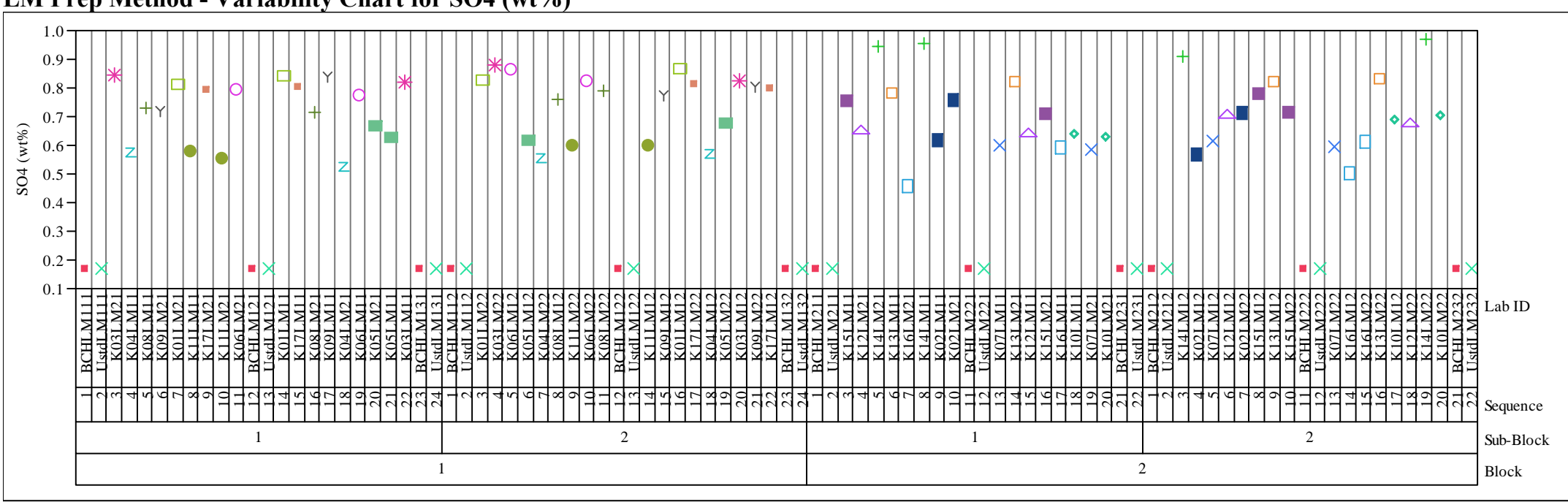

LM Prep Method - Variability Chart for TiO2 (wt\%)

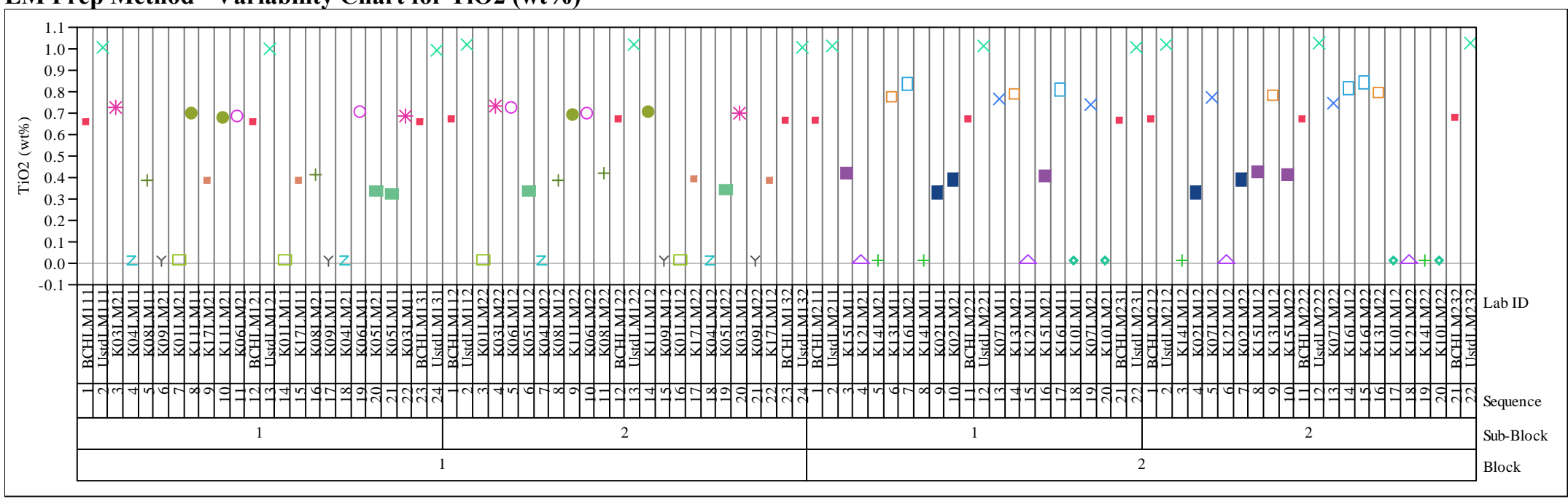


Exhibit A1. Measurements of Initial Glasses in Analytical Sequence for Samples by Prep Method by Oxide

LM Prep Method - Variability Chart for U3O8 (wt\%)

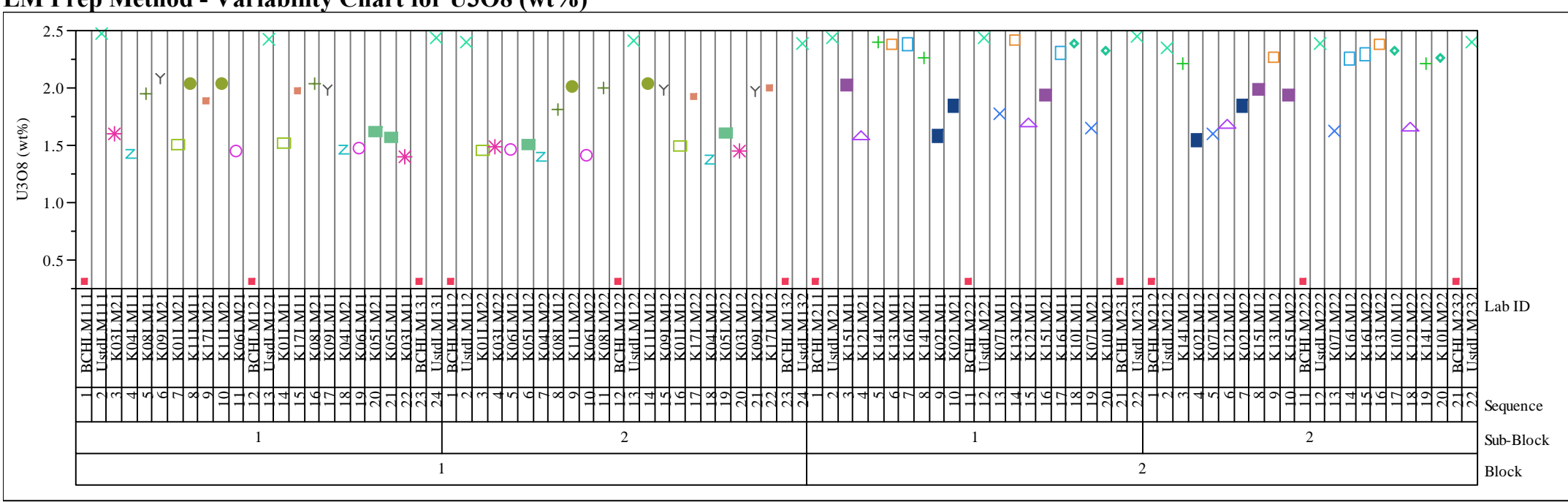

LM Prep Method - Variability Chart for ZnO (wt\%)

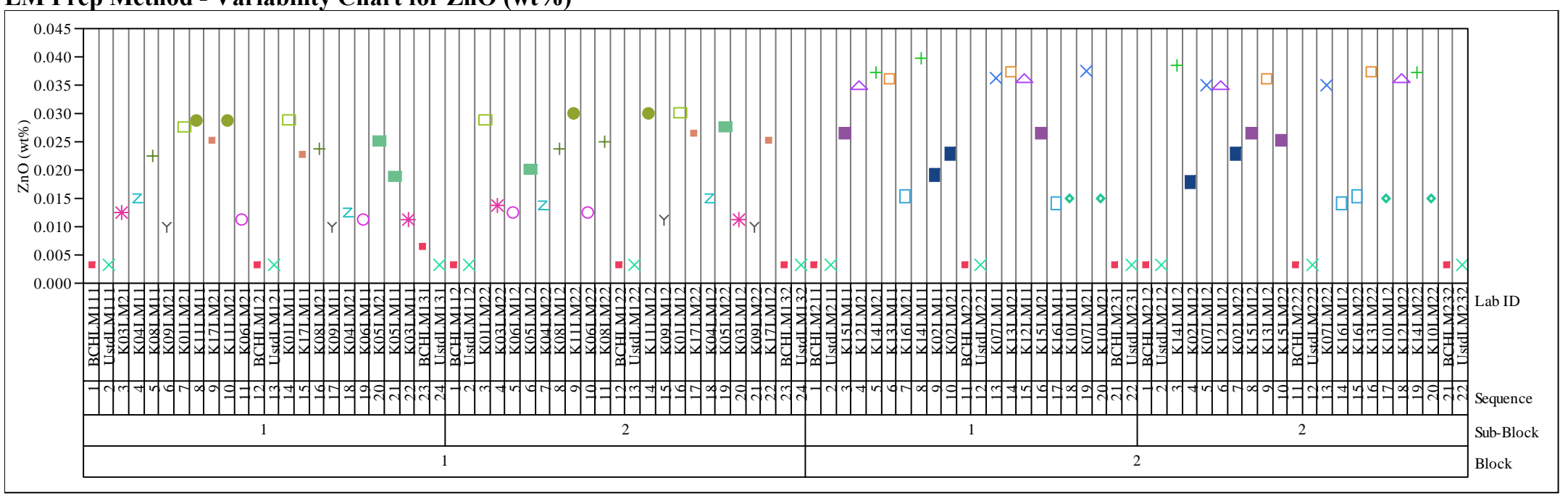




\section{Exhibit A1. Measurements of Initial Glasses in Analytical Sequence for Samples by Prep Method by Oxide}

\section{LM Prep Method - Variability Chart for ZrO2 (wt\%)}

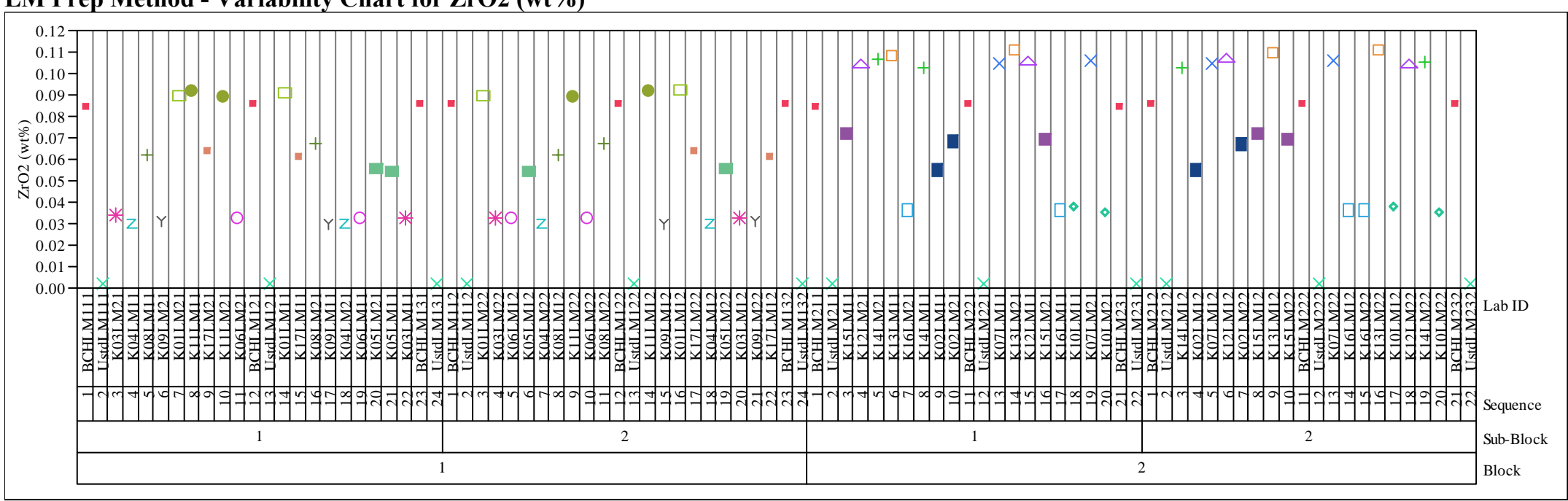

\section{PF Prep Method - Variability Chart for Al2O3 (wt\%)}

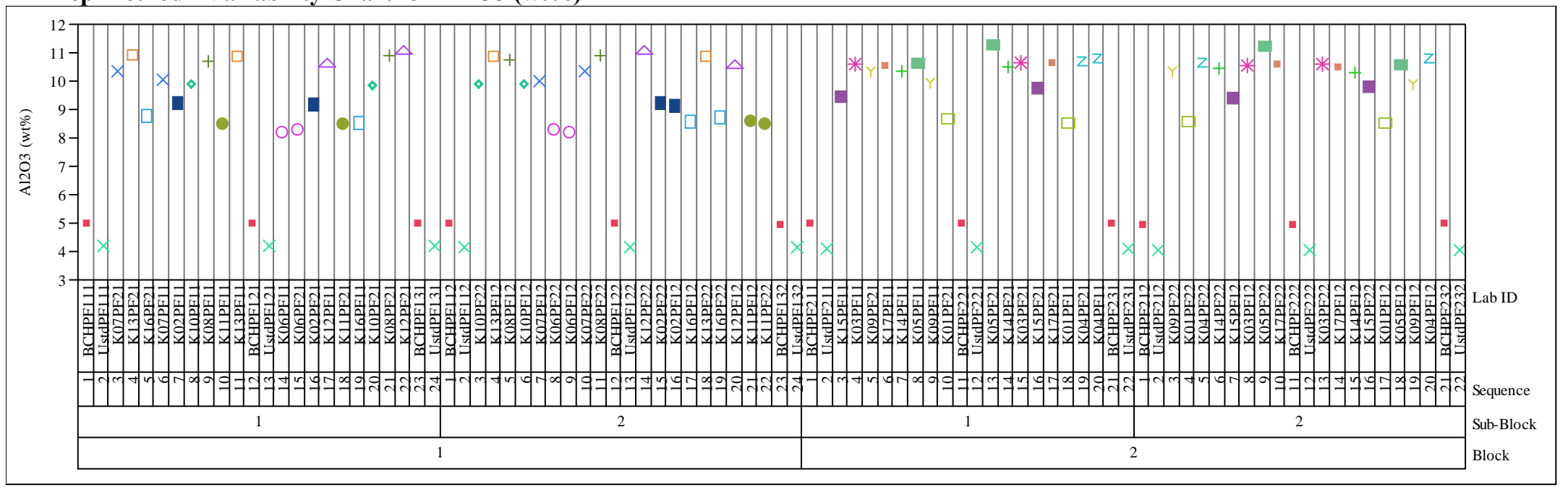




\section{Exhibit A1. Measurements of Initial Glasses in Analytical Sequence for Samples by Prep Method by Oxide}

PF Prep Method - Variability Chart for B2O3 (wt\%)

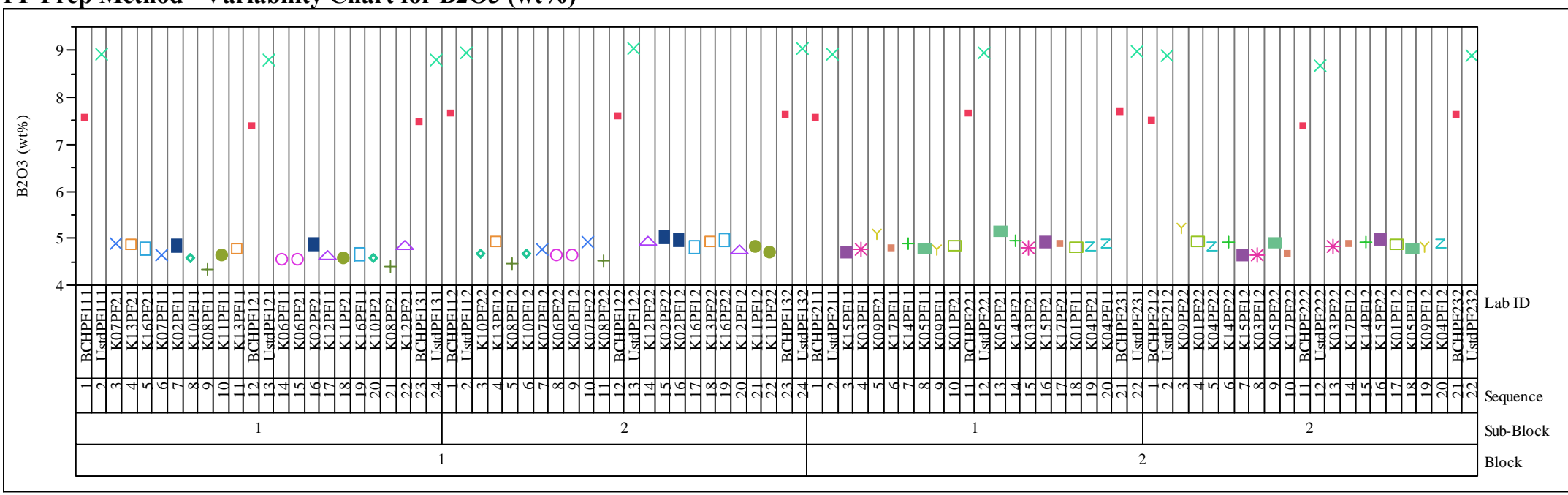

PF Prep Method - Variability Chart for Fe2O3 (wt\%)

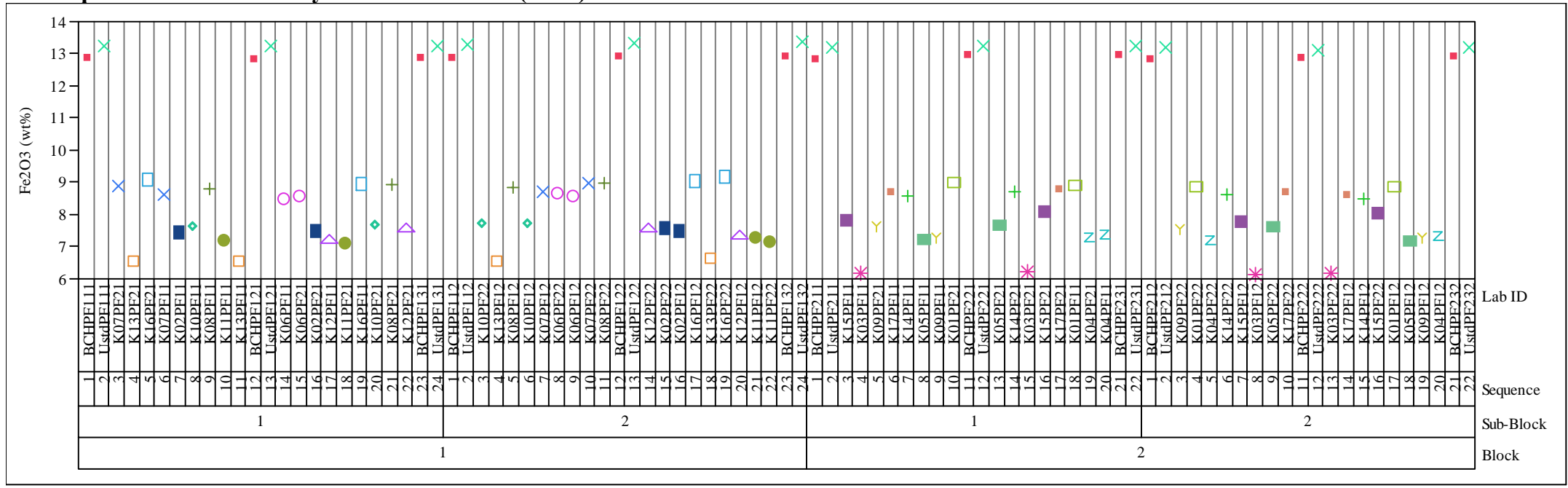


Exhibit A1. Measurements of Initial Glasses in Analytical Sequence for Samples by Prep Method by Oxide

PF Prep Method - Variability Chart for Li2O (wt\%)

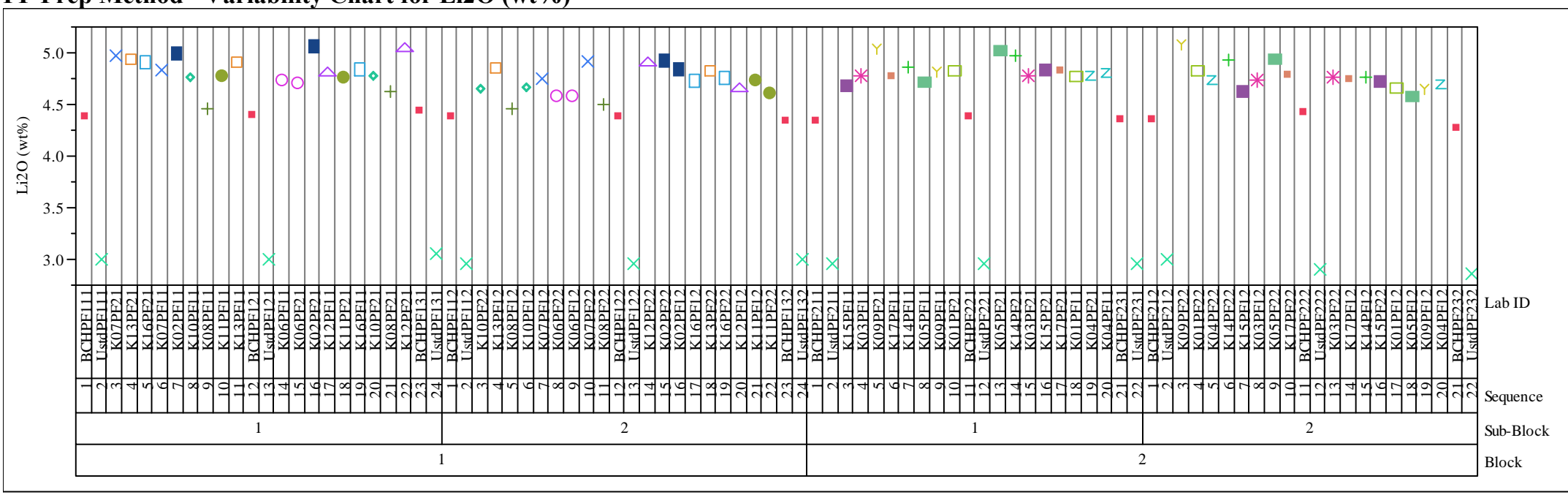

PF Prep Method - Variability Chart for SiO2 (wt\%)

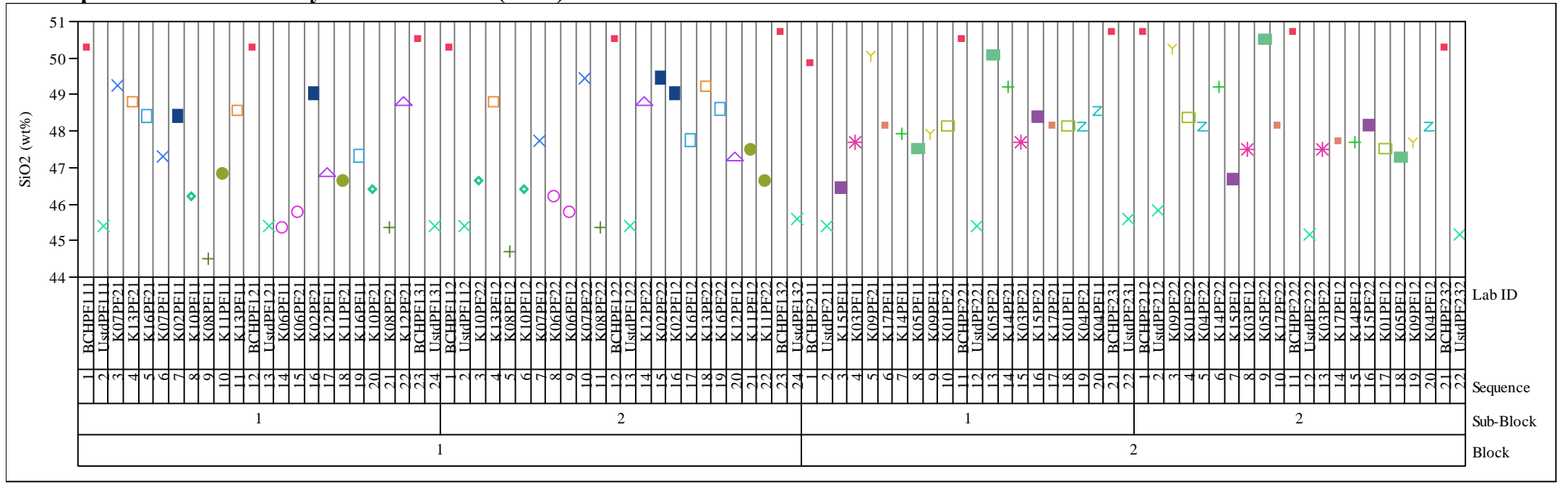




\section{Exhibit A2. Measurements by Lab ID for Initial Glasses by Targeted Concentration for Each Oxide}

\section{Oxide $=\mathrm{Al} 2 \mathrm{O} 3(\mathrm{wt} \%)$}

Variability Chart for Measured

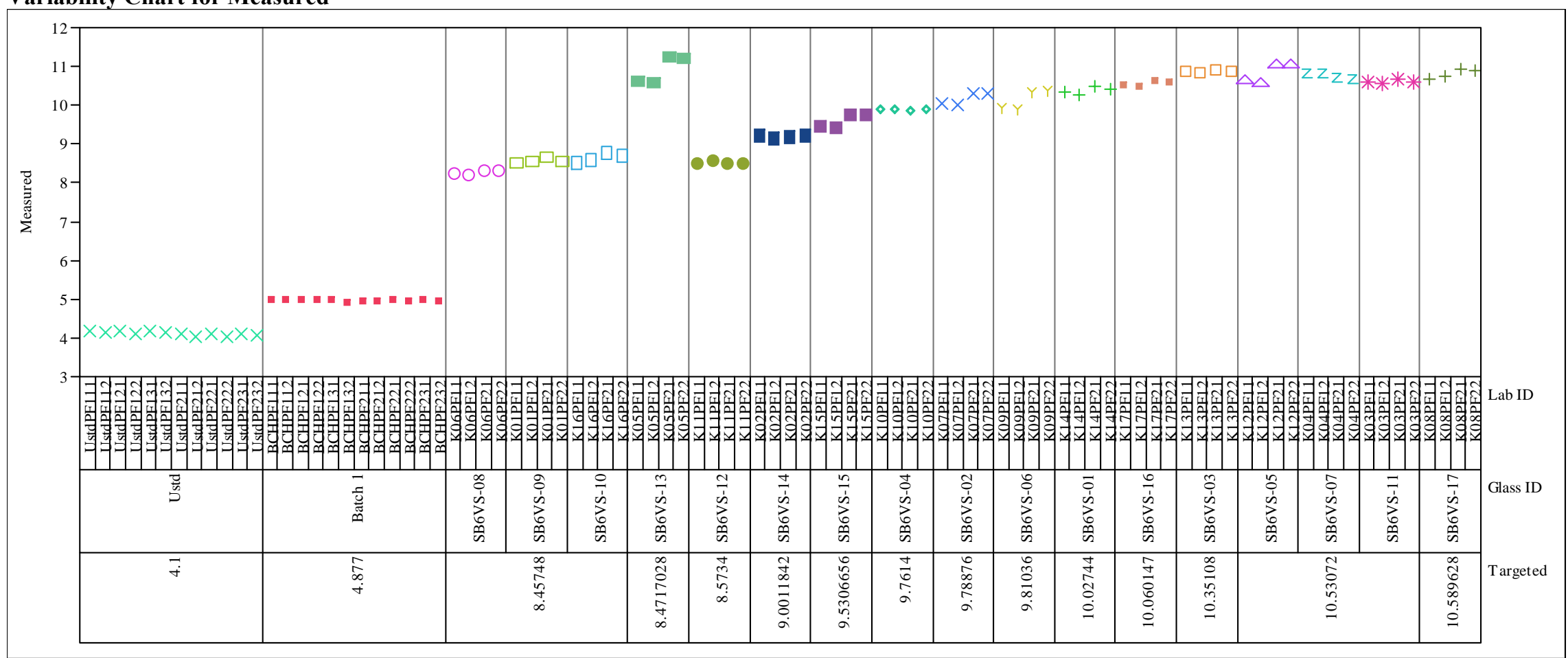




\section{Exhibit A2. Measurements by Lab ID for Initial Glasses by Targeted Concentration for Each Oxide}

\section{Oxide $=\mathbf{B 2 O 3}($ wt $\%)$}

Variability Chart for Measured

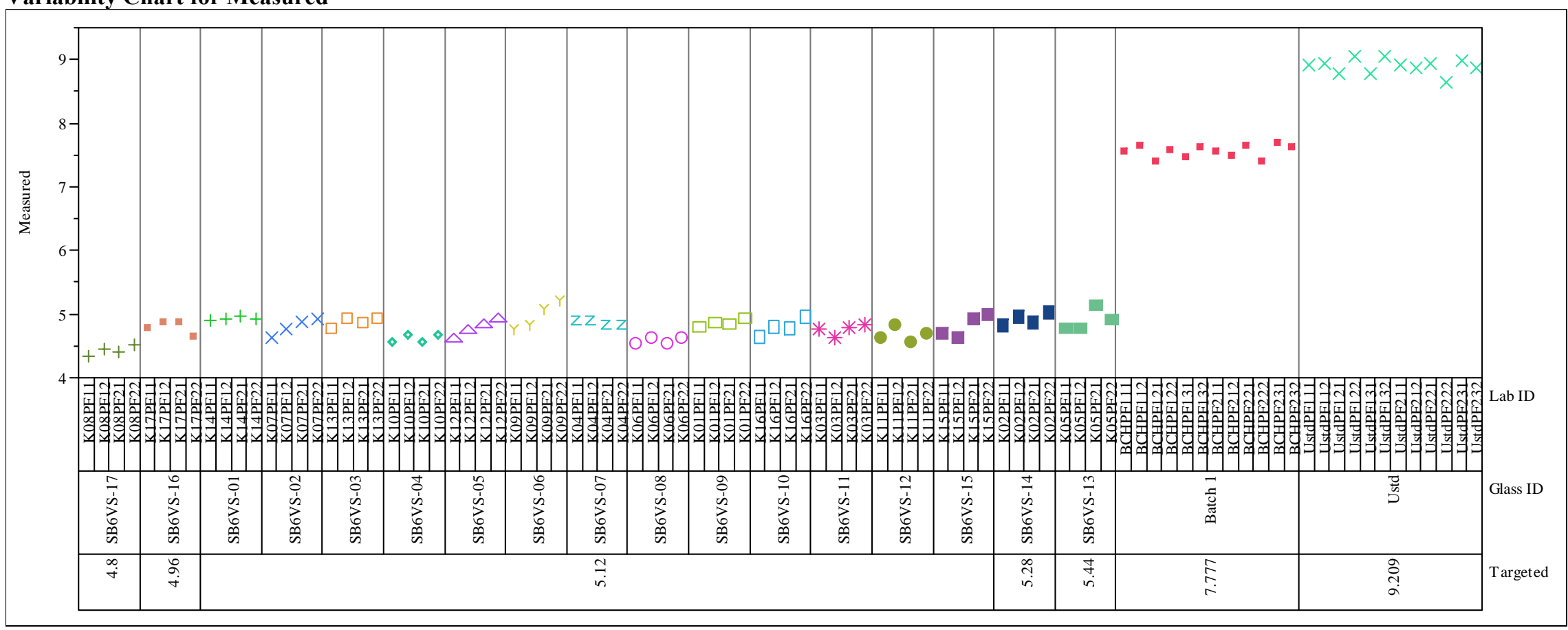




\section{Exhibit A2. Measurements by Lab ID for Initial Glasses by Targeted Concentration for Each Oxide}

\section{Oxide $=\mathrm{BaO}(\mathrm{wt} \%)$}

Variability Chart for Measured

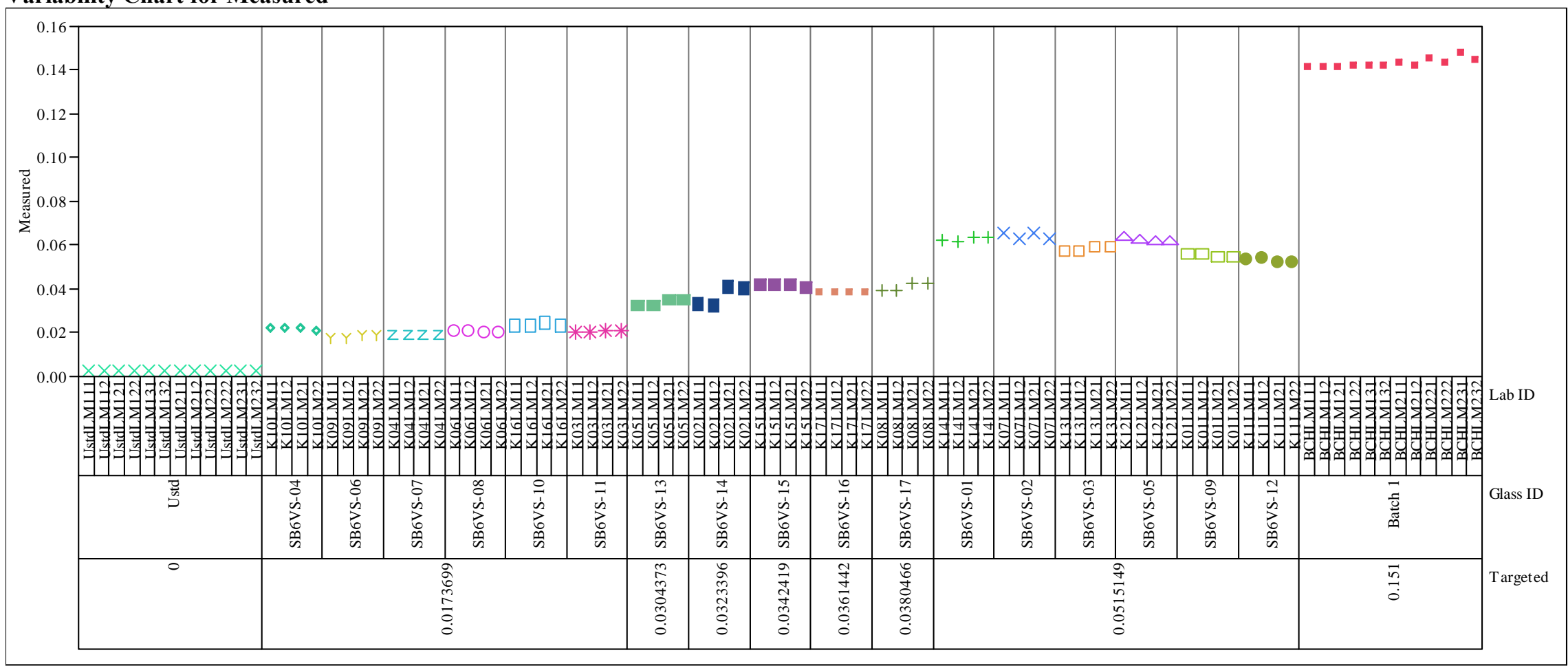




\section{Exhibit A2. Measurements by Lab ID for Initial Glasses by Targeted Concentration for Each Oxide}

\section{Oxide $=\mathrm{CaO}(\mathrm{wt} \%)$}

Variability Chart for Measured

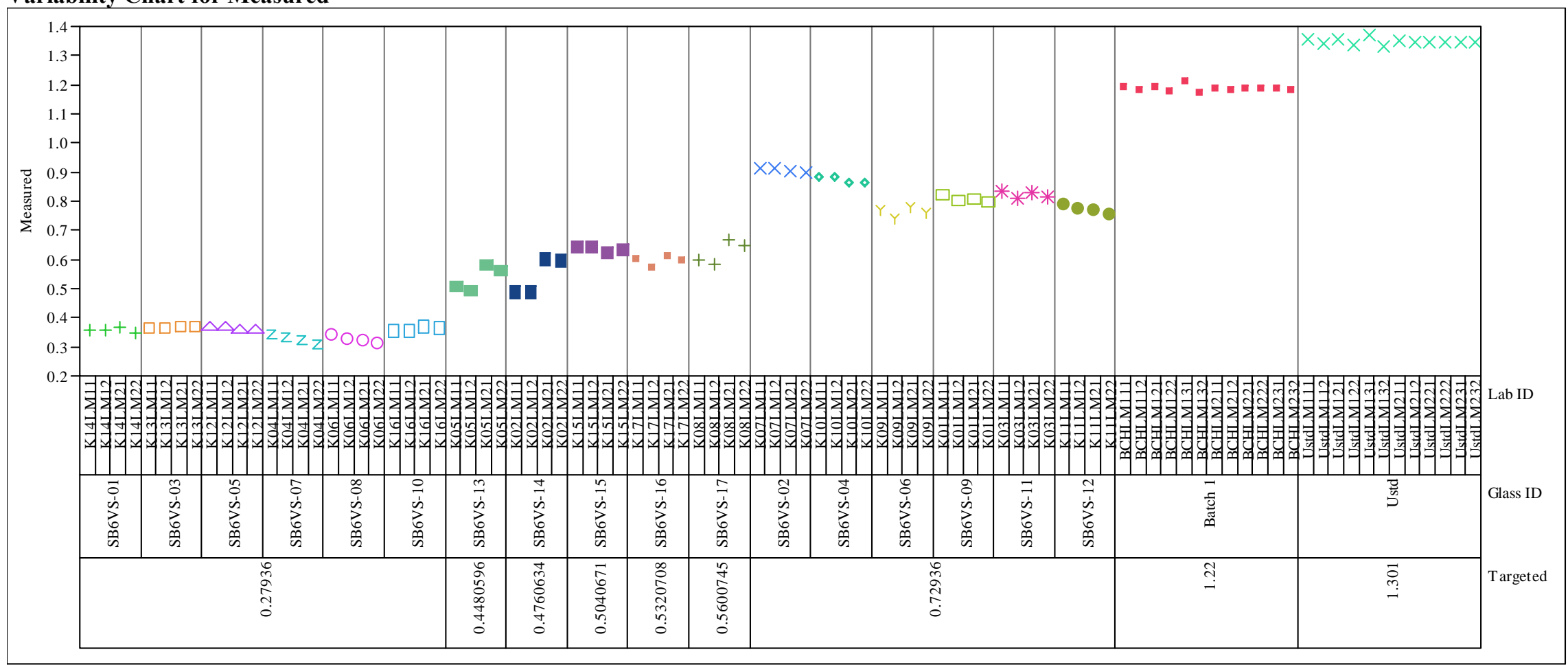




\section{Exhibit A2. Measurements by Lab ID for Initial Glasses by Targeted Concentration for Each Oxide}

\section{Oxide $=\mathbf{C d O}($ wt $\%)$}

Variability Chart for Measured

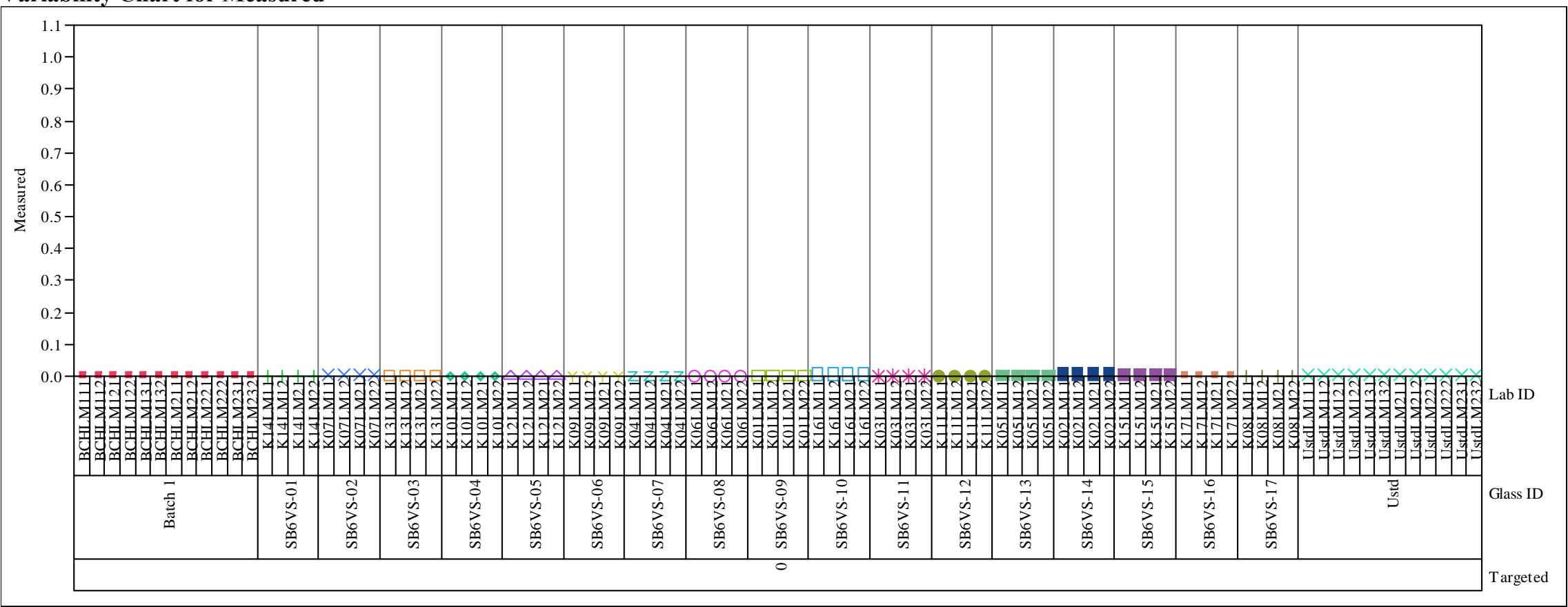




\section{Exhibit A2. Measurements by Lab ID for Initial Glasses by Targeted Concentration for Each Oxide}

\section{Oxide $=\mathrm{Ce} 2 \mathrm{O} 3(\mathrm{wt} \%)$}

Variability Chart for Measured

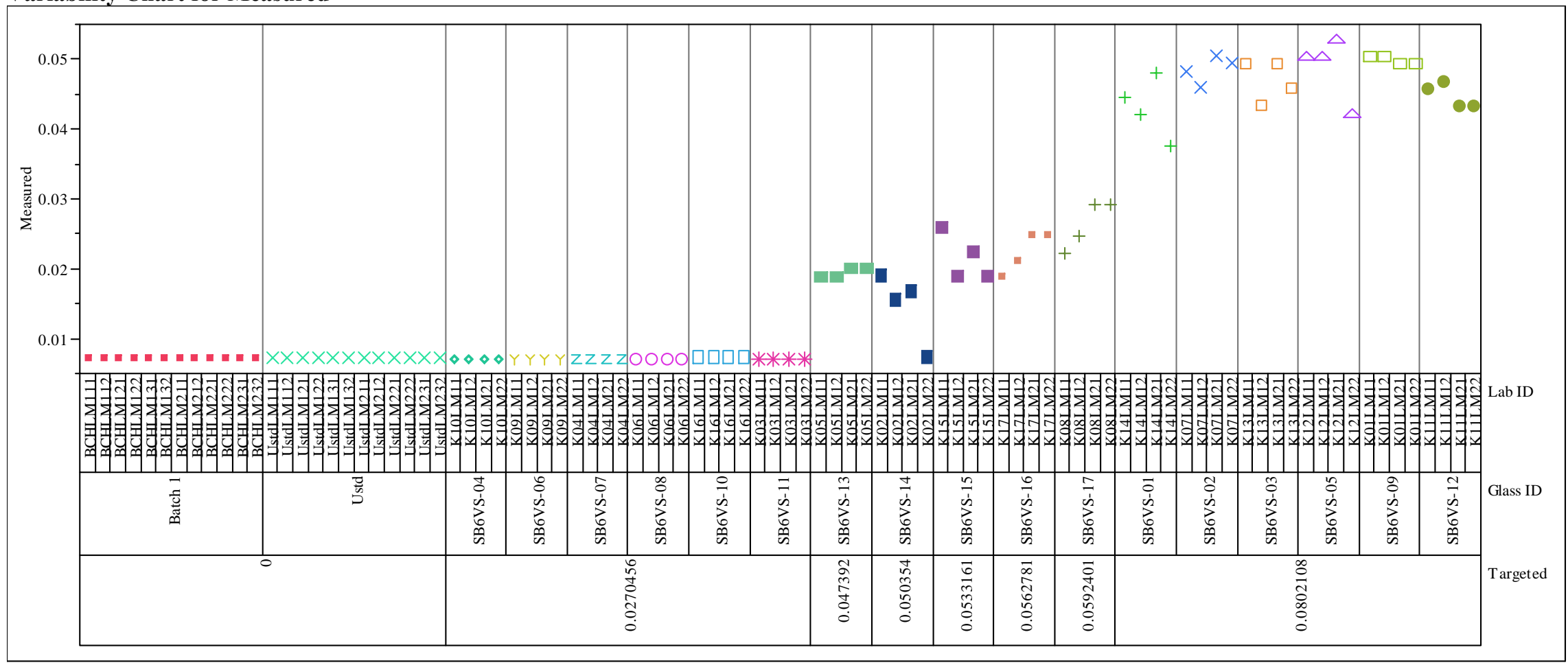




\section{Exhibit A2. Measurements by Lab ID for Initial Glasses by Targeted Concentration for Each Oxide}

\section{Oxide $=\mathbf{C r 2 O 3}(\mathbf{w t} \%)$}

Variability Chart for Measured

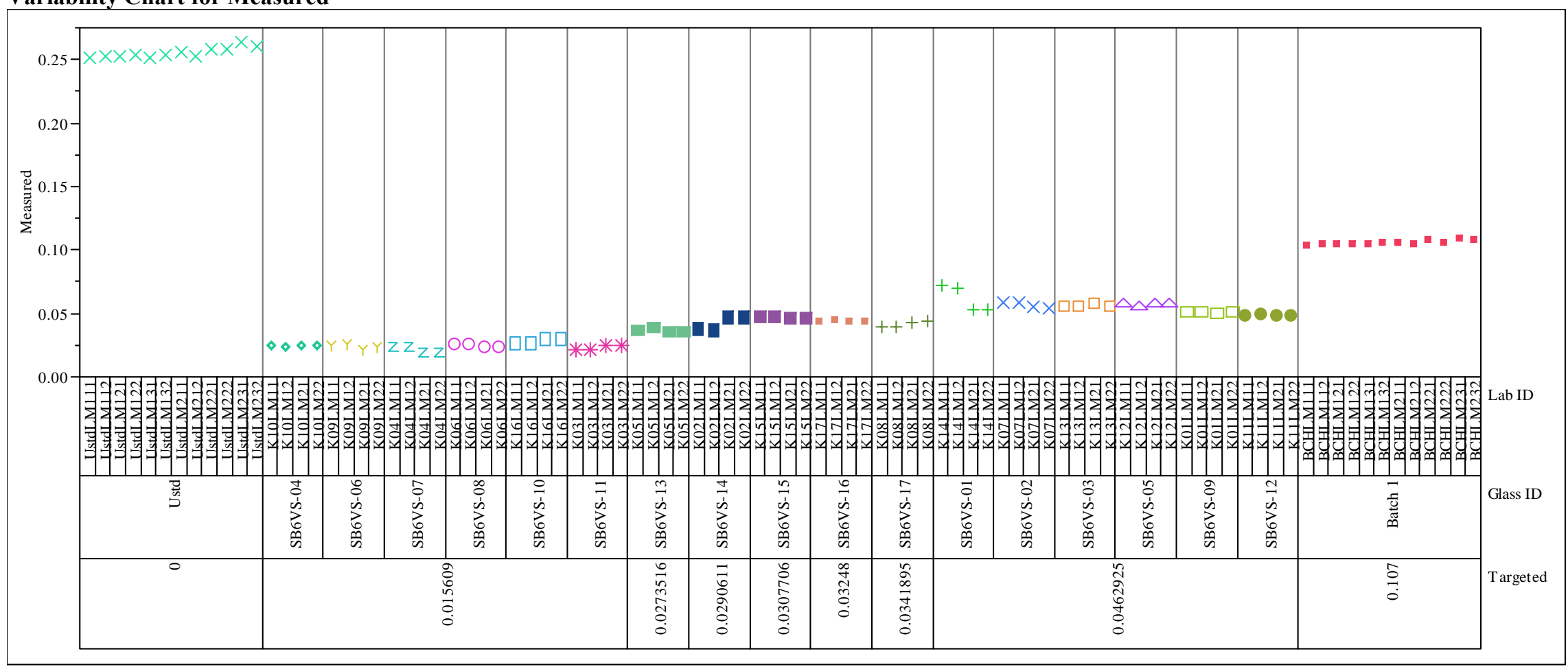




\section{Exhibit A2. Measurements by Lab ID for Initial Glasses by Targeted Concentration for Each Oxide}

\section{Oxide $=\mathrm{CuO}(\mathrm{wt} \%)$}

Variability Chart for Measured

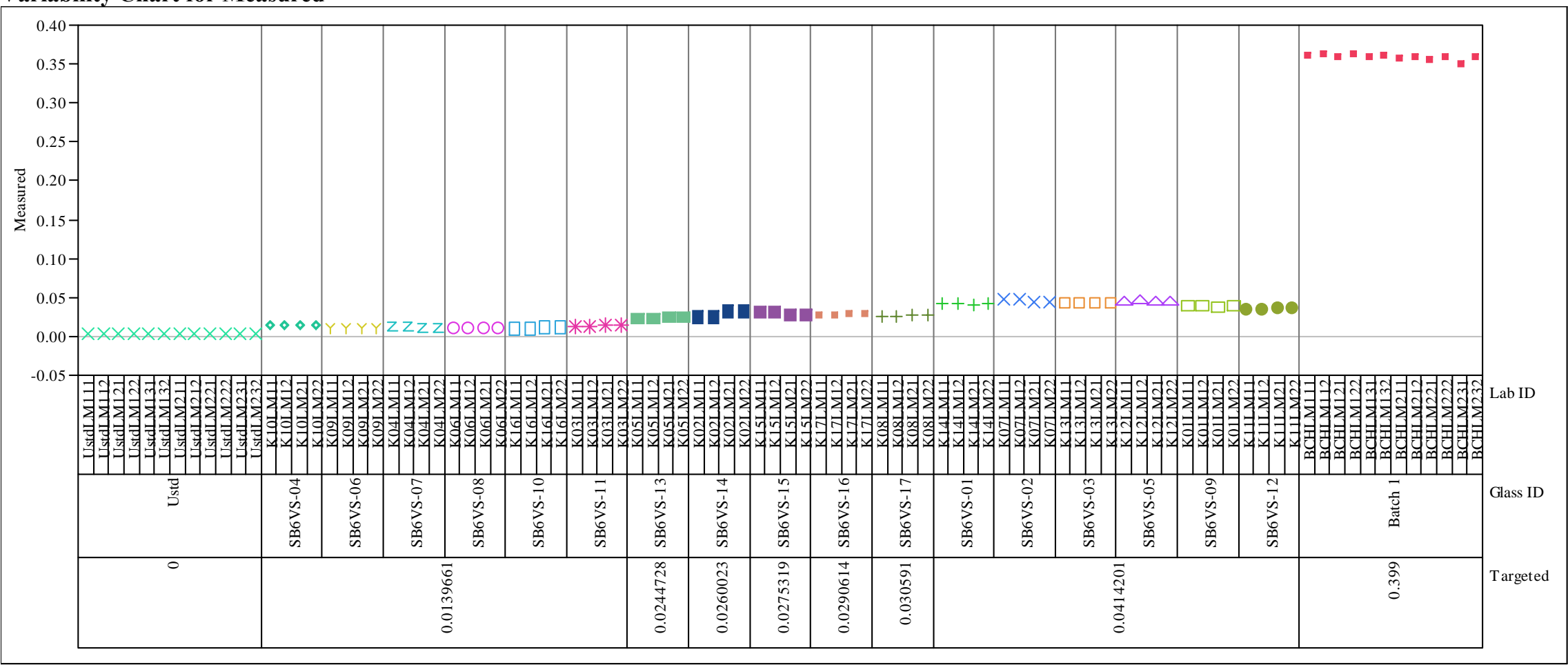




\section{Exhibit A2. Measurements by Lab ID for Initial Glasses by Targeted Concentration for Each Oxide}

\section{Oxide $=\mathrm{Fe} 2 \mathrm{O3}(\mathrm{wt} \%)$}

Variability Chart for Measured

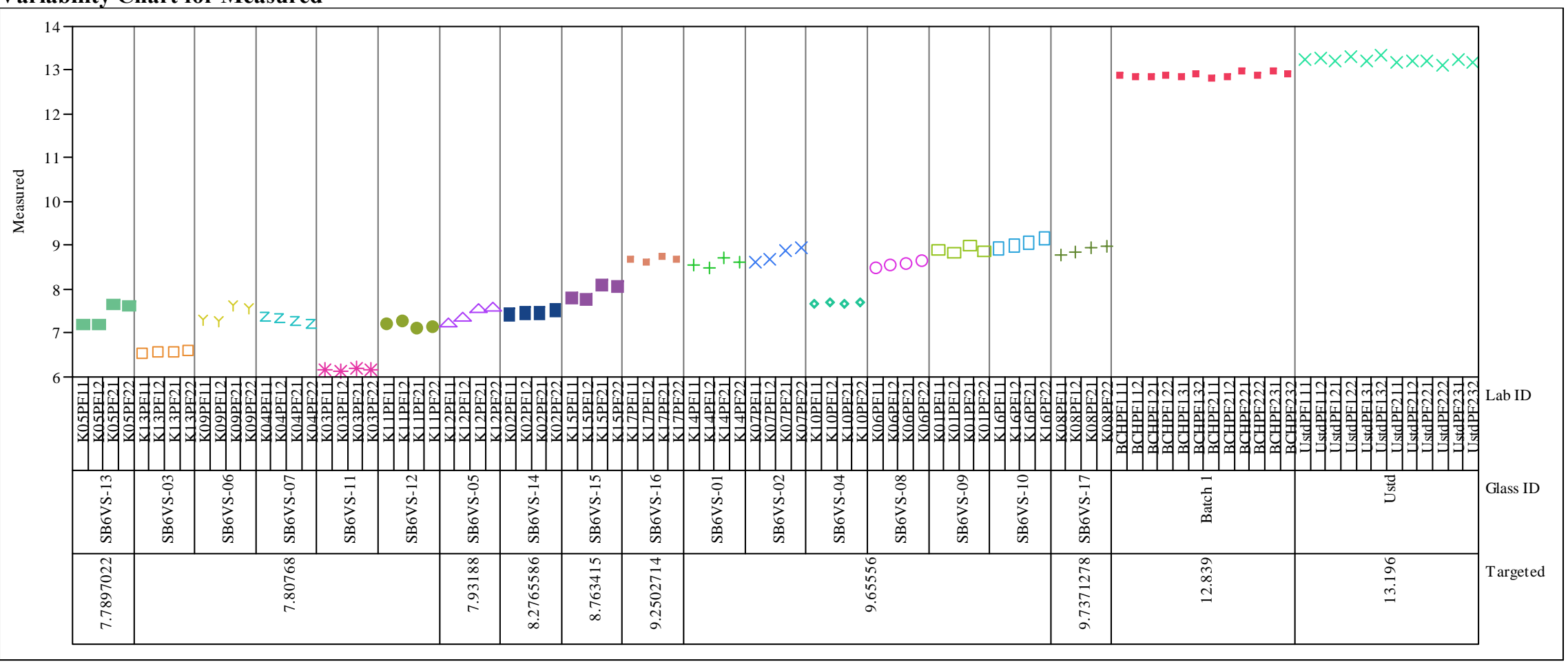




\section{Exhibit A2. Measurements by Lab ID for Initial Glasses by Targeted Concentration for Each Oxide}

\section{Oxide $=\mathrm{K2O}($ wt \%)}

Variability Chart for Measured

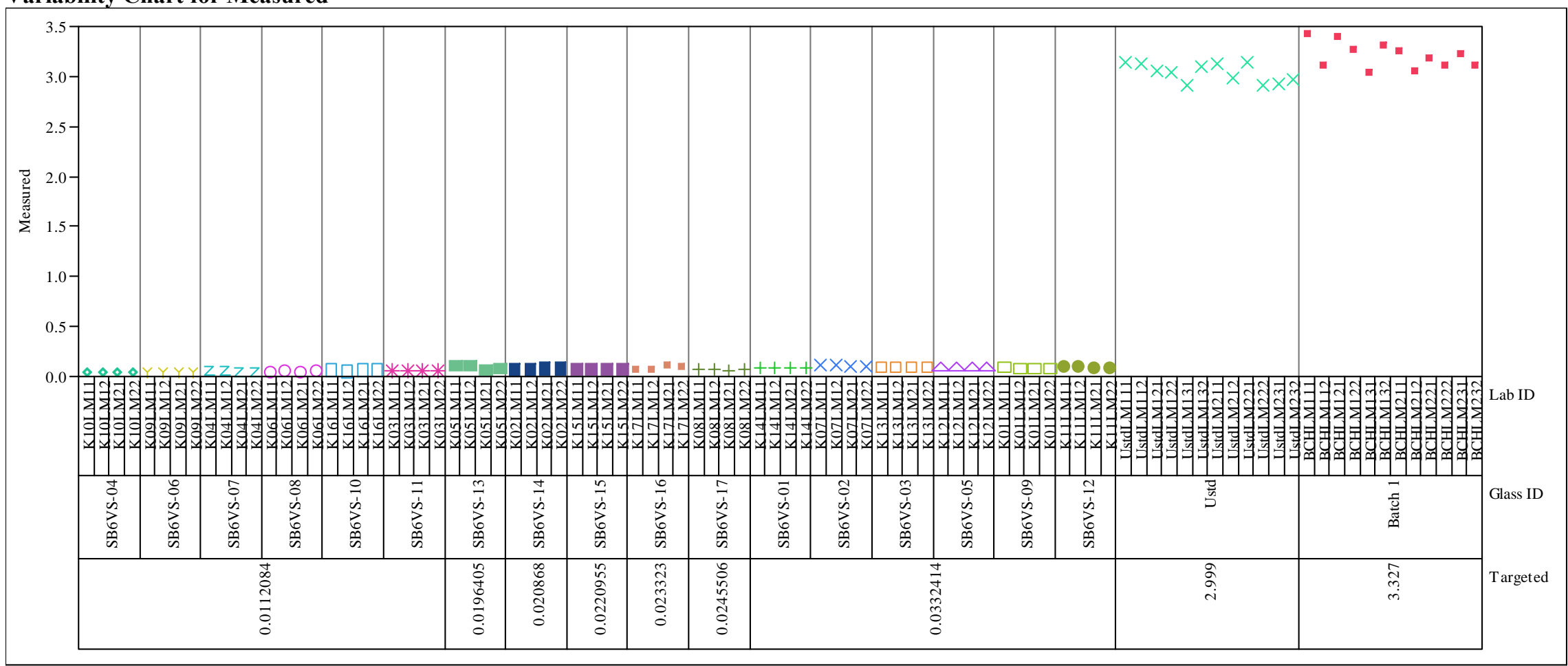




\section{Exhibit A2. Measurements by Lab ID for Initial Glasses by Targeted Concentration for Each Oxide}

\section{Oxide $=\mathbf{L a 2 O 3}(\mathrm{wt} \%)$}

Variability Chart for Measured

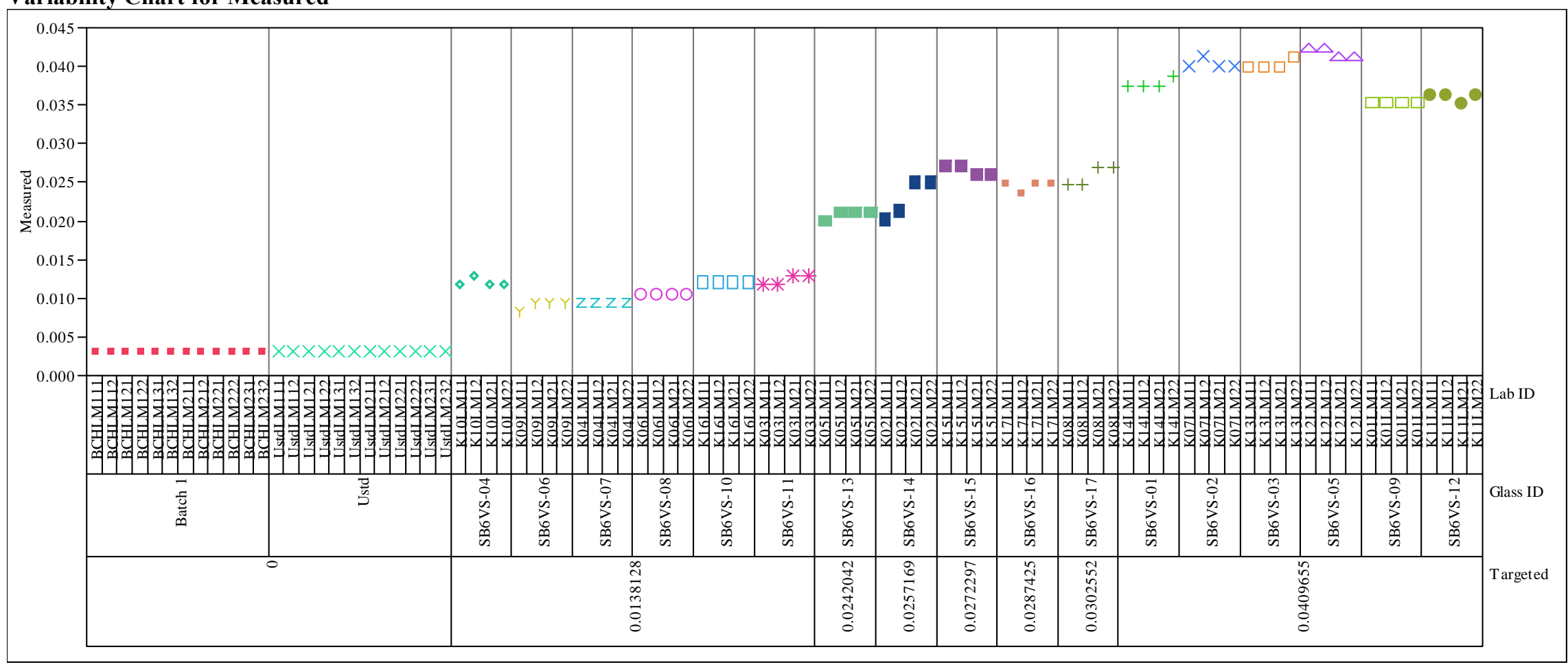




\section{Exhibit A2. Measurements by Lab ID for Initial Glasses by Targeted Concentration for Each Oxide}

\section{Oxide $=$ Li2O $($ wt $\%)$}

Variability Chart for Measured

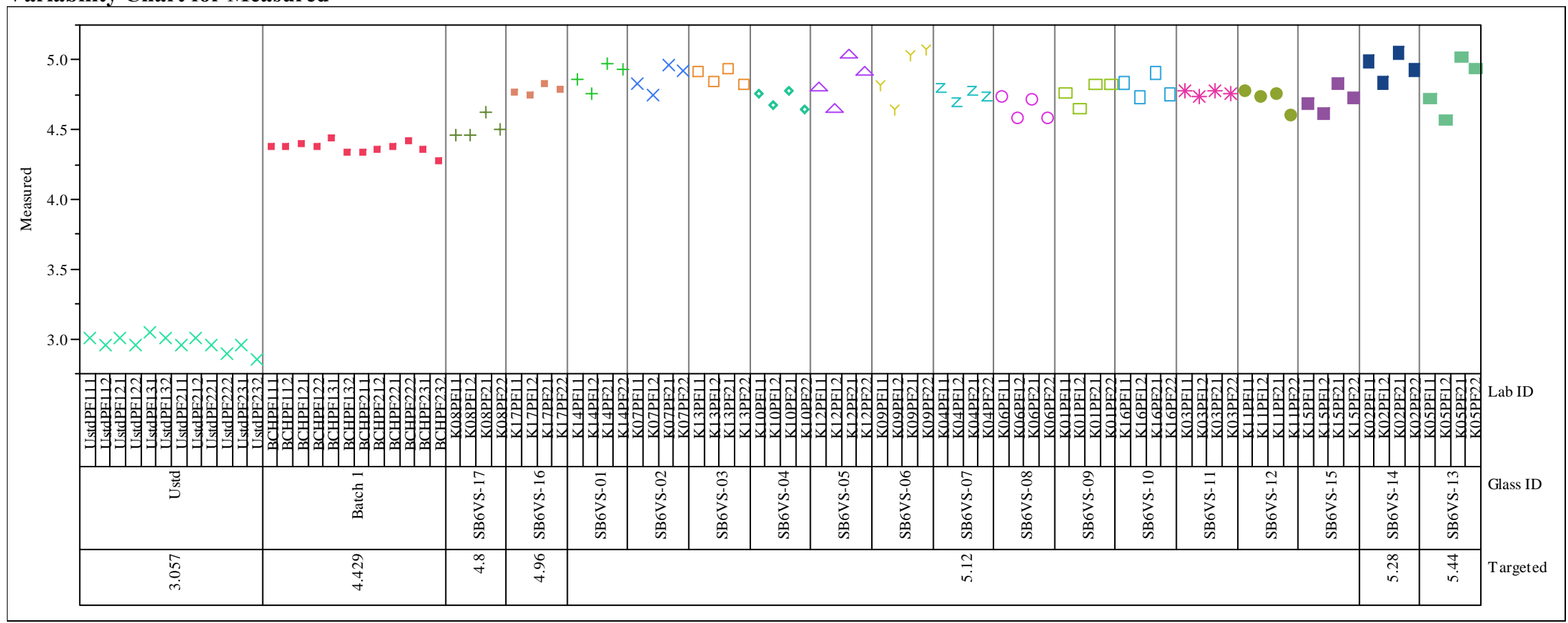




\section{Exhibit A2. Measurements by Lab ID for Initial Glasses by Targeted Concentration for Each Oxide}

\section{Oxide $=\mathrm{MgO}(\mathrm{wt} \%)$}

Variability Chart for Measured

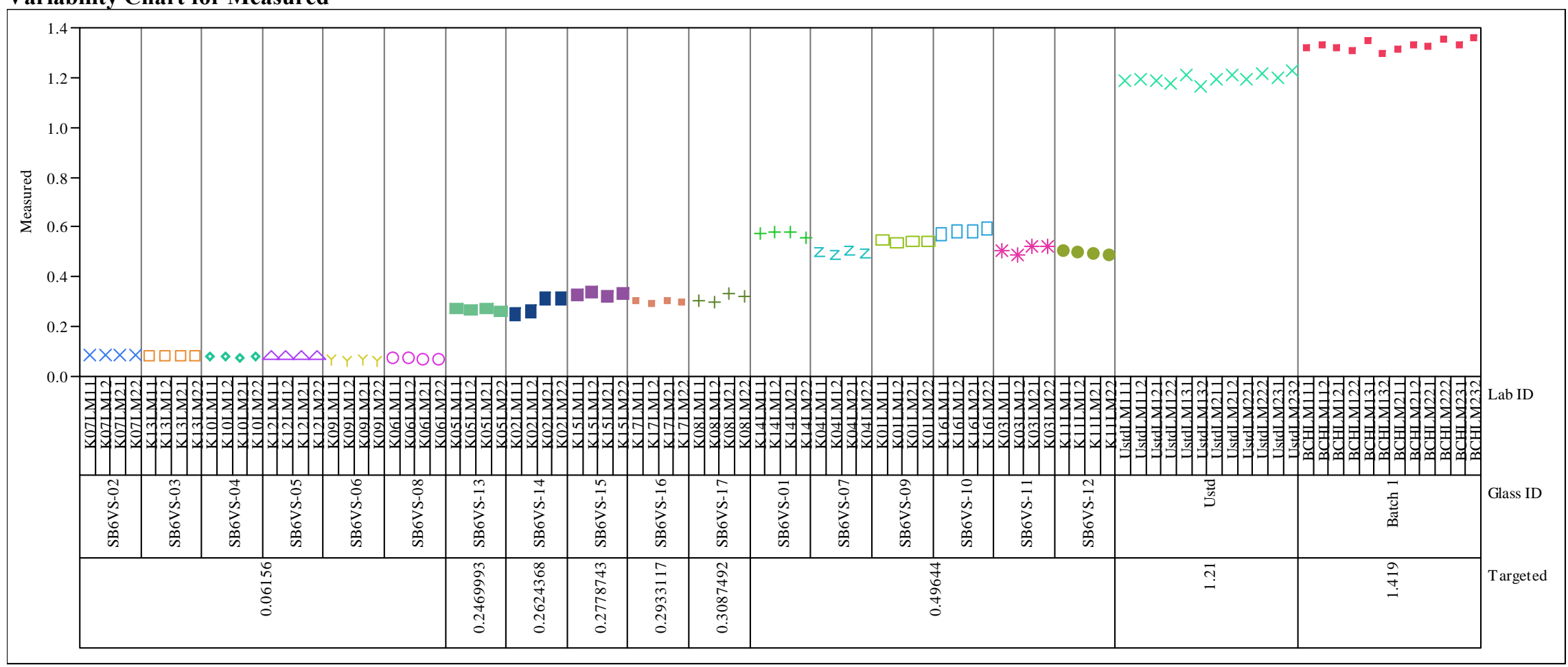




\section{Exhibit A2. Measurements by Lab ID for Initial Glasses by Targeted Concentration for Each Oxide}

\section{Oxide $=\operatorname{MnO}($ wt \%)}

Variability Chart for Measured

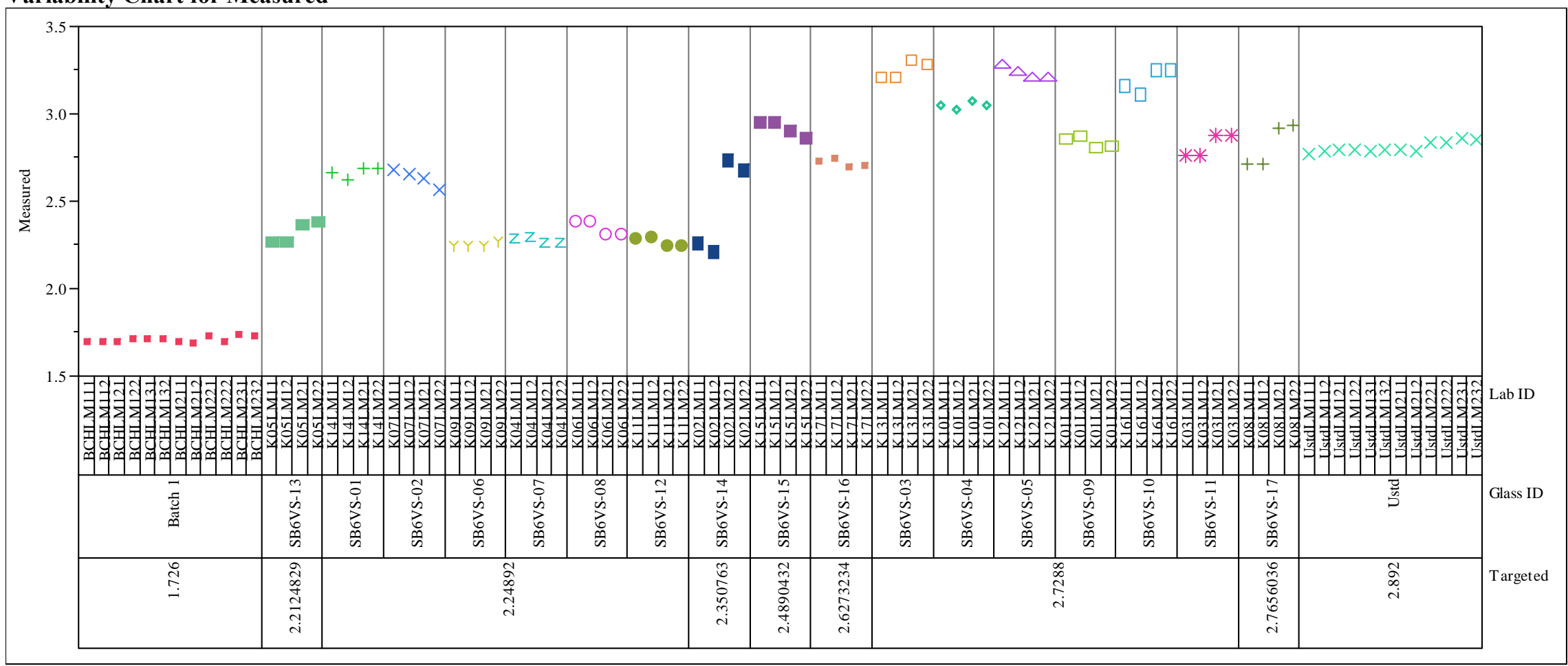




\section{Exhibit A2. Measurements by Lab ID for Initial Glasses by Targeted Concentration for Each Oxide}

\section{Oxide $=\mathrm{Na2O}(\mathrm{wt} \%)$}

Variability Chart for Measured

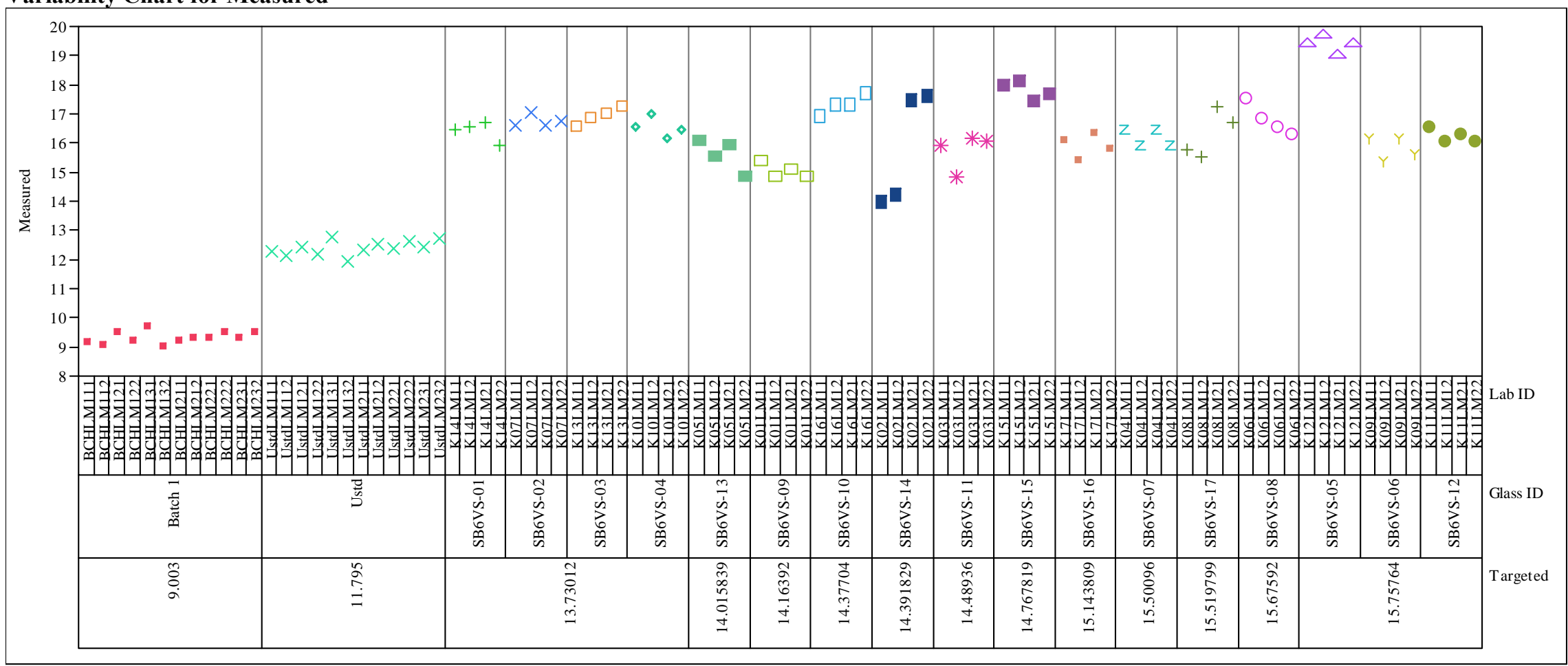




\section{Exhibit A2. Measurements by Lab ID for Initial Glasses by Targeted Concentration for Each Oxide}

\section{Oxide $=\mathrm{NiO}(\mathrm{wt} \%)$}

Variability Chart for Measured

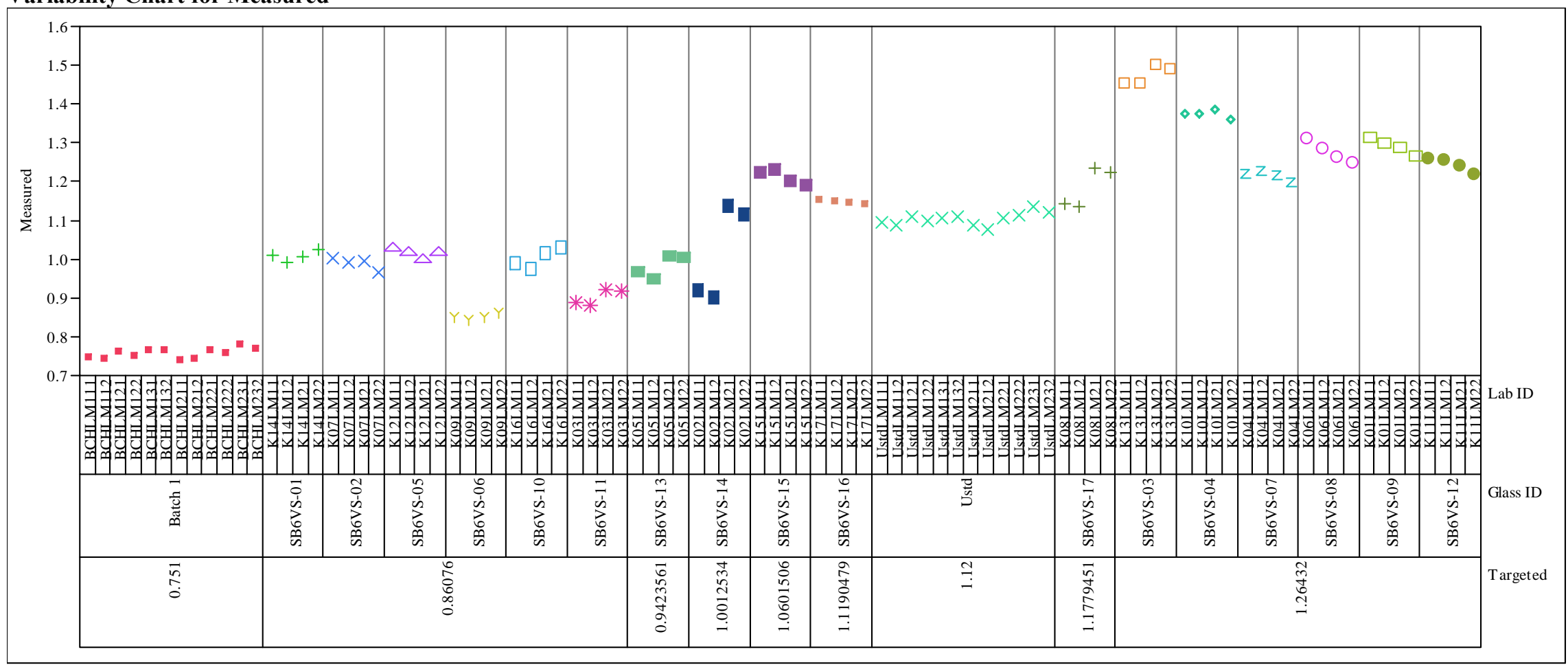




\section{Exhibit A2. Measurements by Lab ID for Initial Glasses by Targeted Concentration for Each Oxide}

\section{Oxide $=\mathbf{P 2 O 5}($ wt \%)}

Variability Chart for Measured

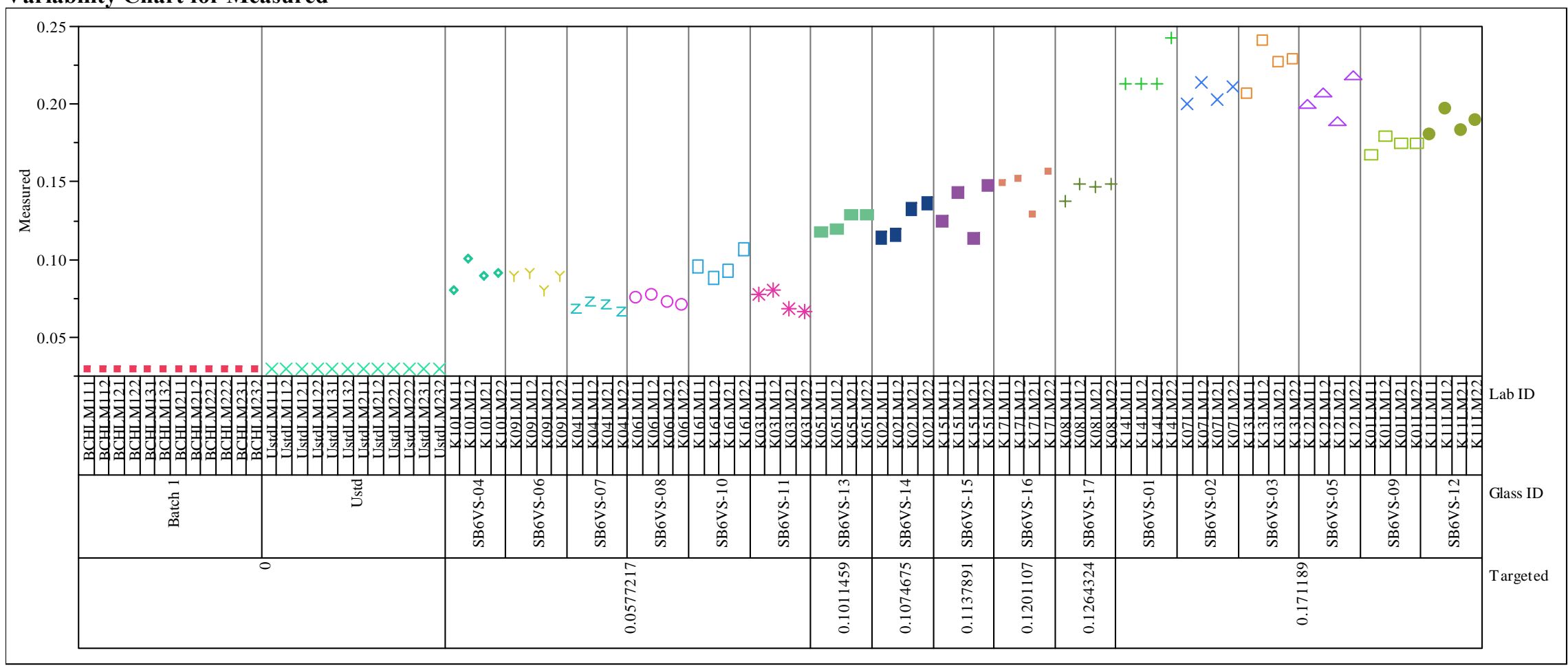




\section{Exhibit A2. Measurements by Lab ID for Initial Glasses by Targeted Concentration for Each Oxide}

\section{Oxide $=$ PbO $($ wt $\%)$}

Variability Chart for Measured

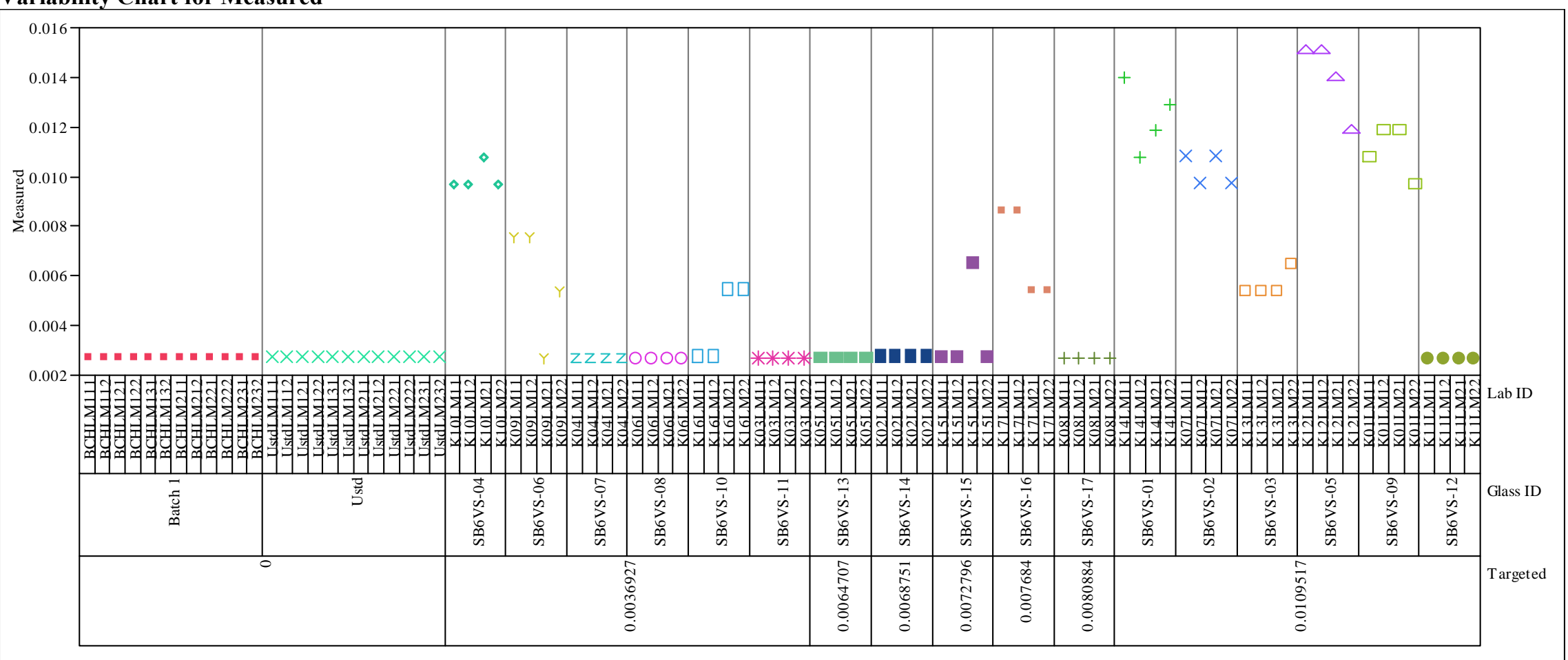




\section{Exhibit A2. Measurements by Lab ID for Initial Glasses by Targeted Concentration for Each Oxide}

\section{Oxide $=\mathrm{SiO} 2($ wt $\%)$}

Variability Chart for Measured

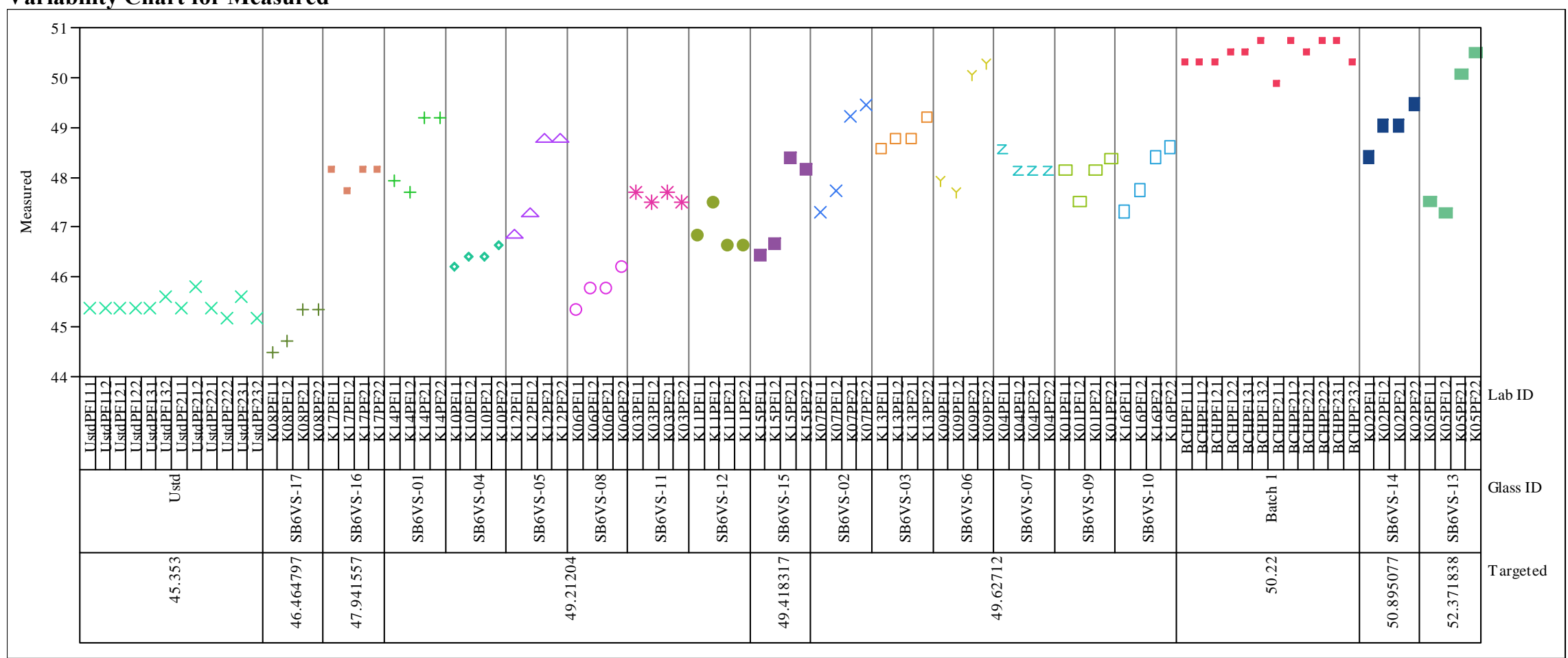




\section{Exhibit A2. Measurements by Lab ID for Initial Glasses by Targeted Concentration for Each Oxide}

\section{Oxide=SO4 (wt\%)}

Variability Chart for Measured

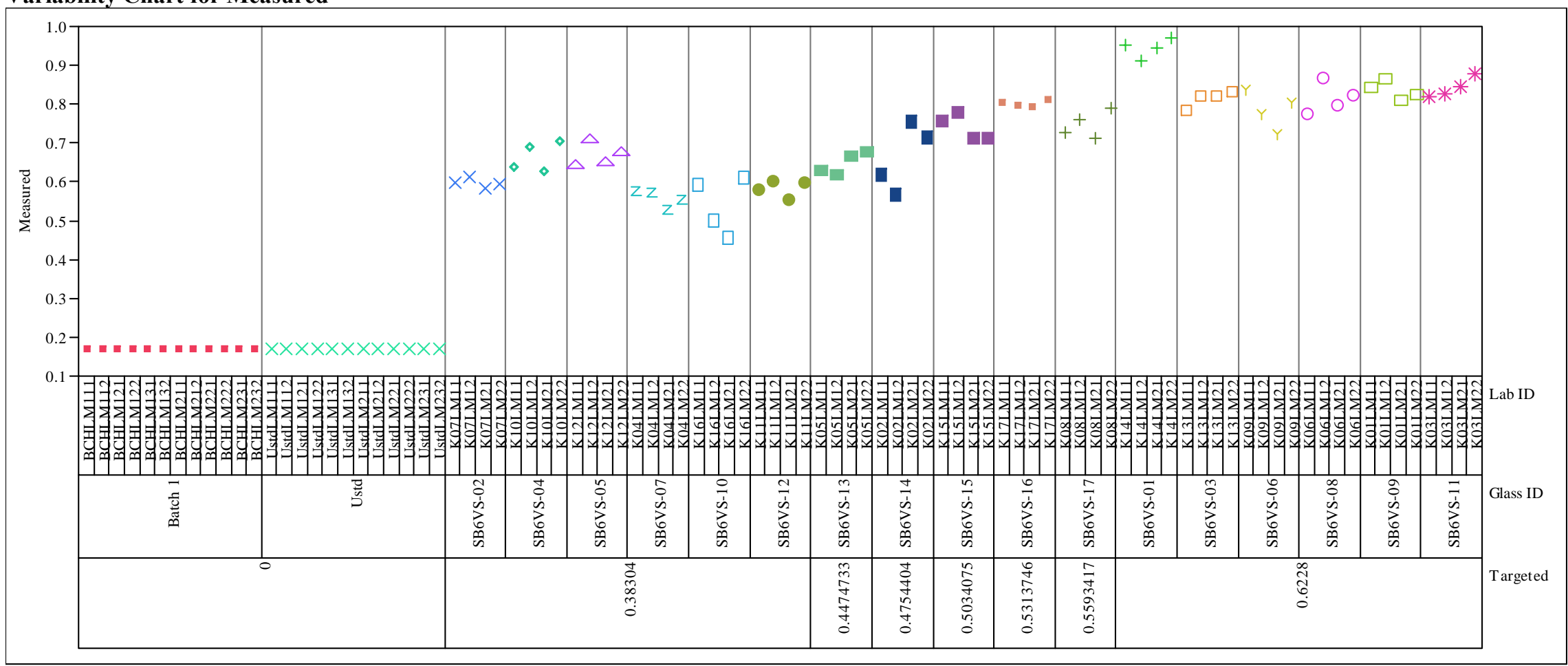




\section{Exhibit A2. Measurements by Lab ID for Initial Glasses by Targeted Concentration for Each Oxide}

\section{Oxide $=\mathrm{TiO} 2(\mathrm{wt} \%)$}

Variability Chart for Measured

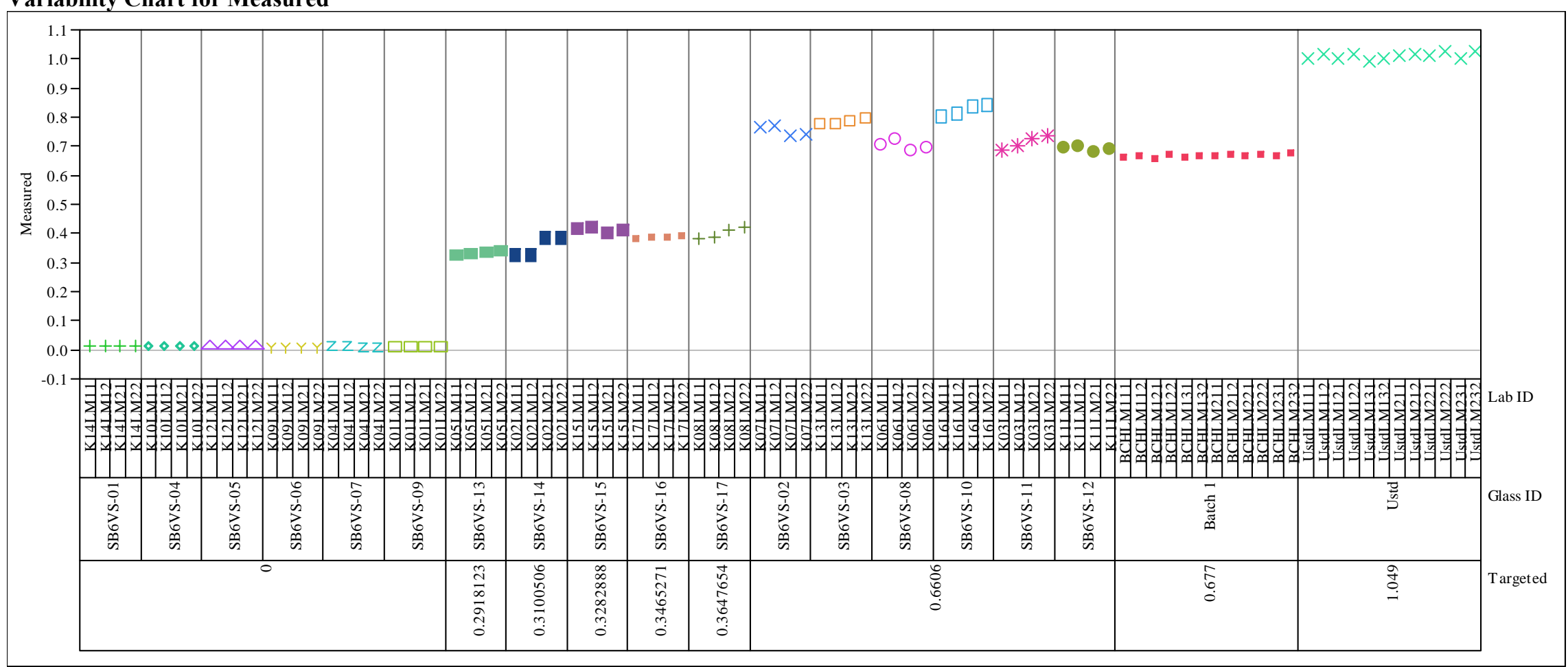




\section{Exhibit A2. Measurements by Lab ID for Initial Glasses by Targeted Concentration for Each Oxide}

\section{Oxide $=\mathbf{U} 308($ wt $\%)$}

Variability Chart for Measured

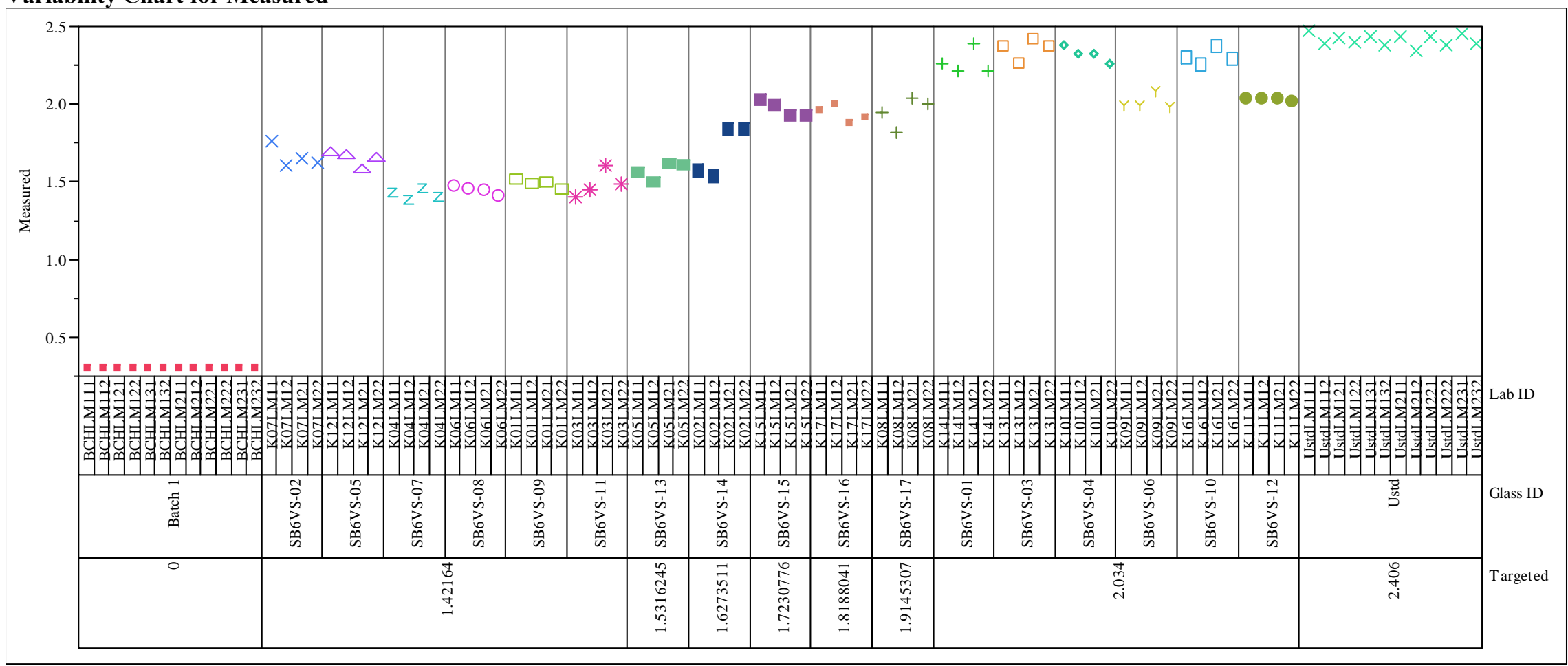




\section{Exhibit A2. Measurements by Lab ID for Initial Glasses by Targeted Concentration for Each Oxide}

\section{Oxide $=\mathrm{ZnO}($ wt $\%)$}

Variability Chart for Measured

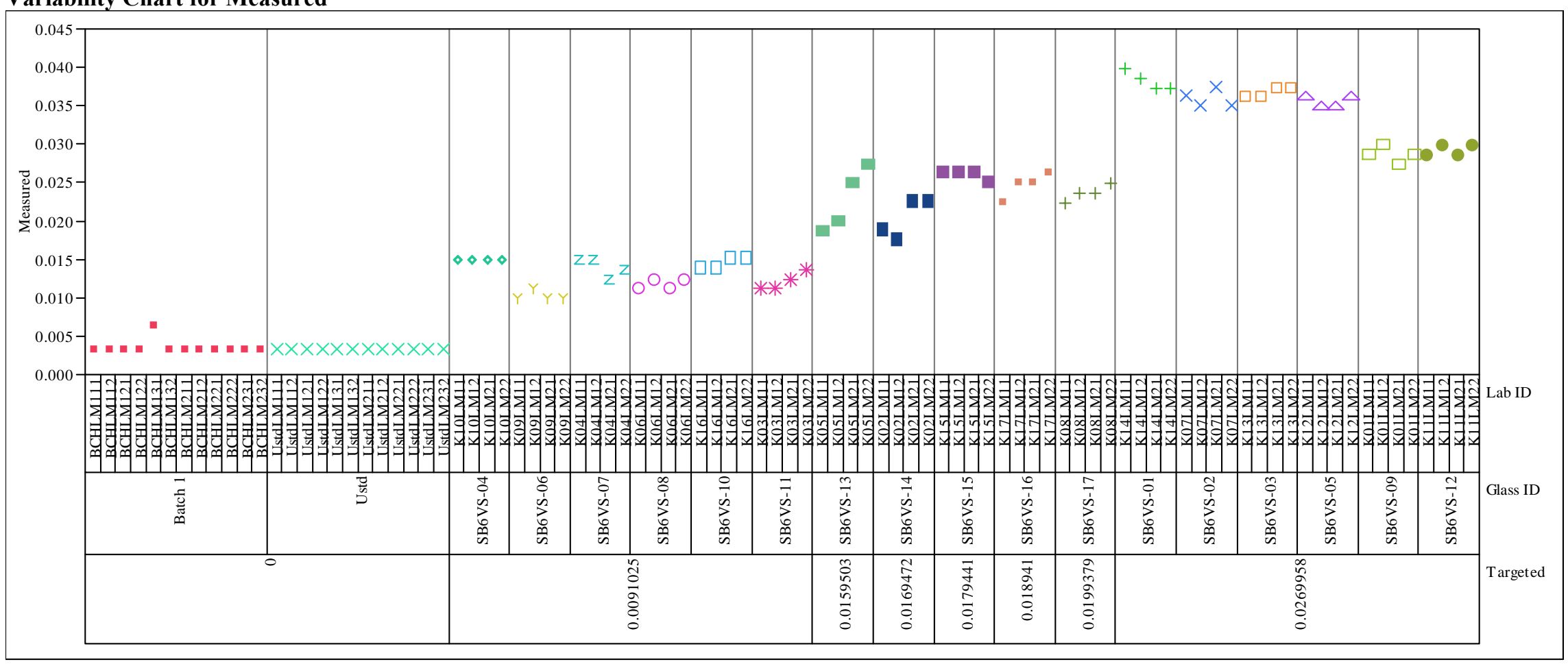




\section{Exhibit A2. Measurements by Lab ID for Initial Glasses by Targeted Concentration for Each Oxide}

\section{Oxide $=\mathrm{ZrO2}(\mathrm{wt} \%)$}

Variability Chart for Measured

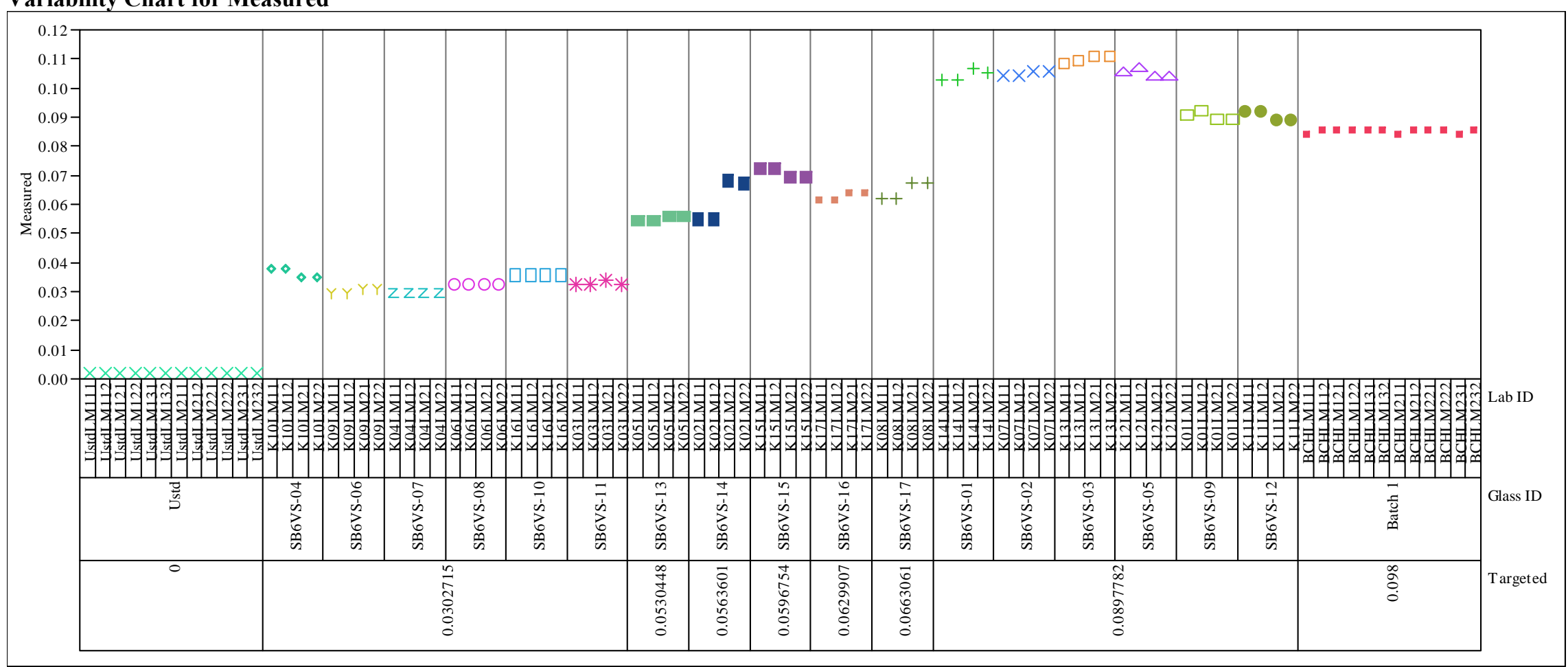




\section{Exhibit A3. Measurements by Block and Sub-Block for Samples of the Batch 1 and Ustd Standards with the Initial Glasses by Oxide}

Oneway Analysis of Measured By Block/Sub-Block Type=Batch 1, Oxide $=\mathbf{A l 2 O 3}(\mathbf{w t} \%)$

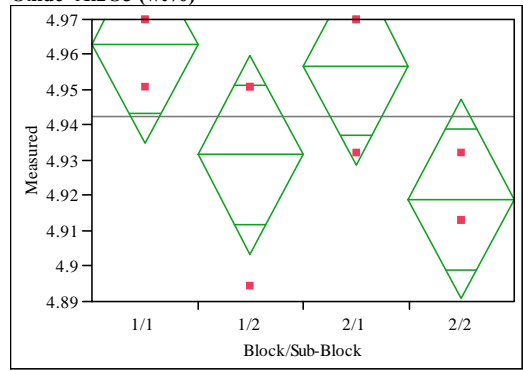

Oneway Anova

Summary of Fit

Rsquare

Adj Rsquare

Observations (or Sum Wgts)

Analysis of Variance
Source DF Sum of Squares Mean Square F Ratio Prob $>$ F

$\begin{array}{lrrrrr}\text { Source } & \text { DF } & \text { Sum of Squares } & \text { Mean Square } & \text { F Ratio } & \text { Prob }>\text { F } \\ \text { Block/Sub-Block } & 3 & 0.00389748 & 0.001299 & 2.9111 & 0.1008\end{array}$

Error

C. Total

$\begin{array}{lll}0.001299 & 2.9111 & 0.1008 \\ 0.000446 & & \end{array}$

Means for Oneway Anova

Level Number Mean Std Error Lower 95\% Upper 95\%

$\begin{array}{llllll}1 / 1 & 3 & 4.96309 & 0.01220 & 4.9350 & 4.9912 \\ 1 / 2 & 3 & 4.93160 & 0.01220 & 4.9035 & 4.9597\end{array}$

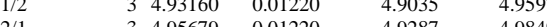

$\begin{array}{llllll}2 / 1 & 3 & 4.95679 & 0.01220 & 4.9287 & 4.9849\end{array}$

Std Error uses a pooled estimate of error variance

Oneway Analysis of Measured By Block/Sub-Block Type=Batch 1, Oxide $=\mathbf{B 2 O 3}(\mathbf{w t} \%)$

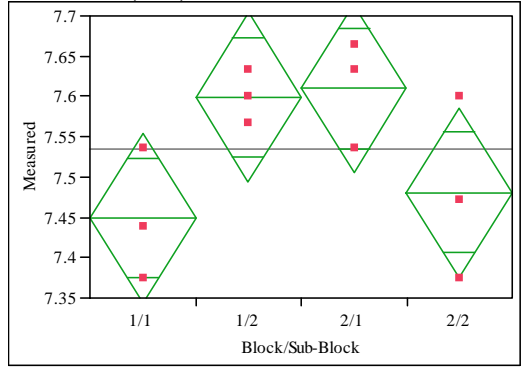

Oneway Anova

Summary of Fit

Rsquare

Adj Rsquare

Root Mean Square Error $\quad 0.078871$

Mean of Response $\quad 7.534566$

Observations (or Sum Wgts) 12

Analysis of Variance

Source DF Sum of Squares Mean Square F Ratio Prob $>$ F

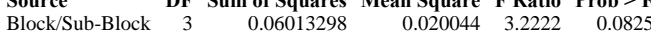

Error 8 - 0.04976523

$\begin{array}{lll}\text { C. Total } & 11 & 0.10989821\end{array}$

Means for Oneway Anova

Level Number Mean Std Error Lower 95\% Upper 95\%

$\begin{array}{llllll}1 / 1 & 3 & 7.44870 & 0.04554 & 7.3437 & 7.5537\end{array}$

$\begin{array}{llllll}1 / 2 & 3 & 7.59896 & 0.04554 & 7.4940 & 7.7040\end{array}$

$\begin{array}{llllll}2 / 1 & 3 & 7.60970 & 0.04554 & 7.5047 & 7.7147\end{array}$

Std Error uses a pooled estimate of error variance
Oneway Analysis of Measured By Block/Sub-Block Type=Batch 1, Oxide $=\mathrm{BaO}(\mathrm{wt} \%)$

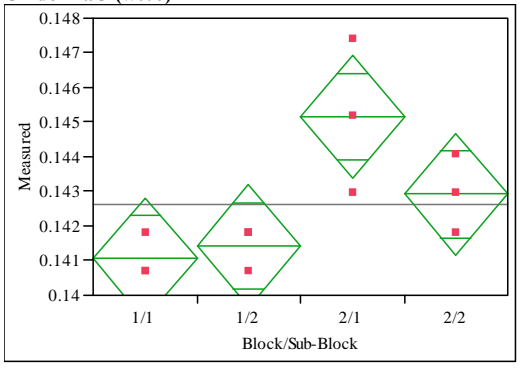

Oneway Anova

Summary of Fit

$\begin{array}{lr}\text { Rsquare } & 0.687356 \\ \text { Adj Rsquare } & 0.570115 \\ \text { Root Mean Square Error } & 0.001329 \\ \text { Mean of Response } & 0.142633 \\ \text { Observations (or Sum Wgts) } & 12\end{array}$

Analysis of Variance

Source DF Sum of Squares Mean Square F Ratio Prob $>$ F $\begin{array}{llllll}\text { Block/Sub-Block } & 3 & 0.00003106 & 0.000010 & 5.8627 & 0.0203\end{array}$

$\begin{array}{lrrr}\text { Error } & 8 & 0.00001413 & 1.766 \mathrm{e}-6\end{array}$

$\begin{array}{lll}\text { C. Total } & 11 & 0.00004519\end{array}$

Means for Oneway Anova

Level Number Mean Std Error Lower 95\% Upper 95\%

$\begin{array}{lrrrrr}1 / 1 & 3 & 0.141051 & 0.00077 & 0.13928 & 0.14282\end{array}$

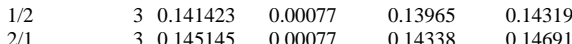

$\begin{array}{llllll}2 / 2 & 3 & 0.142912 & 0.00077 & 0.14114 & 0.14468\end{array}$

Std Error uses a pooled estimate of error variance

Oneway Analysis of Measured By Block/Sub-Block Type=Batch 1, Oxide $=\mathrm{CaO}\left(\mathrm{wt}_{\mathrm{t}} \%\right)$

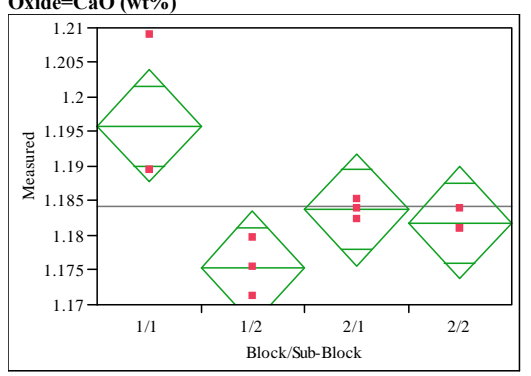

Oneway Anova

Summary of Fit

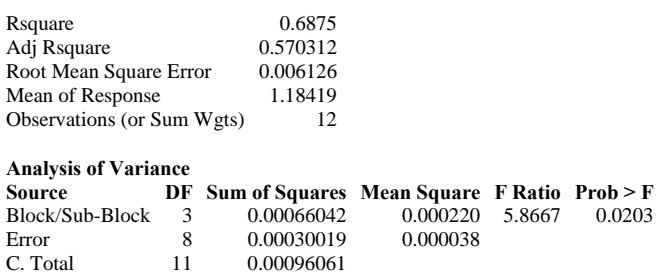

$\begin{array}{lll}\text { C. Total } & 11 & 0.0009606\end{array}$

Means for Oneway Anova

Level Number Mean Std Error Lower 95\% Upper 95\%

$\begin{array}{lrrrrr}1 / 1 & 3 & 1.19585 & 0.00354 & 1.1877 & 1.2040 \\ 1 / 2 & 3 & 1.1753 & 0.00354 & 1.1672 & 1.1835\end{array}$

$\begin{array}{llllll}1 / 1 & 3 & 1.19585 & 0.00354 & 1.1877 & 1.2040 \\ 1 / 2 & 3 & 1.17533 & 0.00354 & 1.1672 & 1.1835\end{array}$

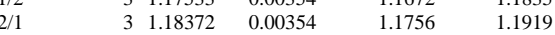

Std Error uses a pooled estimate of error variance 


\section{Exhibit A3. Measurements by Block and Sub-Block for Samples of the Batch 1 and Ustd Standards with the Initial Glasses by Oxide}

Oneway Analysis of Measured By Block/Sub-Block Type=Batch 1, Oxide $=\mathbf{C d O}(\mathrm{wt} \%)$

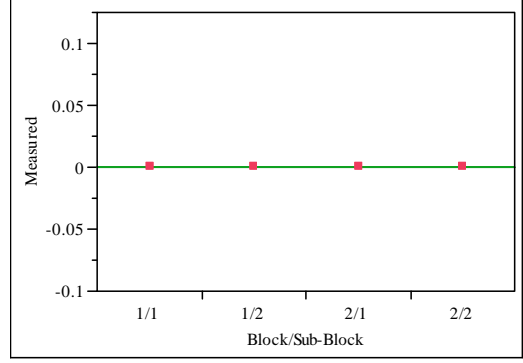

Oneway Anova

Summary of Fit

Rsquare

Adj Rsquare

Root Mean Square Erro

Mean of Response

Observations (or Sum Wgts) $\quad 12$

Analysis of Variance

Source DF Sum of Squares Mean Square F Ratio Prob $>$ F

Block/Sub-Block 3

Error

C. Total

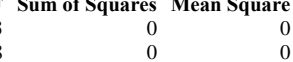

Means for Oneway Anova

Level Number Mean Std Error Lower 95\% Upper 95\%

\begin{tabular}{lrrrrr} 
Level & Number & Mean & Std Error & Lower 95\% & Upper 95\% \\
$1 / 1$ & 3 & 0.000571 & 0 & 0.00057 & 0.00057 \\
$1 / 2$ & 3 & 0.000571 & 0 & 0.00057 & 0.00057 \\
$2 / 1$ & 3 & 0.000571 & 0 & 0.00057 & 0.00057 \\
\hline & 3 & 0.000571 & 0 & 0.00057 & 0.00057
\end{tabular}

30.000571

0.00057

Std Error uses a pooled estimate of error variance

Oneway Analysis of Measured By Block/Sub-Block Type=Batch 1, Oxide $=\mathrm{Ce} 2 \mathrm{O3}(\mathrm{wt} \%)$

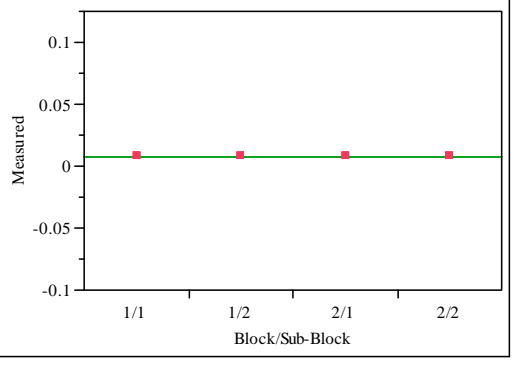

Oneway Anova

Summary of Fit

Rsquare

Adj Rsquare

Mean of Response

Observations (or Sum Wgts) $\quad 12$

Analysis of Variance

Source DF Sum of Squares Mean Square F Ratio Prob $>$ F

Block/Sub-Block 3

Error $\quad 11$

$\begin{array}{cc}0 & 0 \\ 0 & 0 \\ 0 & 0\end{array}$

Means for Oneway Anova

Level Number Mean Std Error Lower 95\% Upper 95\%

Lever Mean Std Error Lower 95\% Upper 95\%

$\begin{array}{llllll}1 / 2 & 3 & 0.007028 & 0 & 0.00703 & 0.00703 \\ 1 / 2 & 3 & 0.007028 & 0 & 0.00703 & 0.00703\end{array}$

$\begin{array}{llllll}1 / 2 & 3 & 0.007028 & 0 & 0.00703 & 0.00703 \\ 2 / 2 & 3 & 0.007028 & 0 & 0.00703 & 0.00703\end{array}$

Std Error uses a pooled estimate of error variance
Oneway Analysis of Measured By Block/Sub-Block Type=Batch 1, Oxide $=\mathbf{C r 2 O 3}(\mathbf{w t} \%)$

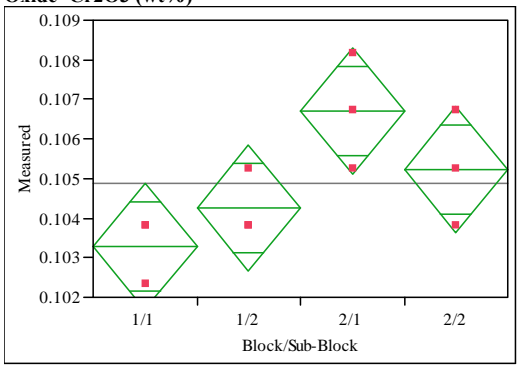

Oneway Anova

Summary of Fit

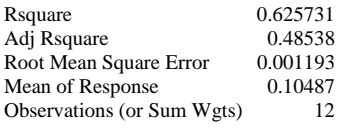

Observations (or Sum Wgts)

Analysis of Variance

Source DF Sum of Squares Mean Square F Ratio Prob $>$ F

$\begin{array}{llllll}\text { Block/Sub-Block } & 3 & 0.00001905 & 6.3495 \mathrm{e}-6 & 4.4583 & 0.0404\end{array}$

$\begin{array}{llll}\text { Error } & 8 & 0.00001139 & 1.4242 \mathrm{e}-6\end{array}$

$\begin{array}{lll}\text { C. Total } & 11 & 0.00003044\end{array}$

Means for Oneway Anova

Level Number Mean Std Error Lower 95\% Upper 95\%

$\begin{array}{llllll}1 / 1 & 3 & 0.103286 & 0.00069 & 0.10170 & 0.10488\end{array}$

$\begin{array}{llllll}1 / 2 & 3 & 0.104261 & 0.00069 & 0.10267 & 0.10585\end{array}$

$\begin{array}{llllll}2 / 1 & 3 & 0.106697 & 0.00069 & 0.10511 & 0.10829 \\ 2 / 2 & 3 & 0.105235 & 0.00069 & 0.10365 & 0.10682\end{array}$

Std Error uses a pooled estimate of error variance

Oneway Analysis of Measured By Block/Sub-Block Type=Batch 1, Oxide $=\mathrm{CuO}($ wt $\%)$

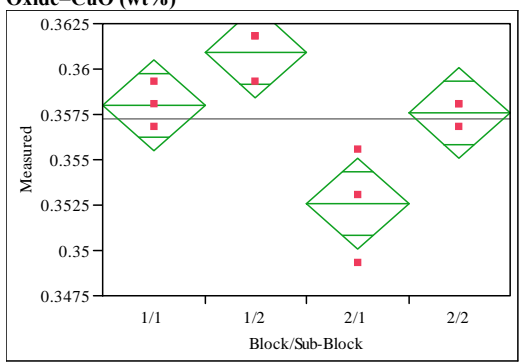

Oneway Anova

Summary of Fit

Rsquare

Root Mean Square Error $\quad 0.001878$

Mean of Response $\quad 0.357285$

Observations (or Sum Wgts) 12

Analysis of Variance

$\begin{array}{lllll}\text { Source DF Sum of Squares Mean Square F Ratio Prob }>\text { F } & \end{array}$

$\begin{array}{lrrrrr}\text { Source } & \text { DF } & \text { Sum of Squares } & \text { Mean Square } & \text { F Ratio } & \text { Prob }>\text { F } \\ \text { Block/Sub-Block } & 3 & 0.00010799 & 0.000036 & 10.2099 & 0.0041\end{array}$

$\begin{array}{lrrr}\text { Block/Sub-Block } & 3 & 0.00010799 & 0.000036 \\ \text { Error } & 8 & 0.00002821 & 3.526 \mathrm{e}-6\end{array}$

$\begin{array}{lrr}\text { C. Total } & 11 & 0.00013620\end{array}$

Means for Oneway Anova

Level Number Mean Std Error Lower 95\% Upper 95\%

$\begin{array}{llllll}1 / 1 & 3 & 0.358015 & 0.00108 & 0.35551 & 0.36051\end{array}$

$\begin{array}{llllll}1 / 2 & 3 & 0.360936 & 0.00108 & 0.35844 & 0.36344\end{array}$

$\begin{array}{llllll}2 / 1 & 3 & 0.352590 & 0.00108 & 0.35009 & 0.35509\end{array}$

$\begin{array}{llllll}2 / 2 & 3 & 0.357598 & 0.00108 & 0.35510 & 0.36010\end{array}$

Std Error uses a pooled estimate of error variance 


\section{Exhibit A3. Measurements by Block and Sub-Block for Samples of the Batch 1 and Ustd Standards with the Initial Glasses by Oxide}

Oneway Analysis of Measured By Block/Sub-Block Type=Batch 1, Oxide $=\mathrm{Fe} 2 \mathrm{O3}(\mathrm{w} \%$

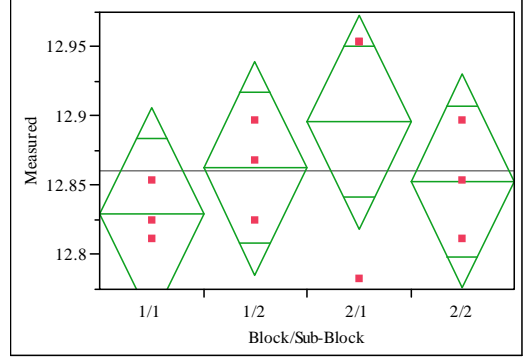

Oneway Anova

Summary of Fit

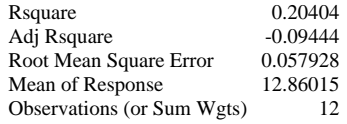

Observations (or Sum Wgts)

Analysis of Variance

Source DF Sum of Squares Mean Square F Ratio Prob $>$ F

$\begin{array}{llllll}\text { Block/Sub-Block } & 3 & 0.00688161 & 0.002294 & 0.6836 & 0.5866\end{array}$

$\begin{array}{llll}\text { Error } & 8 & 0.02684509 & 0.003356\end{array}$

$\begin{array}{lrr}\text { C. Total } & 11 & 0.03372669\end{array}$

Means for Oneway Anova

Level Number Mean Std Error Lower 95\% Upper 95\%

$\begin{array}{lllrrr}1 / 1 & 3 & 12.8292 & 0.03344 & 12.752 & 12.906\end{array}$

$\begin{array}{llllll}1 / 2 & 3 & 12.8625 & 0.03344 & 12.785 & 12.940 \\ 2 / 1 & 3 & 12.8959 & 0.03344 & 12.819 & 12.973\end{array}$

$\begin{array}{lllll}3 & 12.8959 & 0.03344 & 12.819 & 12.973 \\ 3 & 12.8530 & 0.03344 & 12.776 & 12.930\end{array}$

Std Error uses a pooled estimate of error variance

Oneway Analysis of Measured By Block/Sub-Block Type=Batch 1, Oxide $=\mathbf{K 2 O}(\mathbf{w t} \%)$

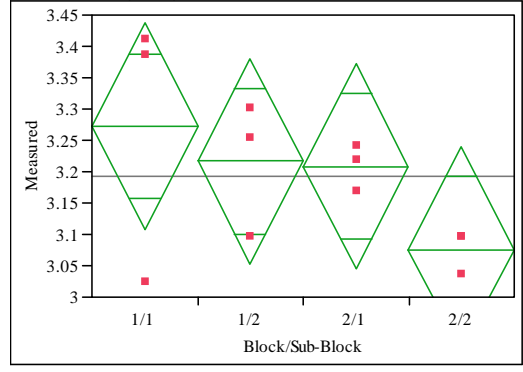

Oneway Anova

Summary of Fit

Rsquare $\quad 0.34013$

Adj Rsquare $\quad 0.092679$

Root Mean Square Error $\quad 0.123141$

Mean of Response 3.193194

Observations (or Sum Wgts) 12

Analysis of Variance

$\begin{array}{lrrrr}\text { Source } & \text { DF } & \text { Sum of Squares Mean Square F Ratio Prob }>\text { F } \\ \text { Block/Sub-Block } & 3 & 0.06252864 & 0.020843 & \end{array}$

$\begin{array}{lll}\text { Brock/Sub-Block } & 3 & 0.06252864 \\ \text { Error } & 8 & 0.12130871 \\ \text { C. } & 11 & 0.18383736\end{array}$

$\begin{array}{lll}\text { C. Total } & 11 & 0.18383736\end{array}$

Means for Oneway Anova

Level Number Mean Std Error Lower 95\% Upper 95\%

$\begin{array}{lrrrrr}1 / 1 & 3 & 3.27250 & 0.07110 & 3.1086 & 3.4364\end{array}$

$\begin{array}{llllll}1 / 2 & 3 & 3.21628 & 0.07110 & 3.0523 & 3.3802\end{array}$

$\begin{array}{llllll}2 / 1 & 3 & 3.20825 & 0.07110 & 3.0443 & 3.3722 \\ 2 / 2 & 3 & 3.07575 & 0.07110 & 2.9118 & 3.2397\end{array}$

Std Error uses a pooled estimate of error variance
Oneway Analysis of Measured By Block/Sub-Block Type=Batch 1, Oxide=La2O3 $(\mathbf{w t} \%)$

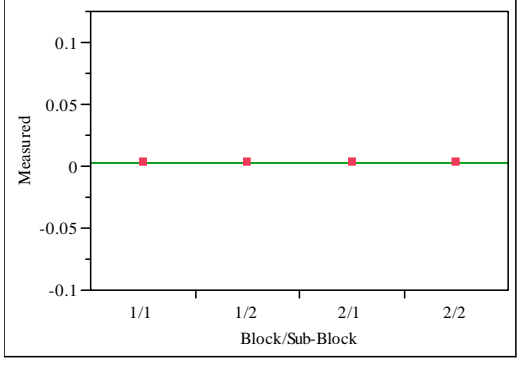

Oneway Anova

Summary of Fit

Rsquare

Adj Rsquare

$\begin{array}{lr}\text { Root Mean Square Error } & \dot{0} \\ \text { Mean of Response } & 0.002932\end{array}$

Observations (or Sum Wgts) $\quad 12$

Analysis of Variance

$\begin{array}{lrrrrr}\text { Source } & \text { DF } & \text { Sum of Squares } & \text { Mean Square } & \text { F Ratio } & \text { Prob }>\text { F } \\ \text { Block/Sub-Block } & 3 & 0 & 0 & . \\ \text { Error } & 8 & 0 & 0 & \\ \text { C. Total } & 11 & 0 & & \end{array}$

Means for Oneway Anova

Level Number Mean Std Error Lower 95\% Upper 95\%

$\begin{array}{lrrrrr}1 / 1 & 3 & 0.002932 & 0 & 0.00293 & 0.00293 \\ 1 / 2 & 3 & 0.002932 & 0 & 0.00293 & 0.00293 \\ 2 / 1 & 3 & 0.002932 & 0 & 0.00293 & 0.00293\end{array}$

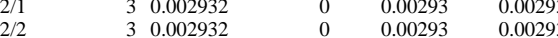

Std Error uses a pooled estimate of error variance

Oneway Analysis of Measured By Block/Sub-Block Type=Batch 1, Oxide $=\mathrm{Li} 20(\mathrm{wt} \%)$

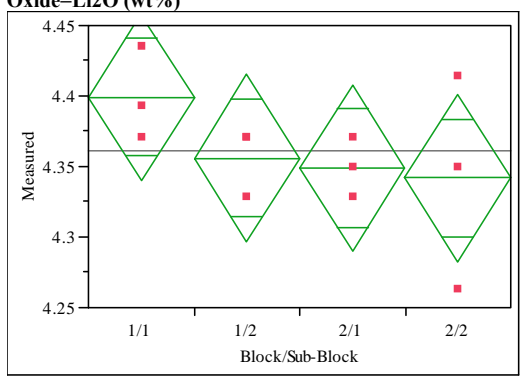

Oneway Anova

Summary of Fit

$\begin{array}{lr}\text { Rsquare } & 0.275311 \\ \text { Adj Rsquare } & 0.003552 \\ \text { Root Mean Square Error } & 0.044383 \\ \text { Mean of Response } & 4.361417 \\ \text { Observations (or Sum Wgts) } & 12\end{array}$

Analysis of Variance

Source DF Sum of Squares Mean Square F Ratio Prob $>$ F $\begin{array}{llllll}\text { Block/Sub-Block } & 3 & 0.00598685 & 0.001996 & 1.0131 & 0.4360\end{array}$ $\begin{array}{lrrr}\text { Error } & 8 & 0.01575893 & 0.001970\end{array}$

$\begin{array}{lrr}\text { Crror } & 11 & 0.02174577\end{array}$

Means for Oneway Anova

Level Number Mean Std Error Lower 95\% Upper 95\%

$\begin{array}{lllrrr}1 / 1 & 3 & 4.39909 & 0.02562 & 4.3400 & 4.4582\end{array}$

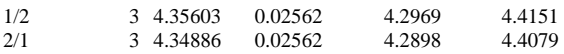

$\begin{array}{llllll}2 / 2 & 3 & 4.34168 & 0.02562 & 4.2826 & 4.4008\end{array}$

Std Error uses a pooled estimate of error variance 


\section{Exhibit A3. Measurements by Block and Sub-Block for Samples of the Batch 1 and Ustd Standards with the Initial Glasses by Oxide}

Oneway Analysis of Measured By Block/Sub-Block Type=Batch 1, Oxide $=\mathbf{M g O}(\mathbf{w t} \%)$

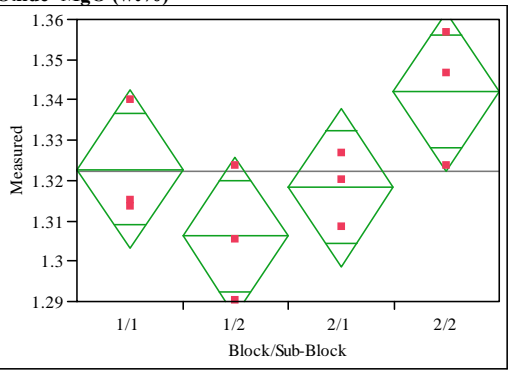

Oneway Anova

Summary of Fit

Rsquare

Adj Rsquare

Observations (or Sum Wgts)

Analysis of Variance
Source DF Sum of Squares Mean Square F Ratio Prob > F

$\begin{array}{lrrrrr}\text { Source } & \text { DF } & \text { Sum of Squares } & \text { Mean Square } & \text { F Ratio } & \text { Prob > F } \\ \text { Block/Sub-Block } & 3 & 0.00200449 & 0.000668 & 3.0659 & 0.0911\end{array}$

$\begin{array}{lrrr}\text { Error } & 8 & 0.00174347 & 0.000218 \\ \text { C. Total } & 11 & 0.00374796 & \end{array}$

Means for Oneway Anova

Level Number Mean Std Error Lower 95\% Upper 95\%

$\begin{array}{llllll}1 / 1 & 3 & 1.32277 & 0.00852 & 1.3031 & 1.3424\end{array}$

$\begin{array}{llllll}1 / 2 & 3 & 1.30619 & 0.00852 & 1.2865 & 1.3258\end{array}$

$\begin{array}{llllll}2 / 1 & 3 & 1.31835 & 0.00852 & 1.2987 & 1.3380\end{array}$

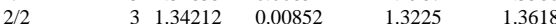

Std Error uses a pooled estimate of error variance

Oneway Analysis of Measured By Block/Sub-Block Type=Batch 1, Oxide $=\mathrm{MnO}(\mathrm{wt} \%)$

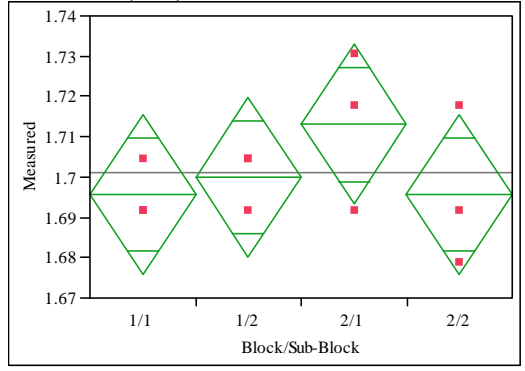

Oneway Anova

Summary of Fit

$\begin{array}{lr}\text { Rsquare } & 0.251462 \\ \text { Adj Rsquare } & -0.02924 \\ \text { Root Mean Square Error } & 0.014909 \\ \text { Mean of Response } & 1.701156 \\ \text { Observations (or Sum Wgts) } & 12\end{array}$

Observations (or Sum Wgts) 12

Analysis of Variance

Source DF Sum of Squares Mean Square F Ratio Prob $>$ F

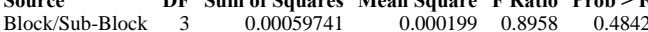

Error -0.00177834

$\begin{array}{lll}\text { C. Total } & 11 & 0.00237576\end{array}$

Means for Oneway Anova

Level Number Mean Std Error Lower 95\% Upper 95\%

$\begin{array}{lrrrrr}1 / 1 & 3 & 1.69578 & 0.00861 & 1.6759 & 1.7156 \\ 1 / 2 & 3 & 1.70008 & 0.00861 & 1.6802 & 1.7199\end{array}$

$\begin{array}{llllll}1 / 2 & 3 & 1.70008 & 0.00861 & 1.6802 & 1.7199 \\ 2 / 1 & 3 & 1.71299 & 0.00861 & 1.6931 & 1.7328\end{array}$

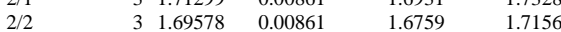

Std Error uses a pooled estimate of error variance
Oneway Analysis of Measured By Block/Sub-Block Type=Batch 1, Oxide $=\mathbf{N a 2 O}(\mathbf{w t} \%)$

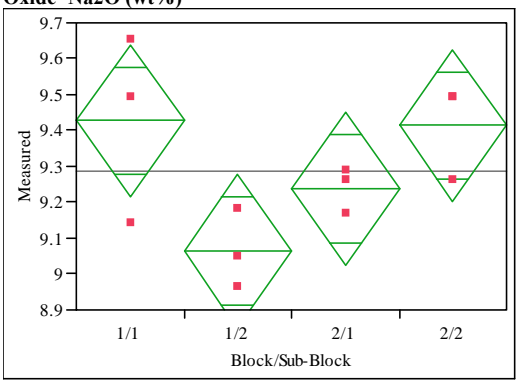

Oneway Anova

Summary of Fit

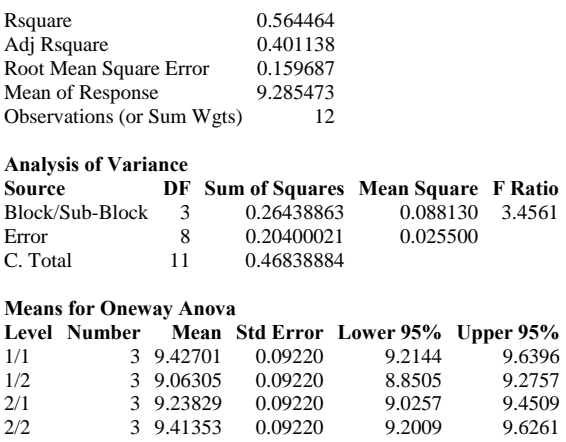

Std Error uses a pooled estimate of error variance

Oneway Analysis of Measured By Block/Sub-Block Type=Batch 1, Oxide $=\mathbf{N i O}(\mathbf{w t} \%)$

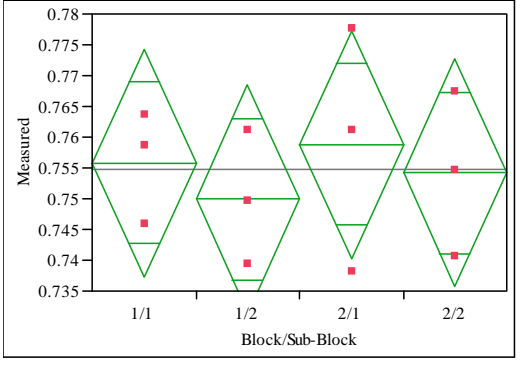

Oneway Anova

Summary of Fit

Rsquare

Root Mean Square Error $\quad 0.013896$

Mean of Response 0.754699

Observations (or Sum Wgts) 12

Analysis of Variance

Source DF Sum of Squares Mean Square F Ratio Prob $>$ F

$\begin{array}{lrrrrr}\text { Block/Sub-Block } & 3 & 0.00012455 & 0.000042 & 0.2150 & 0.8833\end{array}$

$\begin{array}{lll}\text { Error } & 8 & 0.00154477\end{array}$

$\begin{array}{lrr}\text { C. Total } & 11 & 0.00166932\end{array}$

Means for Oneway Anova

Level Number Mean Std Error Lower 95\% Upper 95\%

$\begin{array}{llllll}1 / 1 & 3 & 0.755865 & 0.00802 & 0.73736 & 0.77437\end{array}$

$\begin{array}{llllll}1 / 2 & 3 & 0.749927 & 0.00802 & 0.73143 & 0.76843 \\ & 3 & 0.758834 & 0.00802 & 0.74033 & 0.77733\end{array}$

$\begin{array}{llllll}1 / 2 & 3 & 0.758834 & 0.00802 & 0.74033 & 0.77733 \\ 2 / 1 & 3 & 0.754168 & 0.00802 & 0.73567 & 0.77267\end{array}$

Std Error uses a pooled estimate of error variance 


\section{Exhibit A3. Measurements by Block and Sub-Block for Samples of the Batch 1 and Ustd Standards with the Initial Glasses by Oxide}

Oneway Analysis of Measured By Block/Sub-Block Type=Batch 1, Oxide $=\mathbf{P 2 O 5}(\mathrm{wt} \%)$

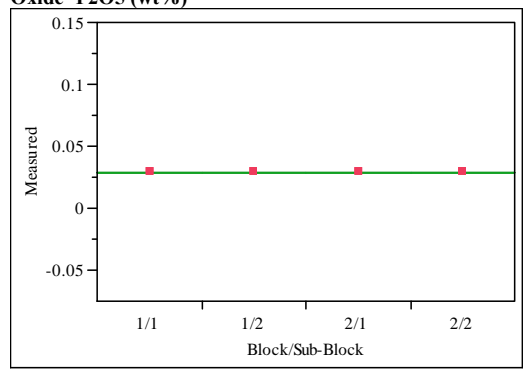

Oneway Anova

Summary of Fit

Rsquare

Adj Rsquare

Root Mean Square Erro

$\begin{array}{lr}\text { Mean of Response } & 0.028643 \\ \text { Observations (or Sum Wgts) } & 12\end{array}$

Analysis of Variance

Source DF Sum of Squares Mean Square F Ratio Prob $>$ F

Block/Sub-Block 3

Error
C.

8
11

Means for Oneway Anova

Level Number Mean Std Error Lower 95\% Upper 95\%

$\begin{array}{lrrrrr}1 / 1 & 3 & 0.028643 & 0 & 0.02864 & 0.02864\end{array}$

$\begin{array}{llllll}1 / 2 & 3 & 0.028643 & 0 & 0.02864 & 0.02864 \\ 2 / 1 & 3 & 0.028643 & 0 & 0.02864 & 0.02864\end{array}$

$\begin{array}{llllll}2 / 1 & 3 & 0.028643 & 0 & 0.02864 & 0.02864 \\ 2 / 2 & 3 & 0.028643 & 0 & 0.02864 & 0.02864\end{array}$

Std Error uses a pooled estimate of error variance

Oneway Analysis of Measured By Block/Sub-Block Type=Batch 1, Oxide $=\mathbf{P b O}(\mathbf{w t} \%)$

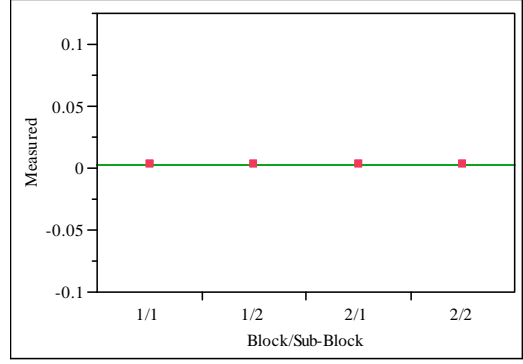

Oneway Anova

Summary of Fit

Rsquare

Adj Rsquare

ror $\quad 5.31 \mathrm{e}-19$

0.002693

Observations (or Sum Wgts) 12

Analysis of Variance

Source DF Sum of Squares Mean Square F Ratio Prob $>$ F

$\begin{array}{rrrrrr}\text { Block/Sub-Block } & 3 & 0 & 0 & 0.0000 & 1.0000\end{array}$

$\begin{array}{lrr}\text { Error } & 8 & 2.2569 \mathrm{e}-36 \\ \text { C. } & 11 & 2.2569 \mathrm{e}-36\end{array}$

Means for Oneway Anova

Level Number Mean Std Error Lower 95\% Upper 95\%

$\begin{array}{lrrrrr}\text { Level } & \text { Number } & \text { Mean } & \text { Std Error } & \text { Lower 95\% } & \text { Upper 95\% } \\ 1 / 1 & 3 & 0.002693 & 3.067 \mathrm{e}-19 & 0.00269 & 0.00269\end{array}$

$\begin{array}{llllll}1 / 1 & 3 & 0.002693 & 3.067 \mathrm{e}-19 & 0.00269 & 0.00269 \\ 1 / 2 & 3 & 0.002693 & 3.067 \mathrm{e}-19 & 0.00269 & 0.00269\end{array}$

$\begin{array}{llllll}1 / 2 & 3 & 0.002693 & 3.067 \mathrm{e}-19 & 0.00269 & 0.00269 \\ 2 / 1 & 3 & 0.002693 & 3.067 \mathrm{e}-19 & 0.00269 & 0.00269\end{array}$

$\begin{array}{llllll}2 / 2 & 3 & 0.002693 & 3.067 \mathrm{e}-19 & 0.00269 & 0.00269\end{array}$

Std Error uses a pooled estimate of error variance

Oneway Analysis of Measured By Block/Sub-Block Type=Batch 1, Oxide $=\mathrm{SiO} 2(\mathrm{wt} \%)$
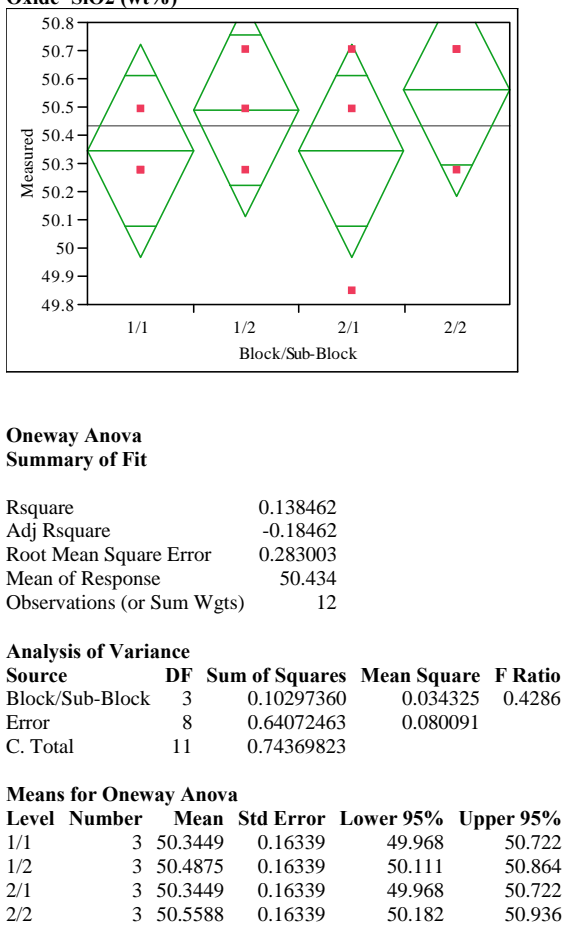

Std Error uses a pooled estimate of error variance

Oneway Analysis of Measured By Block/Sub-Block Type=Batch 1 , Oxide $=\mathrm{SO} 4(\mathrm{wt} \%)$

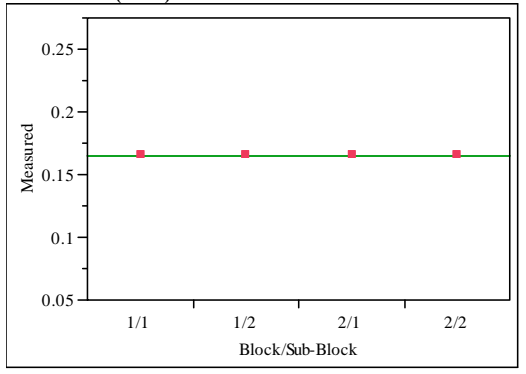

Oneway Anova

Summary of Fit

Rsquare

Adj Rsquare

$\begin{array}{lr}\text { Root Mean Square Error } & 0 \\ \text { Mean of Response } & 0.164775\end{array}$

Observations (or Sum Wgts) $\quad 12$

Analysis of Variance

$\begin{array}{lrrrrr}\text { Source } & \text { DF } & \text { Sum of Squares } & \text { Mean Square } & \text { F Ratio } & \text { Prob }>\text { F } \\ \text { Block/Sub-Block } & 3 & 0 & 0 & . \\ \text { Error } & 8 & 0 & 0 & \\ \text { C. Total } & 11 & 0 & & \end{array}$

Means for Oneway Anova

Level Number Mean Std Error Lower 95\% Upper 95\%

$\begin{array}{llllll}1 / 1 & 3 & 0.164775 & 0 & 0.16477 & 0.16477 \\ 1 / 2 & 3 & 0.164775 & 0 & 0.16477 & 0.16477\end{array}$

$\begin{array}{llllll}1 / 2 & 3 & 0.164775 & 0 & 0.16477 & 0.16477\end{array}$

$\begin{array}{llllll}2 / 1 & 3 & 0.164775 & 0 & 0.16477 & 0.16477\end{array}$

Std Error uses a pooled estimate of error variance 


\section{Exhibit A3. Measurements by Block and Sub-Block for Samples of the Batch 1 and Ustd Standards with the Initial Glasses by Oxide}

Oneway Analysis of Measured By Block/Sub-Block Type=Batch 1, Oxide $=\mathrm{TiO} 2(\mathrm{wt} \%)$

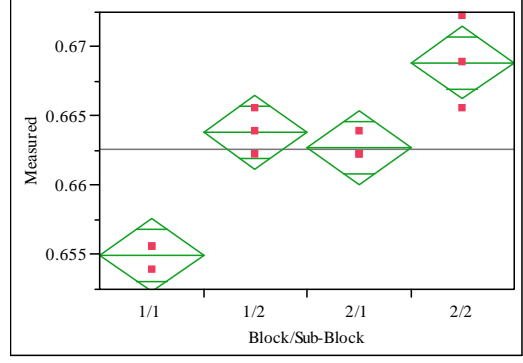

Oneway Anova

Summary of Fit

$\begin{array}{lr}\text { Rsquare } & 0.904158 \\ \text { Adj Rsquare } & 0.868217 \\ \text { Root Mean Square Error } & 0.001985 \\ \text { Mean of Response } & 0.662613 \\ \text { Observations (or Sum Wgts) } & 12\end{array}$

Observations (or Sum Wgts)

Analysis of Variance

Source DF Sum of Squares Mean Square F Ratio Prob $>$ F

$\begin{array}{lllllll}\text { Block/Sub-Block } 3 & 0.00029747 & 0.000099 & 25.1569 & 0.0002\end{array}$

Error

$8-0.00203153$

$3.941 \mathrm{e}-6$

Means for Oneway Anova

Level Number Mean Std Error Lower 95\% Upper 95\%

$\begin{array}{lllrrr}1 / 1 & 3 & 0.654968 & 0.00115 & 0.65232 & 0.65761\end{array}$

$\begin{array}{llllll}1 / 2 & 3 & 0.663864 & 0.00115 & 0.66122 & 0.66651 \\ 2 / 1 & 3 & 0.662752 & 0.00115 & 0.66011 & 0.66540\end{array}$

$\begin{array}{llllll}2 / 2 & 3 & 0.668868 & 0.00115 & 0.66622 & 0.67151\end{array}$

Std Error uses a pooled estimate of error variance

Oneway Analysis of Measured By Block/Sub-Block Type=Batch 1, Oxide $=\mathbf{U} 308(\mathrm{wt} \%)$

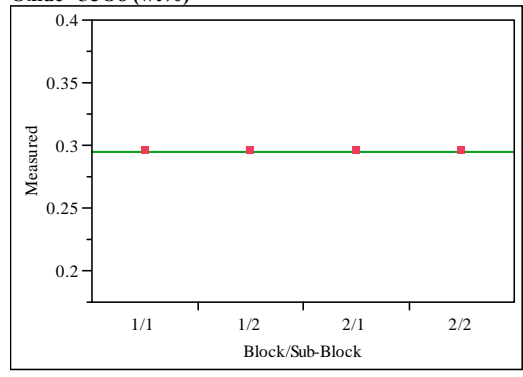

Oneway Anova

Summary of Fit

Rsquare

Adj Rsquare

Root Mean Square Error

Mean of Response

0.2948
Observations (or Sum Wgts)

Analysis of Variance

Source DF

Error

Sum of Squares Mean Square F Ratio Prob $>$ F

C. Tota

0

Means for Oneway Anova

Level Number Mean Std Error Lower 95\% Upper 95\%

$\begin{array}{lrrrrr}1 / 1 & 3 & 0.294800 & 0 & 0.29480 & 0.29480\end{array}$

$\begin{array}{llllll}1 / 2 & 3 & 0.294800 & 0 & 0.29480 & 0.29480\end{array}$

$\begin{array}{llllll}2 / 1 & 3 & 0.294800 & 0 & 0.29480 & 0.29480 \\ 2 / 2 & 3 & 0.294800 & 0 & 0.29480 & 0.29480\end{array}$

Std Error uses a pooled estimate of error variance
Oneway Analysis of Measured By Block/Sub-Block Type=Batch 1, Oxide $=\mathrm{ZnO}(\mathrm{wt} \%)$

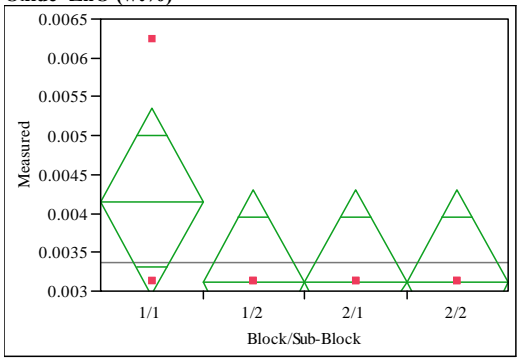

Oneway Anova Summary of Fit

Adj Rsquare

$2.22 \mathrm{e}-16$

Root Mean Square Error $\quad 0.000898$

Mean of Response

Observations (or Sum Wgts) 12

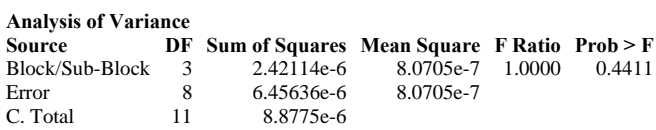

C. Total

$8.8775 \mathrm{e}-6$

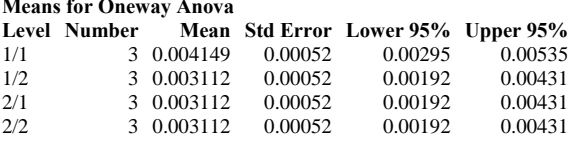

Std Error uses a pooled estimate of error variance

Oneway Analysis of Measured By Block/Sub-Block Type=Batch 1, Oxide $=\mathrm{ZrO2}(\mathrm{wt} \%)$

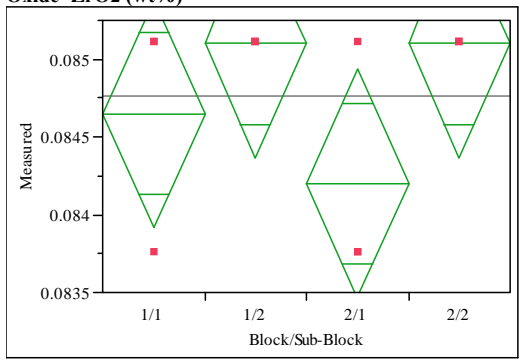

Oneway Anova

Summary of Fit

$\begin{array}{lr}\text { Rsquare } & 0.407407 \\ \text { Adj Rsquare } & 0.185185 \\ \text { Root Mean Square Error } & 0.000551 \\ \text { Mean of Response } & 0.084763 \\ \text { Observations (or Sum Wgts) } & 12\end{array}$

Analysis of Variance

Source DF Sum of Squares Mean Square F Ratio Prob $>$ F

$\begin{array}{llll}\text { Error } & 8 & 2.43288 \mathrm{e}-6 & 3.0411 \mathrm{e}-7\end{array}$

$\begin{array}{lll}\text { C. Total } & 11 & 4.10549 \mathrm{e}-6\end{array}$

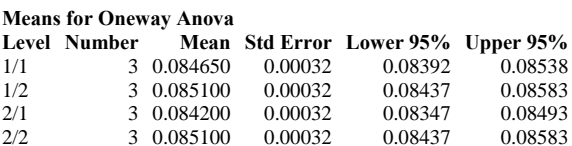

Std Error uses a pooled estimate of error variance 


\section{Exhibit A3. Measurements by Block and Sub-Block for Samples of the Batch 1 and Ustd Standards with the Initial Glasses by Oxide}

Oneway Analysis of Measured By Block/Sub-Block Type=Ustd, Oxide $=\mathbf{A l} 203(\mathbf{w t} \%)$

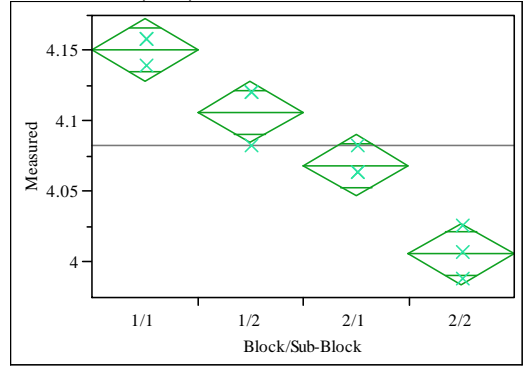

Oneway Anova

Summary of Fit

Rsquare

Adj Rsquare

Observations (or Sum Wgts)

$\begin{array}{lllll}\text { Analysis of Variance } \\ \text { Source } & \text { DF } & \text { Sum of Squares Mean Square F Ratio Prob }>\text { F }\end{array}$

$\begin{array}{lrrrrr}\text { Source } & \text { D F } & \text { Sum of Squares } & \text { Mean Square } & \text { F Ratio } & \text { Prob }>\text { r } \\ \text { Block/Sub-Block } & 3 & 0.03388725 & 0.011296 & 42.1852 & <.0001\end{array}$

$\begin{array}{lll}\text { Error } & 8 & 0.00214213\end{array}$

$\begin{array}{lrr} & 8 & 0.00214213 \\ \text { C. Total } & 11 & 0.03602937\end{array}$

Means for Oneway Anova

Level Number Mean Std Error Lower 95\% Upper 95\%

$\begin{array}{llllll}1 / 1 & 3 & 4.15060 & 0.00945 & 4.1288 & 4.1724\end{array}$

$\begin{array}{llllll}1 / 2 & 3 & 4.10651 & 0.00945 & 4.0847 & 4.1283\end{array}$

$\begin{array}{llllll}2 / 1 & 3 & 4.06872 & 0.00945 & 4.0469 & 4.0905\end{array}$

Std Error uses a pooled estimate of error variance

Oneway Analysis of Measured By Block/Sub-Block Type=Ustd, Oxide=B2O3 (wt \%)

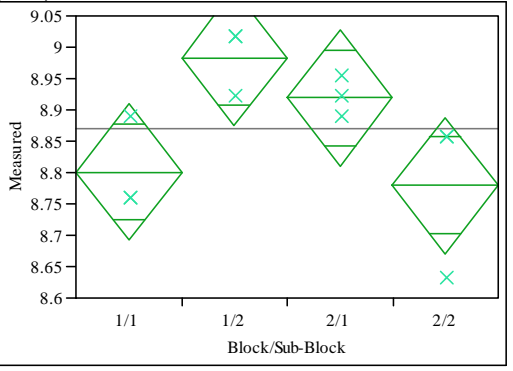

Oneway Anova

Summary of Fit

$\begin{array}{lr}\text { Rsquare } & 0.614035 \\ \text { Adj Rsquare } & 0.469298 \\ \text { Root Mean Square Error } & 0.081564 \\ \text { Mean of Response } & 8.870825 \\ \text { Observations (or Sum Wgts) } & 12\end{array}$

Analysis of Variance

Source DF Sum of Squares Mean Square F Ratio Prob > F

$\begin{array}{lrrrrr}\text { Block/Sub-Block } & 3 & 0.08467001 & 0.028223 & 4.2424 & 0.0454\end{array}$

$\begin{array}{llll}\text { Error } & 8 & 0.05322115 & 0.006653\end{array}$

$\begin{array}{lll}\text { C. Total } & 11 & 0.13789115\end{array}$

Means for Oneway Anova

Level Number Mean Std Error Lower 95\% Upper 95\%

$\begin{array}{llllll}1 / 1 & 3 & 8.80106 & 0.04709 & 8.6925 & 8.9097\end{array}$

$\begin{array}{llllll}1 / 2 & 3 & 8.98352 & 0.04709 & 8.8749 & 9.0921\end{array}$

$\begin{array}{llllll}2 / 1 & 3 & 8.91912 & 0.04709 & 8.8105 & 9.0277\end{array}$

Std Error uses a pooled estimate of error variance
Oneway Analysis of Measured By Block/Sub-Block Type $=$ Ustd, Oxide $=\mathbf{B a O}$ (wt\%)

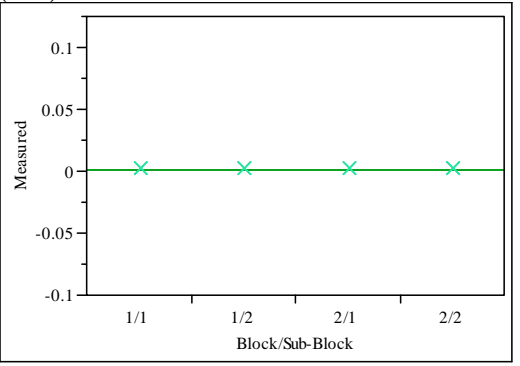

Oneway Anova

Summary of Fit

Rsquare

Adj Rsquare

$\begin{array}{lr}\text { Root Mean Square Error } & \dot{0} \\ \text { Mean of Response } & 0.001675 \\ \text { Observations (or Sum Wgts) } & 12\end{array}$

Observations (or Sum Wgts) $\quad 12$

Analysis of Variance

Source DF Sum of Squares Mean Square F Ratio Prob $>$ F

Block/Sub-Block 3

Error $\quad 8$

$\begin{array}{lll}3 & 0 & 0 \\ 8 & 0 & 0\end{array}$

Means for Oneway Anova

$\begin{array}{lrrrrr}\text { Level } & \text { Number } & \text { Mean } & \text { Std Error } & \text { Lower 95\% } & \text { Upper 95\% } \\ 1 / 1 & 3 & 0.001675 & 0 & 0.00167 & 0.00167 \\ 1 / 2 & 3 & 0.001675 & 0 & 0.00167 & 0.00167 \\ 2 / 1 & 3 & 0.001675 & 0 & 0.00167 & 0.00167 \\ 2 / 2 & 3 & 0.001675 & 0 & 0.00167 & 0.00167\end{array}$

0.001675

0.00167

Std Error uses a pooled estimate of error variance

Oneway Analysis of Measured By Block/Sub-Block Type $=$ Ustd, Oxide $=\mathrm{CaO}$ $(\mathrm{wt} \%)$

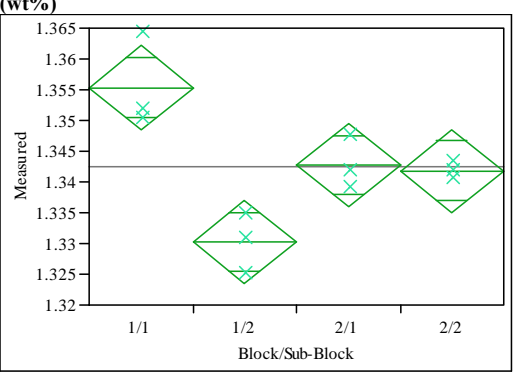

Oneway Anova

Summary of Fit

Adj Rsquare 0.821248

Root Mean Square Error $\quad 0.005093$

Observations (or Sum Wgts)

$\begin{array}{lrrrrr}\begin{array}{l}\text { Analysis of Variance } \\ \text { Source }\end{array} & \text { DF } & \text { Sum of Squares } & \text { Mean Square } & \text { F Ratio } & \text { Prob }>\text { F } \\ \text { Block/Sub-Block } & 3 & 0.00095343 & 0.000318 & 12.2516 & 0.0023 \\ \text { Error } & 8 & 0.00020752 & 0.000026 & & \\ \text { C. Total } & 11 & 0.00116095 & & & \end{array}$

Means for Oneway Anova

Level Number Mean Std Error Lower 95\% Upper 95\%

$\begin{array}{llrrrr}1 / 1 & 3 & 1.35536 & 0.00294 & 1.3486 & 1.3621\end{array}$

$\begin{array}{llllll}1 / 2 & 3 & 1.33017 & 0.00294 & 1.3234 & 1.3370\end{array}$

$\begin{array}{llllll}2 / 1 & 3 & 1.34277 & 0.00294 & 1.3360 & 1.3495\end{array}$

Std Error uses a pooled estimate of error variance 


\section{Exhibit A3. Measurements by Block and Sub-Block for Samples of the Batch 1 and Ustd Standards with the Initial Glasses by Oxide}

Oneway Analysis of Measured By Block/Sub-Block Type $=$ Ustd, Oxide $=$ CdO

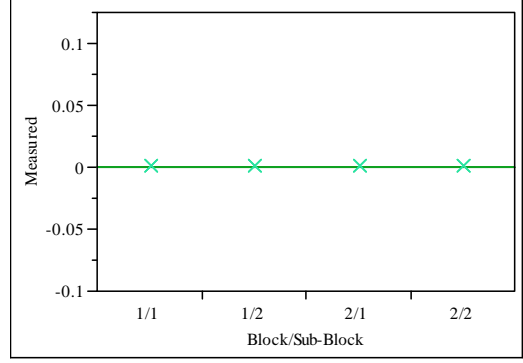

Oneway Anova

Summary of Fit

Rsquare

Adj Rsquare

Root Mean Square Error

Mean of Response

Observations (or Sum Wgts) $\begin{array}{r}0.000571 \\ \hline\end{array}$

Analysis of Variance

Source DF Sum of Squares Mean Square F Ratio Prob $>$ F

Block/Sub-Block 3

Error

C. Total

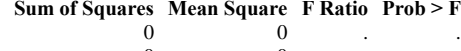

Means for Oneway Anova

Level Number Mean Std Error Lower 95\% Upper 95\%

\begin{tabular}{lrrrrr} 
Level Number & Mean & Std Error & Lower $\mathbf{9 5 \%}$ & Upper 95\% \\
$1 / 1$ & 3 & 0.000571 & 0 & 0.00057 & 0.00057 \\
$1 / 2$ & 3 & 0.000571 & 0 & 0.00057 & 0.00057 \\
$2 / 1$ & 3 & 0.000571 & 0 & 0.00057 & 0.00057 \\
\hline & 3 & 0.000571 & 0 & 0.00057 & 0.00057
\end{tabular}

30.000571

$0.00057 \quad 0.00057$

Std Error uses a pooled estimate of error variance

Oneway Analysis of Measured By Block/Sub-Block Type=Ustd, Oxide $=\mathrm{Ce} 2 \mathrm{O3}(\mathrm{wt} \%)$

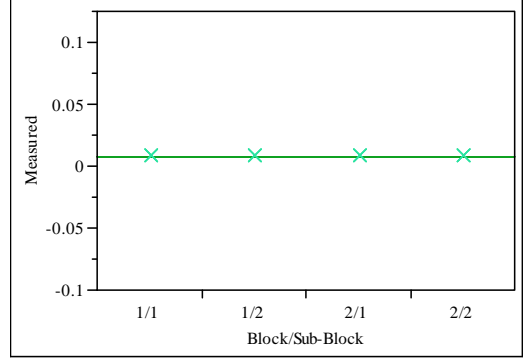

Oneway Anova

Summary of Fit

Rsquare

Adj Rsquare

Mean of Response

Observations (or Sum Wgts) $\quad 12$

Analysis of Variance

Source DF Sum of Squares Mean Square F Ratio Prob $>$ F

Block/Sub-Block 3

Error $\quad 11$

$\begin{array}{ll}0 & 0 \\ 0 & 0 \\ 0 & \end{array}$

Means for Oneway Anova

Level Number Mean Std Error Lower 95\% Upper 95\%

$1 / 1$ Number Mean Std Error Lower $95 \%$ Upper 95\%

$\begin{array}{llllll}1 / 2 & 3 & 0.007028 & 0 & 0.00703 & 0.00703 \\ 1 / 2 & 3 & 0.007028 & 0 & 0.00703 & 0.00703\end{array}$

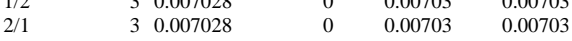

$\begin{array}{llllll}2 / 2 & 3 & 0.007028 & 0 & 0.00703 & 0.00703\end{array}$

Std Error uses a pooled estimate of error variance
Oneway Analysis of Measured By Block/Sub-Block Type=Ustd, Oxide $=\mathbf{C r 2 O 3}(\mathbf{w t} \%)$

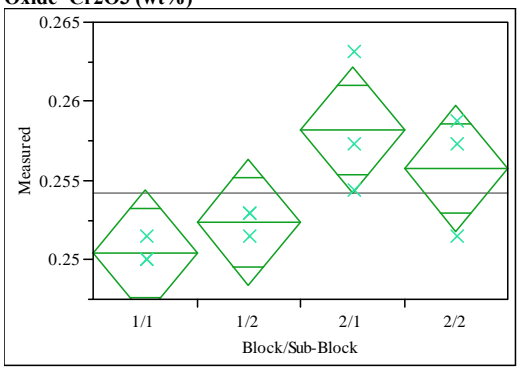

Oneway Anova

Summary of Fit

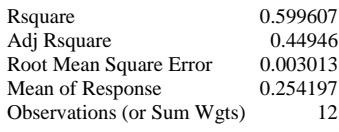

Observations (or Sum Wgts)

Analysis of Variance

Source DF Sum of Squares Mean Square F Ratio Prob $>$ F

$\begin{array}{llllll}\text { Block/Sub-Block } & 3 & 0.00010877 & 0.000036 & 3.9935 & 0.0521\end{array}$

$\begin{array}{llll}\text { Error } & 8 & 0.00007263 & 9.079 \mathrm{e}-6\end{array}$

$\begin{array}{lll}\text { C. Total } & 11 & 0.00018141\end{array}$

Means for Oneway Anova

Level Number Mean Std Error Lower 95\% Upper 95\%

$\begin{array}{llllll}1 / 1 & 3 & 0.250421 & 0.00174 & 0.24641 & 0.25443\end{array}$

$\begin{array}{llllll}1 / 2 & 3 & 0.252370 & 0.00174 & 0.24836 & 0.25638 \\ 2 / 1 & 3 & 0.258216 & 0.00174 & 0.25420 & 0.26223\end{array}$

$\begin{array}{llllll}2 / 2 & 3 & 0.255780 & 0.00174 & 0.25177 & 0.25979\end{array}$

Std Error uses a pooled estimate of error variance

Oneway Analysis of Measured By Block/Sub-Block Type $=$ Ustd, Oxide $=\mathrm{CuO}$ (wt\%)

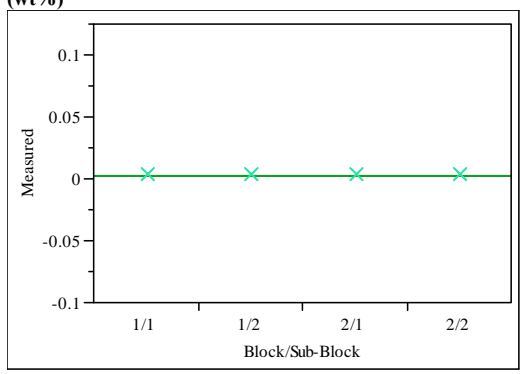

Oneway Anova

Summary of Fit

Rsquare

Adj Rsquare

Root Mean Square Error $\quad 0$

$\begin{array}{lr}\text { Mean of Response } & 0.001878 \\ \text { Observations (or Sum Wgts) } & 12\end{array}$

$\begin{array}{lrrrrr}\begin{array}{l}\text { Analysis of Variance } \\ \text { Source }\end{array} & \text { DF } & \text { Sum of Squares } & \text { Mean Square } & \text { F Ratio } & \text { Prob }>\text { F } \\ \text { Block/Sub-Block } & 3 & 0 & 0 & . \\ \text { Error } & 8 & 0 & 0 & \\ \text { C. Total } & 11 & 0 & & & \end{array}$

Means for Oneway Anova

Level Number Mean Std Error Lower 95\% Upper 95\%

$\begin{array}{llllll}1 / 1 & 3 & 0.001878 & 0 & 0.00188 & 0.00188 \\ 1 / 2 & 3 & 0.001878 & 0 & 0.00188 & 0.00188\end{array}$

$\begin{array}{llllll}2 / 1 & 3 & 0.001878 & 0 & 0.00188 & 0.00188\end{array}$

Std Error uses a pooled estimate of error variance 


\section{Exhibit A3. Measurements by Block and Sub-Block for Samples of the Batch 1 and Ustd Standards with the Initial Glasses by Oxide}

Oneway Analysis of Measured By Block/Sub-Block Type=Ustd, Oxide $=\mathrm{Fe} 2 \mathrm{O3}(\mathrm{wt} \%)$

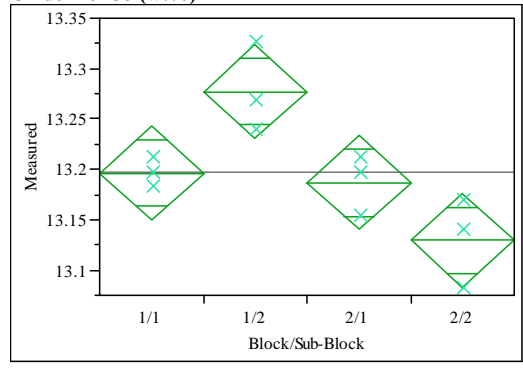

Oneway Anova

Summary of Fi

$\begin{array}{lr}\text { Rsquare } & 0.772422 \\ \text { Adj Rsquare } & 0.68708 \\ \text { Root Mean Square Error } & 0.03502 \\ \text { Mean of Response } & 13.19732 \\ \text { Observations (or Sum Wgts) } & 12\end{array}$

Analysis of Variance

Source DF Sum of Squares Mean Square F Ratio Prob $>$ F

$\begin{array}{llllll}\text { Block/Sub-Block } & 3 & 0.03330085 & 0.011100 & 9.0509 & 0.0060\end{array}$

Error

$0.00981140 \quad 0.00122$

$\begin{array}{lll}\text { C. Total } & 11 & 0.04311225\end{array}$

Means for Oneway Anova

Level Number Mean Std Error Lower 95\% Upper 95\%

$\begin{array}{lrrrrr}1 / 1 & 3 & 13.1961 & 0.02022 & 13.150 & 13.243\end{array}$

$\begin{array}{llllll}1 / 2 & 3 & 13.2771 & 0.02022 & 13.231 & 13.324 \\ 2 / 1 & 3 & 13.1866 & 0.02022 & 13.140 & 13.233\end{array}$

$\begin{array}{lllll}3 & 13.1866 & 0.02022 & 13.140 & 13.233 \\ 3 & 13.1294 & 0.02022 & 13.083 & 13.176\end{array}$

Std Error uses a pooled estimate of error variance

Oneway Analysis of Measured By Block/Sub-Block Type $=$ Ustd, Oxide $=$ K2O (wit\%)

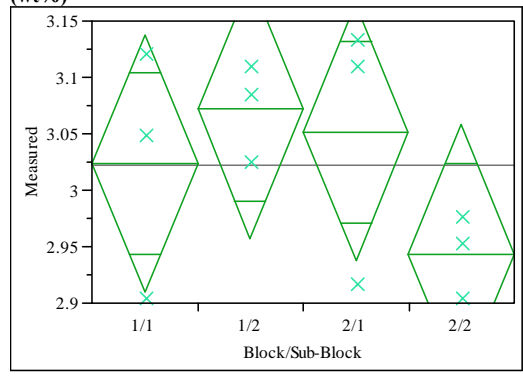

Oneway Anova

Summary of Fit

Rsquare

0.326629

Adj Rsquare

0.074115

Root Mean Square Error $\quad 0.085955$

Mean of Response 3.022542

Observations (or Sum Wgts) 12

Analysis of Variance

$\begin{array}{lrrrrr}\text { Source } & \text { DF } & \text { Sum of Squares } & \text { Mean Square } & \text { F Ratio } & \text { Prob > F } \\ \text { Block/Sub-Block } & 3 & 0.02867055 & 0.009557 & 1.2935 & 0.3414\end{array}$

Block/Sub-Block $3 \quad 0.02867055$

$\begin{array}{lrr}\text { Error } & 8 & 0.05910656\end{array}$

0.007388

Means for Oneway Anova

Level Number Mean Std Error Lower 95\% Upper 95\%

$\begin{array}{lrrrrr}1 / 1 & 3 & 3.02355 & 0.04963 & 2.9091 & 3.1380\end{array}$

$\begin{array}{lllll}3 & 3.07173 & 0.04963 & 2.9573 & 3.1862\end{array}$

$\begin{array}{llllll}2 / 1 & 3 & 3.05165 & 0.04963 & 2.9372 & 3.1661 \\ 2 / 2 & 3 & 2.94324 & 0.04963 & 2.8288 & 3.0577\end{array}$

Std Error uses a pooled estimate of error variance
Oneway Analysis of Measured By Block/Sub-Block Type=Ustd, Oxide $=\mathbf{L a 2 O 3}(\mathbf{w t} \%)$

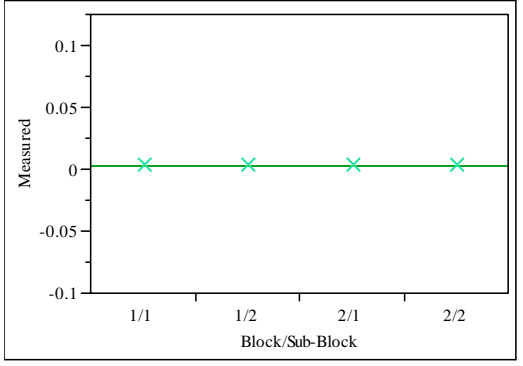

Oneway Anova

Summary of Fit

Rsquare

Adj Rsquare

$\begin{array}{lr}\text { Root Mean Square Error } & 0 \\ \text { Mean of Response } & 0.002932\end{array}$

Observations (or Sum Wgts) $\quad 12$

Analysis of Variance

$\begin{array}{lrrrrr}\text { Source } & \text { DF } & \text { Sum of Squares } & \text { Mean Square } & \text { F Ratio } & \text { Prob }>\text { F } \\ \text { Block/Sub-Block } & 3 & 0 & 0 & . \\ \text { Error } & 8 & 0 & 0 & \\ \text { C. Total } & 11 & 0 & & \end{array}$

Means for Oneway Anova

$\begin{array}{lrrrrr}\text { Level } & \text { Number } & \text { Mean } & \text { Std Error } & \text { Lower 95\% } & \text { Upper 95\% } \\ 1 / 1 & 3 & 0.002932 & 0 & 0.00293 & 0.00293 \\ 1 / 2 & 3 & 0.002932 & 0 & 0.00293 & 0.00293 \\ 2 / 1 & 3 & 0.002932 & 0 & 0.00293 & 0.00293 \\ 2 / 2 & 3 & 0.002932 & 0 & 0.00293 & 0.00293\end{array}$

Std Error uses a pooled estimate of error variance

Oneway Analysis of Measured By Block/Sub-Block Type=Ustd, Oxide=Li2O $(\mathrm{wt} \%)$

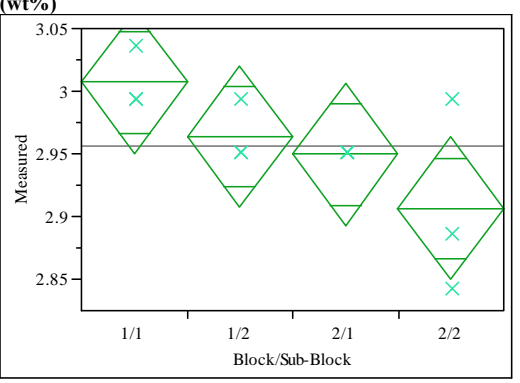

Oneway Anova

Summary of Fit

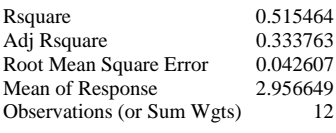

Observations (or Sum Wgts)

Analysis of Variance

Source DF Sum of Squares Mean Square F Ratio Prob $>$ F

$\begin{array}{llllll}\text { Block/Sub-Block } & 3 & 0.01544993 & 0.005150 & 2.8369 & 0.1059\end{array}$

$\begin{array}{llll}\text { Error } & 8 & 0.01452293 & 0.001815\end{array}$

$\begin{array}{lll}\text { C. Total } & 11 \quad 0.02997286\end{array}$

Means for Oneway Anova

Level Number Mean Std Error Lower 95\% Upper 95\%

$\begin{array}{lllrrr}1 / 1 & 3 & 3.00688 & 0.02460 & 2.9502 & 3.0636\end{array}$

$\begin{array}{llllll}1 / 2 & 3 & 2.96383 & 0.02460 & 2.9071 & 3.0206 \\ 2 / 1 & 3 & 2.94947 & 0.02460 & 2.8927 & 3.0062\end{array}$

$\begin{array}{llllll}2 / 2 & 3 & 2.90642 & 0.02460 & 2.8497 & 2.9631\end{array}$

Std Error uses a pooled estimate of error variance 


\section{Exhibit A3. Measurements by Block and Sub-Block for Samples of the Batch 1 and Ustd Standards with the Initial Glasses by Oxide}

Oneway Analysis of Measured By Block/Sub-Block Type=Ustd, Oxide=MgO

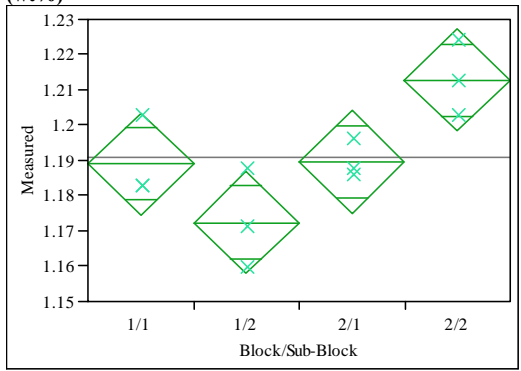

Oneway Anova

Summary of Fit

Rsquare

Adj Rsquare

Observations (or Sum Wgts)

$\begin{array}{llll}\text { Analysis of Variance } & \\ \text { Source DF Sum of Squares Mean Square F Ratio Prob > F }\end{array}$

$\begin{array}{lrrrrr} & \text { DF } & \text { Sum of Squares } & \text { Mean Square } & \text { F Ratio } & \text { Prob }>\text { F } \\ \text { Block/Sub-Block } & 3 & 0.00247588 & 0.000825 & 6.9123 & 0.0130\end{array}$

$\begin{array}{lll}\text { Error } & 8 & 0.00095515 \\ \text { C. Tola } & 11 & 0.00343103\end{array}$

$\begin{array}{lrr}\text { C. Total } & 11 & 0.00343103\end{array}$

Means for Oneway Anova

Level Number Mean Std Error Lower 95\% Upper 95\%

$\begin{array}{llllll}1 / 1 & 3 & 1.18900 & 0.00631 & 1.1745 & 1.2035 \\ 1 / 2 & 3 & 1.17242 & 0.00631 & 1.1579 & 1.1870\end{array}$

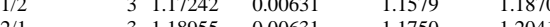

$\begin{array}{llllll}2 / 1 & 3 & 1.18955 & 0.00631 & 1.1750 & 1.2041\end{array}$

Std Error uses a pooled estimate of error variance

Oneway Analysis of Measured By Block/Sub-Block Type $=$ Ustd, Oxide $=\mathbf{M n O}$ $(\mathrm{wt} \%)$

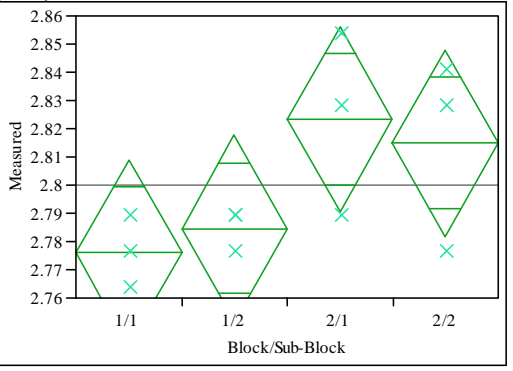

Oneway Anova

Summary of Fit

$\begin{array}{lr}\text { Rsquare } & 0.491329 \\ \text { Adj Rsquare } & 0.300578 \\ \text { Root Mean Square Error } & 0.024725 \\ \text { Mean of Response } & 2.799752 \\ \text { Observations (or Sum Wgts) } & 12\end{array}$

Analysis of Variance

Source DF Sum of Squares Mean Square F Ratio Prob > F

$\begin{array}{lrrrrr}\text { Block/Sub-Block } & 3 & 0.00472373 & 0.001575 & 2.5758 & 0.1266\end{array}$

$\begin{array}{llll}\text { Error } & 8 & 0.00489045 & 0.000611\end{array}$

$\begin{array}{lll}\text { C. Total } & 11 & 0.00961417\end{array}$

Means for Oneway Anova

Level Number Mean Std Error Lower 95\% Upper 95\%

$\begin{array}{llllll}1 / 1 & 3 & 2.77608 & 0.01427 & 2.7432 & 2.8090\end{array}$

$\begin{array}{llllll}1 / 2 & 3 & 2.78469 & 0.01427 & 2.7518 & 2.8176\end{array}$

$\begin{array}{llllll}2 / 1 & 3 & 2.82342 & 0.01427 & 2.7905 & 2.8563\end{array}$

$\begin{array}{llllll}2 / 2 & 3 & 2.81482 & 0.01427 & 2.7819 & 2.8477\end{array}$

Std Error uses a pooled estimate of error variance
Oneway Analysis of Measured By Block/Sub-Block Type $=$ Ustd, Oxide $=\mathbf{N a 2 O}$ (wt\%)
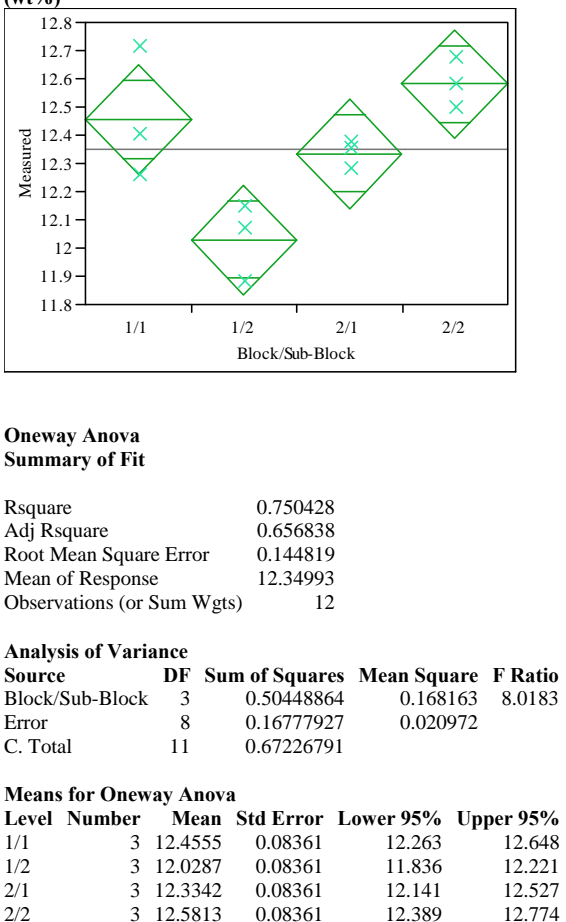

Std Error uses a pooled estimate of error variance

Oneway Analysis of Measured By Block/Sub-Block Type=Ustd, Oxide=NiO (wt\%)

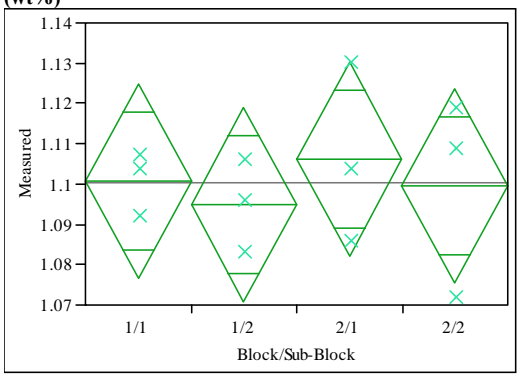

Oneway Anova

Summary of Fit

Rsquare

Root Mean Square Error $\quad 0.018104$

Mean of Response 1.100288

Observations (or Sum Wgts) 12

Analysis of Variance

Source DF Sum of Squares Mean Square F Ratio Prob $>$ F

$\begin{array}{lrrrrr}\text { Block/Sub-Block } & 3 & 0.00019971 & 0.000067 & 0.2031 & 0.8914\end{array}$

$\begin{array}{llll}\text { Error } & 8 & 0.00262212 & 0.000328\end{array}$

$\begin{array}{lll}\text { C. Total } & 11 & 0.00282182\end{array}$

Means for Oneway Anova

Level Number Mean Std Error Lower 95\% Upper 95\%

$\begin{array}{llllll}1 / 1 & 3 & 1.10071 & 0.01045 & 1.0766 & 1.1248\end{array}$

$\begin{array}{llllll}1 / 2 & 3 & 1.09477 & 0.01045 & 1.0707 & 1.1189\end{array}$

\begin{tabular}{llllll}
$1 / 2$ & 3 & 1.10623 & 0.01045 & 1.0821 & 1.1303 \\
\hline & 3 & 1.09944 & 0.01045 & 1.0753 & 1.1235
\end{tabular}

Std Error uses a pooled estimate of error variance 


\section{Exhibit A3. Measurements by Block and Sub-Block for Samples of the Batch 1 and Ustd Standards with the Initial Glasses by Oxide}

Oneway Analysis of Measured By Block/Sub-Block Type=Ustd, Oxide=P2O5

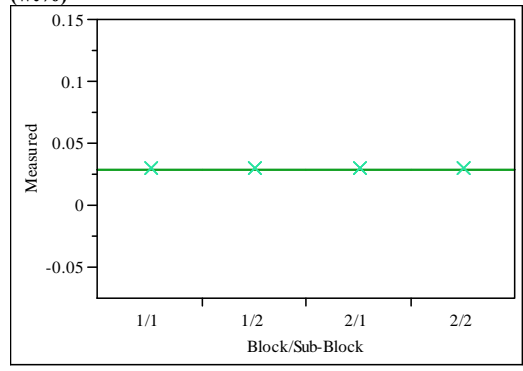

Oneway Anova

Summary of Fit

Rsquare

Adj Rsquare

Root Mean Square Erro

$\begin{array}{lr}\text { Mean of Response } & 0.028643 \\ \text { Observations (or Sum Wgts) } & 12\end{array}$

Analysis of Variance

$\begin{array}{lrrrrr}\text { Source } & \text { DF } & \text { Sum of Squares } & \text { Mean Square } & \text { F Ratio } & \text { Prob }>\text { F } \\ \text { Block/Sub-Block } & 3 & 0 & 0 & . \\ \text { Error } & 8 & 0 & 0 & \\ \text { C. Total } & 11 & 0 & & \end{array}$

Means for Oneway Anova

Level Number Mean Std Error Lower 95\% Upper 95\%

\begin{tabular}{lrrrrr} 
Level & Number & Mean & Std Error & Lower 95\% & Upper 95\% \\
$1 / 1$ & 3 & 0.028643 & 0 & 0.02864 & 0.02864 \\
$1 / 2$ & 3 & 0.028643 & 0 & 0.02864 & 0.02864 \\
$2 / 1$ & 3 & 0.028643 & 0 & 0.02864 & 0.02864 \\
\hline & 3 & 0.028643 & 0 & 0.02864 & 0.02864
\end{tabular}

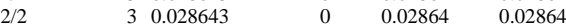

Std Error uses a pooled estimate of error variance

Oneway Analysis of Measured By Block/Sub-Block Type $=$ Ustd, Oxide $=$ PbO (wt\%)

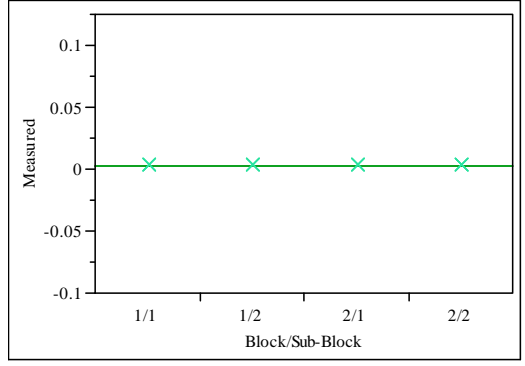

Oneway Anova

Summary of Fit

Rsquare

Adj Rsquare

ror $\quad 5.31 \mathrm{e}-19$

0.002693

Observations (or Sum Wgts) 12

Analysis of Variance

Source DF Sum of Squares Mean Square F Ratio Prob $>$ F

$\begin{array}{rrrrrr}\text { Block/Sub-Block } & 3 & 0 & 0 & 0.0000 & 1.0000\end{array}$

\begin{tabular}{lrr} 
Error & 8 & $2.2569 \mathrm{e}-36$ \\
\hline & 11 & $2.2569 \mathrm{e}-36$
\end{tabular}

\section{Means for Oneway Anova}

Level Number Mean Std Error Lower 95\% Upper 95\%

$\begin{array}{lrrrrr}1 / 1 & 3 & 0.002693 & 3.067 \mathrm{e}-19 & 0.00269 & 0.00269\end{array}$

$\begin{array}{llllll}1 / 2 & 3 & 0.002693 & 3.067 \mathrm{e}-19 & 0.00269 & 0.00269\end{array}$

$\begin{array}{llllll}2 / 1 & 3 & 0.002693 & 3.067 \mathrm{e}-19 & 0.00269 & 0.00269 \\ 2 / 2 & 3 & 0.002693 & 3.067 \mathrm{e}-19 & 0.00269 & 0.00269\end{array}$

Std Error uses a pooled estimate of error variance
Oneway Analysis of Measured By Block/Sub-Block Type=Ustd, Oxide=SiO2 (wt\%)
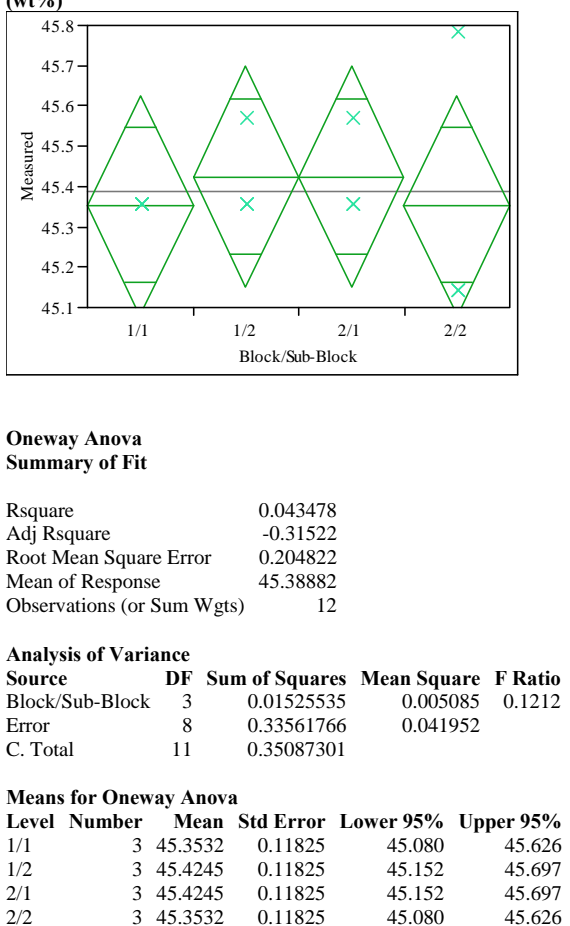

Std Error uses a pooled estimate of error variance

Oneway Analysis of Measured By Block/Sub-Block Type=Ustd, Oxide=SO4 (wt\%)

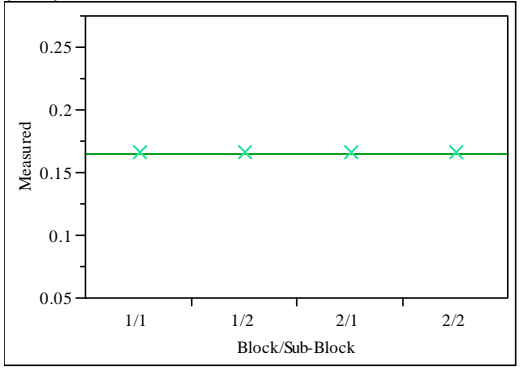

Oneway Anova

Summary of Fit

Rsquare

Adj Rsquare

$\begin{array}{lr}\text { Root Mean Square Error } & 0 \\ \text { Mean of Response } & 0.164775\end{array}$

Observations (or Sum Wgts) $\quad 12$

Analysis of Variance

Source DF Sum of Squares Mean Square F Ratio Prob $>$ F

Block/Sub-Block

Error

C. Total

$\begin{array}{lll}3 & 0 & 0 \\ 8 & 0 & 0\end{array}$

Means for Oneway Anova

Level Number Mean Std Error Lower 95\% Upper 95\%

$\begin{array}{llllll}1 / 1 & 3 & 0.164775 & 0 & 0.16477 & 0.16477\end{array}$

$\begin{array}{llllll}1 / 2 & 3 & 0.164775 & 0 & 0.16477 & 0.16477\end{array}$

$\begin{array}{llllll}2 / 1 & 3 & 0.164775 & 0 & 0.16477 & 0.16477\end{array}$

Std Error uses a pooled estimate of error variance 


\section{Exhibit A3. Measurements by Block and Sub-Block for Samples of the Batch 1 and Ustd Standards with the Initial Glasses by Oxide}

Oneway Analysis of Measured By Block/Sub-Block Type=Ustd, Oxide=TiO2

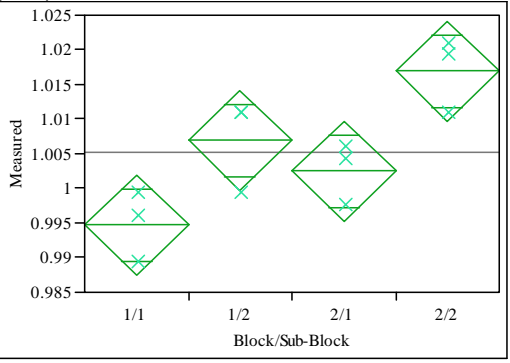

Oneway Anova

Summary of Fi

$\begin{array}{lr}\text { Rsquare } & 0.764168 \\ \text { Adj Rsquare } & 0.675731 \\ \text { Root Mean Square Error } & 0.005469 \\ \text { Mean of Response } & 1.005248 \\ \text { Observations (or Sum Wgts) } & 12\end{array}$

Observations (or Sum Wgts)

Analysis of Variance

Source DF Sum of Squares Mean Square F Ratio Prob $>$ F

$\begin{array}{llllll}\text { Block/Sub-Block } & 3 & 0.00077531 & 0.000258 & 8.6408 & 0.0069\end{array}$

Error

0.00077531

0.000030

$\begin{array}{lll}\text { C. Total } & 11 & 0.00101458\end{array}$

Means for Oneway Anova

Level Number Mean Std Error Lower 95\% Upper 95\%

$\begin{array}{lrrrrr}1 / 1 & 3 & 0.99468 & 0.00316 & 0.9874 & 1.002\end{array}$

$\begin{array}{llllll}1 / 2 & 3 & 1.00692 & 0.00316 & 0.9996 & 1.0142 \\ 2 / 1 & 3 & 1.00247 & 0.00316 & 0.9952 & 1.0097\end{array}$

$\begin{array}{lllll}3 & 1.00247 & 0.00316 & 0.9952 & 1.0097\end{array}$

Std Error uses a pooled estimate of error variance

Oneway Analysis of Measured By Block/Sub-Block Type $=$ Ustd, Oxide=U308 (wt \%)

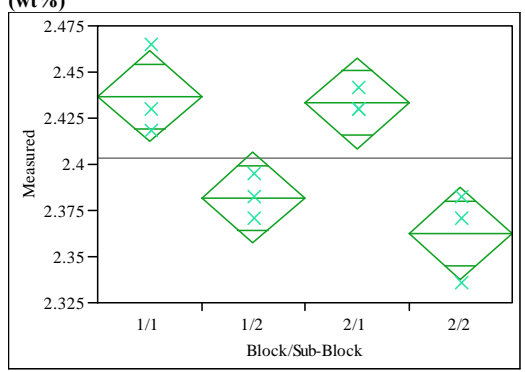

Oneway Anova

Summary of Fit

Adj Rsquare

2.403603

Observations (or Sum Wgts) 12

Analysis of Variance

Source DF Sum of Squares Mean Square F Ratio Prob $>$ F

$\begin{array}{lrrrrr}\text { Block/Sub-Block } & 3 & 0.01246826 & 0.004156 & 11.9556 & 0.0025\end{array}$

Error

0.00278103

0.000348

Means for Oneway Anova

Level Number Mean Std Error Lower 95\% Upper 95\%

Level Number Mean Std Error Lower 95\% Upper 95\%

$\begin{array}{llllll}1 / 1 & 3 & 2.43701 & 0.01076 & 2.4122 & 2.4618 \\ 1 / 2 & 3 & 2.38198 & 0.01076 & 2.3572 & 2.4068\end{array}$

$\begin{array}{llllll}2 / 1 & 3 & 2.43308 & 0.01076 & 2.4083 & 2.4579\end{array}$

Std Error uses a pooled estimate of error variance
Oneway Analysis of Measured By Block/Sub-Block Type $=$ Ustd, Oxide $=\mathbf{Z n O}$ (wt\%)

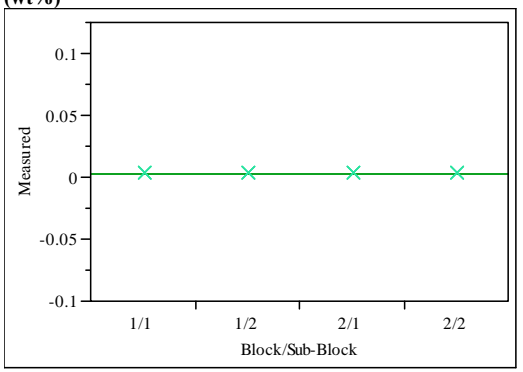

Oneway Anova

Summary of Fit

Rsquare

Adj Rsquare

$\begin{array}{lr}\text { Root Mean Square Error } & 0 \\ \text { Mean of Response } & 0.003112 \\ \text { Observations (or Sum Wgts) } & 12\end{array}$

Analysis of Variance

Source DF Sum of Squares Mean Square F Ratio Prob $>$ F

Block/Sub-Block 3

Error $\quad 8$

$\begin{array}{lll}3 & 0 & 0 \\ 8 & 0 & 0\end{array}$

Means for Oneway Anova

Level Number Mean Std Error Lower 95\% Upper 95\%

$\begin{array}{lrrrrr}1 / 1 & 3 & 0.003112 & 0 & 0.00311 & 0.00311 \\ 1 / 2 & 3 & 0.003112 & 0 & 0.00311 & 0.00311 \\ 2 / 1 & 3 & 0.003112 & 0 & 0.00311 & 0.00311\end{array}$

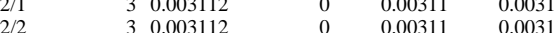

Std Error uses a pooled estimate of error variance

Oneway Analysis of Measured By Block/Sub-Block Type $=$ Ustd, Oxide $=\mathrm{ZrO} 2$ (wt\%)

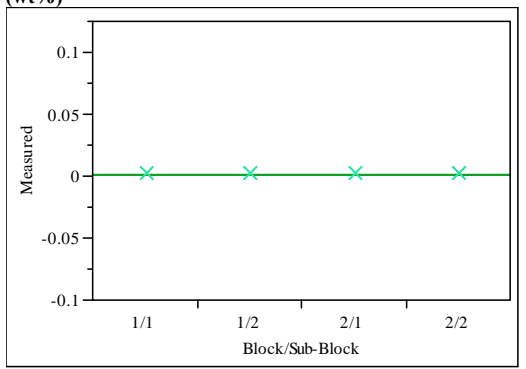

Oneway Anova

Summary of Fit

Rsquare

Adj Rsquare

Root Mean Square Error $\quad 0$

Mean of Response 0.001351

Observations (or Sum Wgts)

Analysis of Variance

$\begin{array}{lrrrrr}\begin{array}{l}\text { Analysis of Variance } \\ \text { Source }\end{array} & \text { DF } & \text { Sum of Squares } & \text { Mean Square } & \text { F Ratio } & \text { Prob }>\text { F } \\ \text { Block/Sub-Block } & 3 & 0 & 0 & . \\ \text { Error } & 8 & 0 & 0 & \\ \text { C. Total } & 11 & 0 & & \end{array}$

Means for Oneway Anova

Level Number Mean Std Error Lower 95\% Upper 95\%

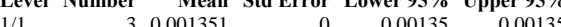

$\begin{array}{llllll}1 / 2 & 3 & 0.001351 & 0 & 0.00135 & 0.00135 \\ 2 & 3 & 0.001351 & 0 & 0.00135 & 0.00135\end{array}$

$\begin{array}{llllll}2 / 1 & 3 & 0.001351 & 0 & 0.00135 & 0.00135 \\ 2 / 2 & 3 & 0.001351 & 0 & 0.00135 & 0.00135\end{array}$

Std Error uses a pooled estimate of error variance 


\section{Exhibit A4. Average Measured Versus Targeted Compositions by Glass ID by Oxide for the Initial SB6 VS Glasses}

Overlay Plot Oxide $=\mathrm{Al} 2 \mathrm{O3}($ wt $\%)$

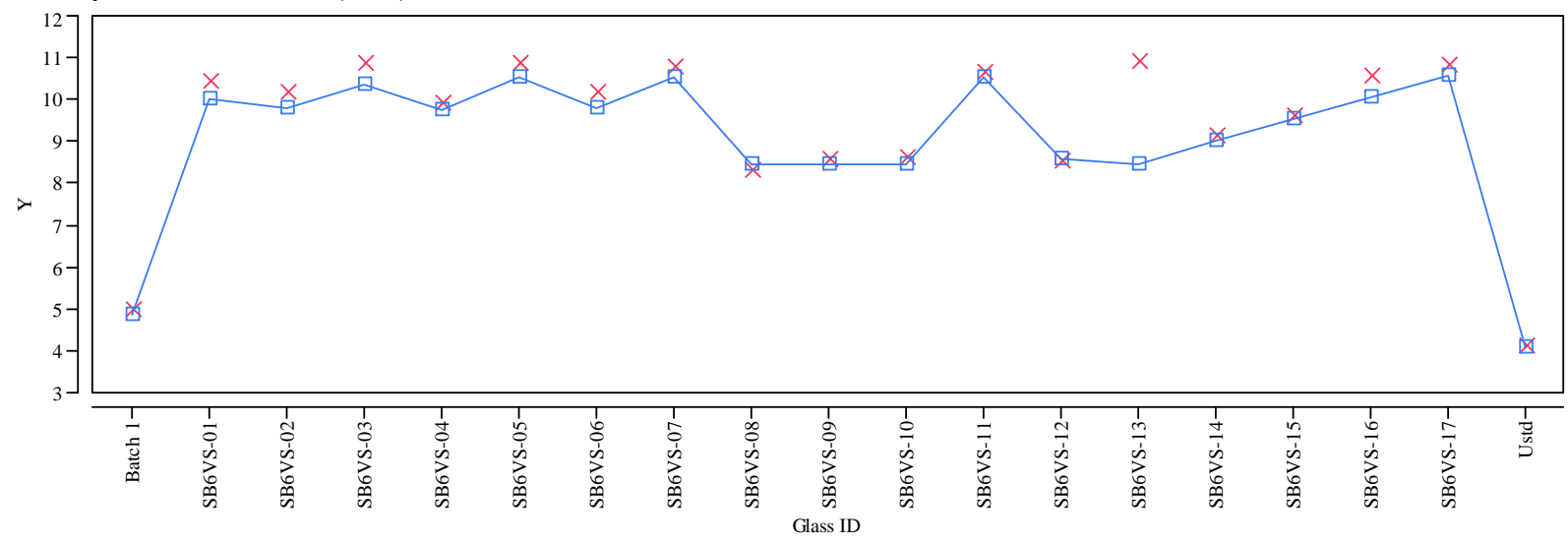

Overlay Plot Oxide=B2O3 (wt \%)

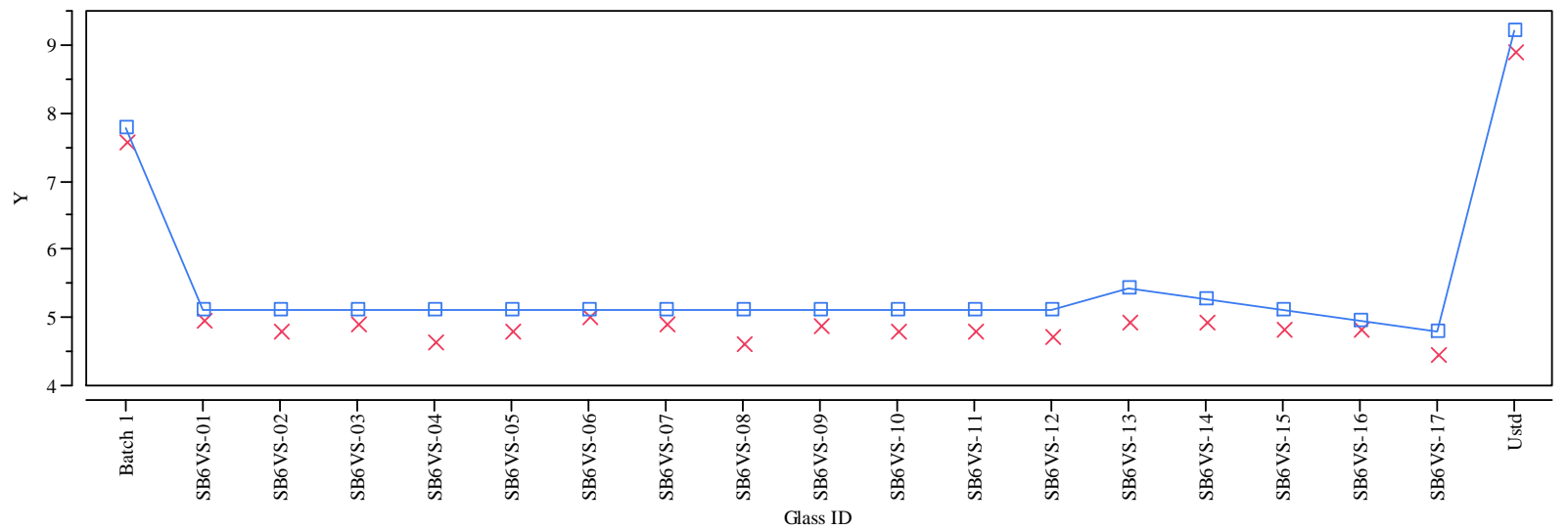

Overlay Plot Oxide $=\mathrm{BaO}($ wt $\%)$

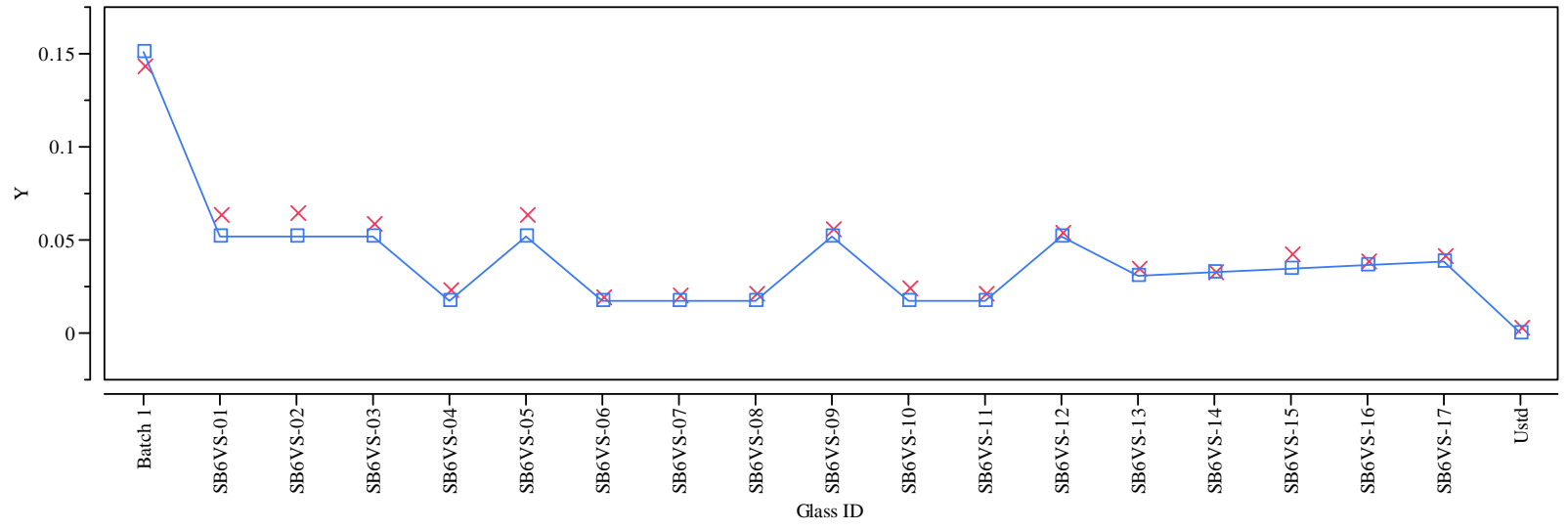

Y $\times$ Measured $\square$ T Targeted 


\section{Exhibit A4. Average Measured Versus Targeted Compositions by Glass ID by Oxide for the Initial SB6 VS Glasses}

Overlay Plot Oxide $=\mathrm{CaO}(w \mathrm{t} \%)$

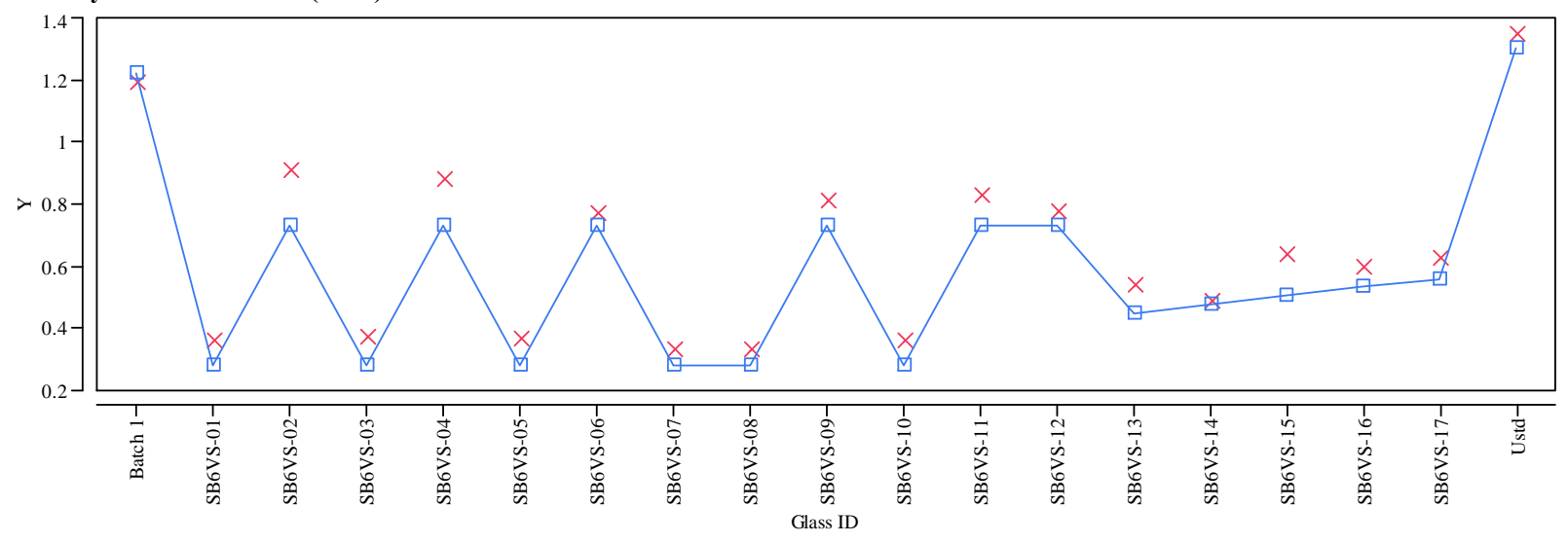

Overlay Plot Oxide $=\mathbf{C d O}(\mathbf{w t} \%)$

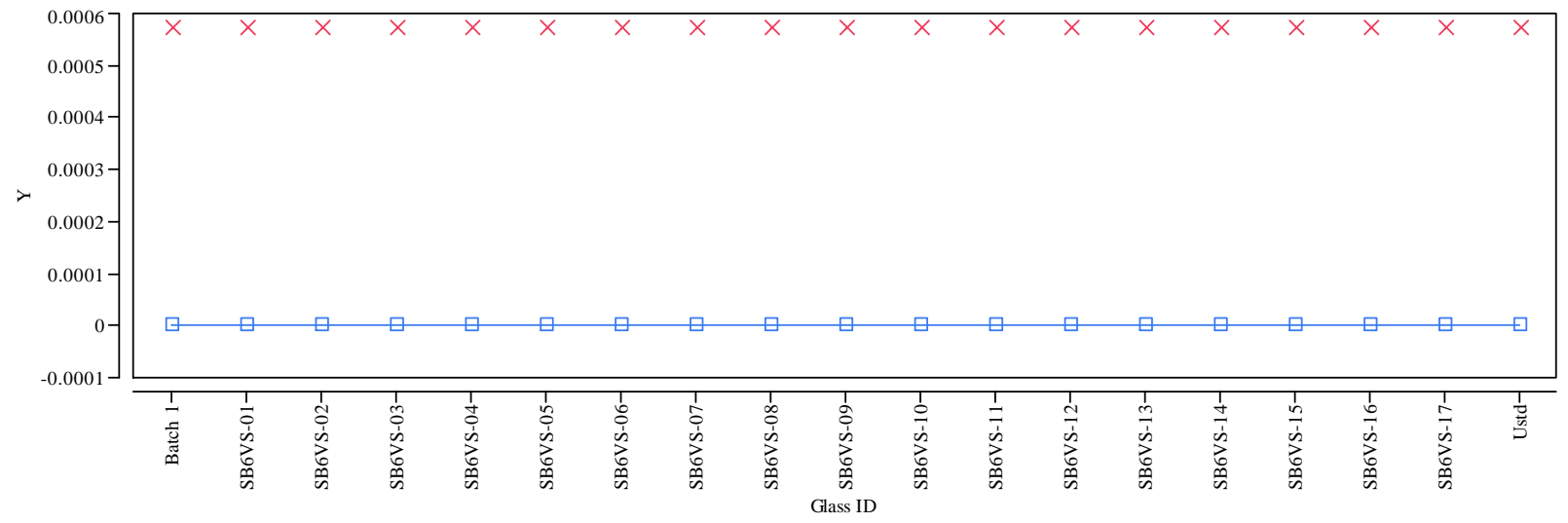

Overlay Plot Oxide $=\mathrm{Ce} 2 \mathrm{O3}(\mathrm{wt} \%)$

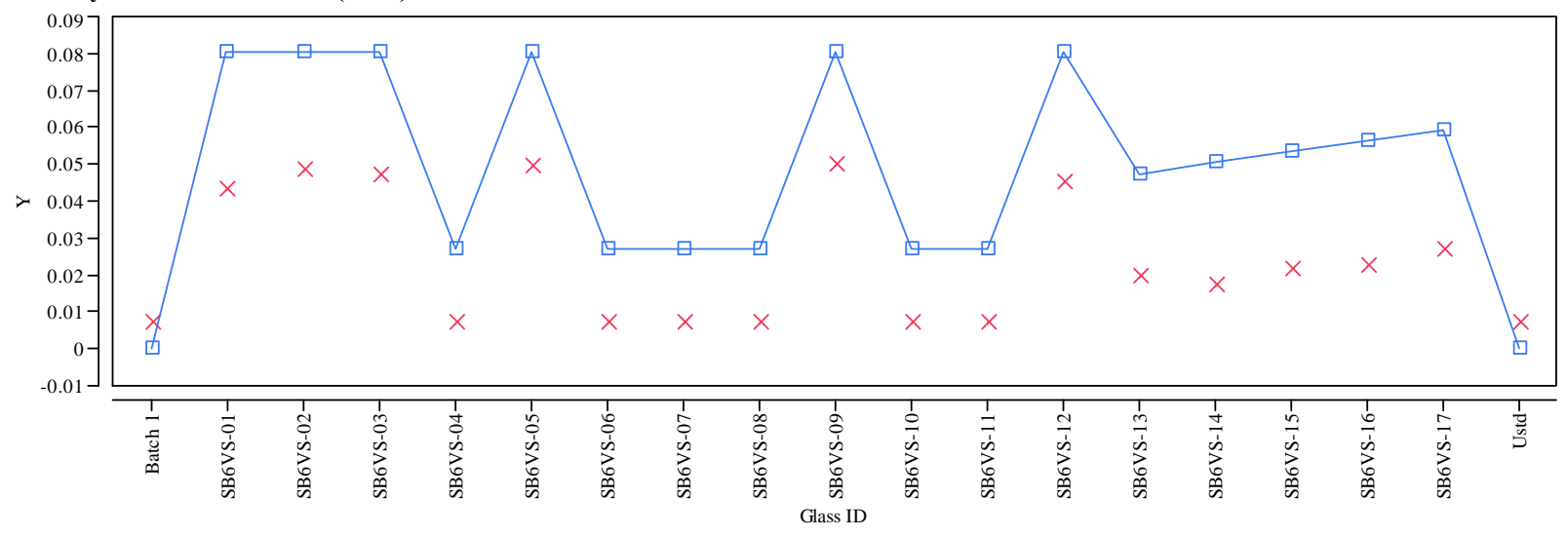

Y $\times$ Measured $\square$ Targeted 
Exhibit A4. Average Measured Versus Targeted Compositions by Glass ID by Oxide for the Initial SB6 VS Glasses

Overlay Plot Oxide $=\mathrm{Cr} 2 \mathrm{O} 3(\mathrm{wt} \%)$

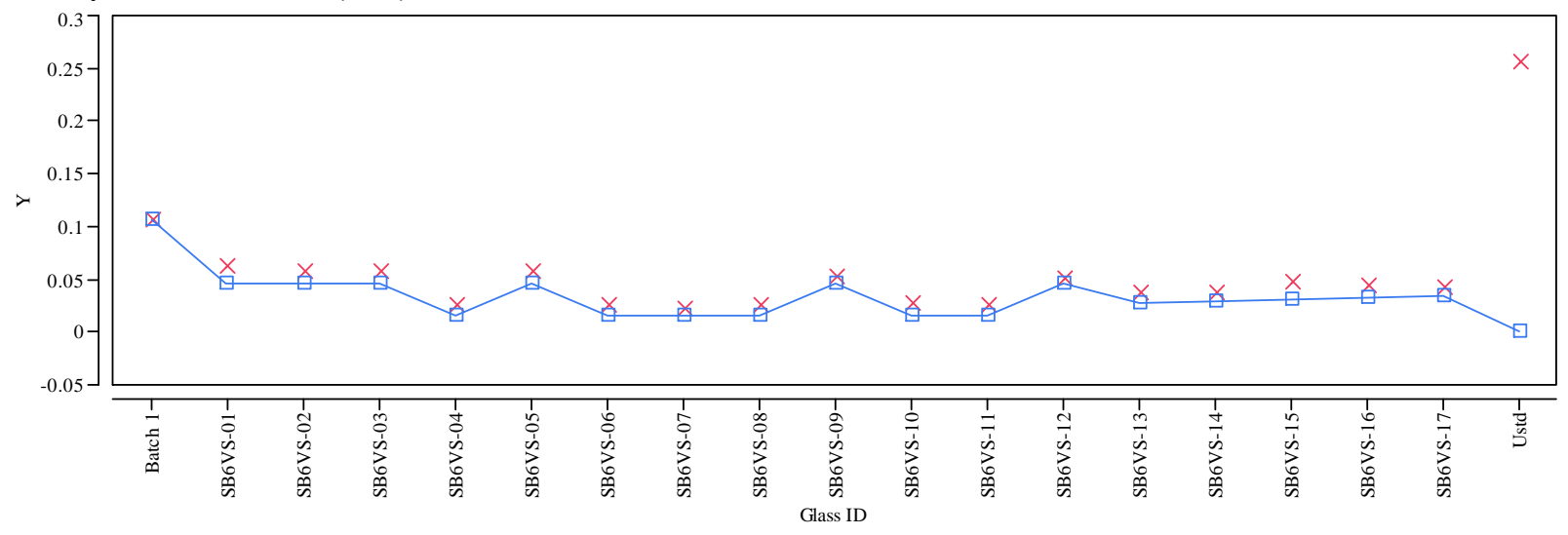

Overlay Plot Oxide $=\mathrm{CuO}(\mathrm{wt} \%)$

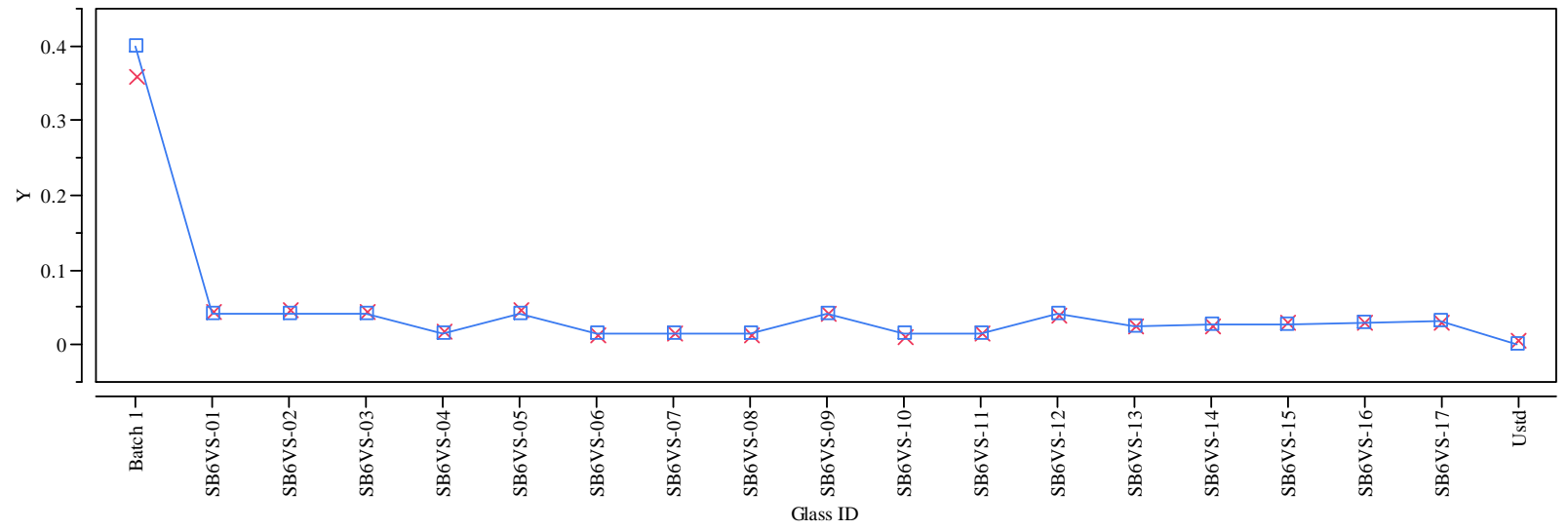

Overlay Plot Oxide $=\mathrm{Fe} 2 \mathrm{O} 3(\mathrm{wt} \%)$

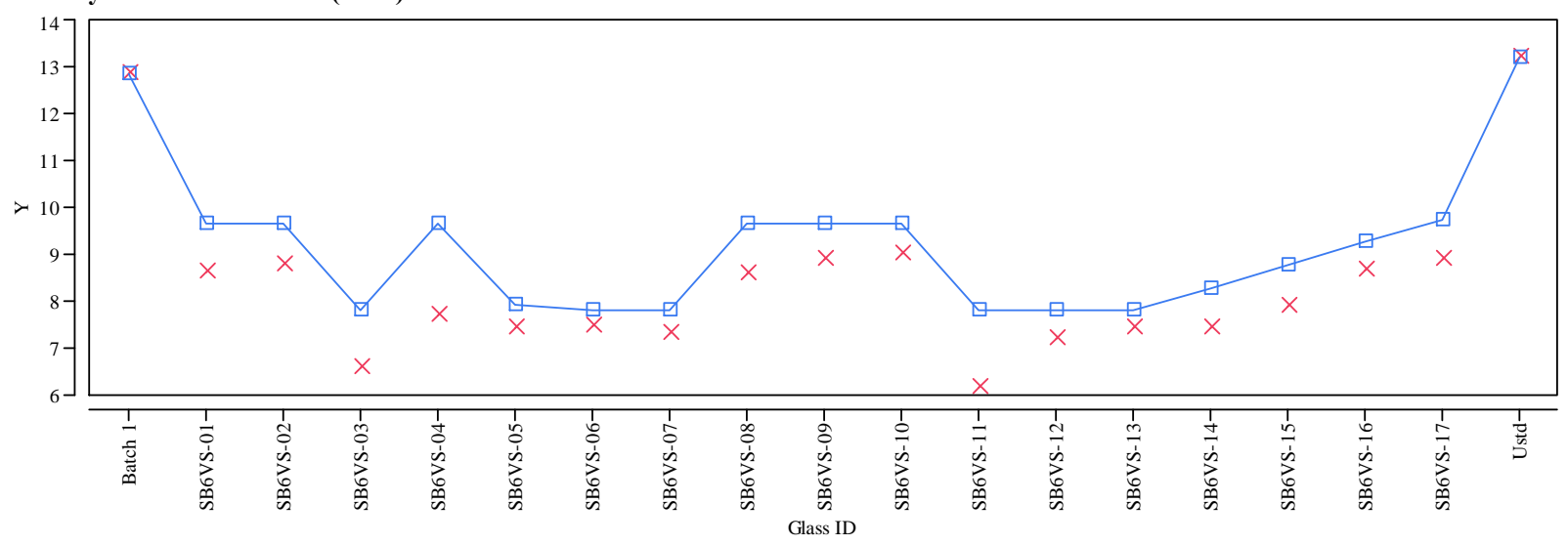

Y $\times$ Measured $\square$ Targeted 


\section{Exhibit A4. Average Measured Versus Targeted Compositions by Glass ID by Oxide for the Initial SB6 VS Glasses}

Overlay Plot Oxide $=$ K2O (wt \%)

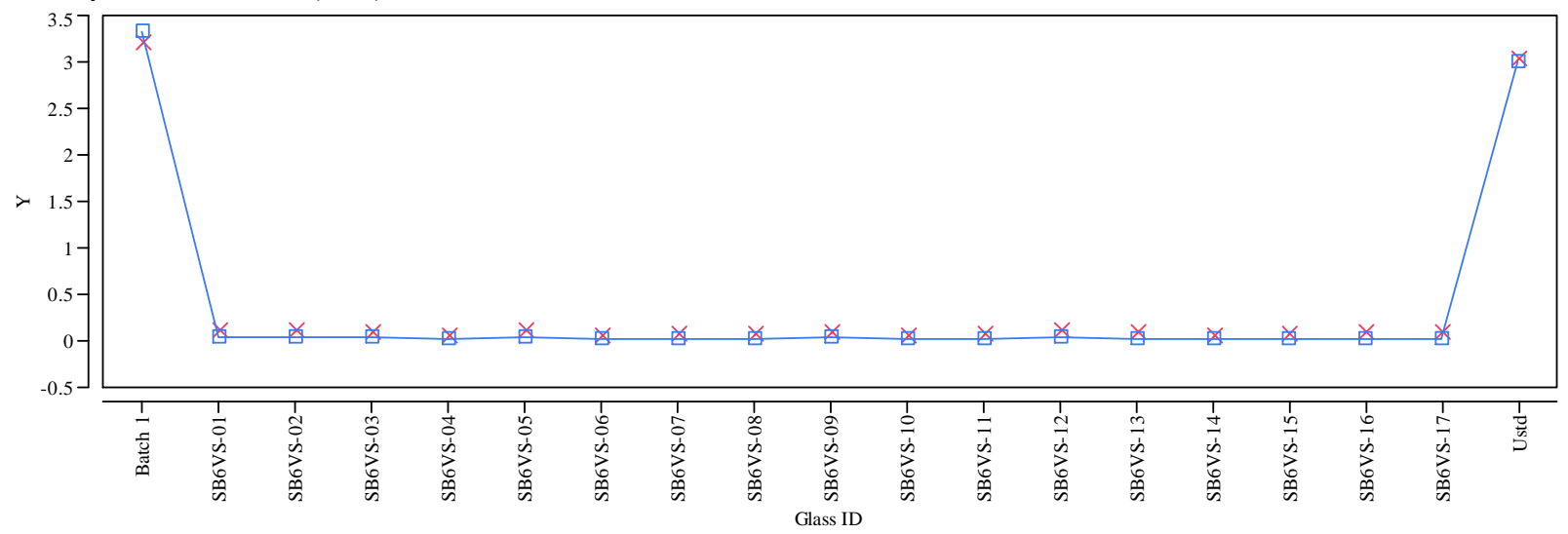

Overlay Plot Oxide $=\mathrm{La2O3}(\mathrm{wt} \%)$

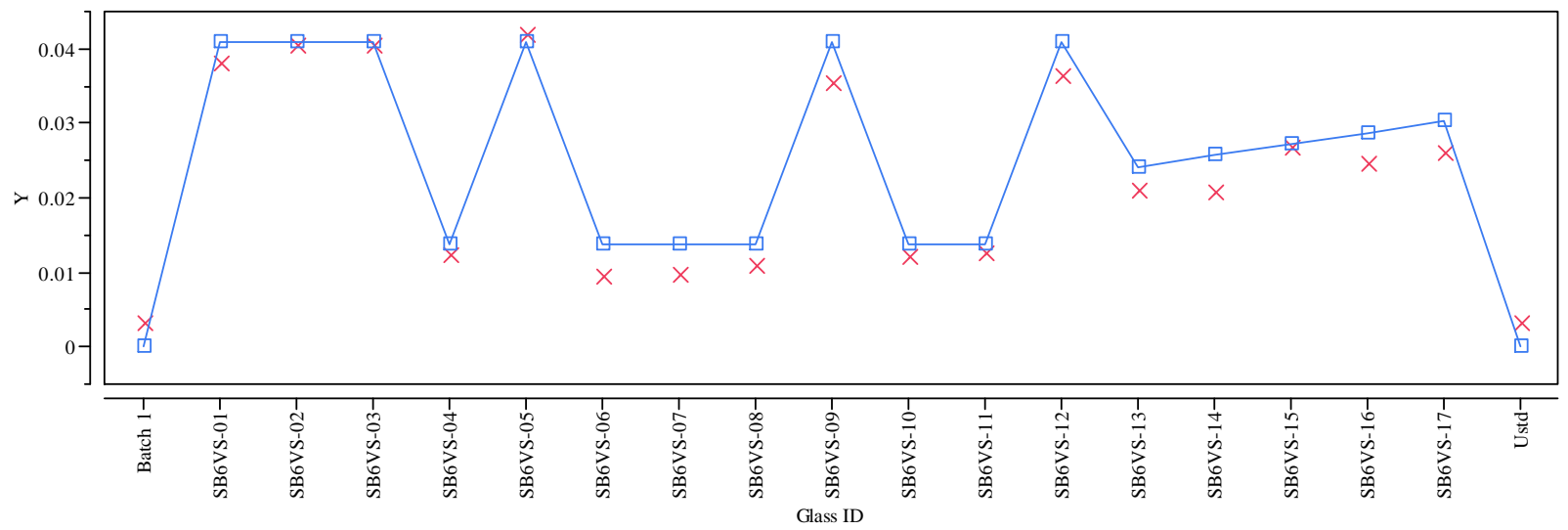

Overlay Plot Oxide=Li2O $($ wt $\%)$

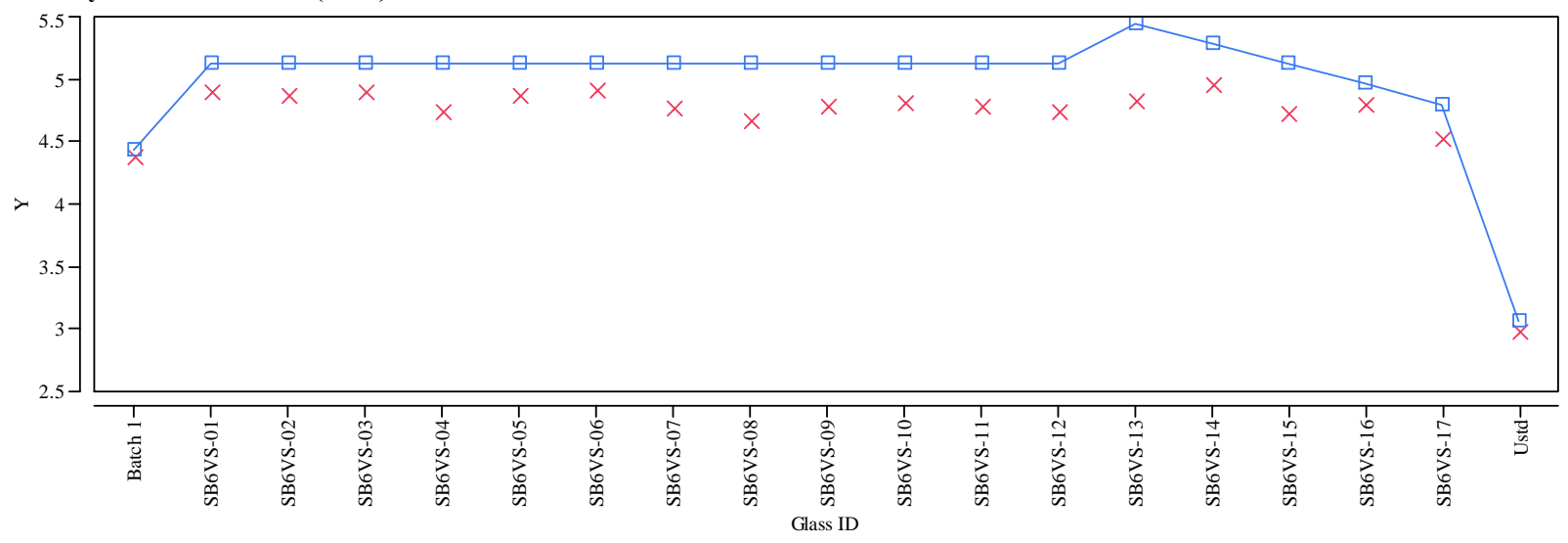

Y $\times$ Measured $\square$ T Targeted 


\section{Exhibit A4. Average Measured Versus Targeted Compositions by Glass ID by Oxide for the Initial SB6 VS Glasses}
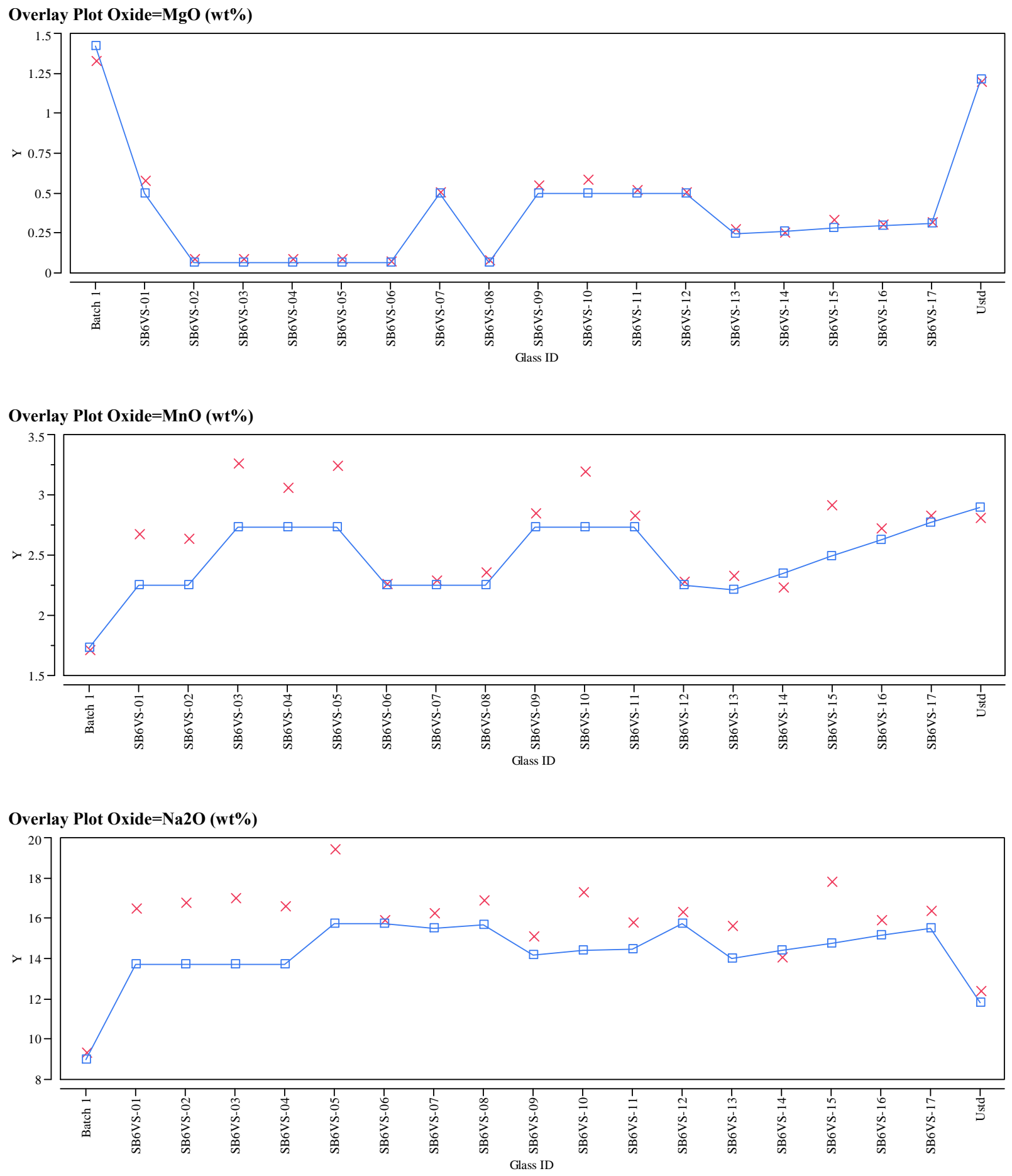

Y $\times$ Measured $\square$ Targeted 


\section{Exhibit A4. Average Measured Versus Targeted Compositions by Glass ID by Oxide for the Initial SB6 VS Glasses}

Overlay Plot Oxide $=\mathrm{NiO}(\mathrm{wt} \%)$
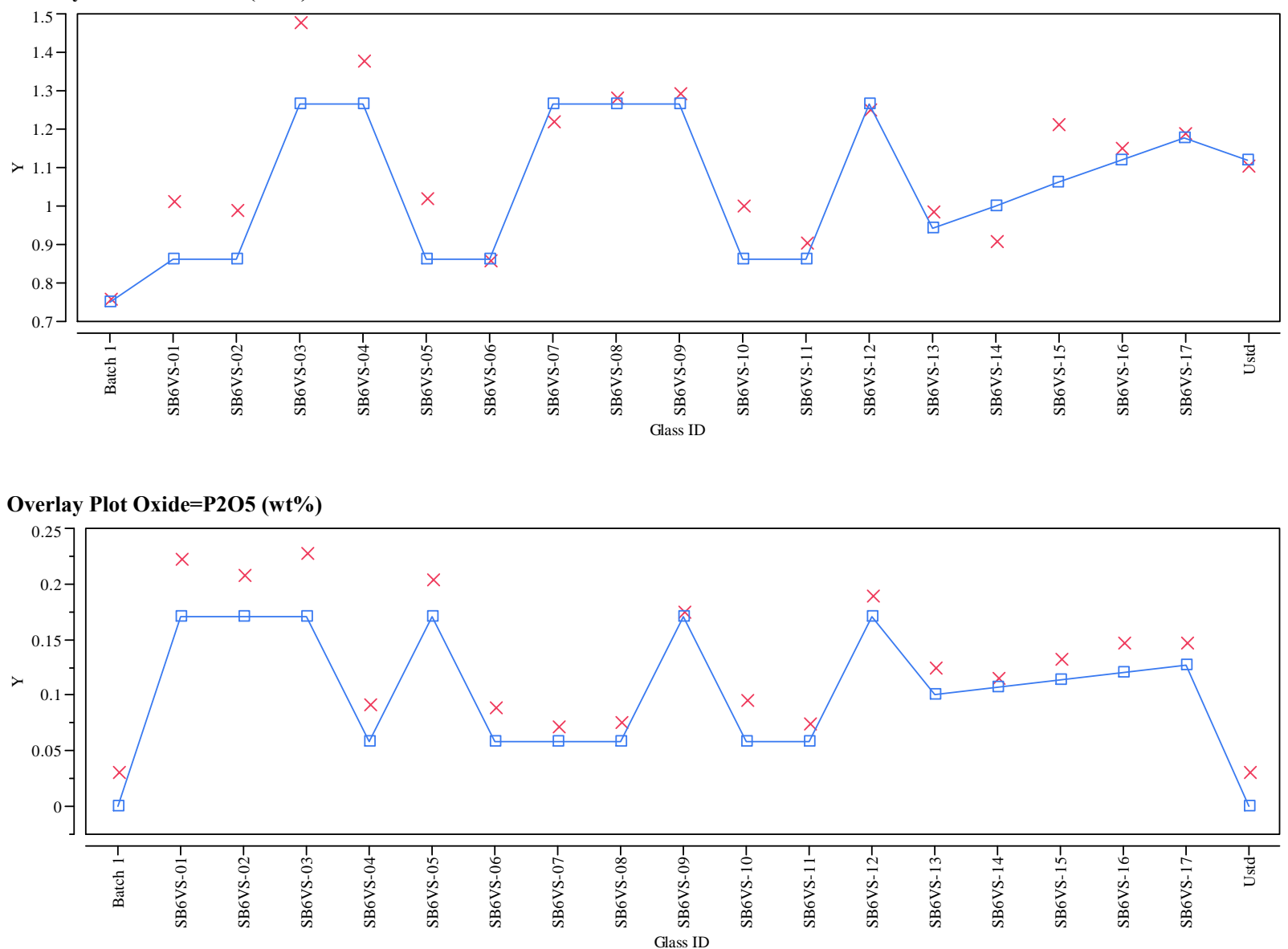

Overlay Plot Oxide $=\mathbf{P b O}(\mathbf{w t} \%)$

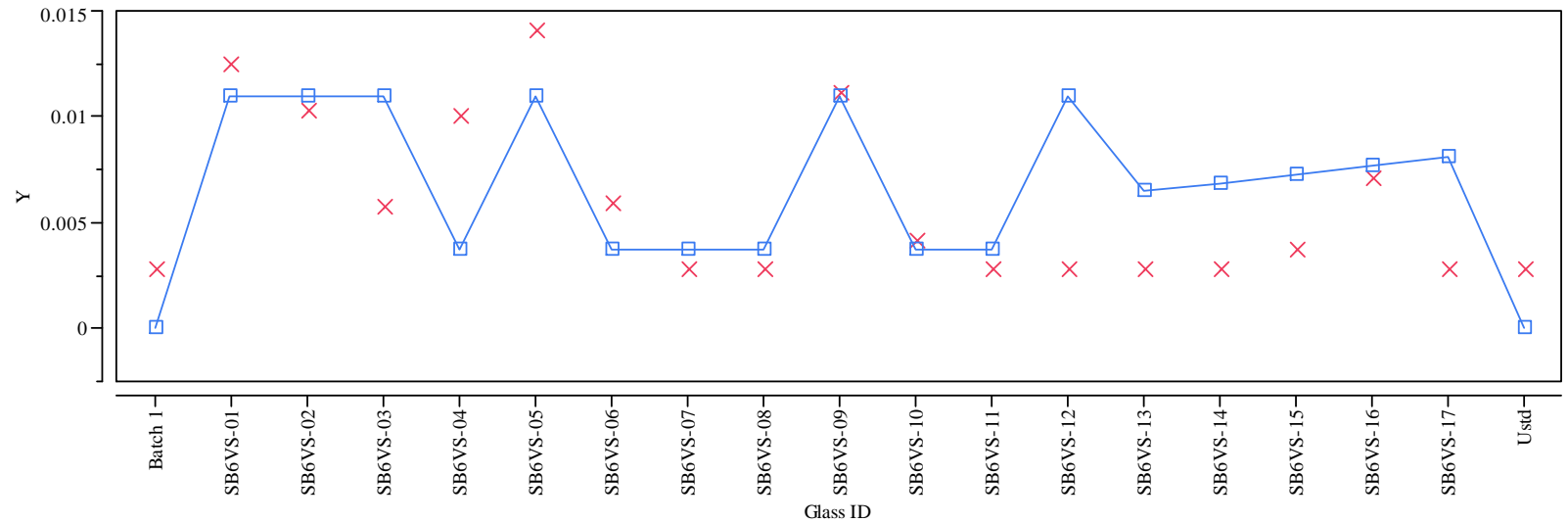

Y $\times$ Measured $\square$ - Targeted 


\section{Exhibit A4. Average Measured Versus Targeted Compositions by Glass ID by Oxide for the Initial SB6 VS Glasses}

Overlay Plot Oxide=SiO2 (wt\%)

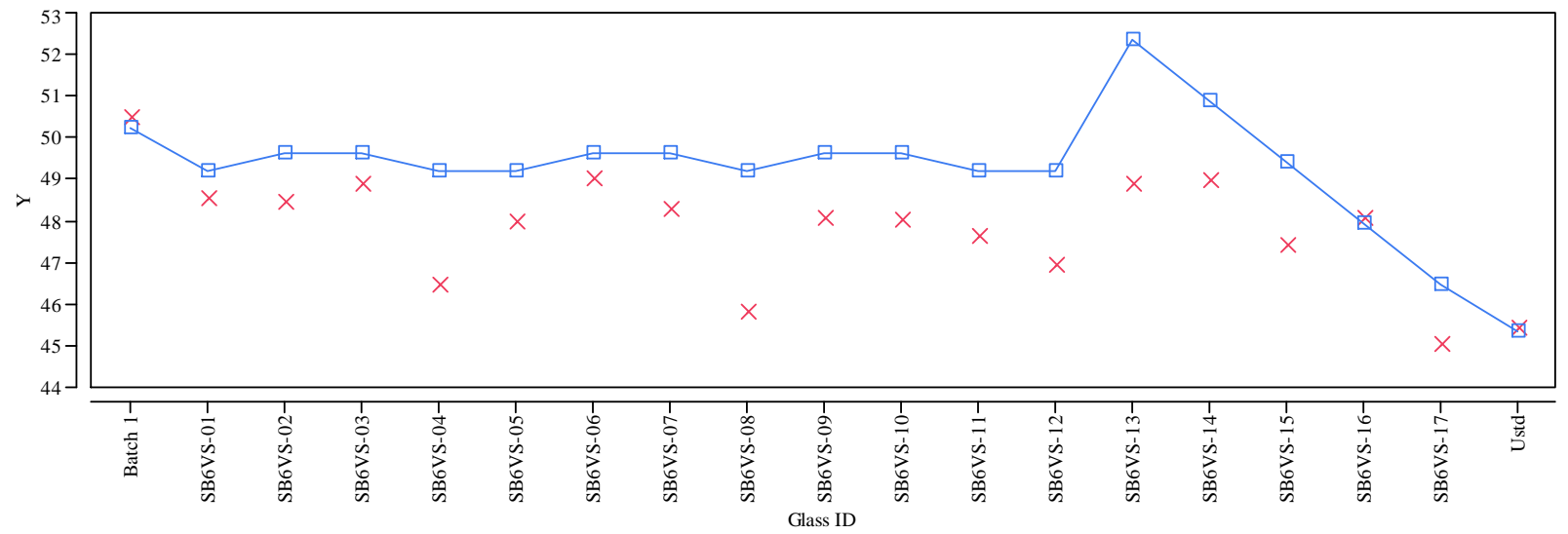

Overlay Plot Oxide=SO4 (wt \%)

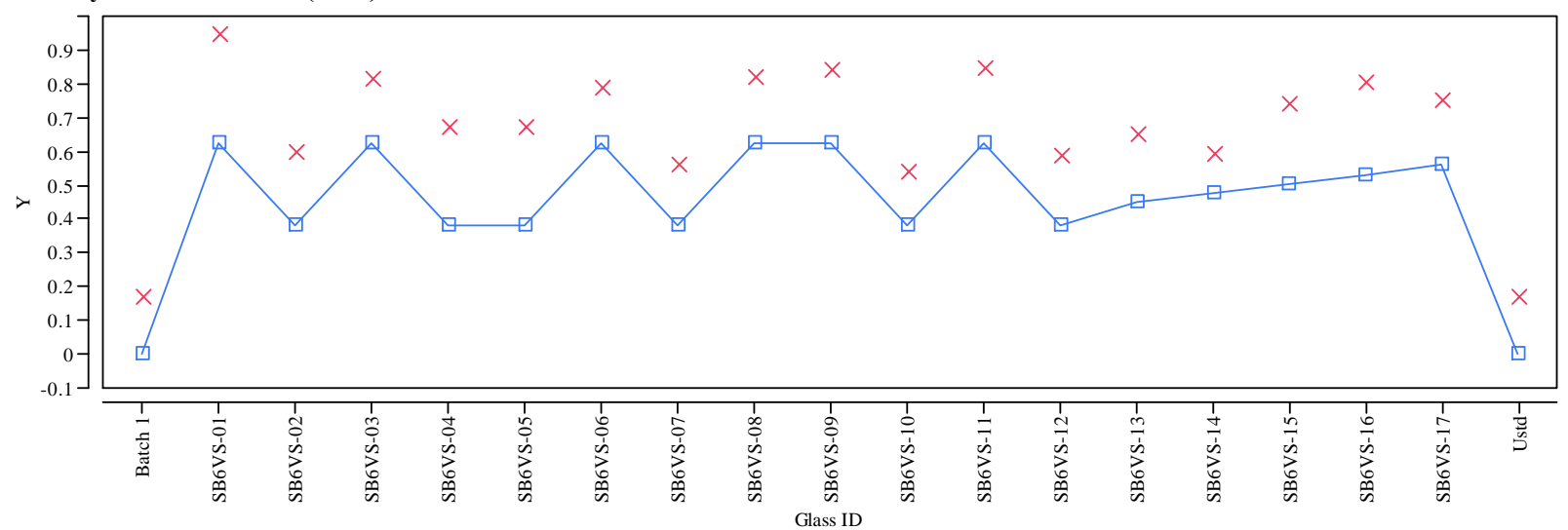

Overlay Plot Oxide=TiO2 $(w t \%)$

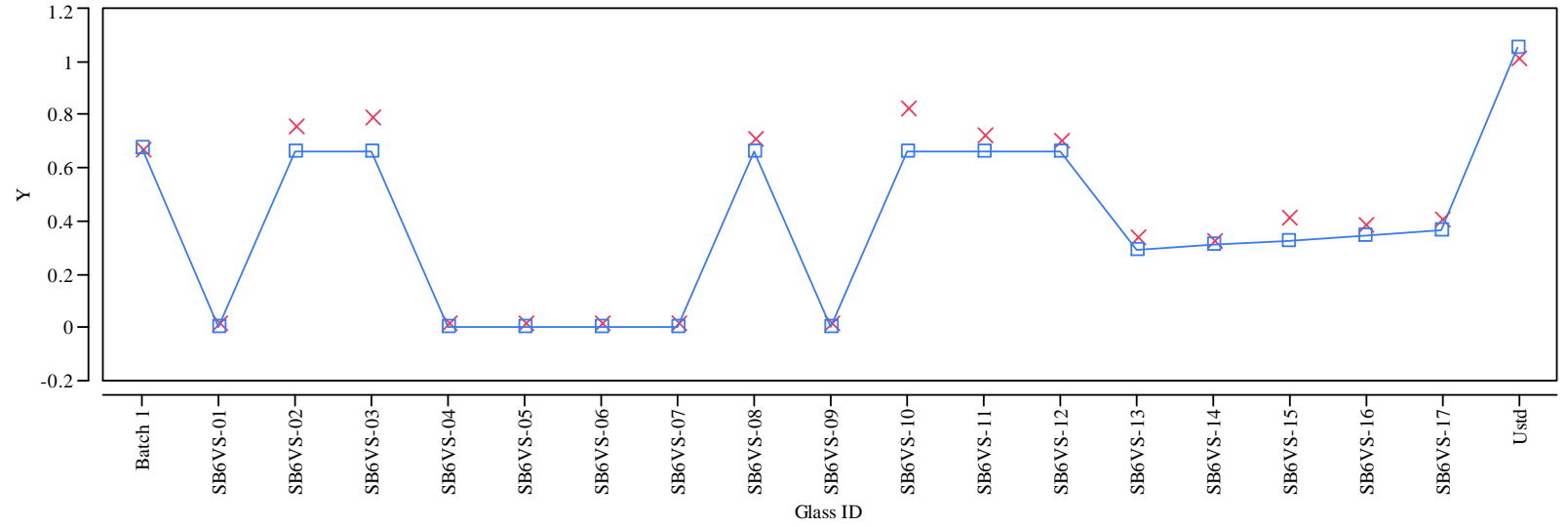

Y $\times$ Measured $\square$ - Targeted 


\section{Exhibit A4. Average Measured Versus Targeted Compositions by Glass ID by Oxide for the Initial SB6 VS Glasses}

Overlay Plot Oxide $=$ U3O8 $($ wt $\%)$
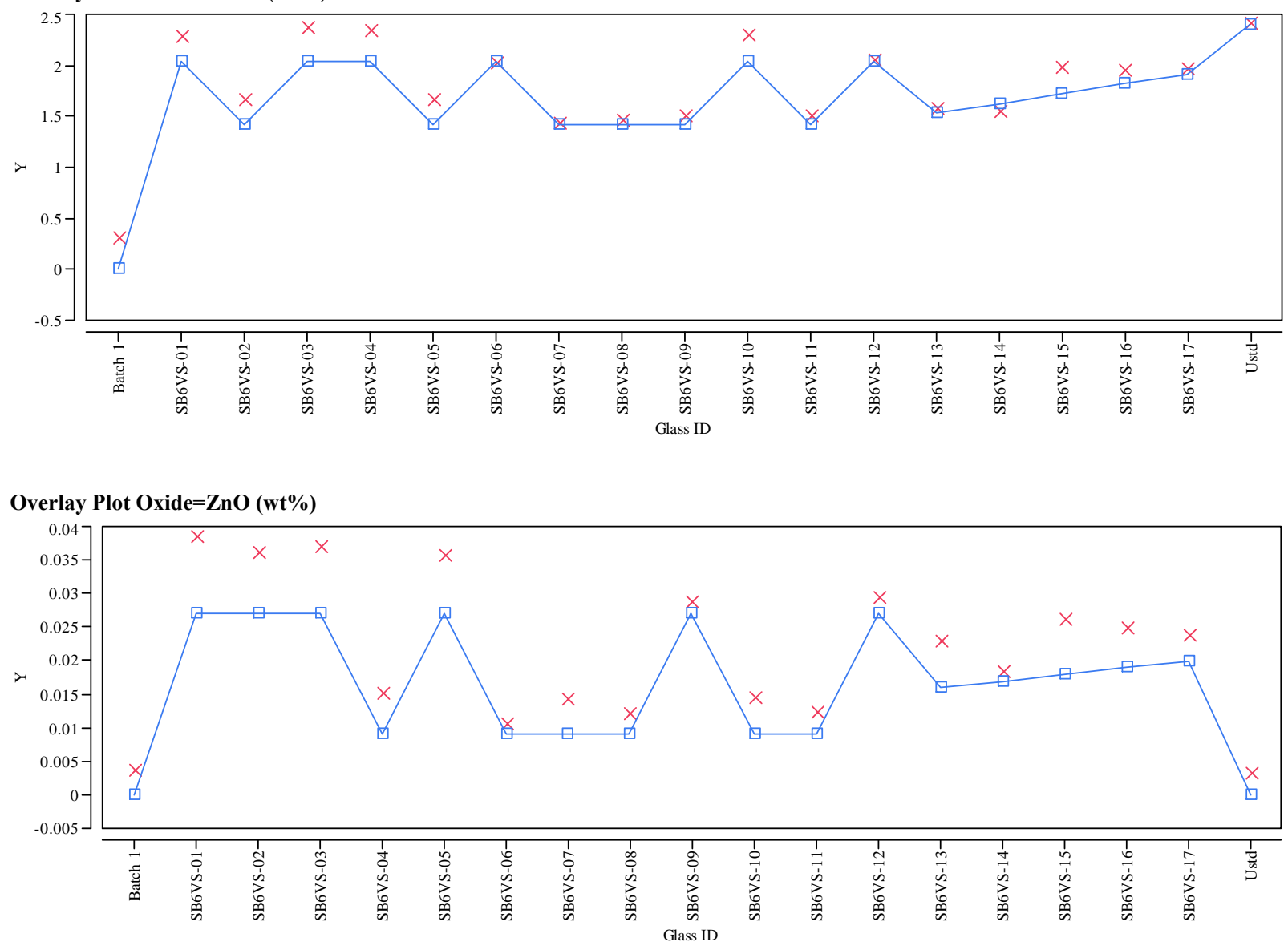

Overlay Plot Oxide $=\mathrm{ZrO} 2(\mathrm{wt} \%)$

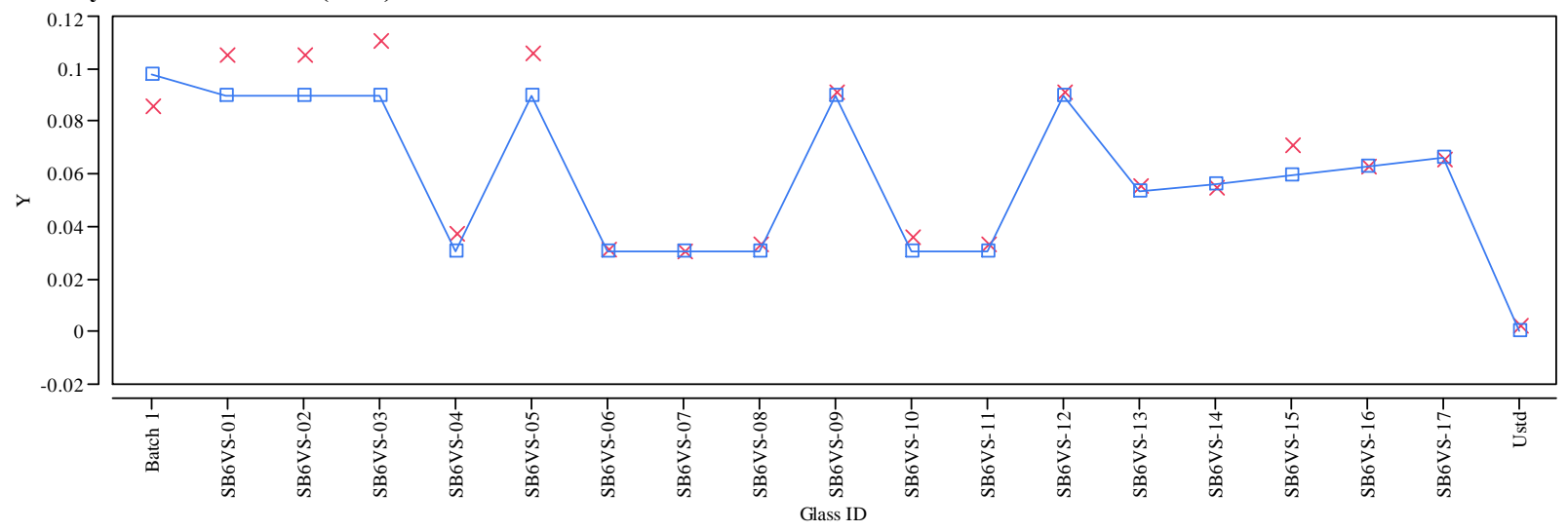

Y $\times$ Measured $\square$ Targeted 


\section{Exhibit A4. Average Measured Versus Targeted Compositions by Glass ID by Oxide for the Initial SB6 VS Glasses}

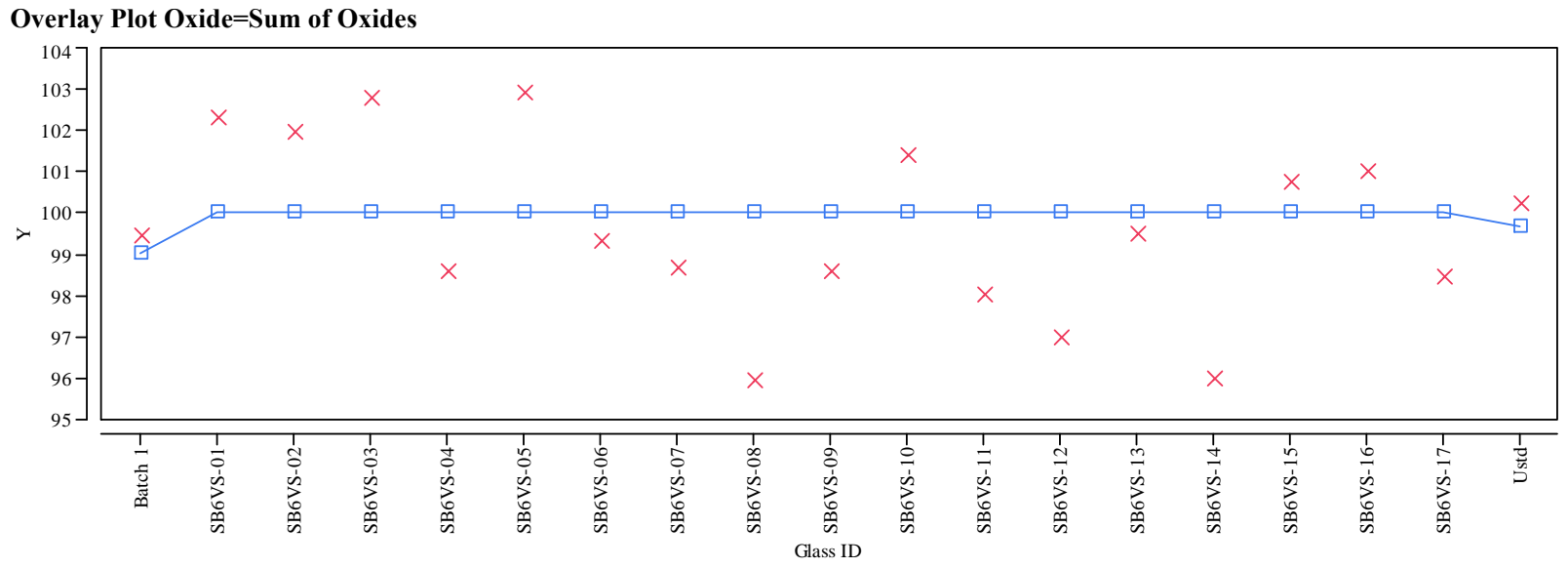

Y $\times$ Measured $\square$ Targeted 


\section{Appendix B:}

\section{Tables and Exhibits Supporting the Analysis of the PCT Results for the Initial (Non- Thorium) Study Glasses}


SRNL-STI-2010-00242

Revision 0

This page intentionally left blank. 
Table B1. Analytical Development's Measurements of the PCT Solutions As-Received (ar) and After Appropriate Adjustments (in ppm)

\begin{tabular}{|c|c|c|c|c|c|c|c|c|c|c|c|c|c|}
\hline Set & \begin{tabular}{|l|} 
Glass \\
ID \\
\end{tabular} & \begin{tabular}{|l|} 
Heat \\
Treatment
\end{tabular} & Block & Seq & Lab ID & B ar & Li ar & $\mathrm{Na}$ ar & Si ar & $\begin{array}{r}\mathbf{B} \\
(\mathbf{p p m}) \\
\end{array}$ & $\begin{array}{r}\mathbf{L i} \\
(\mathbf{p p m})\end{array}$ & $\begin{array}{r}\mathrm{Na} \\
(\mathrm{ppm}) \\
\end{array}$ & $\begin{array}{r}\mathbf{S i} \\
(\mathbf{p p m})\end{array}$ \\
\hline 1 & Soln Std & ref & 1 & 1 & std-11 & 20.0 & \begin{tabular}{|l|l}
9.89 \\
\end{tabular} & 82.5 & 51.0 & 20.000 & 9.890 & 82.500 & 51.000 \\
\hline 1 & SB6VS-02 & quenched & 1 & 2 & M58 & 5.82 & 10.3 & 48.1 & 72.5 & 9.700 & 17.167 & 80.168 & 120.836 \\
\hline 1 & SB6VS-06 & CCC & 1 & 3 & M18 & 6.36 & 11.5 & 66.8 & 81.6 & 10.600 & 19.167 & 111.336 & 136.003 \\
\hline 1 & EA & ref & 1 & 4 & M05 & 34.8 & 10.9 & 97.3 & 54.0 & 580.001 & 181.667 & 1621.670 & 900.002 \\
\hline 1 & SB6VS-09 & quenched & 1 & 5 & M55 & 7.05 & 11.5 & 58.8 & 77.1 & 11.750 & 19.167 & 98.002 & 128.503 \\
\hline 1 & SB6VS-07 & quenched & 1 & 6 & M44 & 6.28 & 10.3 & 64.9 & 77.8 & 10.467 & 17.167 & 108.169 & 129.669 \\
\hline 1 & SB6VS-01 & CCC & 1 & 7 & M50 & 5.90 & 10.3 & 45.4 & 72.4 & 9.834 & 17.167 & 75.668 & 120.669 \\
\hline 1 & SB6VS-04 & quenched & 1 & 8 & M40 & 5.71 & 9.79 & 47.6 & 70.3 & 9.517 & 16.317 & 79.335 & 117.169 \\
\hline 1 & SB6VS-08 & CCC & 1 & 9 & M20 & 6.90 & 11.9 & 68.1 & 84.9 & 11.500 & 19.834 & 113.502 & 141.503 \\
\hline 1 & SB6VS-07 & CCC & 1 & 10 & M57 & 6.49 & 11.4 & 63.2 & 80.1 & 10.817 & 19.000 & 105.335 & 133.503 \\
\hline 1 & SB6VS-01 & quenched & 1 & 11 & M52 & 6.07 & 10.1 & 48.1 & \begin{tabular}{|l|}
72.9 \\
\end{tabular} & 10.117 & 16.834 & 80.168 & 121.502 \\
\hline 1 & SB6VS-03 & quenched & 1 & 12 & M28 & 5.31 & 9.35 & 46.3 & 67.9 & 8.850 & 15.584 & 77.168 & 113.169 \\
\hline 1 & Soln Std & ref & 1 & 13 & std-12 & 19.9 & 9.85 & 82.2 & 50.9 & 19.900 & 9.850 & 82.200 & 50.900 \\
\hline 1 & SB6VS-04 & $\mathrm{CCC}$ & 1 & 14 & M02 & 5.73 & 10.3 & 45.5 & 70.6 & 9.550 & 17.167 & 75.835 & 117.669 \\
\hline 1 & SB6VS-02 & $\mathrm{CCC}$ & 1 & 15 & M06 & 5.43 & 10.0 & 43.9 & 69.6 & 9.050 & 16.667 & 73.168 & 116.002 \\
\hline 1 & SB6VS-03 & $\mathrm{cCC}$ & 1 & 16 & M53 & 5.36 & 9.53 & 43.3 & 68.1 & 8.934 & 15.884 & 72.168 & 113.502 \\
\hline 1 & SB6VS-09 & CCC & 1 & 17 & M35 & 6.32 & \begin{tabular}{|l|}
10.4 \\
\end{tabular} & 49.6 & 69.6 & 10.534 & 17.334 & 82.668 & 116.002 \\
\hline 1 & \begin{tabular}{|l|} 
SB6VS-06 \\
\end{tabular} & quenched & 1 & 18 & M10 & 6.62 & 11.5 & 75.2 & 84.9 & 11.034 & 19.167 & 125.336 & 141.503 \\
\hline 1 & SB6VS-05 & CCC & 1 & 19 & M19 & 6.15 & 11.0 & 62.8 & 76.1 & 10.250 & 18.334 & 104.669 & 126.836 \\
\hline 1 & SB6VS-05 & quenched & 1 & 20 & M12 & 6.05 & 10.2 & 67.3 & 77.7 & 10.084 & 17.000 & 112.169 & 129.503 \\
\hline 1 & ARM-1 & ref & 1 & 21 & M56 & 9.91 & 7.83 & 20.9 & 35.7 & 16.517 & 13.050 & 34.834 & 59.501 \\
\hline 1 & blank & ref & 1 & 22 & M62 & $<0.176$ & $<0.173$ & $<0.863$ & $<0.231$ & \begin{tabular}{l|l|}
0.147 \\
\end{tabular} & 0.144 & 0.719 & 0.193 \\
\hline 1 & SB6VS-08 & quenched & 1 & 23 & M04 & 7.20 & 12.0 & 76.5 & 86.6 & 12.000 & 20.000 & 127.503 & 144.336 \\
\hline 1 & Soln Std & ref & 1 & 24 & std-13 & 20.0 & 9.93 & \begin{tabular}{|l|}
82.7 \\
\end{tabular} & 50.9 & 20.000 & 9.930 & 82.700 & 50.900 \\
\hline 1 & Soln Std & ref & 2 & 1 & std-21 & 20.3 & 10.1 & 82.7 & 51.6 & 20.300 & 10.100 & 82.700 & 51.600 \\
\hline 1 & SB6VS-05 & CCC & 2 & 2 & M36 & 6.10 & 10.8 & 61.6 & 76.5 & 10.167 & 18.000 & 102.669 & 127.503 \\
\hline 1 & SB6VS-06 & quenched & 2 & 3 & M34 & 6.55 & 11.3 & 72.7 & 80.0 & 10.917 & 18.834 & 121.169 & 133.336 \\
\hline 1 & SB6VS-04 & quenched & 2 & 4 & M47 & 5.83 & 9.98 & 47.8 & 72.8 & 9.717 & 16.634 & 79.668 & 121.336 \\
\hline 1 & SB6VS-04 & CCC & 2 & 5 & M30 & 5.76 & 10.4 & 45.0 & 70.9 & 9.600 & 17.334 & 75.002 & 118.169 \\
\hline 1 & SB6VS-05 & quenched & 2 & 6 & M51 & 5.91 & 9.89 & 65.2 & 73.9 & 9.850 & 16.484 & 108.669 & 123.169 \\
\hline 1 & SB6VS-01 & quenched & 2 & 7 & M27 & 6.44 & 11.0 & 50.3 & 75.0 & 10.734 & 18.334 & 83.835 & 125.003 \\
\hline 1 & SB6VS-03 & quenched & 2 & 8 & M09 & 5.31 & 9.39 & 44.2 & 67.2 & 8.850 & 15.650 & 73.668 & 112.002 \\
\hline 1 & SB6VS-06 & CCC & 2 & 9 & M29 & 6.41 & \begin{tabular}{|l|}
11.5 \\
\end{tabular} & 66.7 & \begin{tabular}{|l|}
78.7 \\
\end{tabular} & 10.684 & 19.167 & 111.169 & 131.169 \\
\hline 1 & SB6VS-09 & quenched & 2 & 10 & M17 & 6.93 & 11.3 & 57.3 & 75.6 & 11.550 & 18.834 & 95.502 & 126.003 \\
\hline 1 & SB6VS-03 & CCC & 2 & 11 & M23 & 5.29 & 9.56 & 42.4 & 67.7 & 8.817 & 15.934 & 70.668 & 112.836 \\
\hline 1 & SB6VS-07 & quenched & 2 & 12 & M26 & 6.65 & 10.8 & 68.2 & 80.4 & 11.084 & 18.000 & 113.669 & 134.003 \\
\hline 1 & Soln Std & ref & 2 & 13 & std-22 & 20.0 & 10.0 & 82.3 & 50.8 & 20.000 & 10.000 & 82.300 & 50.800 \\
\hline 1 & ARM-1 & ref & 2 & 14 & M22 & 9.58 & 7.69 & 20.6 & 35.2 & 15.967 & 12.817 & 34.334 & 58.668 \\
\hline 1 & EA & ref & 2 & 15 & M13 & 35.5 & 10.9 & 97.5 & 52.1 & 591.668 & 181.667 & 1625.003 & 868.335 \\
\hline 1 & SB6VS-08 & quenched & 2 & 16 & M46 & 7.45 & 12.2 & \begin{tabular}{|l|}
77.0 \\
\end{tabular} & 85.3 & 12.417 & 20.334 & 128.336 & 142.170 \\
\hline 1 & SB6VS-01 & CCC & 2 & 17 & M11 & 5.70 & 10.0 & 43.6 & 69.0 & 9.500 & 16.667 & 72.668 & 115.002 \\
\hline 1 & SB6VS-07 & CCC & 2 & 18 & M41 & 6.28 & 11.1 & 60.6 & 75.6 & 10.467 & 18.500 & 101.002 & 126.003 \\
\hline 1 & SB6VS-02 & quenched & 2 & 19 & M24 & 6.01 & 10.7 & 49.8 & 73.9 & 10.017 & 17.834 & 83.002 & 123.169 \\
\hline 1 & SB6VS-08 & CCC & 2 & 20 & M45 & 7.21 & 12.6 & 70.1 & 84.5 & 12.017 & 21.000 & 116.836 & 140.836 \\
\hline 1 & SB6VS-09 & CCC & 2 & 21 & M25 & 6.26 & 10.4 & 48.9 & 68.7 & 10.434 & 17.334 & 81.502 & 114.502 \\
\hline 1 & SB6VS-02 & CCC & 2 & 22 & M15 & 5.44 & 10.0 & 43.5 & 68.8 & 9.067 & 16.667 & 72.501 & 114.669 \\
\hline 1 & Soln Std & ref & 2 & 23 & std-23 & 19.9 & 9.93 & 81.8 & 50.4 & 19.900 & 9.930 & 81.800 & 50.400 \\
\hline 1 & Soln Std & ref & 3 & 1 & std-31 & 20.0 & 9.93 & 82.5 & 50.6 & 20.000 & 9.930 & 82.500 & 50.600 \\
\hline 1 & SB6VS-02 & quenched & 3 & 2 & M16 & 5.91 & 10.6 & 49.1 & 73.6 & 9.850 & 17.667 & 81.835 & 122.669 \\
\hline 1 & SB6VS-09 & CCC & 3 & 3 & M08 & 6.53 & 10.9 & 51.4 & 71.3 & 10.884 & 18.167 & 85.668 & 118.836 \\
\hline 1 & SB6VS-01 & quenched & 3 & 4 & M03 & 6.19 & 10.5 & 48.4 & 72.4 & 10.317 & 17.500 & 80.668 & 120.669 \\
\hline 1 & SB6VS-05 & CCC & 3 & 5 & M48 & 6.05 & \begin{tabular}{|l|}
10.9 \\
\end{tabular} & 61.7 & 77.6 & 10.084 & 18.167 & 102.835 & 129.336 \\
\hline 1 & \begin{tabular}{|l|} 
SB6VS-08 \\
\end{tabular} & quenched & 3 & 6 & M54 & 7.12 & 11.6 & 75.0 & 82.8 & 11.867 & 19.334 & 125.003 & 138.003 \\
\hline 1 & SB6VS-03 & CCC & 3 & 7 & M42 & 5.32 & 9.67 & 42.5 & 67.0 & 8.867 & 16.117 & 70.835 & 111.669 \\
\hline 1 & \begin{tabular}{|l|} 
SB6VS-02 \\
\end{tabular} & CCC & 3 & 8 & M01 & 5.36 & 9.86 & 42.9 & 68.4 & 8.934 & 16.434 & 71.501 & 114.002 \\
\hline 1 & SB6VS-09 & quenched & 3 & 9 & M49 & 6.84 & 11.3 & 57.1 & 75.4 & 11.400 & 18.834 & 95.169 & 125.669 \\
\hline 1 & SB6VS-04 & quenched & 3 & 10 & M43 & 5.72 & 9.94 & 48.1 & 69.2 & 9.534 & 16.567 & 80.168 & 115.336 \\
\hline 1 & SB6VS-06 & quenched & 3 & 11 & M39 & 6.56 & 11.6 & 74.8 & 80.7 & 10.934 & 19.334 & 124.669 & 134.503 \\
\hline 1 & SB6VS-07 & CCC & 3 & 12 & M60 & 6.54 & 11.6 & 63.9 & 78.3 & 10.900 & 19.334 & 106.502 & 130.503 \\
\hline 1 & Soln Std & ref & 3 & 13 & std-32 & 20.0 & 9.95 & 82.3 & 50.3 & 20.000 & 9.950 & 82.300 & 50.300 \\
\hline 1 & SB6VS-08 & CCC & 3 & 14 & M21 & 6.84 & 11.8 & 67.4 & 81.4 & 11.400 & 19.667 & 112.336 & 135.669 \\
\hline 1 & SB6VS-01 & CCC & 3 & 15 & M32 & 5.55 & 9.80 & 42.4 & 67.5 & 9.250 & 16.334 & 70.668 & 112.502 \\
\hline 1 & EA & ref & 3 & 16 & M14 & 32.7 & 10.6 & 92.5 & 52.2 & 545.001 & 176.667 & 1541.670 & 870.002 \\
\hline 1 & SB6VS-07 & quenched & 3 & 17 & M38 & 6.56 & 10.9 & 67.7 & \begin{tabular}{|l|}
79.0 \\
\end{tabular} & 10.934 & 18.167 & 112.836 & 131.669 \\
\hline 1 & ARM-1 & ref & 3 & 18 & M59 & 9.39 & \begin{tabular}{|l|}
7.61 \\
\end{tabular} & 19.6 & 33.9 & 15.650 & 12.684 & 32.667 & 56.501 \\
\hline 1 & blank & ref & 3 & 19 & M37 & $<0.176$ & $<0.173$ & $<0.863$ & $<0.231$ & 0.147 & 0.144 & 0.719 & 0.193 \\
\hline 1 & SB6VS-03 & quenched & 3 & 20 & M31 & 5.49 & 9.78 & 45.8 & 70.4 & 9.150 & 16.300 & 76.335 & 117.336 \\
\hline 1 & SB6VS-05 & quenched & 3 & 21 & M07 & 5.89 & 9.92 & 65.5 & 72.5 & 9.817 & 16.534 & 109.169 & 120.836 \\
\hline 1 & SB6VS-06 & CCC & 3 & 22 & M61 & 6.22 & 11.4 & 66.1 & 77.0 & 10.367 & 19.000 & 110.169 & 128.336 \\
\hline
\end{tabular}


Table B1. Analytical Development's Measurements of the PCT Solutions As-Received (ar) and After Appropriate Adjustments (in ppm)

\begin{tabular}{|c|c|c|c|c|c|c|c|c|c|c|c|c|c|}
\hline Set & \begin{tabular}{|l|} 
Glass \\
ID \\
\end{tabular} & \begin{tabular}{|l|} 
Heat \\
Treatment \\
\end{tabular} & Block & Seq & Lab ID & B ar & Li ar & Na ar & Si ar & $\begin{array}{r}\mathbf{B} \\
(\mathbf{p p m})\end{array}$ & $\begin{array}{r}\mathbf{L i} \\
(\mathbf{p p m})\end{array}$ & $\begin{array}{r}\mathrm{Na} \\
(\mathrm{ppm}) \\
\end{array}$ & $\begin{array}{r}\mathbf{S i} \\
(\mathbf{p p m}) \\
\end{array}$ \\
\hline 1 & SB6VS-04 & CCC & 3 & 23 & M33 & 5.74 & 10.4 & 45.6 & 69.4 & 9.567 & 17.334 & 76.002 & 115.669 \\
\hline 1 & Soln Std & ref & 3 & 24 & std-33 & 19.9 & 10.1 & 82.8 & 49.8 & 19.900 & 10.100 & 82.800 & 49.800 \\
\hline 2 & Soln Std & ref & 1 & 1 & std-11 & 20.1 & 10.0 & 82.9 & 51.1 & 20.100 & 10.000 & 82.900 & 51.100 \\
\hline 2 & SB6VS-14 & quenched & 1 & 2 & N30 & 6.46 & 11.6 & 57.6 & 81.9 & 10.767 & 19.334 & 96.002 & 136.503 \\
\hline 2 & SB6VS-13 & CCC & 1 & 3 & N33 & 5.96 & 10.9 & 46.3 & 75.0 & 9.934 & 18.167 & 77.168 & 125.003 \\
\hline 2 & SB6VS-13 & quenched & 1 & 4 & N46 & 6.16 & 11.3 & 51.3 & 77.6 & 10.267 & 18.834 & 85.502 & 129.336 \\
\hline 2 & SB6VS-15 & CCC & 1 & 5 & N13 & 6.82 & \begin{tabular}{|l|}
12.2 \\
\end{tabular} & \begin{tabular}{|l|}
61.7 \\
\end{tabular} & 85.3 & 11.367 & 20.334 & 102.835 & 142.170 \\
\hline 2 & SB6VS-15 & quenched & 1 & 6 & N25 & 6.94 & \begin{tabular}{|l|}
12.2 \\
\end{tabular} & 66.1 & 86.5 & 11.567 & 20.334 & 110.169 & 144.170 \\
\hline 2 & SB6VS-17 & CCC & 1 & 7 & N45 & 6.70 & 11.0 & 66.2 & 79.0 & 11.167 & 18.334 & 110.336 & 131.669 \\
\hline 2 & SB6VS-17 & quenched & 1 & 8 & N53 & 7.09 & \begin{tabular}{|l|}
11.4 \\
\end{tabular} & 75.9 & 82.4 & 11.817 & 19.000 & 126.503 & 137.336 \\
\hline 2 & ARM-1 & ref & 1 & 9 & N29 & 10.9 & 8.70 & 22.7 & \begin{tabular}{|l|}
38.7 \\
\end{tabular} & 18.167 & 14.500 & 37.834 & 64.501 \\
\hline 2 & SB6VS-11 & quenched & 1 & 10 & N41 & 6.23 & 11.1 & 58.4 & 77.3 & 10.384 & 18.500 & 97.335 & 128.836 \\
\hline 2 & SB6VS-14 & CCC & 1 & 11 & N54 & 7.04 & 12.9 & 59.6 & 89.9 & 11.734 & 21.500 & 99.335 & 149.836 \\
\hline 2 & Soln Std & ref & 1 & 12 & std-12 & 20.1 & \begin{tabular}{|l|}
10.1 \\
\end{tabular} & \begin{tabular}{|l|}
82.2 \\
\end{tabular} & 50.9 & 20.100 & 10.100 & 82.200 & 50.900 \\
\hline 2 & blank & ref & 1 & 13 & N05 & $<0.176$ & $<0.173$ & $<0.863$ & $<0.231$ & 0.147 & 0.144 & 0.719 & 0.193 \\
\hline 2 & SB6VS-10 & quenched & 1 & 14 & N38 & 7.94 & 13.0 & 65.4 & 94.0 & 13.234 & 21.667 & 109.002 & 156.670 \\
\hline 2 & EA & ref & 1 & 15 & N17 & 24.2 & 8.40 & \begin{tabular}{|l|}
70.1 \\
\end{tabular} & 42.3 & 403.334 & 140.000 & 1168.336 & 705.001 \\
\hline 2 & \begin{tabular}{|l|} 
SB6VS-12 \\
\end{tabular} & CCC & 1 & 16 & N34 & 7.24 & 12.7 & 71.7 & \begin{tabular}{|l|}
83.7 \\
\end{tabular} & 12.067 & 21.167 & 119.502 & 139.503 \\
\hline 2 & SB6VS-11 & CCC & 1 & 17 & N06 & 6.02 & 11.0 & 53.2 & 74.0 & 10.034 & 18.334 & 88.668 & 123.336 \\
\hline 2 & SB6VS-10 & CCC & 1 & 18 & N19 & 6.87 & 12.1 & 56.8 & 87.1 & 11.450 & 20.167 & 94.669 & 145.170 \\
\hline 2 & SB6VS-16 & quenched & 1 & 19 & N50 & 6.87 & \begin{tabular}{|l|}
11.7 \\
\end{tabular} & 69.8 & 82.4 & 11.450 & 19.500 & 116.336 & 137.336 \\
\hline 2 & SB6VS-12 & quenched & 1 & 20 & N02 & 7.66 & 13.0 & 81.2 & 86.4 & 12.767 & 21.667 & 135.336 & 144.003 \\
\hline 2 & SB6VS-16 & CCC & 1 & 21 & N40 & 6.93 & \begin{tabular}{|l|}
12.1 \\
\end{tabular} & 65.8 & 86.0 & 11.550 & 20.167 & 109.669 & 143.336 \\
\hline 2 & Soln Std & ref & 1 & 22 & std-13 & 20.0 & \begin{tabular}{|l|}
10.1 \\
\end{tabular} & 82.5 & 50.4 & 20.000 & 10.100 & 82.500 & 50.400 \\
\hline 2 & Soln Std & ref & 2 & 1 & std-21 & 19.8 & 9.92 & 82.2 & 50.5 & 19.800 & 9.920 & 82.200 & 50.500 \\
\hline 2 & SB6VS-15 & quenched & 2 & 2 & N26 & 6.87 & 11.8 & 64.1 & 85.1 & 11.450 & 19.667 & 106.835 & 141.836 \\
\hline 2 & SB6VS-13 & CCC & 2 & 3 & N31 & 6.09 & 11.2 & 48.1 & 78.2 & 10.150 & 18.667 & 80.168 & 130.336 \\
\hline 2 & SB6VS-15 & CCC & 2 & 4 & N37 & 6.88 & 12.1 & 61.1 & 83.9 & 11.467 & 20.167 & 101.835 & 139.836 \\
\hline 2 & SB6VS-17 & CCC & 2 & 5 & N42 & 6.50 & \begin{tabular}{|l|}
10.7 \\
\end{tabular} & 64.3 & \begin{tabular}{|l|}
75.7 \\
\end{tabular} & 10.834 & 17.834 & 107.169 & 126.169 \\
\hline 2 & SB6VS-11 & CCC & 2 & 6 & N44 & 5.70 & \begin{tabular}{|l|}
10.0 \\
\end{tabular} & \begin{tabular}{|l|}
49.7 \\
\end{tabular} & 69.4 & 9.500 & 16.667 & 82.835 & 115.669 \\
\hline 2 & SB6VS-14 & quenched & 2 & 7 & N49 & 6.29 & 11.1 & 56.2 & 78.9 & 10.484 & 18.500 & 93.669 & 131.503 \\
\hline 2 & SB6VS-10 & CCC & 2 & 8 & N22 & 6.83 & 11.9 & 55.8 & 84.3 & 11.384 & 19.834 & 93.002 & 140.503 \\
\hline 2 & SB6VS-16 & CCC & 2 & 9 & N03 & 6.72 & 11.3 & 62.2 & 81.9 & 11.200 & 18.834 & 103.669 & 136.503 \\
\hline 2 & SB6VS-12 & CCC & 2 & 10 & N15 & 7.21 & \begin{tabular}{|l|}
12.7 \\
\end{tabular} & 71.0 & 83.4 & 12.017 & 21.167 & 118.336 & 139.003 \\
\hline 2 & SB6VS-13 & quenched & 2 & 11 & N48 & 6.44 & 12.0 & 53.5 & 79.9 & 10.734 & 20.000 & 89.168 & 133.169 \\
\hline 2 & Soln Std & ref & 2 & 12 & std-22 & \begin{tabular}{|l|}
19.7 \\
\end{tabular} & 9.94 & \begin{tabular}{|l|}
81.7 \\
\end{tabular} & 50.2 & 19.700 & 9.940 & 81.700 & 50.200 \\
\hline 2 & EA & \begin{tabular}{|l} 
ref \\
\end{tabular} & 2 & 13 & N08 & \begin{tabular}{|l|}
23.7 \\
\end{tabular} & \begin{tabular}{|l|}
8.37 \\
\end{tabular} & 68.6 & \begin{tabular}{|l|}
42.7 \\
\end{tabular} & 395.001 & 139.500 & 1143.336 & 711.668 \\
\hline 2 & ARM-1 & ref & 2 & 14 & N04 & 9.92 & 8.26 & 21.6 & 36.8 & 16.534 & 13.767 & 36.001 & 61.335 \\
\hline 2 & SB6VS-17 & quenched & 2 & 15 & N24 & 6.99 & 11.1 & 73.7 & 82.6 & 11.650 & 18.500 & 122.836 & 137.669 \\
\hline 2 & SB6VS-12 & quenched & 2 & 16 & N07 & 7.67 & 12.9 & 80.4 & 88.7 & 12.784 & 21.500 & 134.003 & 147.836 \\
\hline 2 & SB6VS-11 & quenched & 2 & 17 & N35 & 6.32 & 11.2 & 58.5 & 78.7 & 10.534 & 18.667 & 97.502 & 131.169 \\
\hline 2 & SB6VS-16 & quenched & 2 & 18 & N32 & 6.96 & \begin{tabular}{|l|}
11.7 \\
\end{tabular} & \begin{tabular}{|l|}
70.3 \\
\end{tabular} & 84.4 & 11.600 & 19.500 & 117.169 & 140.669 \\
\hline 2 & SB6VS-10 & quenched & 2 & 19 & N09 & 7.60 & \begin{tabular}{|l|}
12.2 \\
\end{tabular} & 61.6 & 88.6 & 12.667 & 20.334 & 102.669 & 147.670 \\
\hline 2 & SB6VS-14 & CCC & 2 & 20 & N27 & 6.73 & 12.5 & 57.6 & 86.5 & 11.217 & 20.834 & 96.002 & 144.170 \\
\hline 2 & Soln Std & ref & 2 & 21 & std-23 & 19.8 & 10.1 & 81.5 & 50.5 & 19.800 & 10.100 & 81.500 & 50.500 \\
\hline 2 & Soln Std & ref & 3 & 1 & std-31 & 20.0 & 9.95 & 80.8 & 50.6 & 20.000 & 9.950 & 80.800 & 50.600 \\
\hline 2 & SB6VS-15 & $\mathrm{CCC}$ & 3 & 2 & N56 & 7.26 & 12.5 & 62.8 & 88.9 & 12.100 & 20.834 & 104.669 & 148.170 \\
\hline 2 & SB6VS-14 & CCC & 3 & 3 & N01 & 6.81 & 12.4 & 57.4 & 83.4 & 11.350 & 20.667 & 95.669 & 139.003 \\
\hline 2 & SB6VS-17 & CCC & 3 & 4 & N12 & 6.80 & \begin{tabular}{|l|}
10.7 \\
\end{tabular} & 64.1 & 76.2 & 11.334 & 17.834 & 106.835 & 127.003 \\
\hline 2 & EA & ref & 3 & 5 & N23 & 22.6 & 7.85 & 65.2 & 41.2 & 376.667 & 130.834 & 1086.669 & 686.668 \\
\hline 2 & SB6VS-11 & quenched & 3 & 6 & N20 & 6.41 & \begin{tabular}{|l|}
11.1 \\
\end{tabular} & 58.4 & 76.3 & 10.684 & 18.500 & 97.335 & 127.169 \\
\hline 2 & blank & ref & 3 & 7 & N11 & $<0.176$ & $<0.173$ & $<0.863$ & $<0.231$ & \begin{tabular}{l|l|}
0.147 \\
\end{tabular} & 0.144 & 0.719 & 0.193 \\
\hline 2 & ARM-1 & ref & 3 & 8 & N10 & 10.2 & 8.28 & 22.3 & 37.1 & 17.000 & 13.800 & 37.167 & 61.835 \\
\hline 2 & SB6VS-13 & CCC & 3 & 9 & N18 & 6.18 & \begin{tabular}{|l|}
11.3 \\
\end{tabular} & \begin{tabular}{|l|}
47.7 \\
\end{tabular} & 81.4 & 10.300 & 18.834 & 79.502 & 135.669 \\
\hline 2 & SB6VS-12 & CCC & 3 & 10 & N47 & 7.28 & 12.5 & 70.2 & 82.3 & 12.134 & 20.834 & 117.002 & 137.169 \\
\hline 2 & SB6VS-15 & quenched & 3 & 11 & N28 & 6.94 & \begin{tabular}{|l|}
11.8 \\
\end{tabular} & 64.4 & 83.7 & 11.567 & 19.667 & 107.335 & 139.503 \\
\hline 2 & Soln Std & ref & 3 & 12 & std-32 & 19.8 & 9.99 & 80.7 & 50.4 & 19.800 & 9.990 & 80.700 & 50.400 \\
\hline 2 & SB6VS-11 & CCC & 3 & 13 & N16 & 6.01 & \begin{tabular}{|l|}
10.7 \\
\end{tabular} & 51.0 & 72.6 & 10.017 & 17.834 & 85.002 & 121.002 \\
\hline 2 & SB6VS-10 & CCC & 3 & 14 & N51 & 6.98 & \begin{tabular}{|l|}
11.9 \\
\end{tabular} & 56.3 & 85.0 & 11.634 & 19.834 & 93.835 & 141.670 \\
\hline 2 & SB6VS-14 & quenched & 3 & 15 & N43 & 6.29 & \begin{tabular}{|l|}
11.1 \\
\end{tabular} & 55.4 & 81.9 & 10.484 & 18.500 & 92.335 & 136.503 \\
\hline 2 & SB6VS-13 & quenched & 3 & 16 & N36 & 6.15 & 11.2 & 51 & 75.9 & 10.250 & 18.667 & 85.002 & 126.503 \\
\hline 2 & SB6VS-10 & quenched & 3 & 17 & N39 & $\begin{array}{l}7.77 \\
\end{array}$ & \begin{tabular}{|l|}
12.4 \\
\end{tabular} & \begin{tabular}{|l|}
62.1 \\
\end{tabular} & 86.8 & 12.950 & 20.667 & 103.502 & 144.670 \\
\hline 2 & SB6VS-16 & CCC & 3 & 18 & N55 & 6.77 & 11.1 & 61.7 & 79.0 & 11.284 & 18.500 & 102.835 & 131.669 \\
\hline 2 & SB6VS-12 & quenched & 3 & 19 & N52 & 7.77 & 13.1 & 80.5 & \begin{tabular}{|l|}
86.7 \\
\end{tabular} & 12.950 & 21.834 & 134.169 & 144.503 \\
\hline 2 & SB6VS-16 & quenched & 3 & 20 & N21 & 7.00 & \begin{tabular}{|l|}
11.7 \\
\end{tabular} & 68.9 & 81.9 & 11.667 & 19.500 & 114.836 & 136.503 \\
\hline 2 & SB6VS-17 & quenched & 3 & 21 & N14 & 6.85 & 10.6 & 70.5 & 77.2 & 11.417 & 17.667 & 117.502 & 128.669 \\
\hline 2 & Soln Std & ref & 3 & 22 & std-33 & 19.8 & 10.1 & 81.1 & 50.5 & 19.800 & 10.100 & 81.100 & 50.500 \\
\hline
\end{tabular}


Table B2. Initial Study Glasses with Unpredictable Durability Responses for Boron Release

(Glasses whose rows are shaded in yellow have unpredictable boron releases.)

\begin{tabular}{|c|c|c|c|c|c|c|c|c|c|}
\hline $\begin{array}{c}\text { Glass } \\
\text { ID }\end{array}$ & $\begin{array}{c}\text { Heat } \\
\text { Treatment }\end{array}$ & $\begin{array}{c}\text { Composition } \\
\text { View }\end{array}$ & $\begin{array}{c}\text { NL } \\
{[B(g / L)]}\end{array}$ & $\begin{array}{l}\text { Del } \\
\text { Gp }\end{array}$ & $\begin{array}{c}\log \\
\operatorname{NL}[\mathbf{B}(\mathrm{g} / \mathrm{L})]\end{array}$ & $\begin{array}{c}\text { Lower 95\% } \\
\text { Indiv } \log \mathrm{NL}[\mathrm{B}(\mathrm{g} / \mathrm{L})] \\
\end{array}$ & $\begin{array}{c}\text { Pred Formula } \\
\log \mathrm{NL}[\mathrm{B}(\mathrm{g} / \mathrm{L})]\end{array}$ & $\begin{array}{c}\text { Upper } 95 \% \\
\text { Indiv } \log \mathrm{NL}[\mathrm{B}(\mathrm{g} / \mathrm{L})] \\
\end{array}$ & \begin{tabular}{|c} 
LM \\
Prep Block
\end{tabular} \\
\hline EA & & reference & 16.30 & -15.5186 & 1.212065 & 0.47178733 & 0.91057981 & 1.34937228 & . \\
\hline EA & & reference & 11.16 & -15.5186 & 1.047504 & 0.47178733 & 0.91057981 & 1.34937228 & . \\
\hline SB6VS-05 & CCC & measured & 0.69 & -14.6796 & -0.16382 & 0.32251251 & 0.75856007 & 1.19460763 & 2 \\
\hline SB6VS-05 & quenched & measured & 0.67 & -14.6796 & -0.17465 & 0.32251251 & 0.75856007 & 1.19460763 & 2 \\
\hline SB6VS-15 & CCC & measured & 0.78 & -13.5161 & -0.10577 & 0.11470753 & 0.54773009 & 0.98075266 & 2 \\
\hline SB6VS-15 & quenched & measured & 0.78 & -13.5161 & -0.10998 & 0.11470753 & 0.54773009 & 0.98075266 & 2 \\
\hline SB6VS-10 & CCC & measured & 0.78 & -13.3413 & -0.10926 & 0.08339879 & 0.51604635 & 0.94869391 & 2 \\
\hline SB6VS-10 & quenched & measured & 0.88 & -13.3413 & -0.05732 & 0.08339879 & 0.51604635 & 0.94869391 & 2 \\
\hline SB6VS-08 & $\mathrm{CCC}$ & measured & 0.82 & -12.764 & -0.08801 & -0.0201245 & 0.41143402 & 0.84299259 & 1 \\
\hline SB6VS-08 & quenched & measured & 0.85 & -12.764 & -0.0713 & -0.0201245 & 0.41143402 & 0.84299259 & 1 \\
\hline SB6VS-04 & cCC & measured & 0.67 & -12.5591 & -0.17583 & -0.0569247 & 0.3743026 & 0.80552993 & 2 \\
\hline SB6VS-04 & quenched & measured & 0.67 & -12.5591 & -0.17509 & -0.0569247 & 0.3743026 & 0.80552993 & 2 \\
\hline SB6VS-12 & $\mathrm{CCC}$ & measured & 0.83 & -12.5278 & -0.08107 & -0.0625384 & 0.36864098 & 0.79982036 & 1 \\
\hline SB6VS-12 & quenched & measured & 0.88 & -12.5278 & -0.05452 & -0.0625384 & 0.36864098 & 0.79982036 & 1 \\
\hline SB6VS-03 & CCC & measured & 0.59 & -12.4797 & -0.23166 & -0.0711779 & 0.35992904 & 0.79103595 & 2 \\
\hline SB6VS-03 & quenched & measured & 0.59 & -12.4797 & -0.22792 & -0.0711779 & 0.35992904 & 0.79103595 & 2 \\
\hline SB6VS-02 & CCC & measured & 0.61 & -12.2808 & -0.21595 & -0.1069341 & 0.32389012 & 0.7547143 & 2 \\
\hline SB6VS-02 & quenched & measured & 0.66 & -12.2808 & -0.17734 & -0.1069341 & 0.32389012 & 0.7547143 & 2 \\
\hline SB6VS-01 & CCC & measured & 0.62 & -12.1009 & -0.20583 & -0.1393175 & 0.29127452 & 0.72186649 & 2 \\
\hline SB6VS-01 & quenched & measured & 0.68 & -12.1009 & -0.16825 & -0.1393175 & 0.29127452 & 0.72186649 & 2 \\
\hline SB6VS-17 & cCC & measured & 0.81 & -11.6559 & -0.09261 & -0.2194625 & 0.21065224 & 0.64076702 & 1 \\
\hline SB6VS-17 & quenched & measured & 0.85 & -11.6559 & -0.07284 & -0.2194625 & 0.21065224 & 0.64076702 & 1 \\
\hline SB6VS-09 & CCC & measured & 0.70 & -11.6218 & -0.15233 & -0.225624 & 0.20445981 & 0.63454365 & 1 \\
\hline SB6VS-09 & quenched & measured & 0.77 & \begin{tabular}{|l|}
-11.6218 \\
\end{tabular} & -0.11507 & -0.225624 & 0.20445981 & 0.63454365 & 1 \\
\hline SB6VS-06 & CCC & measured & 0.68 & -11.6003 & $\begin{array}{l}-0.1657 \\
\end{array}$ & -0.2294987 & 0.2005661 & 0.6306309 & 1 \\
\hline SB6VS-06 & quenched & measured & 0.71 & -11.6003 & -0.14907 & -0.2294987 & 0.2005661 & 0.6306309 & 1 \\
\hline SB6VS-11 & cCC & measured & 0.67 & \begin{tabular}{|l|}
-11.55 \\
\end{tabular} & -0.17622 & -0.2385644 & 0.19145712 & 0.62147864 & 1 \\
\hline SB6VS-11 & quenched & measured & 0.71 & -11.55 & -0.14698 & -0.2385644 & 0.19145712 & 0.62147864 & 1 \\
\hline SB6VS-07 & CCC & measured & 0.71 & -11.5483 & -0.14853 & -0.2388711 & 0.19114896 & 0.62116904 & 1 \\
\hline SB6VS-07 & quenched & measured & 0.72 & -11.5483 & -0.14456 & -0.2388711 & 0.19114896 & 0.62116904 & 1 \\
\hline SB6VS-16 & $\mathrm{CCC}$ & measured & 0.76 & -11.4299 & -0.11697 & -0.2602338 & 0.16969146 & 0.5996167 & 1 \\
\hline SB6VS-16 & quenched & measured & 0.78 & -11.4299 & -0.10831 & -0.2602338 & 0.16969146 & 0.5996167 & 1 \\
\hline SB6VS-13 & CCC & measured & 0.67 & -10.9773 & -0.17565 & -0.3419707 & 0.08768248 & 0.51733562 & 1 \\
\hline SB6VS-13 & quenched & measured & 0.69 & -10.9773 & -0.16349 & -0.3419707 & 0.08768248 & 0.51733562 & 1 \\
\hline SB6VS-14 & CCC & measured & 0.75 & -10.3192 & -0.12303 & -0.4610855 & -0.0315721 & 0.39794125 & 2 \\
\hline SB6VS-14 & quenched & measured & 0.70 & -10.3192 & -0.15676 & -0.4610855 & -0.0315721 & 0.39794125 & 2 \\
\hline ARM & & reference & 0.46 & -9.79971 & -0.34001 & -0.5553188 & -0.1257013 & 0.30391612 & . \\
\hline ARM & & reference & 0.49 & -9.79971 & -0.3092 & -0.5553188 & -0.1257013 & 0.30391612 & \\
\hline
\end{tabular}


Exhibit B1. PCT Measurements (as Common Logarithms) in Analytical Sequence by Analytical Set for Initial Glasses

Set $=1$

Variability Chart for $\log [\mathrm{B}$ ppm]

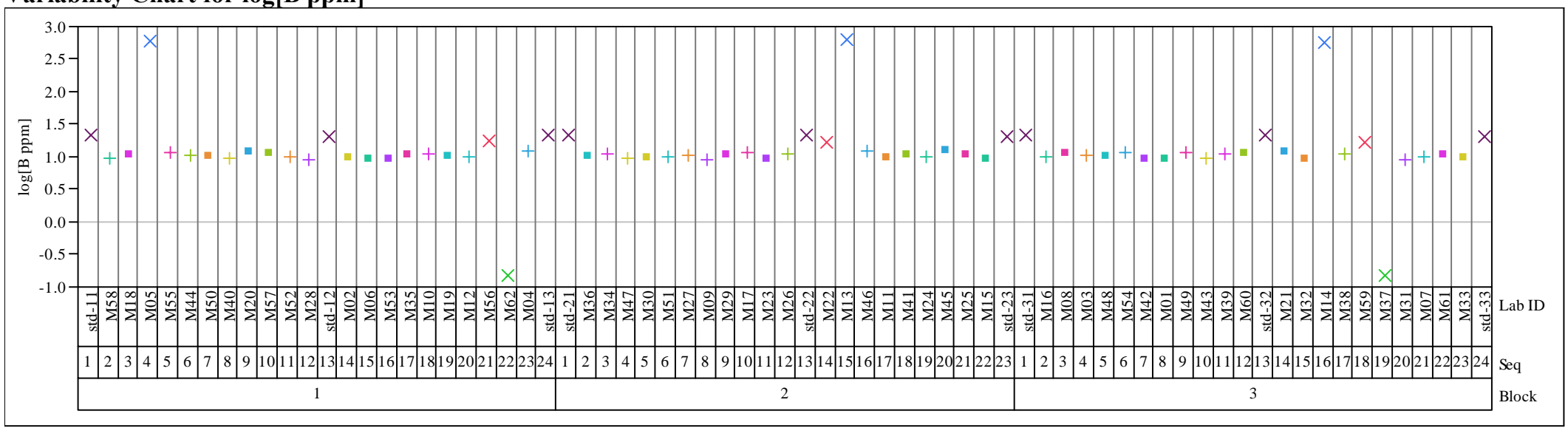

\section{Set $=1$}

Variability Chart for $\log [\mathrm{Li}$ ppm]

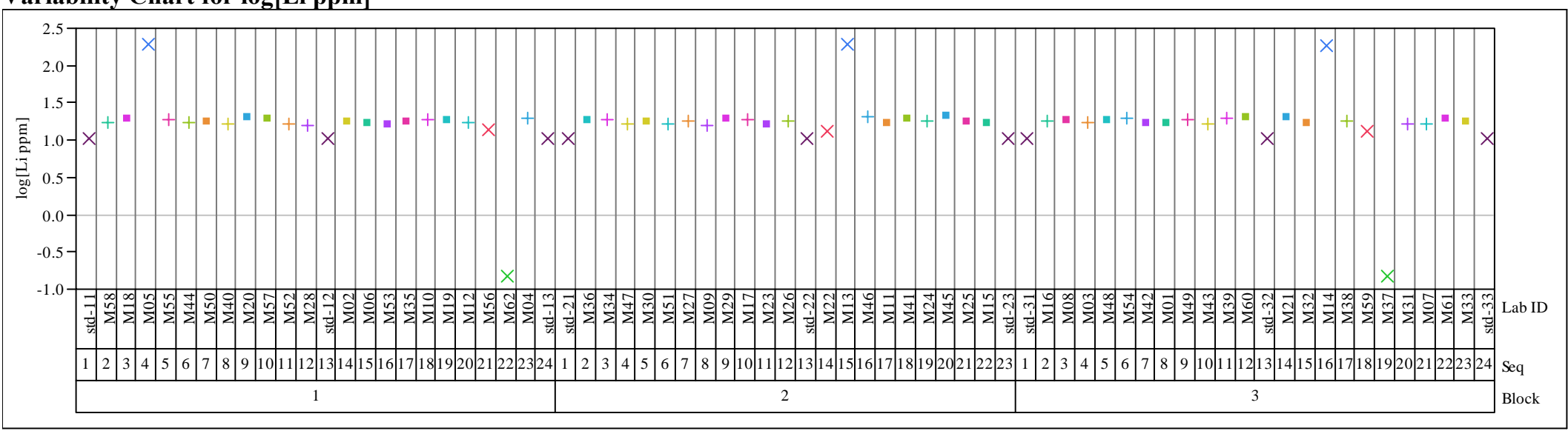


Exhibit B1. PCT Measurements (as Common Logarithms) in Analytical Sequence by Analytical Set for Initial Glasses

Set $=1$

Variability Chart for $\log [\mathrm{Na}$ ppm]

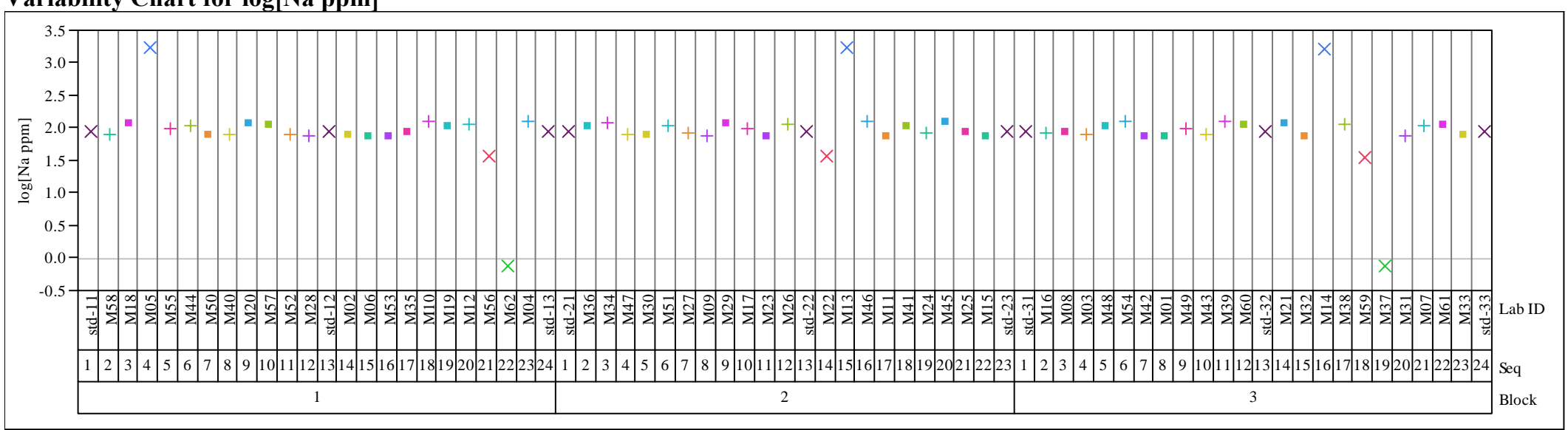

\section{Set $=1$}

Variability Chart for log[Si ppm]

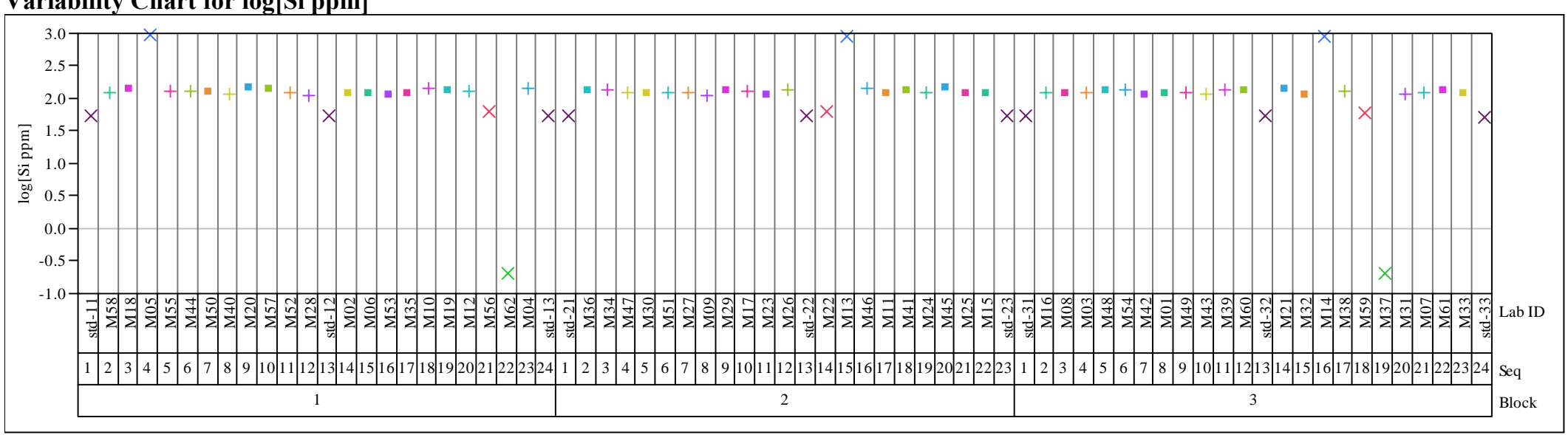


Exhibit B1. PCT Measurements (as Common Logarithms) in Analytical Sequence by Analytical Set for Initial Glasses

Set $=2$

Variability Chart for $\log [\mathrm{B}$ ppm]

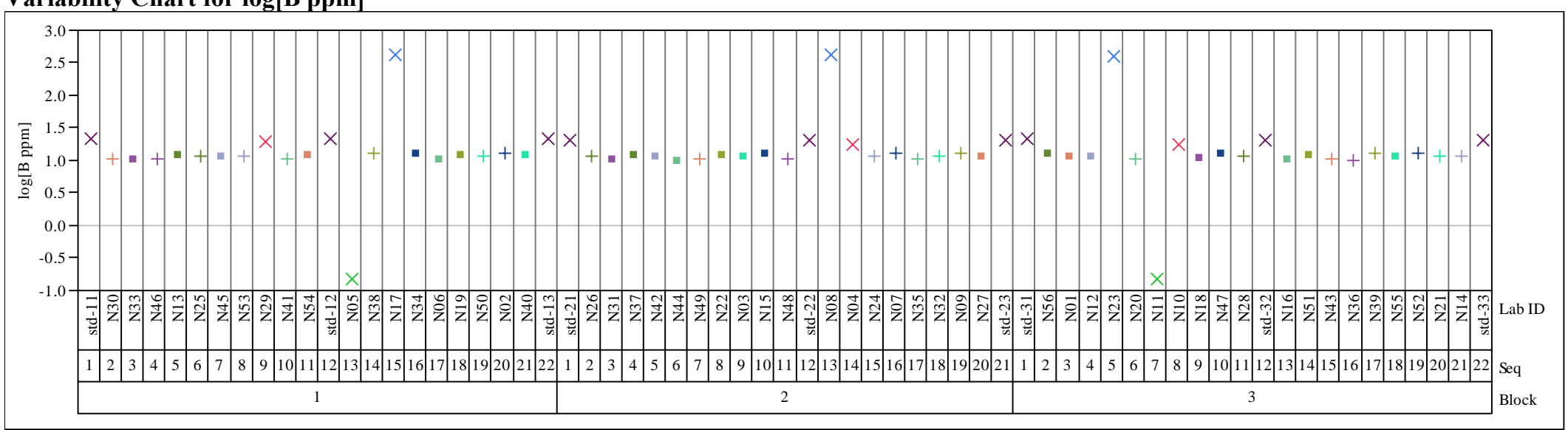

Set $=2$

Variability Chart for $\log [\mathrm{Li}$ ppm]

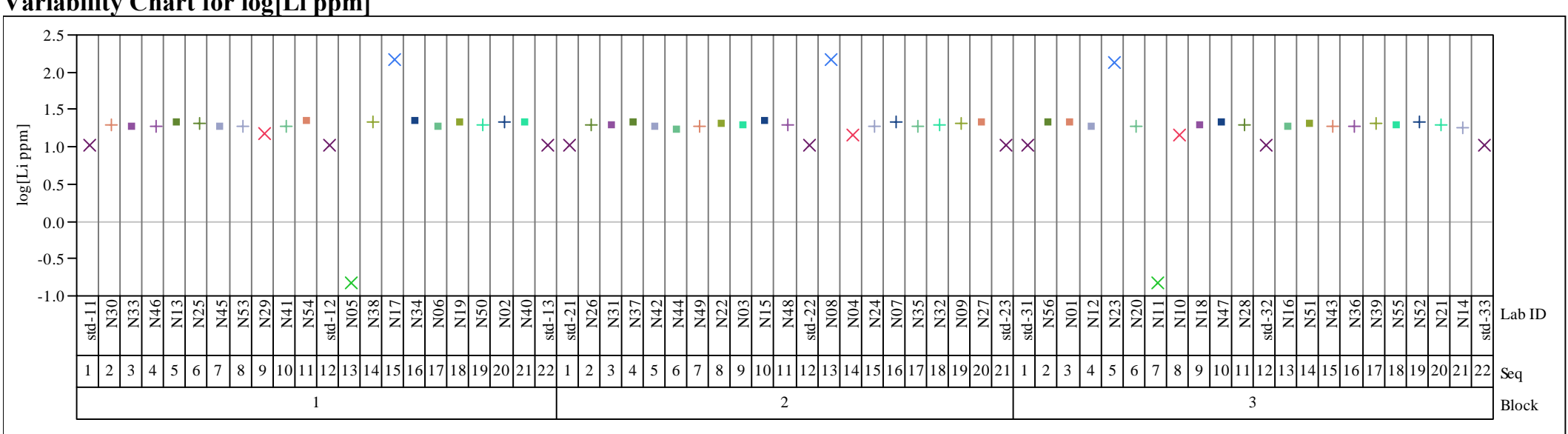


Exhibit B1. PCT Measurements (as Common Logarithms) in Analytical Sequence by Analytical Set for Initial Glasses

Set $=2$

Variability Chart for log[Na ppm]

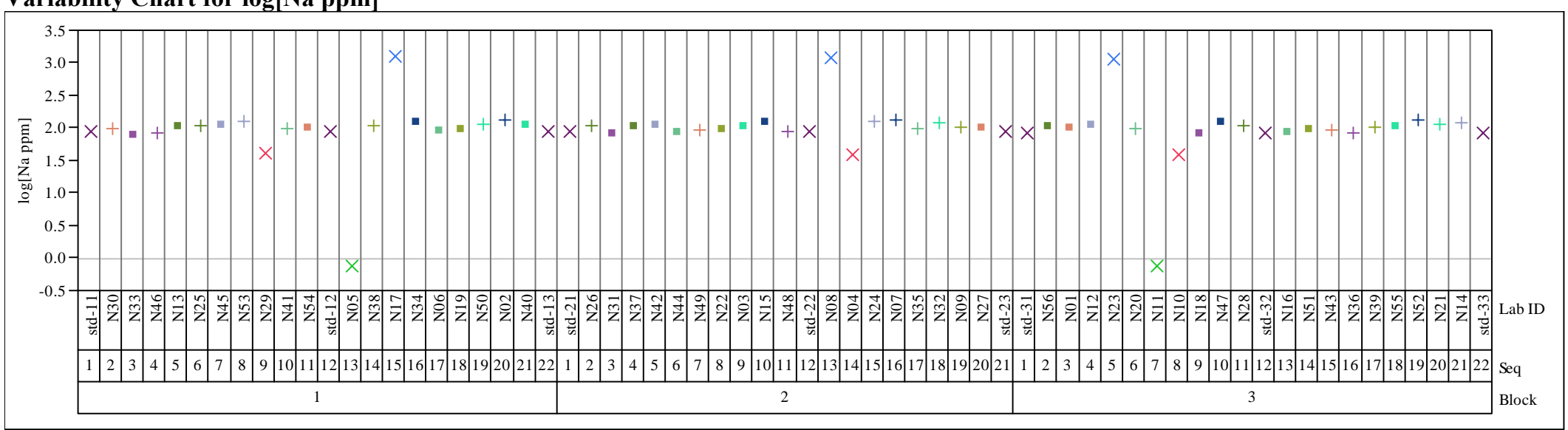

Set $=2$

Variability Chart for $\log [\mathrm{Si}$ ppm]

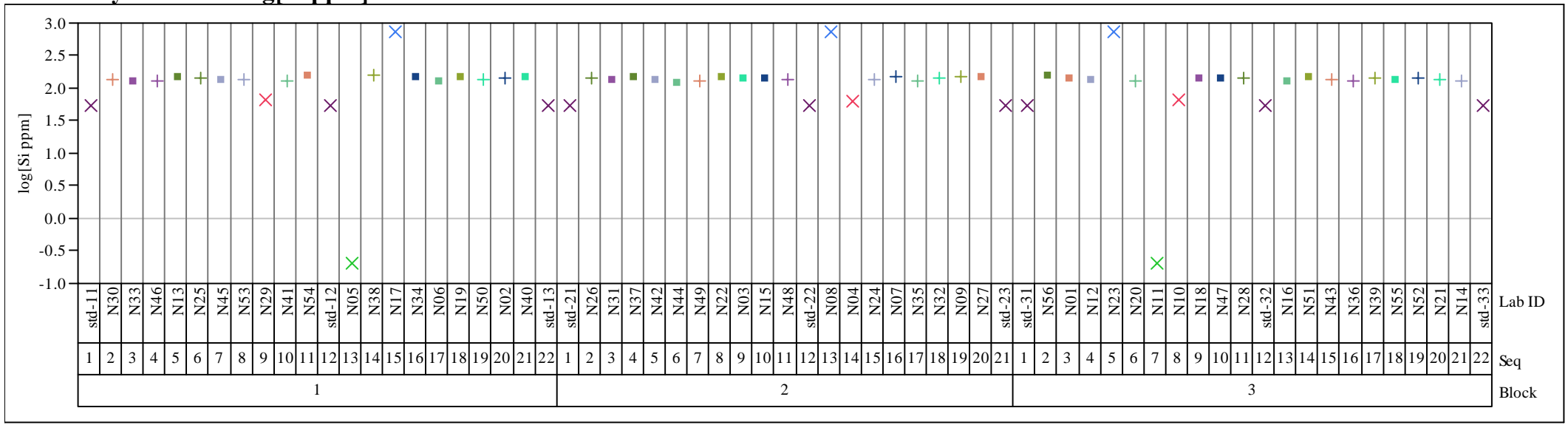




\section{Exhibit B2. Measurements of the Multi-Element Solution Standard by ICP Block for Initial Glasses}

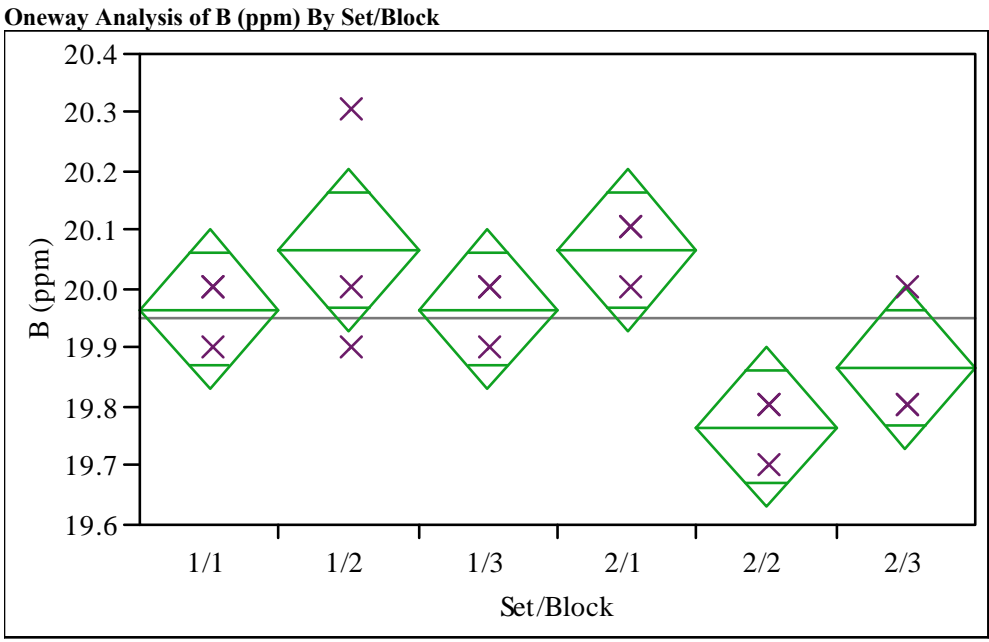

\section{Oneway Anova}

\section{Summary of}

$\begin{array}{lr}\text { Rsquare } & 0.594203 \\ \text { Adj Rsquare } & 0.425121 \\ \text { Root Mean Square Error } & 0.108012 \\ \text { Mean of Response } & 19.95 \\ \text { Observations (or Sum Wgts) } & 18\end{array}$

Analysis of Variance

Source DF Sum of Squares Mean Square F Ratio Prob $>$ F

Set/Block $\quad 5 \quad 0.20500000 \quad 0.041000 \quad 3.5143 \quad 0.0346$

$\begin{array}{lrrr}\text { Error } & 12 & 0.14000000 & 0.011667 \\ \text { C. Total } & 17 & 0.34500000 & \end{array}$

Means for Oneway Anova

Level Number Mean Std Error Lower 95\% Upper 95\%

$\begin{array}{llllll}1 / 1 & 3 & 19.9667 & 0.06236 & 19.831 & 20.103 \\ 1 / 2 & 3 & 20.0667 & 0.06236 & 19.931 & 20.203 \\ 1 / 3 & 3 & 19.9667 & 0.06236 & 19.831 & 20.103 \\ 2 / 1 & 3 & 20.0667 & 0.06236 & 19.931 & 20.203 \\ 2 / 2 & 3 & 19.7667 & 0.06236 & 19.631 & 19.903 \\ 2 / 3 & 3 & 19.8667 & 0.06236 & 19.731 & 20.003\end{array}$

Std Error uses a pooled estimate of error variance

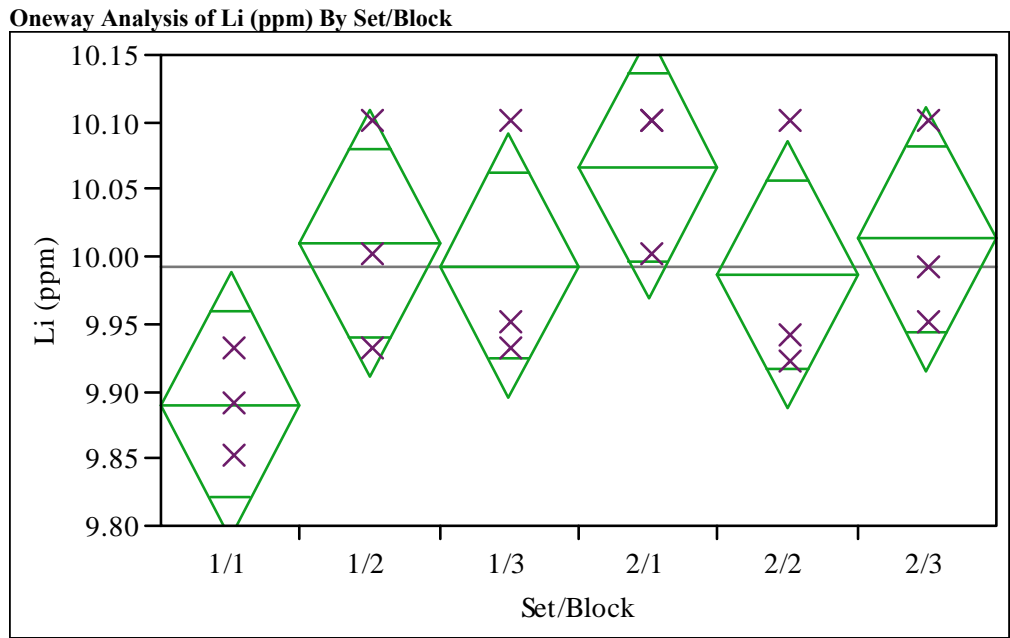

Oneway Anova

Summary of Fit

$\begin{array}{lr}\text { Rsquare } & 0.407228 \\ \text { Adj Rsquare } & 0.160239 \\ \text { Root Mean Square Error } & 0.078138 \\ \text { Mean of Response } & 9.993333 \\ \text { Observations (or Sum Wgts) } & 18\end{array}$

Analysis of Variance

Source DF Sum of Squares Mean Square F Ratio Prob $>$ F

$\begin{array}{llllll}\text { Set/Block } & 5 & 0.05033333 & 0.010067 & 1.6488 & 0.2213\end{array}$

\begin{tabular}{lrr} 
Error & 12 & 0.07326667 \\
\hline
\end{tabular}

Means for Oneway Anova

Level Number Mean Std Error Lower 95\% Upper 95\%

$\begin{array}{lrrrrr}1 / 1 & 3 & 9.8900 & 0.04511 & 9.7917 & 9.988 \\ 1 / 2 & 3 & 10.0100 & 0.04511 & 9.9117 & 10.108\end{array}$

$\begin{array}{rrrrrr}1 / 2 & 3 & 10.0100 & 0.04511 & 9.9117 & 10.108 \\ 1 / 3 & 3 & 9.9933 & 0.04511 & 9.8950 & 10.092\end{array}$

$\begin{array}{llllll}2 / 1 & 3 & 10.0667 & 0.04511 & 9.9684 & 10.165\end{array}$

$\begin{array}{rrrrrr}2 / 2 & 3 & 9.9867 & 0.04511 & 9.8884 & 10.085 \\ 2 / 3 & 3 & 10.0133 & 0.04511 & 9.9150 & 10.112\end{array}$

Std Error uses a pooled estimate of error variance 


\section{Exhibit B2. Measurements of the Multi-Element Solution Standard by ICP Block for Initial Glasses}

Oneway Analysis of Na (ppm) By Set/Block

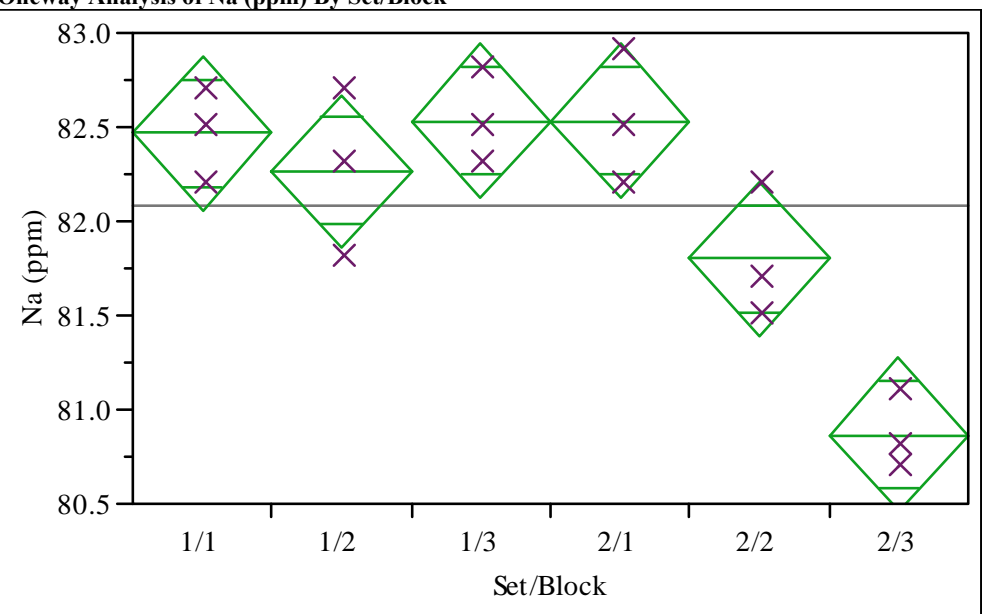

Oneway Anova

Summary of Fit

$\begin{array}{lr}\text { Rsquare } & 0.837041 \\ \text { Adj Rsquare } & 0.769142 \\ \text { Root Mean Square Error } & 0.323179 \\ \text { Mean of Response } & 82.07778 \\ \text { Observations (or Sum Wgts) } & 18\end{array}$

Analysis of Variance

Source DF Sum of Squares Mean Square F Ratio Prob $>$ F

$\begin{array}{llllll}\text { Set/Block } & 5 & 6.4377778 & 1.28756 & 12.3277 & 0.0002\end{array}$

Error $\quad 12 \quad 1.2533333$

0.10444

C. Total $17 \quad 7.6911111$

Level Number Mean Std Error Lower 95\% Upper 95\%

$\begin{array}{llllll}1 / 1 & 3 & 82.4667 & 0.18659 & 82.060 & 82.873\end{array}$

$\begin{array}{llllll}1 / 2 & 3 & 82.2667 & 0.18659 & 81.860 & 82.673 \\ 1 / 3 & 3 & 82.5333 & 0.18659 & 82.127 & 82.940\end{array}$

$\begin{array}{llllll}1 / 3 & 3 & 82.5333 & 0.18659 & 82.127 & 82.940 \\ 2 / 1 & 3 & 81.8000 & 0.1659 & 81.393 & 82.207\end{array}$

$\begin{array}{llllll}2 / 2 & 3 & 81.8000 & 0.18659 & 82.127 & 82.940 \\ 2 / 3 & 3 & 80.8667 & 0.18659 & 80.460 & 82.207 \\ & & & & & \end{array}$

Std Error uses a pooled estimate of error variance

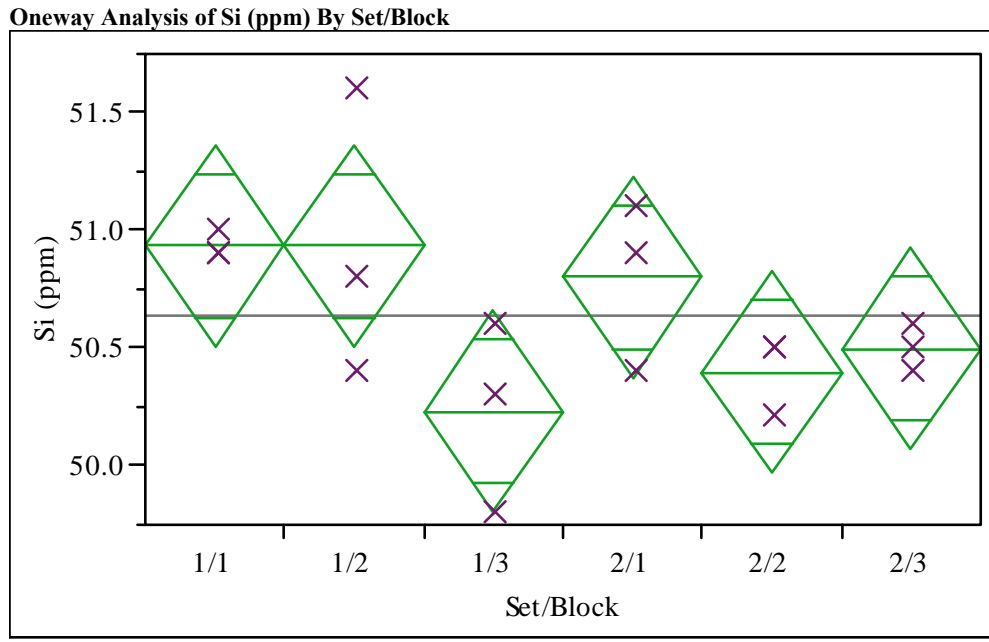

Oneway Anova

Summary of Fit

$\begin{array}{lr}\text { Rsquare } & 0.481752 \\ \text { Adj Rsquare } & 0.265815 \\ \text { Root Mean Square Error } & 0.343996 \\ \text { Mean of Response } & 50.63333 \\ \text { Observations (or Sum Wgts) } & 18\end{array}$

Analysis of Variance

Source DF Sum of Squares Mean Square F Ratio Prob $>$ F

$\begin{array}{llllll}\text { Set/Block } & 5 & 1.3200000 & 0.264000 & 2.2310 & 0.1183\end{array}$

$\begin{array}{lrrr}\text { Error } & 12 & 1.4200000 & 0.118333\end{array}$

Means for Oneway Anova

Level Number Mean Std Error Lower 95\% Upper 95\%

$\begin{array}{lllllr}1 / 1 & 3 & 50.9333 & 0.19861 & 50.501 & 51.366 \\ 1 / 2 & 3 & 50.9333 & 0.19861 & 50.501 & 51.366\end{array}$

$\begin{array}{llllll}1 / 2 & 3 & 50.9333 & 0.19861 & 50.501 & 51.366 \\ 1 / 3 & 3 & 50.2333 & 0.19861 & 49.801 & 50.666\end{array}$

$\begin{array}{llllll}1 / 3 & 3 & 50.8000 & 0.19861 & 50.367 & 51.233\end{array}$

$\begin{array}{llllll}2 / 2 & 3 & 50.4000 & 0.19861 & 49.967 & 50.833 \\ 2 / 3 & 3 & 50.5000 & 0.19861 & 50.067 & 50.933\end{array}$

Std Error uses a pooled estimate of error variance 
Exhibit B3. Laboratory PCT Measurements by Glass Identifier for Initial Study Glasses and Standards

Variability Chart for B (ppm)

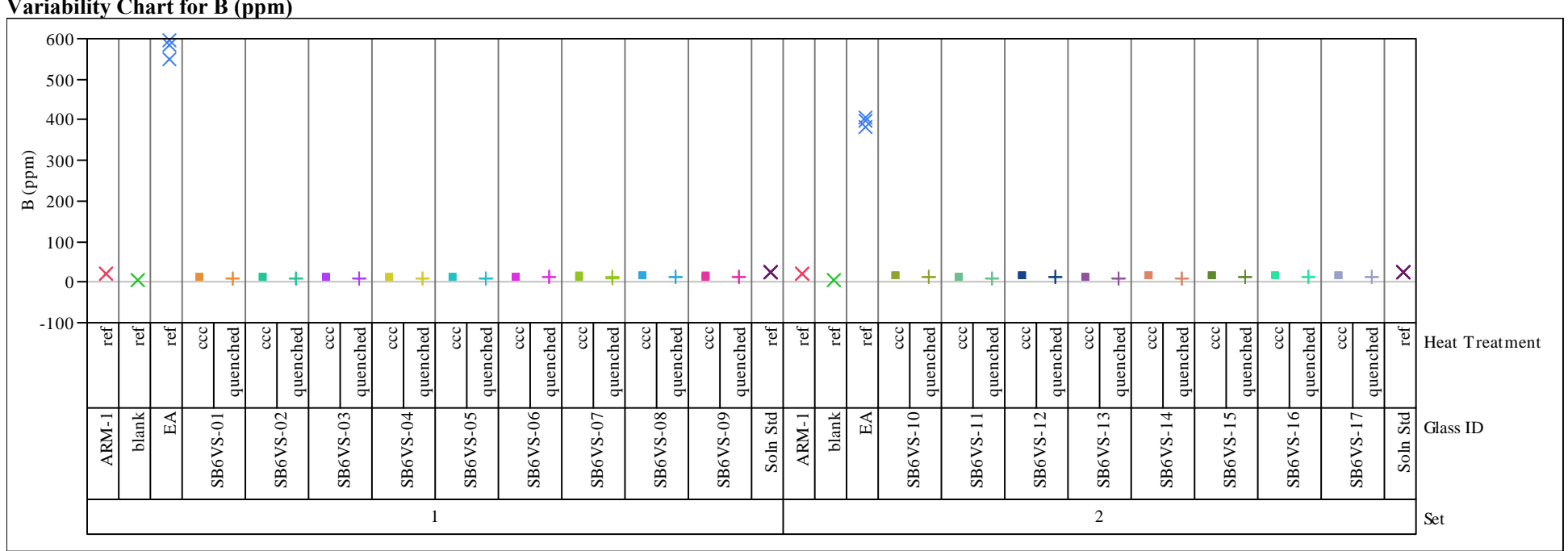

Variability Chart for Li (ppm)

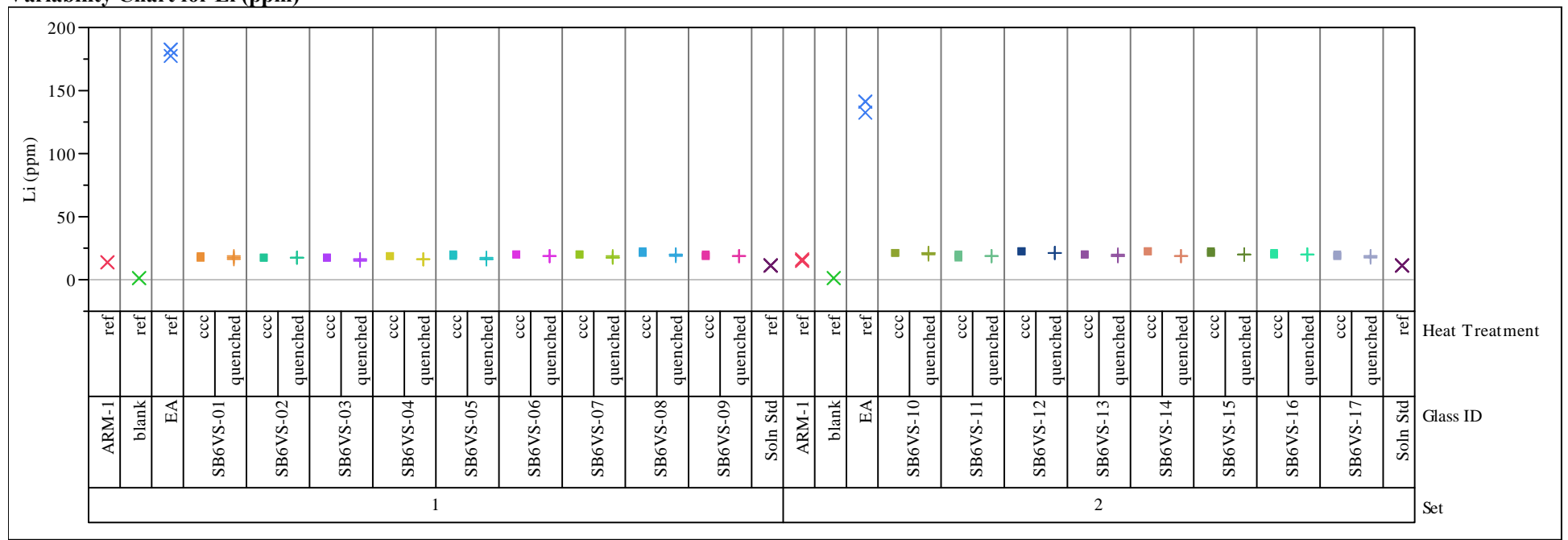




\section{Exhibit B3. Laboratory PCT Measurements by Glass Identifier for Initial Study Glasses and Standards}

Variability Chart for Na (ppm)

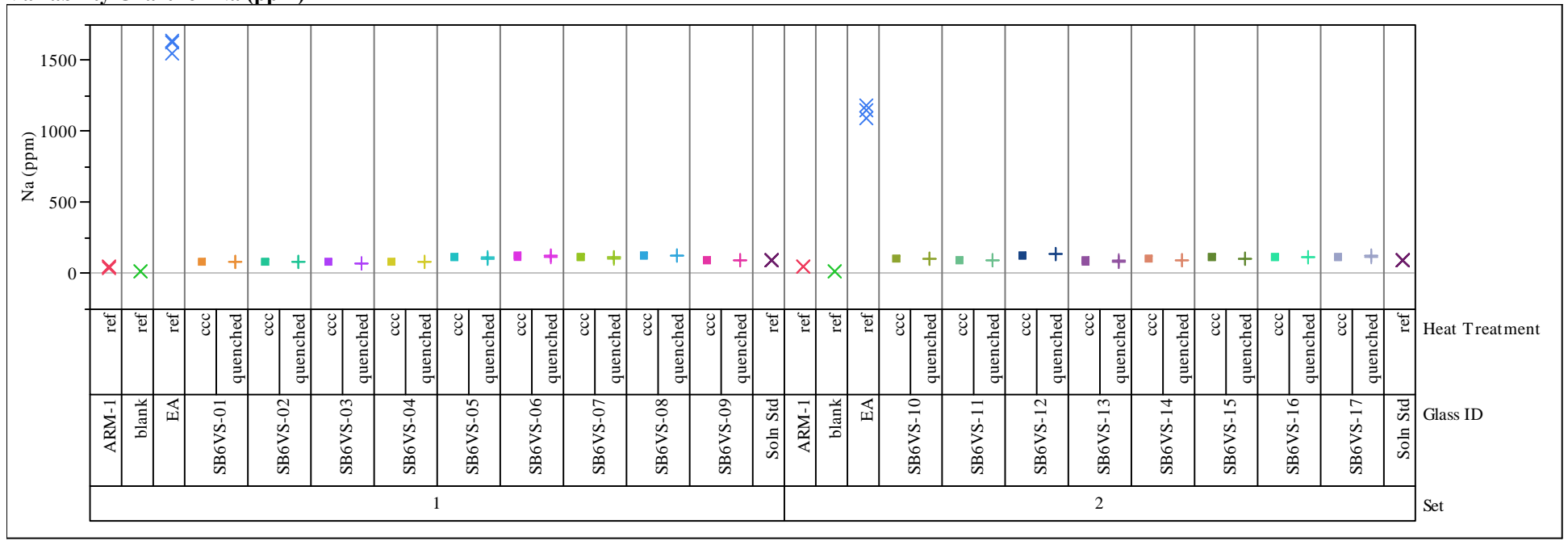

Variability Chart for Si (ppm)

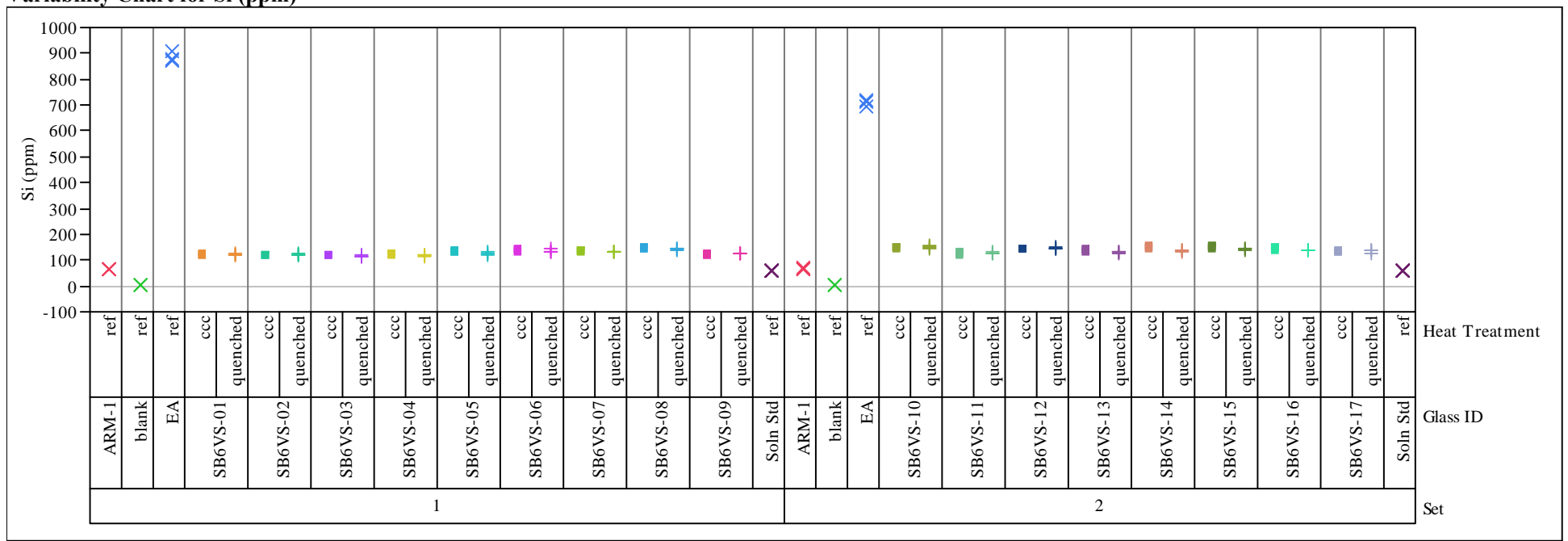


Exhibit B3. Laboratory PCT Measurements by Glass Identifier for Initial Study Glasses and Standards

Variability Chart for $\log [\mathrm{B}$ ppm]

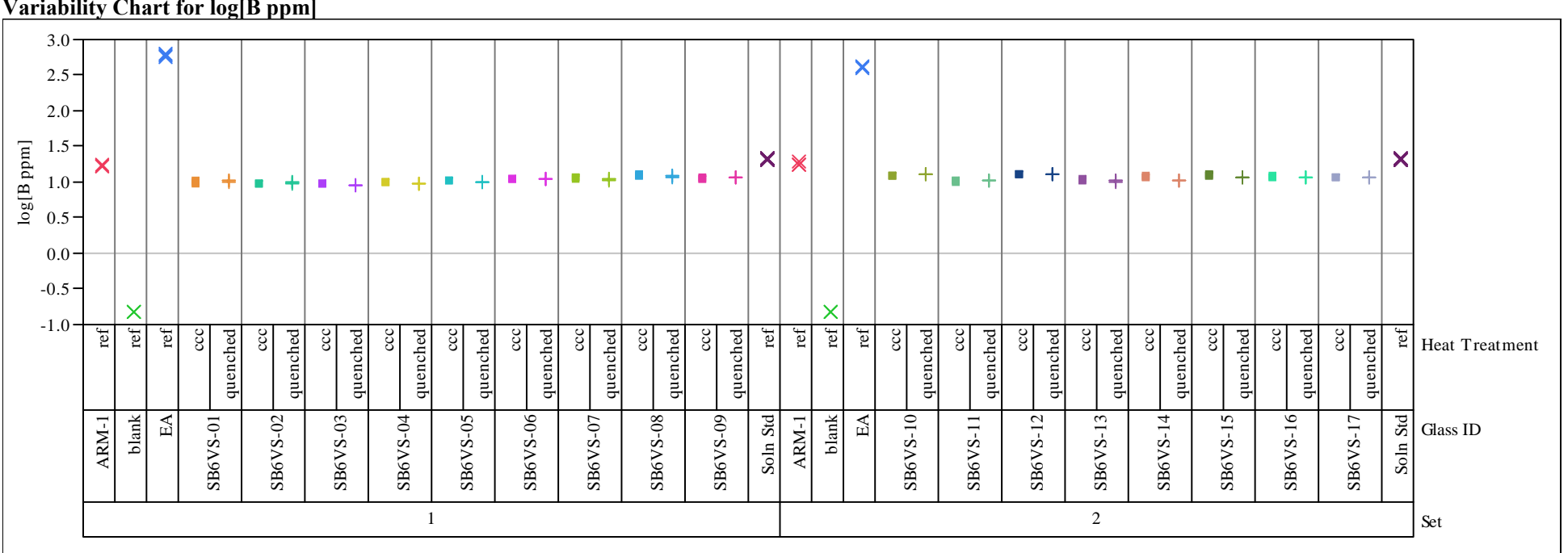

Variability Chart for $\log [\mathrm{Li}$ ppm]

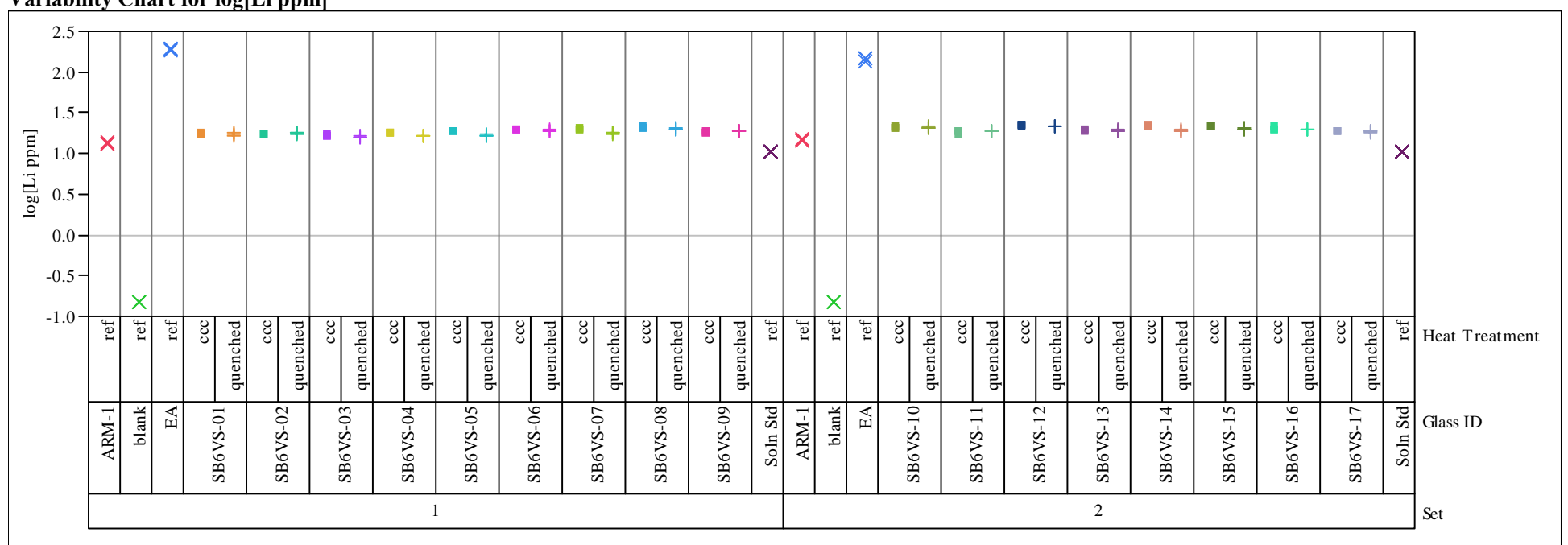




\section{Exhibit B3. Laboratory PCT Measurements by Glass Identifier for Initial Study Glasses and Standards}

Variability Chart for $\log [\mathbf{N a} \mathrm{ppm}]$

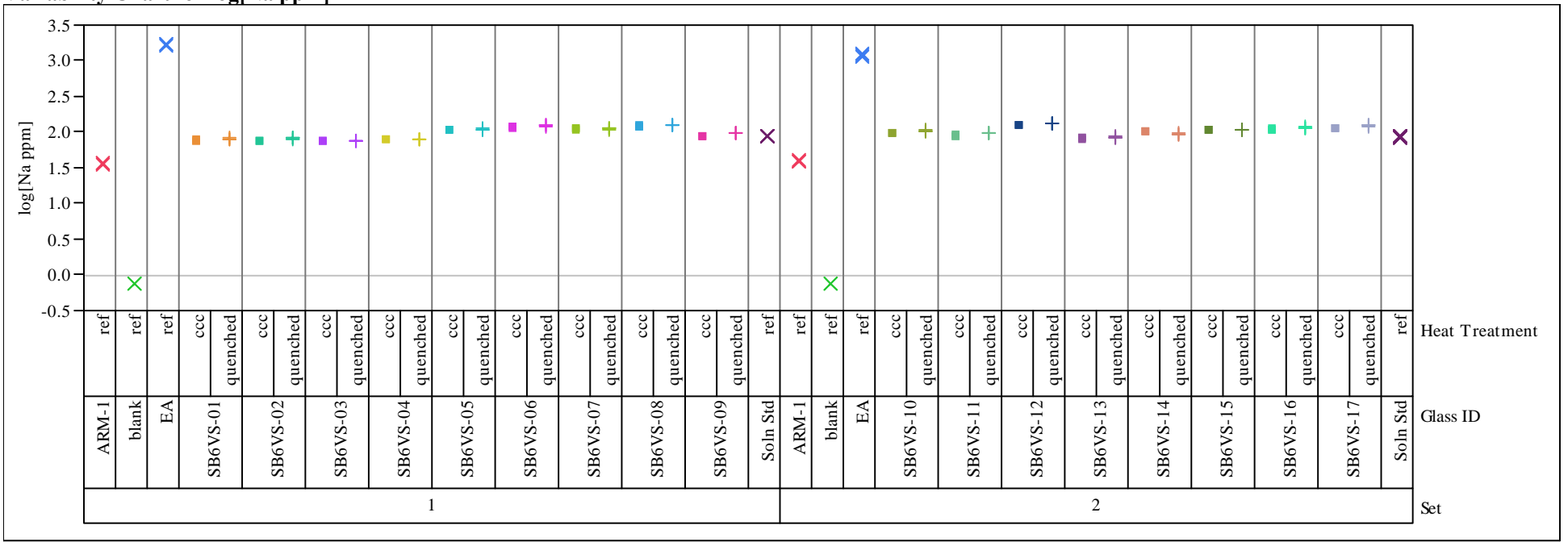

Variability Chart for $\log [$ Si ppm]

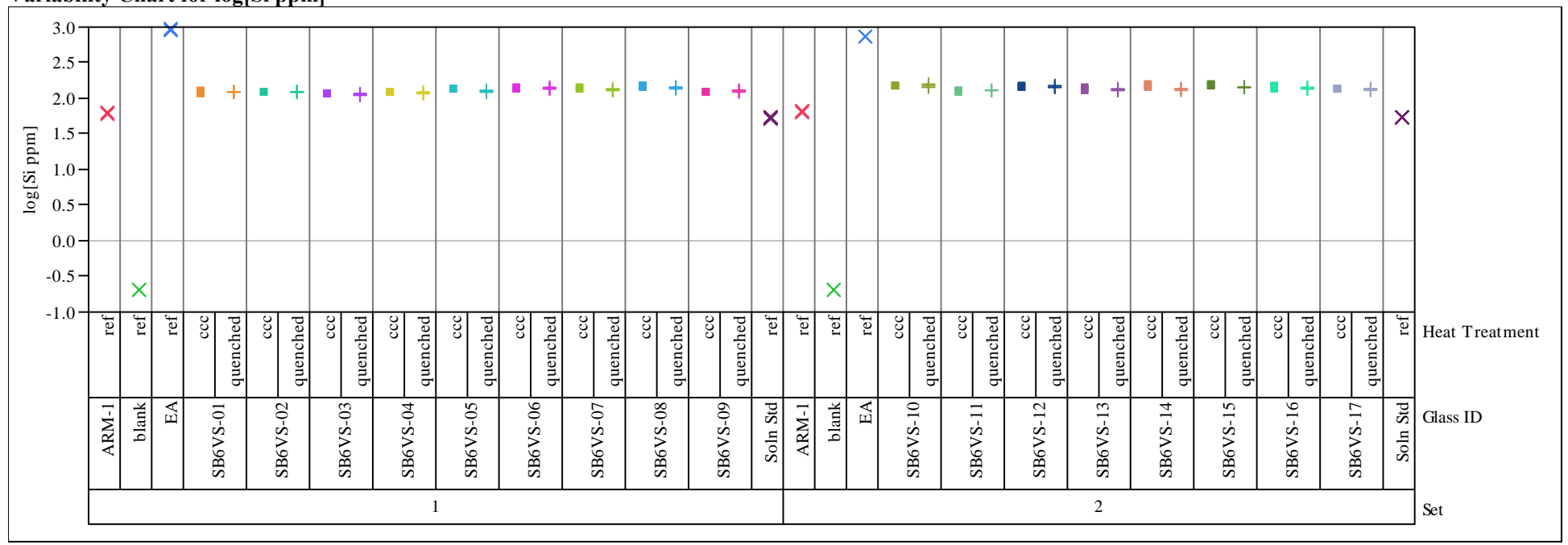


Exhibit B4. Correlations and Scatter Plots of Normalized PCTs Over All Compositional Views and Heat Treatments for All Initial Study Glasses
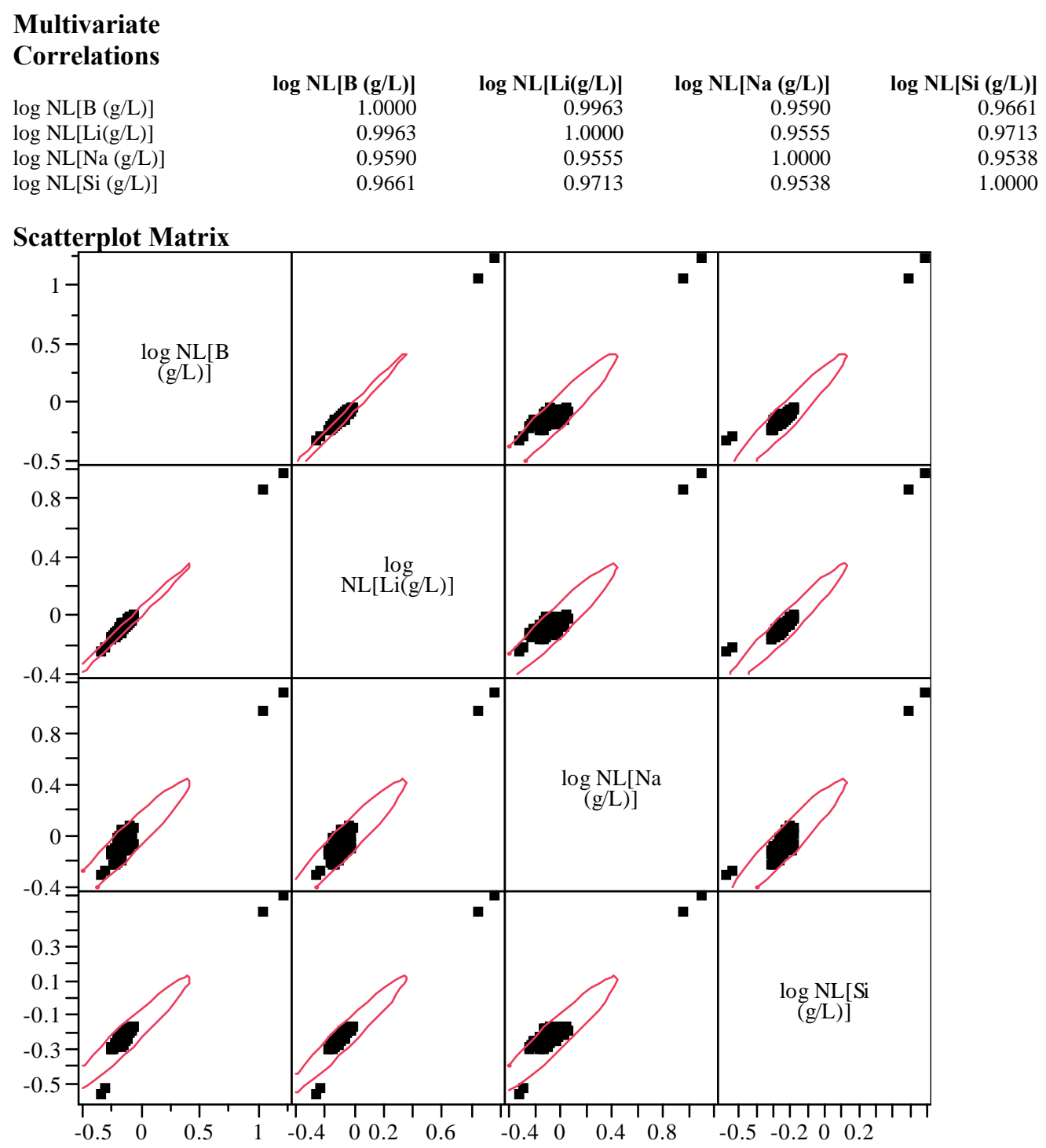
Exhibit B5. Effects of Heat Treatment for Initial Study Glasses by Compositional View

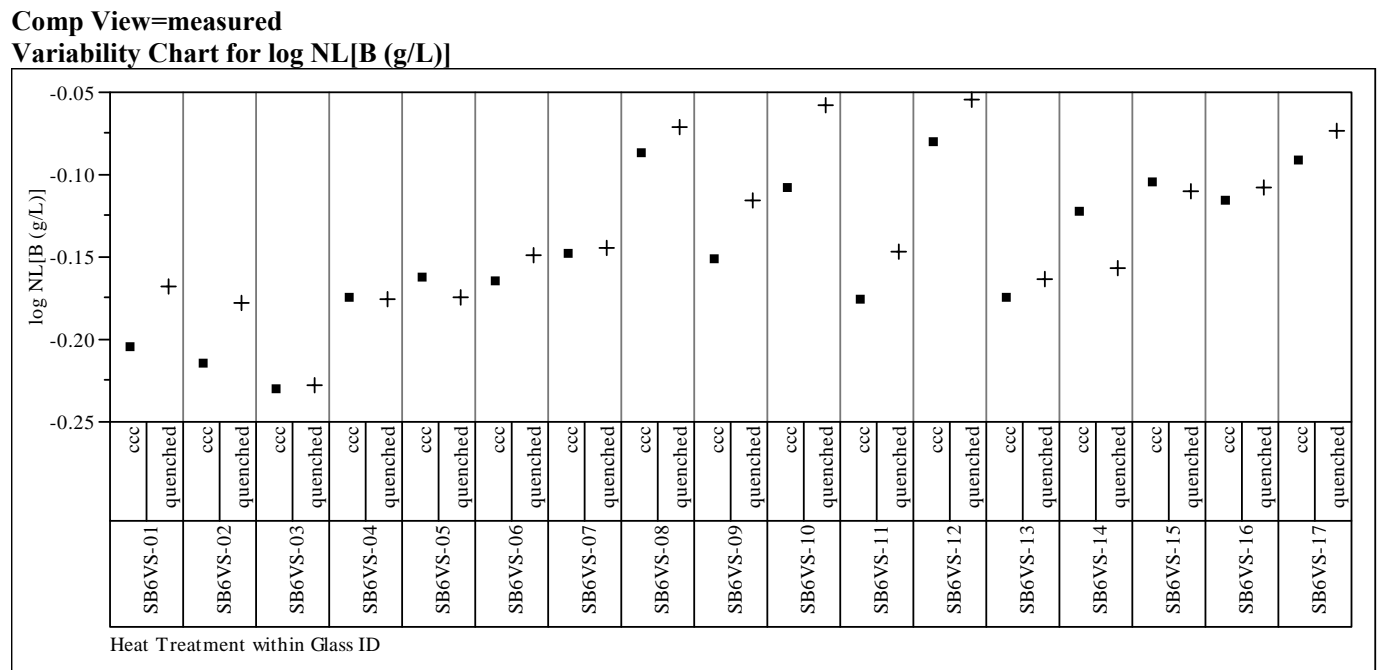

Comp View $=$ measured

Variability Chart for $\log \mathrm{NL}[\mathrm{Li}(\mathrm{g} / \mathrm{L})]$

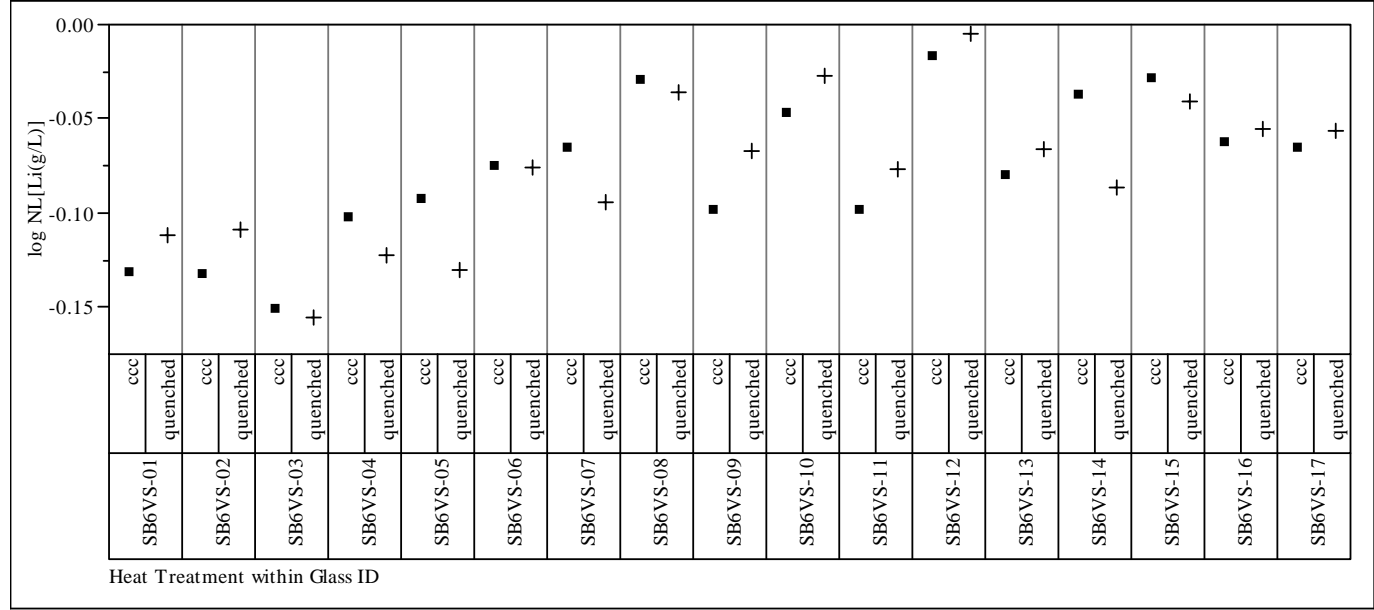

Comp View=measured

Variability Chart for $\log \mathrm{NL}[\mathrm{Na}(\mathrm{g} / \mathrm{L})]$

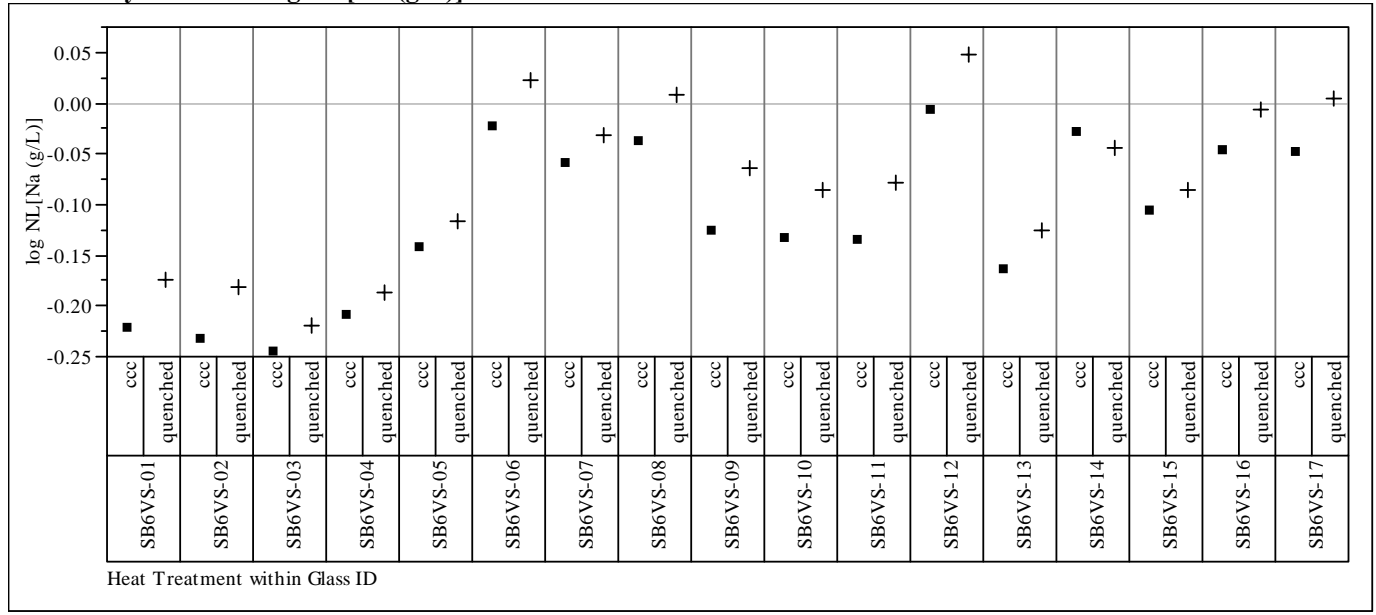




\section{Exhibit B5. Effects of Heat Treatment for Initial Study Glasses by Compositional View}

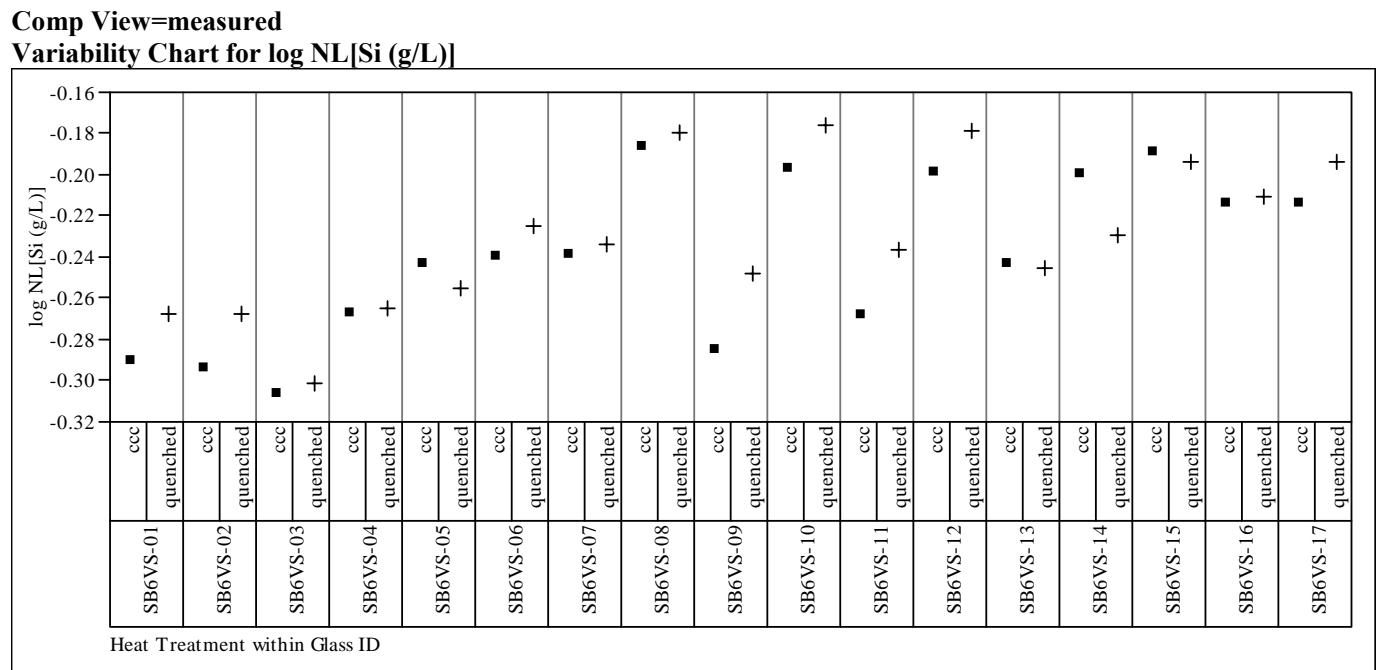

Comp View=targeted

Variability Chart for $\log$ NL[B (g/L)]

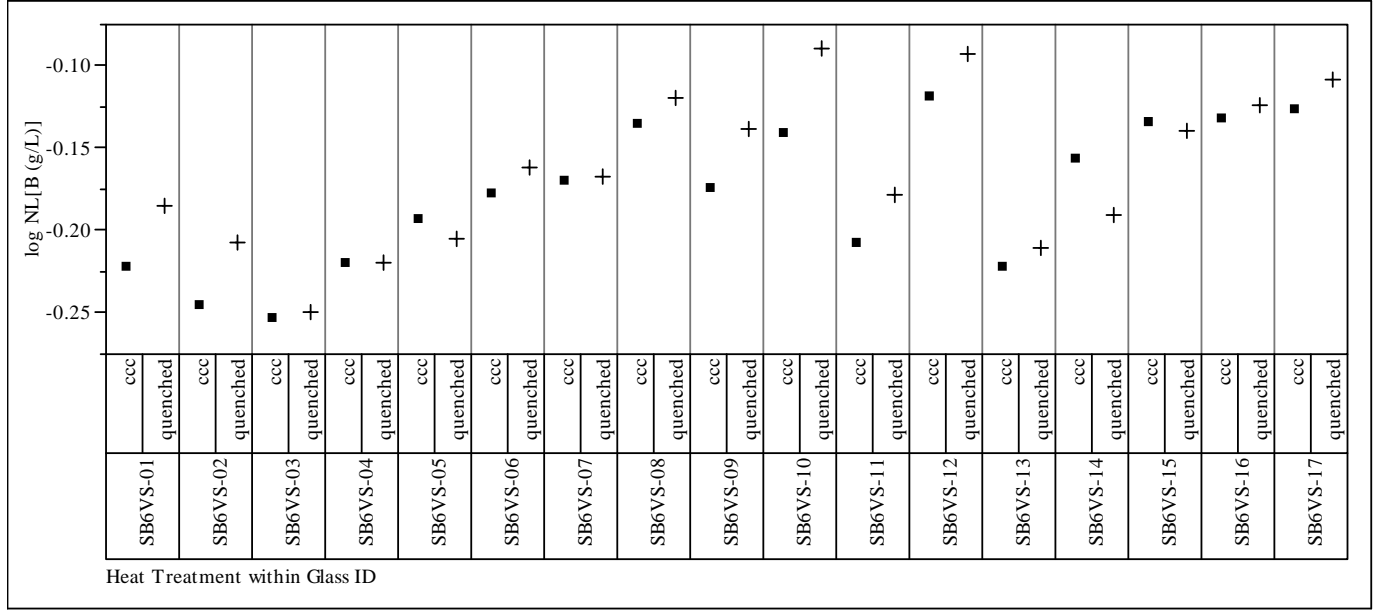

Comp View $=$ targeted

Variability Chart for log NL[Li(g/L)]

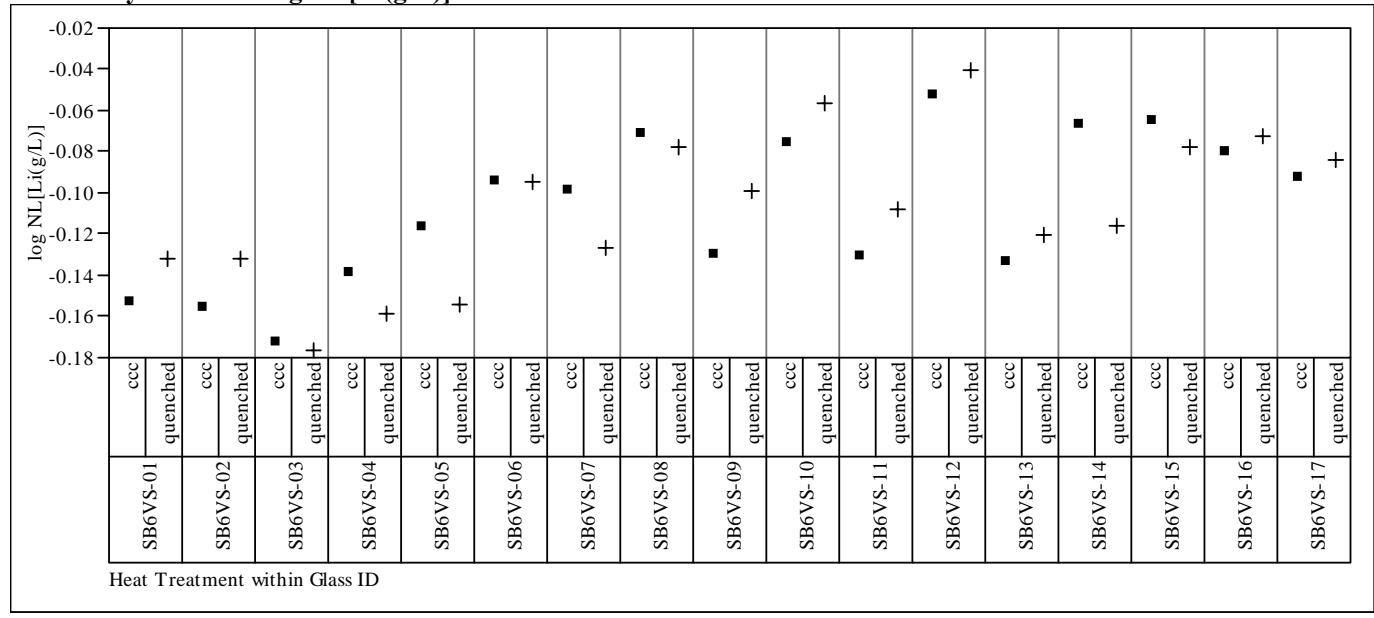


Exhibit B5. Effects of Heat Treatment for Initial Study Glasses by Compositional View

Comp View=targeted

Variability Chart for $\log \mathrm{NL}[\mathrm{Na}(\mathrm{g} / \mathrm{L})]$

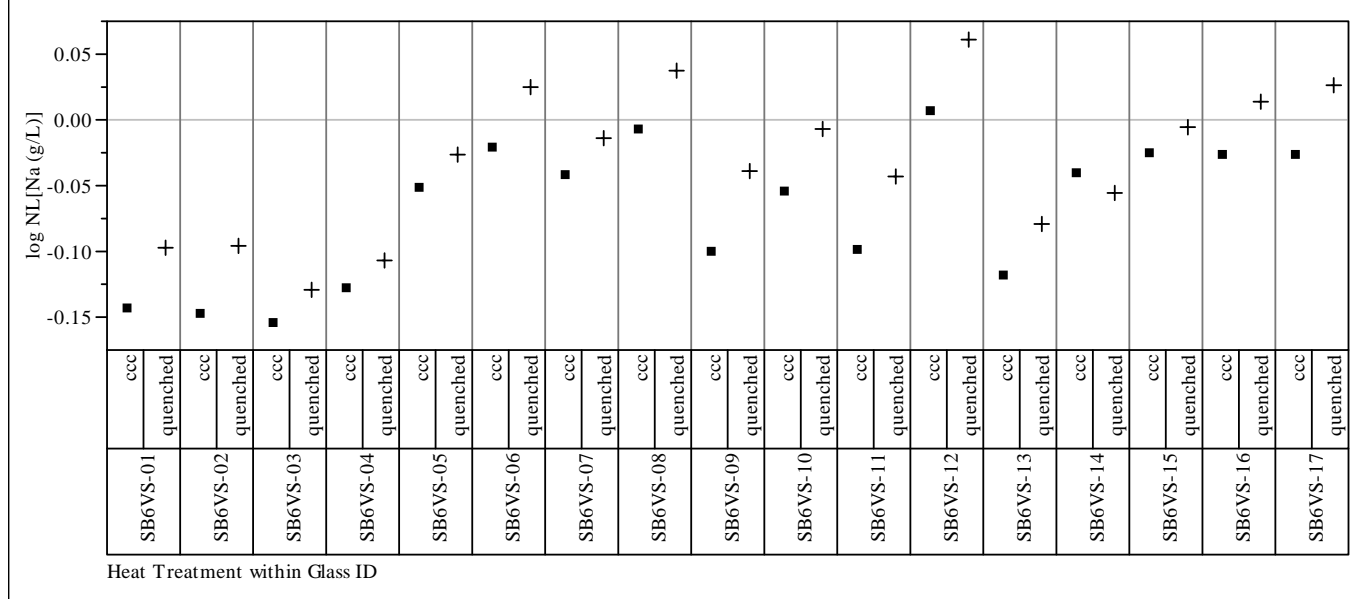

Comp View $=$ targeted

Variability Chart for $\log \mathrm{NL}[\mathrm{Si}(\mathrm{g} / \mathrm{L})]$

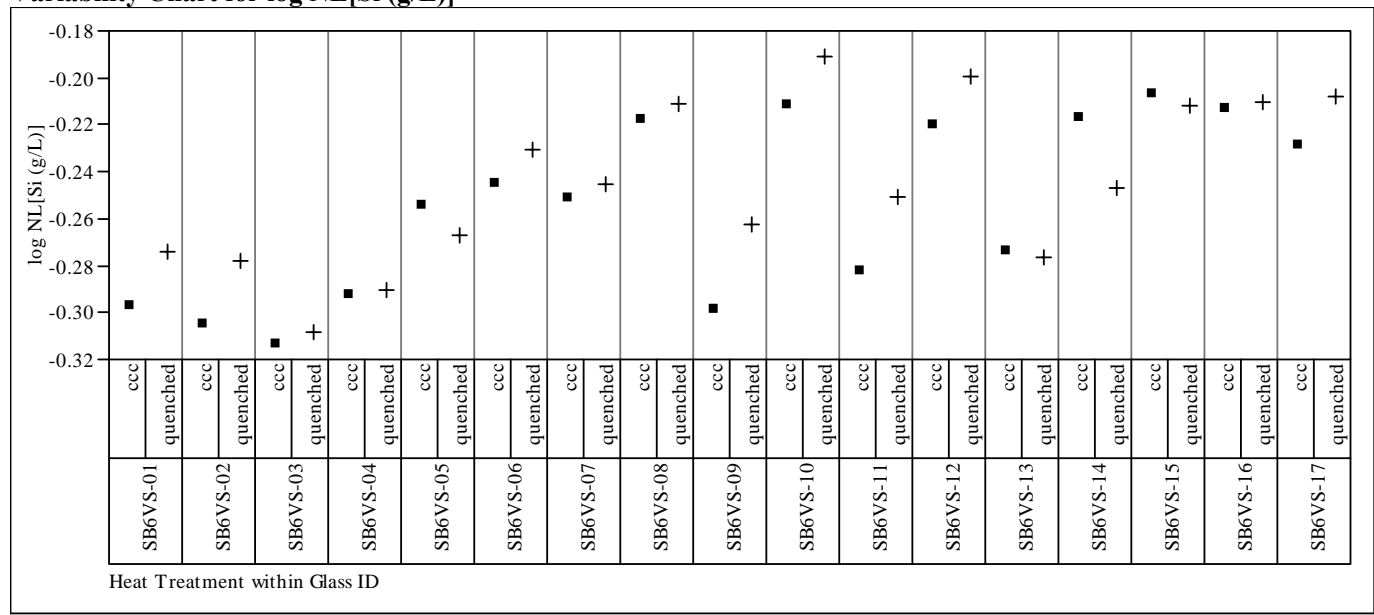


Exhibit B6. del $G p\left(\Delta G_{p}\right)$ Predictions versus the Common Logarithm of the Normalized Leachate $(\log \mathrm{NL}[]$.$) for \mathrm{B}, \mathrm{Li}, \mathrm{Na}$ and $\mathrm{Si}$ for Targeted Compositions for the Initial Study Glasses for Both Heat Treatments

\begin{tabular}{|c|c|}
\multicolumn{1}{c}{ Legend } \\
\hline Symbol & $\begin{array}{c}\text { Standard/ } \\
\text { Comp View-Heat Treatment }\end{array}$ \\
\hline$z$ & EA \\
\hline$\diamond$ & ARM \\
\hline$\times$ & Targeted-ccc \\
\hline$\bullet$ & Targeted-quenched \\
\hline
\end{tabular}

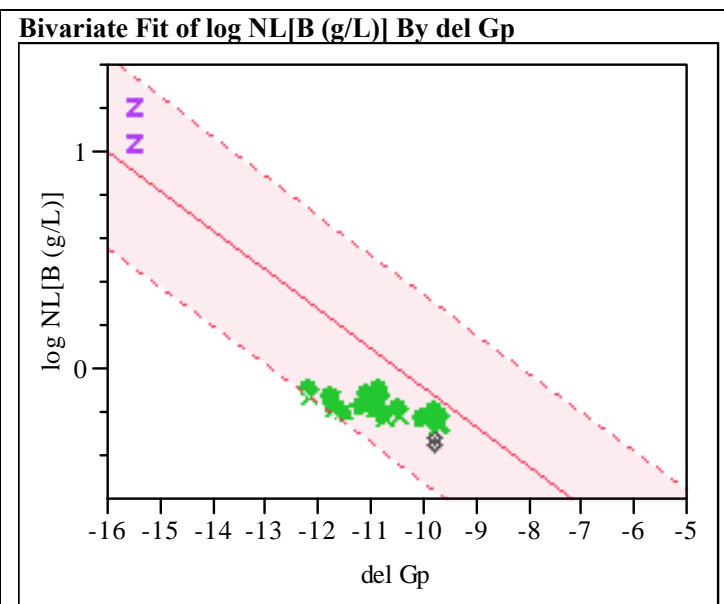

Bivariate Fit of $\log \mathrm{NL}[\mathrm{Li}(\mathrm{g} / \mathrm{L})]$ By del Gp

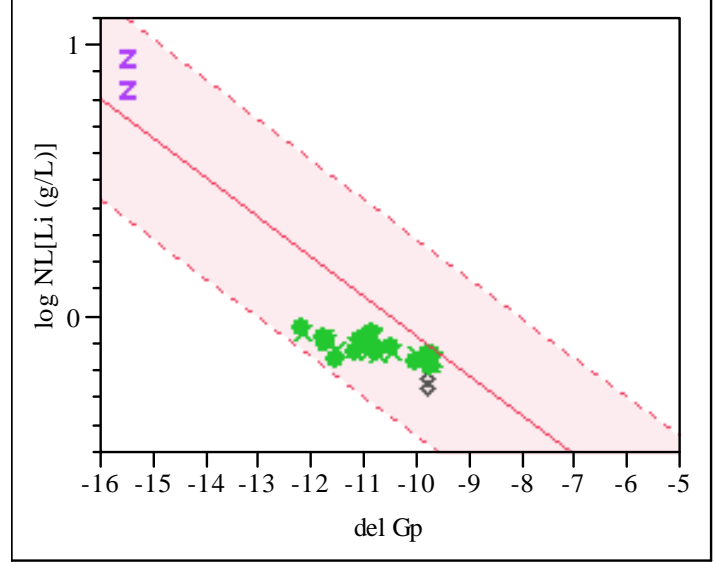

Bivariate Fit of $\log \mathrm{NL}[\mathrm{Na}(\mathrm{g} / \mathrm{L})]$ By del Gp

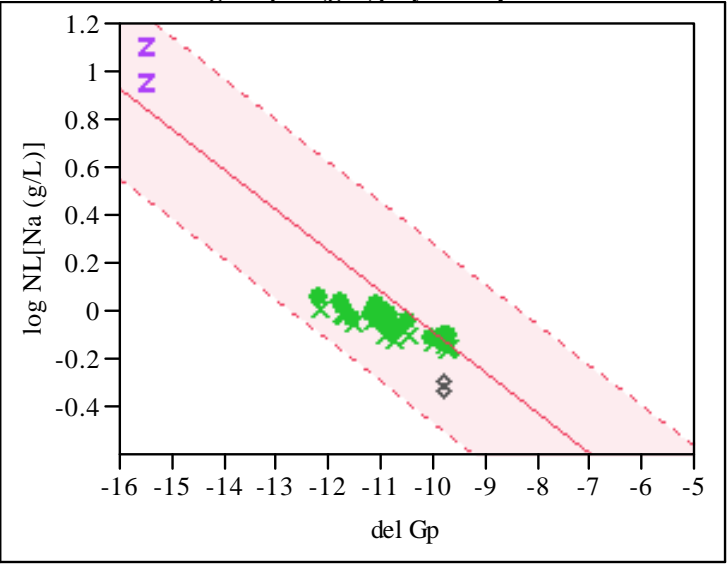

Bivariate Fit of $\log$ NL[Si (g/L)] By del Gp

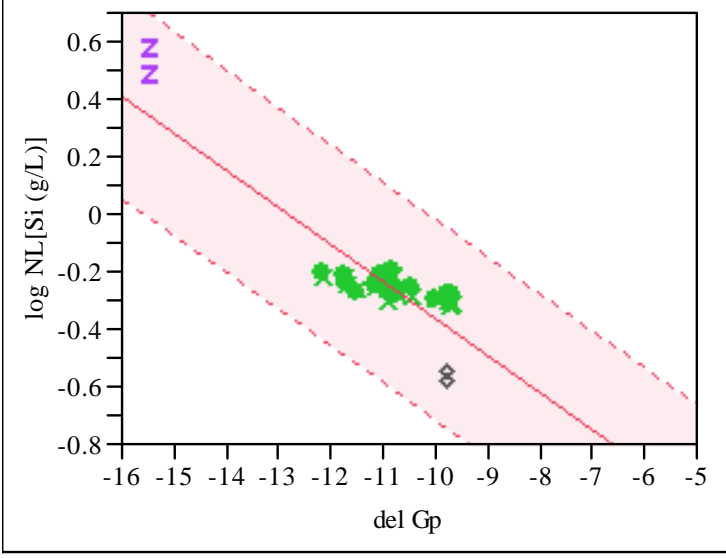


Exhibit B6. del $G p\left(\Delta G_{p}\right)$ Predictions versus the Common Logarithm of the Normalized Leachate $(\log \mathrm{NL}[]$.$) for B, Li, Na and Si for Measured Compositions for the Initial Study$ Glasses for Both Heat Treatments

\begin{tabular}{|c|c|}
\multicolumn{2}{c}{ Legend } \\
Symbol & $\begin{array}{c}\text { Standard/ } \\
\text { Comp View-Heat Treatment }\end{array}$ \\
\hline$z$ & EA \\
\hline$\diamond$ & ARM \\
\hline$\times$ & Measured-ccc \\
\hline$\bullet$ & Measured-quenched \\
\hline
\end{tabular}

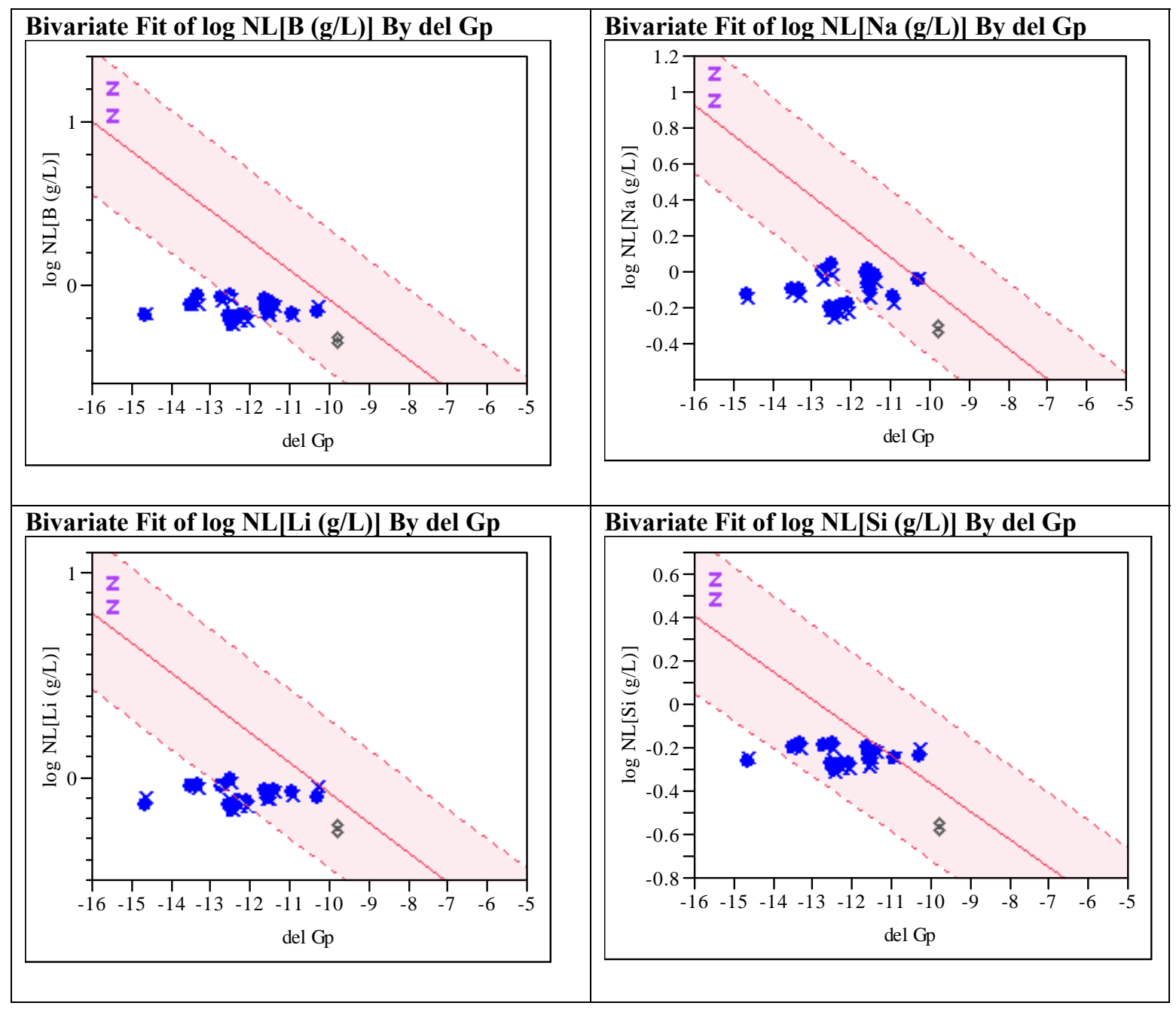




\section{Appendix C:}

\section{Tables and Exhibits Supporting the Analysis of the Chemical Composition Measurements of the SB6 VS Glasses with Thorium}


SRNL-STI-2010-00242

Revision 0

This page intentionally left blank. 
Table C1. Measured Elemental Concentrations (micrograms of element per gram of glass) for the Thorium Study Glasses Prepared Using a 10X Dilution Factor with Mixed Acid Preparation Method

\begin{tabular}{|c|c|c|c|c|c|c|c|c|c|c|c|c|c|c|c|}
\hline $\begin{array}{c}\text { Prep } \\
\text { Method }\end{array}$ & \begin{tabular}{c|} 
Dilution \\
Factor \\
\end{tabular} & Glass ID & Block & Seq. & Lab ID & $\begin{array}{c}\text { Al } \\
(\mathrm{ug} / \mathrm{g})\end{array}$ & $\begin{array}{c}\text { Ba } \\
(\mathrm{ug} / \mathrm{g})\end{array}$ & $\begin{array}{c}\mathrm{Cu} \\
(\mathrm{ug} / \mathrm{g})\end{array}$ & $\begin{array}{c}\mathrm{Fe} \\
(\mathrm{ug} / \mathrm{g})\end{array}$ & $\begin{array}{c}\mathrm{La} \\
(\mathrm{ug} / \mathrm{g})\end{array}$ & $\begin{array}{c}\mathbf{L i} \\
(\mathbf{u g} / \mathbf{g})\end{array}$ & $\begin{array}{c}\text { Mn } \\
\text { (ug/g) }\end{array}$ & $\begin{array}{c}\mathrm{Na} \\
(\mathrm{ug} / \mathrm{g})\end{array}$ & $\begin{array}{c}\mathbf{S i} \\
(\mathrm{ug} / \mathrm{g})\end{array}$ & $\begin{array}{c}\begin{array}{c}\text { Th } \\
\text { (ug/g) }\end{array} \\
\end{array}$ \\
\hline MA & $10 \mathrm{X}$ & ARG-1 & 1 & 1 & 300272391 & 26100 & 845 & $<138$ & 106000 & $<96.9$ & 14900 & 15200 & 89000 & 242000 & $<2290$ \\
\hline MA & $10 \mathrm{X}$ & U-std & 1 & 2 & 300272392 & 21300 & $<115$ & $<139$ & 93100 & $<97.7$ & 13000 & 21000 & 84900 & 216000 & $<2310$ \\
\hline MA & $10 \mathrm{X}$ & SB6VS20 & 1 & 3 & 300272393 & 46800 & 372 & 247 & 54300 & 264 & 20700 & 16800 & 96900 & 221000 & 5630 \\
\hline MA & $10 \mathrm{X}$ & SB6VS18 & 1 & 4 & 300272394 & 45000 & 294 & 251 & 51400 & 154 & 23700 & 16100 & 98400 & 249000 & 3000 \\
\hline MA & $10 \mathrm{X}$ & SB6VS22 & 1 & 5 & 300272395 & 52900 & 366 & 275 & 61800 & 171 & 20200 & 19300 & 103000 & 216000 & 3370 \\
\hline MA & $10 \mathrm{X}$ & SB6VS19 & 1 & 6 & 300272396 & 48200 & 354 & 261 & 55500 & 142 & 23400 & 17400 & 102000 & 247000 & 2810 \\
\hline MA & $10 \mathrm{X}$ & SB6VS22 & 1 & 7 & 300272397 & 48300 & 348 & 255 & 56500 & 230 & 18500 & 17600 & 94400 & 201000 & 5370 \\
\hline MA & $10 \mathrm{X}$ & ARG-1 & 1 & 8 & 300272398 & 26400 & 853 & $<138$ & 107000 & $<97.3$ & 15400 & 15400 & 89800 & 247000 & $<2300$ \\
\hline MA & $10 \mathrm{X}$ & U-std & 1 & 9 & 300272399 & 23300 & $<117$ & $<141$ & 102000 & $<99.1$ & 14800 & 23100 & 92000 & 240000 & $<2340$ \\
\hline MA & $10 X$ & SB6VS19 & 1 & 10 & 300272400 & 49700 & 360 & 262 & 57300 & 143 & 24600 & 17900 & 104000 & 254000 & 2640 \\
\hline MA & $10 \mathrm{X}$ & SB6VS18 & 1 & 11 & 300272401 & 42100 & 296 & 219 & 48600 & 190 & 22700 & 15200 & 92000 & 235000 & 4250 \\
\hline MA & $10 \mathrm{X}$ & SB6VS21 & 1 & 12 & 300272402 & 53200 & 477 & 292 & 61700 & 364 & 22300 & 19200 & 105000 & 232000 & 8300 \\
\hline MA & $10 \mathrm{X}$ & SB6VS21 & 1 & 13 & 300272403 & 57700 & 508 & 333 & 66500 & 357 & 24100 & 20700 & 114000 & 248000 & 8260 \\
\hline MA & $10 \mathrm{X}$ & SB6VS20 & 1 & 14 & 300272404 & 46300 & 359 & 262 & 53800 & 254 & 20800 & 16800 & 95000 & 219000 & 5310 \\
\hline MA & $10 \mathrm{X}$ & ARG-1 & 1 & 15 & 300272405 & 24700 & 798 & $<139$ & 99900 & $<97.9$ & 14400 & 14400 & 83800 & 232000 & $<2310$ \\
\hline MA & $10 \mathrm{X}$ & BLANK & 1 & 16 & 300272407 & $<533$ & $<118$ & $<142$ & 121 & $<100$ & $<1640$ & $<13$ & $<863$ & 24700 & $<2360$ \\
\hline MA & $10 \mathrm{X}$ & U-std & 1 & 17 & 300272406 & 21400 & $<116$ & $<139$ & 93000 & $<98.2$ & 13200 & 21000 & 83900 & 218000 & $<2320$ \\
\hline MA & $10 \mathrm{X}$ & ARG-1 & 2 & 1 & 300272391 & 26200 & 843 & $<138$ & 105000 & $<96.9$ & 15300 & 15100 & 89800 & 237000 & $<2290$ \\
\hline MA & $10 \mathrm{X}$ & U-std & 2 & 2 & 300272392 & 21500 & $<115$ & $<139$ & 92400 & $<97.7$ & 13400 & 21000 & 85600 & 211000 & $<2310$ \\
\hline MA & $10 \mathrm{X}$ & SB6VS20 & 2 & 3 & 300272393 & 47100 & 382 & 257 & 54100 & 352 & 21200 & 16900 & 97800 & 216000 & 7110 \\
\hline MA & $10 \mathrm{X}$ & SB6VS18 & 2 & 4 & 300272394 & 45000 & 300 & 223 & 51300 & 172 & 24200 & 16000 & 99200 & 241000 & 3550 \\
\hline MA & $10 \mathrm{X}$ & SB6VS22 & 2 & 5 & 300272395 & 52900 & 369 & 276 & 61400 & 189 & 20300 & 19200 & 105000 & 208000 & 3710 \\
\hline MA & $10 \mathrm{X}$ & SB6VS19 & 2 & 6 & 300272396 & 48200 & 358 & 255 & 54900 & 171 & 23700 & 17300 & 103000 & 238000 & 3450 \\
\hline MA & $10 \mathrm{X}$ & SB6VS22 & 2 & 7 & 300272397 & 48200 & 357 & 252 & 55900 & 293 & 18500 & 17500 & 95300 & 191000 & 6180 \\
\hline MA & $10 \mathrm{X}$ & ARG-1 & 2 & 8 & 300272398 & 26600 & 855 & $<138$ & 106000 & $<97.3$ & 15700 & 15400 & 90800 & 240000 & $<2300$ \\
\hline MA & $10 \mathrm{X}$ & U-std & 2 & 9 & 300272399 & 23300 & $<117$ & $<141$ & 102000 & $<99.1$ & 14800 & 23000 & 93000 & 232000 & $<2340$ \\
\hline MA & $10 \mathrm{X}$ & SB6VS19 & 2 & 10 & 300272400 & 49700 & 364 & 261 & 56900 & 146 & 24600 & 17900 & 106000 & 247000 & 3070 \\
\hline MA & $10 \mathrm{X}$ & SB6VS18 & 2 & 11 & 300272401 & 42400 & 293 & 238 & 48400 & 206 & 22700 & 15100 & 92900 & 228000 & 4220 \\
\hline MA & $10 \mathrm{X}$ & SB6VS21 & 2 & 12 & 300272402 & 53300 & 478 & 312 & 61400 & 408 & 22300 & 19100 & 107000 & 226000 & 8930 \\
\hline MA & $10 \mathrm{X}$ & SB6VS21 & 2 & 13 & 300272403 & 57600 & 521 & 323 & 66400 & 408 & 23900 & 20700 & 115000 & 242000 & 9220 \\
\hline MA & $10 \mathrm{X}$ & SB6VS20 & 2 & 14 & 300272404 & 46400 & 373 & 244 & 53600 & 289 & 21000 & 16700 & 96200 & 214000 & 5980 \\
\hline MA & $10 \mathrm{X}$ & ARG-1 & 2 & 15 & 300272405 & 24500 & 800 & $<139$ & 99000 & $<97.9$ & 14600 & 14300 & 84400 & 225000 & $<2310$ \\
\hline MA & $10 \mathrm{X}$ & BLANK & 2 & 16 & 300272407 & $<533$ & $<118$ & $<142$ & 111 & $<100$ & $<756$ & $<13$ & $<863$ & 14600 & $<2360$ \\
\hline MA & $10 \mathrm{X}$ & U-std & 2 & 17 & 300272406 & 21500 & $<116$ & $<139$ & 92000 & $<98.2$ & 13500 & 21000 & 84900 & 212000 & $<2320$ \\
\hline
\end{tabular}


Table C2. Measured Elemental Concentrations (micrograms of element per gram of glass) for the Thorium Study Glasses Prepared Using a 2X Dilution Factor with Mixed Acid Preparation Method

\begin{tabular}{|c|c|c|c|c|c|c|c|c|c|c|c|c|c|c|c|c|c|c|c|}
\hline $\begin{array}{c}\text { Prep } \\
\text { Method }\end{array}$ & $\begin{array}{c}\text { Dilution } \\
\text { Factor }\end{array}$ & \begin{tabular}{|l|} 
Glass ID \\
\end{tabular} & Block & Seq. & Lab ID & Ca (ug/g) & Cd (ug/g) & $\mathrm{Ce}(\mathrm{ug} / \mathrm{g})$ & $\operatorname{Cr}(\mathrm{ug} / \mathrm{g})$ & K (ug/g) & Mg (ug/g) & \begin{tabular}{|l|}
$\mathrm{Ni}(\mathrm{ug} / \mathrm{g})$ \\
\end{tabular} & P (ug/g) & Pb (ug/g) & \begin{tabular}{|l|} 
S (ug/g) \\
\end{tabular} & \begin{tabular}{|l|} 
Ti (ug/g) \\
\end{tabular} & U (ug/g) & Zn (ug/g) & $\operatorname{Zr}(\mathbf{u g} / \mathrm{g})$ \\
\hline MA & $2 \mathrm{X}$ & ARG-1 & 1 & 1 & 300272391 & 10700 & $<24.8$ & $<342$ & 682 & 23000 & 5490 & 8130 & 1220 & $<142$ & $<2180$ & 7150 & $<1680$ & 171 & 1010 \\
\hline MA & $2 \mathrm{X}$ & U-std & 1 & 2 & \begin{tabular}{|l|}
300272392 \\
\end{tabular} & 9110 & $<25$ & $<345$ & 1620 & 23400 & 7120 & 7830 & $<166$ & $<143$ & $<2210$ & 5610 & 18800 & $<29.5$ & 16.4 \\
\hline MA & $2 \mathrm{X}$ & SB6VS20 & 1 & 3 & \begin{tabular}{|l|}
300272393 \\
\end{tabular} & 3340 & $<25.3$ & $<349$ & 225 & $<396$ & 1570 & 6680 & 494 & $<145$ & $<2220$ & 1800 & 12600 & 186 & 568 \\
\hline MA & $2 \mathrm{X}$ & SB6VS18 & 1 & 4 & 300272394 & 3200 & $<25.3$ & $<350$ & 219 & $<396$ & 1500 & 6500 & 456 & $<145$ & $<2220$ & 1720 & 11800 & 193 & 529 \\
\hline MA & $2 \mathrm{X}$ & SB6VS22 & 1 & 5 & 300272395 & 3800 & $<25.1$ & $<346$ & 261 & $<392$ & 1770 & 7680 & 543 & $<143$ & $<2210$ & 2040 & 14100 & 216 & 639 \\
\hline MA & $2 \mathrm{X}$ & SB6VS19 & 1 & 6 & \begin{tabular}{|l|}
300272396 \\
\end{tabular} & 3430 & $<25.2$ & $<348$ & 229 & $<394$ & 1620 & 7010 & 492 & $<144$ & $<2220$ & 1840 & 12600 & 195 & 569 \\
\hline MA & $2 \mathrm{X}$ & SB6VS22 & 1 & 7 & \begin{tabular}{|l|}
300272397 \\
\end{tabular} & 3450 & $<25.4$ & $<351$ & 242 & $<398$ & 1620 & 7050 & 524 & $<145$ & $<2240$ & 1850 & 12700 & 195 & 564 \\
\hline MA & $2 \mathrm{X}$ & \begin{tabular}{|l|} 
ARG-1 \\
\end{tabular} & 1 & 8 & 300272398 & 10900 & $<24.9$ & $<344$ & 705 & 23300 & 5600 & 8330 & 1230 & $<142$ & $<2190$ & 7260 & $<1690$ & 181 & 1030 \\
\hline MA & $2 \mathrm{X}$ & U-std & 1 & 9 & \begin{tabular}{|l|l|}
300272399 \\
\end{tabular} & 9890 & $<25.4$ & $<350$ & 1790 & 25600 & 7720 & 8590 & $<168$ & $<145$ & $<2240$ & 6150 & 20100 & $<29.9$ & 22.8 \\
\hline MA & $2 \mathrm{X}$ & \begin{tabular}{|l|} 
SB6VS19 \\
\end{tabular} & 1 & 10 & 300272400 & 3530 & $<25.2$ & $<347$ & 235 & $<394$ & 1660 & 7180 & 524 & $<144$ & $<2220$ & 1900 & 13100 & 208 & 571 \\
\hline MA & $2 \mathrm{X}$ & \begin{tabular}{|l|} 
SB6VS18 \\
\end{tabular} & 1 & 11 & \begin{tabular}{|l|}
300272401 \\
\end{tabular} & 3010 & $<25.2$ & $<347$ & 199 & $<394$ & 1410 & 6100 & 479 & $<144$ & $<2220$ & 1610 & 11200 & 182 & 498 \\
\hline MA & $2 \mathrm{X}$ & \begin{tabular}{|l|} 
SB6VS21 \\
\end{tabular} & 1 & 12 & 300272402 & 3800 & $<25.2$ & $<348$ & 225 & $<394$ & 1780 & 7630 & 562 & $<144$ & $<2220$ & 2010 & 14100 & 207 & 633 \\
\hline MA & $2 \mathrm{X}$ & \begin{tabular}{|l|} 
SB6VS21 \\
\end{tabular} & 1 & 13 & \begin{tabular}{|l|}
300272403 \\
\end{tabular} & 4110 & $<25.3$ & $<349$ & 238 & $<396$ & 1910 & 8200 & 621 & $<144$ & 2230 & 2180 & 15300 & 230 & 663 \\
\hline MA & $2 \mathrm{X}$ & \begin{tabular}{|l|} 
SB6VS20 \\
\end{tabular} & 1 & 14 & \begin{tabular}{|l|}
300272404 \\
\end{tabular} & 3330 & $<25$ & $<346$ & 219 & $<392$ & 1570 & 6600 & 485 & $<143$ & $<2210$ & 1770 & 12300 & 188 & 529 \\
\hline MA & $2 \mathrm{X}$ & ARG-1 & 1 & 15 & \begin{tabular}{|l|}
300272405 \\
\end{tabular} & 10200 & $<25$ & $<346$ & 651 & 21400 & 5160 & 7640 & 1110 & $<143$ & $<2210$ & 6750 & $<1700$ & 162 & 947 \\
\hline MA & $2 \mathrm{X}$ & BLANK & 1 & 16 & 300272407 & 18.4 & $<25.6$ & $<353$ & $<27.8$ & $<400$ & 5.4 & $<186$ & $<170$ & $<146$ & $<2250$ & $<3.4$ & $<1740$ & $<30.2$ & $<9.4$ \\
\hline MA & $2 \mathrm{X}$ & U-std & 1 & 17 & 300272406 & 9110 & $<25.1$ & $<347$ & 1610 & 23000 & 7000 & 7770 & $<167$ & $<144$ & $<2210$ & 5560 & 18400 & $<29.7$ & 25.5 \\
\hline MA & $2 \mathrm{X}$ & ARG-1 & 2 & 1 & 300272391 & 10800 & $<24.8$ & $<342$ & 684 & 23200 & 5510 & 8160 & 1270 & $<142$ & $<2180$ & 7150 & $<1680$ & 167 & 1020 \\
\hline MA & $2 \mathrm{X}$ & U-std & 2 & 2 & \begin{tabular}{|l|}
300272392 \\
\end{tabular} & 9100 & $<25$ & $<345$ & 1620 & 23800 & 7120 & 7850 & $<166$ & $<143$ & $<2210$ & 5600 & 18400 & $<29.5$ & 17 \\
\hline MA & $2 \mathrm{X}$ & \begin{tabular}{|l|} 
SB6VS20 \\
\end{tabular} & 2 & 3 & \begin{tabular}{|l|}
300272393 \\
\end{tabular} & 3320 & $<25.3$ & $<349$ & 224 & $<396$ & 1570 & 6710 & 523 & $<145$ & $<2220$ & 1800 & 12500 & 184 & 548 \\
\hline MA & $2 \mathrm{X}$ & \begin{tabular}{|l|} 
SB6VS18 \\
\end{tabular} & 2 & 4 & \begin{tabular}{|l|}
300272394 \\
\end{tabular} & 3180 & $<25.3$ & $<350$ & 215 & $<396$ & 1510 & 6470 & 529 & $<145$ & $<2240$ & 1720 & 11900 & 188 & 525 \\
\hline MA & $2 \mathrm{X}$ & \begin{tabular}{|l|} 
SB6VS22 \\
\end{tabular} & 2 & 5 & \begin{tabular}{|l|}
300272395 \\
\end{tabular} & 3770 & $<25.1$ & $<346$ & 258 & $<392$ & 1780 & 7680 & 598 & $<143$ & $<2210$ & 2030 & 14100 & 209 & 610 \\
\hline MA & $2 \mathrm{X}$ & \begin{tabular}{|l|} 
SB6VS199 \\
\end{tabular} & 2 & 6 & 300272396 & 3420 & $<25.2$ & $<348$ & 229 & $<394$ & 1610 & 6960 & 545 & $<144$ & $<2220$ & 1830 & 12800 & 192 & 559 \\
\hline MA & $2 \mathrm{X}$ & \begin{tabular}{|l|} 
SB6VS22 \\
\end{tabular} & 2 & 7 & \begin{tabular}{|l|}
300272397 \\
\end{tabular} & 3440 & $<25.4$ & $<351$ & 243 & $<398$ & 1620 & 7030 & 551 & $<145$ & $<2240$ & 1840 & 12800 & 190 & 560 \\
\hline MA & $2 \mathrm{X}$ & ARG-1 & 2 & 8 & \begin{tabular}{|l|}
300272398 \\
\end{tabular} & 10900 & $<24.9$ & $<344$ & 689 & 23300 & 5560 & 8260 & 1260 & $<142$ & $<2190$ & 7230 & $<1690$ & 176 & 1030 \\
\hline MA & $2 \mathrm{X}$ & U-std & 2 & 9 & \begin{tabular}{|l|}
300272399 \\
\end{tabular} & 9880 & $<25.4$ & $<350$ & 1760 & 25800 & 7660 & 8600 & $<168$ & $<145$ & $<2240$ & 6120 & 20300 & $<29.9$ & 22.2 \\
\hline MA & $2 \mathrm{X}$ & \begin{tabular}{|l|} 
SB6VS19 \\
\end{tabular} & 2 & 10 & 300272400 & 3530 & $<25.2$ & $<347$ & 233 & $<394$ & 1650 & 7160 & 540 & $<144$ & $<2210$ & 1890 & 13200 & 202 & 569 \\
\hline MA & $2 \mathrm{X}$ & \begin{tabular}{|l|} 
SB6VS18 \\
\end{tabular} & 2 & 11 & \begin{tabular}{|l|}
300272401 \\
\end{tabular} & 3000 & $<25.2$ & $<347$ & 199 & $<394$ & 1410 & 6130 & 519 & $<144$ & $<2220$ & 1610 & 11300 & 178 & 499 \\
\hline MA & $2 \mathrm{X}$ & \begin{tabular}{|l|} 
SB6VS21 \\
\end{tabular} & 2 & 12 & 300272402 & 3790 & $<25.2$ & $<348$ & 222 & $<394$ & 1780 & 7690 & 632 & $<144$ & $<2220$ & 2000 & 14300 & 203 & 624 \\
\hline MA & $2 \mathrm{X}$ & \begin{tabular}{|l|} 
SB6VS21 \\
\end{tabular} & 2 & 13 & \begin{tabular}{|l|}
300272403 \\
\end{tabular} & 4090 & $<25.3$ & $<349$ & 239 & $<396$ & 1920 & 8290 & 645 & $<144$ & $<2220$ & 2180 & 15500 & 226 & 657 \\
\hline MA & $2 \mathrm{X}$ & \begin{tabular}{|l|} 
SB6VS20 \\
\end{tabular} & 2 & 14 & 300272404 & 3320 & $<25$ & $<346$ & 219 & $<392$ & 1580 & 6700 & 532 & $<143$ & $<2210$ & 1760 & 12300 & 192 & 530 \\
\hline MA & $2 \mathrm{X}$ & ARG-1 & 2 & 15 & 300272405 & 10200 & $<25$ & $<346$ & 642 & 21900 & 5200 & 7750 & 1180 & $<143$ & $<2210$ & 6740 & $<1700$ & 160 & 945 \\
\hline MA & $2 \mathrm{X}$ & BLANK & 2 & 16 & 300272407 & 20.2 & $<25.6$ & $<353$ & $<27.8$ & $<400$ & 6.6 & $<186$ & $<170$ & $<146$ & $<2250$ & $<3.4$ & $<1740$ & $<30.2$ & $<9.4$ \\
\hline MA & $2 \mathrm{X}$ & U-std & 2 & 17 & 300272406 & 9060 & $<25.1$ & $<347$ & 1590 & 23500 & 7040 & 7830 & $<167$ & $<144$ & $<2210$ & 5550 & 18500 & $<29.7$ & 25.5 \\
\hline
\end{tabular}


Table C3. Measured Elemental Concentrations (micrograms of element per gram of glass) for the Thorium Study Glasses Prepared Using a 10X Dilution Factor with Peroxide Fusion Preparation Method (part 1)

\begin{tabular}{|c|c|c|c|c|c|c|c|c|c|c|c|c|c|c|c|c|c|}
\hline $\begin{array}{l}\text { Prep } \\
\text { Method }\end{array}$ & \begin{tabular}{|l|} 
Dilution \\
Factor
\end{tabular} & Glass ID & Block & Seq & Lab ID & \begin{tabular}{|l|}
$\mathrm{Al}$ \\
$(\mathbf{u g} / \mathrm{g})$
\end{tabular} & \begin{tabular}{|l|l} 
B \\
$(\mathbf{u g} / \mathrm{g})$
\end{tabular} & \begin{tabular}{|l|}
$\mathrm{Ba}$ \\
$(\mathrm{ug} / \mathrm{g})$
\end{tabular} & \begin{tabular}{|l|} 
Ca \\
(ug/g)
\end{tabular} & Cd (ug/g) & \begin{tabular}{|l|}
$\mathbf{C e}$ \\
(ug/g)
\end{tabular} & \begin{tabular}{|l|}
$\mathbf{C r}$ \\
$(\mathrm{ug} / \mathrm{g})$
\end{tabular} & $\mathrm{Cu}(\mathrm{ug} / \mathrm{g})$ & 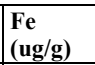 & \begin{tabular}{|l|}
$\mathrm{K}$ \\
$(\mathrm{ug} / \mathrm{g})$
\end{tabular} & $\begin{array}{l}\mathbf{L a} \\
(\mathrm{ug} / \mathrm{g})\end{array}$ & \begin{tabular}{|l|l}
$\mathbf{L i}$ \\
(ug/g)
\end{tabular} \\
\hline $\mathrm{PF}$ & $10 \mathrm{X}$ & ARG-1 & 1 & 1 & 300272374 & 25600 & 25400 & 784 & 11300 & $<155$ & $<1590$ & 653 & $<172$ & 97200 & 22900 & $<131$ & 14600 \\
\hline $\mathrm{PF}$ & $10 \mathrm{X}$ & U-std & 1 & 2 & 300272375 & 21300 & 27300 & $<117$ & 10100 & $<127$ & $<1300$ & 1650 & $<141$ & 92200 & 25000 & $<107$ & 13900 \\
\hline $\mathrm{PF}$ & $10 \mathrm{X}$ & \begin{tabular}{|l|} 
SB6VS20 \\
\end{tabular} & 1 & 3 & 300272376 & 50000 & 15200 & 424 & 4340 & $<127$ & $<1300$ & 259 & 291 & 57700 & 3050 & 246 & 23200 \\
\hline $\mathrm{PF}$ & $10 \mathrm{X}$ & \begin{tabular}{|l|} 
SB6VS18 \\
\end{tabular} & 1 & 4 & \begin{tabular}{|l|}
300272377 \\
\end{tabular} & 44900 & 16200 & 348 & 3950 & $<127$ & $<1300$ & 220 & 285 & 51300 & $\begin{array}{l}<<1980 \\
\end{array}$ & 219 & 24700 \\
\hline $\mathrm{PF}$ & $10 \mathrm{X}$ & \begin{tabular}{|l|} 
SB6VS22 \\
\end{tabular} & 1 & 5 & \begin{tabular}{|l|}
300272378 \\
\end{tabular} & 55300 & 14300 & 442 & 5000 & $<127$ & $<1300$ & 282 & 324 & 63900 & $<1990$ & 257 & 21700 \\
\hline $\mathrm{PF}$ & $10 \mathrm{X}$ & \begin{tabular}{|l|} 
SB6VS22 \\
\end{tabular} & 1 & 6 & \begin{tabular}{|l|}
300272379 \\
\end{tabular} & 55400 & 14300 & 452 & 4910 & $<127$ & $\begin{array}{l}<1300 \\
\end{array}$ & 293 & 334 & 64100 & 2070 & 284 & 21800 \\
\hline $\mathrm{PF}$ & $10 \mathrm{X}$ & \begin{tabular}{|l|} 
SB6VS19 \\
\end{tabular} & 1 & 7 & \begin{tabular}{|l|}
300272380 \\
\end{tabular} & \begin{tabular}{|l|}
47700 \\
\end{tabular} & 15800 & 400 & \begin{tabular}{|l|}
4090 \\
\end{tabular} & $<126$ & $<1290$ & \begin{tabular}{|l|}
257 \\
\end{tabular} & 292 & 54500 & 2980 & 230 & 23800 \\
\hline $\mathrm{PF}$ & $10 \mathrm{X}$ & ARG-1 & 1 & 8 & 300272381 & 25700 & 25700 & 795 & 11100 & $<129$ & $<1320$ & 667 & $<143$ & 97600 & 23400 & $<109$ & 14600 \\
\hline $\mathrm{PF}$ & $10 \mathrm{X}$ & U-std & 1 & 9 & 300272382 & 21600 & 27500 & $<116$ & 10200 & $<126$ & \begin{tabular}{|l|}
$<1290$ \\
\end{tabular} & 1630 & $<140$ & 92400 & 26300 & $<107$ & 13700 \\
\hline $\mathrm{PF}$ & $10 \mathrm{X}$ & \begin{tabular}{|l|} 
SB6VS21 \\
\end{tabular} & 1 & 10 & \begin{tabular}{|l|}
300272383 \\
\end{tabular} & 53600 & 14800 & \begin{tabular}{|l|}
491 \\
\end{tabular} & 4710 & $<127$ & $<1300$ & 239 & 315 & 60900 & 2660 & 238 & 22400 \\
\hline $\mathrm{PF}$ & $10 \mathrm{X}$ & \begin{tabular}{|l|} 
SB6VS18 \\
\end{tabular} & 1 & 11 & \begin{tabular}{|l|}
300272384 \\
\end{tabular} & 44800 & 16200 & 342 & 4080 & $<127$ & $<1300$ & 241 & 276 & 50700 & 2410 & 230 & 24400 \\
\hline $\mathrm{PF}$ & $10 \mathrm{X}$ & \begin{tabular}{|l} 
SB6VS19 \\
\end{tabular} & 1 & 12 & 300272385 & 47500 & 15600 & 400 & 4100 & $<126$ & $<1290$ & 244 & 283 & 54100 & 3070 & 222 & 23600 \\
\hline $\mathrm{PF}$ & $10 \mathrm{X}$ & \begin{tabular}{|l|} 
SB6VS21 \\
\end{tabular} & 1 & 13 & 300272386 & 53400 & 14700 & 480 & 4720 & $<127$ & $<1300$ & 247 & 320 & 60800 & 3040 & 230 & 22100 \\
\hline $\mathrm{PF}$ & $10 \mathrm{X}$ & \begin{tabular}{|l|} 
SB6VS20 \\
\end{tabular} & 1 & 14 & 300272387 & 50700 & 15400 & 415 & 4350 & $<127$ & $<1300$ & 244 & 299 & 57900 & 3530 & 226 & 22800 \\
\hline $\mathrm{PF}$ & $10 \mathrm{X}$ & \begin{tabular}{|l|} 
ARG-1 \\
\end{tabular} & 1 & 15 & 300272388 & 25600 & 25600 & 776 & 11100 & $<137$ & \begin{tabular}{|l|l|}
$<1410$ \\
\end{tabular} & 620 & $<152$ & 96900 & 23200 & $<116$ & 14500 \\
\hline $\mathrm{PF}$ & $10 \mathrm{X}$ & BLANK & 1 & 16 & 300272390 & 1140 & $<176$ & $<118$ & 1400 & $<128$ & \begin{tabular}{|l|}
$<1310$ \\
\end{tabular} & $<139$ & $<142$ & 221 & $\begin{array}{l}<2000 \\
\end{array}$ & $<108$ & $<756$ \\
\hline PF & $10 \mathrm{X}$ & U-std & 1 & 17 & 300272389 & 21700 & 27400 & $<116$ & 9840 & $<126$ & $<1290$ & 1620 & $<140$ & 92200 & 25800 & $<106$ & 13600 \\
\hline $\mathrm{PF}$ & $10 \mathrm{X}$ & ARG-1 & 2 & 1 & \begin{tabular}{|l|}
300272374 \\
\end{tabular} & 25400 & 25800 & 783 & 11400 & $<155$ & $<1590$ & 650 & $<172$ & 97700 & 26200 & $\mid<131$ & 14900 \\
\hline $\mathrm{PF}$ & $10 \mathrm{X}$ & U-std & 2 & 2 & \begin{tabular}{|l|}
300272375 \\
\end{tabular} & 21200 & 27400 & $<117$ & 10100 & $<127$ & $<1300$ & 1660 & $<141$ & 92100 & 28700 & $<107$ & 14000 \\
\hline $\mathrm{PF}$ & $10 \mathrm{X}$ & \begin{tabular}{|l|} 
SB6VS20 \\
\end{tabular} & 2 & 3 & \begin{tabular}{|l|}
300272387 \\
\end{tabular} & 50900 & 15400 & 418 & 4400 & $<127$ & $<1300$ & 255 & 288 & 57900 & 3800 & 234 & 23700 \\
\hline $\mathrm{PF}$ & $10 \mathrm{X}$ & \begin{tabular}{|l|} 
SB6VS21 \\
\end{tabular} & 2 & 4 & \begin{tabular}{|l|l|}
300272383 \\
\end{tabular} & 53600 & 14700 & 485 & 4730 & $<127$ & $<1300$ & 265 & 320 & 60900 & 3600 & 232 & 23000 \\
\hline $\mathrm{PF}$ & $10 \mathrm{X}$ & \begin{tabular}{|l|} 
SB6VS22 \\
\end{tabular} & 2 & 5 & 300272379 & 55900 & 14300 & 442 & 4940 & $<127$ & $<1300$ & 301 & 322 & 64000 & 3440 & 258 & 22300 \\
\hline $\mathrm{PF}$ & $10 \mathrm{X}$ & \begin{tabular}{|l|} 
SB6VS19 \\
\end{tabular} & 2 & 6 & \begin{tabular}{|l|}
300272380 \\
\end{tabular} & 47800 & 15700 & 387 & 4100 & $<126$ & $<1290$ & 253 & 277 & 54000 & $\begin{array}{l}<<1970 \\
\end{array}$ & 213 & 24300 \\
\hline $\mathrm{PF}$ & $10 \mathrm{X}$ & \begin{tabular}{|l|l} 
SB6VS20 \\
\end{tabular} & 2 & 7 & 300272376 & 50000 & 15200 & 416 & 4350 & $<127$ & $<1300$ & 251 & 295 & 57200 & $<1990$ & 242 & 23500 \\
\hline $\mathrm{PF}$ & $10 \mathrm{X}$ & ARG-1 & 2 & 8 & 300272381 & 25900 & 25600 & 779 & 11200 & $<129$ & \begin{tabular}{|l|}
$<1320$ \\
\end{tabular} & 662 & $<143$ & 97200 & 23400 & $<109$ & 14800 \\
\hline $\mathrm{PF}$ & $10 \mathrm{X}$ & U-std & 2 & 9 & 300272382 & 21800 & 27600 & $<116$ & 10400 & $<126$ & $<1290$ & 1700 & $<140$ & 93600 & 25700 & $<107$ & 14000 \\
\hline $\mathrm{PF}$ & $10 \mathrm{X}$ & \begin{tabular}{|l|} 
SB6VS18 \\
\end{tabular} & 2 & 10 & 300272384 & 44800 & 16400 & 346 & 4240 & $<127$ & $<1300$ & 223 & 256 & 51900 & $<1990$ & 220 & 24800 \\
\hline $\mathrm{PF}$ & $10 \mathrm{X}$ & \begin{tabular}{|l|} 
SB6VS21 \\
\end{tabular} & 2 & 11 & 300272386 & 53800 & 14800 & 493 & 4900 & $<127$ & $<1300$ & 247 & 316 & 62100 & $<1980$ & 226 & 22700 \\
\hline $\mathrm{PF}$ & $10 \mathrm{X}$ & \begin{tabular}{|l|} 
SB6VS22 \\
\end{tabular} & 2 & 12 & \begin{tabular}{|l|}
300272378 \\
\end{tabular} & 56200 & 14500 & 446 & 4900 & $<127$ & $<1300$ & 299 & 318 & 65300 & $<1990$ & 263 & 21800 \\
\hline $\mathrm{PF}$ & $10 \mathrm{X}$ & \begin{tabular}{|l|} 
SB6VS19 \\
\end{tabular} & 2 & 13 & \begin{tabular}{|l|}
300272385 \\
\end{tabular} & 48000 & 16000 & 405 & 4340 & $<126$ & $<1290$ & 277 & 271 & 55700 & $<1980$ & 238 & 24100 \\
\hline PF & $10 \mathrm{X}$ & \begin{tabular}{|l|} 
SB6VS18 \\
\end{tabular} & 2 & 14 & \begin{tabular}{|l|}
300272377 \\
\end{tabular} & 45300 & 16500 & 350 & \begin{tabular}{|l|l|}
4130 \\
\end{tabular} & $<127$ & $<1300$ & 227 & 263 & 52500 & $<1980$ & 199 & 24700 \\
\hline $\mathrm{PF}$ & $10 \mathrm{X}$ & \begin{tabular}{|l|} 
ARG-1 \\
\end{tabular} & 2 & 15 & \begin{tabular}{|l|}
300272388 \\
\end{tabular} & 25700 & 26000 & 808 & 11700 & $<137$ & $<1410$ & 686 & $<152$ & 100000 & 22500 & $<116$ & 14500 \\
\hline $\mathrm{PF}$ & $10 \mathrm{X}$ & BLANK & 2 & 16 & 300272390 & 962 & $<375$ & $<118$ & 1460 & $<128$ & \begin{tabular}{|l|}
$<1310$ \\
\end{tabular} & $<139$ & $<142$ & 240 & $<2000$ & $<108$ & $<173$ \\
\hline $\mathrm{PF}$ & $10 \mathrm{X}$ & U-std & 2 & 17 & 300272389 & 22000 & 28200 & $<116$ & 10500 & $<126$ & $<1290$ & 1710 & $<140$ & 96900 & 24300 & $<106$ & 13700 \\
\hline
\end{tabular}


Table C4. Measured Elemental Concentrations (micrograms of element per gram of glass) for the Thorium Study Glasses Prepared Using a 10X Dilution Factor with Peroxide Fusion Preparation Method (part 2)

\begin{tabular}{|c|c|c|c|c|c|c|c|c|c|c|c|c|c|c|c|c|}
\hline $\begin{array}{c}\text { Prep } \\
\text { Method }\end{array}$ & $\begin{array}{c}\text { Dilution } \\
\text { Factor }\end{array}$ & Glass ID & Block & Seq & Lab ID & $\operatorname{Mg}(u g / g)$ & Mn (ug/g) & $\mathrm{Ni}(\mathrm{ug} / \mathrm{g})$ & P (ug/g) & $\mathrm{Pb}(\mathrm{ug} / \mathrm{g})$ & S (ug/g) & $\mathbf{S i}(\mathrm{ug} / \mathrm{g})$ & Th (ug/g) & Ti (ug/g) & $\mathrm{U}(\mathbf{u g} / \mathrm{g})$ & $\mathrm{Zn}(\mathrm{ug} / \mathrm{g})$ \\
\hline PF & $10 \mathrm{X}$ & ARG-1 & 1 & 1 & 300272374 & 5140 & 14200 & 8030 & $<1030$ & $<885$ & $<9080$ & 228000 & $<2860$ & 6980 & $<9080$ & $<183$ \\
\hline $\mathrm{PF}$ & $10 \mathrm{X}$ & U-std & 1 & 2 & 300272375 & 7350 & 21200 & 8220 & $<841$ & $<724$ & $<7430$ & 218000 & $<2340$ & 5890 & 19900 & $<150$ \\
\hline $\mathrm{PF}$ & $10 \mathrm{X}$ & $\begin{array}{l}\text { SB6VS20 } \\
\end{array}$ & 1 & 3 & 300272376 & 1640 & 18000 & 7220 & $<842$ & $<725$ & $<7440$ & 237000 & 8710 & 2000 & 12800 & 212 \\
\hline $\mathrm{PF}$ & $10 \mathrm{X}$ & $\begin{array}{l}\text { SB6VS18 } \\
\end{array}$ & 1 & 4 & 300272377 & 1470 & 16200 & 6650 & $<842$ & $<725$ & $<7430$ & 251000 & 7610 & 1820 & 11600 & 366 \\
\hline $\mathrm{PF}$ & $10 \mathrm{X}$ & SB6VS22 & 1 & 5 & 300272378 & 1830 & 20400 & 8230 & $<843$ & $<726$ & $<7440$ & 223000 & 9830 & 2230 & 15600 & 198 \\
\hline $\mathrm{PF}$ & $10 \mathrm{X}$ & $\begin{array}{l}\text { SB6VS22 } \\
\end{array}$ & 1 & 6 & 300272379 & 1840 & 20400 & 8250 & $<843$ & $<726$ & $<7450$ & 224000 & 10000 & 2240 & 15100 & 214 \\
\hline $\mathrm{PF}$ & $10 \mathrm{X}$ & $\begin{array}{l}\text { SB6VS19 } \\
\end{array}$ & 1 & 7 & 300272380 & 1550 & 17400 & 7080 & $<837$ & $<721$ & $<7400$ & 246000 & 7920 & 1940 & 11900 & 205 \\
\hline $\mathrm{PF}$ & $10 \mathrm{X}$ & ARG-1 & 1 & 8 & 300272381 & 5170 & 14100 & 7940 & 1290 & $<738$ & $<7570$ & 232000 & $<2380$ & 7060 & $<7570$ & 173 \\
\hline $\mathrm{PF}$ & $10 \mathrm{X}$ & U-std & 1 & 9 & 300272382 & 7330 & 21400 & 8050 & $<838$ & $<721$ & $<7400$ & 220000 & $<2330$ & 5930 & 20500 & $<149$ \\
\hline $\mathrm{PF}$ & $10 \mathrm{X}$ & $\begin{array}{l}\text { SB6VS21 } \\
\end{array}$ & 1 & 10 & 300272383 & 1730 & 19100 & 7710 & $<840$ & $<723$ & $<7420$ & 232000 & 9410 & 2120 & 14300 & 204 \\
\hline $\mathrm{PF}$ & $10 \mathrm{X}$ & SB6VS18 & 1 & 11 & 300272384 & 1470 & 16100 & 6560 & $<843$ & $<726$ & $<7450$ & 251000 & 7870 & 1810 & 11900 & 172 \\
\hline $\mathrm{PF}$ & $10 \mathrm{X}$ & SB6VS19 & 1 & 12 & 300272385 & 1540 & 17200 & 7040 & $<838$ & $<722$ & $<7410$ & 246000 & 8230 & 1910 & 12700 & 167 \\
\hline $\mathrm{PF}$ & $10 \mathrm{X}$ & SB6VS21 & 1 & 13 & 300272386 & 1730 & 19300 & 7690 & $<841$ & $<724$ & $<7430$ & 231000 & 9490 & 2120 & 15400 & 185 \\
\hline $\mathrm{PF}$ & $10 \mathrm{X}$ & SB6VS20 & 1 & 14 & 300272387 & 1640 & 18400 & 7530 & $<840$ & $<723$ & $<7420$ & 239000 & 8520 & 2020 & 14400 & 185 \\
\hline $\mathrm{PF}$ & $10 \mathrm{X}$ & ARG-1 & 1 & 15 & 300272388 & 5120 & 14200 & 7660 & $<911$ & $<785$ & $<8050$ & 231000 & $<2540$ & 7070 & $<8050$ & $<162$ \\
\hline $\mathrm{PF}$ & $10 \mathrm{X}$ & BLANK & 1 & 16 & 300272390 & $<25$ & $<110$ & $<930$ & $<849$ & $<731$ & $<7500$ & $<231$ & $<2360$ & $<110$ & $<7500$ & $<151$ \\
\hline $\mathrm{PF}$ & $10 \mathrm{X}$ & U-std & 1 & 17 & 300272389 & 7280 & 21300 & 8140 & $<836$ & $<720$ & $<7390$ & 219000 & $<2330$ & 5940 & 20700 & $<149$ \\
\hline $\mathrm{PF}$ & $10 \mathrm{X}$ & ARG-1 & 2 & 1 & 300272374 & 5110 & 14100 & 7680 & $<1030$ & $<885$ & $<9080$ & 230000 & $<2860$ & 6940 & $<9080$ & $<183$ \\
\hline PF & $10 \mathrm{X}$ & U-std & 2 & 2 & 300272375 & 7240 & 20900 & 7870 & $<841$ & $<724$ & $<7430$ & 217000 & $<2340$ & 5830 & 20400 & $<150$ \\
\hline $\mathrm{PF}$ & $10 \mathrm{X}$ & SB6VS20 & 2 & 3 & 300272387 & 1620 & 17900 & 7140 & $<840$ & $<723$ & $<7420$ & 237000 & 8500 & 1980 & 14000 & 216 \\
\hline $\mathrm{PF}$ & $10 \mathrm{X}$ & SB6VS21 & 2 & 4 & 300272383 & 1710 & 18600 & 7760 & $<840$ & $<723$ & $<7420$ & 231000 & 9290 & 2080 & 14700 & 198 \\
\hline $\mathrm{PF}$ & $10 \mathrm{X}$ & $\begin{array}{l}\text { SB6VS22 } \\
\end{array}$ & 2 & 5 & 300272379 & 1810 & 20100 & 8090 & $<843$ & $<726$ & $<7450$ & 223000 & 10000 & 2210 & 15400 & 220 \\
\hline $\mathrm{PF}$ & $10 \mathrm{X}$ & $\begin{array}{l}\text { SB6VS19 } \\
\end{array}$ & 2 & 6 & 300272380 & 1510 & 16900 & 6810 & $<837$ & $<721$ & $<7400$ & 243000 & 7880 & 1880 & 12200 & 201 \\
\hline $\mathrm{PF}$ & $10 \mathrm{X}$ & SB6VS20 & 2 & 7 & 300272376 & 1610 & 17800 & 7190 & $<842$ & $<725$ & $<7440$ & 236000 & 8770 & 1960 & 13200 & 217 \\
\hline $\mathrm{PF}$ & $10 \mathrm{X}$ & ARG-1 & 2 & 8 & 300272381 & 5070 & 13900 & 7640 & 910 & $<738$ & $<7570$ & 230000 & $<2380$ & 6970 & $<7570$ & $\begin{array}{l}170 \\
\end{array}$ \\
\hline $\mathrm{PF}$ & $10 \mathrm{X}$ & U-std & 2 & 9 & 300272382 & 7270 & 21200 & 8020 & $<838$ & $<721$ & $<7400$ & 220000 & $<2330$ & 5880 & 19100 & $<149$ \\
\hline $\mathrm{PF}$ & $10 \mathrm{X}$ & SB6VS18 & 2 & 10 & 300272384 & 1470 & 16100 & 6620 & $<843$ & $<726$ & $<7450$ & 254000 & 7780 & 1760 & 13000 & 217 \\
\hline $\mathrm{PF}$ & $10 \mathrm{X}$ & $\begin{array}{l}\text { SB6VS21 } \\
\end{array}$ & 2 & 11 & 300272386 & 1730 & 19100 & 7800 & $<841$ & $<724$ & $<7430$ & 233000 & 9290 & 2060 & 15000 & 220 \\
\hline $\mathrm{PF}$ & $10 \mathrm{X}$ & $\begin{array}{l}\text { SB6VS22 } \\
\end{array}$ & 2 & 12 & 300272378 & 1830 & 20500 & 8090 & $<843$ & $<726$ & $<7440$ & 226000 & 9710 & 2230 & 15400 & 233 \\
\hline $\mathrm{PF}$ & $10 \mathrm{X}$ & SB6VS19 & 2 & 13 & 300272385 & 1560 & 17300 & 7100 & $<838$ & $<722$ & $<7410$ & 250000 & 8040 & 1910 & 12400 & 190 \\
\hline $\mathrm{PF}$ & $10 \mathrm{X}$ & SB6VS18 & 2 & 14 & 300272377 & 1480 & 16300 & 6700 & $<842$ & $<725$ & $<7430$ & 255000 & 7460 & 1810 & 12000 & 199 \\
\hline $\mathrm{PF}$ & $10 \mathrm{X}$ & ARG-1 & 2 & 15 & 300272388 & 5170 & 14300 & 7960 & 1060 & $<785$ & $<8050$ & 235000 & $<2540$ & 7050 & $<8050$ & $<162$ \\
\hline $\mathrm{PF}$ & $10 \mathrm{X}$ & BLANK & 2 & 16 & 300272390 & $<25$ & $<110$ & $<930$ & $<849$ & $<731$ & $<7500$ & $<231$ & $<2360$ & $<110$ & $<7500$ & $<151$ \\
\hline $\mathrm{PF}$ & $10 \mathrm{X}$ & U-std & 2 & 17 & 300272389 & 7420 & 21700 & 8380 & $<837$ & $<721$ & $<7390$ & 226000 & $<2330$ & 5920 & 19200 & $<149$ \\
\hline
\end{tabular}


SRNL-STI-2009-xxxxx, Revision 0

Table C5. Average Measured Chemical Compositions Versus Targeted Compositions by Oxide by Glass ID for Thorium Glasses

\begin{tabular}{|c|c|c|c|c|c|}
\hline & & Measured & Targeted & Difference of & $\%$ Difference of \\
\hline Glass ID & Oxide & $(w t \%)$ & $(w t \%)$ & Measured versus Targeted & Measured versus Targeted \\
\hline ARG-1 & $\mathrm{Al}_{2} \mathrm{O}_{3}$ & 4.8466 & 4.7300 & 0.1166 & $2.5 \%$ \\
\hline ARG-1 & $\mathrm{B}_{2} \mathrm{O}_{3}$ & 8.2698 & 8.6700 & -0.4002 & $-4.6 \%$ \\
\hline ARG-1 & $\mathrm{BaO}$ & 0.0879 & 0.0900 & -0.0021 & $-2.3 \%$ \\
\hline ARG-1 & $\mathrm{CaO}$ & 1.4855 & 1.4300 & 0.0555 & $3.9 \%$ \\
\hline ARG-1 & $\mathrm{Ce}_{2} \mathrm{O}_{3}$ & 0.0843 & 0.0000 & 0.0843 & \\
\hline ARG-1 & $\mathrm{Cr}_{2} \mathrm{O}_{3}$ & 0.0959 & 0.0900 & 0.0059 & $6.6 \%$ \\
\hline ARG-1 & $\mathrm{CuO}$ & 0.0097 & 0.0000 & 0.0097 & \\
\hline ARG-1 & $\mathrm{Fe}_{2} \mathrm{O}_{3}$ & 13.9777 & 14.0000 & -0.0223 & $-0.2 \%$ \\
\hline ARG-1 & $\mathrm{K}_{2} \mathrm{O}$ & 2.8429 & 2.7100 & 0.1329 & $4.9 \%$ \\
\hline ARG-1 & $\mathrm{La}_{2} \mathrm{O}_{3}$ & 0.0070 & 0.0000 & 0.0070 & \\
\hline ARG-1 & $\mathrm{Li}_{2} \mathrm{O}$ & 3.1540 & 3.2100 & -0.0560 & $-1.7 \%$ \\
\hline ARG-1 & $\mathrm{MgO}$ & 0.8507 & 0.8600 & -0.0093 & $-1.1 \%$ \\
\hline ARG-1 & $\mathrm{MnO}$ & 1.8249 & 2.3100 & -0.4851 & $-21.0 \%$ \\
\hline ARG-1 & $\mathrm{Na}_{2} \mathrm{O}$ & 11.8534 & 11.5000 & 0.3534 & $3.1 \%$ \\
\hline ARG-1 & $\mathrm{NiO}$ & 0.9949 & 1.0500 & -0.0551 & $-5.2 \%$ \\
\hline ARG-1 & $\mathrm{P}_{2} \mathrm{O}_{5}$ & 0.1812 & 0.2200 & -0.0388 & $-17.6 \%$ \\
\hline ARG-1 & $\mathrm{PbO}$ & 0.0432 & 0.0000 & 0.0432 & \\
\hline ARG-1 & $\mathrm{SiO}_{2}$ & 49.4178 & 47.9000 & 1.5178 & $3.2 \%$ \\
\hline ARG-1 & $\mathrm{SO}_{4}$ & 1.2333 & 0.0000 & 1.2333 & \\
\hline ARG-1 & $\mathrm{ThO}_{2}$ & 0.1475 & 0.0000 & 0.1475 & \\
\hline ARG-1 & $\mathrm{TiO}_{2}$ & 1.1695 & 1.1500 & 0.0195 & $1.7 \%$ \\
\hline ARG-1 & $\mathrm{U}_{3} \mathrm{O}_{8}$ & 0.4854 & 0.0000 & 0.4854 & \\
\hline ARG-1 & $\mathrm{ZnO}$ & 0.0143 & 0.0200 & -0.0057 & $-28.6 \%$ \\
\hline ARG-1 & $\mathrm{ZrO}_{2}$ & 0.1347 & 0.1300 & 0.0047 & $3.6 \%$ \\
\hline ARG-1 & Sum & 103.3159 & 100.0700 & 3.2459 & $3.2 \%$ \\
\hline SB6VS18 & $\mathrm{Al}_{2} \mathrm{O}_{3}$ & 8.4933 & 8.1160 & 0.3773 & $4.6 \%$ \\
\hline SB6VS18 & $\mathrm{B}_{2} \mathrm{O}_{3}$ & 5.2565 & 5.4400 & -0.1835 & $-3.4 \%$ \\
\hline SB6VS18 & $\mathrm{BaO}$ & 0.0387 & 0.0420 & -0.0033 & $-7.9 \%$ \\
\hline SB6VS18 & $\mathrm{CaO}$ & 0.4334 & 0.4390 & -0.0056 & $-1.3 \%$ \\
\hline SB6VS18 & $\mathrm{Ce}_{2} \mathrm{O}_{3}$ & 0.0761 & 0.0650 & 0.0111 & $17.1 \%$ \\
\hline SB6VS18 & $\mathrm{Cr}_{2} \mathrm{O}_{3}$ & 0.0333 & 0.0380 & -0.0047 & $-12.4 \%$ \\
\hline SB6VS18 & $\mathrm{CuO}$ & 0.0338 & 0.0330 & 0.0008 & $2.4 \%$ \\
\hline SB6VS18 & $\mathrm{Fe}_{2} \mathrm{O}_{3}$ & 7.3773 & 7.5090 & -0.1317 & $-1.8 \%$ \\
\hline SB6VS18 & $\mathrm{K}_{2} \mathrm{O}$ & 0.1622 & 0.0270 & 0.1352 & $500.6 \%$ \\
\hline SB6VS18 & $\mathrm{La}_{2} \mathrm{O}_{3}$ & 0.0254 & 0.0330 & -0.0076 & $-22.9 \%$ \\
\hline SB6VS18 & $\mathrm{Li}_{2} \mathrm{O}$ & 5.3069 & 5.4400 & -0.1331 & $-2.4 \%$ \\
\hline SB6VS18 & $\mathrm{MgO}$ & 0.2442 & 0.2440 & 0.0002 & $0.1 \%$ \\
\hline SB6VS18 & $\mathrm{MnO}$ & 2.0885 & 2.1370 & -0.0485 & $-2.3 \%$ \\
\hline SB6VS18 & $\mathrm{Na}_{2} \mathrm{O}$ & 12.8903 & 13.6920 & -0.8018 & $-5.9 \%$ \\
\hline SB6VS18 & $\mathrm{NiO}$ & 0.8440 & 0.9160 & -0.0720 & $-7.9 \%$ \\
\hline SB6VS18 & $\mathrm{P}_{2} \mathrm{O}_{5}$ & 0.0965 & 0.1390 & -0.0425 & $-30.6 \%$ \\
\hline SB6VS18 & $\mathrm{PbO}$ & 0.0391 & 0.0090 & 0.0301 & $334.2 \%$ \\
\hline SB6VS18 & $\mathrm{SiO}_{2}$ & 54.0708 & 52.3530 & 1.7178 & $3.3 \%$ \\
\hline SB6VS18 & $\mathrm{SO}_{4}$ & 1.1145 & 0.4320 & 0.6825 & $158.0 \%$ \\
\hline SB6VS18 & $\mathrm{ThO}_{2}$ & 0.8739 & 1.0110 & -0.1371 & $-13.6 \%$ \\
\hline
\end{tabular}


SRNL-STI-2009-xxxxx, Revision 0

Table C5. Average Measured Chemical Compositions Versus Targeted Compositions by Oxide by Glass ID for Thorium Glasses

\begin{tabular}{|c|c|c|c|c|c|}
\hline & & Measured & Targeted & Difference of & \% Difference of \\
\hline Glass ID & Oxide & (wt\%) & $(w t \%)$ & Measured versus Targeted & Measured versus Targeted \\
\hline SB6VS18 & $\mathrm{TiO}_{2}$ & 0.3002 & 0.2940 & 0.0062 & $2.1 \%$ \\
\hline SB6VS18 & $\mathrm{U}_{3} \mathrm{O}_{8}$ & 1.4298 & 1.4980 & -0.0682 & $-4.6 \%$ \\
\hline SB6VS18 & $\mathrm{ZnO}$ & 0.0297 & 0.0220 & 0.0077 & $34.9 \%$ \\
\hline SB6VS18 & $\mathrm{ZrO}_{2}$ & 0.0693 & 0.0720 & -0.0027 & $-3.8 \%$ \\
\hline SB6VS18 & Sum & 101.4751 & 100.0010 & 1.4741 & $1.5 \%$ \\
\hline SB6VS19 & $\mathrm{Al}_{2} \mathrm{O}_{3}$ & 9.0224 & 8.6230 & 0.3994 & $4.6 \%$ \\
\hline SB6VS19 & $\mathrm{B}_{2} \mathrm{O}_{3}$ & 5.0794 & 5.2800 & -0.2006 & $-3.8 \%$ \\
\hline SB6VS19 & $\mathrm{BaO}$ & 0.0444 & 0.0440 & 0.0004 & $1.0 \%$ \\
\hline SB6VS19 & $\mathrm{CaO}$ & 0.4866 & 0.4670 & 0.0196 & $4.2 \%$ \\
\hline SB6VS19 & $\mathrm{Ce}_{2} \mathrm{O}_{3}$ & 0.0755 & 0.0690 & 0.0065 & $9.5 \%$ \\
\hline SB6VS19 & $\mathrm{Cr}_{2} \mathrm{O}_{3}$ & 0.0377 & 0.0400 & -0.0023 & $-5.8 \%$ \\
\hline SB6VS19 & $\mathrm{CuO}$ & 0.0351 & 0.0360 & -0.0009 & $-2.4 \%$ \\
\hline SB6VS19 & $\mathrm{Fe}_{2} \mathrm{O}_{3}$ & 7.8026 & 7.9780 & -0.1754 & $-2.2 \%$ \\
\hline SB6VS19 & $\mathrm{K}_{2} \mathrm{O}$ & 0.2417 & 0.0290 & 0.2127 & $733.4 \%$ \\
\hline SB6VS19 & $\mathrm{La}_{2} \mathrm{O}_{3}$ & 0.0265 & 0.0350 & -0.0085 & $-24.4 \%$ \\
\hline SB6VS19 & $\mathrm{Li}_{2} \mathrm{O}$ & 5.1562 & 5.2800 & -0.1238 & $-2.3 \%$ \\
\hline SB6VS19 & $\mathrm{MgO}$ & 0.2554 & 0.2590 & -0.0036 & $-1.4 \%$ \\
\hline SB6VS19 & $\mathrm{MnO}$ & 2.2209 & 2.2700 & -0.0491 & $-2.2 \%$ \\
\hline SB6VS19 & $\mathrm{Na}_{2} \mathrm{O}$ & 13.9855 & 14.0480 & -0.0625 & $-0.4 \%$ \\
\hline SB6VS19 & $\mathrm{NiO}$ & 0.8917 & 0.9730 & -0.0813 & $-8.4 \%$ \\
\hline SB6VS19 & $\mathrm{P}_{2} \mathrm{O}_{5}$ & 0.0960 & 0.1480 & -0.0520 & $-35.2 \%$ \\
\hline SB6VS19 & $\mathrm{PbO}$ & 0.0389 & 0.0100 & 0.0289 & $288.6 \%$ \\
\hline SB6VS19 & $\mathrm{SiO}_{2}$ & 52.6803 & 50.8750 & 1.8053 & $3.5 \%$ \\
\hline SB6VS19 & $\mathrm{SO}_{4}$ & 1.1092 & 0.4590 & 0.6502 & $141.7 \%$ \\
\hline SB6VS19 & $\mathrm{ThO}_{2}$ & 0.9123 & 1.0740 & -0.1617 & $-15.1 \%$ \\
\hline SB6VS19 & $\mathrm{TiO}_{2}$ & 0.3186 & 0.3130 & 0.0056 & $1.8 \%$ \\
\hline SB6VS19 & $\mathrm{U}_{3} \mathrm{O}_{8}$ & 1.4504 & 1.5910 & -0.1406 & $-8.8 \%$ \\
\hline SB6VS19 & $\mathrm{ZnO}$ & 0.0237 & 0.0230 & 0.0007 & $3.2 \%$ \\
\hline SB6VS19 & $\mathrm{ZrO}_{2}$ & 0.0766 & 0.0770 & -0.0004 & $-0.5 \%$ \\
\hline SB6VS19 & Sum & 102.1698 & 100.0010 & 2.1688 & $2.2 \%$ \\
\hline SB6VS20 & $\mathrm{Al}_{2} \mathrm{O}_{3}$ & 9.5231 & 9.1300 & 0.3931 & $4.3 \%$ \\
\hline SB6VS20 & $\mathrm{B}_{2} \mathrm{O}_{3}$ & 4.9264 & 5.1200 & -0.1936 & $-3.8 \%$ \\
\hline SB6VS20 & $\mathrm{BaO}$ & 0.0467 & 0.0470 & -0.0003 & $-0.6 \%$ \\
\hline SB6VS20 & $\mathrm{CaO}$ & 0.4656 & 0.4940 & -0.0284 & $-5.8 \%$ \\
\hline SB6VS20 & $\mathrm{Ce}_{2} \mathrm{O}_{3}$ & 0.0761 & 0.0730 & 0.0031 & $4.3 \%$ \\
\hline SB6VS20 & $\mathrm{Cr}_{2} \mathrm{O}_{3}$ & 0.0369 & 0.0430 & -0.0061 & $-14.3 \%$ \\
\hline SB6VS20 & $\mathrm{CuO}$ & 0.0367 & 0.0380 & -0.0013 & $-3.4 \%$ \\
\hline SB6VS20 & $\mathrm{Fe}_{2} \mathrm{O}_{3}$ & 8.2458 & 8.4470 & -0.2012 & $-2.4 \%$ \\
\hline SB6VS20 & $\mathrm{K}_{2} \mathrm{O}$ & 0.3426 & 0.0300 & 0.3126 & $1041.9 \%$ \\
\hline SB6VS20 & $\mathrm{La}_{2} \mathrm{O}_{3}$ & 0.0278 & 0.0370 & -0.0092 & $-24.9 \%$ \\
\hline SB6VS20 & $\mathrm{Li}_{2} \mathrm{O}$ & 5.0163 & 5.1200 & -0.1037 & $-2.0 \%$ \\
\hline SB6VS20 & $\mathrm{MgO}$ & 0.2699 & 0.2750 & -0.0051 & $-1.9 \%$ \\
\hline SB6VS20 & $\mathrm{MnO}$ & 2.3274 & 2.4040 & -0.0766 & $-3.2 \%$ \\
\hline SB6VS20 & $\mathrm{Na}_{2} \mathrm{O}$ & 13.0048 & 14.4040 & -1.3992 & $-9.7 \%$ \\
\hline SB6VS20 & $\mathrm{NiO}$ & 0.9251 & 1.0300 & -0.1049 & $-10.2 \%$ \\
\hline
\end{tabular}


SRNL-STI-2009-xxxxx, Revision 0

Table C5. Average Measured Chemical Compositions Versus Targeted Compositions by Oxide by Glass ID for Thorium Glasses

\begin{tabular}{|c|c|c|c|c|c|}
\hline & & Measured & Targeted & Difference of & \% Difference of \\
\hline Glass ID & Oxide & (wt\%) & $(w t \%)$ & Measured versus Targeted & Measured versus Targeted \\
\hline SB6VS20 & $\mathrm{P}_{2} \mathrm{O}_{5}$ & 0.0964 & 0.1560 & -0.0596 & $-38.2 \%$ \\
\hline SB6VS20 & $\mathrm{PbO}$ & 0.0390 & 0.0100 & 0.0290 & $289.9 \%$ \\
\hline SB6VS20 & $\mathrm{SiO}_{2}$ & 50.7549 & 49.3970 & 1.3579 & $2.7 \%$ \\
\hline SB6VS20 & $\mathrm{SO}_{4}$ & 1.1130 & 0.4860 & 0.6270 & $129.0 \%$ \\
\hline SB6VS20 & $\mathrm{ThO}_{2}$ & 0.9814 & 1.1370 & -0.1556 & $-13.7 \%$ \\
\hline SB6VS20 & $\mathrm{TiO}_{2}$ & 0.3319 & 0.3310 & 0.0009 & $0.3 \%$ \\
\hline SB6VS20 & $\mathrm{U}_{3} \mathrm{O}_{8}$ & 1.6037 & 1.6850 & -0.0813 & $-4.8 \%$ \\
\hline SB6VS20 & $\mathrm{ZnO}$ & 0.0258 & 0.0250 & 0.0008 & $3.3 \%$ \\
\hline SB6VS20 & $\mathrm{ZrO}_{2}$ & 0.0734 & 0.0810 & -0.0076 & $-9.3 \%$ \\
\hline SB6VS20 & Sum & 100.4424 & 100.0000 & 0.4424 & $0.4 \%$ \\
\hline SB6VS21 & $\mathrm{Al}_{2} \mathrm{O}_{3}$ & 10.1277 & 9.6370 & 0.4907 & $5.1 \%$ \\
\hline SB6VS21 & $\mathrm{B}_{2} \mathrm{O}_{3}$ & 4.7494 & 4.9600 & -0.2106 & $-4.2 \%$ \\
\hline SB6VS21 & $\mathrm{BaO}$ & 0.0544 & 0.0500 & 0.0044 & $8.8 \%$ \\
\hline SB6VS21 & $\mathrm{CaO}$ & 0.5523 & 0.5220 & 0.0303 & $5.8 \%$ \\
\hline SB6VS21 & $\mathrm{Ce}_{2} \mathrm{O}_{3}$ & 0.0761 & 0.0770 & -0.0009 & $-1.1 \%$ \\
\hline SB6VS21 & $\mathrm{Cr}_{2} \mathrm{O}_{3}$ & 0.0365 & 0.0450 & -0.0085 & $-19.0 \%$ \\
\hline SB6VS21 & $\mathrm{CuO}$ & 0.0398 & 0.0400 & -0.0002 & $-0.6 \%$ \\
\hline SB6VS21 & $\mathrm{Fe}_{2} \mathrm{O}_{3}$ & 8.7462 & 8.9170 & -0.1708 & $-1.9 \%$ \\
\hline SB6VS21 & $\mathrm{K}_{2} \mathrm{O}$ & 0.3099 & 0.0320 & 0.2779 & $868.4 \%$ \\
\hline SB6VS21 & $\mathrm{La}_{2} \mathrm{O}_{3}$ & 0.0272 & 0.0390 & -0.0118 & $-30.4 \%$ \\
\hline SB6VS21 & $\mathrm{Li}_{2} \mathrm{O}$ & 4.8548 & 4.9600 & -0.1052 & $-2.1 \%$ \\
\hline SB6VS21 & $\mathrm{MgO}$ & 0.2861 & 0.2900 & -0.0039 & $-1.4 \%$ \\
\hline SB6VS21 & $\mathrm{MnO}$ & 2.4565 & 2.5370 & -0.0805 & $-3.2 \%$ \\
\hline SB6VS21 & $\mathrm{Na}_{2} \mathrm{O}$ & 14.8617 & 14.7600 & 0.1017 & $0.7 \%$ \\
\hline SB6VS21 & $\mathrm{NiO}$ & 0.9849 & 1.0880 & -0.1031 & $-9.5 \%$ \\
\hline SB6VS21 & $\mathrm{P}_{2} \mathrm{O}_{5}$ & 0.0963 & 0.1650 & -0.0687 & $-41.6 \%$ \\
\hline SB6VS21 & $\mathrm{PbO}$ & 0.0390 & 0.0110 & 0.0280 & $254.3 \%$ \\
\hline SB6VS21 & $\mathrm{SiO}_{2}$ & 49.5783 & 47.9190 & 1.6593 & $3.5 \%$ \\
\hline SB6VS21 & $\mathrm{SO}_{4}$ & 1.1122 & 0.5130 & 0.5992 & $116.8 \%$ \\
\hline SB6VS21 & $\mathrm{ThO}_{2}$ & 1.0662 & 1.2000 & -0.1338 & $-11.1 \%$ \\
\hline SB6VS21 & $\mathrm{TiO}_{2}$ & 0.3494 & 0.3500 & -0.0006 & $-0.2 \%$ \\
\hline SB6VS21 & $\mathrm{U}_{3} \mathrm{O}_{8}$ & 1.7511 & 1.7780 & -0.0269 & $-1.5 \%$ \\
\hline SB6VS21 & $\mathrm{ZnO}$ & 0.0251 & 0.0260 & -0.0009 & $-3.4 \%$ \\
\hline SB6VS21 & $\mathrm{ZrO}_{2}$ & 0.0870 & 0.0860 & 0.0010 & $1.2 \%$ \\
\hline SB6VS21 & Sum & 102.3897 & 100.0020 & 2.3877 & $2.4 \%$ \\
\hline SB6VS22 & $\mathrm{Al}_{2} \mathrm{O}_{3}$ & 10.5245 & 10.1450 & 0.3795 & $3.7 \%$ \\
\hline SB6VS22 & $\mathrm{B}_{2} \mathrm{O}_{3}$ & 4.6206 & 4.8000 & -0.1794 & $-3.7 \%$ \\
\hline SB6VS22 & $\mathrm{BaO}$ & 0.0497 & 0.0520 & -0.0023 & $-4.3 \%$ \\
\hline SB6VS22 & $\mathrm{CaO}$ & 0.5058 & 0.5490 & -0.0432 & $-7.9 \%$ \\
\hline SB6VS22 & $\mathrm{Ce}_{2} \mathrm{O}_{3}$ & 0.0761 & 0.0810 & -0.0049 & $-6.0 \%$ \\
\hline SB6VS22 & $\mathrm{Cr}_{2} \mathrm{O}_{3}$ & 0.0429 & 0.0470 & -0.0041 & $-8.7 \%$ \\
\hline SB6VS22 & $\mathrm{CuO}$ & 0.0406 & 0.0420 & -0.0014 & $-3.3 \%$ \\
\hline SB6VS22 & $\mathrm{Fe}_{2} \mathrm{O}_{3}$ & 9.1965 & 9.3860 & -0.1895 & $-2.0 \%$ \\
\hline SB6VS22 & $\mathrm{K}_{2} \mathrm{O}$ & 0.2259 & 0.0340 & 0.1919 & $564.3 \%$ \\
\hline SB6VS22 & $\mathrm{La}_{2} \mathrm{O}_{3}$ & 0.0311 & 0.0410 & -0.0099 & $-24.1 \%$ \\
\hline
\end{tabular}


SRNL-STI-2009-xxxxx, Revision 0

Table C5. Average Measured Chemical Compositions Versus Targeted Compositions by Oxide by Glass ID for Thorium Glasses

\begin{tabular}{|c|c|c|c|c|c|}
\hline & & Measured & Targeted & Difference of & \% Difference of \\
\hline Glass ID & Oxide & (wt\%) & (wt\%) & Measured versus Targeted & Measured versus Targeted \\
\hline SB6VS22 & $\mathrm{Li}_{2} \mathrm{O}$ & 4.7149 & 4.8000 & -0.0851 & $-1.8 \%$ \\
\hline SB6VS22 & $\mathrm{MgO}$ & 0.3031 & 0.3050 & -0.0019 & $-0.6 \%$ \\
\hline SB6VS22 & $\mathrm{MnO}$ & 2.6276 & 2.6710 & -0.0434 & $-1.6 \%$ \\
\hline SB6VS22 & $\mathrm{Na}_{2} \mathrm{O}$ & 13.4025 & 15.1160 & -1.7135 & $-11.3 \%$ \\
\hline SB6VS22 & $\mathrm{NiO}$ & 1.0390 & 1.1450 & -0.1060 & $-9.3 \%$ \\
\hline SB6VS22 & $\mathrm{P}_{2} \mathrm{O}_{5}$ & 0.0966 & 0.1740 & -0.0774 & $-44.5 \%$ \\
\hline SB6VS22 & $\mathrm{PbO}$ & 0.0391 & 0.0110 & 0.0281 & $255.5 \%$ \\
\hline SB6VS22 & $\mathrm{SiO}_{2}$ & 47.9203 & 46.4410 & 1.4793 & $3.2 \%$ \\
\hline SB6VS22 & $\mathrm{SO}_{4}$ & 1.1152 & 0.5400 & 0.5752 & $106.5 \%$ \\
\hline SB6VS22 & $\mathrm{ThO}_{2}$ & 1.1248 & 1.2630 & -0.1382 & $-10.9 \%$ \\
\hline SB6VS22 & $\mathrm{TiO}_{2}$ & 0.3715 & 0.3680 & 0.0035 & $1.0 \%$ \\
\hline SB6VS22 & $\mathrm{U}_{3} \mathrm{O}_{8}$ & 1.8130 & 1.8720 & -0.0590 & $-3.2 \%$ \\
\hline SB6VS22 & $\mathrm{ZnO}$ & 0.0269 & 0.0270 & -0.0001 & $-0.3 \%$ \\
\hline SB6VS22 & $\mathrm{ZrO}_{2}$ & 0.0801 & 0.0900 & -0.0099 & $-11.0 \%$ \\
\hline SB6VS22 & Sum & 100.1808 & 100.0000 & 0.1808 & $0.2 \%$ \\
\hline U-std & $\mathrm{Al}_{2} \mathrm{O}_{3}$ & 4.0813 & 4.1000 & -0.0187 & $-0.5 \%$ \\
\hline U-std & $\mathrm{B}_{2} \mathrm{O}_{3}$ & 8.8762 & 9.2090 & -0.3328 & $-3.6 \%$ \\
\hline U-std & $\mathrm{BaO}$ & 0.0065 & 0.0000 & 0.0065 & \\
\hline U-std & $\mathrm{CaO}$ & 1.3094 & 1.3010 & 0.0084 & $0.6 \%$ \\
\hline U-std & $\mathrm{Ce}_{2} \mathrm{O}_{3}$ & 0.0757 & 0.0000 & 0.0757 & \\
\hline U-std & $\mathrm{Cr}_{2} \mathrm{O}_{3}$ & 0.2429 & 0.0000 & 0.2429 & \\
\hline U-std & $\mathrm{CuO}$ & 0.0088 & 0.0000 & 0.0088 & \\
\hline U-std & $\mathrm{Fe}_{2} \mathrm{O}_{3}$ & 13.3296 & 13.1960 & 0.1336 & $1.0 \%$ \\
\hline U-std & $\mathrm{K}_{2} \mathrm{O}$ & 3.1279 & 2.9990 & 0.1289 & $4.3 \%$ \\
\hline U-std & $\mathrm{La}_{2} \mathrm{O}_{3}$ & 0.0063 & 0.0000 & 0.0063 & \\
\hline U-std & $\mathrm{Li}_{2} \mathrm{O}$ & 2.9746 & 3.0570 & -0.0824 & $-2.7 \%$ \\
\hline U-std & $\mathrm{MgO}$ & 1.2130 & 1.2100 & 0.0030 & $0.3 \%$ \\
\hline U-std & $\mathrm{MnO}$ & 2.7481 & 2.8920 & -0.1439 & $-5.0 \%$ \\
\hline U-std & $\mathrm{Na}_{2} \mathrm{O}$ & 11.7793 & 11.7950 & -0.0157 & $-0.1 \%$ \\
\hline U-std & $\mathrm{NiO}$ & 1.0324 & 1.1200 & -0.0876 & $-7.8 \%$ \\
\hline U-std & $\mathrm{P}_{2} \mathrm{O}_{5}$ & 0.0961 & 0.0000 & 0.0961 & \\
\hline U-std & $\mathrm{PbO}$ & 0.0389 & 0.0000 & 0.0389 & \\
\hline U-std & $\mathrm{SiO}_{2}$ & 47.0646 & 45.3530 & 1.7116 & $3.8 \%$ \\
\hline U-std & $\mathrm{SO}_{4}$ & 1.1095 & 0.0000 & 1.1095 & \\
\hline U-std & $\mathrm{ThO}_{2}$ & 0.1328 & 0.0000 & 0.1328 & \\
\hline U-std & $\mathrm{TiO}_{2}$ & 0.9838 & 1.0490 & -0.0652 & $-6.2 \%$ \\
\hline U-std & $\mathrm{U}_{3} \mathrm{O}_{8}$ & 2.3545 & 2.4060 & -0.0515 & $-2.1 \%$ \\
\hline U-std & $\mathrm{ZnO}$ & 0.0093 & 0.0000 & 0.0093 & \\
\hline U-std & $\mathrm{ZrO}_{2}$ & 0.0029 & 0.0000 & 0.0029 & \\
\hline U-std & Sum & 102.7279 & 99.6870 & 3.0409 & $3.1 \%$ \\
\hline
\end{tabular}




\section{Exhibit C1. Measurements of Thorium Glasses in Analytical Sequence for Samples by Prep Method by Oxide}

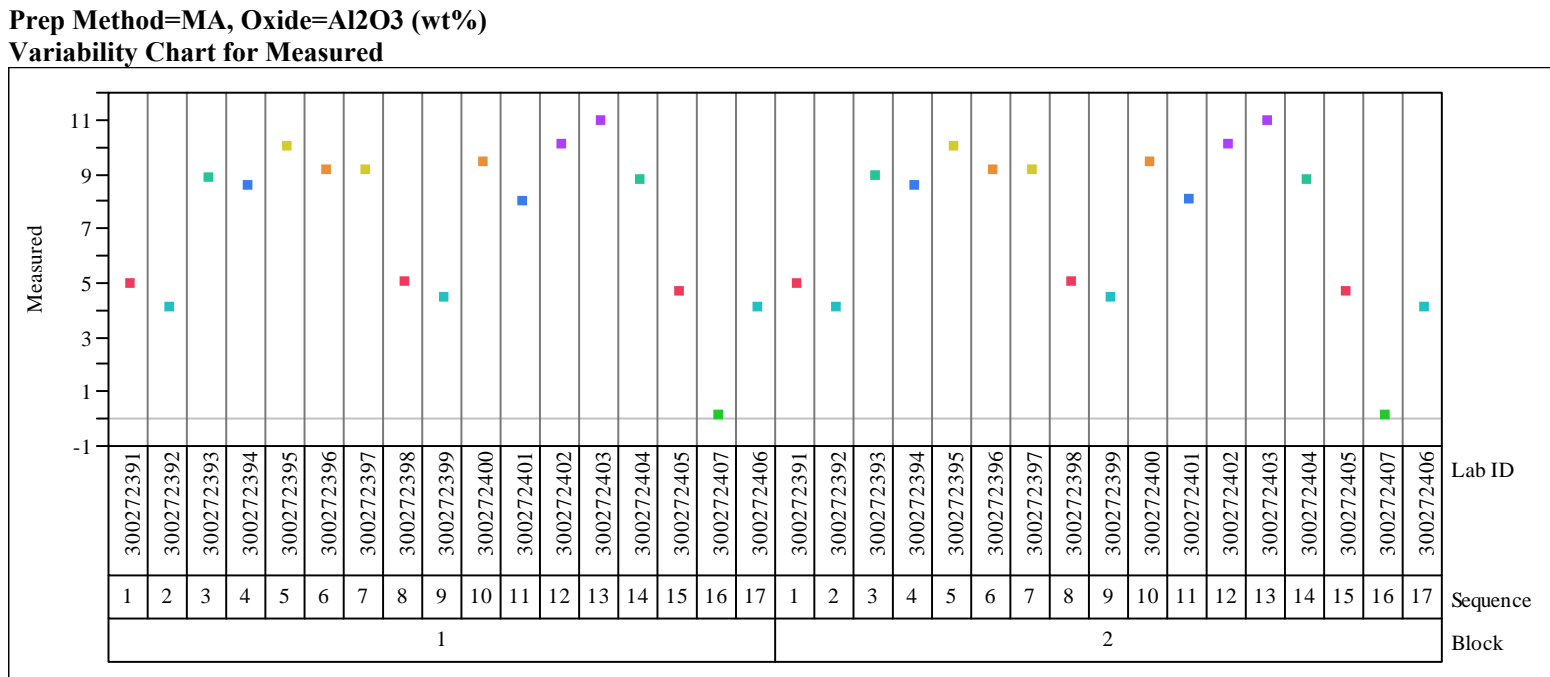

Prep Method $=$ MA, Oxide $=\mathrm{BaO}\left(\mathrm{wt}_{\mathrm{t}} \%\right)$

Variability Chart for Measured

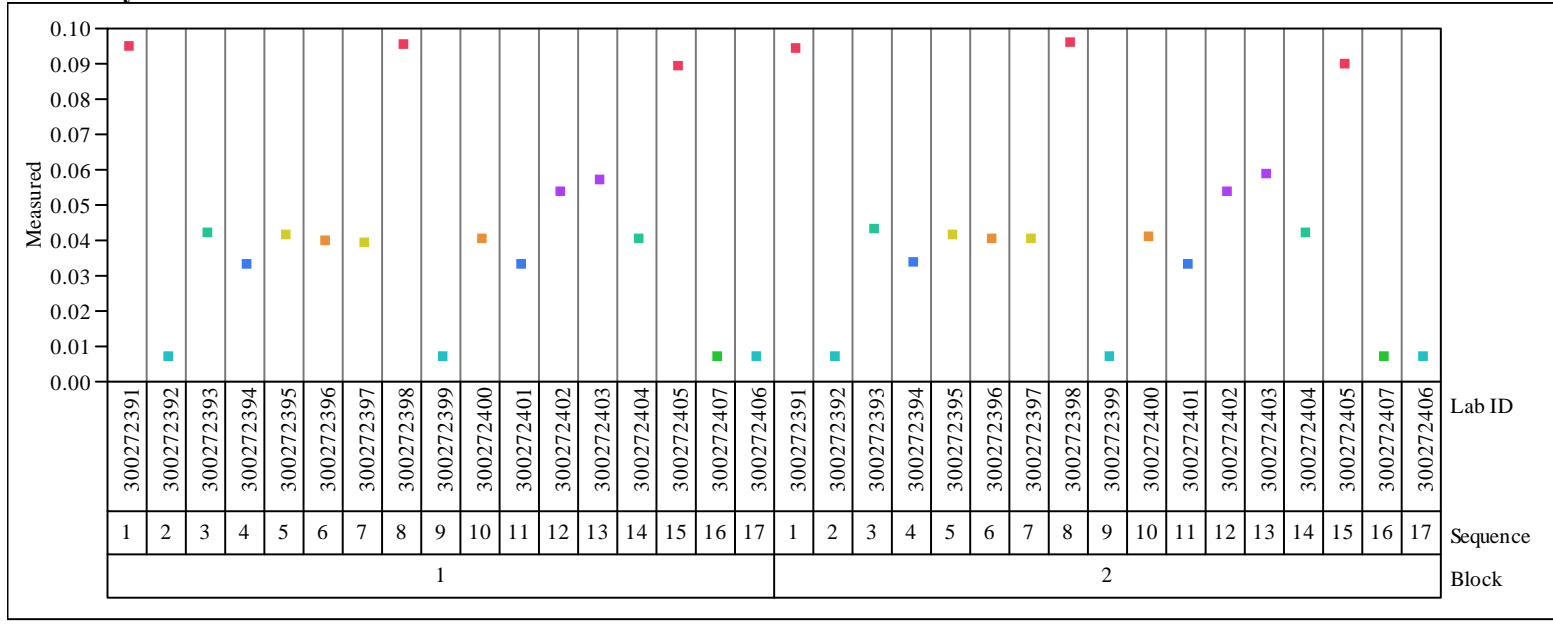

Prep Method $=$ MA, Oxide $=\mathrm{CaO}(\mathrm{wt} \%)$

Variability Chart for Measured

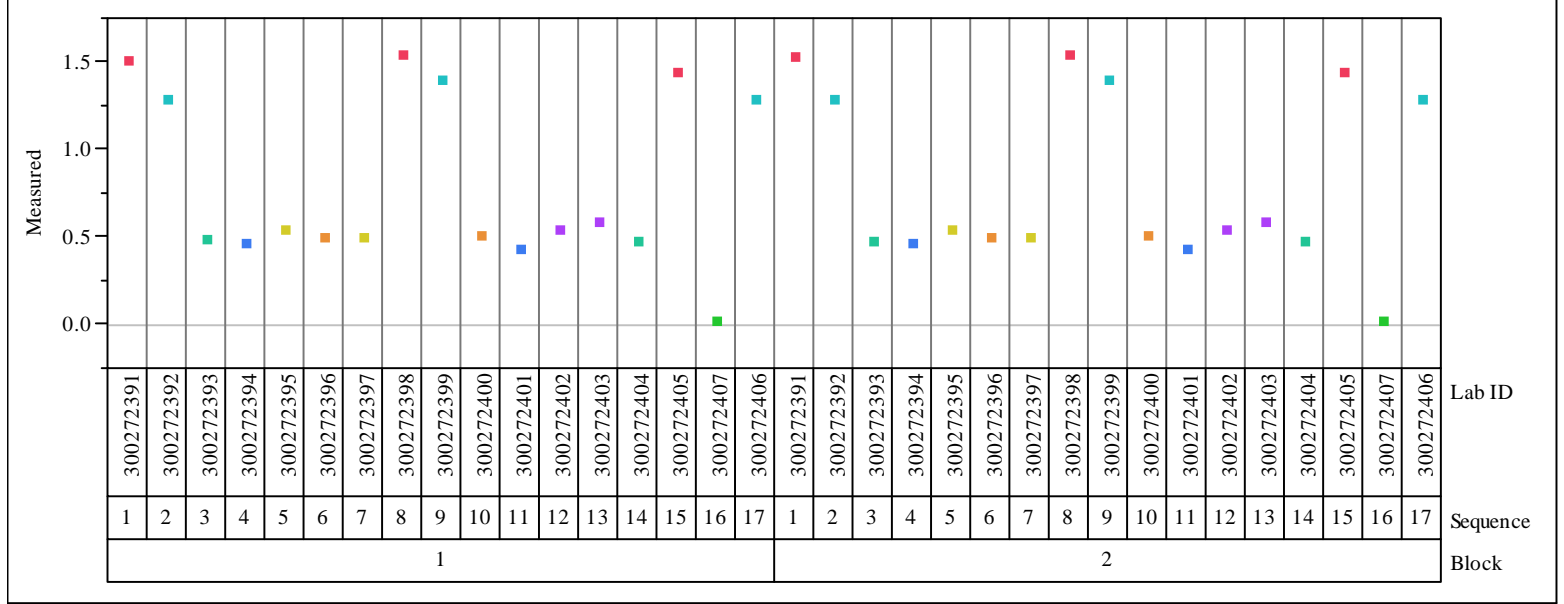




\section{Exhibit C1. Measurements of Thorium Glasses in Analytical Sequence for Samples by Prep Method by Oxide}

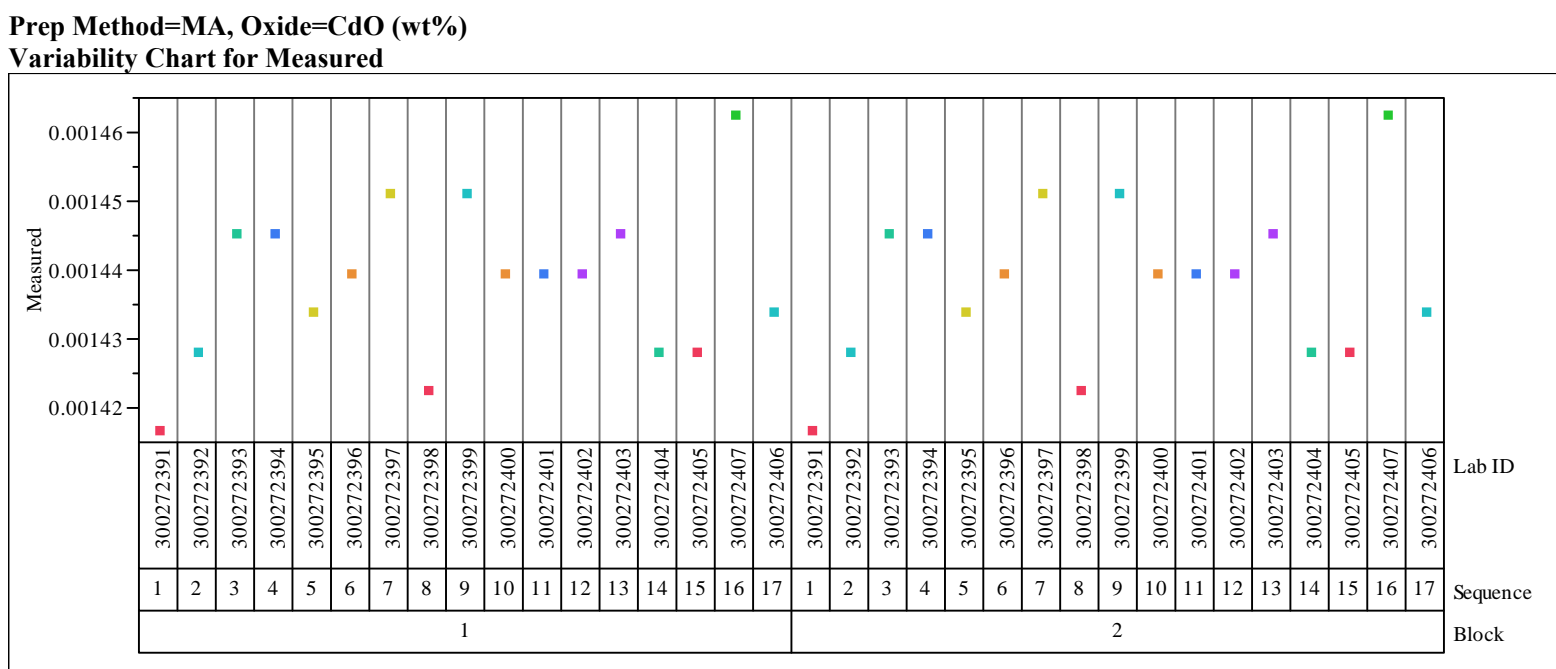

Prep Method=MA, Oxide $=\mathrm{Ce} 2 \mathrm{O3}(\mathrm{wt} \%)$

Variability Chart for Measured

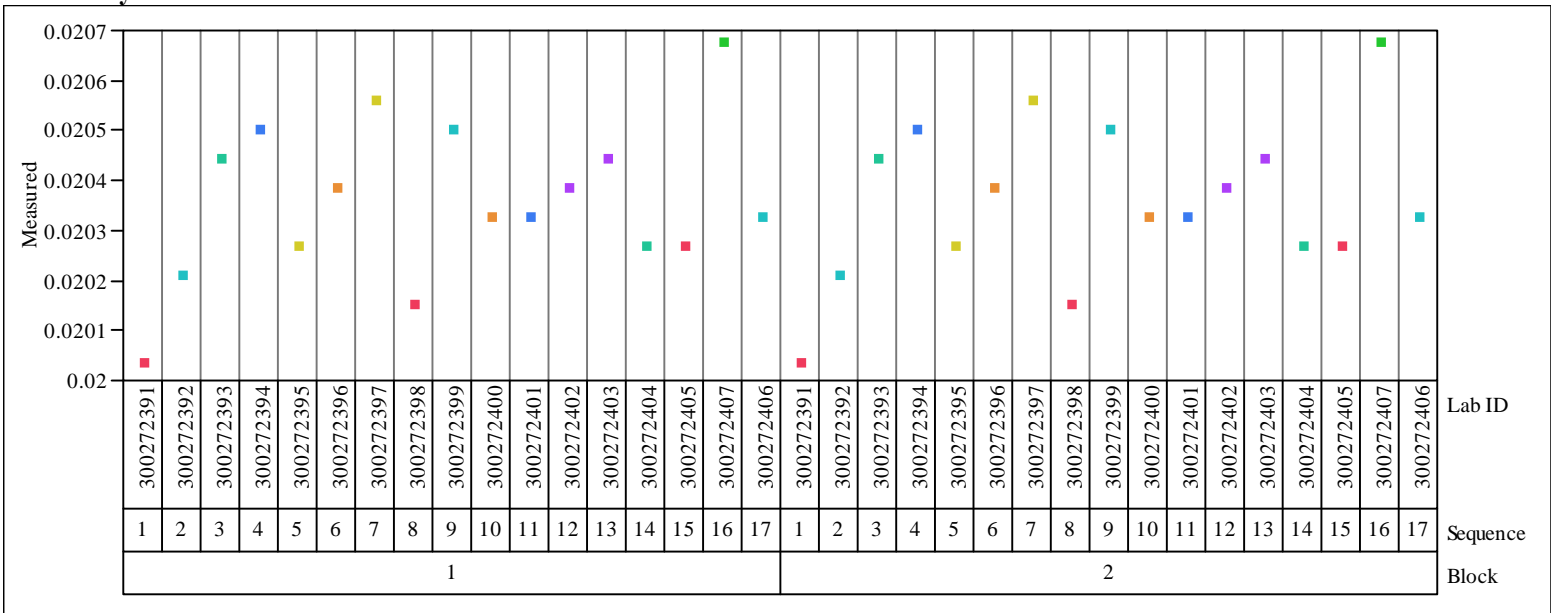




\section{Exhibit C1. Measurements of Thorium Glasses in Analytical Sequence for Samples by Prep Method by Oxide}
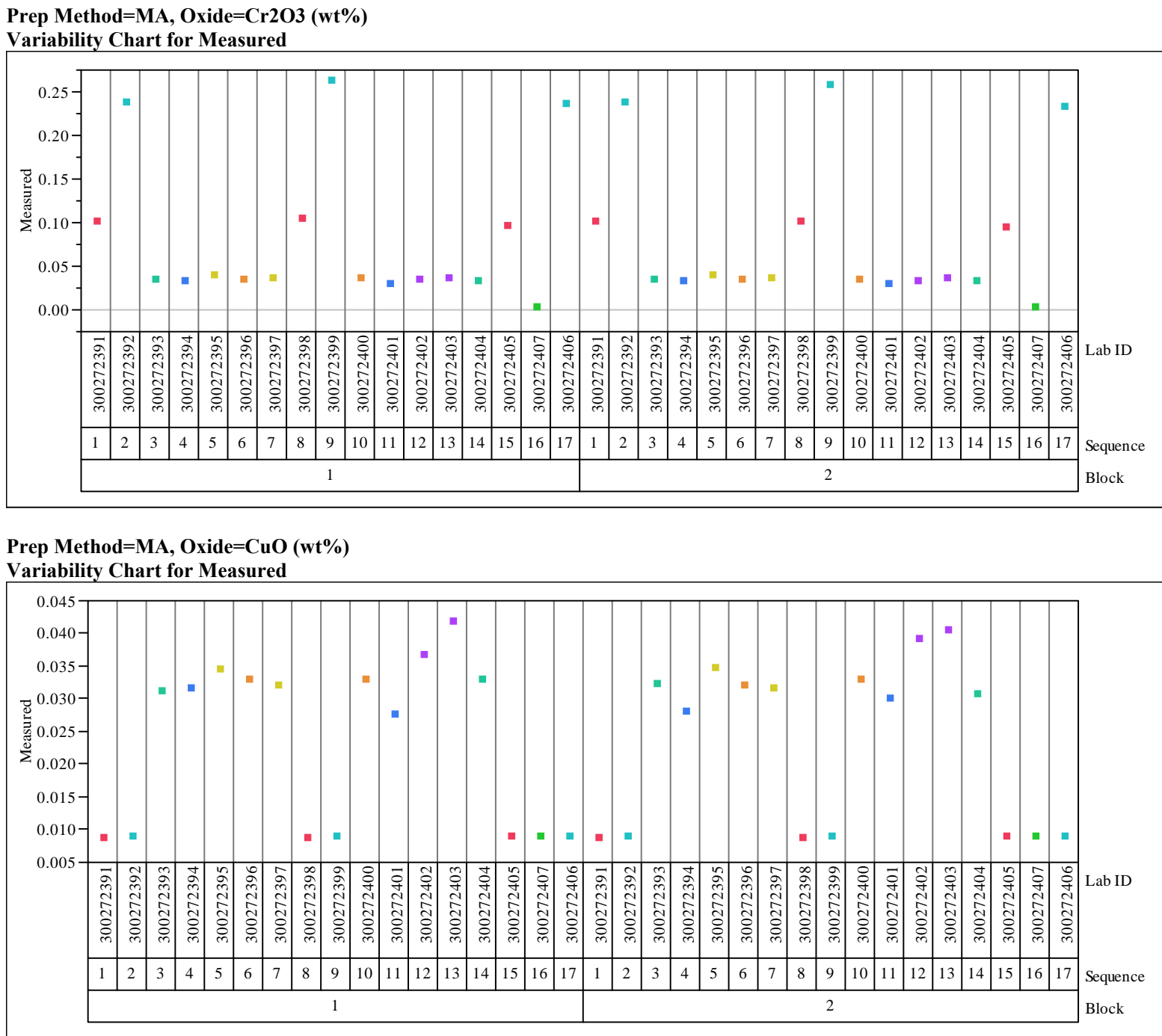

Prep Method=MA, Oxide=Fe2O3 (wt \%)

Variability Chart for Measured

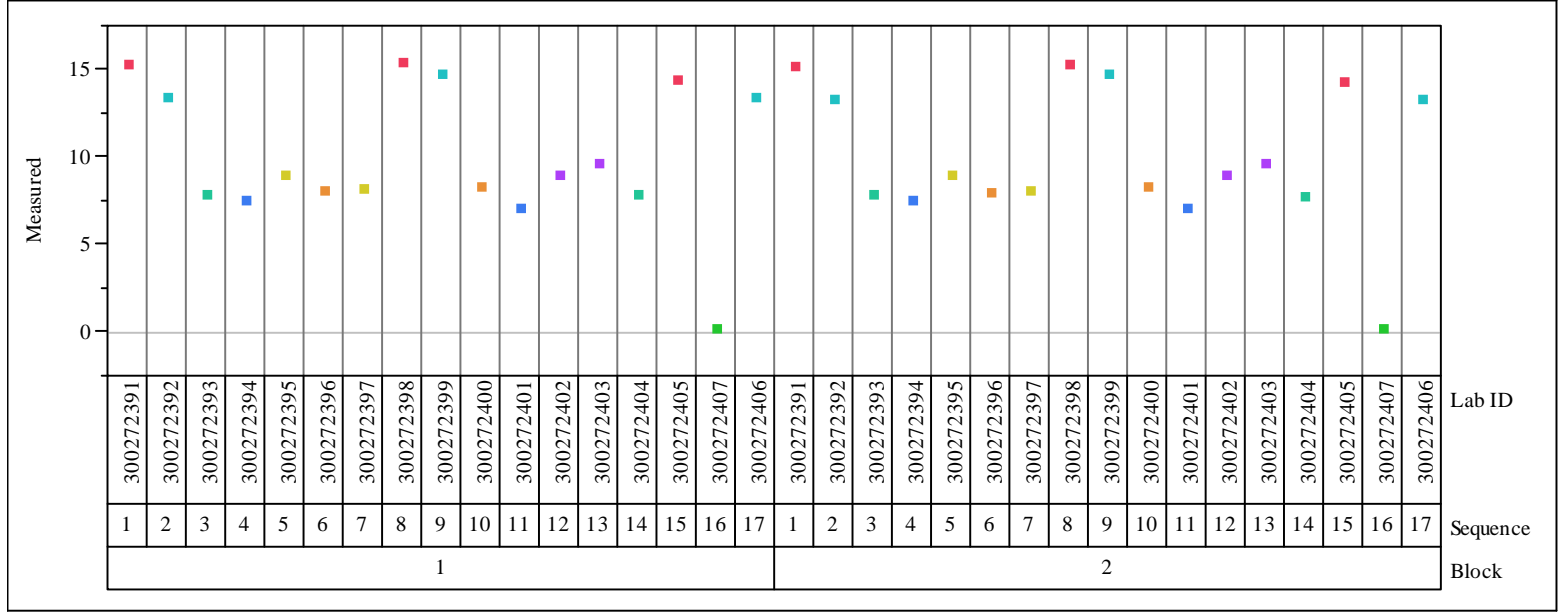




\section{Exhibit C1. Measurements of Thorium Glasses in Analytical Sequence for Samples by Prep Method by Oxide}

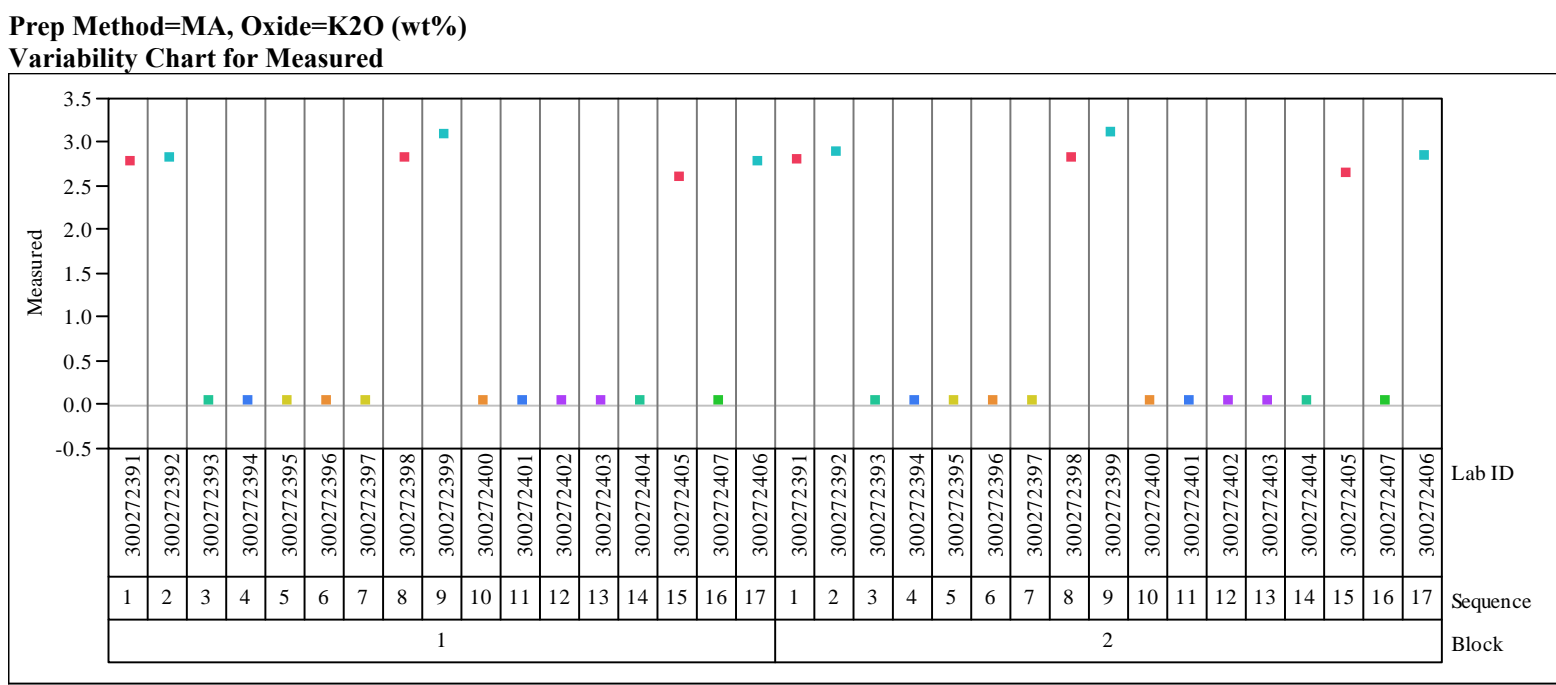

Prep Method=MA, Oxide $=\mathbf{L a 2 O 3}(\mathbf{w t} \%)$

Variability Chart for Measured

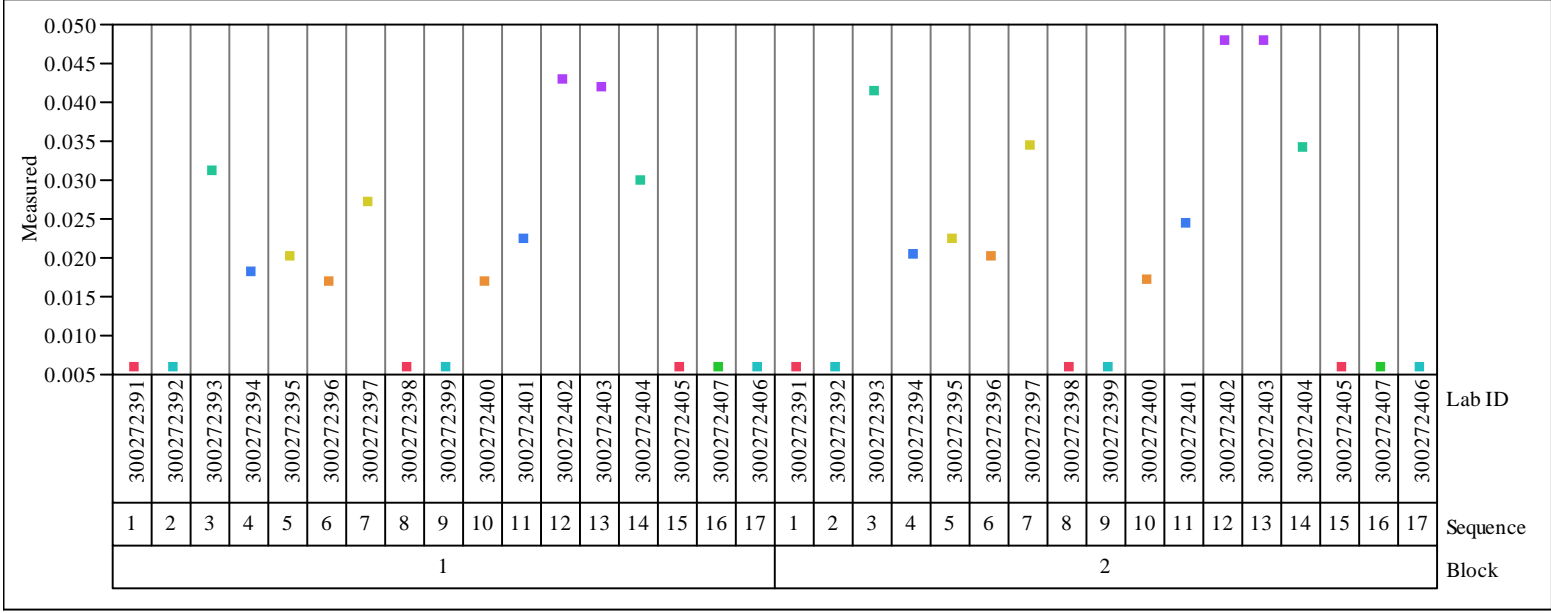




\section{Exhibit C1. Measurements of Thorium Glasses in Analytical Sequence for Samples by Prep Method by Oxide}

Prep Method $=$ MA, Oxide $=$ Li2O $(w t \%)$

Variability Chart for Measured

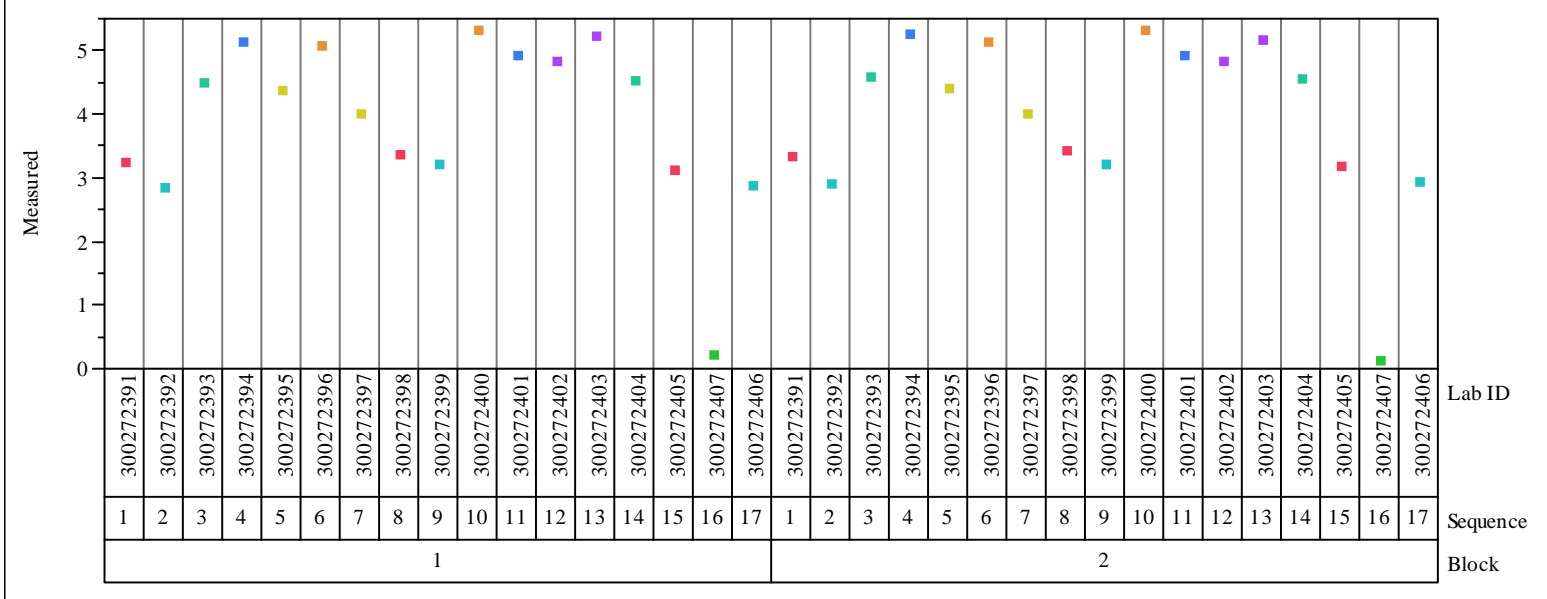

Prep Method=MA, Oxide $=$ MgO $(w t \%)$

Variability Chart for Measured

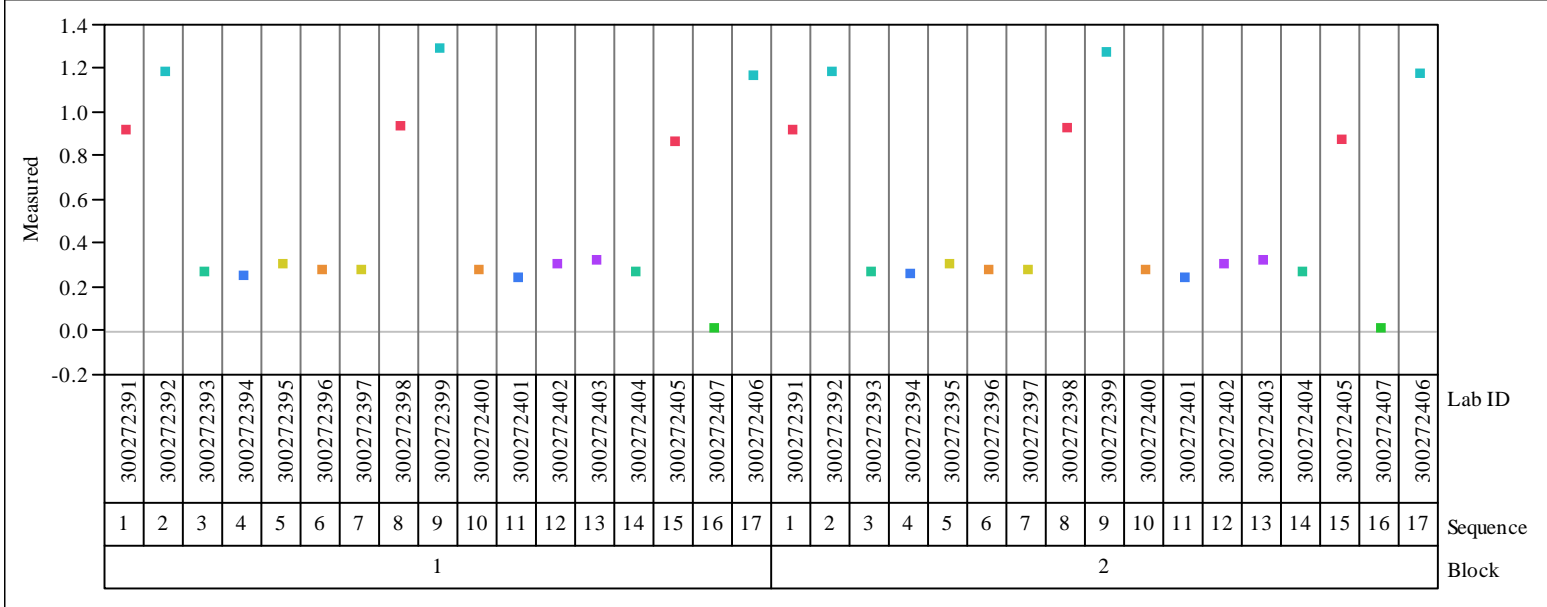

Prep Method $=$ MA, Oxide $=\mathrm{MnO}(\mathrm{wt} \%)$

Variability Chart for Measured

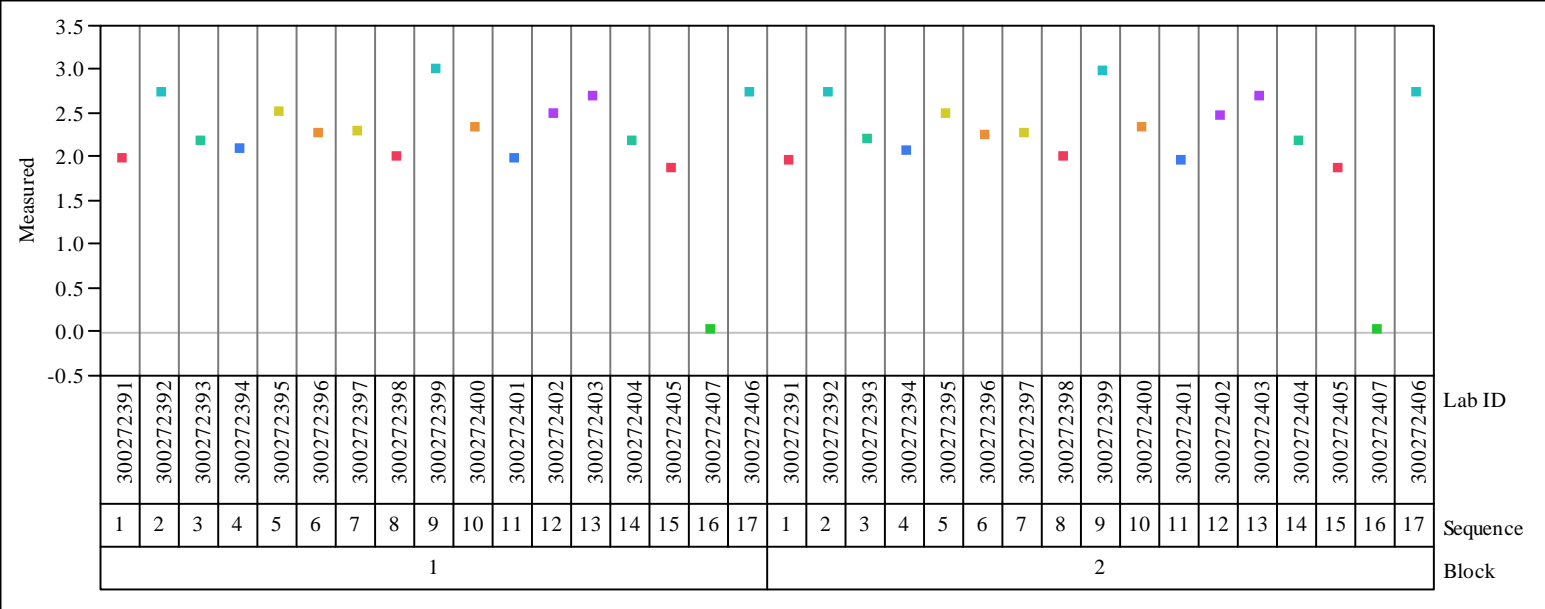




\section{Exhibit C1. Measurements of Thorium Glasses in Analytical Sequence for Samples by Prep Method by Oxide}

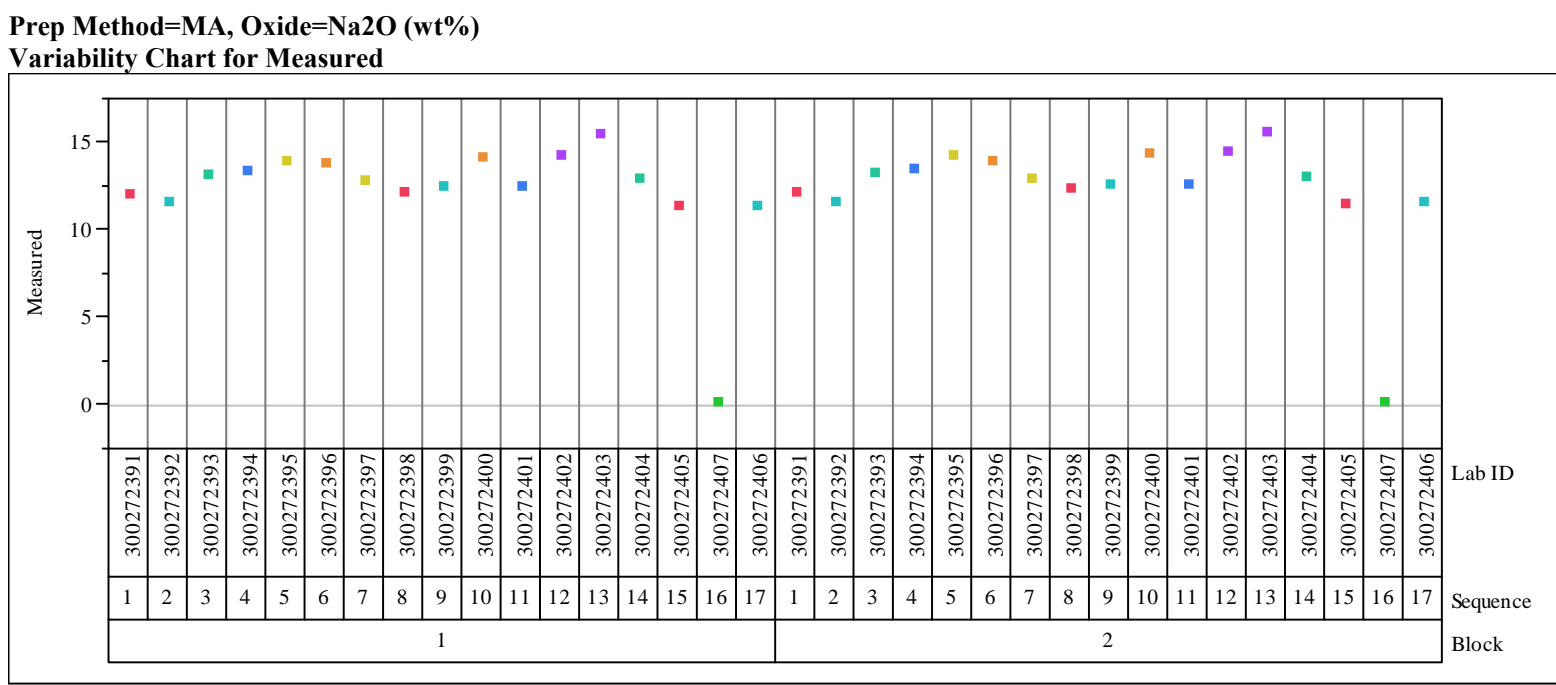

Prep Method=MA, Oxide=NiO $($ wt $\%)$

Variability Chart for Measured

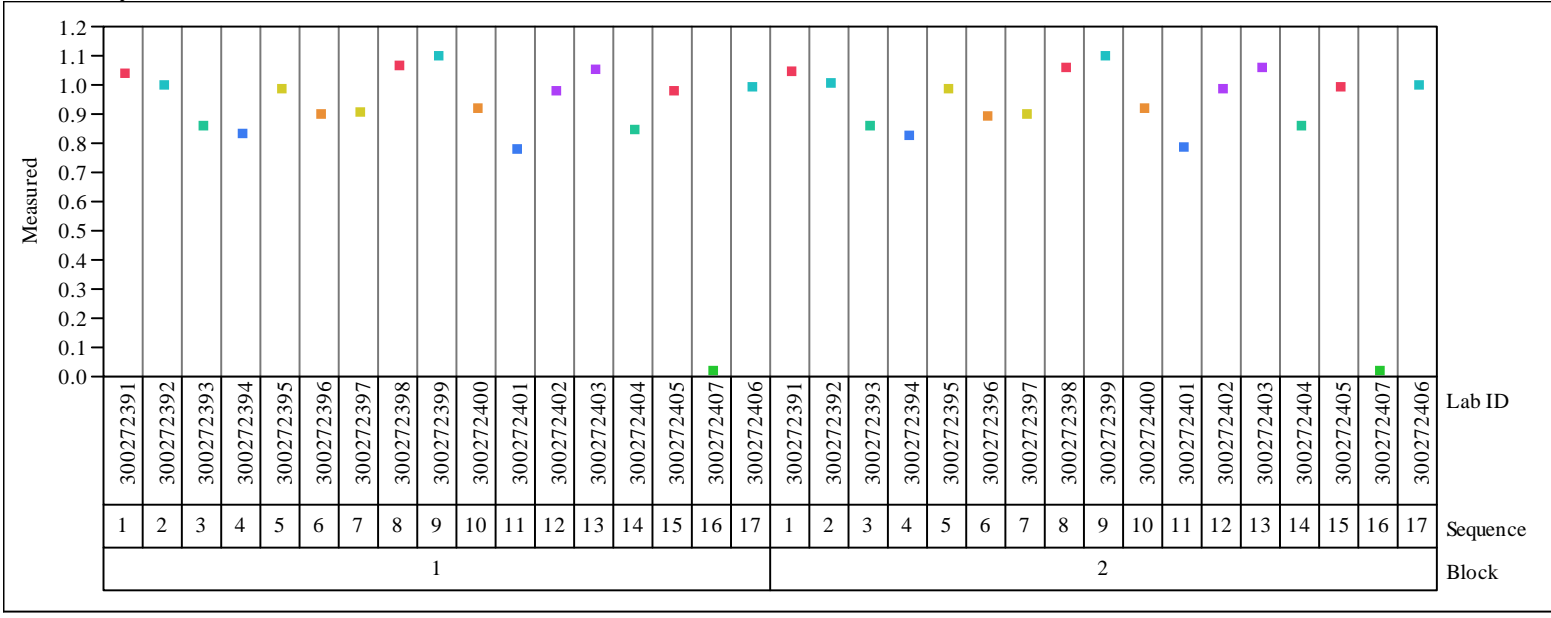




\section{Exhibit C1. Measurements of Thorium Glasses in Analytical Sequence for Samples by Prep Method by Oxide}

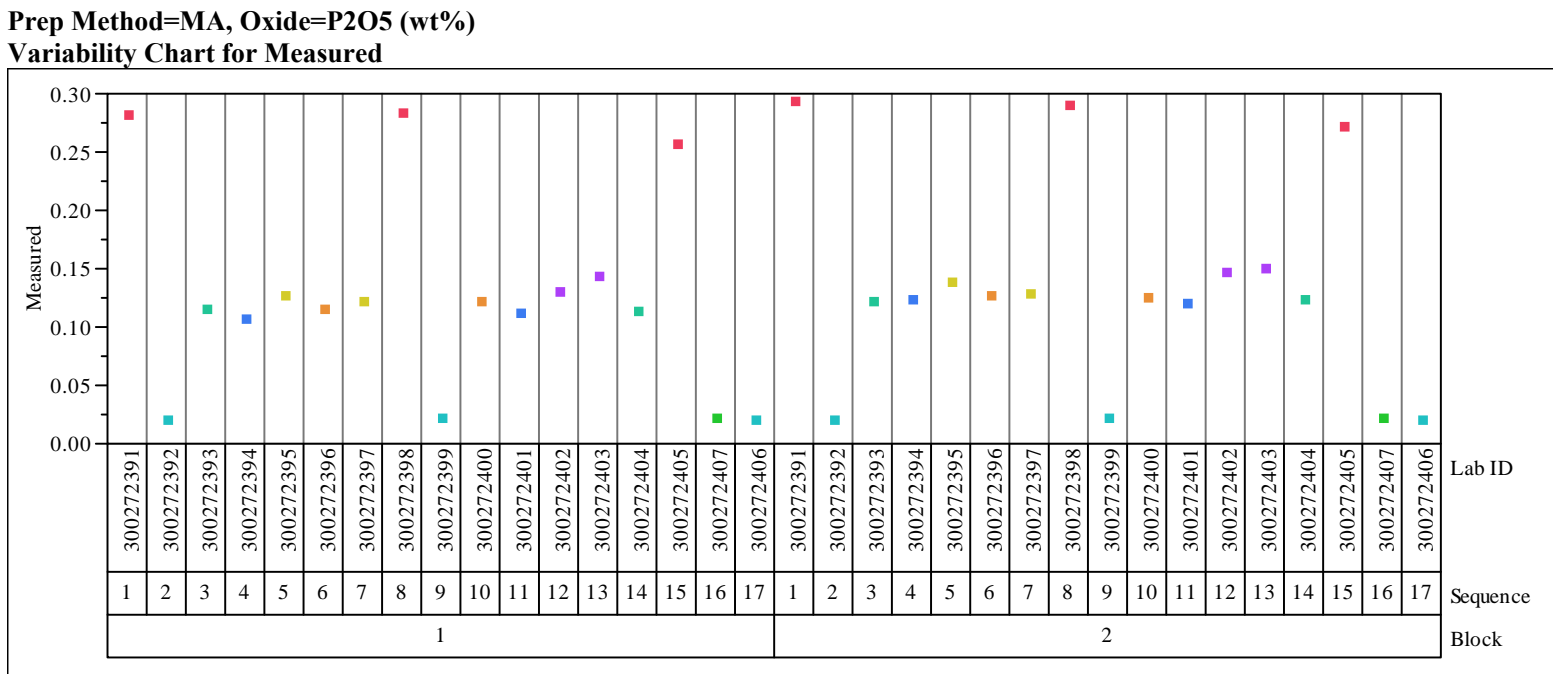

Prep Method $=$ MA, Oxide $=$ PbO $($ wt $\%)$

Variability Chart for Measured

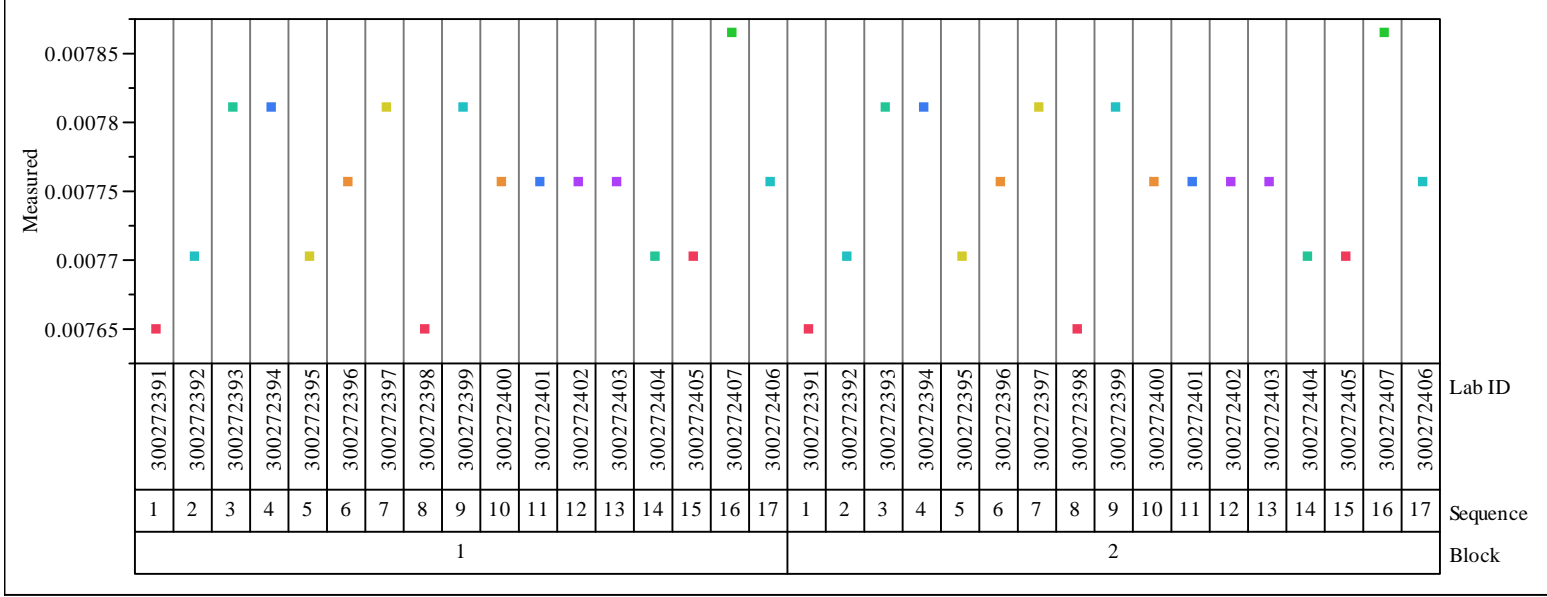

Prep Method=MA, Oxide $=\mathrm{SiO} 2(\mathrm{wt} \%)$

Variability Chart for Measured

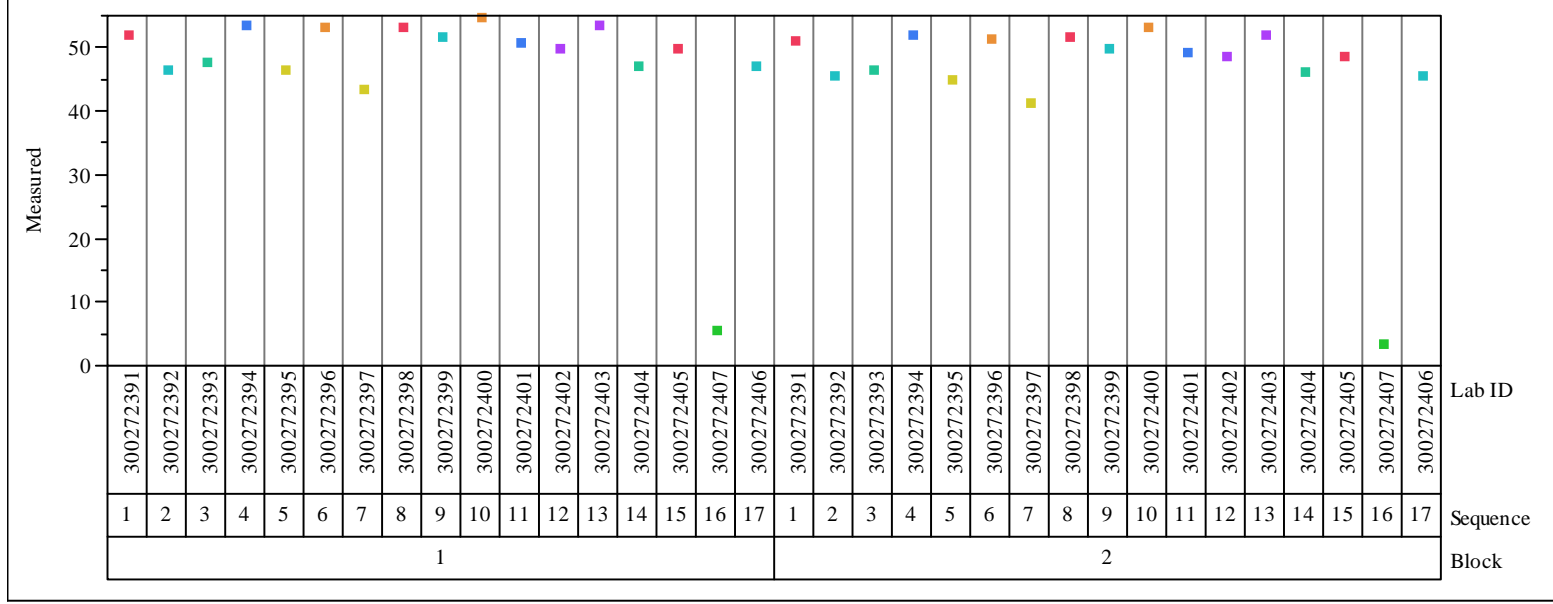




\section{Exhibit C1. Measurements of Thorium Glasses in Analytical Sequence for Samples by Prep Method by Oxide}

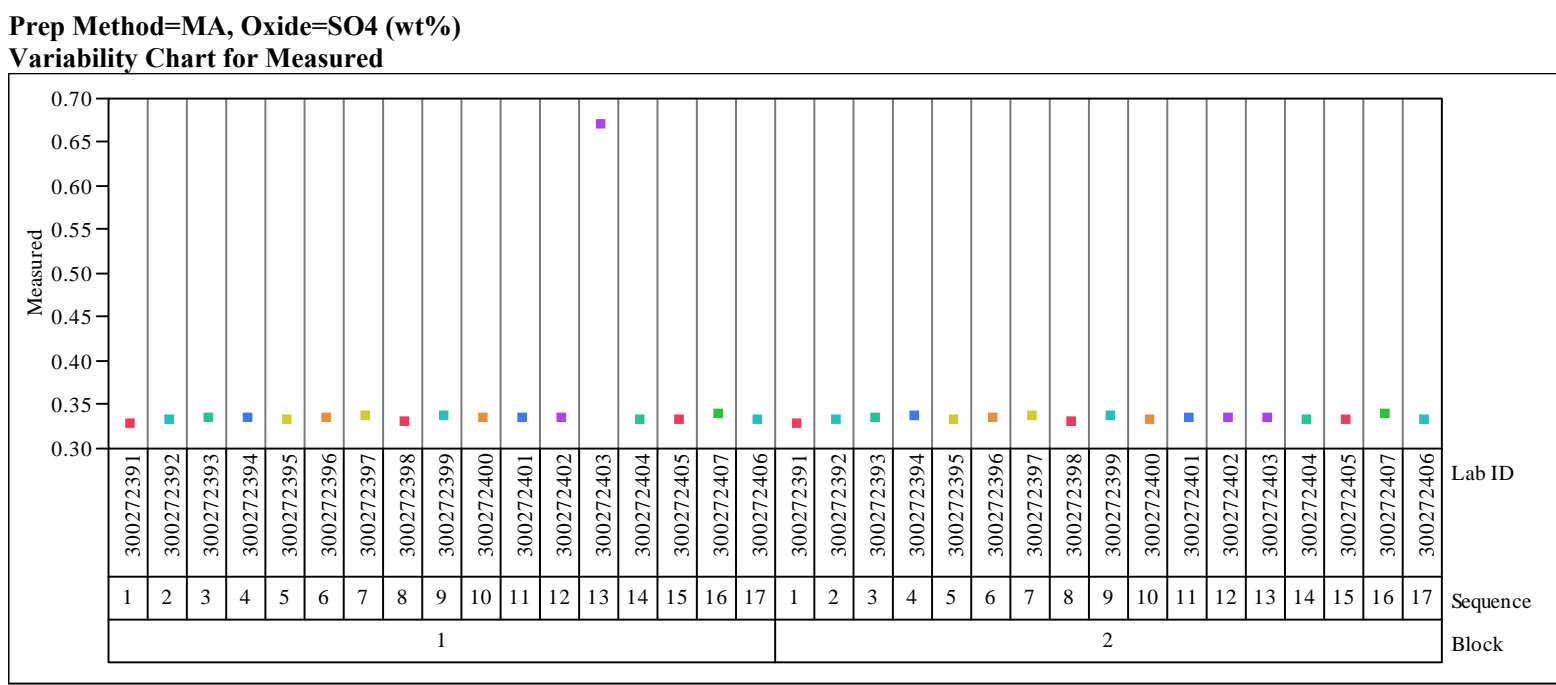

Prep Method=MA, Oxide $=\mathrm{ThO} 2(\mathrm{wt} \%)$

Variability Chart for Measured

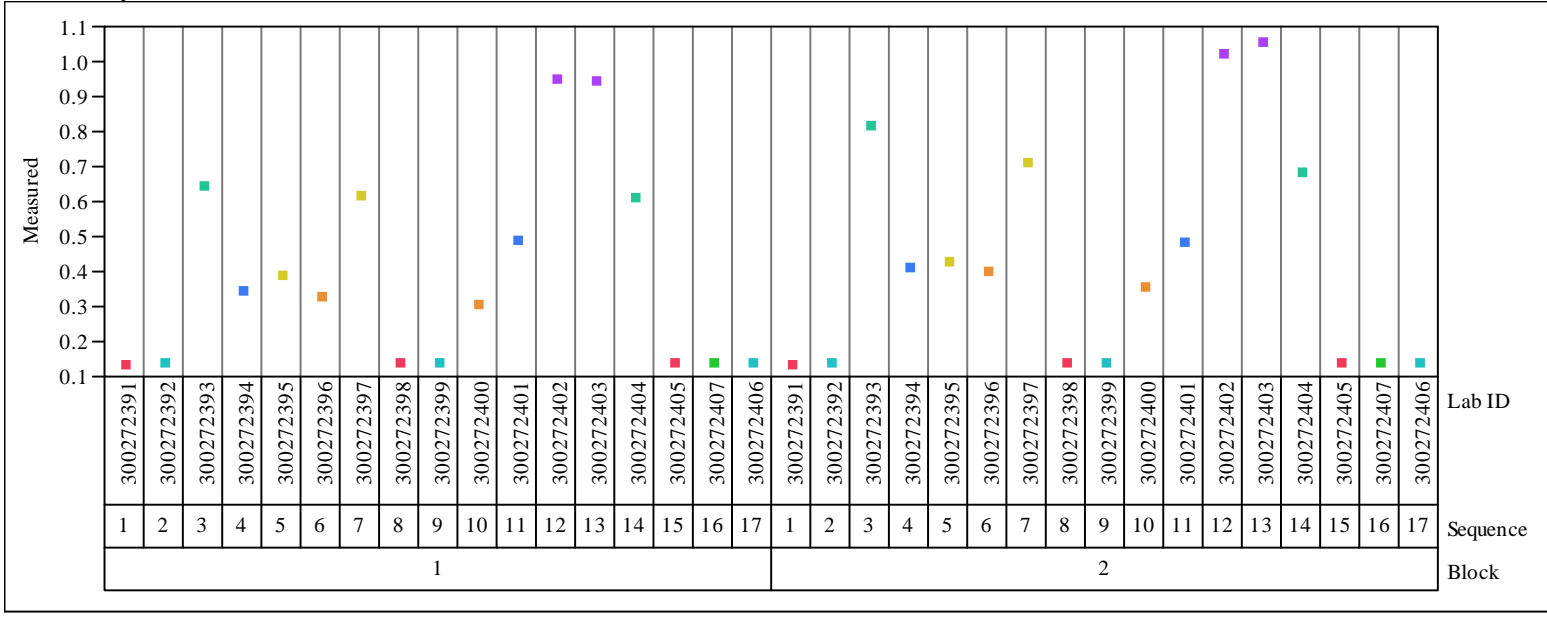




\section{Exhibit C1. Measurements of Thorium Glasses in Analytical Sequence for Samples by Prep Method by Oxide}
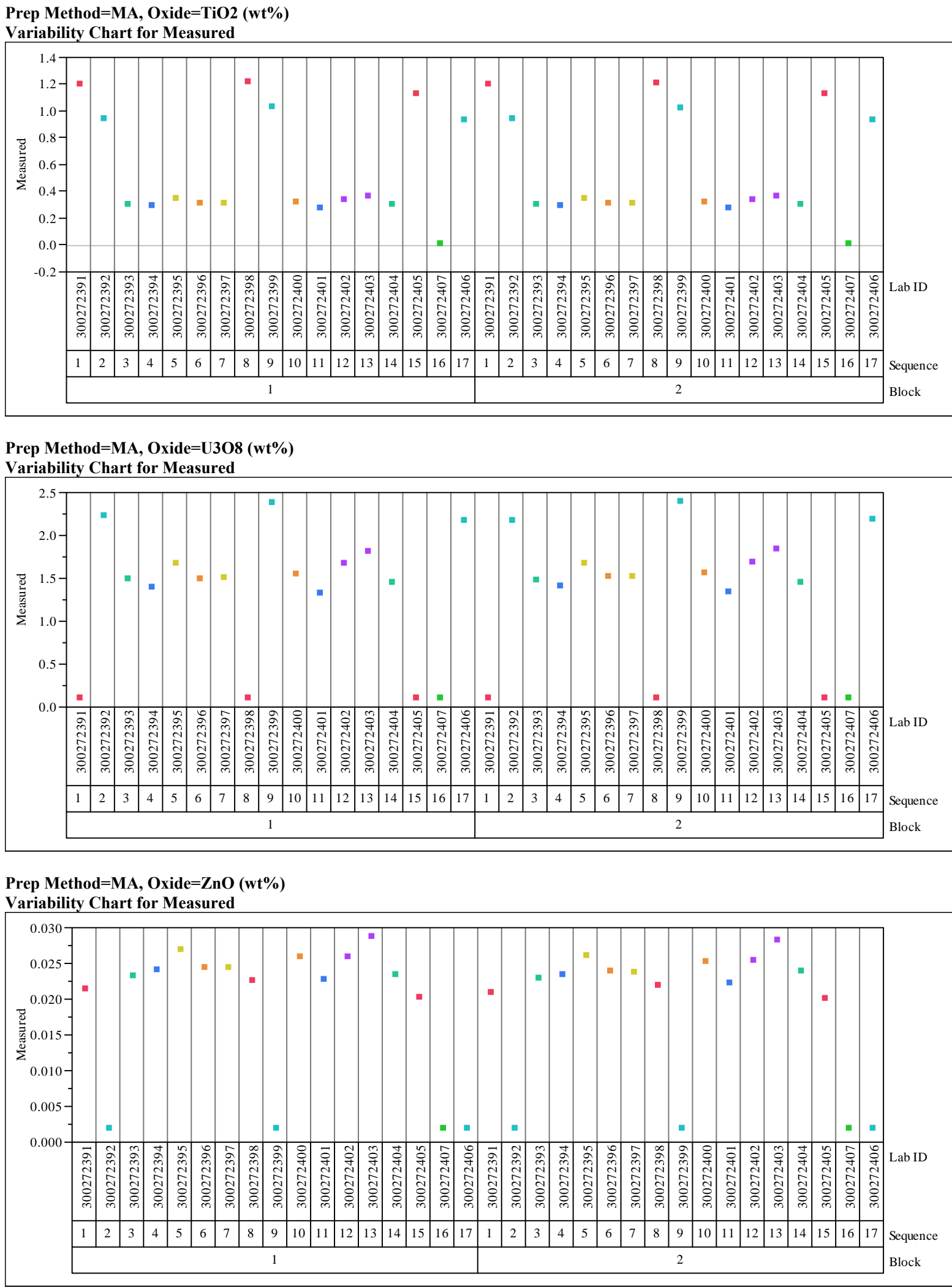


\section{Exhibit C1. Measurements of Thorium Glasses in Analytical Sequence for Samples by Prep Method by Oxide}

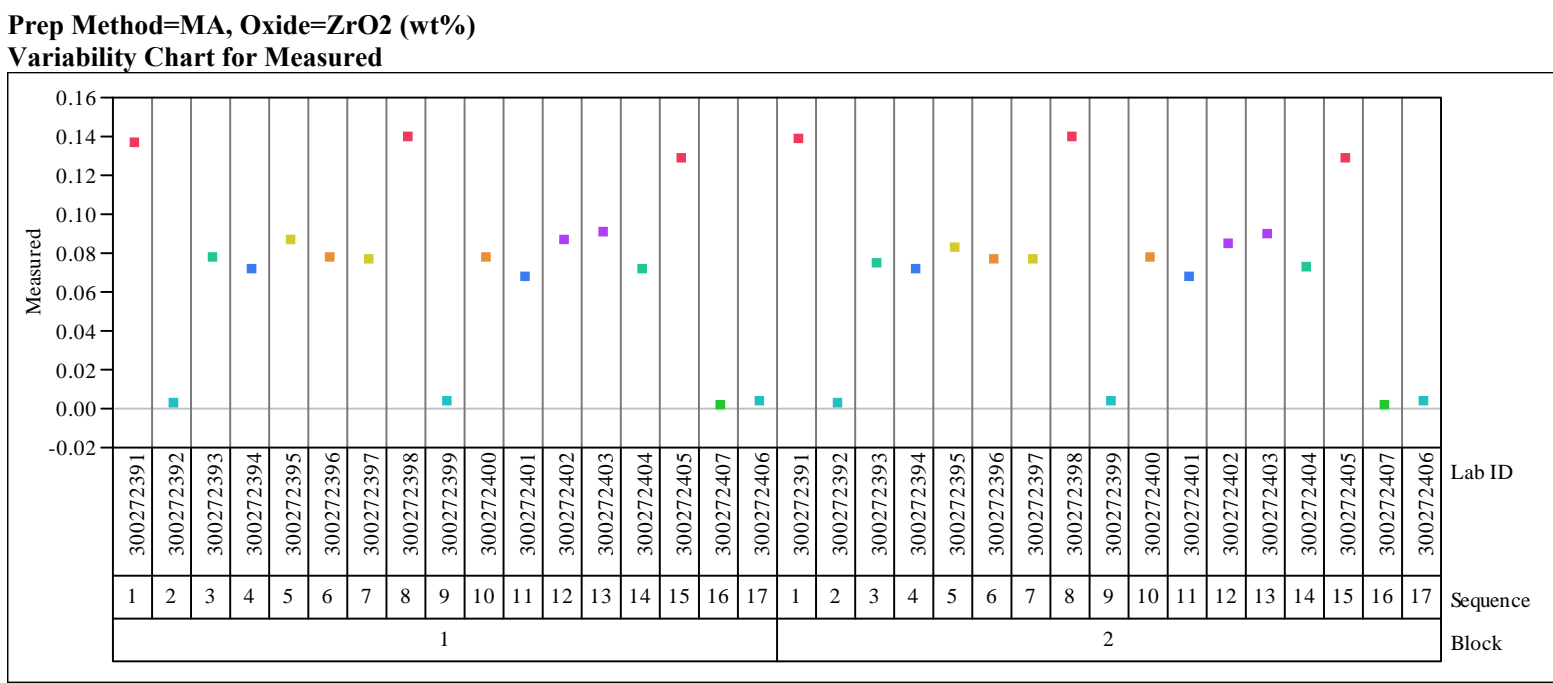

Prep Method=PF, Oxide=Al2O3 (wt\%)

Variability Chart for Measured

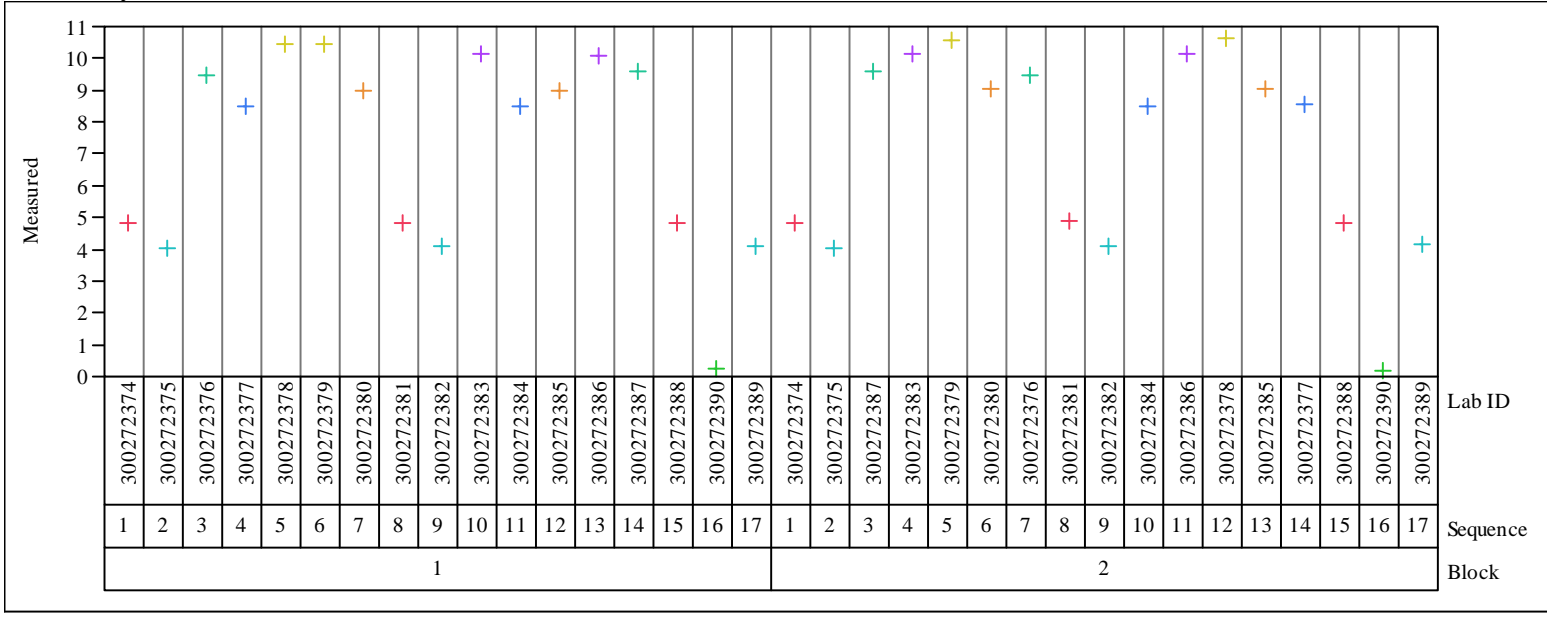




\section{Exhibit C1. Measurements of Thorium Glasses in Analytical Sequence for Samples by Prep Method by Oxide}
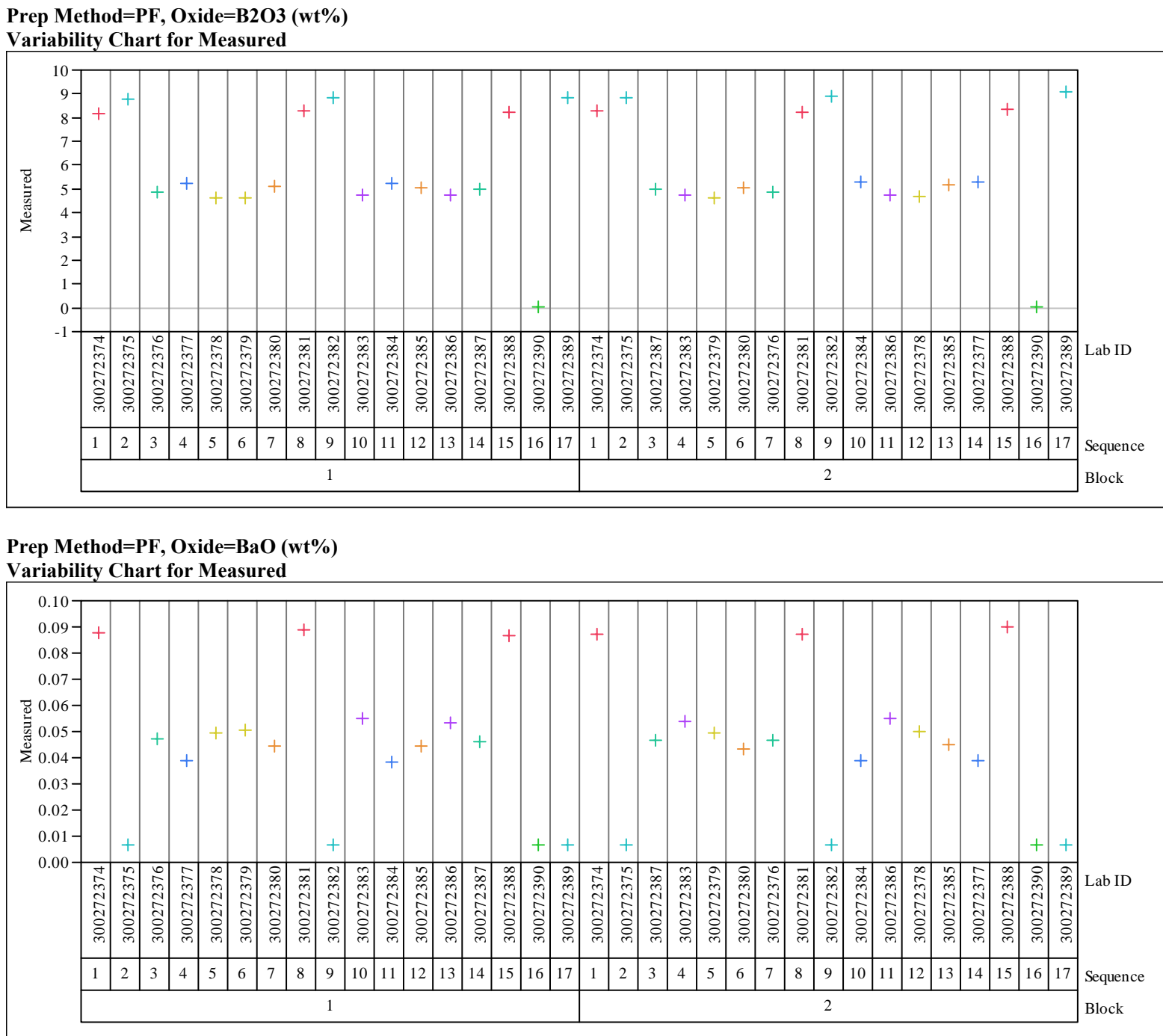

Prep Method=PF, Oxide $=\mathrm{CaO}(\mathbf{w t} \%)$

Variability Chart for Measured

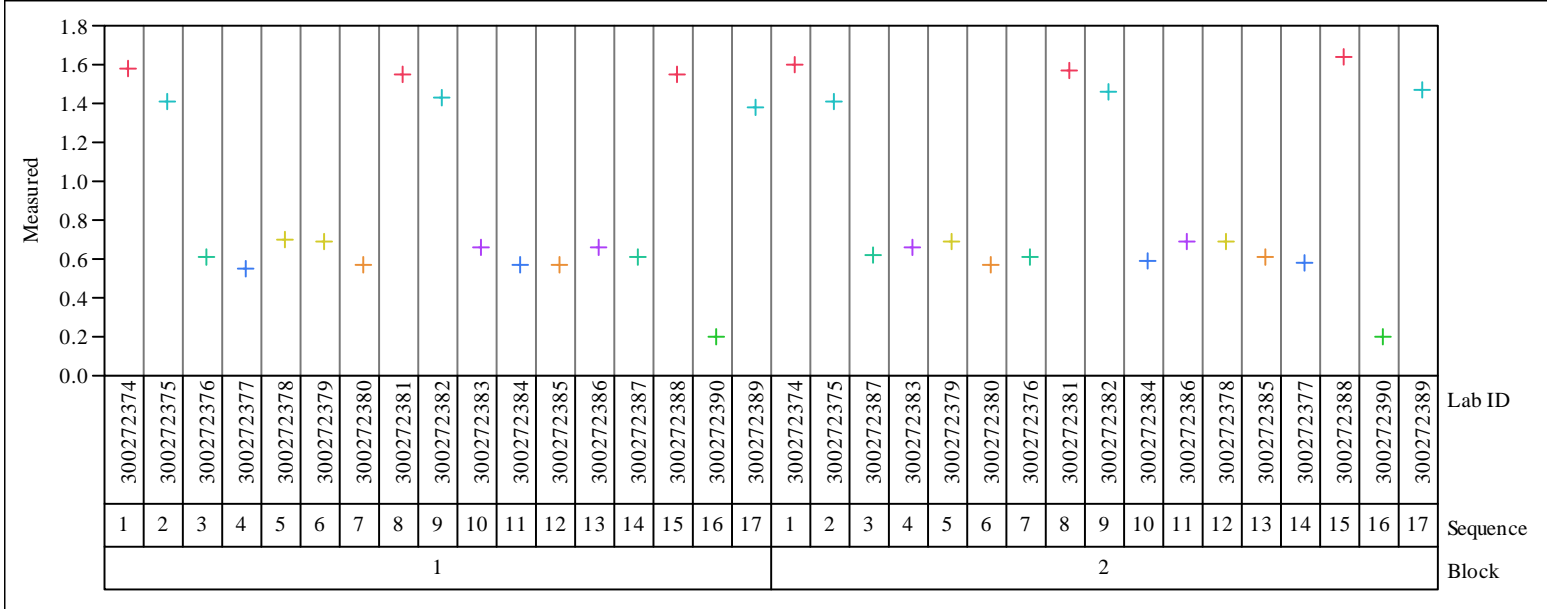




\section{Exhibit C1. Measurements of Thorium Glasses in Analytical Sequence for Samples by Prep Method by Oxide}

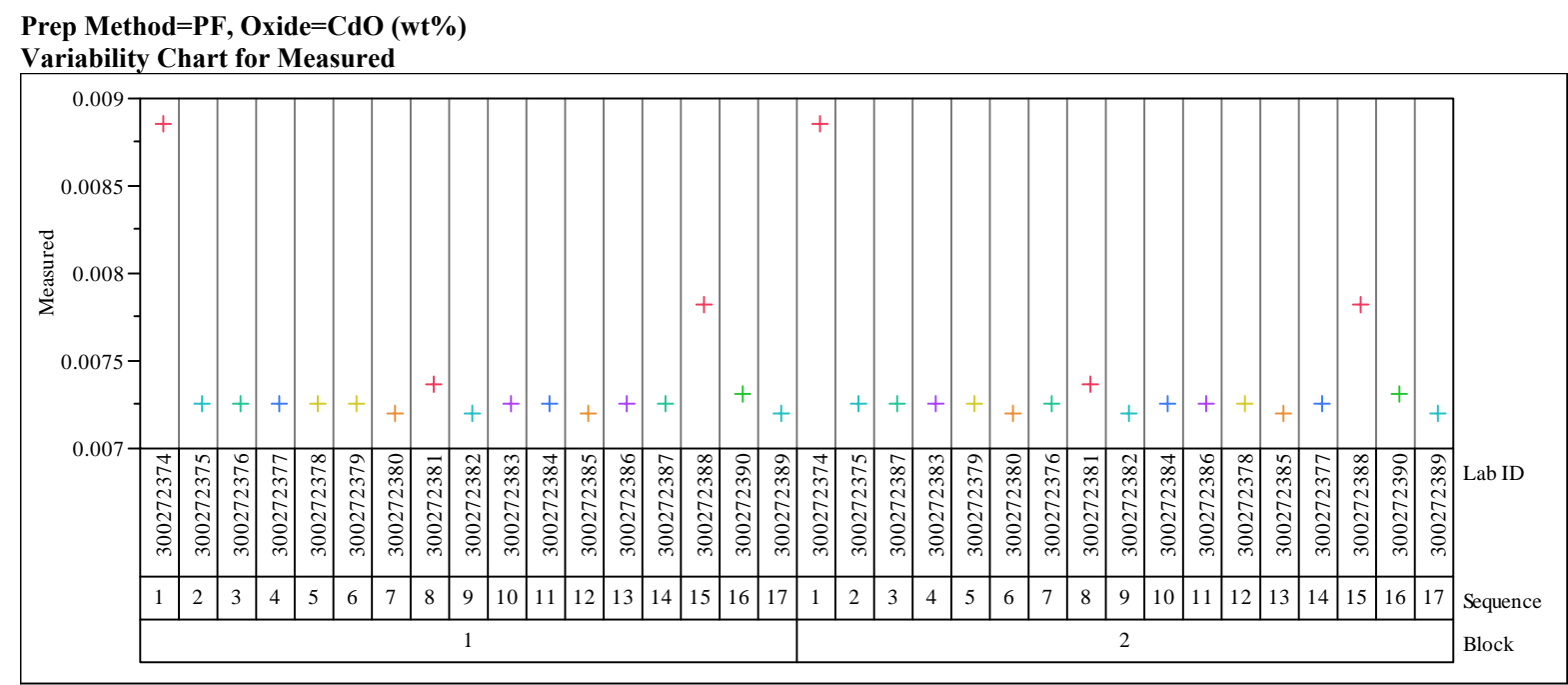

Prep Method=PF, Oxide $=\mathrm{Ce} 2 \mathrm{O3}(\mathrm{wt} \%)$

Variability Chart for Measured

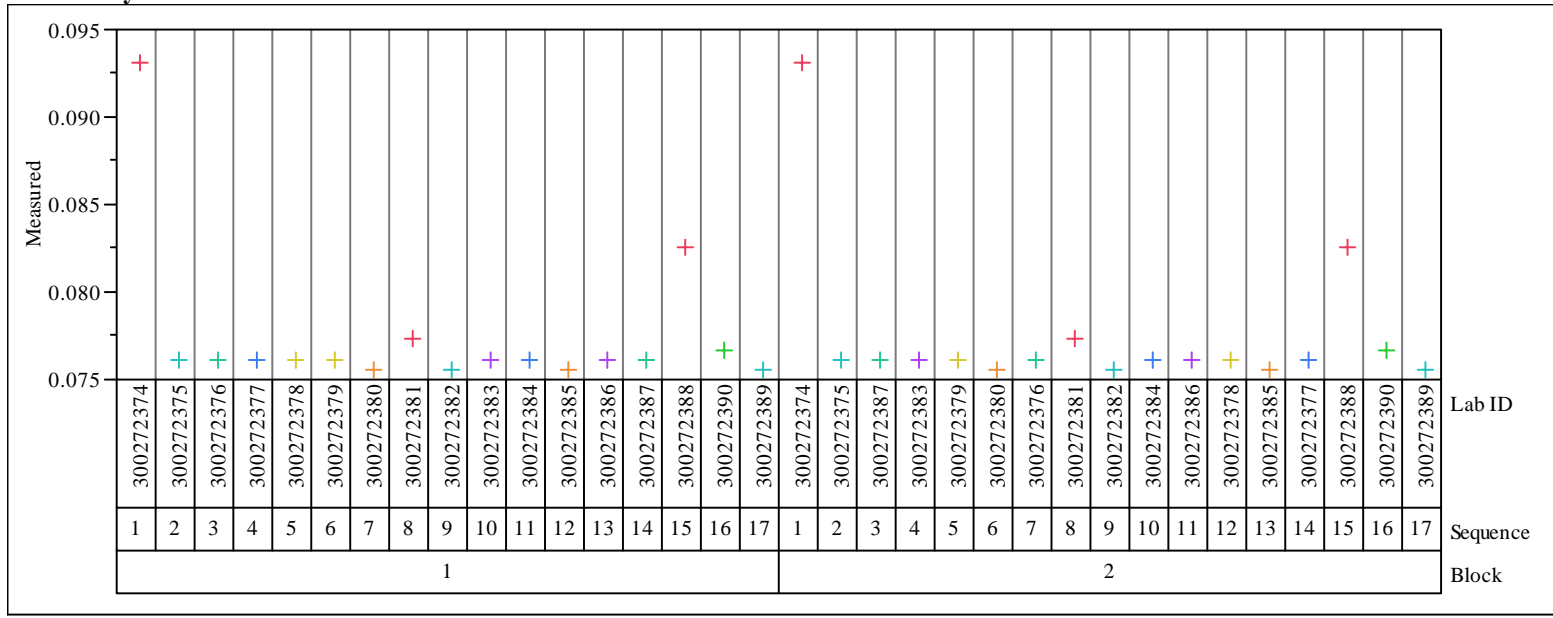




\section{Exhibit C1. Measurements of Thorium Glasses in Analytical Sequence for Samples by Prep Method by Oxide}
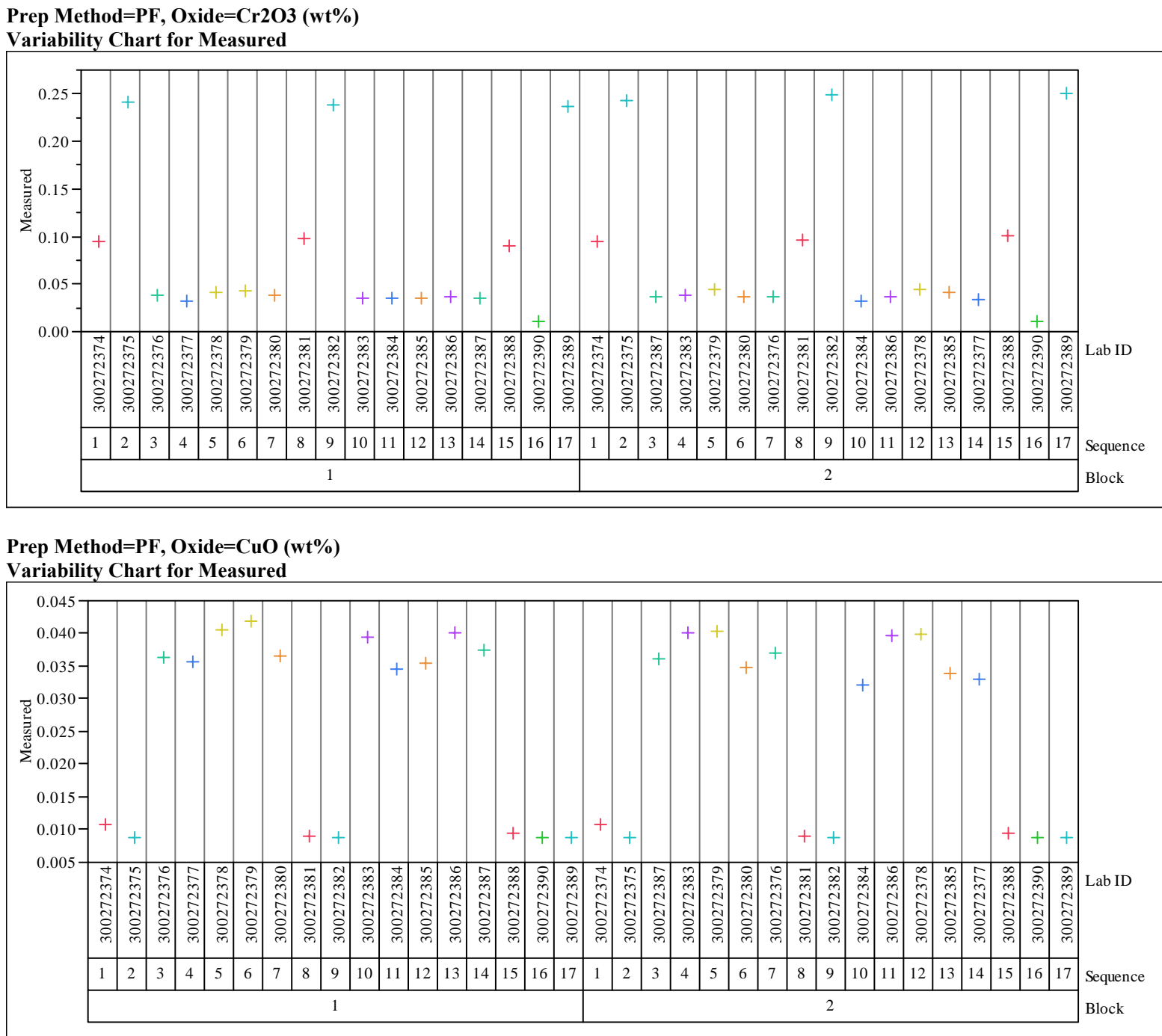

Prep Method=PF, Oxide=Fe2O3 (wt\%)

Variability Chart for Measured

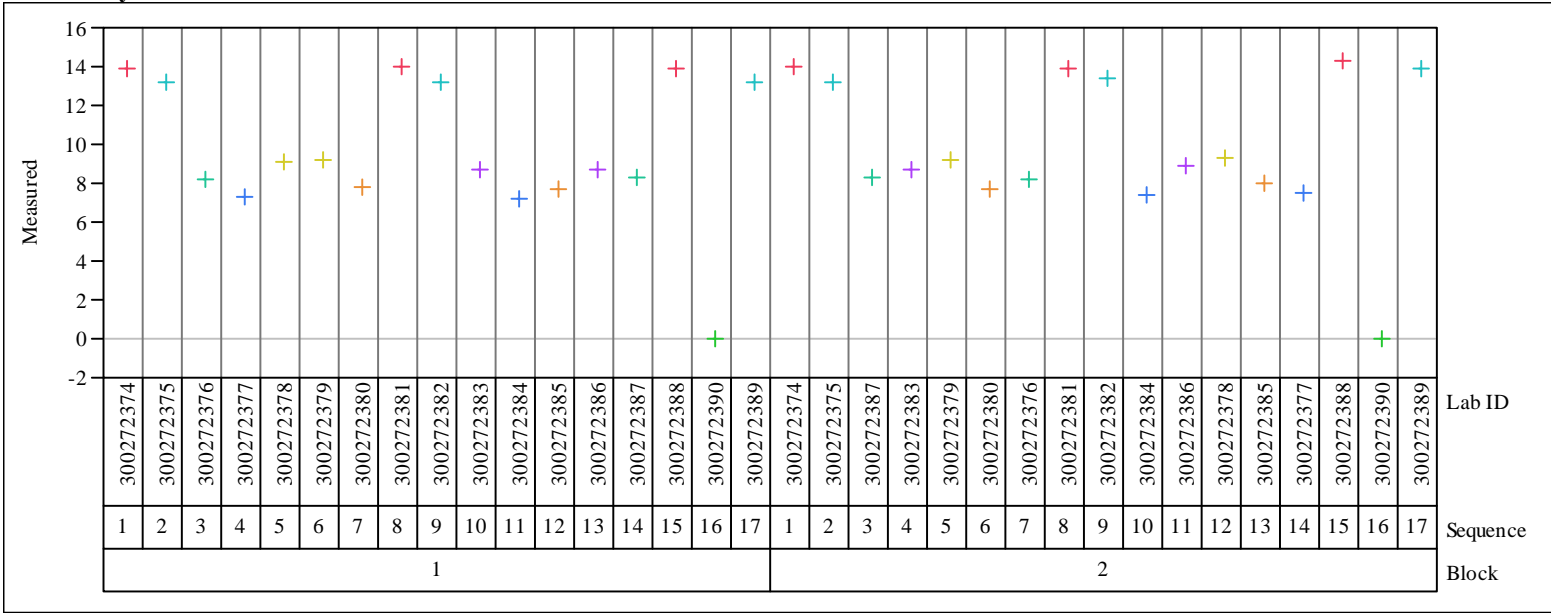




\section{Exhibit C1. Measurements of Thorium Glasses in Analytical Sequence for Samples by Prep Method by Oxide}

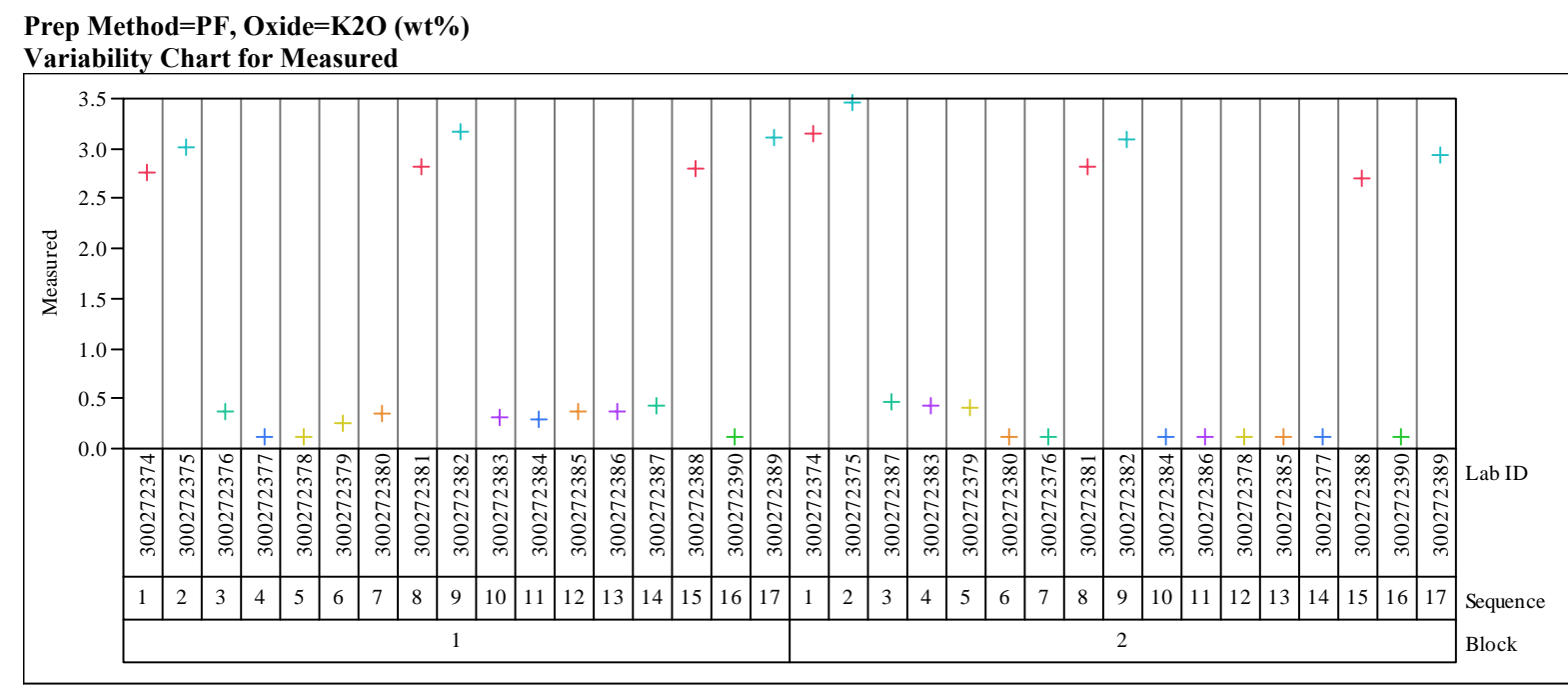

Prep Method=PF, Oxide=La2O3 $(\mathrm{wt} \%)$

Variability Chart for Measured

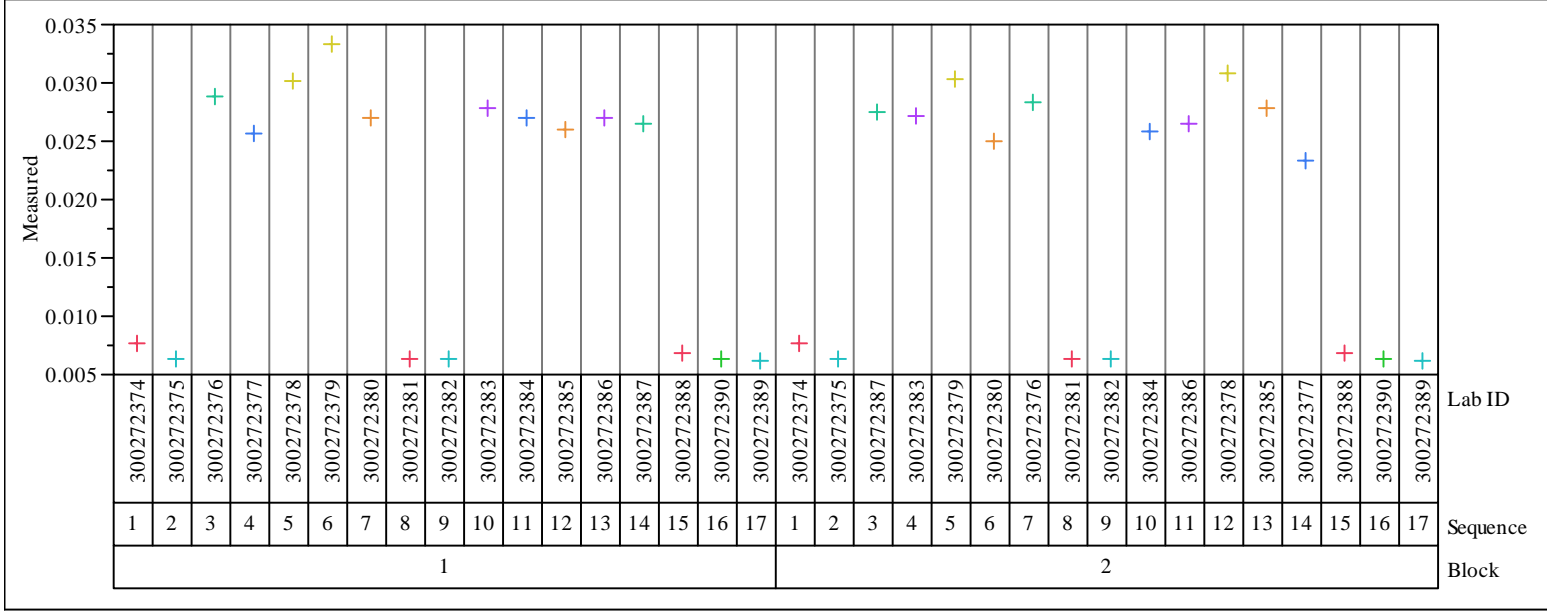




\section{Exhibit C1. Measurements of Thorium Glasses in Analytical Sequence for Samples by Prep Method by Oxide}
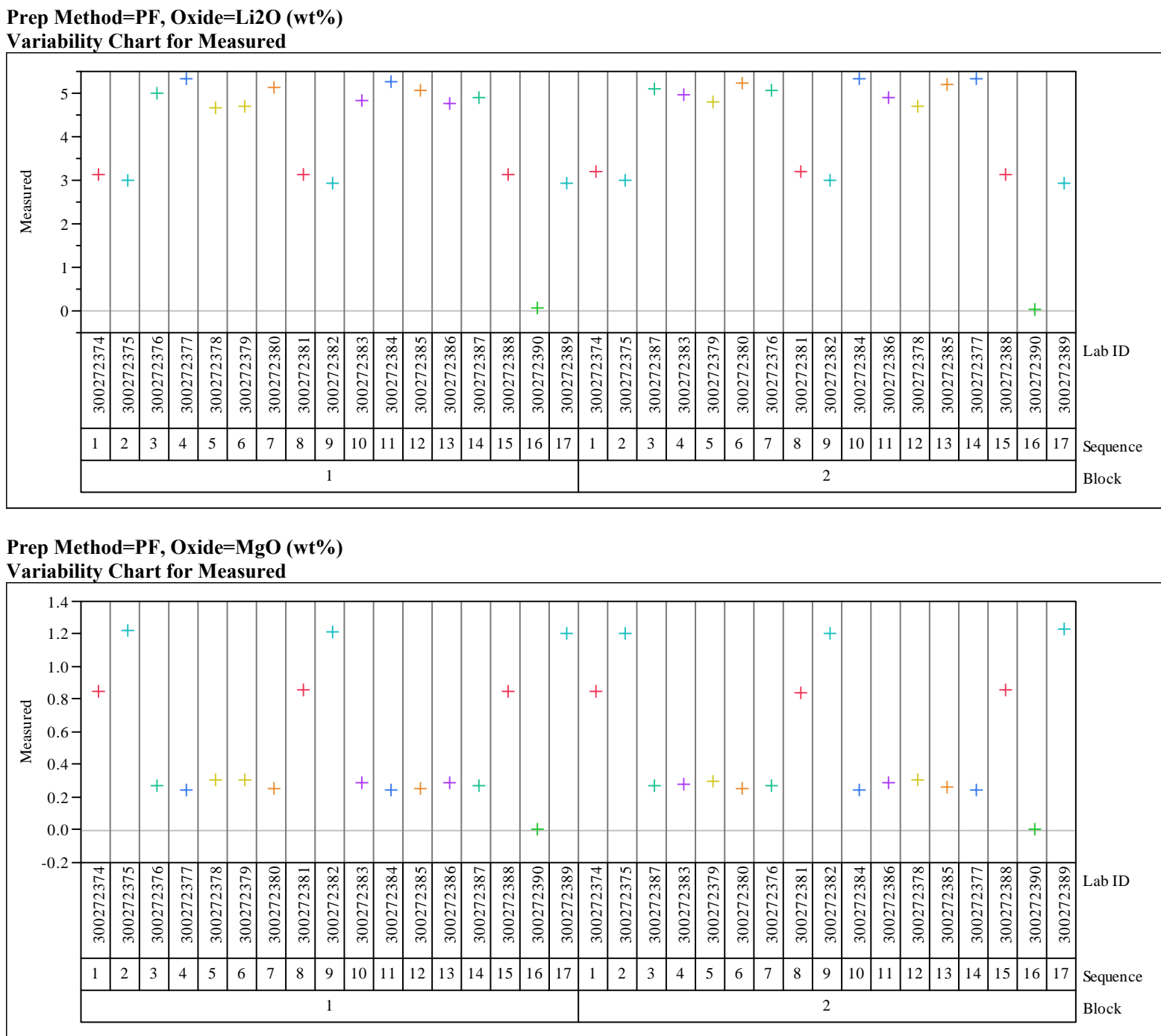

Prep Method $=$ PF, Oxide $=$ MnO $(w t \%)$

Variability Chart for Measured

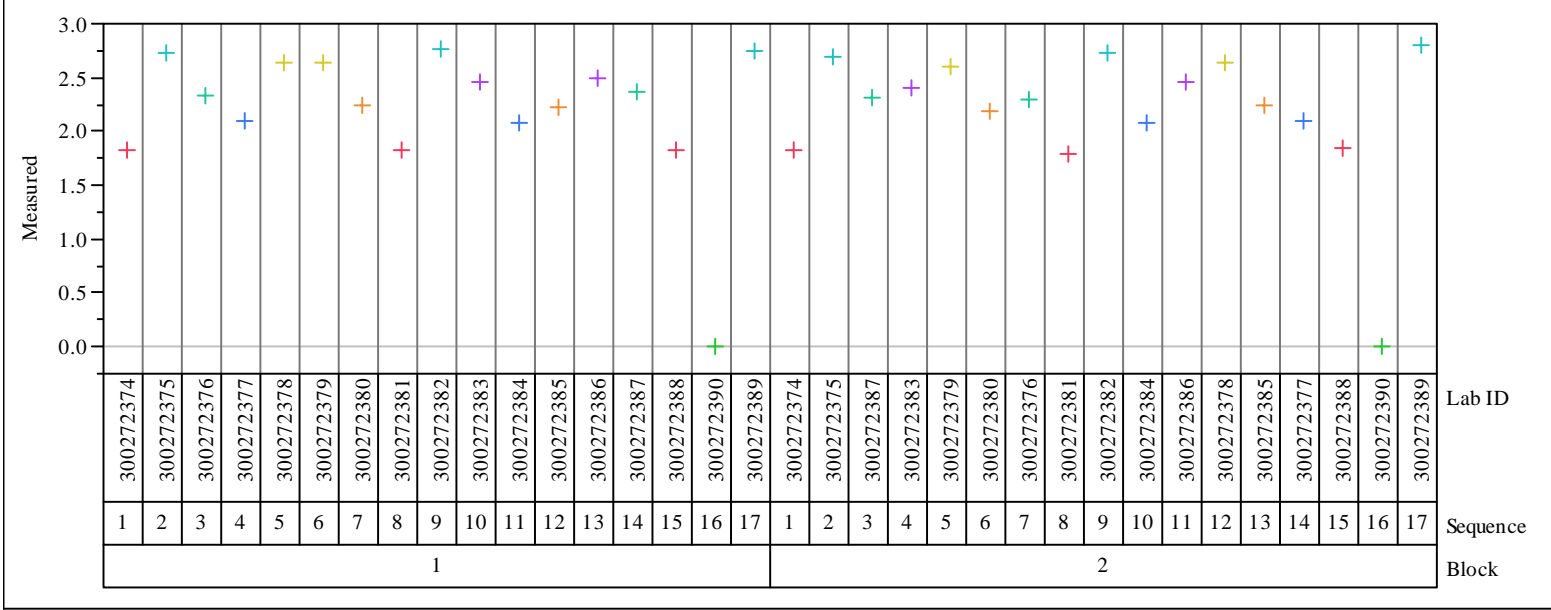




\section{Exhibit C1. Measurements of Thorium Glasses in Analytical Sequence for Samples by Prep Method by Oxide}

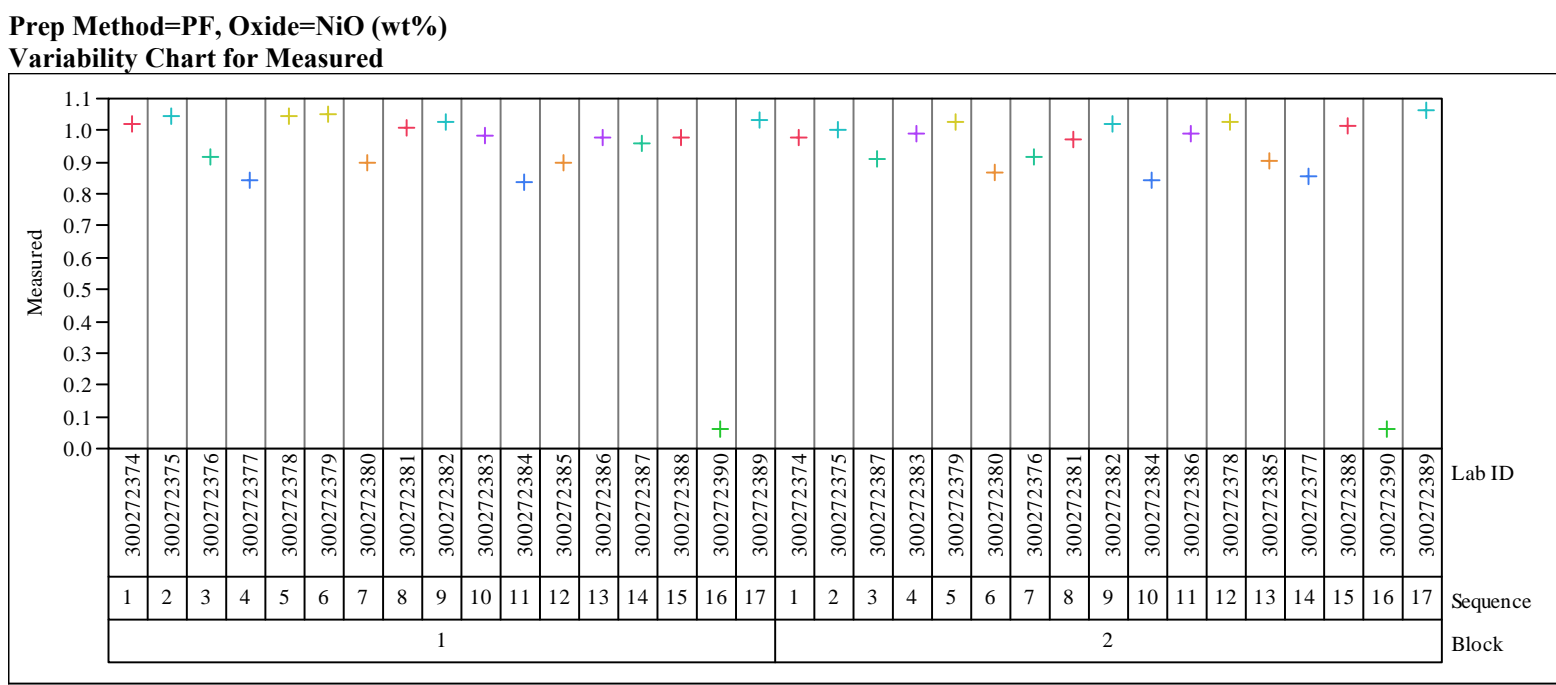

Prep Method=PF, Oxide=P2O5 (wt\%)

Variability Chart for Measured

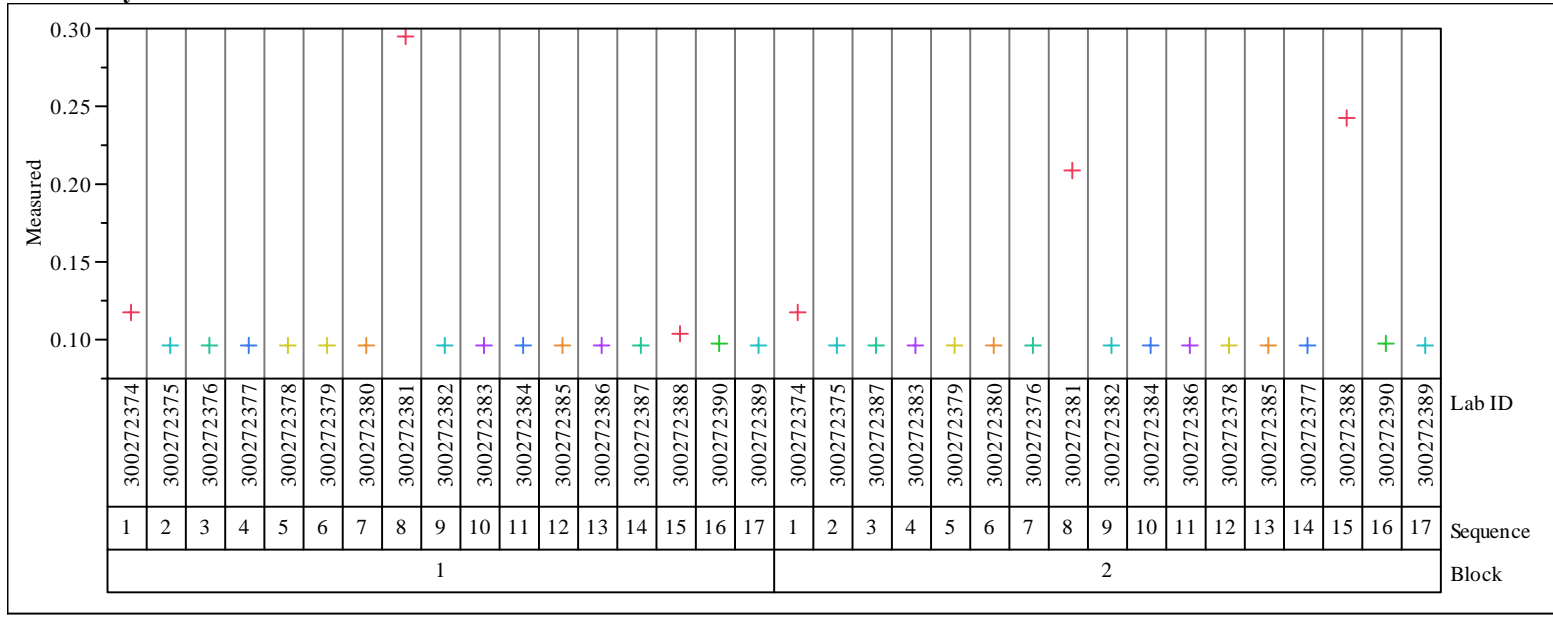




\section{Exhibit C1. Measurements of Thorium Glasses in Analytical Sequence for Samples by Prep Method by Oxide}

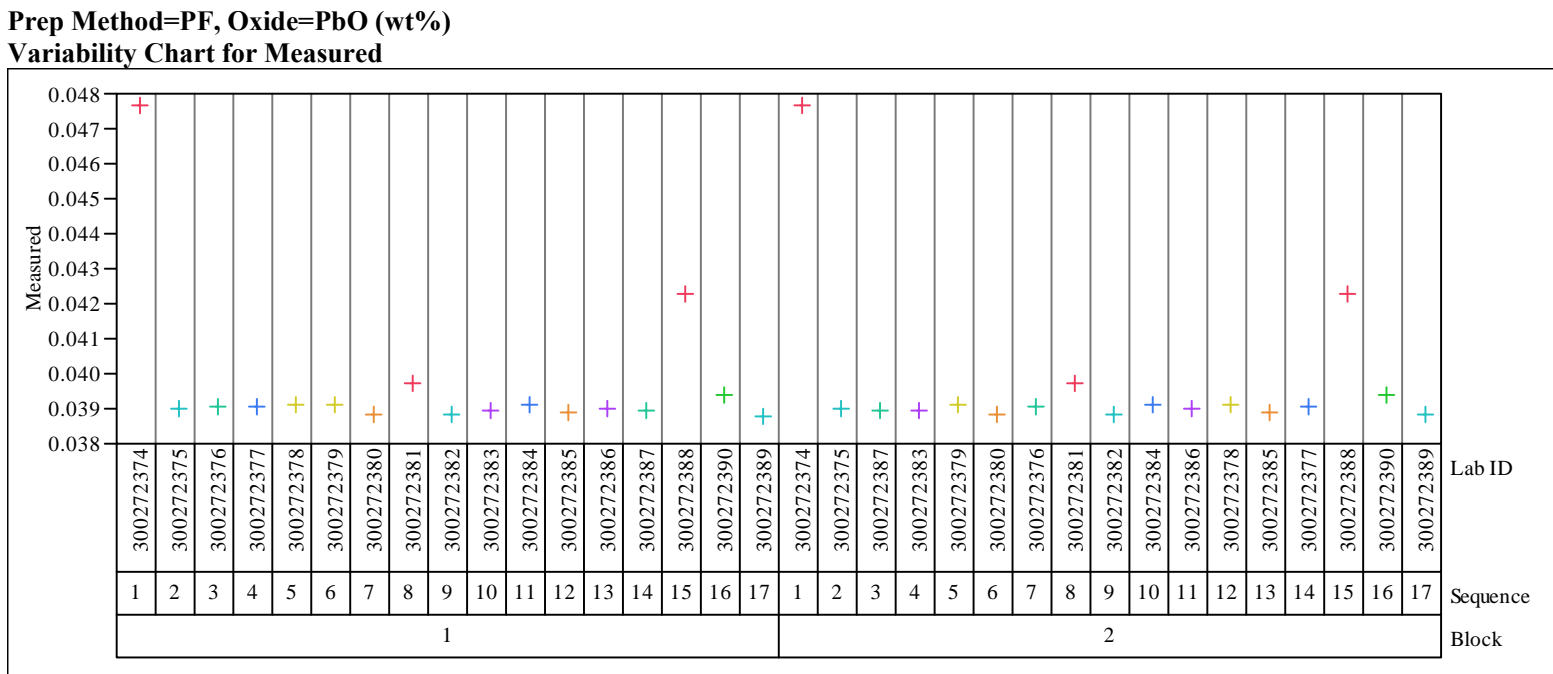

Prep Method=PF, Oxide=SiO2 $(w t \%)$

Variability Chart for Measured

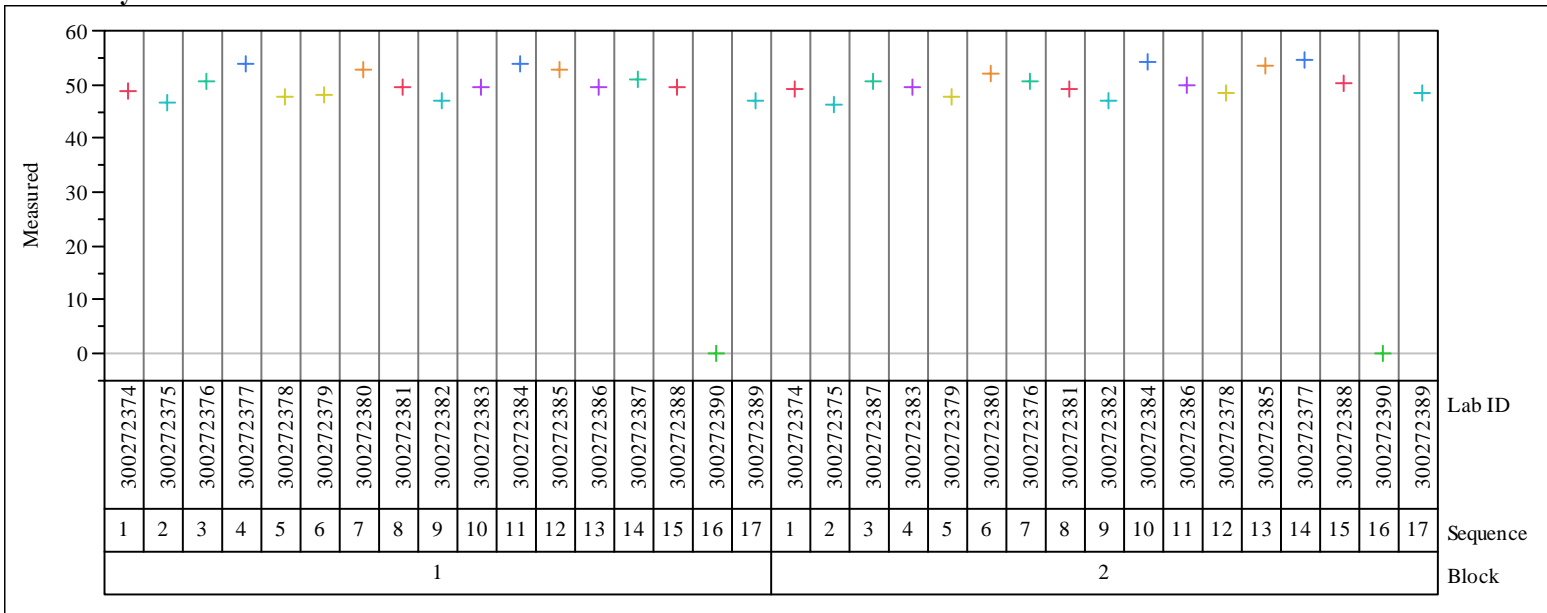

Prep Method=PF, Oxide $=$ SO4 $(w t \%)$

Variability Chart for Measured

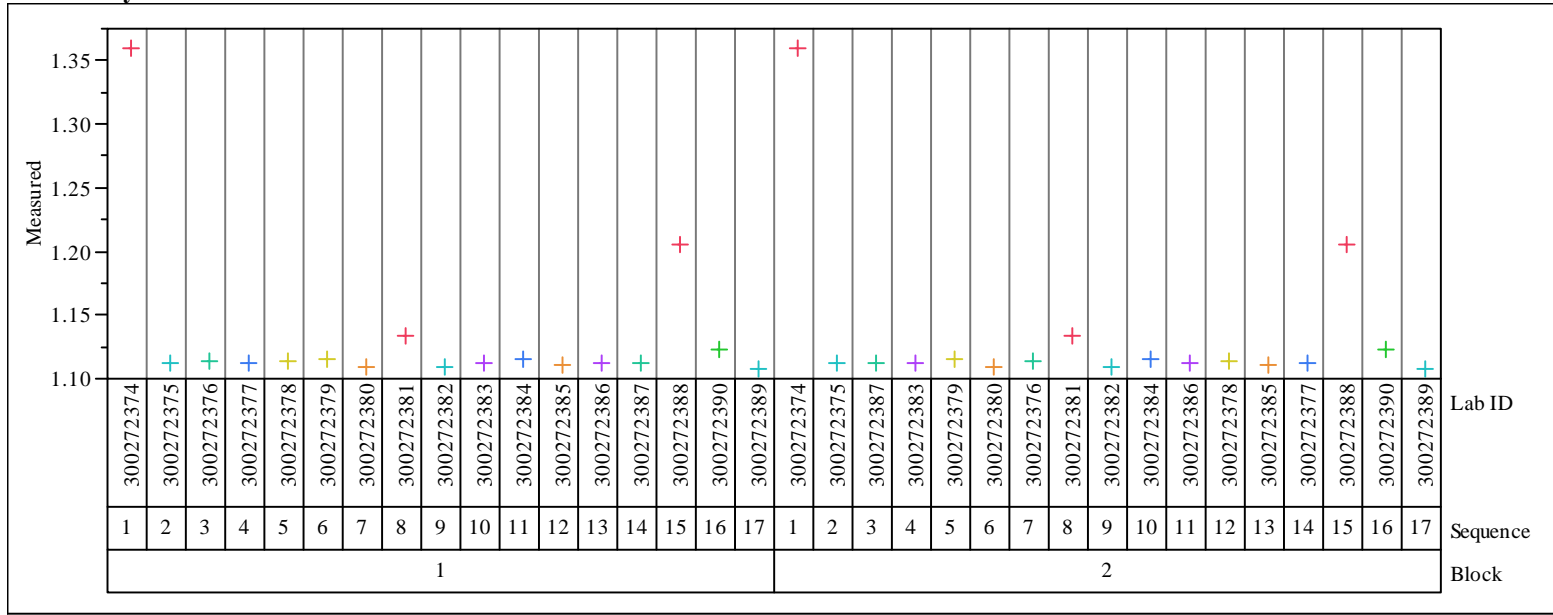




\section{Exhibit C1. Measurements of Thorium Glasses in Analytical Sequence for Samples by Prep Method by Oxide}

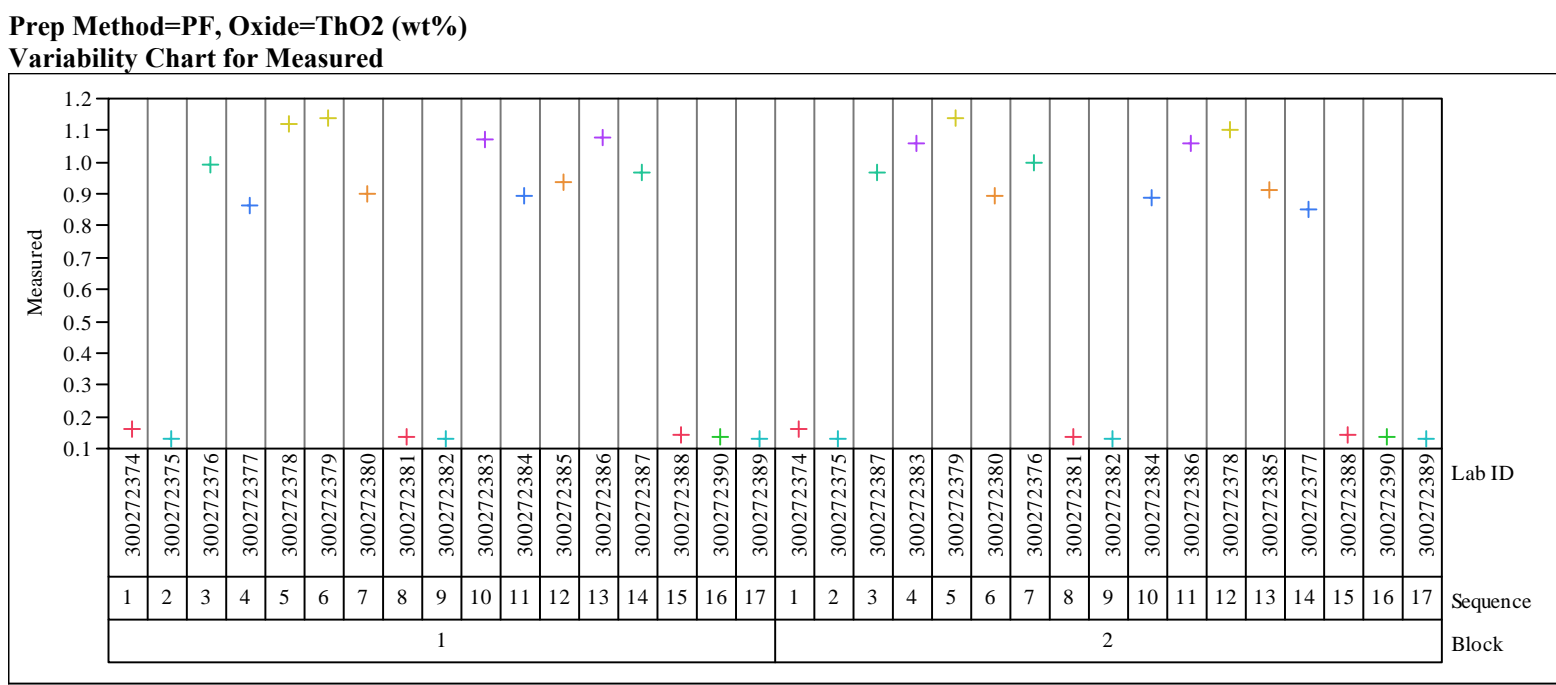

Prep Method=PF, Oxide $=\mathrm{TiO} 2($ wt $\%)$

Variability Chart for Measured

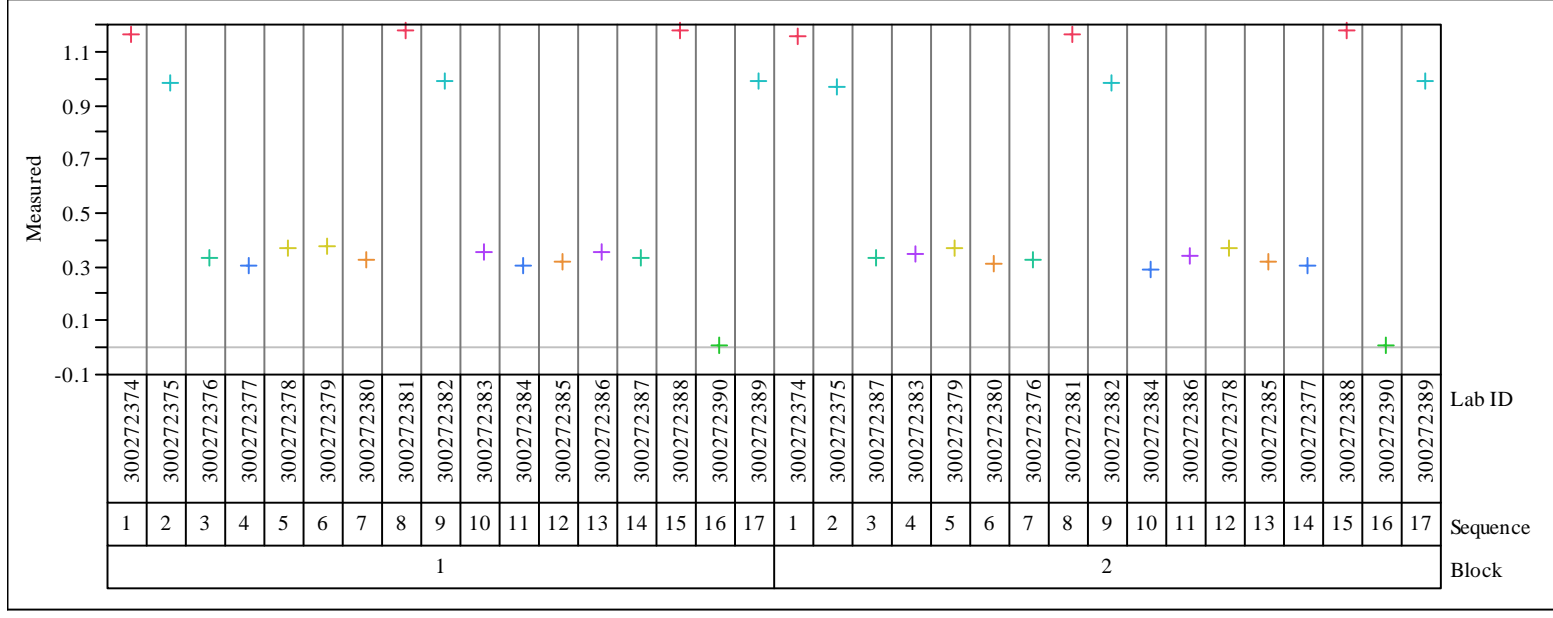




\section{Exhibit C1. Measurements of Thorium Glasses in Analytical Sequence for Samples by Prep Method by Oxide}

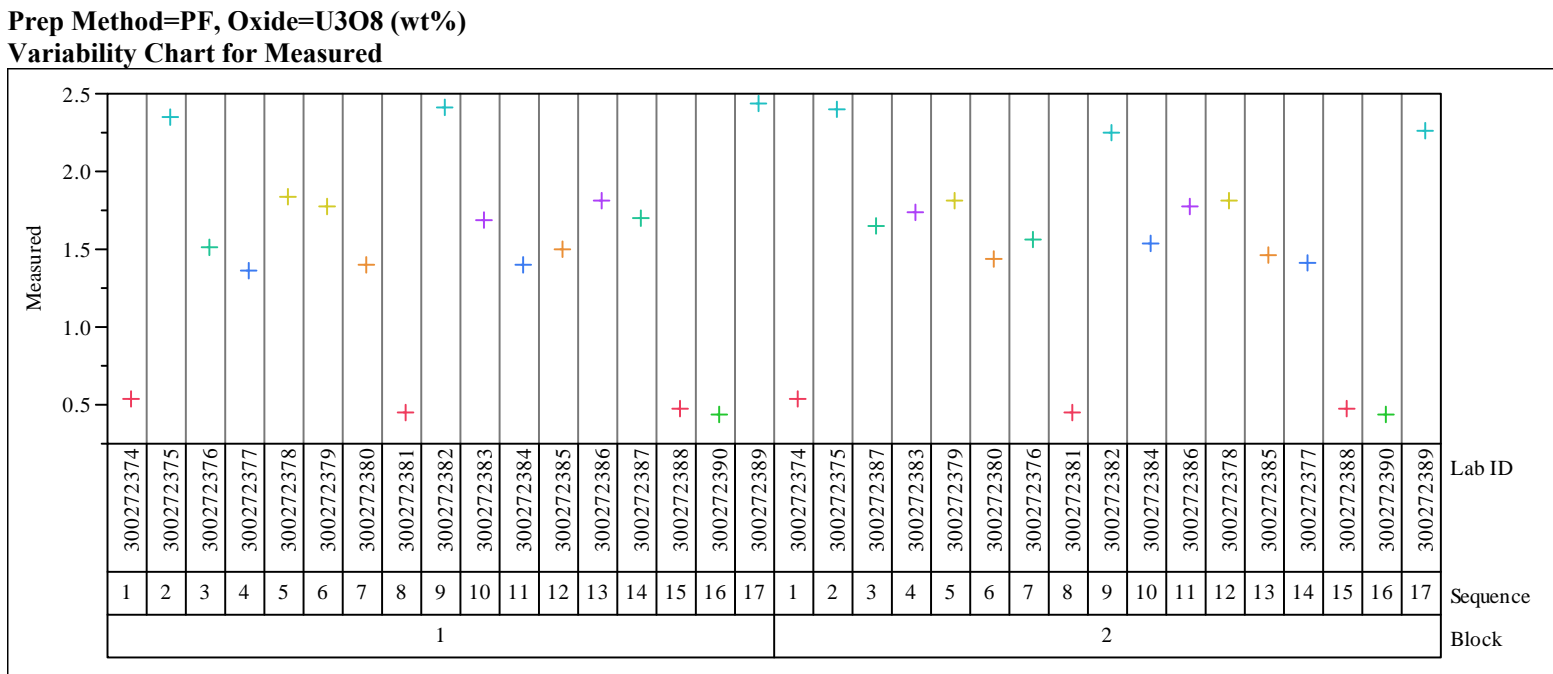

Prep Method=PF, Oxide $=\mathrm{ZnO}(\mathrm{wt} \%)$

Variability Chart for Measured

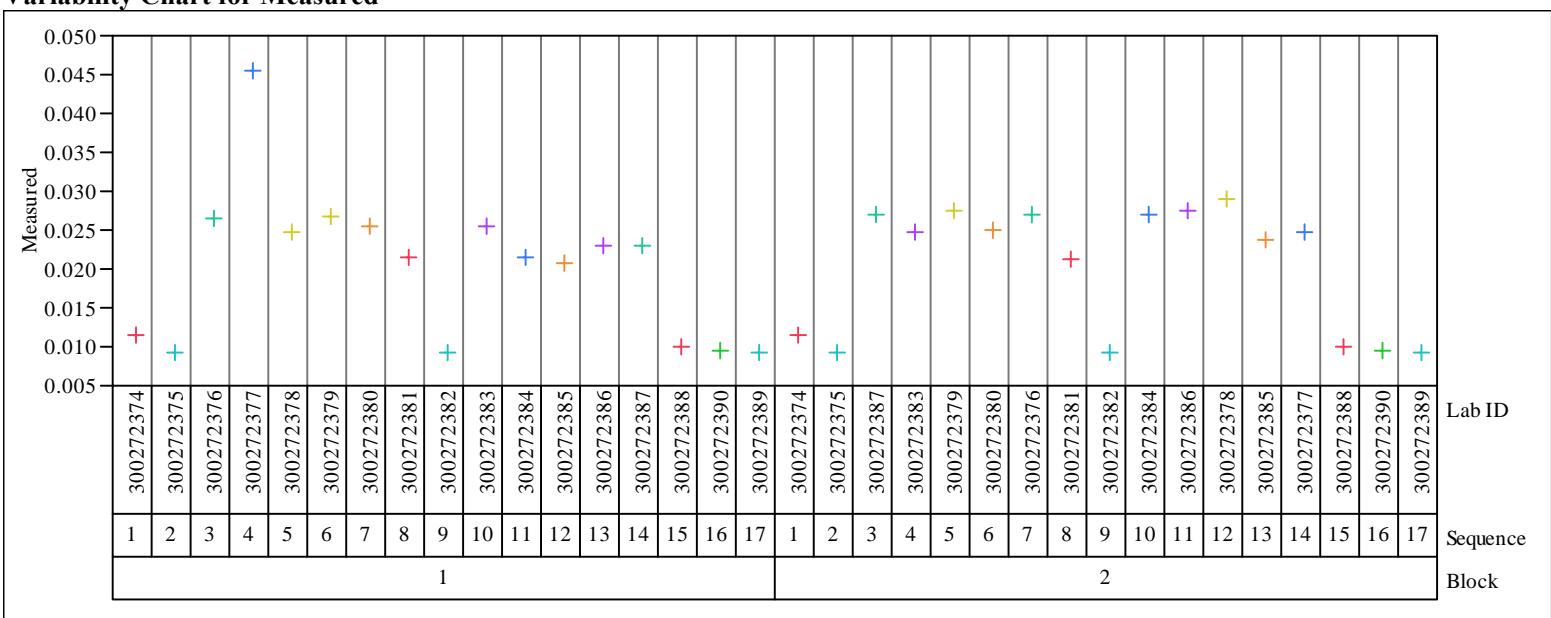


Exhibit C2. Measurements by Lab ID for Thorium Glasses by Targeted Concentration for Each Oxide

Oxide $=\mathrm{Al2O} 3(\mathbf{w t} \%)$

Variability Chart for Measured

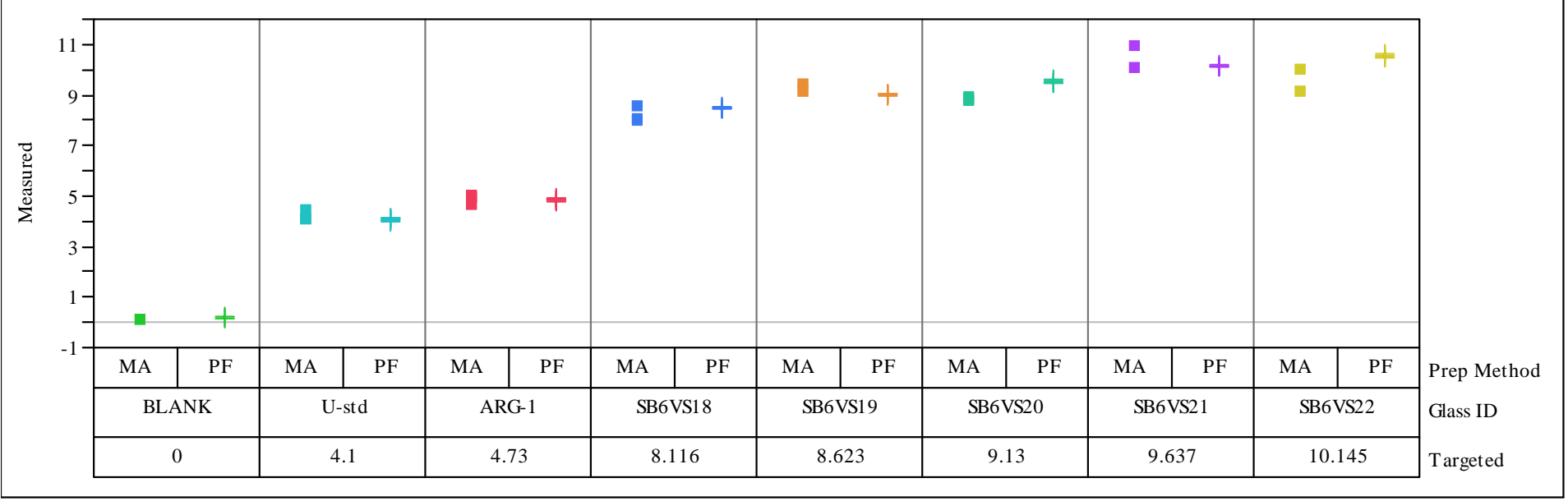

Oxide $=$ B2O3 (wt $\%$ )

Variability Chart for Measured

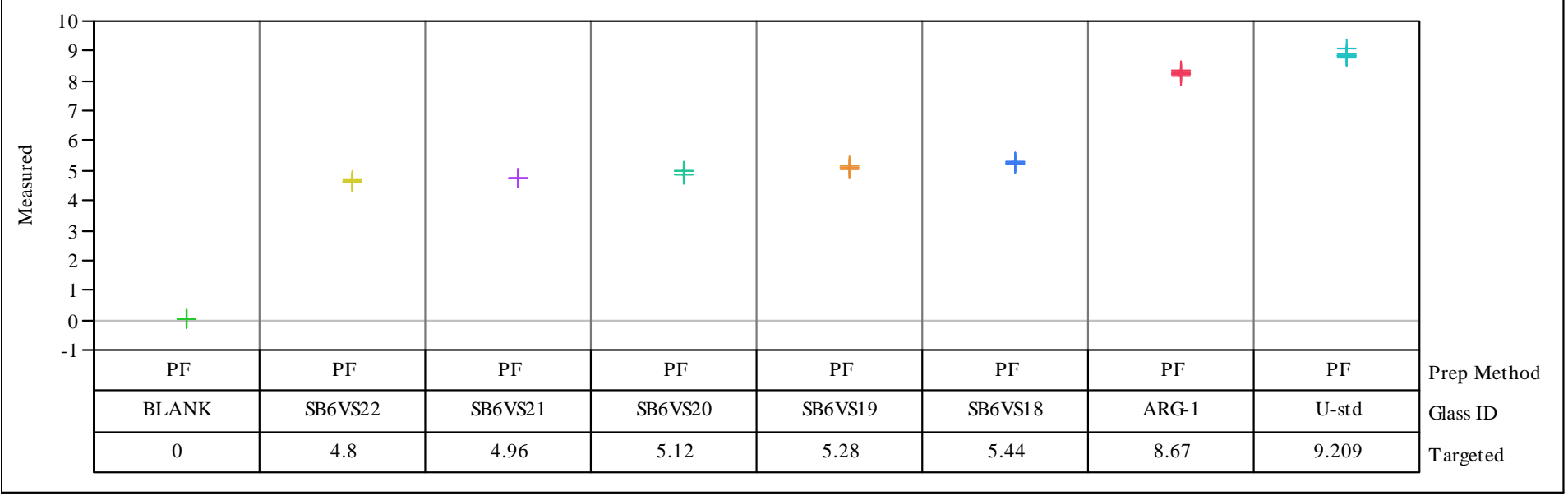


Exhibit C2. Measurements by Lab ID for Thorium Glasses by Targeted Concentration for Each Oxide

Oxide=BaO (wt\%)

Variability Chart for Measured

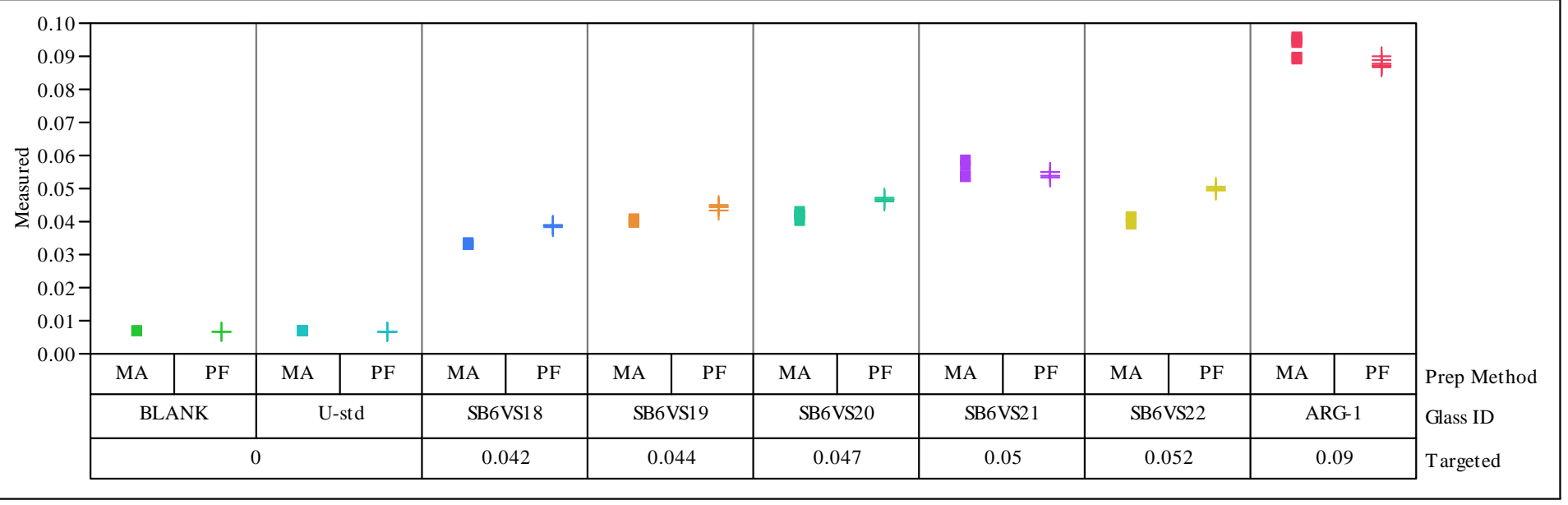

Oxide $=\mathrm{CaO}($ wt $\%)$

Variability Chart for Measured

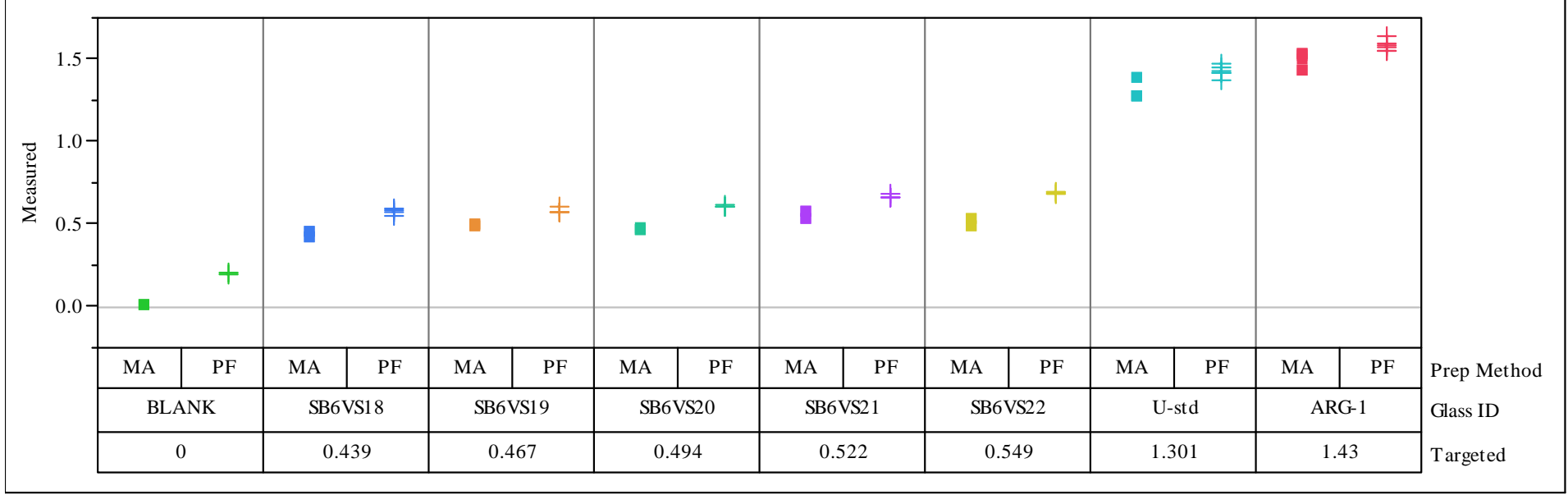


Exhibit C2. Measurements by Lab ID for Thorium Glasses by Targeted Concentration for Each Oxide

Oxide $=\mathrm{CdO}(\mathrm{wt} \%)$

Variability Chart for Measured

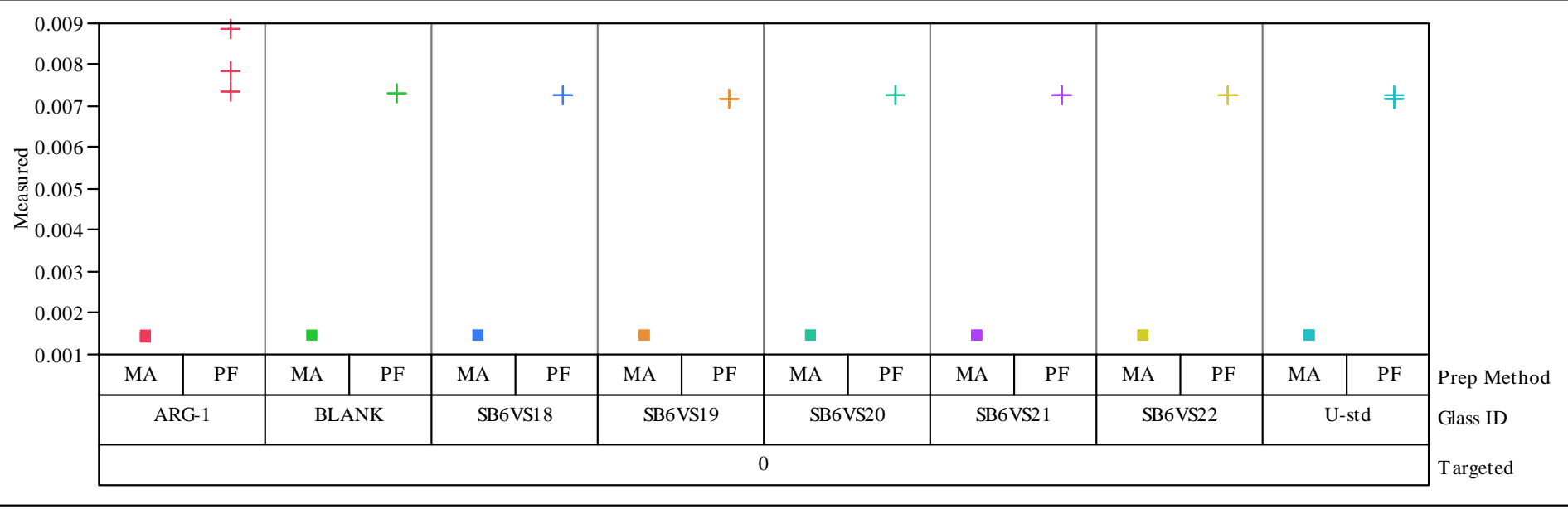

Oxide $=\mathrm{Ce} 2 \mathrm{O3}(\mathrm{wt} \%)$

Variability Chart for Measured

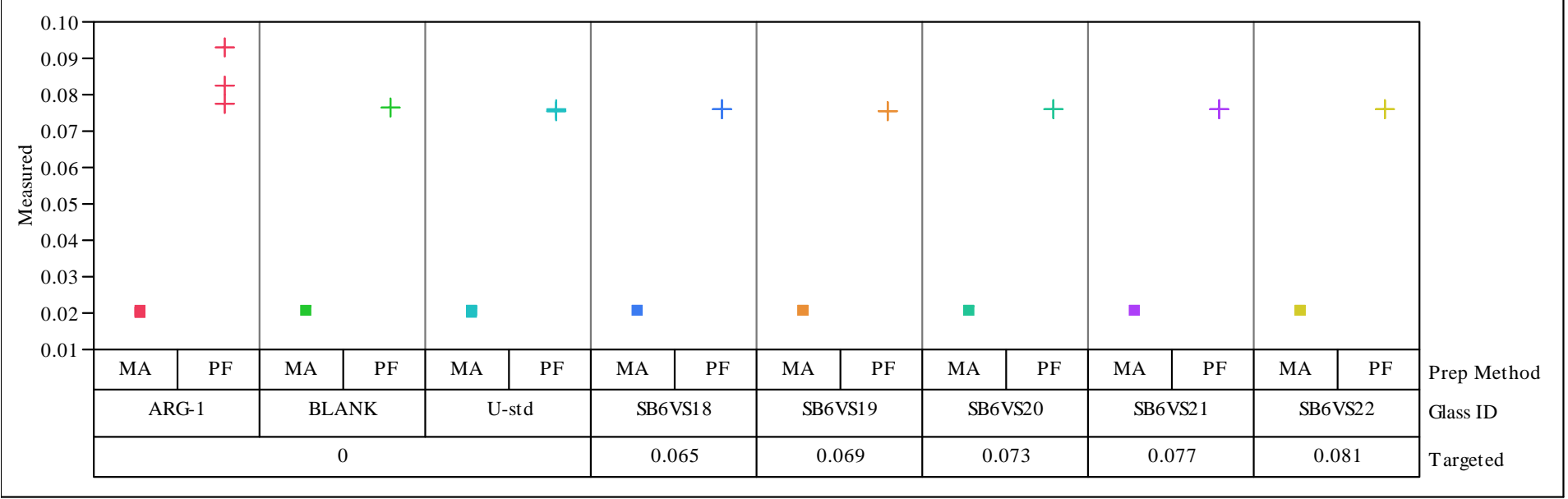


Exhibit C2. Measurements by Lab ID for Thorium Glasses by Targeted Concentration for Each Oxide

Oxide $=\mathbf{C r 2 O 3}(\mathbf{w t} \%)$

Variability Chart for Measured

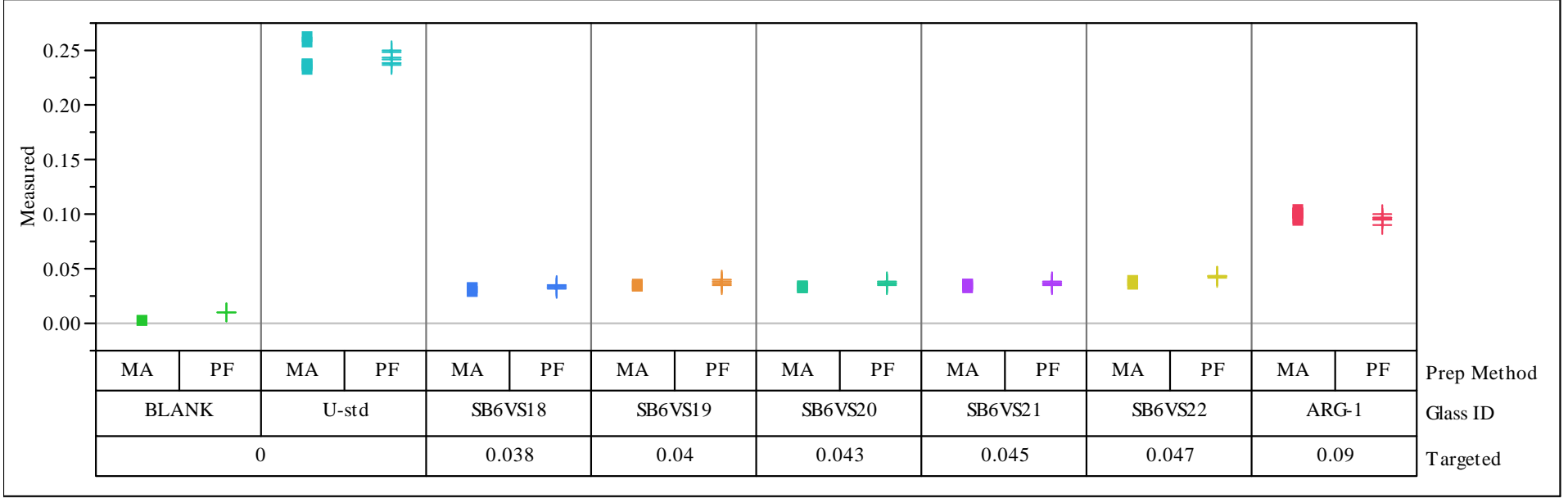

Oxide $=\mathrm{CuO}(\mathrm{wt} \%)$

Variability Chart for Measured

\begin{tabular}{|c|c|c|c|c|c|c|c|c|c|c|c|c|c|c|c|c|c|}
\hline 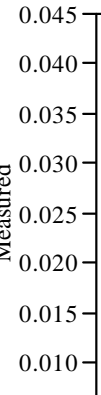 & - & $\neq$ & - & + & - & + & E & 丰 & - & 奉 & 1 & $\neq$ & 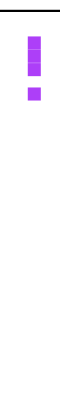 & $\neq$ & \multicolumn{2}{|l|}{ 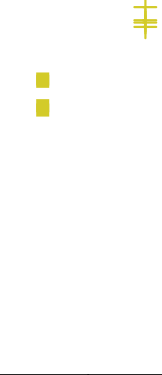 } & \multirow{4}{*}{$\begin{array}{l}\text { Prep Method } \\
\text { Glass ID }\end{array}$} \\
\hline \multirow[t]{3}{*}{$0.005-$} & MA & $\mathrm{PF}$ & MA & $\mathrm{PF}$ & MA & $\mathrm{PF}$ & MA & $\mathrm{PF}$ & MA & $\mathrm{PF}$ & MA & $\mathrm{PF}$ & MA & $\mathrm{PF}$ & MA & $\mathrm{PF}$ & \\
\hline & \multicolumn{2}{|c|}{ ARG-1 } & \multicolumn{2}{|c|}{ BLANK } & \multicolumn{2}{|c|}{ U-std } & \multicolumn{2}{|c|}{ SB6VS18 } & \multicolumn{2}{|c|}{ SB6VS19 } & \multicolumn{2}{|c|}{ SB6VS20 } & \multicolumn{2}{|c|}{ SB6VS21 } & \multicolumn{2}{|c|}{ SB6VS22 } & \\
\hline & \multicolumn{6}{|c|}{0} & \multicolumn{2}{|c|}{0.033} & \multicolumn{2}{|c|}{0.036} & \multicolumn{2}{|c|}{0.038} & \multicolumn{2}{|c|}{0.04} & \multicolumn{2}{|c|}{0.042} & \\
\hline
\end{tabular}


Exhibit C2. Measurements by Lab ID for Thorium Glasses by Targeted Concentration for Each Oxide

Oxide $=\mathrm{Fe} 2 \mathrm{O3}(\mathrm{wt} \%)$

Variability Chart for Measured

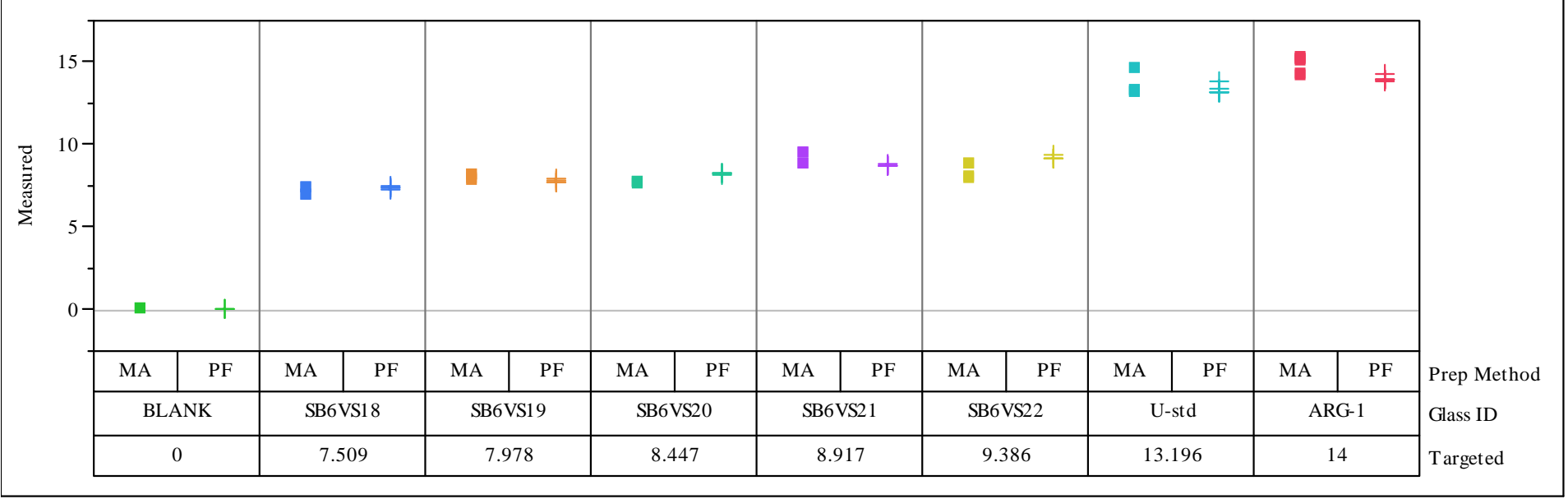

Oxide=K2O (wt\%)

Variability Chart for Measured

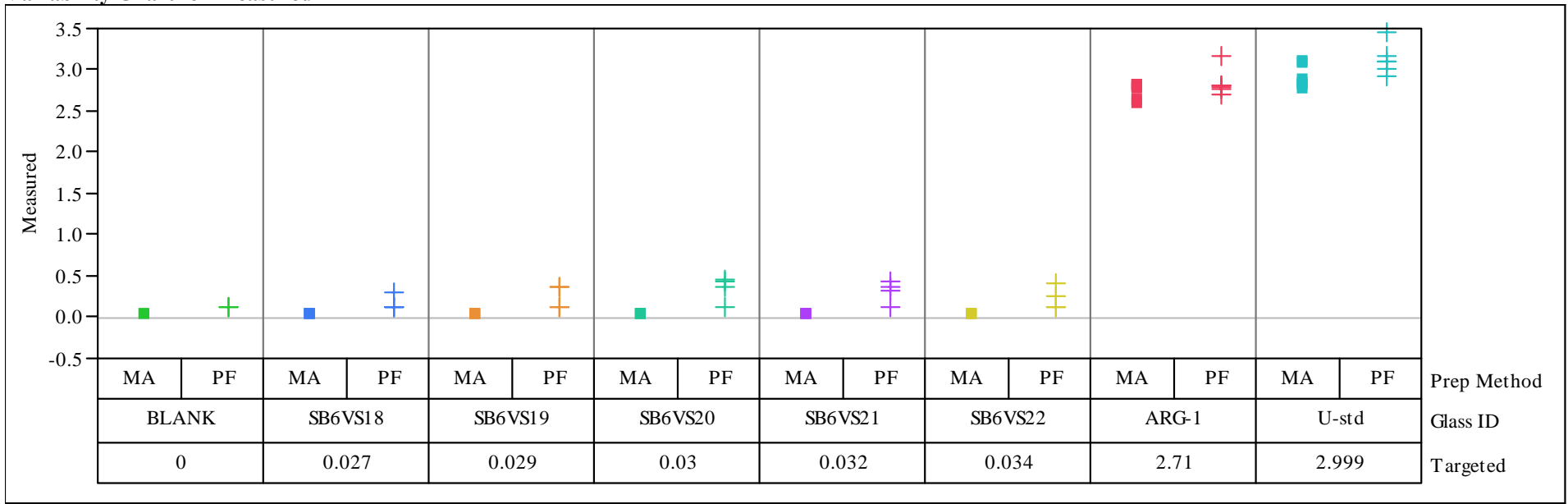


Exhibit C2. Measurements by Lab ID for Thorium Glasses by Targeted Concentration for Each Oxide

Oxide $=\mathbf{L a 2 O 3}(\mathbf{w t} \%)$

Variability Chart for Measured

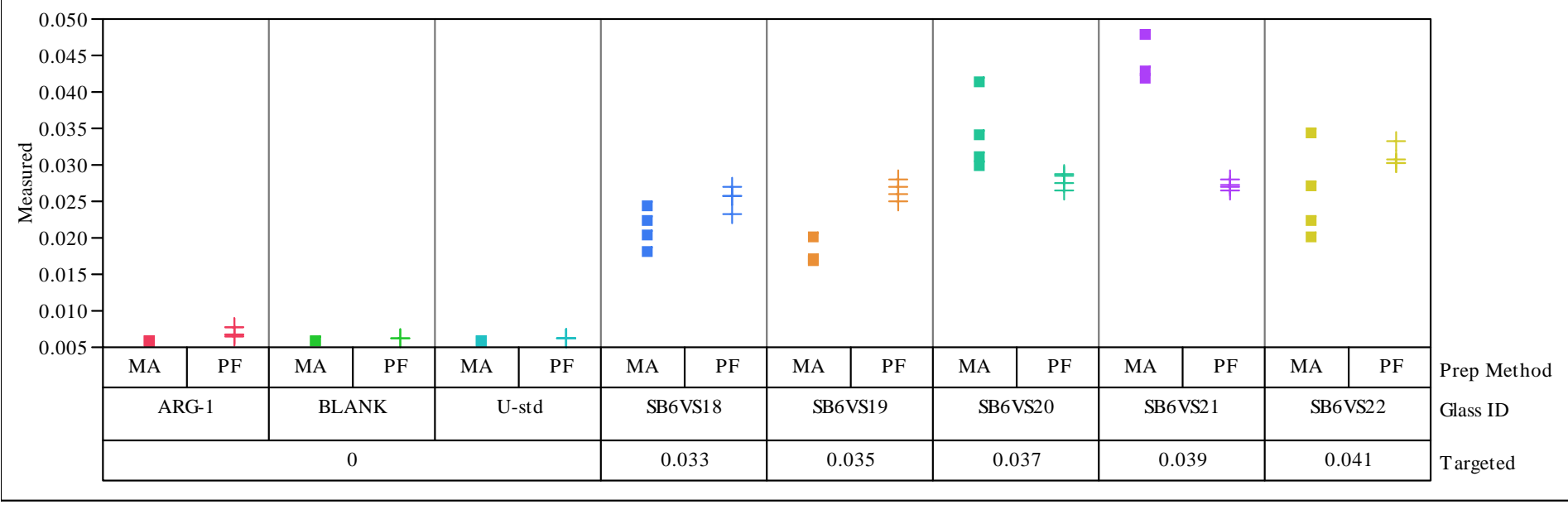

Oxide=Li2O (wt \%)

Variability Chart for Measured

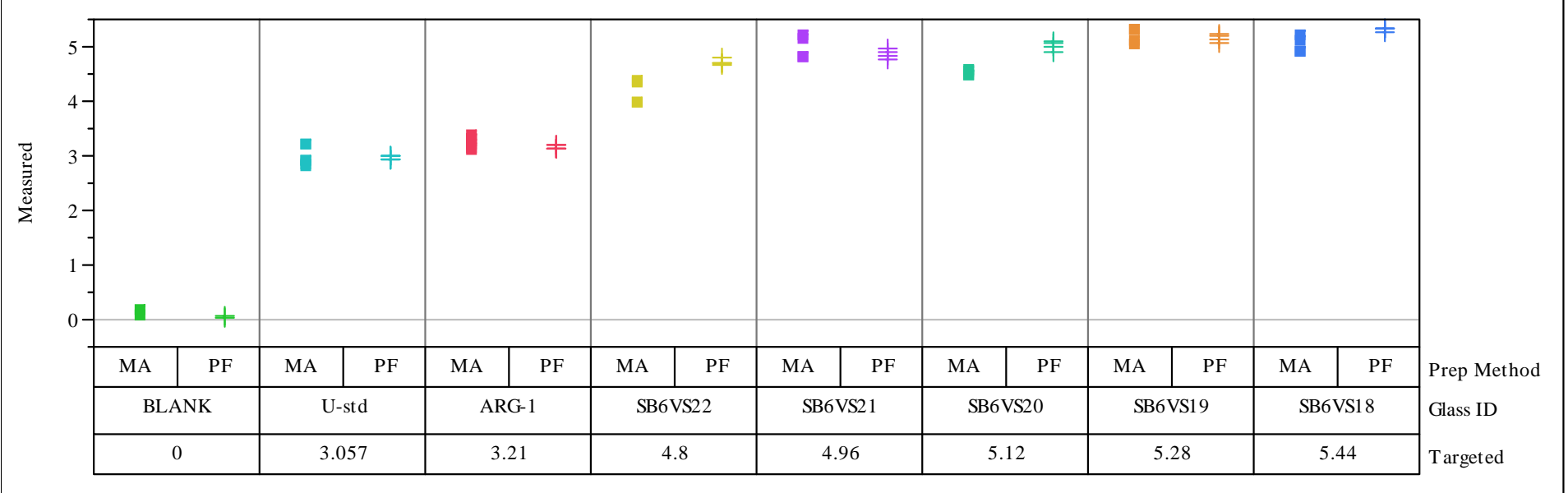


Exhibit C2. Measurements by Lab ID for Thorium Glasses by Targeted Concentration for Each Oxide

Oxide $=\operatorname{MgO}(w t \%)$

Variability Chart for Measured

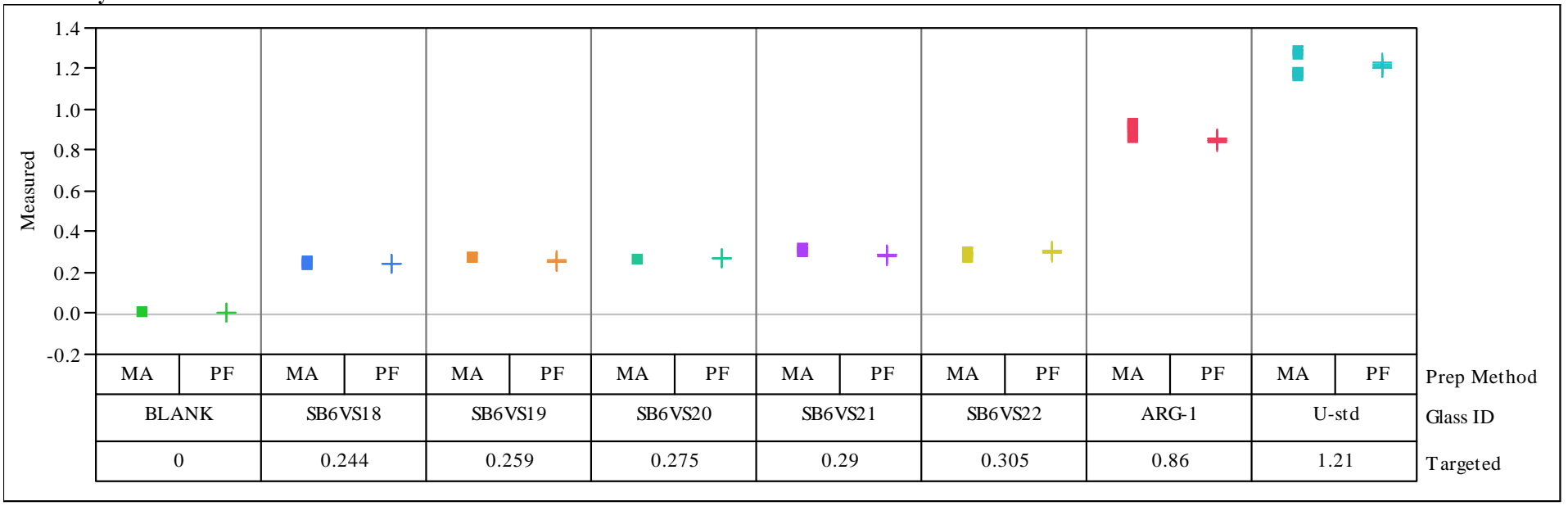

Oxide $=\operatorname{MnO}(\mathbf{w t} \%)$

Variability Chart for Measured

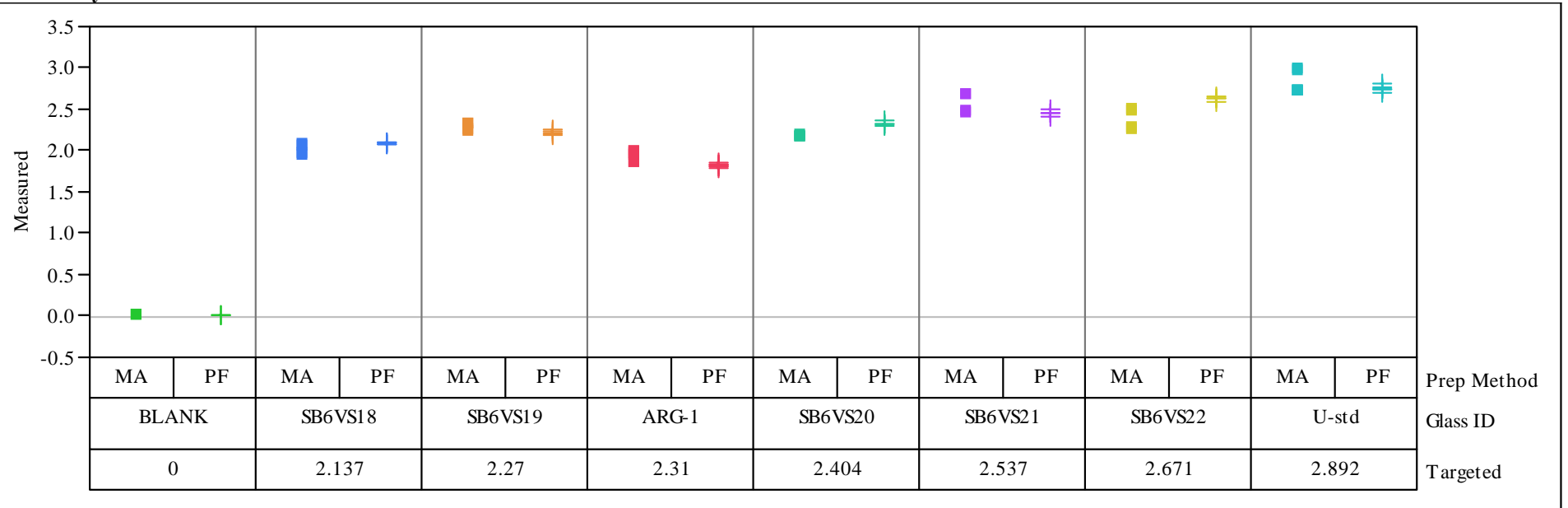


Exhibit C2. Measurements by Lab ID for Thorium Glasses by Targeted Concentration for Each Oxide

Oxide $=\mathbf{N a 2 O}(\mathrm{wt} \%)$

Variability Chart for Measured

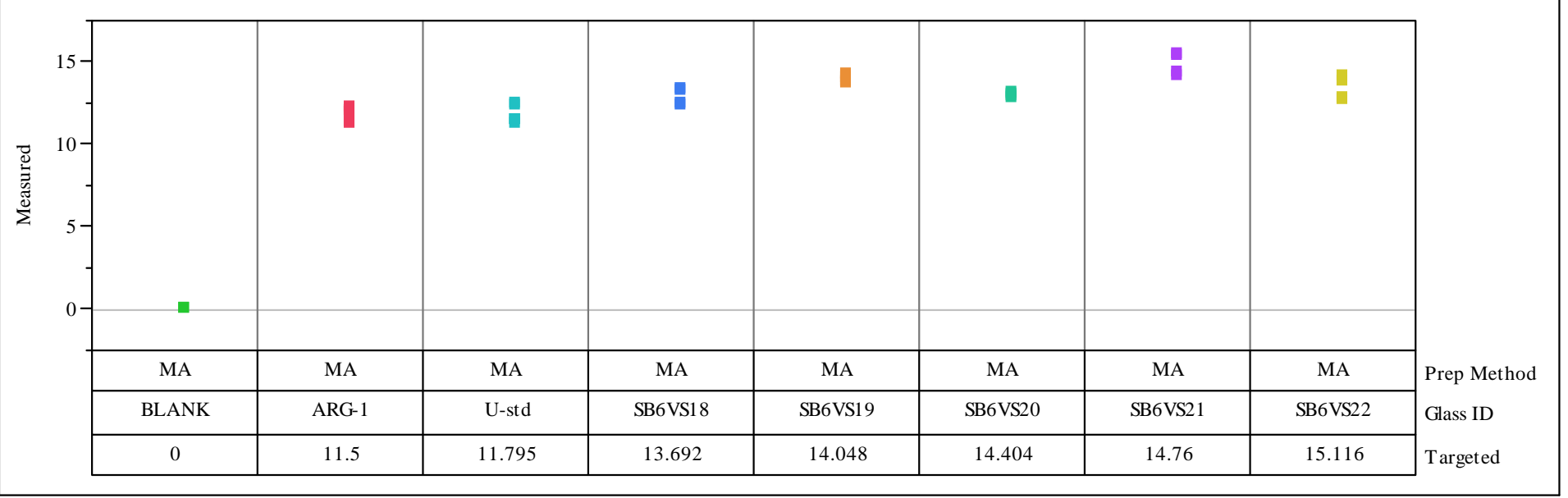

Oxide $=\mathrm{NiO}(\mathrm{wt} \%)$

Variability Chart for Measured

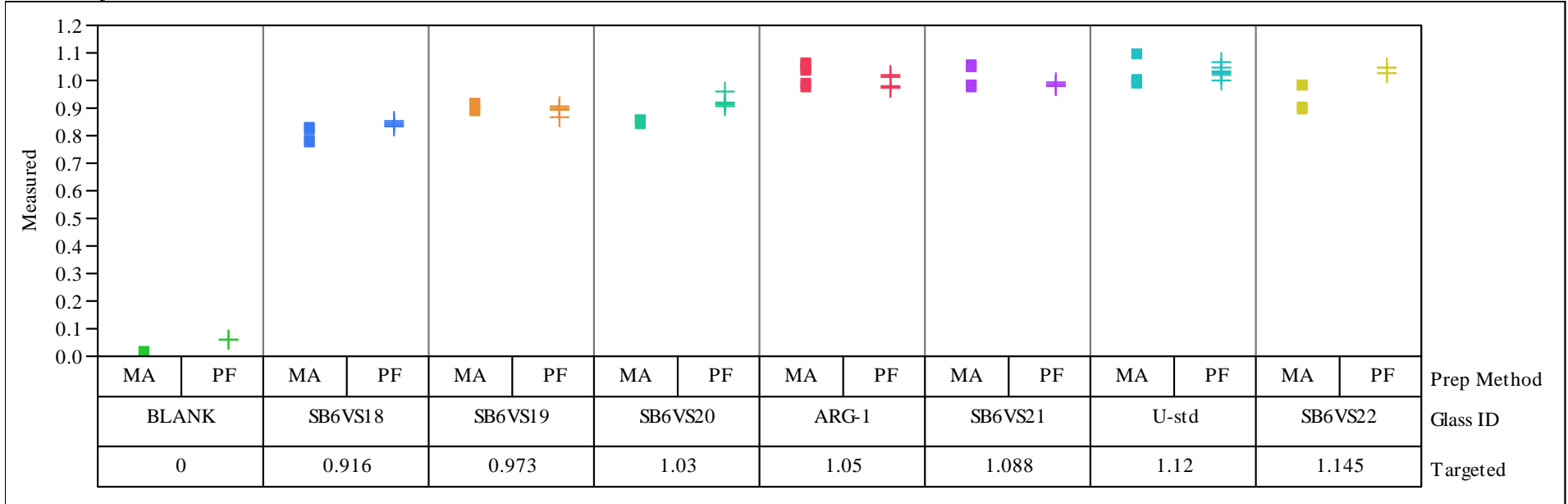


Exhibit C2. Measurements by Lab ID for Thorium Glasses by Targeted Concentration for Each Oxide

Oxide=P2O5 (wt\%)

Variability Chart for Measured

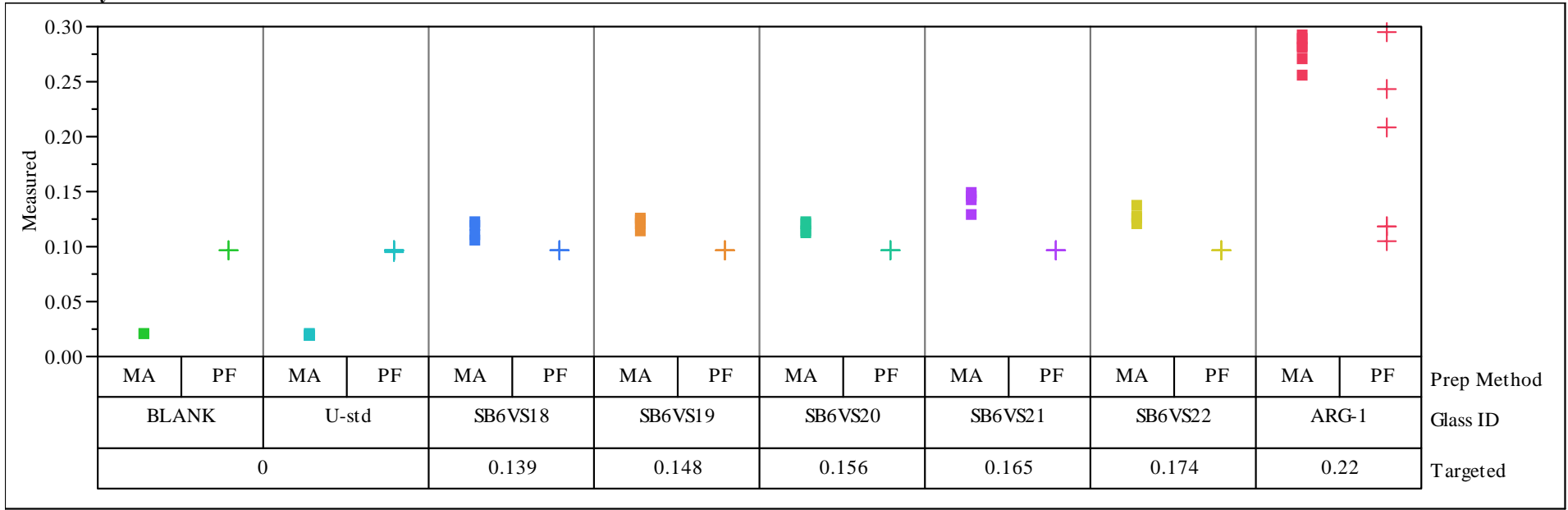

Oxide $=\mathbf{P b O}(\mathbf{w t} \%)$

Variability Chart for Measured

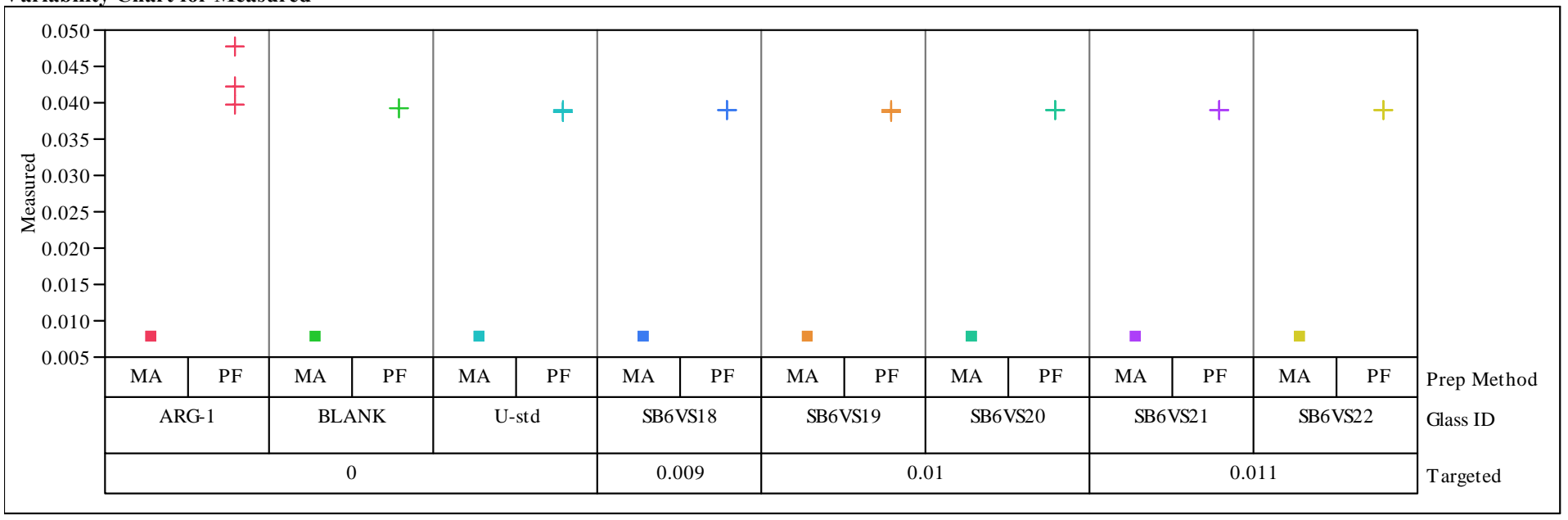


Exhibit C2. Measurements by Lab ID for Thorium Glasses by Targeted Concentration for Each Oxide

Oxide $=\mathrm{SiO} 2(\mathrm{wt} \%)$

Variability Chart for Measured

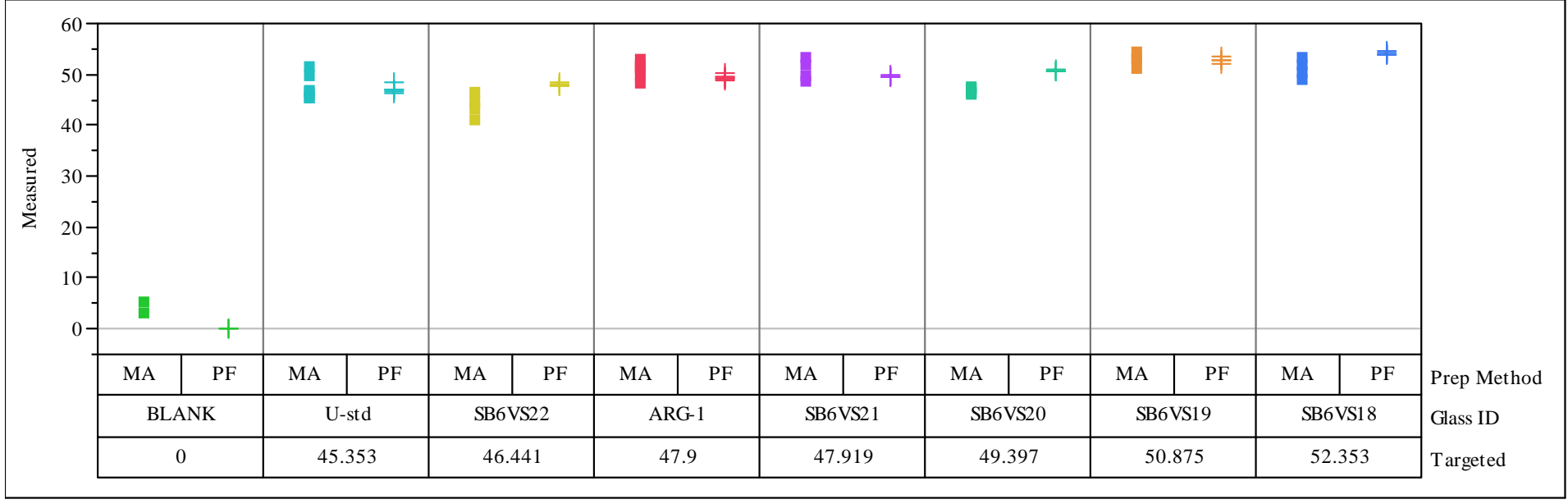

Oxide=SO4 (wt\%)

Variability Chart for Measured

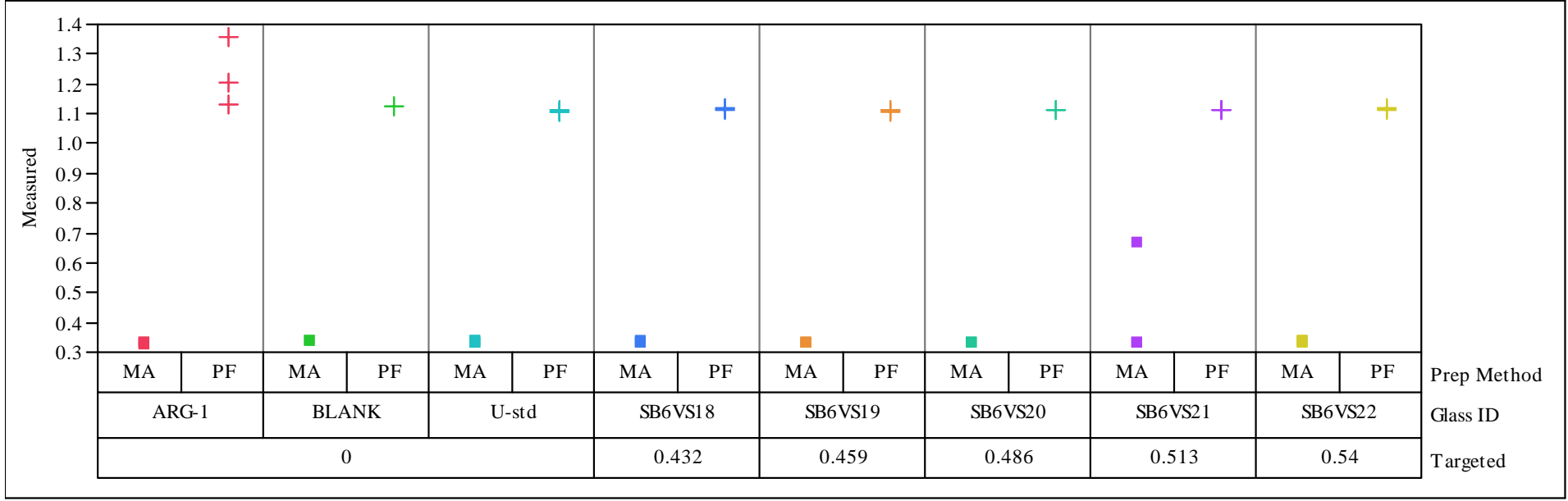


Exhibit C2. Measurements by Lab ID for Thorium Glasses by Targeted Concentration for Each Oxide

Oxide $=$ ThO2 $($ wt $\%)$

Variability Chart for Measured

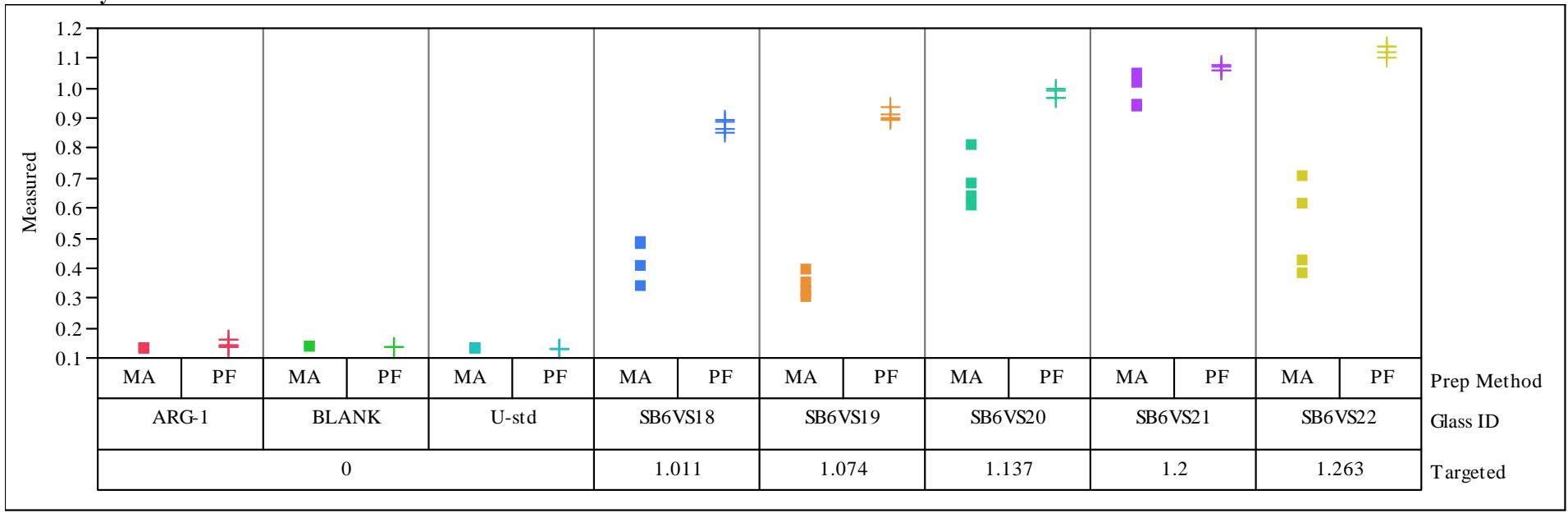

Oxide $=\mathrm{TiO} 2(\mathrm{wt} \%)$

Variability Chart for Measured

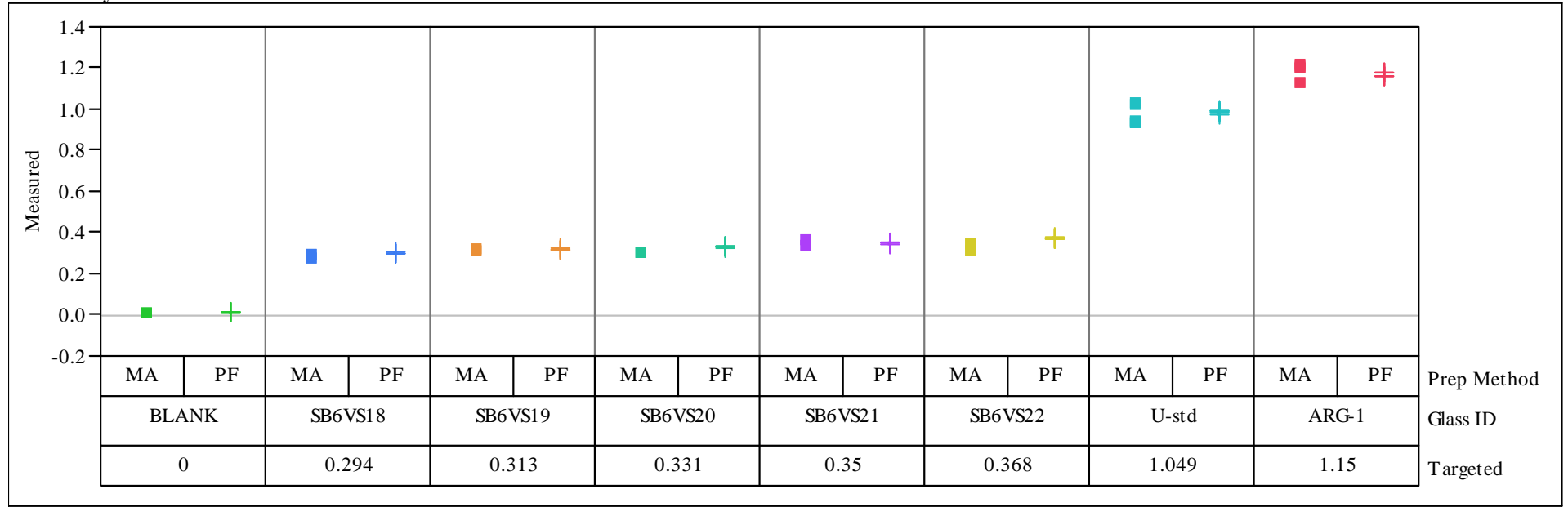


Exhibit C2. Measurements by Lab ID for Thorium Glasses by Targeted Concentration for Each Oxide

Oxide $=\mathbf{U 3 O 8}(\mathrm{wt} \%)$

Variability Chart for Measured

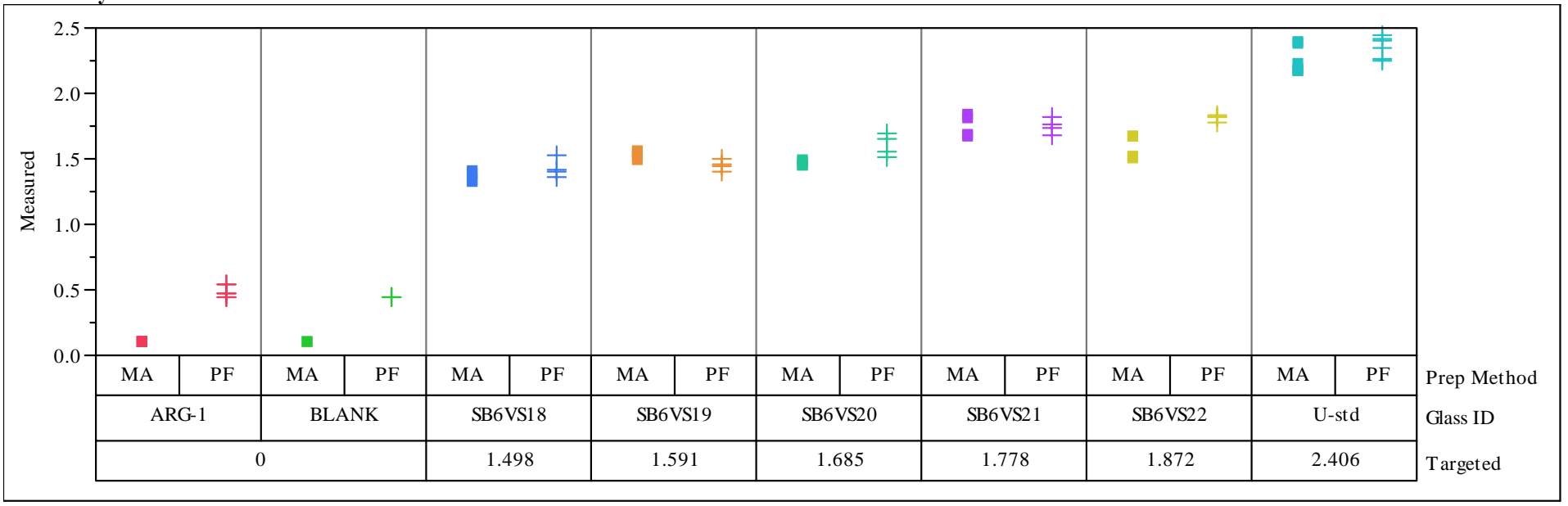

Oxide $=\mathrm{ZnO}(\mathrm{wt} \%)$

Variability Chart for Measured

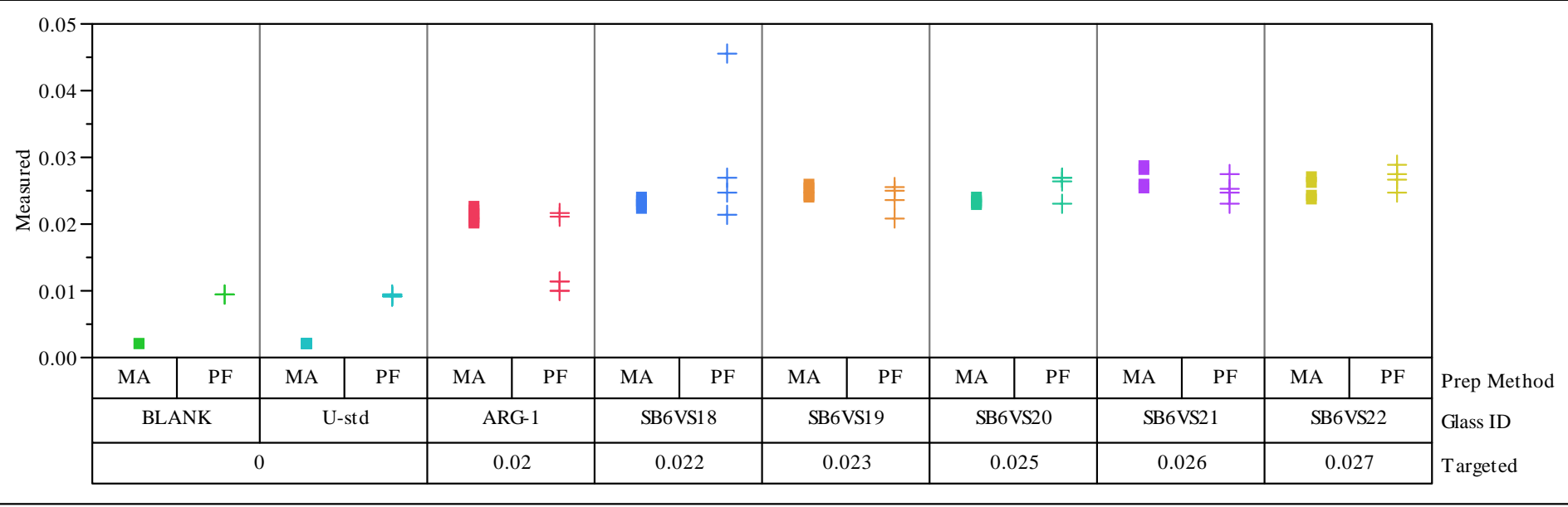


Exhibit C2. Measurements by Lab ID for Thorium Glasses by Targeted Concentration for Each Oxide

Oxide $=\mathrm{ZrO} 2(\mathrm{wt} \%)$

Variability Chart for Measured

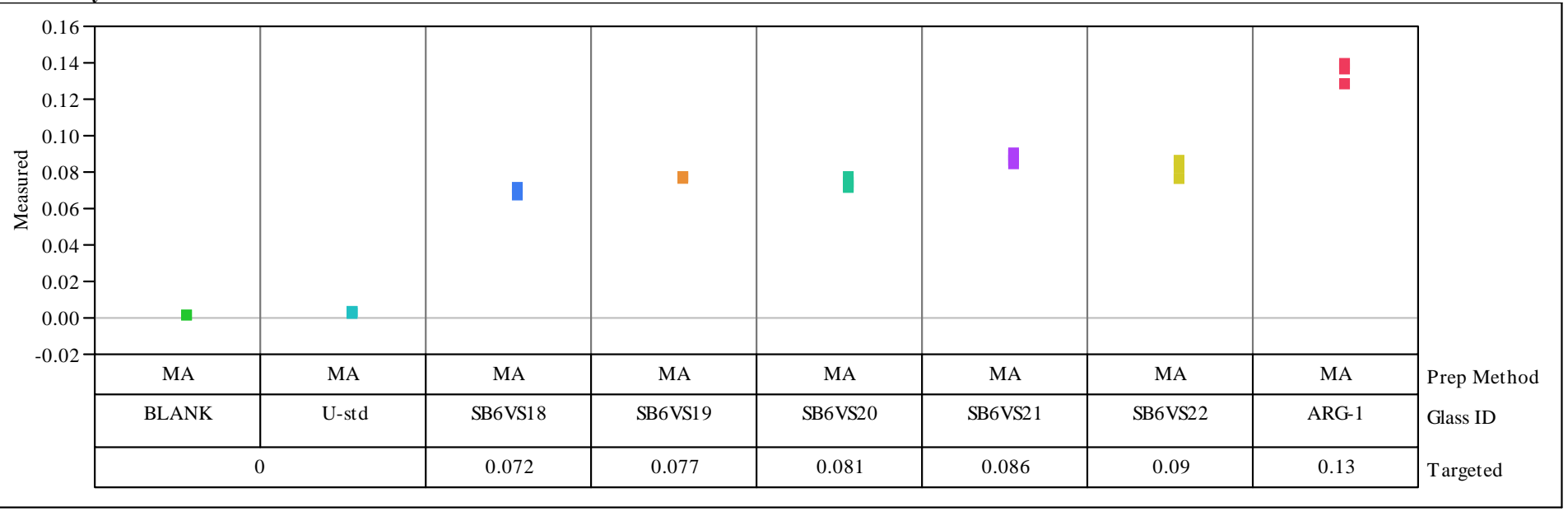




\section{Exhibit C3. Measurements by Block and Sub-Block for Samples of the ARG-1 and Ustd Standards with the Thorium Glasses by Oxide}

Oneway Analysis of Measured By Block Prep Method=MA, Glass ID=ARG-

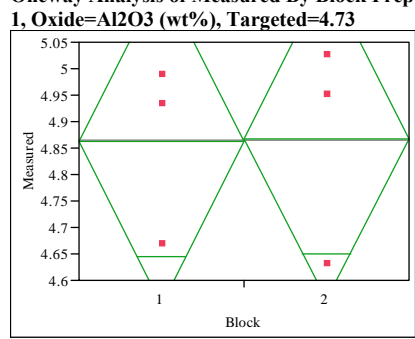

Oneway Anova

Summary of Fit

Rsquare

Adj Rsquare

0.000403

$-0.2495$

0.192073

4.865463

Mean of Response

Observations (or Sum Wgts)

t Test

2-1

Assuming equal variances

Difference $\quad 0.00630$ t Ratio $\quad 0.040161$

Std Err Dif $\quad 0.15683$ DF

Upper CL Dif 0.44172 Prob $>|t| \quad 0.9699$

$\begin{array}{lll}\text { Lower CL Dif }-0.42912 \text { Prob }>t & 0.4849\end{array}$

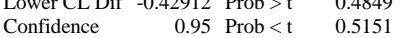

Means for Oneway Anova

Level Number Mean Std Error Lower 95\% Upper 95\%

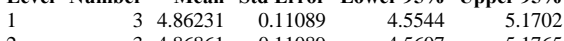

Std Error uses

Oneway Analysis of Measured By Block Prep Method=MA, Glass ID=ARG1, Oxide $=\mathrm{BaO}(\mathrm{wt} \%)$, Targeted $=\mathbf{0 . 0 9}$

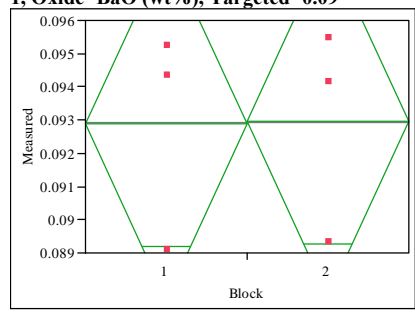

Oneway Anova
Summary of Fit

Rsquare

Adj Rsquare

0.000194

Root Mean Sq

$-0.24976$

0.003274

Mean of Response

Observations (or Sum Wgts) $\quad 6$

t Test

2-1

Assuming equal variances

Difference $\quad 7.443 e-5$ t Ratio 0.027848

Std Err Dif $\quad 0.00267$ DF $\quad 4$

Upper CL Dif 0.00750 Prob $>|t| \quad 0.979$

Lower CL Dif -0.00735 Prob $>\mathrm{t} \quad 0.4896$

$\begin{array}{lrl}\text { Confidence } & 0.95 \text { Prob }<\mathrm{t} & 0.5104\end{array}$

Means for Oneway Anova

Level Number Mean Std Error Lower 95\% Upper 95\%

$\begin{array}{lllllr}1 & 3 & 0.092893 & 0.00189 & 0.08765 & 0.09814 \\ 2 & 3 & 0.092967 & 0.00189 & 0.08772 & 0.09821\end{array}$

Std Error uses a pooled estimate of error variance
Oneway Analysis of Measured By Block Prep Method=MA, Glass ID=ARG1, Oxide $=\mathrm{CaO}(\mathrm{wt} \%)$, Targeted $=1.43$

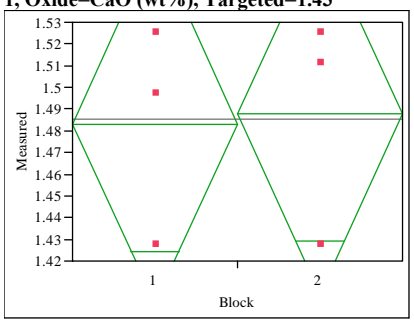

Oneway Anova

Rsquare

Adj Rsquare 0.00304

Root Mean Square Error $\quad 0.051726$

Mean of Response

Observations (or Sum Wgts)

1.485484

t Test

$2-1$

Assuming equal variances

Difference $\quad 0.00466$ t Ratio $\quad 0.110432$

Std Err Dif $\quad 0.04223$ DF

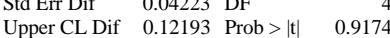

$\begin{array}{lll}\text { Lower CL Dif } & -0.11260 \text { Prob }>t \quad 0.4587\end{array}$

$\begin{array}{lrl}\text { Confidence } & 0.95 \text { Prob }<\mathrm{t} & 0.5413\end{array}$

Means for Oneway Anova

Level Number Mean Std Error Lower 95\% Upper 95\%

$\begin{array}{lllllr}1 & 3 & 1.48315 & 0.02986 & 1.4002 & 1.5661\end{array}$

Std Error uses a pooled estimate of error variance

Oneway Analysis of Measured By Block Prep Method=MA, Glass ID=ARG1, Oxide $=\mathbf{C d O}($ wt $\%)$, Targeted $=0$

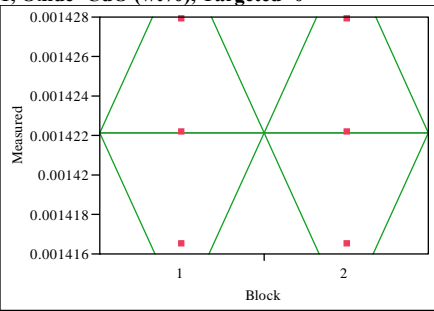

Oneway Anova

Summary of Fi

Adj Rsquare

$-0.25$

Mean of Rin

Observations (or Sum Wgts)

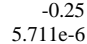

$5.711 \mathrm{e}-6$

0.001422

t Test

Assuming equal variances

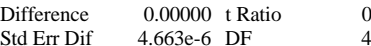

$\begin{array}{lrlr}\text { Std Err Dif } & 4.663 e-6 & \text { DF } & 4 \\ \text { Upper CL Dif } & 0.000013 & \text { Prob }>|t| & 1.0000\end{array}$

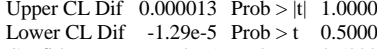

$\begin{array}{lrl}\text { Confidence } & 0.95 \text { Prob }<\mathrm{t} \quad 0.5000\end{array}$

Means for Oneway Anova

Level Number Mean Std Error Lower 95\% Upper 95\%

$\begin{array}{lrrrrr}\text { Level } & \text { Number } & \text { Mean } & \text { Std Error } & \text { Lower 95\% } & \text { Upper 95\% } \\ 1 & 3 & 0.001422 & 3.2975 \mathrm{e}-6 & 0.00141 & 0.00143\end{array}$

$\begin{array}{llllll}1 & 3 & 0.001422 & 3.2975 \mathrm{e}-6 & 0.00141 & 0.00143 \\ 2 & 3 & 0.001422 & 3.2975 \mathrm{e}-6 & 0.00141 & 0.00143\end{array}$

Std Error uses a pooled estimate of error variance 


\section{Exhibit C3. Measurements by Block and Sub-Block for Samples of the ARG-1 and Ustd Standards with the Thorium Glasses by Oxide}

Oneway Analysis of Measured By Block Prep Method=MA, Glass ID=ARG1, Oxide $=\mathrm{Ce} 2 \mathrm{O} 3(\mathrm{wt} \%)$, Targeted $=0$

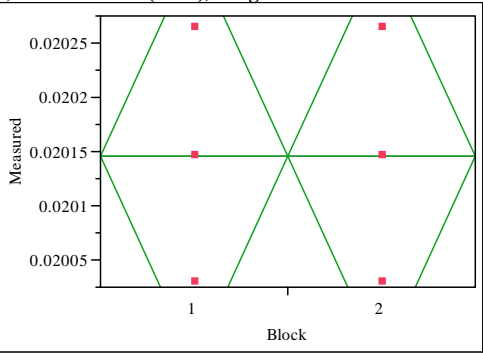

Oneway Anova

Summary of Fit

Rsquare

$-0.25$

Root Mean Squr

Observations (or Sum Wgts)

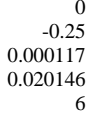

t Test

2-1

Assuming equal variances

Difference $\quad 0.00000$ t Ratio $\quad 0$

Std Err Dif 9.564e-5 DF 4

Upper CL Dif 0.00027 Prob $>|\mathrm{t}| 1.0000$

Lower CL Dif -0.00027 Prob $>t \quad 0.5000$

$\begin{array}{lrl}\text { Confidence } & 0.95 \text { Prob }<\mathrm{t} \quad 0.5000\end{array}$

Means for Oneway Anova

Level Number Mean Std Error Lower 95\% Upper 95\%

$\begin{array}{lllrrr}1 & 3 & 0.020146 & 6.76 \mathrm{e}-5 & 0.01996 & 0.02033\end{array}$

Std Error uses a pool

Oneway Analysis of Measured By Block Prep Method=MA, Glass ID=ARG-

1, Oxide $=\mathrm{Cr} 2 \mathrm{O} 3(\mathrm{wt} \%)$, Targeted $=\mathbf{0 . 0 9}$

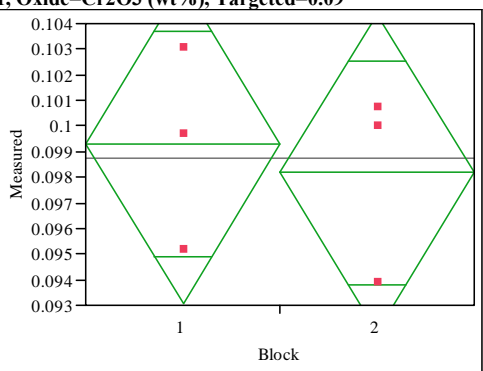

Oneway Anova

Summary of Fit

Rsquare

Adj Rsquare

Root Mean Square Eror $\quad-0.21186$

Mean of Response $\quad 0.098731$

Observations (or Sum Wgts) 6

t Test

2-1

Assuming equal variances

Difference $\quad-0.00112$ t Ratio $\quad-0.35481$

Std Err Dif $\quad 0.00316$ DF $\quad 4$

Upper CL Dif 0.00765 Prob $>|t| \quad 0.7406$

Lower CL Dif -0.00989 Prob $>t \quad 0.6297$

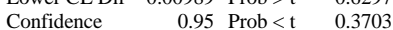

Means for Oneway Anova

Level Number Mean Std Error Lower 95\% Upper 95\%

$\begin{array}{llllll}1 & 3 & 0.099291 & 0.00223 & 0.09309 & 0.10549 \\ 2 & 3 & 0.098171 & 0.00223 & 0.09197 & 0.10437\end{array}$

Std Error uses a pooled estimate of error variance
Oneway Analysis of Measured By Block Prep Method=MA, Glass ID=ARG1, Oxide $=\mathrm{CuO}(\mathrm{wt} \%)$, Targeted $=\mathbf{0}$

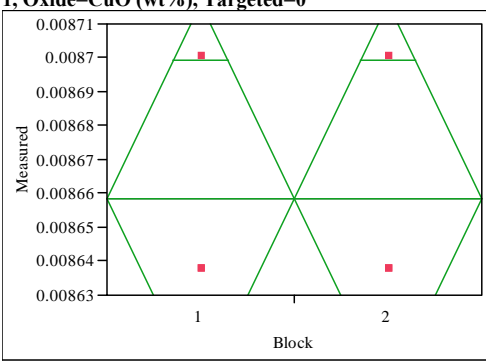

Oneway Anova

Rsquare

Adj Rsquare

$\begin{array}{lr}\text { Adj Rsquare } & -0.25 \\ \text { Root Mean Square Error } \quad 3.614 \mathrm{e}-5\end{array}$

Mean of Response

Observations (or Sum Wgts)

0.008658

t Test

2-1

Assuming equal variances

Difference $\quad 0.00000$ t Ratio $\quad 0$

Std Err Dif 2.951e-5 DF 4

Upper CL Dif 0.000082 Prob $>|t| 1.0000$

Lower CL Dif $-8.19 \mathrm{e}-5$ Prob $>\mathrm{t} \quad 0.5000$

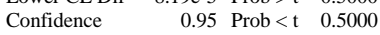

Means for Oneway Anova

Level Number Mean Std Error Lower 95\% Upper 95\%

$\begin{array}{llllll}1 & 3 & 0.008658 & 0.00002 & 0.00860 & 0.00872\end{array}$

Std Error uses a pooled estimate of error variance

Oneway Analysis of Measured By Block Prep Method=MA, Glass ID=ARG1, Oxide $=\mathrm{Fe} 2 \mathrm{O} 3(\mathrm{wt} \%)$, Targeted $=14$

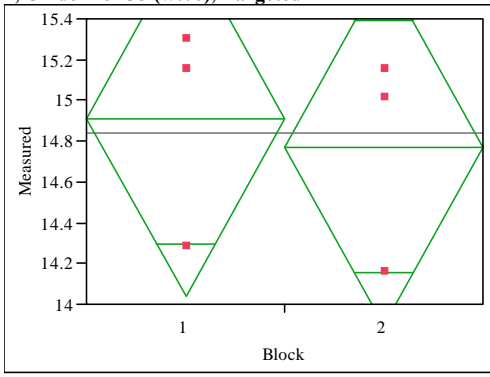

Oneway Anova

Summary of Fit

Adj Rsquare $\quad-0.2206$

0.545383

Mean of Respons

14.84267

t Test

2-1

Assuming equal variances

Difference $\quad-0.1382$ t Ratio $\quad-0.31036$

Std Err Dif $\quad 0.4453$ DF 4

Upper CL Dif 1.0982 Prob $>|t| \quad 0.7718$

Lower CL Dif -1.3746 Prob $>t \quad 0.6141$

$\begin{array}{lrl}\text { Confidence } & 0.95 \text { Prob }<\mathrm{t} & 0.3859\end{array}$

Means for Oneway Anova

Level Number Mean Std Error Lower 95\% Upper 95\%

$\begin{array}{llllll}1 & 3 & 14.9118 & 0.31488 & 14.038 & 15.786\end{array}$

$\begin{array}{llllll}2 & 3 & 14.7736 & 0.31488 & 13.899 & 15.648\end{array}$

Std Error uses a pooled estimate of error variance 


\section{Exhibit C3. Measurements by Block and Sub-Block for Samples of the ARG-1 and Ustd Standards with the Thorium Glasses by Oxide}

Oneway Analysis of Measured By Block Prep Method=MA, Glass ID=ARG1, Oxide $=\mathrm{K} 2 \mathrm{O}(\mathrm{wt} \%)$, Targeted $=\mathbf{2 . 7 1}$

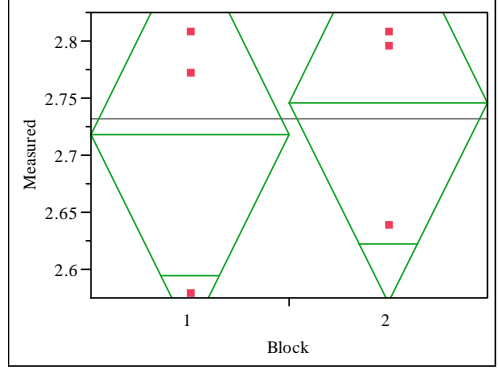

Oneway Anova
Summary of Fit

Rsquare

Adj Rsquare

0.024102

$-0.21987$

Root Mean Square Error $\quad 0.109524$

Mean of Response $\quad 2.732434$

Observations (or Sum Wgts)

t Test

2-1

Assuming equal variances

Difference $\quad 0.02811$ t Ratio 0.314309

Std Err Dif $\quad 0.08943$ DF $\quad 4$

$\begin{array}{rr}4 \\ \text { Upper CL Dif } 0.27639 \text { Prob }>|t| & 0.7690\end{array}$

Lower CL Dif -0.22018 Prob $>t \quad 0.3845$

$\begin{array}{lr}\text { Confidence } & 0.95 \text { Prob }<\mathrm{t} \quad 0.6155\end{array}$

Means for Oneway Anova

Level Number Mean Std Error Lower 95\% Upper 95\%

$\begin{array}{lrrrrr}1 & 3 & 2.71838 & 0.06323 & 2.5428 & 2.8939 \\ 2 & 3 & 2.74649 & 0.06323 & 2.5709 & 2.9221\end{array}$

Std Error uses a pooled estimate of error variance

Oneway Analysis of Measured By Block Prep Method=MA, Glass ID=ARG1, Oxide $=\mathrm{La2O3}(\mathrm{wt} \%)$, Targeted $=0$

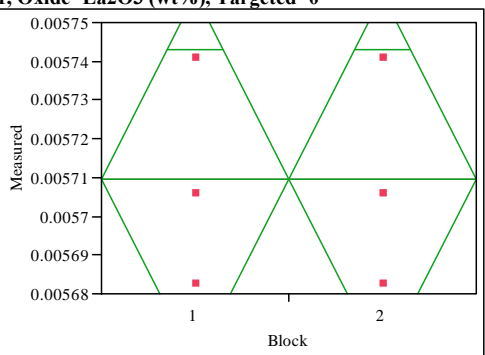

Oneway Anova

Summary of Fit

Rsquare

Adj Rsquare

Mean of Response

Observations (or Sum Wgts)

t Test

Assuming equal variances

$\begin{array}{lrll}\text { Difference } & 0.00000 & \text { t Ratio } & 0 \\ \text { Std Err Dif } & 0.000024 & \text { DF } & 4\end{array}$

$\begin{array}{lrlr}\text { Std Err Dif } & 0.000024 & \text { DF } & 4 \\ \text { Upper CL Dif } & 0.000067 & \text { Prob }>|t| & 1.0000\end{array}$

$\begin{array}{lrll}\text { Upper CL Dif } & 0.000067 & \text { Prob }>|t| & 1.0000 \\ \text { Lower CL Dif } & -6.69 e-5 & \text { Prob }>t & 0.5000\end{array}$

$\begin{array}{lrl}\text { Confidence } & 0.95 \text { Prob }<\mathrm{t} \quad 0.5000\end{array}$

Means for Oneway Anova

Level Number Mean Std Error Lower 95\% Upper 95\%

$\begin{array}{lrrrrr}\text { Level } & \text { Number } & \text { Mean } & \text { Std Error } & \text { Lower } 95 \% & \text { Upper 95\% } \\ 1 & 3 & 0.005710 & 1.7 \mathrm{e}-5 & 0.00566 & 0.00576\end{array}$

$\begin{array}{llllll}1 & 3 & 0.005710 & 1.7 \mathrm{e}-5 & 0.00566 & 0.00576 \\ 2 & 3 & 0.005710 & 1.7 \mathrm{e}-5 & 0.00566 & 0.00576\end{array}$
Std Error uses a pooled estimate of error variance

Oneway Analysis of Measured By Block Prep Method=MA, Glass ID=ARG1, Oxide $=$ Li2O $($ wt $\%)$, Targeted $=3.2$

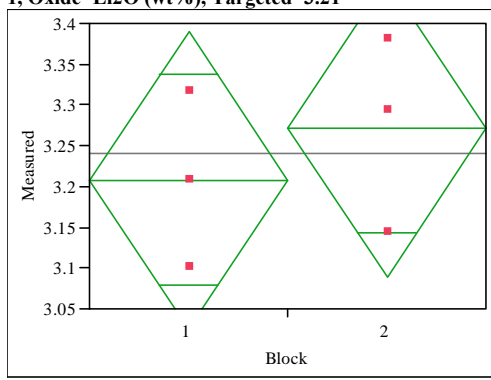

Oneway Anova

Summary of Fit

Rsquare

\begin{tabular}{lll}
-0.11554 \\
\hline
\end{tabular}

Mean of Respons

Mean of Respons

3.240115

t Test

2-1

Assuming equal variances

$\begin{array}{lrlr}\text { Difference } & 0.06459 & \mathrm{t} \text { Ratio } & 0.694365 \\ \text { Std Err Dif } & 0.09302 & \text { DF } & 4 \\ \text { Upper CL Dif } & 0.32284 & \text { Prob }>|t| & 0.5257 \\ \text { Lower CL Dif } & -0.19367 & \text { Prob }>t & 0.2628 \\ \text { Confidence } & 0.95 & \text { Prob }<t & 0.7372\end{array}$

Means for Oneway Anova

Level Number Mean Std Error Lower 95\% Upper 95\%

$\begin{array}{llrrrr}1 & 3 & 3.20782 & 0.06577 & 3.0252 & 3.3904 \\ 2 & 3 & 3.27241 & 0.06577 & 3.0898 & 3.4550\end{array}$

Std Error uses a pooled estimate of error variance

Oneway Analysis of Measured By Block Prep Method=MA, Glass ID=ARG1, Oxide $=\mathrm{MgO}(\mathrm{wt} \%)$, Targeted $=0.86$

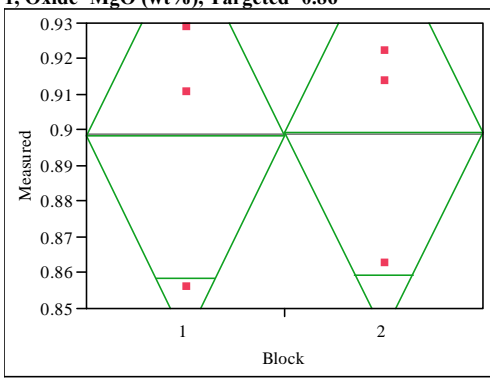

Oneway Anova

Summary of Fit

Rsquare

Adj Rsquare $\quad-0.2495$

Root Mean Square Error $\quad 0.035269$

Mean of Respons

Observations (or Sum Wgts)

t Test

Assuming equal variances

Difference $\quad 0.00111$ t Ratio 0.038391

Std Err Dif $\quad 0.02880 \mathrm{DF} \quad 0.038391$

Upper CL Dif $\quad 0.08106$ Prob $>|t| \quad 0.9712$

$\begin{array}{lrll}\text { Upper CL Dif } & 0.08106 & \text { Prob }>|t| & 0.9712 \\ \text { Lower CL Dif } & -0.07885 & \text { Prob }>t & 0.4856\end{array}$

$\begin{array}{lrl}\text { Confidence } & 0.95 \text { Prob }<\mathrm{t} \quad 0.5144\end{array}$

Means for Oneway Anova

\begin{tabular}{rrrrr} 
Level Number & Mean & Std Error & Lower 95\% & Upper 95\% \\
\hline & 0.898246 & 0.02036 & 0.84171 & 0.95478
\end{tabular} 


\section{Exhibit C3. Measurements by Block and Sub-Block for Samples of the ARG-1 and Ustd Standards with the Thorium Glasses by Oxide}

Level Number Mean Std Error Lower 95\% Upper 95\% $\begin{array}{llllll}2 & 3 & 0.899351 & 0.02036 & 0.84282 & 0.95589\end{array}$ Std Error uses a pooled estimate of error variance

Oneway Analysis of Measured By Block Prep Method=MA, Glass ID=ARG1, Oxide $=\mathrm{MnO}(\mathrm{wt} \%)$, Targeted $=\mathbf{2 . 3 1}$

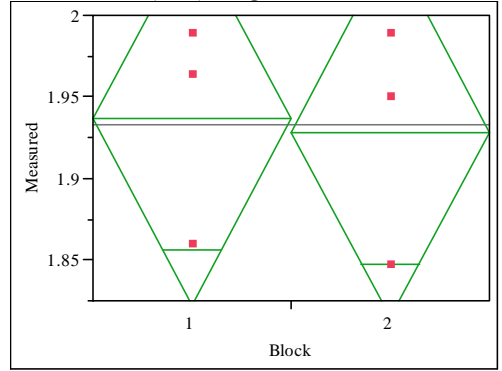

Oneway Anova

Summary of Fit

$\begin{array}{lr}\text { Rsquare } & 0.005495 \\ \text { Adj Rsquare } & -0.24313 \\ \text { Root Mean Square Error } & 0.070918 \\ \text { Mean of Response } & 1.932496 \\ \text { Observations (or Sum Wgts) } & 6\end{array}$

t Test

Assuming equal variances

Difference $\quad-0.00861$ t Ratio $\quad-0.14866$

$\begin{array}{lrlr}\text { Std Err Dif } & 0.05790 & \text { DF } & 4 \\ \text { Upper CL Dif } & 0.15216 & \text { Prob }>|t| & 0.8890\end{array}$

$\begin{array}{lrlr}\text { Upper CL Dif } & 0.15216 & \text { Prob }>|t| & 0.8890 \\ \text { Lower CL Dif } & -0.16938 & \text { Prob }>t & 0.5555\end{array}$

$\begin{array}{lll}\text { Confidence } & 0.95 \text { Prob }<\mathrm{t} \quad 0.4445\end{array}$

Means for Oneway Anova

Level Number Mean Std Error Lower 95\% Upper 95\%

$\begin{array}{lrrrrr}\text { Level } & \text { Number } & \text { Mean } & \text { Std Error } & \text { Lower 95\% } & \text { Upper 95\% } \\ 1 & 3 & 1.93680 & 0.04094 & 1.8231 & 2.0505\end{array}$

$\begin{array}{llllll}2 & 3 & 1.92819 & 0.04094 & 1.8145 & 2.0419\end{array}$

Std Error uses a pooled estimate of error variance

Oneway Analysis of Measured By Block Prep Method=MA, Glass ID=ARG1, Oxide $=\mathrm{Na2O}(\mathrm{wt} \%)$, Targeted $=11.5$

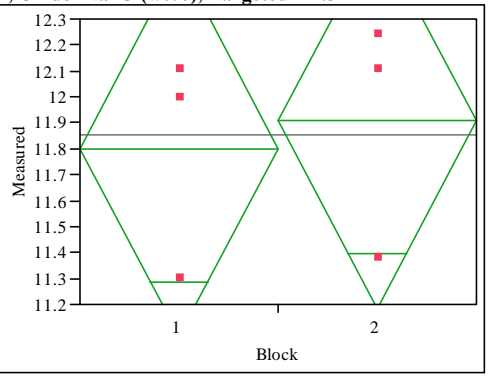

Oneway Anova

Summary of Fit

Rsquare $\quad 0.020918$

Adj Rsquare $\quad-0.22385$

Root Mean Square Error $\quad 0.451798$

$\begin{array}{lr}\text { Mean of Response } & 11.85341 \\ \text { Observations (or Sum Wgts) } & 6\end{array}$

t Test

$2-1$

Assuming equal variances

Difference $\quad 0.1078$ t Ratio $\quad 0.292335$

Std Err Dif $\quad 0.3689$ DF

Upper CL Dif 1.1320 Prob $>|t| \quad 0.7846$

Lower CL Dif -0.9164 Prob $>t \quad 0.3923$

$\begin{array}{lrl}\text { Confidence } & 0.95 \text { Prob }<\mathrm{t} \quad 0.6077\end{array}$

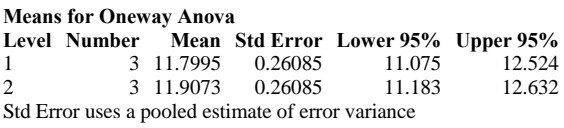

Oneway Analysis of Measured By Block Prep Method=MA, Glass ID=ARG1 , Oxide $=\mathrm{NiO}(\mathrm{wt} \%)$, Targeted $=1.05$

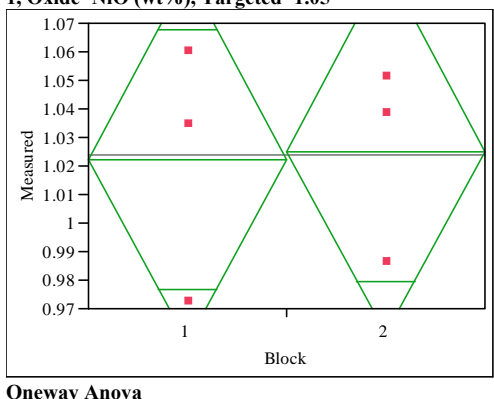

Oneway Anova

$\begin{array}{lr}\text { Rsquare } & 0.002047 \\ \text { Adj Rsquare } & -0.24744 \\ \text { Root Mean Square Error } & 0.040146 \\ \text { Mean of Response } & 1.023726 \\ \text { Observations (or Sum Wgts) } & 6\end{array}$

t Test

Assuming equal variances

Difference $\quad 0.00297$ t Ratio 0.090581

Std Err Dif $\quad 0.03278$ D

Upper CL Dif 0.09398 Prob $>|t| \quad 0.9322$

Lower CL Dif -0.08804 Prob $>t \quad 0.4661$

$\begin{array}{lll}\text { Confidence } & 0.95 \text { Prob }<\mathrm{t} \quad 0.5339\end{array}$

Means for Oneway Anova

Level Number Mean Std Error Lower 95\% Upper 95\%

$\begin{array}{lrrrrr}1 & 3 & 1.02224 & 0.02318 & 0.95789 & 1.0866 \\ 2 & 3 & 1.02521 & 0.02318 & 0.96086 & 1.0896\end{array}$

Std Error uses a pooled estimate of error variance

Oneway Analysis of Measured By Block Prep Method=MA, Glass ID=ARG$\mathbf{1}$, Oxide $=\mathbf{P 2 O}$ ( wt $\%)$, Targeted $=\mathbf{0 . 2 2}$

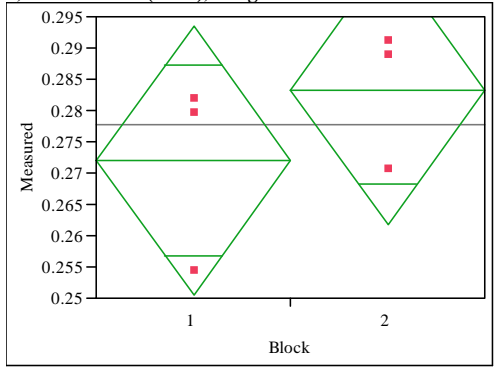

Oneway Anova

Summary of Fit

Rsquare

0.018112

0.013426

$\begin{array}{lr}\text { Mean of Response } & 0.277641 \\ \text { Observations (or Sum Wgts) } & 6\end{array}$

t Test

$2-1$

Assuming equal variances

Difference $\quad 0.01146$ t Ratio 1.0451

Std Err Dif $\quad 0.01096$ DF $\quad 4$

Upper CL Dif 0.04189 Prob $>|t| 0.3550$

Lower CL Dif -0.01898 Prob $>$ t 0.1775

Confidence $\quad 0.95$ Prob $<\mathrm{t} \quad 0.8225$ 


\section{Exhibit C3. Measurements by Block and Sub-Block for Samples of the ARG-1 and Ustd Standards with the Thorium Glasses by Oxide}

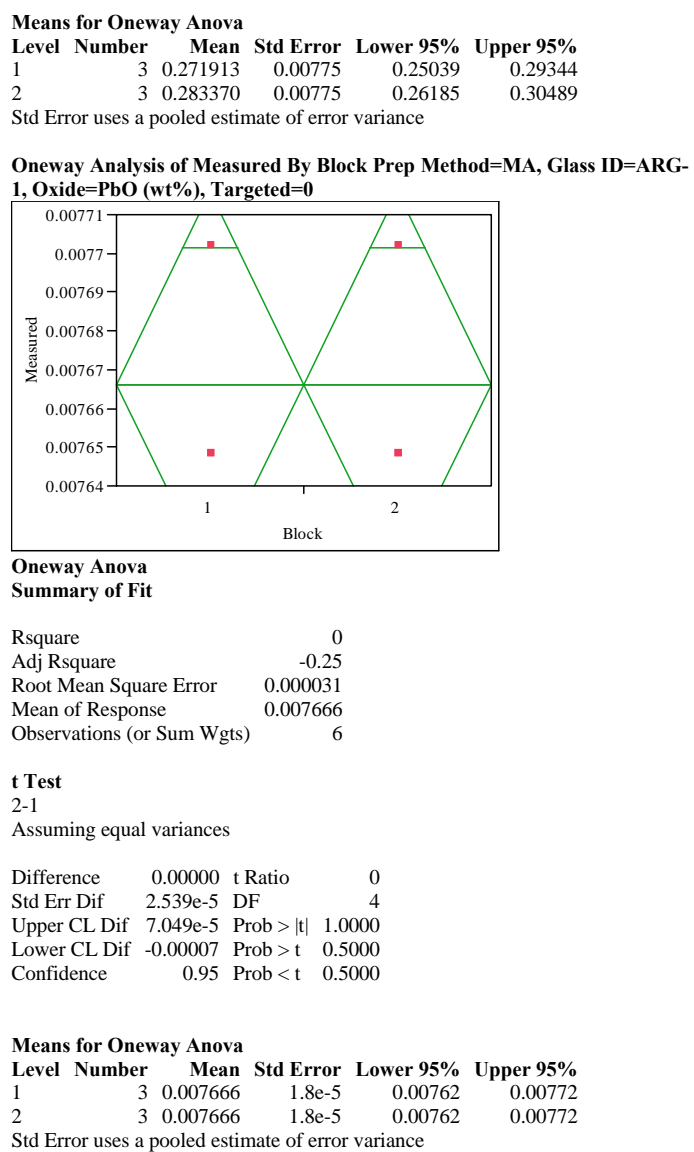

Oneway Analysis of Measured By Block Prep Method=MA, Glass ID=ARG1, Oxide $=\mathrm{SiO} 2(\mathrm{wt} \%)$, Targeted $=47.9$

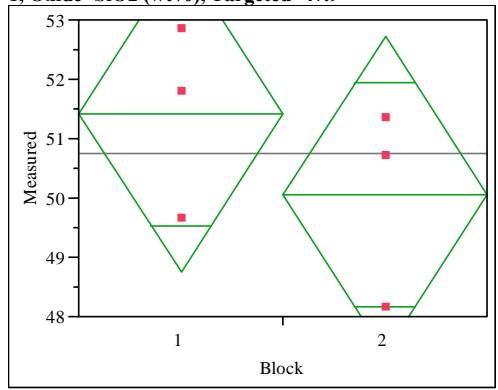

Oneway Anova

Summary of Fit

Adj Rsquare

50.73707

t Test

Assuming equal variances

Difference $\quad-1.3549$ t Ratio $\quad-0.99587$

Std Err Dif 1.3605 D

Upper CL Dif 2.4225 Prob $>|t| \quad 0.3757$

Lower CL Dif -5.1323 Prob $>t \quad 0.8122$

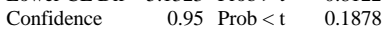

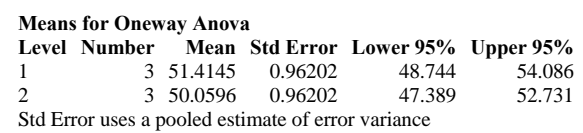

Oneway Analysis of Measured By Block Prep Method=MA, Glass ID=ARG1, Oxide $=\mathrm{SO4}(\mathrm{wt} \%)$, Targeted $=0$

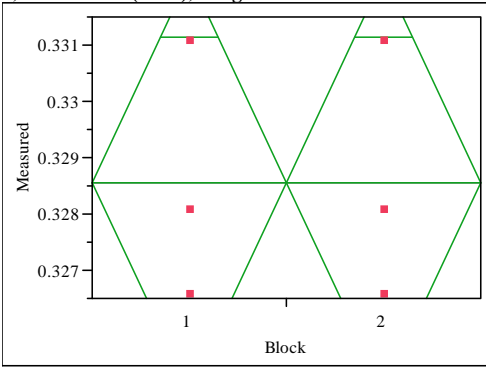

Oneway Anova

Summary of Fit

Rsquare

Adj Rsquare

Root Mean Square Error $\quad-0.25$

Mean of Response $\quad 0.002288$

Observations (or Sum Wgts)

t Test

2-1

Assuming equal variances

$\begin{array}{lrlr}\text { Difference } & 0.00000 & \text { t Ratio } & 0 \\ \text { Std Err Dif } & 0.00187 & \text { DF } & 4 \\ \text { Upper CL Dif } & 0.00519 & \text { Prob }>|t| & 1.0000 \\ \text { Lower CL Dif } & -0.00519 & \text { Prob }>\text { t } & 0.5000 \\ \text { Confidence } & 0.95 & \text { Prob }<t & 0.5000\end{array}$

Means for Oneway Anova

Level Number Mean Std Error Lower 95\% Upper 95\%

$\begin{array}{lrrrrr}1 & 3 & 0.328550 & 0.00132 & 0.32488 & 0.33222 \\ 2 & 3 & 0.328550 & 0.00132 & 0.32488 & 0.33222\end{array}$

Std Error uses a pooled estimate of error variance

Oneway Analysis of Measured By Block Prep Method=MA, Glass ID=ARG1 , Oxide $=$ ThO2 $($ wt $\%)$, Targeted $=0$

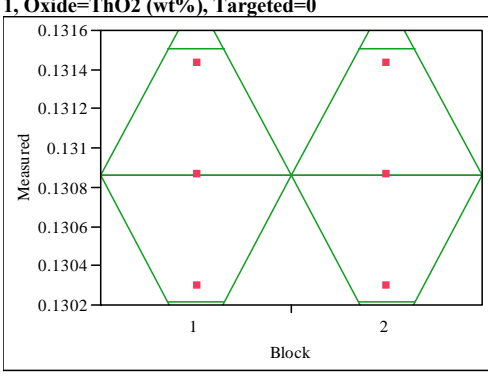

Oneway Anova

Rsquare

Adj Rsquare

Root Mean Square Error $\quad \begin{array}{rr}-0.25 \\ \quad 0.000569\end{array}$

Observations (or Sum Wgts) 6

t Test

2-1

Assuming equal variances

Difference $\quad 0.00000$ t Ratio 0

Upper CL Dif 0.00129 Prob $>|t| 1.0000$ 


\section{Exhibit C3. Measurements by Block and Sub-Block for Samples of the ARG-1 and Ustd Standards with the Thorium Glasses by Oxide}

Lower CL Dif -0.00129 Prob $>t \quad 0.5000$

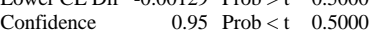

Means for Oneway Anova

Level Number Mean Std Error Lower 95\% Upper 95\%

$\begin{array}{lllrrr}1 & 3 & 0.130859 & 0.00033 & 0.12995 & 0.13177 \\ 2 & 3 & 0.130859 & 0.00033 & 0.12995 & 0.13177\end{array}$

Std Error uses a pooled estimate of error variance

Oneway Analysis of Measured By Block Prep Method=MA, Glass ID=ARG1, Oxide $=$ TiO2 $($ wt $\%)$, Targeted $=1.15$

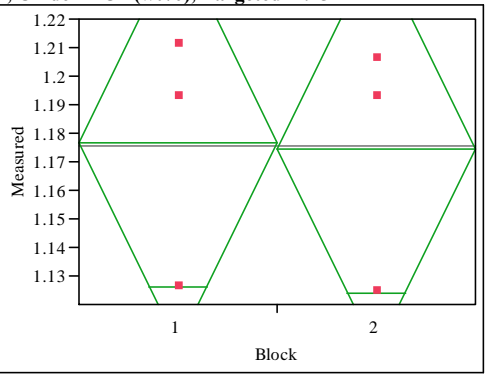

Oneway Anova

Summary of Fit

Rsquare

0.000944

Adj Rsquare

$-0.24882$

0.24882

Root Mean Square Error $\quad 0.0443$

Mean of Response

1.175384
6

t Test

2-1

Assuming equal variances

Difference $\quad-0.00222$ t Ratio $\quad-0.06147$

Std Err Dif $\quad 0.03618$ DF 4

Upper CL Dif 0.09822 Prob $>|t| \quad 0.9539$

Lower CL Dif -0.10267 Prob $>\mathrm{t} \quad 0.5230$

$\begin{array}{lrr}\text { Confidence } & 0.95 \text { Prob }<\mathrm{t} & 0.4770\end{array}$

Means for Oneway Anova

Level Number Mean Std Error Lower 95\% Upper 95\%

$\begin{array}{llrrrr}1 & 3 & 1.17650 & 0.02558 & 1.1055 & 1.2475 \\ 2 & 3 & 1.17427 & 0.02558 & 1.1032 & 1.2453\end{array}$

$2 \quad 3 \quad 1.17427 \quad 0.02558 \quad 1.1032$

Oneway Analysis of Measured By Block Prep Method=MA, Glass ID=ARG1, Oxide $=$ U3O8 $($ wt $\%)$, Targeted $=0$

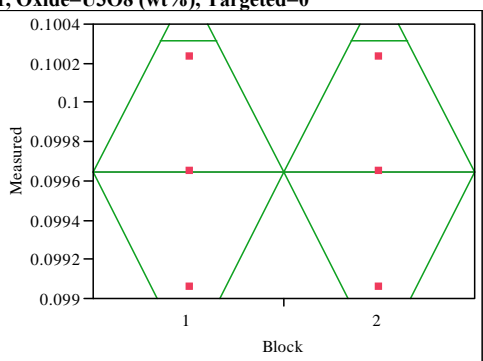

Summary of Fit

Rsquare

Adj Rsquare

Root Mean Square Error

Mean of Response

0.00059
-0.099642

Observations (or Sum Wgts)

t Test

$2-1$

Difference $\quad 0.00000$ t Ratio

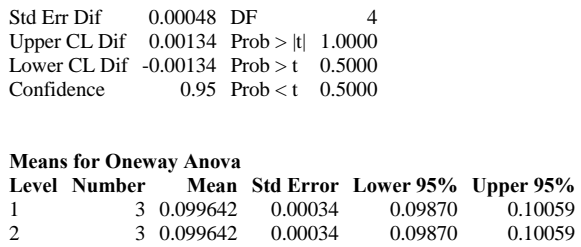

Means for Oneway Anova

Level Number Mean Std Error Lower 95\% Upper 95\%

$\begin{array}{lrrrrr}1 & 3 & 0.099642 & 0.00034 & 0.09870 & 0.10059\end{array}$

$\begin{array}{llllll}1 & 3 & 0.099642 & 0.00034 & 0.09870 & 0.10059 \\ 2 & 3 & 0.099642 & 0.00034 & 0.09870 & 0.10059\end{array}$

Std Error uses a pooled estimate of error variance

Oneway Analysis of Measured By Block Prep Method=MA, Glass ID=ARG1, Oxide $=\mathrm{ZnO}(\mathrm{wt} \%)$, Targeted $=\mathbf{0 . 0 2}$

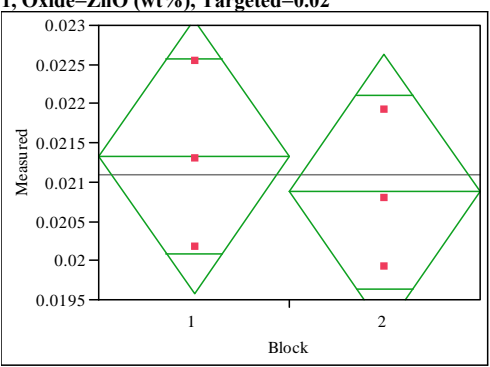

Oneway Anova

Summary of Fit

Rsquare

Rsquare

Root Mean Square Error $\quad 0.001095$

Mean of Response 0.021099

Observations (or Sum Wgts)

t Test

Assuming equal variances

Difference $\quad-0.00046$ t Ratio $\quad-0.51066$ Std Err Dif $\quad 0.00089$ DF

Upper CL Dif 0.00203 Prob $>|t| \quad 0.6365$

Lower CL Dif -0.00294 Prob $>t \quad 0.6818$

$\begin{array}{lrl}\text { Confidence } & 0.95 \text { Prob }<\mathrm{t} \quad 0.3182\end{array}$

Means for Oneway Anova

Level Number Mean Std Error Lower 95\% Upper 95\%

$\begin{array}{lrrrrr}1 & 3 & 0.021328 & 0.00063 & 0.01957 & 0.02308 \\ 2 & 3 & 0.020871 & 0.00063 & 0.01912 & 0.02263\end{array}$

Std Error uses a pooled estimate of error variance

Oneway Analysis of Measured By Block Prep Method=MA, Glass ID=ARG1, Oxide $=\mathrm{ZrO} 2(\mathrm{wt} \%)$, Targeted $=\mathbf{0 . 1 3}$

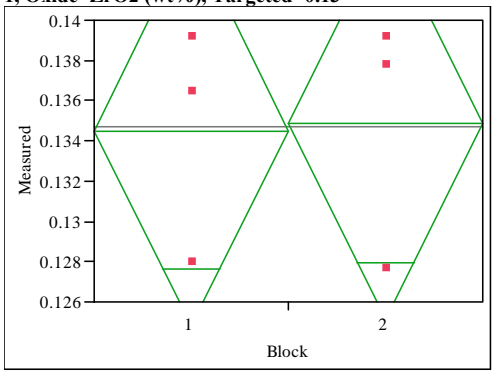

Summary of Fit

Rsquare

Adj Rsquare

Root Mean Square Error $\quad-0.24835$

Observations (or Sum Wgts)

t Test

Assuming equal variances 


\section{Exhibit C3. Measurements by Block and Sub-Block for Samples of the ARG-1 and Ustd Standards with the Thorium Glasses by Oxide}

$\begin{array}{lrlr}\text { Difference } & 0.00036 & \mathrm{t} \text { Ratio } & 0.072715 \\ \text { Std Err Dif } & 0.00495 \text { DF } & 4 \\ \text { Upper CL Dif } & 0.01411 \text { Prob }>|t| & 0.9455 \\ \text { Lower CL Dif } & -0.01339 \text { Prob }>\mathrm{t} & 0.4728 \\ \text { Confidence } & 0.95 \text { Prob }<\mathrm{t} & 0.5272\end{array}$

Means for Oneway Anova
Level Number Mean Std Error Lower $95 \%$ Upper $95 \%$

$\begin{array}{lrrrrr}\text { Level } & \text { Number } & \text { Mean } & \text { Std Error } & \text { Lower 95\% } & \text { Upper 95\% } \\ 1 & 3 & 0.134495 & 0.00350 & 0.12477 & 0.14422\end{array}$

$\begin{array}{llllll}2 & 3 & 0.134855 & 0.00350 & 0.12513 & 0.14458\end{array}$

Std Error uses a pooled estimate of error variance

Oneway Analysis of Measured By Block Prep Method=MA, Glass ID=U-std, Oxide $=\mathrm{Al} 2 \mathrm{O} 3(\mathrm{wt} \%)$, Targeted $=4.1$

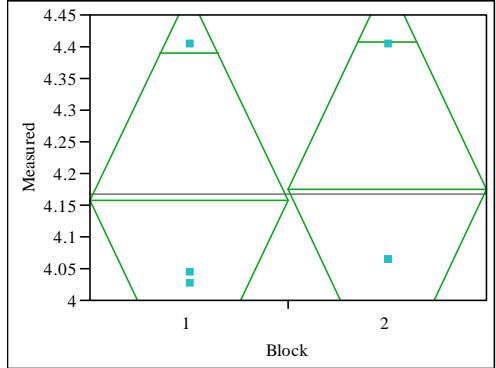

Oneway Anova

Summary of Fit

Rsquare $\quad 0.003181$

Adj Rsquare $\quad-0.24602$

Root Mean Square Error $\quad 0.204817$

$\begin{array}{lr}\text { Mean of Response } & 4.166348 \\ \text { Observations (or Sum Wgts) } & 6\end{array}$

t Test

t T

Assuming equal variances

Difference $\quad 0.01889$ t Ratio $\quad 0.112987$

$\begin{array}{llr}\text { Std Err Dif } & 0.16723 \text { DF } & 4\end{array}$

Upper CL Dif 0.48321 Prob $>|t| \quad 0.9155$

$\begin{array}{lll}\text { Lower CL Dif }-0.44542 \text { Prob }>t & 0.4577\end{array}$

$\begin{array}{lrl}\text { Confidence } & 0.95 \text { Prob }<\mathrm{t} \quad 0.5423\end{array}$

Means for Oneway Anova

Level Number Mean Std Error Lower 95\% Upper 95\%

$\begin{array}{lrrrrr}\text { Level } & \text { Number } & \text { Mean } & \text { Std Error } & \text { Lower 95\% } & \text { Upper 95\% } \\ 1 & 3 & 4.15690 & 0.11825 & 3.8286 & 4.4852\end{array}$

$\begin{array}{llllll}1 & 3 & 4.17579 & 0.11825 & 3.8475 & 4.5041\end{array}$

Std Error uses a pooled estimate of error variance

Oneway Analysis of Measured By Block Prep Method=MA, Glass ID=U-std, Oxide $=\mathrm{BaO}\left(\mathrm{wt}^{\mathrm{O}} \mathrm{)}\right)$, Targeted $=\mathbf{0}$

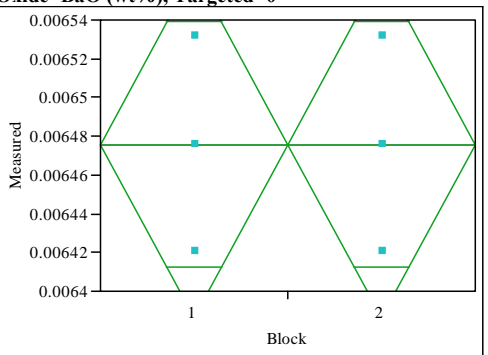

Oneway Anova

Summary of Fit

Rsquare

Adj Rsquare

Root Mean Squat $\quad-0.25$

Observations (or Sum Wgts)

t Test
$2-1$
Assuming equal variances

$\begin{array}{lrlr}\text { Difference } & 0.00000 & \mathrm{t} \text { Ratio } & 0 \\ \text { Std Err Dif } & 4.558 \mathrm{e}-5 & \text { DF } & 4 \\ \text { Upper CL Dif } & 0.00013 & \text { Prob }>|t| & 1.0000 \\ \text { Lower CL Dif } & -0.00013 & \text { Prob }>\mathrm{t} & 0.5000 \\ \text { Confidence } & 0.95 & \text { Prob }<\mathrm{t} & 0.5000\end{array}$

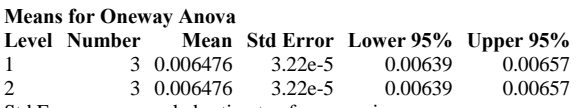

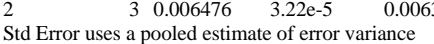

Oneway Analysis of Measured By Block Prep Method=MA, Glass ID=U-std, Oxide $=\mathbf{C a O}(\mathbf{w t} \%)$, Targeted $=\mathbf{1 . 3 0 1}$

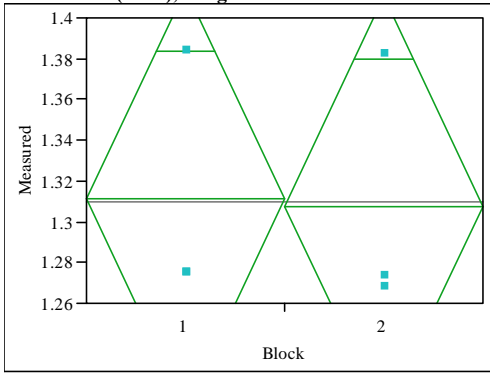

way Anova

Summary of Fit

Rsquare

Root Mean Square Error $\quad 0.063854$

Mean of Response$$
\text { ) }
$$

t Test

Assuming equal variances

Difference $\quad-0.00326$ t Ratio -0.06262

Std Err Dif 0.05214 DF

Upper CL Dif 0.14149 Prob $>|t| r \mid \begin{array}{r}4 \\ 0.9531\end{array}$

Lower CL Dif -0.14802 Prob $>t \quad 0.5235$

$\begin{array}{lrl}\text { Confidence } & 0.95 \text { Prob }<\mathrm{t} & 0.4765\end{array}$

Means for Oneway Anova

Level Number Mean Std Error Lower 95\% Upper 95\%

$\begin{array}{lrrrrr}1 & 3 & 1.31105 & 0.03687 & 1.2087 & 1.4134 \\ 2 & 3 & 1.30779 & 0.03687 & 1.2054 & 1.4101\end{array}$

Std Error uses a pooled estimate of error variance

Oneway Analysis of Measured By Block Prep Method=MA, Glass ID=U-std, Oxide $=\mathbf{C d O}($ wt $\%)$, Targeted $=0$

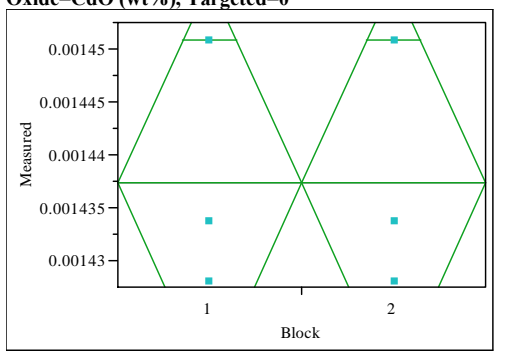

Oneway Anova

Summary of Fit

Rsquare

$\begin{array}{lr}\text { Adj Rsquare } & -0.25 \\ \text { Root Mean Square Error } & 1.189 \mathrm{e}-5\end{array}$

Mean of Respons

Observations (or Sum

0.001437

t Test

2-1 


\section{Exhibit C3. Measurements by Block and Sub-Block for Samples of the ARG-1 and Ustd Standards with the Thorium Glasses by Oxide}

Assuming equal variances

$\begin{array}{lrlr}\text { Difference } & 0.00000 & \text { t Ratio } & 0 \\ \text { Std Err Dif } & 9.708 \mathrm{e}-6 & \text { DF } & 4 \\ \text { Upper CL Dif } & 0.000027 & \text { Prob }>|t| & 1.0000 \\ \text { Lower CL Dif } & -2.7 \mathrm{e}-5 & \text { Prob }>\mathrm{t} & 0.5000 \\ \text { Confidence } & 0.95 & \text { Prob }<\mathrm{t} & 0.5000\end{array}$

Means for Oneway Anova

Level Number Mean Std Error Lower 95\% Upper 95\%

$\begin{array}{lrrrrr}1 & 3 & 0.001437 & 6.8644 \mathrm{e}-6 & 0.00142 & 0.00146\end{array}$

$\begin{array}{llllll}1 & 3 & 0.001437 & 6.8644 \mathrm{e}-6 & 0.00142 & 0.00146\end{array}$

Std Error uses a pooled estimate of error variance

Oneway Analysis of Measured By Block Prep Method=MA, Glass ID=U-std, Oxide $=\mathrm{Ce} 2 \mathrm{O} 3(\mathrm{wt} \%)$, Targeted $=0$

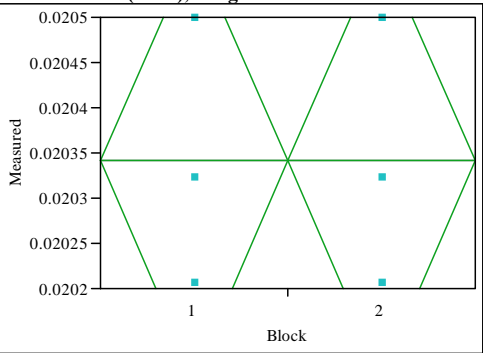

Oneway Anova

Summary of Fit

Rsquare

Adj Rsquare

Root Mean Square Eror $\quad-0.25$

Mean of Response $\quad 0.000147$

Observations (or Sum Wgts)

t Test

2-1

Assuming equal variances

Difference $\quad 0.00000$ t Ratio 0 Std Err Dif $\quad 0.00012$ DF

Upper CL Dif 0.00033 Prob $>|t| 1.0000$

Lower CL Dif -0.00033 Prob $>t \quad 0.5000$

Means for Oneway Anova

Level Number Mean Std Error Lower 95\% Upper 95\%

$\begin{array}{lrrrrr}1 & 3 & 0.020342 & 8.51 \mathrm{e}-5 & 0.02011 & 0.02058 \\ 2 & 3 & 0.020342 & 8.51 \mathrm{e}-5 & 0.02011 & 0.02058\end{array}$

Std Error uses a pooled estimate of error variance

Oneway Analysis of Measured By Block Prep Method=MA, Glass ID=U-std, Oxide $=\mathbf{C r 2 O 3}(\mathrm{wt} \%)$, Targeted $=0$

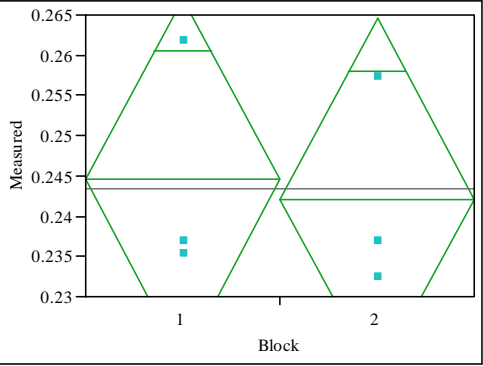

Oneway Anova

Summary of Fit

Rsquare

Adj Rsquare

Ad Rsqur

Mean of Response $\quad 0.243356$

Observations (or Sum Wgts) 6

t Test

$2-1$
Assuming equal variances

$\begin{array}{lrlr}\text { Difference } & -0.00244 & \text { t Ratio } & -0.21243 \\ \text { Std Err Dif } & 0.01147 & \text { DF } & 4 \\ \text { Upper CL Dif } & 0.02940 & \text { Prob }>|t| & 0.8422 \\ \text { Lower CL Dif } & -0.03427 & \text { Prob }>\mathrm{t} & 0.5789 \\ \text { Confidence } & 0.95 & \text { Prob }<\mathrm{t} & 0.4211\end{array}$

\begin{tabular}{lrrrrr}
\multicolumn{2}{l}{$\begin{array}{l}\text { Means for Oneway Anova } \\
\text { Level Number }\end{array}$} & Mean & Std Error & Lower 95\% & Upper 95\% \\
1 & 3 & 0.244574 & 0.00811 & 0.22206 & 0.26709 \\
2 & 3 & 0.242138 & 0.00811 & 0.21963 & 0.26465 \\
Std Error uses a pooled estimate of error variance &
\end{tabular}

Oneway Analysis of Measured By Block Prep Method=MA, Glass ID=U-std, Oxide $=\mathbf{C u O}\left(w^{\circ} \%\right)$, Targeted $=0$

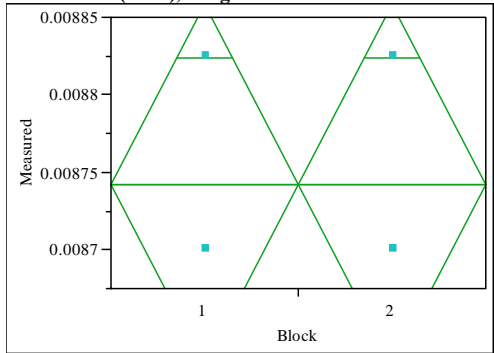

Oneway Anova

Summary of Fit

Rsquare

$\begin{array}{lr}\text { Adj Rsquare } & -0.25 \\ \text { Root Mean Square Error } & 7.227 \mathrm{e}-5\end{array}$

Mean of Response $\quad 0.008742$

Observations (or Sum Wgts)

t Test

2-1

Assuming equal variances

Difference $\quad 0.00000$ t Ratio 0 Std Err Dif $\quad 0.000059$ D

Upper CL Dif 0.00016 Prob $>|t| 1.0000$

Lower CL Dif -0.00016 Prob $>t \quad 0.5000$

Confidence

Means for Oneway Anova

Level Number Mean Std Error Lower 95\% Upper 95\%

$\begin{array}{lllllr}1 & 3 & 0.008742 & 4.17 \mathrm{e}-5 & 0.00863 & 0.00886 \\ 2 & 3 & 0.008742 & 4.17 \mathrm{e}-5 & 0.00863 & 0.00886\end{array}$

Std Error uses a pooled estimate of error variance

Oneway Analysis of Measured By Block Prep Method=MA, Glass ID=U-std, Oxide $=\mathrm{Fe} 2 \mathrm{O} 3(\mathrm{wt} \%)$, Targeted $=13.196$

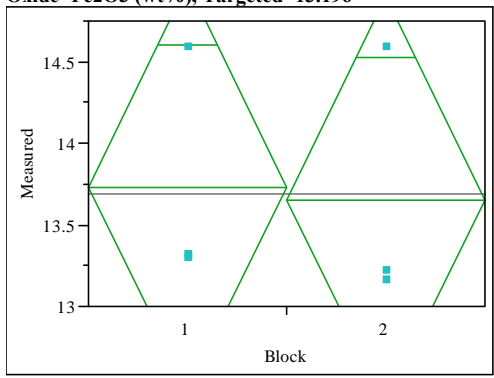

Oneway Anova

Summary of Fit

Rsquare

$\begin{array}{lr}\text { Adj Rsquare } & -0.2449 \\ \text { Root Mean Square Error } \quad 0.774923\end{array}$

0.004082

Mean of Response 13.68938

Observations (or Sum Wgts) 


\section{Exhibit C3. Measurements by Block and Sub-Block for Samples of the ARG-1 and Ustd Standards with the Thorium Glasses by Oxide}

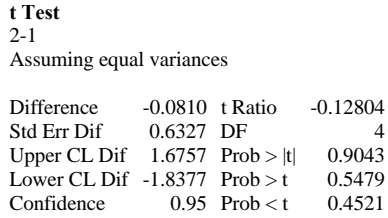

Oneway Analysis of Measured By Block Prep Method=MA, Glass ID=U-std, Oxide $=$ K2O $(w \mathbf{w} \%)$, Targeted $=2.999$

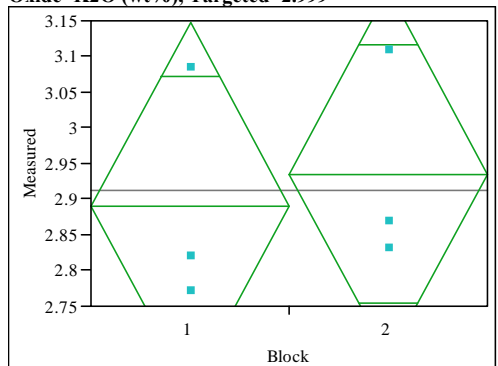

Oneway Anova

Summary of Fit

Rsquare

Adj Rsquare

0.159884

2.913124

Observations (or Sum Wgts)

t Test

2-1

Assuming equal variances

Difference $\quad 0.04417$ t Ratio 0.33834 Std Err Dif $\quad 0.13054$ DF

Upper CL Dif 0.40662 Prob $>|t| \quad 0.7521$

Lower CL Dif -0.31828 Prob $>t \quad 0.3761$

Confidence $\quad 0.95$ Prob $<\mathrm{t} \quad 0.6239$

Means for Oneway Anova

Level Number Mean Std Error Lower 95\% Upper 95\%

$\begin{array}{lrrrrr}1 & 3 & 2.89104 & 0.09231 & 2.6347 & 3.1473 \\ 2 & 3 & 2.93521 & 0.09231 & 2.6789 & 3.1915\end{array}$

Std Error uses a pooled estimate of error variance

Oneway Analysis of Measured By Block Prep Method=MA, Glass ID=U-std, Oxide $=\mathrm{La2O3}(\mathrm{wt} \%)$, Targeted $=0$

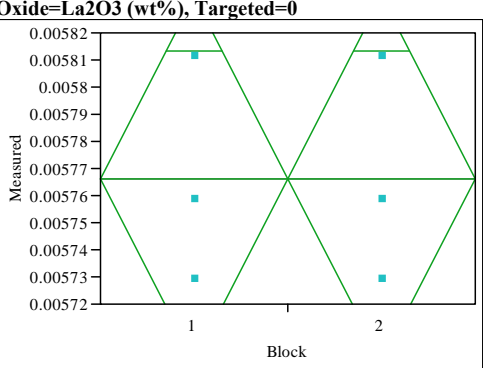

Oneway Anova
Summary of Fit

Rsquare

Adj Rsquare

Root Mean Square Error

Mean of Response

Observations (or Sum Wgts) t Test

Assuming equal variances

$\begin{array}{lrlr}\text { Difference } & 0.00000 & \text { t Ratio } & 0 \\ \text { Std Err Dif } & 0.000034 & \text { DF } & 4 \\ \text { Upper CL Dif } & 9.431 \mathrm{e}-5 & \text { Prob }>|t| & 1.0000 \\ \text { Lower CL Dif } & -9.43 e-5 & \text { Prob }>\text { t } & 0.5000\end{array}$

Means for Oneway Anova

\begin{tabular}{rrrrrr} 
Level & Number & Mean & Std Error & Lower 95\% & Upper 95\% \\
1 & 3 & 0.005766 & $2.4 \mathrm{e}-5$ & 0.00570 & 0.00583 \\
\hline & 3 & 0.00566 & 2.45 & 0.00570 & 0.00583
\end{tabular}

$\begin{array}{llllll}1 & 3 & 0.005766 & 2.4 \mathrm{e}-5 & 0.00570 & 0.00583 \\ 2 & 3 & 0.00570 & 0.00583\end{array}$

Std Error uses a pooled estimate of error variance

Oneway Analysis of Measured By Block Prep Method=MA, Glass ID=U-std, Oxide $=$ Li2O $($ wt $\%)$, Targeted $=\mathbf{3 . 0 5 7}$

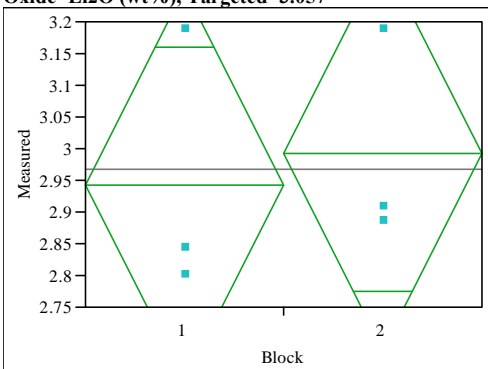

Oneway Anova

Summary of Fit

Adj Rsquare

$-0.21857$

nesponse

2.967414

Observations (or Sum Wgts)

t Test

2-1

Assuming equal variances

Difference $\quad 0.05023$ t Ratio 0.321182 Std Err Dif $\quad 0.15640$ DF

Upper CL Dif 0.48448 Prob $>|t| \quad 0.7642$

Lower CL Dif -0.38401 Prob $>$ t $\quad 0.3821$

$\begin{array}{lrr}\text { Lower CL Dif } & -0.38401 \text { Prob }>t & 0.3821 \\ \text { Confidence } & 0.95 \text { Prob }<\mathrm{t} & 0.6179\end{array}$

Means for Oneway Anova

Level Number Mean Std Error Lower 95\% Upper 95\% $\begin{array}{lrrrrr}1 & 3 & 2.94230 & 0.11059 & 2.6352 & 3.2494 \\ 2 & 3 & 2.99253 & 0.11059 & 2.6855 & 3.2996\end{array}$

Std Error uses a pooled estimate of error variance

Oneway Analysis of Measured By Block Prep Method=MA, Glass ID=U-std, Oxide $=\mathrm{MgO}(\mathrm{wt} \%)$, Targeted $=1.21$

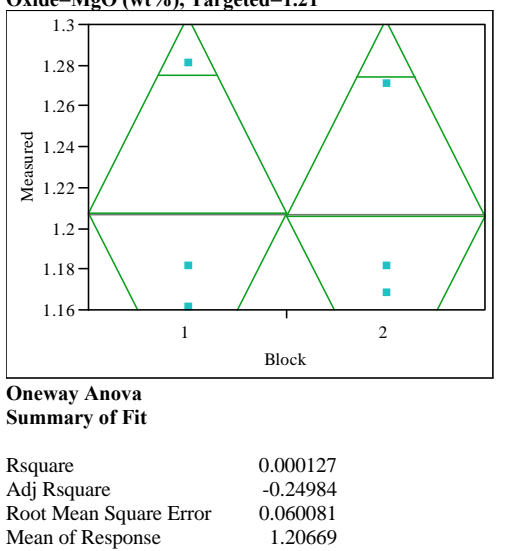




\section{Exhibit C3. Measurements by Block and Sub-Block for Samples of the ARG-1 and Ustd Standards with the Thorium Glasses by Oxide}

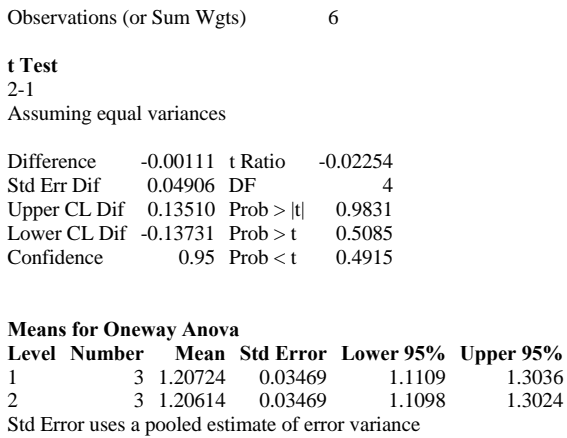

Oneway Analysis of Measured By Block Prep Method=MA, Glass ID=U-std, Oxide $=\mathrm{MnO}(\mathrm{wt} \%)$, Targeted $=\mathbf{2 . 8 9 2}$

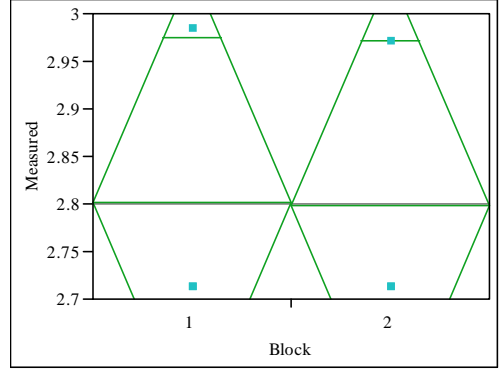

Oneway Anova

$-0.24963$

Error $\quad 0.152868$

Mean of Response 2.799752

Observations (or Sum Wgts)

t Test

2-1

Assuming equal variances

Difference $\quad-0.00430$ t Ratio $\quad-0.03448$

Std Err Dif $\quad 0.12482$ DF $\quad 4$

Upper CL Dif 0.34224 Prob $>|t| \quad 0.9741$

Lower CL Dif -0.35085 Prob $>t \quad 0.5129$

$\begin{array}{lrl}\text { Confidence } & 0.95 \text { Prob }<\mathrm{t} & 0.4871\end{array}$

Means for Oneway Anova

Level Number Mean Std Error Lower 95\% Upper 95\%

$\begin{array}{lllllr}1 & 3 & 2.80190 & 0.08826 & 2.5569 & 3.0469\end{array}$

Std Error uses a pooled estimate of error variance

Oneway Analysis of Measured By Block Prep Method=MA, Glass ID=U-std, Oxide $=\mathrm{Na2O}(\mathrm{wt} \%)$, Targeted $=11.795$

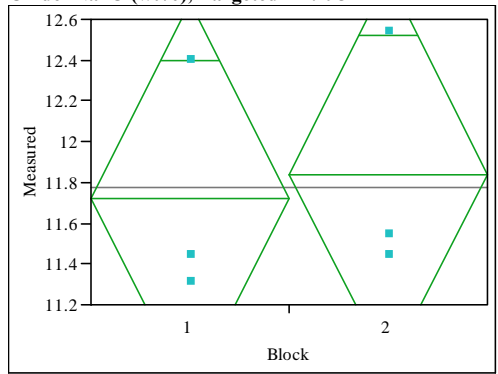

Oneway Anova

Summary of Fit

Rsquare 0.015092

Adj Rsquare $\begin{array}{lr}\text { Root Mean Square Error } & 0.600175 \\ \text { Mean of Response } & 11.77927 \\ \text { Observations (or Sum Wgts) } & 6\end{array}$

t Test

2-1

Assuming equal variances

$\begin{array}{lrlr}\text { Difference } & 0.1213 & \text { t Ratio } & 0.247571 \\ \text { Std Err Dif } & 0.4900 & \text { DF } & 4 \\ \text { Upper CL Dif } & 1.4819 & \text { Prob }>|t| & 0.8167 \\ \text { Lower CL Dif } & -1.2393 & \text { Prob }>\text { t } & 0.4083 \\ \text { Confidence } & 0.95 & \text { Prob }<\mathrm{t} & 0.5917\end{array}$

Means for Oneway Anova

Level Number Mean Std Error Lower 95\% Upper 95\%

$\begin{array}{llllrr}1 & 3 & 11.7186 & 0.34651 & 10.757 & 12.681\end{array}$

Std Error uses a pooled estimate of error variance $\quad 12.802$

Oneway Analysis of Measured By Block Prep Method=MA, Glass ID=U-std, Oxide $=\mathrm{NiO}(\mathrm{wt} \%)$, Targeted $=1.12$

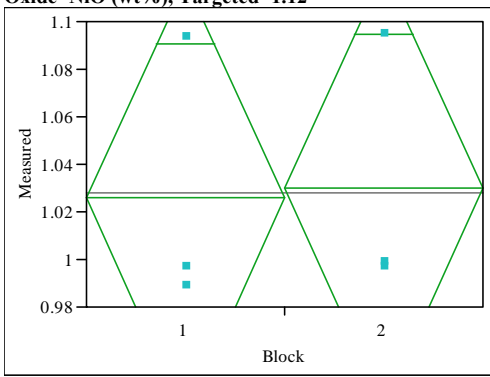

Oneway Anova

Summary of Fi

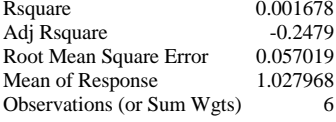

Observations (or Sum Wgts)

t Test

Assuming equal variances

Difference $\quad 0.00382$ t Ratio 0.081998

$\begin{array}{llll}\text { Std Err Dif } & 0.04656 \text { DF } & 4\end{array}$

$\begin{array}{lrr}\text { Std Err Dif } & 0.04656 & \text { DF } \\ \text { Upper CL Dif } & 0.13308 \text { Prob }>|t| r \mid & 0.9386\end{array}$

Lower CL Dif -0.12544 Prob $>\mathrm{t} \quad 0.4693$

$\begin{array}{lrl}\text { Confidence } & 0.95 \text { Prob }<\mathrm{t} \quad 0.5307\end{array}$

Means for Oneway Anova

Level Number Mean Std Error Lower 95\% Upper 95\%

\begin{tabular}{lrrrrr}
1 & 3 & 1.02606 & 0.03292 & 0.93466 & 1.1175 \\
\hline & 3 & 1.02988 & 0.03292 & 0.93848 & 1.1213
\end{tabular}

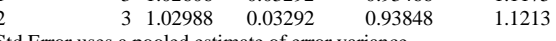

Std Error uses a pooled estimate of error variance

Oneway Analysis of Measured By Block Prep Method=MA, Glass ID=U-std, Oxide $=\mathbf{P 2 O 5}(\mathbf{w t} \%)$, Targeted $=\mathbf{0}$

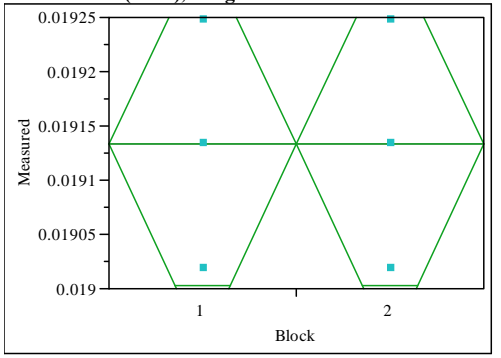

Summary of Fit 


\section{Exhibit C3. Measurements by Block and Sub-Block for Samples of the ARG-1 and Ustd Standards with the Thorium Glasses by Oxide}

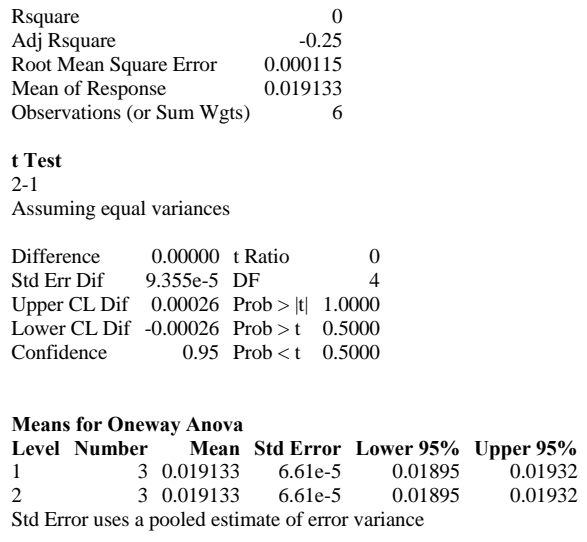

Oneway Analysis of Measured By Block Prep Method=MA, Glass ID=U-std, Oxide $=\mathbf{P b O}(\mathbf{w t} \%)$, Targeted $=\mathbf{0}$

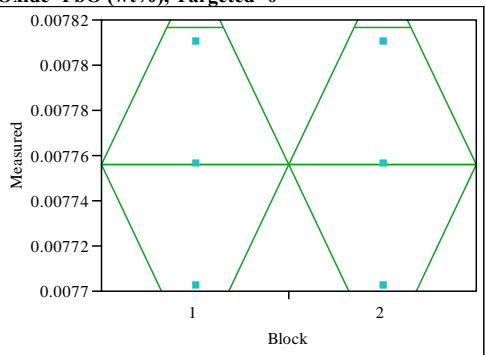

Oneway Anova

Summary of Fit

Rsquare

Adj Rsquare

Root Mean Square Eror $\quad-0.25$

5.386e- 5

Observations (or Sum Wgts)

t Test

2-1

Assuming equal variances

Difference $\quad 0.00000$ t Ratio

Std Err Dif $\quad 0.000044$ DF

Upper CL Dif 0.00012 Prob $>|t| 1.0000$

Lower CL Dif -0.00012 Prob $>t \quad 0.5000$

Confidence $\quad 0.95$ Prob $<t \quad 0.5000$

Means for Oneway Anova

Level Number Mean Std Error Lower 95\% Upper 95\%

$\begin{array}{lllrrr}1 & 3 & 0.007756 & 3.11 \mathrm{e}-5 & 0.00767 & 0.00784 \\ 2 & 3 & 0.007756 & 3.11 \mathrm{e}-5 & 0.00767 & 0.00784\end{array}$

Std Error uses a pooled estimate of error variance

Oneway Analysis of Measured By Block Prep Method=MA, Glass ID=U-std, Oxide $=\mathrm{SiO} 2(\mathrm{wt} \%)$, Targeted $=\mathbf{4 5 . 3 5 3}$

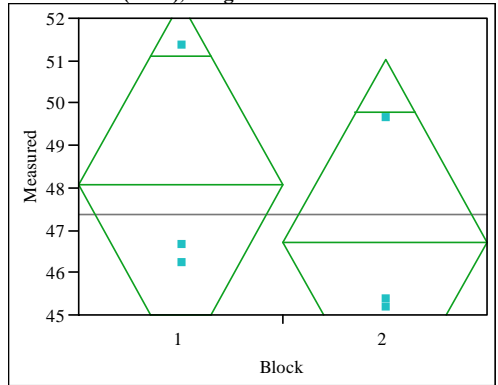

Oneway Anova

Summary of Fit

$\begin{array}{ll}\text { Root Mean Square Error } & 2.69614 \\ \text { Mean of Response } & 47.3855\end{array}$

Observations (or Sum Wgts)

t Test

Assuming equal variances

Difference $\quad-1.3549$ t Ratio $\quad-0.61547$

Std Err Dif $\quad 2.2014$ DF

Upper CL Dif 4.7571 Prob $>|t| \quad 0.5715$

Lower CL Dif -7.4669 Prob $>t \quad 0.7142$

Confidence $\quad 0.95$ Prob $<\mathrm{t} \quad 0.2858$

Means for Oneway Anova

Level Number Mean Std Error Lower 95\% Upper 95\%

$\begin{array}{lrrrrr}\text { Level } & \text { Number } & \text { Mean } & \text { Std Error } & \text { Lower } \mathbf{9 5 \%} & \text { Upper 95\% } \\ 1 & 3 & 48.0629 & 1.5566 & 43.741 & 52.385 \\ 2 & 3 & 46.7081 & 1.5566 & 42.386 & 51.030\end{array}$

Std Error uses a pooled estimate of error variance

Oneway Analysis of Measured By Block Prep Method=MA, Glass ID=U-std, Oxide $=\mathrm{SO} 4($ wt $\%)$, Targeted $=0$

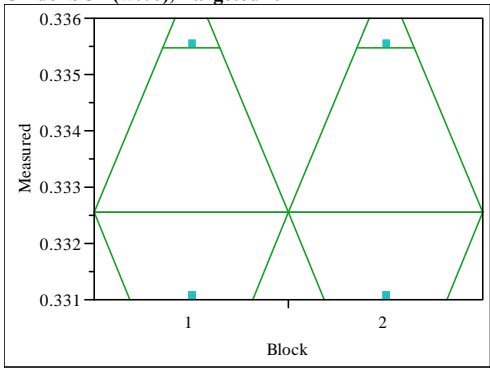

Rsquare

Adj Rsquare

Root Mean Square Error $\quad 0.002595$

Mean of Response

0.332545
6

t Test

Assuming equal variances

Difference $\quad 0.00000$ t Ratio

Std Err Dif $\quad 0.00212$ DF

Lower CL Dif -0.00588 Prob $>t \quad 0.5000$

Confidence $\quad 0.95$ Prob $<\mathrm{t} \quad 0.5000$

Means for Oneway Anova

$\begin{array}{rrrrrr}\text { Level } & \text { Number } & \text { Mean } & \text { Std Error } & \text { Lower 95\% } & \text { Upper 95\% } \\ 1 & 3 & 0.332545 & 0.00150 & 0.32839 & 0.33670\end{array}$

$\begin{array}{llllll}1 & 3 & 0.332545 & 0.00150 & 0.32839 & 0.33670 \\ 2 & 3 & 0.332545 & 0.00150 & 0.32839 & 0.33670\end{array}$

Std Error uses a pooled estimate of error variance 


\section{Exhibit C3. Measurements by Block and Sub-Block for Samples of the ARG-1 and Ustd Standards with the Thorium Glasses by Oxide}

Oneway Analysis of Measured By Block Prep Method=MA, Glass ID=U-std, Oxide $=\mathrm{ThO} 2(\mathrm{wt} \%)$, Targeted $=0$

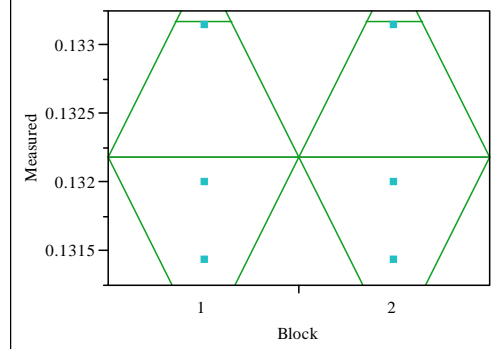

Oneway Anova

Summary of Fit

Rsquare

Adj Rsquare

Root Mean Square Error $\quad-0.25$

Mean of Response

Observations (or Sum Wgts)

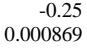
0.132186

t Test

Assuming equal variances

Difference $\quad 0.00000$ t Ratio $\quad 0$ Std Err Dif $\quad 0.00071$ DF

Upper CL Dif 0.00197 Prob $>|t| 1.0000$

Lower CL Dif -0.00197 Prob $>t \quad 0.5000$

$\begin{array}{lll}\text { Confidence } & 0.95 \text { Prob }<\mathrm{t} \quad 0.5000\end{array}$

Means for Oneway Anova

Level Number Mean Std Error Lower 95\% Upper 95\%

$\begin{array}{lrrrrr}\text { Level Number } & \text { Mean } & \text { Std Error } & \text { Lower 95\% } & \text { Upper 95\% } \\ 1 & 3 & 0.132186 & 0.00050 & 0.13079 & 0.13358\end{array}$

$\begin{array}{llllll}1 & 3 & 0.132186 & 0.00050 & 0.13079 & 0.13358\end{array}$

Std Error uses a pooled estimate of error variance

Oneway Analysis of Measured By Block Prep Method=MA, Glass ID=U-std, Oxide $=$ TiO2 $($ wt $\%)$, Targeted $=1.049$

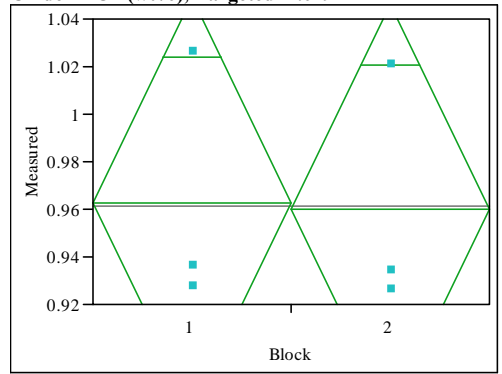

Oneway Anova

Summary of Fit

Rsquare

Adj Rsquare

Root Mean Square Error

$-0.24874$

0.053619

Mean of Response

0.961602
6

t Test

2-1

Assuming equal variances

Difference $\quad-0.00278$ t Ratio $\quad-0.0635$

Std Err Dif $\quad 0.04378$ DF

Upper CL Dif 0.11877 Prob $>|t| \quad 0.9524$

Lower CL Dif -0.12433 Prob $>t \quad 0.5238$

$\begin{array}{lrl}\text { Confidence } & 0.95 \text { Prob }<\mathrm{t} & 0.4762\end{array}$

Means for Oneway Anova
Level Number Mean Std Error Lower 95\% Upper 95\%

$\begin{array}{lrrrrr}\text { Level } & \text { Number } & \text { Mean } & \text { Std Error } & \text { Lower 95\% } & \text { Upper 95\% } \\ 1 & 3 & 0.962992 & 0.03096 & 0.87704 & 1.0489\end{array}$

$\begin{array}{llllll}1 & 3 & 0.960212 & 0.03096 & 0.87426 & 1.0462\end{array}$

Std Error uses a pooled estimate of error variance
Oneway Analysis of Measured By Block Prep Method=MA, Glass ID=U-std, Oxide $=$ U3O8 (wt $\%$ ), Targeted $=\mathbf{2 . 4 0 6}$

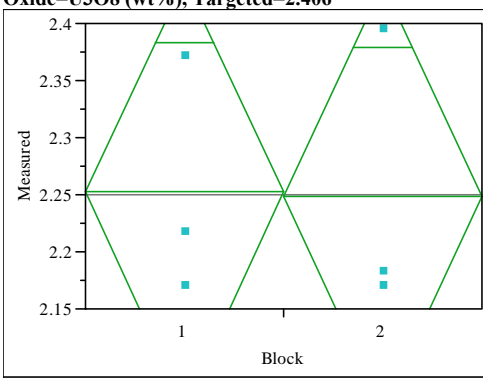

\section{Oneway Anova
Summary of Fit}

Rsquare

Adj Rsquare

0.000431

Root Mean Square Error $\quad 0.115938$

Mean of Response $\quad 2.250307$

Observations (or Sum Wgts)

t Test

$2-1$

Assuming equal variances

Difference -0.00393 t Ratio -0.04152

Std Err Dif $\quad 0.09466$ DF $\quad 4$

Upper CL Dif 0.25890 Prob $>|t| \quad 0.9689$

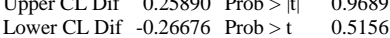

$\begin{array}{lrl}\text { Confidence } & 0.95 \text { Prob }<\mathrm{t} & 0.4844\end{array}$

Means for Oneway Anova

Level Number Mean Std Error Lower 95\% Upper 95\%

$\begin{array}{llllrr}1 & 3 & 2.25227 & 0.06694 & 2.0664 & 2.4381 \\ 2 & 3 & 2.24834 & 0.06694 & 2.0625 & 2.4342\end{array}$

Std Error uses a pooled estimate of error variance

Oneway Analysis of Measured By Block Prep Method=MA, Glass ID=U-std, Oxide $=\mathrm{ZnO}$ (wt $\%)$, Targeted $=\mathbf{0}$

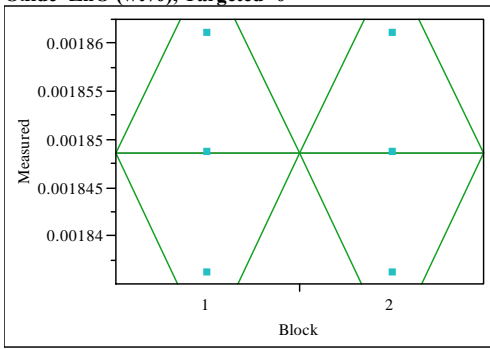

Oneway Anova

Summary of Fit

Rsquare

Adj Rsquare

Root Mean Square Error $\quad \begin{array}{r}-0.25 \\ 1.245 e-5\end{array}$

$1.245 e-5$
0.001849

t Test

2-1

Assuming equal variances

Difference $\quad 0.00000$ t Ratio 0

$\begin{array}{lrr}\text { Std Err Dif } & 1.016 \mathrm{e}-5 \text { DF } & 4 \\ \end{array}$

Lower CL Dif $-2.82 \mathrm{e}-5$ Prob $>\mathrm{t} \quad 0.5000$

Means for Oneway Anova

Level Number Mean Std Error Lower 95\% Upper 95\%

$\begin{array}{lrrrrr}1 & 3 & 0.001849 & 7.1869 \mathrm{e}-6 & 0.00183 & 0.00187 \\ 2 & 3 & 0.001849 & 7.1869 \mathrm{e}-6 & 0.00183 & 0.00187\end{array}$

Std Error uses a pooled estimate of error variance 


\section{Exhibit C3. Measurements by Block and Sub-Block for Samples of the ARG-1 and Ustd Standards with the Thorium Glasses by Oxide}

Oneway Analysis of Measured By Block Prep Method=MA, Glass ID=U-std, Oxide $=\mathrm{ZrO} 2(\mathbf{w t} \%)$, Targeted $=0$

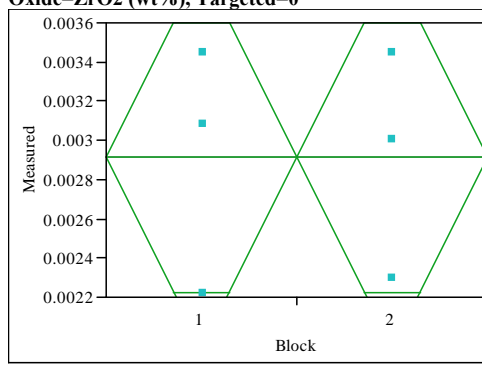

Oneway Anova

Summary of Fit

Rsquare

Adj Rsquare

Root Mean Square Error -0.25

Mean of Respons

-0.25
0.000606 0.002913

t Test

$2-1$

Assuming equal variances

Difference $\quad 0.00000$ t Ratio 0

Std Err Dif $\quad 0.00049$ DF 4

Upper CL Dif 0.00137 Prob $>|t| 1.0000$

$\begin{array}{lll}\text { Lower CL Dif }-0.00137 & \text { Prob }>t & 0.5000\end{array}$

$\begin{array}{llll}\text { Confidence } & 0.95 \text { Prob }<\mathrm{t} \quad 0.5000\end{array}$

Means for Oneway Anova
Level Number Mean Std Error Lower 95\% Upper 95\%

$\begin{array}{lrrrrr}\text { Level } & \text { Number } & \text { Mean } & \text { Std Error } & \text { Lower 95\% } & \text { Upper 95\% } \\ 1 & 3 & 0.002913 & 0.00035 & 0.00194 & 0.00388\end{array}$

$\begin{array}{llllll}1 & 3 & 0.002913 & 0.00035 & 0.00194 & 0.00388\end{array}$

Std Error uses a pooled estimate of error variance

Oneway Analysis of Measured By Block Prep Method=PF, Glass ID=ARG-1, Oxide $=\mathrm{Al} 2 \mathrm{O} 3(\mathrm{wt} \%)$, Targeted $=\mathbf{4 . 7 3}$

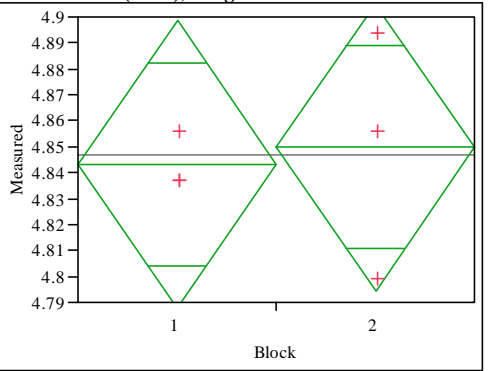

Oneway Anova

Summary of Fit

Rsquare $\quad 0.012346$

Adj Rsquare

$-0.23457$

Root Mean Square Error

0.034497

Mean of Respons 4.846568
6

t Test

2-1

Assuming equal variances

Difference $\quad 0.00630$ t Ratio $\quad 0.223607$

Std Err Dif $\quad 0.02817$ DF

Upper CL Dif 0.08450 Prob $>|t| \quad 0.8340$

Lower CL Dif -0.07191 Prob $>t \quad 0.4170$

$\begin{array}{lrl}\text { Confidence } & 0.95 \text { Prob }<\mathrm{t} & 0.5830\end{array}$

Means for Oneway Anova
Level Number Mean Std Error Lower 95\% Upper 95\%

$\begin{array}{lrrrrr}\text { Level } & \text { Number } & \text { Mean } & \text { Std Error } & \text { Lower 95\% } & \text { Upper 95\% } \\ 1 & 3 & 4.84342 & 0.01992 & 4.7881 & 4.8987\end{array}$

$\begin{array}{llllll}1 & 3 & 4.84342 & 0.01992 & 4.7881 & 4.8987 \\ 2 & 3 & 4.84972 & 0.01992 & 4.7944 & 4.9050\end{array}$
Std Error uses a pooled estimate of error variance

Oneway Analysis of Measured By Block Prep Method=PF, Glass ID=ARG-1, Oxide $=$ B2O3 $($ wt $\%)$, Targeted $=8.67$

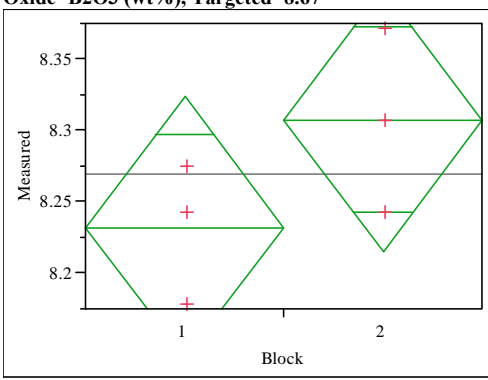

Oneway Anova

Rsquare

Adj Rsquare

Root Mean Square Error $\quad 0.057299$

Mean of Respons er. 8.26777

Observations (or Sum Wgts)

t Test

2-1

Assuming equal variances

$\begin{array}{lrlr}\text { Difference } & 0.07513 & \text { t Ratio } & 1.60591 \\ \text { Std Err Dif } & 0.04678 & \text { DF } & 4 \\ \text { Upper CL Dif } & 0.20502 & \text { Prob }>|t| & 0.1836 \\ \text { Lower CL Dif } & -0.05476 & \text { Prob }>t & 0.0918 \\ \text { Confidence } & 0.95 & \text { Prob }<t & 0.9082\end{array}$

Means for Oneway Anova

Level Number Mean Std Error Lower 95\% Upper 95\%

$\begin{array}{llllrr}1 & 3 & 8.23221 & 0.03308 & 8.1404 & 8.3241 \\ 2 & 3 & 8.30734 & 0.03308 & 8.2155 & 8.3992\end{array}$

Std Error uses a pooled estimate of error variance

Oneway Analysis of Measured By Block Prep Method=PF, Glass ID=ARG-1, Oxide $=\mathrm{BaO}($ wt $\%)$, Targeted $=0.09$

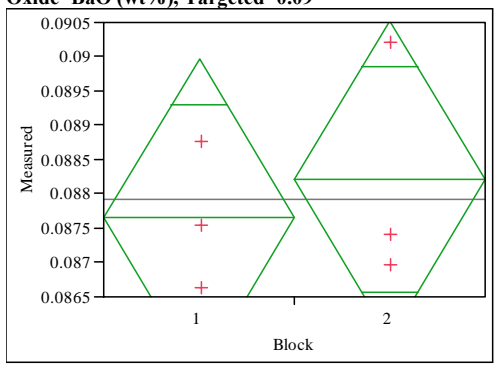

Oneway Anova

Summary of Fit

Rsquare

Adj Rsquare

Root Mean Square Error $\quad 0.001451$

Mean of Response

Observations (or Sum Wgts)

0.087924
6

t Test

Assuming equal variances

Difference $\quad 0.00056$ t Ratio $\quad 0.471056$

Std Err Dif $\quad 0.00119$ DF

Upper CL Dif 0.00385 Prob $>|t| \quad 0.6621$

Lower CL Dif -0.00273 Prob $>t \quad 0.331$

$\begin{array}{lll}\text { Confidence } & 0.95 \text { Prob }<\mathrm{t} \quad 0.6689\end{array}$

Means for Oneway Anova

Level Number Mean Std Error Lower 95\% Upper 95\%

$\begin{array}{rrrrrr}1 & 3 & 0.087645 & 0.00084 & 0.08532 & 0.08997\end{array}$ 


\section{Exhibit C3. Measurements by Block and Sub-Block for Samples of the ARG-1 and Ustd Standards with the Thorium Glasses by Oxide}

$\begin{array}{lrrrrr}\text { Level } & \text { Number } & \text { Mean } & \text { Std Error } & \text { Lower 95\% } & \text { Upper 95\% } \\ 2 & 3 & 0.088204 & 0.00084 & 0.08588 & 0.09053\end{array}$

Std Error uses a pooled estimate of error variance

Oneway Analysis of Measured By Block Prep Method=PF, Glass ID=ARG-1, Oxide $=\mathrm{CaO}(\mathrm{wt} \%)$, Targeted $=\mathbf{1 . 4 3}$

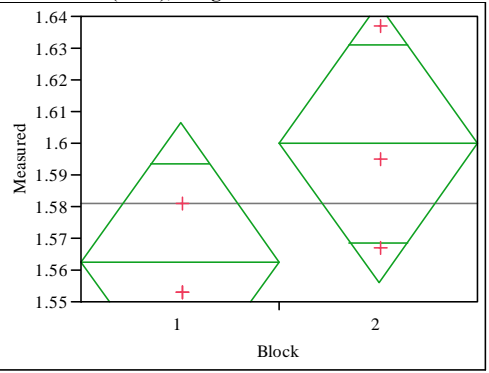

Oneway Anova

Summary of Fit

$\begin{array}{lr}\text { Rsquare } & 0.410256 \\ \text { Adj Rsquare } & 0.262821 \\ \text { Root Mean Square Error } & 0.027395 \\ \text { Mean of Response } & 1.581096 \\ \text { Observations (or Sum Wgts) } & 6\end{array}$

t Test

Assuming equal variances

Difference $\quad 0.03731$ t Ratio $\quad 1.668115$

Std Err Dif 0.02237 DF 4

Upper CL Dif 0.09941 Prob $>|t| \quad 0.1706$

Lower CL Dif -0.02479 Prob $>t \quad 0.0853$

$\begin{array}{lll}\text { Confidence } & 0.95 \text { Prob }<\mathrm{t} \quad 0.9147\end{array}$

Means for Oneway Anova

Level Number Mean Std Error Lower 95\% Upper 95\%

$\begin{array}{lrrrrr}\text { Level } & \text { Number } & \text { Mean } & \text { Std Error } & \text { Lower 95\% } & \text { Upper 95\% } \\ 1 & 3 & 1.56244 & 0.01582 & 1.5185 & 1.6064\end{array}$

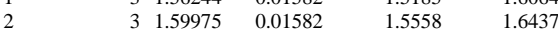

Std Error uses a pooled estimate of error variance

Oneway Analysis of Measured By Block Prep Method=PF, Glass ID=ARG-1, Oxide $=\mathrm{CdO}\left(\mathrm{wt}_{\mathrm{t}} \%\right)$, Targeted $=\mathbf{0}$

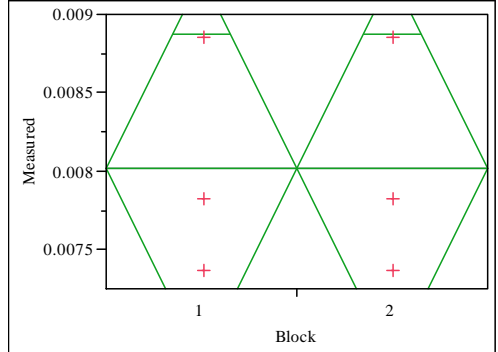

Oneway Anova

Summary of Fit

Rsquare

Adj Rsquare

Mean of Response

0.008015

t Test

$2-1$

Assuming equal variances

Difference $\quad 0.00000 \mathrm{t}$ Ratio

Std Err Dif $\quad 0.00062$ DF

Upper CL Dif 0.00172 Prob $>|t| 1.0000$

Lower CL Dif -0.00172 Prob $>t \quad 0.5000$

Confidence

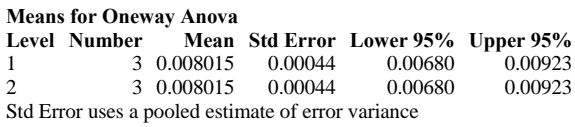

Means for Oneway Anova

Level Number Mean Std Error Lower 95\% Upper 95\%

$\begin{array}{llllrr}1 & 3 & 0.008015 & 0.00044 & 0.00680 & 0.00923\end{array}$

$\begin{array}{llllll}2 & 3 & 0.008015 & 0.00044 & 0.00680 & 0.00923\end{array}$

Std Error uses a pooled estimate of error variance

Oneway Analysis of Measured By Block Prep Method=PF, Glass ID=ARG-1, Oxide $=\mathrm{Ce} 2 \mathrm{O} 3(\mathrm{wt} \%)$, Targeted $=0$

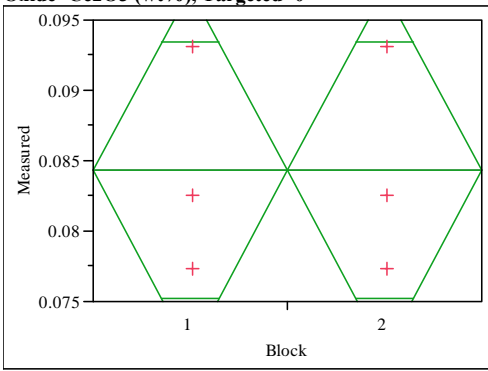

Oneway Anova

Summary of Fit

Rsquare

Root Mean Square Error $\quad 0.00805$

Mean of Respons 0.084334
6

t Test

Assuming equal variances

Difference $\quad 0.00000$ t Ratio $\quad 0$

Std Err Dif $\quad 0.00657$ DF 4

Upper CL Dif 0.01825 Prob $>|t| 1.0000$

Lower CL Dif -0.01825 Prob $>$ t 0.5000

Confidence $\quad 0.95$ Prob $<\mathrm{t} \quad 0.5000$

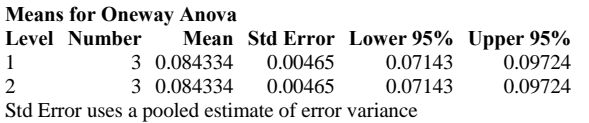

Std Error uses a pooled estimate of error variance

Oneway Analysis of Measured By Block Prep Method=PF, Glass ID=ARG-1, Oxide $=\mathbf{C r 2 O 3}($ wt $\%$ ), Targeted $=\mathbf{0 . 0 9}$

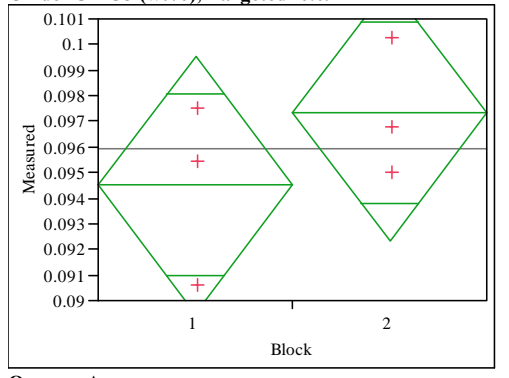

Oneway Anova

Summary of Fit

Rsquare

Adj Rsquare $\quad 0.042339$

$\quad 0.003132$

Mean of Respons

0.09593

t Test

Assuming equal variances

Difference $\quad 0.00283$ t Ratio 1.105013

Std Err Dif $\quad 0.00256$ DF

$\begin{array}{llll} & & \\ \text { Upper CL Dif } & 0.00993 & \text { Prob }>|t| & 0.3311\end{array}$

$\begin{array}{lrll}\text { Upper CL Dif } & 0.00993 & \text { Prob }>|t| & 0.3311 \\ \text { Lower CL Dif } & -0.00427 & \text { Prob }>t & 0.1656\end{array}$

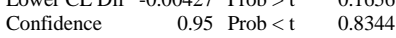




\section{Exhibit C3. Measurements by Block and Sub-Block for Samples of the ARG-1 and Ustd Standards with the Thorium Glasses by Oxide}

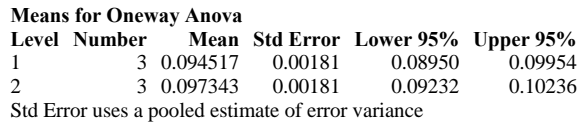

Oneway Analysis of Measured By Block Prep Method=PF, Glass ID=ARG-1, Oxide $=\mathbf{C u O}(\mathbf{w t} \%)$, Targeted $=0$

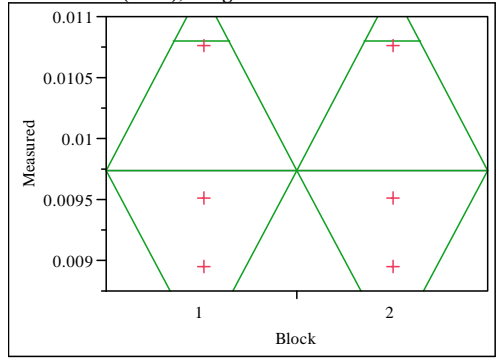

$$
\begin{aligned}
& \text { Oneway Anova } \\
& \text { Summary of Fit }
\end{aligned}
$$

Rsquare

Adj Rsquare

$-0.25$

rror $\quad 0.000929$

Mean of Res

Observations (or Sum Wgts) $\quad 6$

t Test

2-1

Assuming equal variances

Difference $\quad 0.00000$ t Ratio $\quad 0$

Std Err Dif $\quad 0.00076$ DF 4

Upper CL Dif 0.00211 Prob $>|t| 1.0000$

Lower CL Dif -0.00211 Prob $>t \quad 0.5000$

$\begin{array}{lrl}\text { Confidence } & 0.95 \text { Prob }<\mathrm{t} \quad 0.5000\end{array}$

Means for Oneway Anova

Level Number Mean Std Error Lower 95\% Upper 95\%

$\begin{array}{llllll}1 & 3 & 0.009743 & 0.00054 & 0.00825 & 0.01123\end{array}$

Std Error uses a pooled estimate of error variance

Oneway Analysis of Measured By Block Prep Method=PF, Glass ID=ARG-1, Oxide $=\mathrm{Fe} 2 \mathrm{O} 3(\mathrm{wt} \%)$, Targeted $=14$

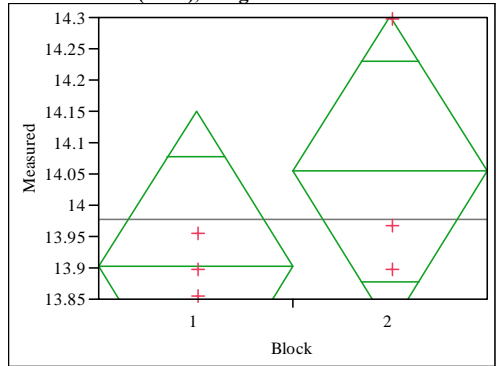

Oneway Anova

Summary of Fit

Rsquare

Adj Rsquare

$\begin{array}{lr}\text { Mean of Response } & 13.9777 \\ \text { Observations (or Sum Wgts) } & 6\end{array}$

t Test

2-1

Assuming equal variances

Difference $\quad 0.15250$ t Ratio 1.204336

Std Err Dif $\quad 0.12663$ DF

$\begin{array}{lll}\text { Upper CL Dif } 0.50407 & \text { Prob }>|t| & 0.2948\end{array}$

Lower CL Dif -0.19907 Prob $>t \quad 0.1474$

0.95 Prob $<\mathrm{t} \quad 0.8526$

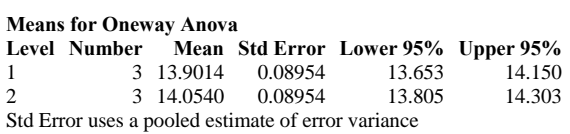

Oneway Analysis of Measured By Block Prep Method=PF, Glass ID=ARG-1, Oxide $=$ K2O $($ wt $\%)$, Targeted $=2.71$

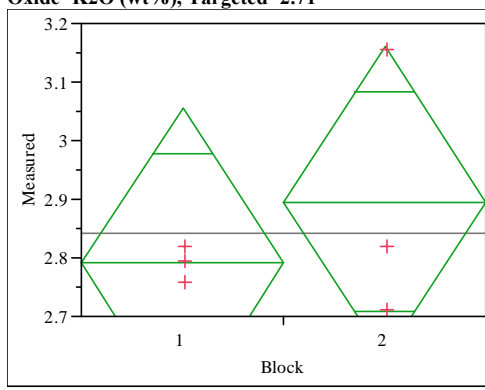

Oneway Anova

Summary of Fit

$\begin{array}{lr}\text { Rsquare } & 0.129502 \\ \text { Adj Rsquare } & -0.08812 \\ \text { Root Mean Square Error } & 0.165751 \\ \text { Mean of Response } & 2.842856 \\ \text { Observations (or Sum Wgts) } & 6\end{array}$

t Test

2-1

Assuming equal variances

Difference $\quad 0.10440$ t Ratio $\quad 0.771408$

Std Err Dif $\quad 0.13534$ DF

Upper CL Dif 0.48015 Prob $>|t| \quad 0.4835$

Lower CL Dif -0.27135 Prob $>t \quad 0.2418$

$\begin{array}{lll}\text { Confidence } & 0.95 \text { Prob }<\mathrm{t} \quad 0.7582\end{array}$

Means for Oneway Anova

Level Number Mean Std Error Lower 95\% Upper 95\%

$\begin{array}{rrrrrr}\text { Level } & \text { Number } & \text { Mean } & \text { Std Error } & \text { Lower 95\% } & \text { Upper 95\% } \\ 1 & 3 & 2.79066 & 0.09570 & 2.5250 & 3.0564\end{array}$

$\begin{array}{llllll}2 & 3 & 2.89506 & 0.09570 & 2.6294 & 3.1608\end{array}$

Std Error uses a pooled estimate of error variance

Oneway Analysis of Measured By Block Prep Method=PF, Glass ID=ARG-1, Oxide $=\mathbf{L a 2 O 3}(\mathrm{wt} \%)$, Targeted $=0$

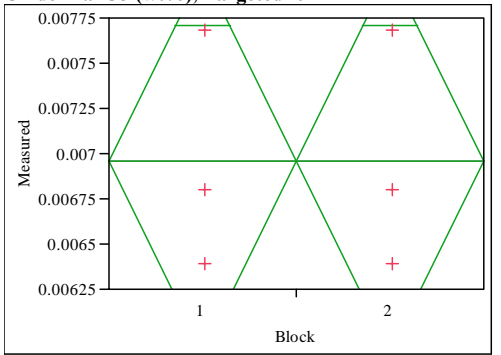

Oneway Anova

Summary of Fit

Rsquare

Adj Rsquare

Root Mean Square Error $\quad 0.000659$

Mean of Response 0.006959

Observations (or Sum Wg

6

t Test

Assuming equal variances

Difference $\quad 0.00000$ t Ratio

Std Err Dif 0.00054 DF

$\begin{array}{lrr}\text { Upper CL Dif } & 0.00149 & \text { Prob }>|t| \\ 1.0000\end{array}$ 


\section{Exhibit C3. Measurements by Block and Sub-Block for Samples of the ARG-1 and Ustd Standards with the Thorium Glasses by Oxide}

Lower CL Dif -0.00149 Prob $>t \quad 0.5000$

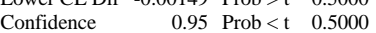

Means for Oneway Anova

Level Number Mean Std Error Lower 95\% Upper 95\%

$\begin{array}{lllrrr}1 & 3 & 0.006959 & 0.00038 & 0.00590 & 0.00802 \\ 2 & 3 & 0.006959 & 0.00038 & 0.00590 & 0.00802\end{array}$

Std Error uses a pooled estimate of error variance

Oneway Analysis of Measured By Block Prep Method=PF, Glass ID=ARG-1, Oxide $=\mathrm{Li} 2 \mathrm{O}(\mathrm{wt} \%)$, Targeted $=3.21$

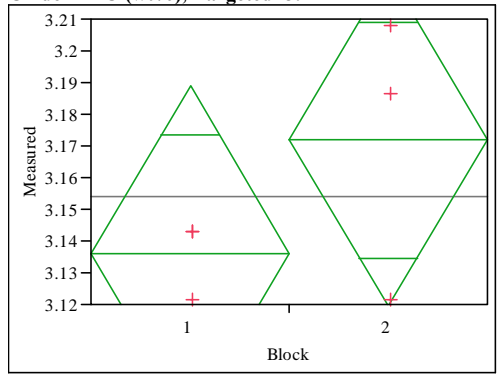

Oneway Anova

Summary of Fi

Rsquare

0.308642

Adj Rsquare

Mean of Res

Observations (or Sum Wgts)

t Test

2-1

Assuming equal variances

Difference $\quad 0.03588$ t Ratio 1.336306

Std Err Dif $\quad 0.02685$ DF

Upper CL Dif 0.11043 Prob $>|t| \quad 0.2524$

Lower CL Dif -0.03867 Prob $>t \quad 0.1262$

$\begin{array}{lrl}\text { Confidence } & 0.95 \text { Prob }<\mathrm{t} \quad 0.8738\end{array}$

Means for Oneway Anova

Level Number Mean Std Error Lower 95\% Upper 95\%

$\begin{array}{lllrrr}1 & 3 & 3.13606 & 0.01899 & 3.0833 & 3.1888\end{array}$

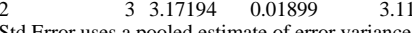

Oneway Analysis of Measured By Block Prep Method=PF, Glass ID=ARG-1, Oxide $=$ MgO $\left(w_{t} \%\right)$, Targeted $=0.86$

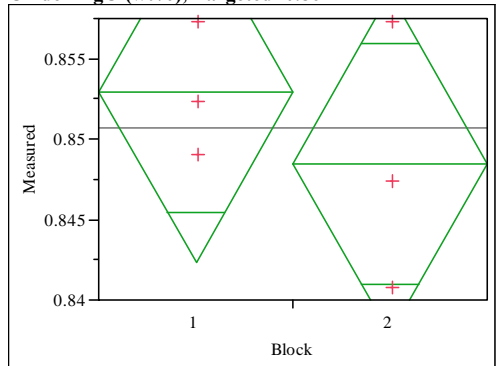

Oneway Anova

Summary of Fit

Rsquare

Adj Rsquare

Mean of Response $\quad 0.850708$

Observations (or Sum Wgts) $\quad 6$

t Test

2-1

Assuming equal variances

Difference $\quad-0.00442$ t Ratio $\quad-0.82078$

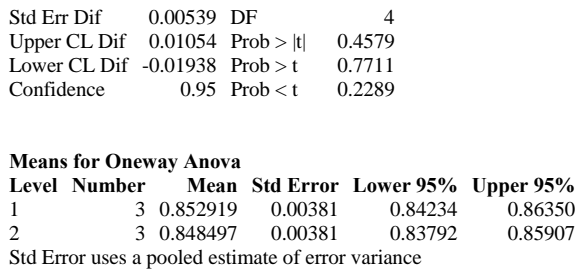

Oneway Analysis of Measured By Block Prep Method=PF, Glass ID=ARG-1, Oxide $=\mathbf{M n O}\left(w_{t} \%\right)$, Targeted $=2.31$

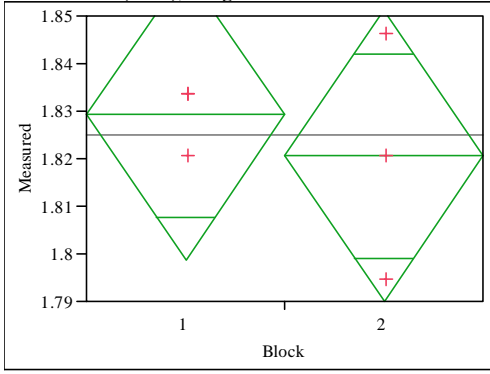

Oneway Anova

Summary of Fi

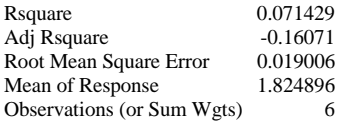

Observations (or Sum Wgts) $\quad 6$

t Test

Assuming equal variances

Difference $\quad-0.00861$ t Ratio $\quad-0.5547$

Std Err Dif $\quad 0.01552$ DF

Upper CL Dif 0.03448 Prob $>|t| \quad 0.6087$

Lower CL Dif -0.05169 Prob $>t \quad 0.6957$

Confidence $\quad 0.95$ Prob $<\mathrm{t} \quad 0.3043$

Means for Oneway Anova

Level Number Mean Std Error Lower 95\% Upper 95\%

$\begin{array}{lrrrrr}\text { Level } & \text { Number } & \text { Mean } & \text { Std Error } & \text { Lower 95\% } & \text { Upper 95\% } \\ 1 & 3 & 1.82920 & 0.01097 & 1.7987 & 1.8597\end{array}$

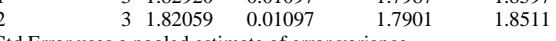

Std Error uses a pooled estimate of error variance

Oneway Analysis of Measured By Block Prep Method=PF, Glass ID=ARG-1, Oxide $=\mathrm{NiO}(\mathrm{wt} \%)$, Targeted $=1.05$

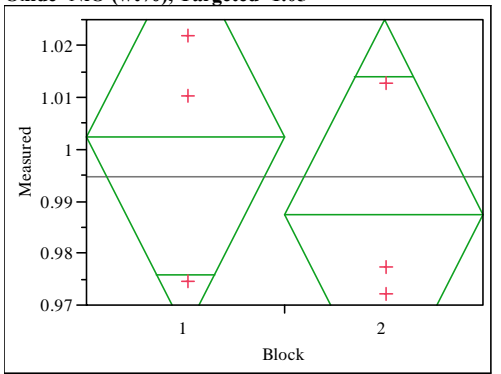

Oneway Anova

Summary of Fit

Rsquare

Adj Rsquare

Root Mean Square Er

Mean of Response

Observations (or Sum Wgts)

t Test

2-1

Assuming equal variances 


\section{Exhibit C3. Measurements by Block and Sub-Block for Samples of the ARG-1 and Ustd Standards with the Thorium Glasses by Oxide}

$\begin{array}{lrlr}\text { Difference } & -0.01485 & \mathrm{t} \text { Ratio } & -0.77701 \\ \text { Std Err Dif } & 0.01911 \text { DF } & 4 \\ \text { Upper CL Dif } & 0.03820 \text { Prob }>|\mathrm{t}| & 0.4805 \\ \text { Lower CL Dif } & -0.06789 \text { Prob }>\mathrm{t} & 0.7597 \\ \text { Confidence } & 0.95 \text { Prob }<\mathrm{t} & 0.2403\end{array}$

Means for Oneway Anova

Level Number Mean Std Error Lower 95\% Upper 95\%

$\begin{array}{rrrrrr}\text { Level Number } & \text { Mean } & \text { Std Error } & \text { Lower 95\% } & \text { Upper 95\% } \\ 1 & 3 & 1.00231 & 0.01351 & 0.96480 & 1.0398\end{array}$

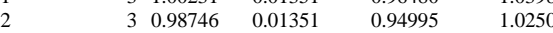

Std Error uses a pooled estimate of error variance

Oneway Analysis of Measured By Block Prep Method=PF, Glass ID=ARG-1, Oxide $=\mathbf{P 2 O 5}(\mathbf{w t} \%)$, Targeted $=\mathbf{0 . 2}$

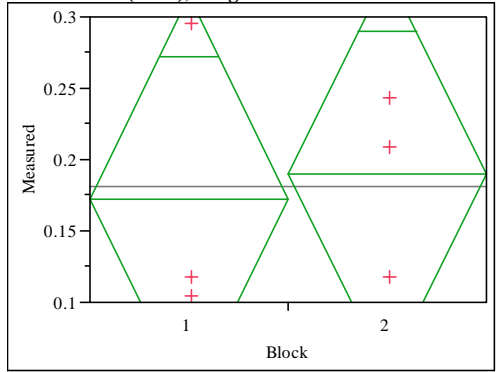

Oneway Anova

Summary of Fit

Rsquare

Adj Rsquare

$\begin{array}{lr}\text { Mean of Response } & 0.181231 \\ \text { Observations (or Sum Wgts) } & 6\end{array}$

t Test

$2-1$

Assuming equal variances

Difference $\quad 0.01715$ t Ratio $\quad 0.23823$

Std Err Dif $\quad 0.07198$ DF

Upper CL Dif 0.21699 Prob $>|t| \quad 0.8234$

$\begin{array}{llll}\text { Lower CL Dif } & -0.18270 \text { Prob }>t & 0.4117\end{array}$

$\begin{array}{lrl}\text { Confidence } & 0.95 \text { Prob }<\mathrm{t} \quad 0.5883\end{array}$

Means for Oneway Anova
Level Number Mean Std Error Lower 95\% Upper 95\%

$\begin{array}{lrrrrr}\text { Level } & \text { Number } & \text { Mean } & \text { Std Error } & \text { Lower 95\% } & \text { Upper 95\% } \\ 1 & 3 & 0.172657 & 0.05090 & 0.03135 & 0.31397\end{array}$

$\begin{array}{llllll}1 & 3 & 0.189804 & 0.05090 & 0.04849 & 0.33111\end{array}$

Std Error uses a pooled estimate of error variance

Oneway Analysis of Measured By Block Prep Method=PF, Glass ID=ARG-1, Oxide $=\mathbf{P b O}(\mathbf{w t} \%)$, Targeted $=\mathbf{0}$

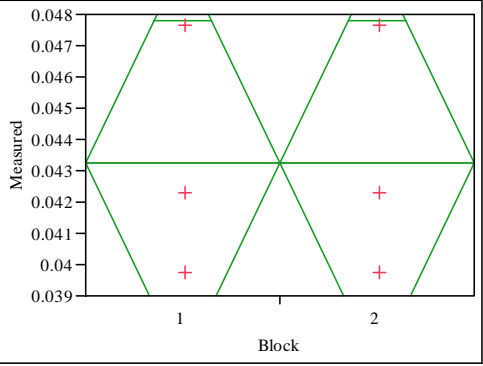

Oneway Anova

Summary of Fit

Rsquare

Adj Rsquare

0.004044

Mean of Response

0.043232

t Test
Assuming equal variances

$\begin{array}{lrlr}\text { Difference } & 0.00000 & \mathrm{t} \text { Ratio } & 0 \\ \text { Std Err Dif } & 0.00330 & \text { DF } & 4 \\ \text { Upper CL Dif } & 0.00917 & \text { Prob }>|t| & 1.0000 \\ \text { Lower CL Dif } & -0.00917 & \text { Prob }>t & 0.5000 \\ \text { Confidence } & 0.95 & \text { Prob }<\mathrm{t} & 0.5000\end{array}$

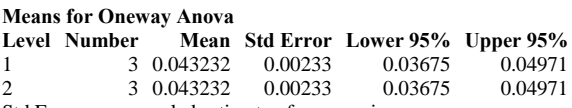

Std Error uses a pooled estimate of error variance

Oneway Analysis of Measured By Block Prep Method=PF, Glass ID=ARG-1, Oxide $=\mathbf{S i O} 2($ wt $\%)$, Targeted $=47.9$

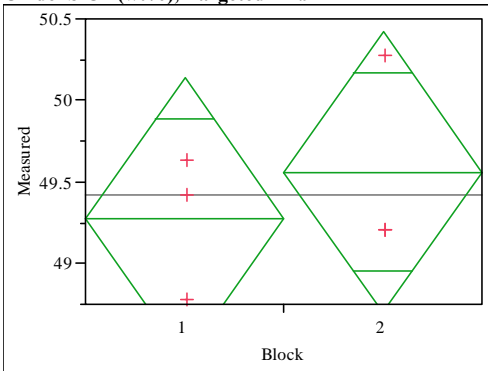

Summary of Fit

Rsquare

0.095238

Root Mean Square Error $\quad 0.538379$

Mean of Response 49.41783

Observations (or Sum Wgts)

t Test

Assuming equal variances

Difference $\quad 0.2852$ t Ratio 0.648886

Std Err Dif 0.4396 DF

Upper CL Dif 1.5057 Prob $>|t| \quad 0.5518$

$\begin{array}{lll}\text { Lower CL Dif }-0.9352 \text { Prob }>t & 0.2759\end{array}$

Confidence $\quad 0.95$ Prob $<\mathrm{t} \quad 0.7241$

Means for Oneway Anova

Level Number Mean Std Error Lower 95\% Upper 95\%

$\begin{array}{lllrrr}1 & 3 & 49.2752 & 0.31083 & 48.412 & 50.138 \\ 2 & 3 & 49.5605 & 0.31083 & 48.697 & 50.423\end{array}$

Std Error uses a pooled estimate of error variance

Oneway Analysis of Measured By Block Prep Method=PF, Glass ID=ARG-1, Oxide $=\mathrm{SO} 4(\mathrm{wt} \%)$, Targeted $=0$

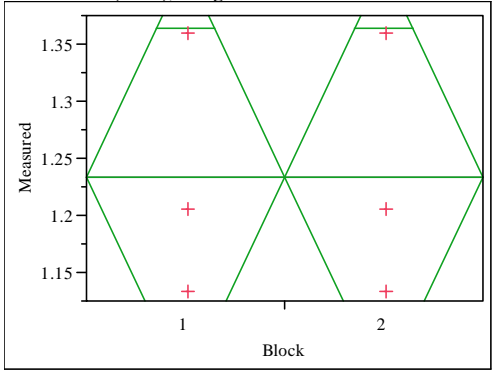

Summary of Fit

Rsquare

Adj Rsquare

Root Mean Square Error $\quad-0.25$

Observations (or Sum Wgts) 1.233312 


\section{Exhibit C3. Measurements by Block and Sub-Block for Samples of the ARG-1 and Ustd Standards with the Thorium Glasses by Oxide}

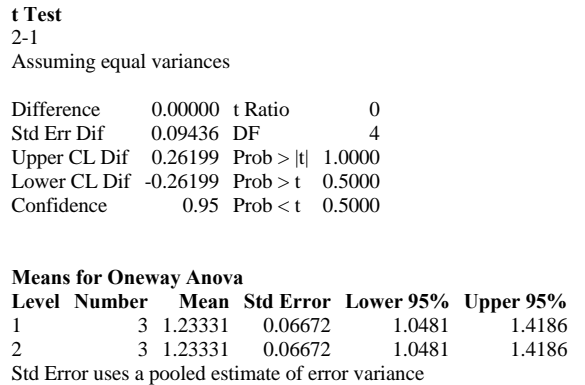

Oneway Analysis of Measured By Block Prep Method=PF, Glass ID=ARG-1, Oxide $=\mathrm{ThO} 2(\mathrm{wt} \%)$, Targeted $=0$

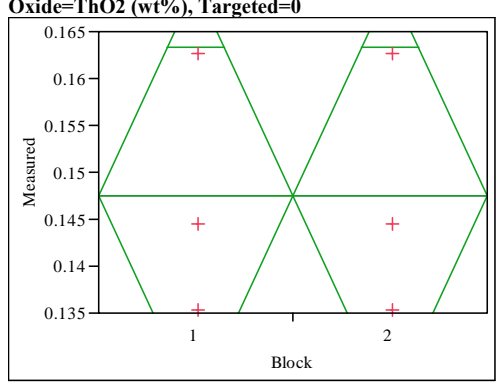

Summary of Fi

Rsquare

Adj Rsquare

Root Mean Square Error $\quad-0.25$

0.013905

Observations (or Sum Wgts) $\quad 6$

t Test

Assuming equal variances

Difference $\quad 0.00000$ t Ratio $\quad 0$

Std Err Dif 0.01135 DF

Upper CL Dif 0.03152 Prob $>|t| 1.0000$

$\begin{array}{lll}\text { Lower CL Dif }-0.03152 \text { Prob }>t & 0.5000\end{array}$

Confidence $\quad 0.95$ Prob $<\mathrm{t} \quad 0.5000$

Means for Oneway Anova

Level Number Mean Std Error Lower 95\% Upper 95\%

$\begin{array}{lllllr}1 & 3 & 0.147548 & 0.00803 & 0.12526 & 0.16984\end{array}$

$\begin{array}{llllll}2 & 3 & 0.147548 & 0.00803 & 0.12526 & 0.16984\end{array}$

Std Error uses a pooled estimate of error variance

Oneway Analysis of Measured By Block Prep Method=PF, Glass ID=ARG-1, Oxide $=\mathrm{TiO} 2(\mathrm{wt} \%)$, Targeted $=1.15$

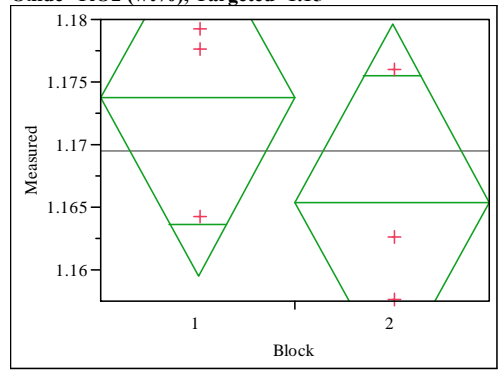

Oneway Anova

Summary of Fit

$\begin{array}{lr}\text { Rsquare } & 0.248619 \\ \text { Adj Rsquare } & 0.060773 \\ \text { Root Mean Square Error } & 0.008879 \\ \text { Mean of Response } & 1.169546 \\ \text { Observations (or Sum Wgts) } & 6\end{array}$

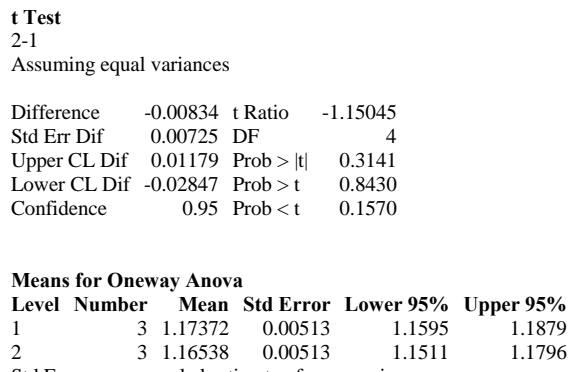

Means for Oneway Anova

$\begin{array}{rrrrrr}\text { Level } & \text { Number } & \text { Mean } & \text { Std Error } & \text { Lower 95\% } & \text { Upper 95\% } \\ 1 & 3 & 1.17372 & 0.00513 & 1.1595 & 1.1879\end{array}$

$\begin{array}{llllll}1 & 3 & 1.16538 & 0.00513 & 1.1595 & 1.1879 \\ 2 & 3 & 0.00513 & 1.1511 & 1.1796\end{array}$

Std Error uses a pooled estimate of error variance

Oneway Analysis of Measured By Block Prep Method=PF, Glass ID=ARG-1, Oxide $=\mathbf{U} 308(\mathrm{wt} \%)$, Targeted $=\mathbf{0}$

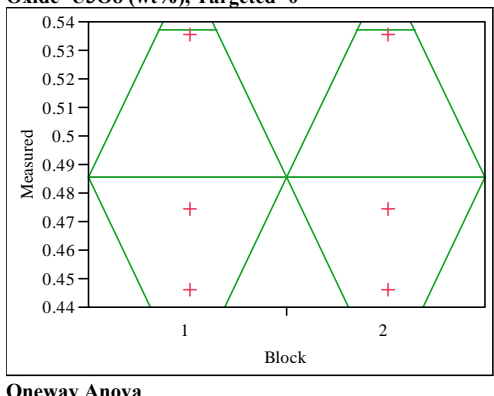

Oneway Anova

Summary of Fit

Rsquare

Adj Rsquare

Root Mean Square Error $\quad-0.25$

0.045488
0.485437

Observations (or Sum Wgts)

t Test

t-1

Assuming equal variances

$\begin{array}{lrlr}\text { Difference } & 0.00000 & \mathrm{t} \text { Ratio } & 0 \\ \text { Std Err Dif } & 0.03714 & \text { DF } & 4 \\ \text { Upper CL Dif } & 0.10312 & \text { Prob }>|t| & 1.0000 \\ \text { Lower CL Dif } & -0.10312 & \text { Prob }>\text { t } & 0.5000 \\ \text { Confidence } & 0.95 & \text { Prob }<\mathrm{t} & 0.5000\end{array}$

Means for Oneway Anova

$\begin{array}{rrrrrr}\text { Level } & \text { Number } & \text { Mean } & \text { Std Error } & \text { Lower 95\% } & \text { Upper 95\% } \\ 1 & 3 & 0.485437 & 0.02626 & 0.41252 & 0.55835\end{array}$

$\begin{array}{llllll}1 & 3 & 0.485437 & 0.02626 & 0.41252 & 0.55835 \\ 2 & 3 & 0.485437 & 0.02626 & 0.41252 & 0.55835\end{array}$

Std Error uses a pooled estimate of error variance

Oneway Analysis of Measured By Block Prep Method=PF, Glass ID=ARG-1, Oxide $=\mathbf{Z n O}(\mathbf{w t} \%)$, Targeted $=\mathbf{0 . 0 2}$

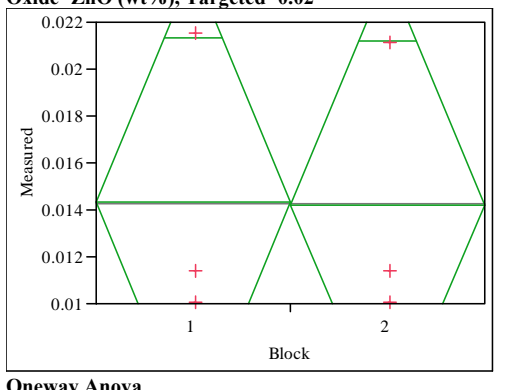

Rsquare

Adj Rsquare

Mean of Response $\quad 0.014274$ 


\section{Exhibit C3. Measurements by Block and Sub-Block for Samples of the ARG-1 and Ustd Standards with the Thorium Glasses by Oxide}

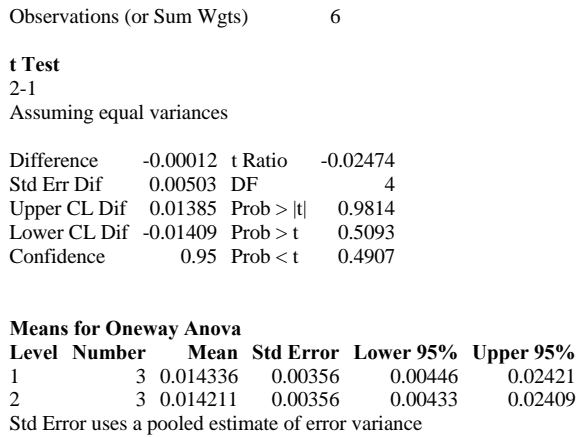

Oneway Analysis of Measured By Block Prep Method=PF, Glass ID=U-std, Oxide $=\mathrm{Al2O3}(\mathrm{wt} \%)$, Targeted $=4.1$

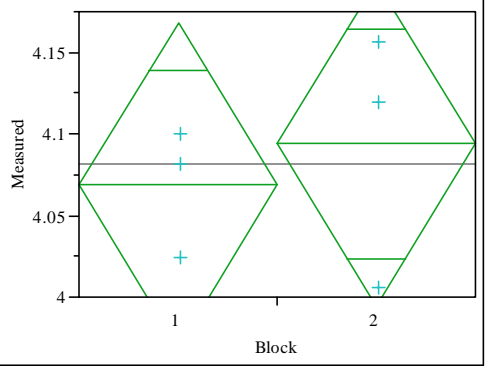

Oneway Anova

Rsquare 057971

Adj Rsquare

$-0.17754$

Root Mean Square Error $\quad 0.062191$

Mean of Response

Observations (or Sum Wgts) $\quad 6$

t Test

2-1

Assuming equal variances

Difference $\quad 0.02519$ t Ratio 0.496139

Std Err Dif $\quad 0.05078$ DF

Upper CL Dif 0.16618 Prob $>|\mathrm{t}| \quad 0.6458$

Lower CL Dif -0.11579 Prob $>t \quad 0.3229$

$\begin{array}{lrl}\text { Confidence } & 0.95 \text { Prob }<\mathrm{t} & 0.6771\end{array}$

Means for Oneway Anova

Level Number Mean Std Error Lower 95\% Upper 95\%

$\begin{array}{llllll}1 & 3 & 4.06872 & 0.03591 & 3.9690 & 4.1684 \\ 2 & 3 & 4.09392 & 0.03591 & 3.9942 & 4.1936\end{array}$

Std Error uses a pooled estimate of error variance

Oneway Analysis of Measured By Block Prep Method=PF, Glass ID=U-std, Oxide $=\mathrm{B} 2 \mathrm{O} 3(\mathrm{wt} \%)$, Targeted $=\mathbf{9 . 2 0 9}$

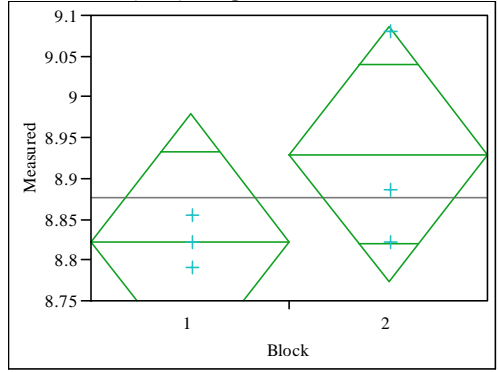

Oneway Anova

Summary of Fit

Rsquare

0.140625

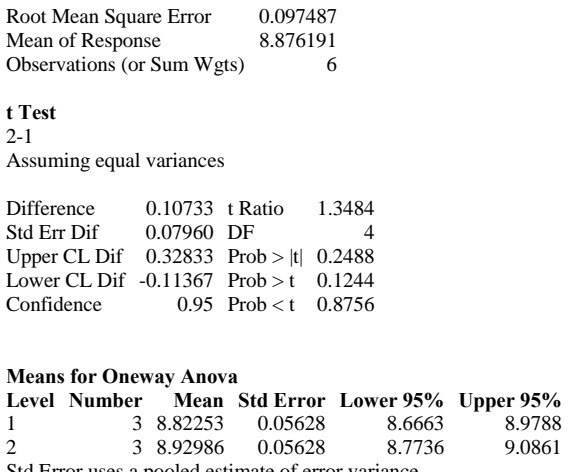

Oneway Analysis of Measured By Block Prep Method=PF, Glass ID=U-std, Oxide $=\mathbf{B a O}\left(w_{t} \%\right)$, Targeted $=\mathbf{0}$
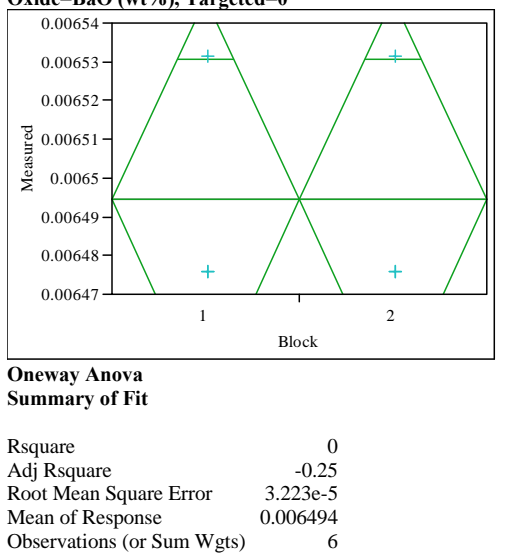

Observations (or Sum Wgts) 6

t Test

Assuming equal variances

Difference $\quad 0.00000$ t Ratio $\quad 0$

Std Err Dif 2.632e-5 DF 4

Upper CL Dif 0.000073 Prob $>|t| 1.0000$

Lower CL Dif $\quad-7.31 \mathrm{e}-5$ Prob $>\mathrm{t} \quad 0.5000$

Confidence $\quad 0.95$ Prob $<\mathrm{t} \quad 0.5000$

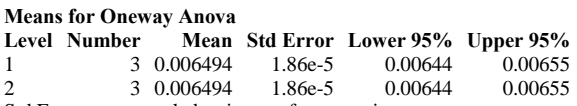

Std Error uses a pooled estimate of error variance

Oneway Analysis of Measured By Block Prep Method=PF, Glass ID=U-std, Oxide $=\mathbf{C a O}\left(w_{t} \%\right)$, Targeted $=1.301$

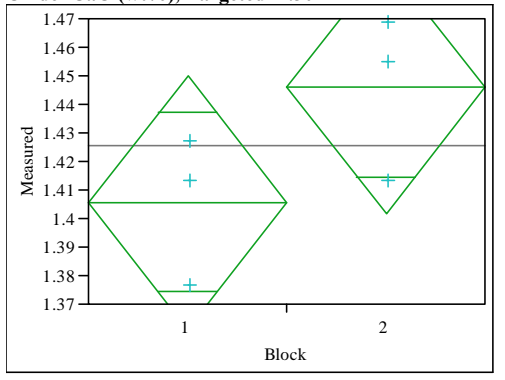

Summary of Fit 


\section{Exhibit C3. Measurements by Block and Sub-Block for Samples of the ARG-1 and Ustd Standards with the Thorium Glasses by Oxide}

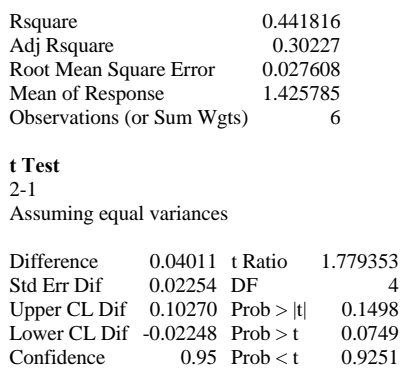

Oneway Analysis of Measured By Block Prep Method=PF, Glass ID $=\mathrm{U}$-std, Oxide $=\mathbf{C d O}\left(w_{t} \%\right)$, Targeted $=0$

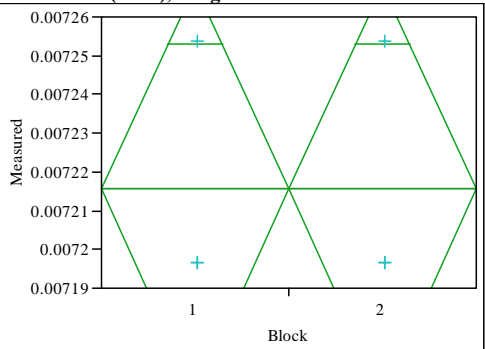

Oneway Anova

Summary of Fit

Rsquare

Adj Rsquare

Root Mean Squte Eror $\quad-0.25$

Mean of Response Error $\quad 0.000033$

Observations (or Sum Wgts)

t Test

2-1

Assuming equal variances

Difference $\quad 0.00000$ t Ratio

Std Err Dif $\quad 0.000027$ DF

Upper CL Dif 7.475e-5 Prob $>|t| 1.0000$

Lower CL Dif $\quad-7.48 \mathrm{e}-5$ Prob $>\mathrm{t} \quad 0.5000$

Confidence $\quad 0.95$ Prob $<\mathrm{t} \quad 0.5000$

Means for Oneway Anova

Level Number Mean Std Error Lower 95\% Upper 95\%

$\begin{array}{lrrrrr}1 & 3 & 0.007216 & 0.00002 & 0.00716 & 0.00727 \\ 2 & 3 & 0.007216 & 0.00002 & 0.00716 & 0.00727\end{array}$

Std Error uses a pooled estimate of error variance

Oneway Analysis of Measured By Block Prep Method=PF, Glass ID=U-std, Oxide $=\mathrm{Ce} 2 \mathrm{O} 3(\mathrm{wt} \%)$, Targeted $=0$

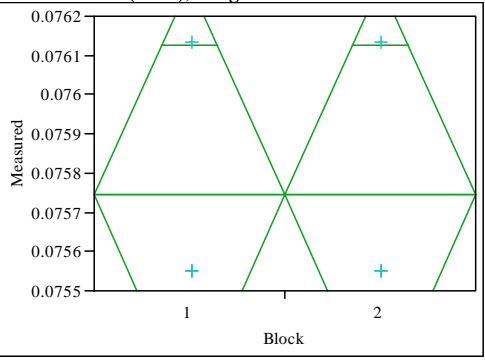

Oneway Anova

Summary of Fit

Rsquare

Adj Rsquare $\quad-0.25$

Root Mean Square Error $\quad 0.000338$

Mean of Response 0.075744

Test

t Test

Assuming equal variances

Difference $\quad 0.00000$ t Ratio

Std Err Dif $\quad 0.00028$ DF

Upper CL Dif 0.00077 Prob $>|t| 1.0000$

Lower CL Dif -0.00077 Prob $>$ t 0.5000

Confidence $\quad 0.95$ Prob $<\mathrm{t} \quad 0.5000$

Means for Oneway Anova

Level Number Mean Std Error Lower 95\% Upper 95\%

$\begin{array}{lrrrrr}1 & 3 & 0.075744 & 0.00020 & 0.07520 & 0.07629 \\ 2 & 3 & 0.075744 & 0.00020 & 0.07520 & 0.07629\end{array}$

Std Error uses a pooled estimate of error variance

Oneway Analysis of Measured By Block Prep Method=PF, Glass ID $=\mathbf{U}$-std, Oxide $=\mathbf{C r 2 O 3}(\mathrm{wt} \%)$, Targeted $=0$

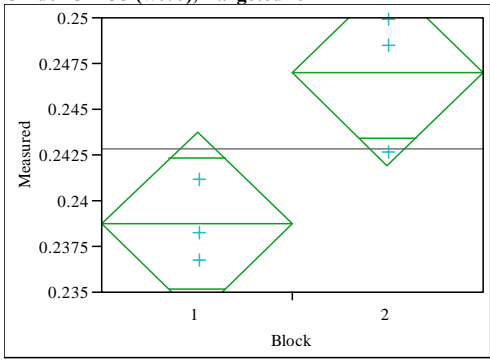

Oneway Anova

Rsquare

Adj Rsquare

0.650873

O.003157

Mean of Response $\quad 0.242869$

Observations (or Sum Wgts) 6

t Test

$2-1$

Assuming equal variances

Difference $\quad 0.008282$ t Ratio 3.212698

Std Err Dif $\quad 0.002578$ DF $\quad 4$

Upper CL Dif 0.015440 Prob $>|t| \quad 0.0325$

Lower CL Dif 0.001125 Prob $>t \quad 0.0163$

$\begin{array}{lrl}\text { Confidence } & 0.95 \text { Prob }<\mathrm{t} & 0.9837\end{array}$

Means for Oneway Anova

Level Number Mean Std Error Lower 95\% Upper 95\%

$\begin{array}{lllllr}1 & 3 & 0.238728 & 0.00182 & 0.23367 & 0.24379 \\ 2 & 3 & 0.247010 & 0.00182 & 0.24195 & 0.25207\end{array}$

Std Error uses a pooled estimate of error variance

Oneway Analysis of Measured By Block Prep Method=PF, Glass ID=U-std, Oxide $=\mathrm{CuO}\left(\mathrm{wt}_{\mathrm{t}} \mathrm{)}\right)$, Targeted $=\mathbf{0}$

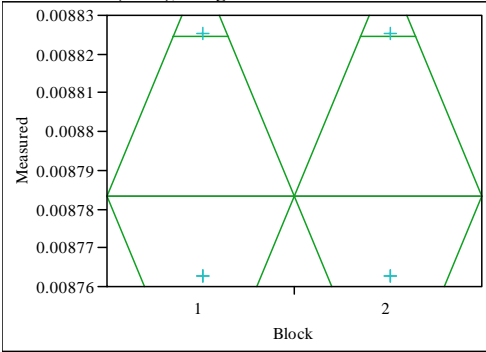




\section{Exhibit C3. Measurements by Block and Sub-Block for Samples of the ARG-1 and Ustd Standards with the Thorium Glasses by Oxide}

Oneway Anova

Summary of Fit

Rsquare

Adj Rsquare

Root Mean Square Error $\quad 3.614 \mathrm{e}-5$

Mean of Response

Observations (or Sum Wgts)

t Test

$2-1$

Assuming equal variances

Difference $\quad 0.00000$ t Ratio

Std Err Dif 2.951e-5 DF

Upper CL Dif 0.000082 Prob $>|t| 1.0000$

Lower CL Dif -8.19e-5 Prob $>t \quad 0.5000$

Confidence $\quad 0.95$ Prob $<t \quad 0.5000$

Means for Oneway Anova

Level Number Mean Std Error Lower 95\% Upper 95\%

$\begin{array}{lrrrrr}1 & 3 & 0.008783 & 0.00002 & 0.00873 & 0.00884 \\ 2 & 3 & 0.008783 & 0.00002 & 0.00873 & 0.00884\end{array}$

Std Error uses a pooled estimate of error variance

Oneway Analysis of Measured By Block Prep Method=PF, Glass ID=U-std, Oxide $=\mathrm{Fe} 2 \mathrm{O3}(\mathrm{wt} \%)$, Targeted $=\mathbf{1 3 . 1 9 6}$

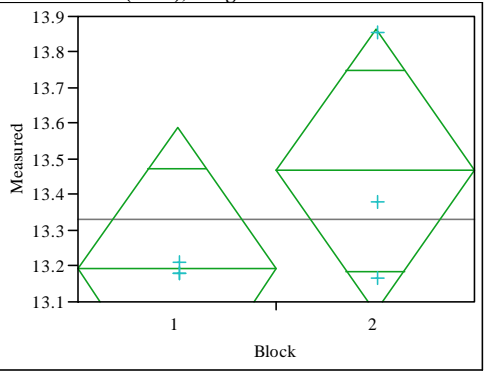

Oneway Anova

Summary of Fit

Rsquare

Adj Rsquare

Mean of Response

Observations (or Sum Wgts)

0.248524

13.32957

t Test

2-1

Assuming equal variances

Difference $\quad 0.27641$ t Ratio 1.36216

Std Err Dif 0.20292 DF

Upper CL Dif 0.83980 Prob $>|t| \quad 0.2448$

Lower CL Dif -0.28698 Prob $>t \quad 0.1224$

Confidence $\quad 0.95$ Prob $<\mathrm{t} \quad 0.8776$

Means for Oneway Anova

Level Number Mean Std Error Lower 95\% Upper 95\%

$\begin{array}{llrrrr}1 & 3 & 13.1914 & 0.14349 & 12.793 & 13.590 \\ 2 & 3 & 13.4678 & 0.14349 & 13.069 & 13.866\end{array}$

Std Error uses a pooled estimate of error variance
Oneway Analysis of Measured By Block Prep Method=PF, Glass ID=U-std, Oxide $=$ K2O $($ wt $\%)$, Targeted $=2.999$

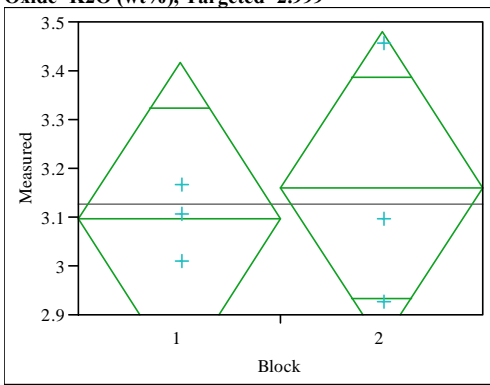

Oneway Anova

Summary of Fit

Rsquare

0.20319
-0.19957

Reot Mean Square Error $\quad 0.199457$

Mean of Respons

3.127945

Observations (or Sum Wgts)

t Test

Assuming equal variances

Difference $\quad 0.06425$ t Ratio 0.394491

Std Err Dif $\quad 0.16286$ DF

Upper CL Dif 0.51641 Prob $>|t| \quad 0.7133$

Lower CL Dif -0.38792 Prob $>t \quad 0.3567$

Confidence $\quad 0.95$ Prob $<\mathrm{t} \quad 0.6433$

Means for Oneway Anova

Level Number Mean Std Error Lower 95\% Upper 95\%

$\begin{array}{llllll}1 & 3 & 3.09582 & 0.11516 & 2.7761 & 3.4155 \\ 2 & 3 & 3.16007 & 0.11516 & 2.8403 & 3.4798\end{array}$

Std Error uses a pooled estimate of error variance

Oneway Analysis of Measured By Block Prep Method=PF, Glass ID=U-std, Oxide $=$ La2O3 $(w t \%)$, Targeted $=0$

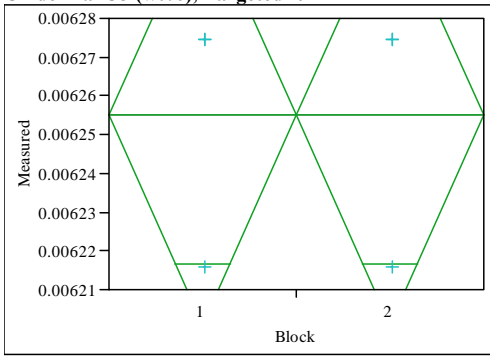

Oneway Anova

Summary of Fit

Rsquare

Adj Rsquare

Root Mean Square Error $\quad-0.25$

Mean of Response $\quad 0.006255$

Observations (or Sum Wgts)

0.006255
6

t Test

Assuming equal variances

Difference $\quad 0.00000$ t Ratio

Std Err Dif 2.764e-5 DF

Upper CL Dif 7.675e-5 Prob >|t 1.0000

Lower CL Dif $-7.67 \mathrm{e}-5$ Prob $>\mathrm{t} \quad 0.5000$

Confidence $\quad 0.95$ Prob $<\mathrm{t} \quad 0.5000$

Means for Oneway Anova

$\begin{array}{rrrrrr}\text { Level Number } & \text { Mean } & \text { Std Error } & \text { Lower 95\% } & \text { Upper 95\% } \\ 1 & 3 & 0.006255 & 0.00002 & 0.00620 & 0.00631\end{array}$

$\begin{array}{llllll}1 & 3 & 0.006255 & 0.00002 & 0.00620 & 0.00631 \\ 2 & 3 & 0.006255 & 0.00002 & 0.00620 & 0.00631\end{array}$

Std Error uses a pooled estimate of error variance 


\section{Exhibit C3. Measurements by Block and Sub-Block for Samples of the ARG-1 and Ustd Standards with the Thorium Glasses by Oxide}

Oneway Analysis of Measured By Block Prep Method=PF, Glass ID=U-std, Oxide $=\mathrm{Li} 2 \mathrm{O}(\mathrm{wt} \%)$, Targeted $=3.057$

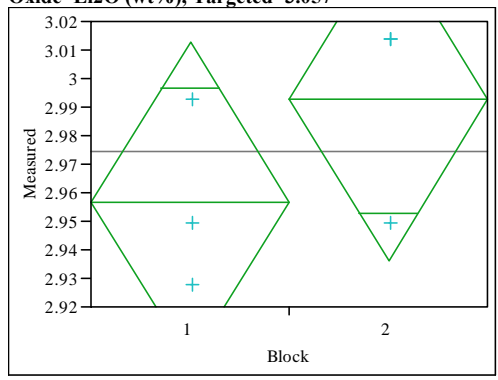

Oneway Anova
Summary of Fit

Rsquare

0.280899

Adj Rsquare

Root Mear Square Error 0.035157

Mean of Response

2.97459

Observations (or Sum Wgts)

t Test

2-1

Assuming equal variances

Difference $\quad 0.03588$ t Ratio $\quad 1.25$

Std Err Dif $\quad 0.02871$ DF 4

Upper CL Dif 0.11558 Prob $>|t| 0.2794$

Lower CL Dif -0.04382 Prob $>t \quad 0.1397$

$\begin{array}{lrl}\text { Confidence } & 0.95 \text { Prob }<\mathrm{t} & 0.8603\end{array}$

Means for Oneway Anova

Level Number Mean Std Error Lower 95\% Upper 95\%

$\begin{array}{llrrrr}1 & 3 & 2.95665 & 0.02030 & 2.9003 & 3.0130 \\ 2 & 3 & 2.99253 & 0.02030 & 2.9362 & 3.0489\end{array}$

Std Error uses a pooled estimate of error variance

Oneway Analysis of Measured By Block Prep Method=PF, Glass ID=U-std, Oxide $=\mathrm{MgO}($ wt $\%)$, Targeted $=1.21$

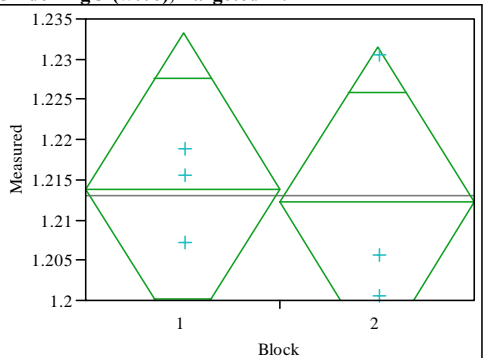

Oneway Anova

Summary of Fit

Rsquare

Adj Rsquare

Mean of Response 1.213046

Observations (or Sum Wgts) 6

t Test

2-1

Assuming equal variances

$\begin{array}{lll}\text { Difference } & -0.00166 \text { t Ratio } \quad-0.16823 \\ \text { Std Ern } & 0.00986 \text { DF } & \end{array}$

Std Err Dif $\quad 0.00986$ DF $\quad 4$

Upper CL Dif 0.02571 Prob $>|t| 0.8746$

Lower CL Dif -0.02903 Prob $>\mathrm{t} \quad 0.5627$

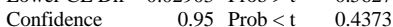

Means for Oneway Anova

Level Number Mean Std Error Lower 95\% Upper 95\%

$\begin{array}{lrrrrr}1 & 3 & 1.21388 & 0.00697 & 1.1945 & 1.2332 \\ 2 & 3 & 1.21222 & 0.00697 & 1.1929 & 1.2316\end{array}$
Std Error uses a pooled estimate of error variance

Oneway Analysis of Measured By Block Prep Method=PF, Glass ID=U-std, Oxide $=\mathbf{M n O}(\mathbf{w t} \%)$, Targeted $=\mathbf{2 . 8 9 2}$

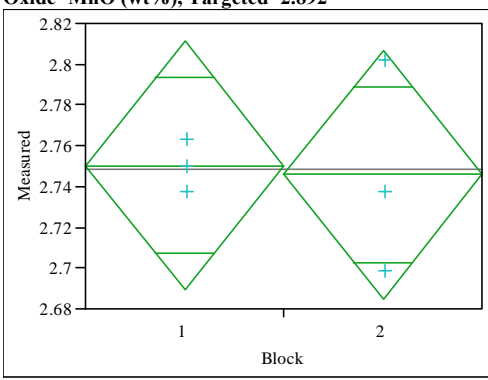

Oneway Anova

Summary of Fit

Adj Rsquare $\quad-0.2440$

Root Mean Square

Mean of Respons

2.748104

t Test

2-1

Assuming equal variances

$\begin{array}{lrlr}\text { Difference } & -0.00430 & \mathrm{t} \text { Ratio } & -0.13868 \\ \text { Std Err Dif } & 0.03104 & \text { DF } & 4 \\ \text { Upper CL Dif } & 0.08187 & \text { Prob }>|t| & 0.8964 \\ \text { Lower CL Dif } & -0.09048 & \text { Prob }>t & 0.5518 \\ \text { Confidence } & 0.95 & \text { Prob }<\mathrm{t} & 0.4482\end{array}$

Means for Oneway Anova

Level Number Mean Std Error Lower 95\% Upper 95\%

$\begin{array}{llllrr}1 & 3 & 2.75026 & 0.02195 & 2.6893 & 2.8112\end{array}$

$\begin{array}{llllll}2 & 3 & 2.74595 & 0.02195 & 2.6850 & 2.8069\end{array}$

Std Error uses a pooled estimate of error variance

Oneway Analysis of Measured By Block Prep Method=PF, Glass ID=U-std, Oxide $=\mathrm{NiO}(\mathrm{wt} \%)$, Targeted $=\mathbf{1 . 1 2}$

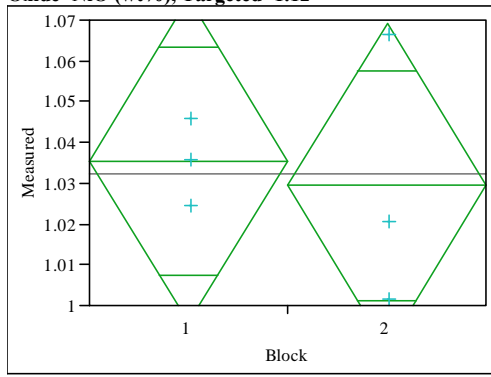

Oneway Anova

Rsquare

Adj Rsquare $\quad-0.22368$

Error $\quad 0.024795$

Mean of Respons

1.032422

t Test

Assuming equal variances

Difference $\quad-0.00594$ t Ratio $\quad-0.29333$

Std Err Dif 0.02024 DF 4

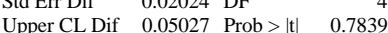

$\begin{array}{lll}\text { Lower CL Dif } & -0.06215 \text { Prob }>t & 0.6081\end{array}$

$\begin{array}{lrl}\text { Confidence } & 0.95 \text { Prob }<\mathrm{t} & 0.3919\end{array}$

Means for Oneway Anova

Level Number Mean Std Error Lower 95\% Upper 95\%

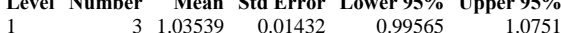




\section{Exhibit C3. Measurements by Block and Sub-Block for Samples of the ARG-1 and Ustd Standards with the Thorium Glasses by Oxide}

Level Number Mean Std Error Lower 95\% Upper 95\%

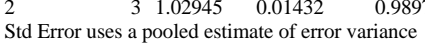

Oneway Analysis of Measured By Block Prep Method=PF, Glass ID=U-std, Oxide $=\mathbf{P 2 O}(\mathbf{w t} \%)$, Targeted $=\mathbf{0}$

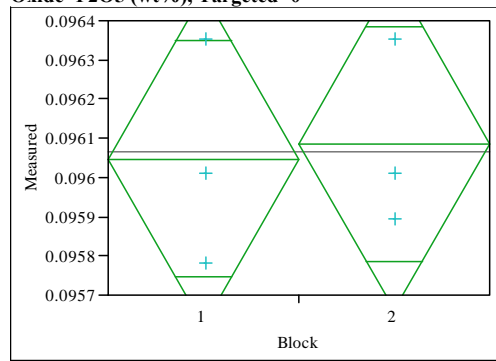

Oneway Anova

Summary of Fit

Adj Rsquare

0.000265

Mean of Response 0.096067

Observations (or Sum Wgts)

t Test

$2-1$

Assuming equal variances

Difference $\quad 3.819 \mathrm{e}-5$ t Ratio 0.176777 Std Err Dif $\quad 0.00022$ DF

Upper CL Dif 0.00064 Prob $>|\mathrm{t}| \quad 0.8683$

Lower CL Dif -0.00056 Prob $>t \quad 0.4341$

Confidence $\quad 0.95$ Prob $<\mathrm{t} \quad 0.5659$

Means for Oneway Anova

Level Number Mean Std Error Lower 95\% Upper 95\%

$\begin{array}{llllll}1 & 3 & 0.096048 & 0.00015 & 0.09562 & 0.09647 \\ 2 & 3 & 0.096086 & 0.00015 & 0.09566 & 0.09651\end{array}$

Std Error uses a pooled estimate of error variance

Oneway Analysis of Measured By Block Prep Method=PF, Glass ID=U-std, Oxide $=\mathbf{P b O}($ wt $\%)$, Targeted $=0$

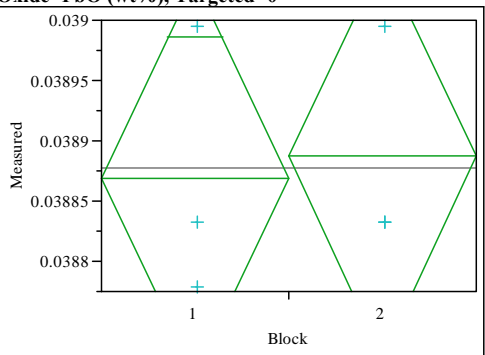

Oneway Anova
Summary of Fit

Rsquare

Adj Rsquare

Root Mean Square Error $\quad-0.23596$

Mean of Response $\quad 0.038878$

Observations (or Sum Wgts) 6

t Test

2-1

Assuming equal variances

Difference $\quad 0.000018$ t Ratio 0.213201

Std Err Dif 8.421e-5 DF $\quad 4$

Upper CL Dif 0.00025 Prob $>|t| \quad 0.8416$

Lower CL Dif -0.00022 Prob $>\mathrm{t} \quad 0.4208$

$\begin{array}{lrl}\text { Confidence } & 0.95 \mathrm{Prob}<\mathrm{t} & 0.5792\end{array}$

Means for Oneway Anova

Level Number Mean Std Error Lower 95\% Upper 95\%

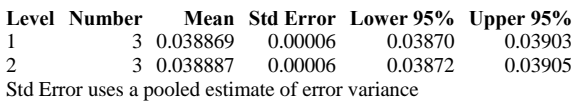

Oneway Analysis of Measured By Block Prep Method=PF, Glass ID=U-std, Oxide $=\mathrm{SiO} 2(\mathrm{wt} \%)$, Targeted $=\mathbf{4 5 . 3 5 3}$

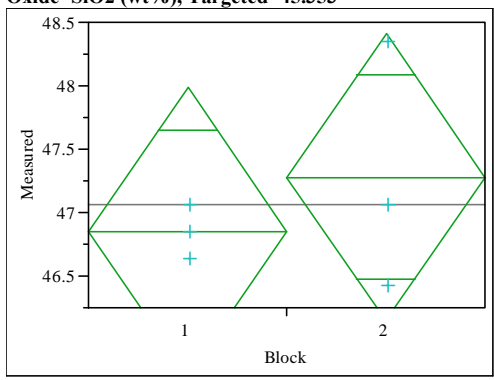

Oneway Anova

Summary of Fit

Rsquare

0.1
Root

0.709526

Observations (or Sum Wgts) $\quad 47.0646$

t Test

Assuming equal variances

Difference $\quad 0.4279$ t Ratio $\quad 0.738549$

$\begin{array}{lrlr}\text { Std Err Dif } & 0.5793 & \text { DF } & 4 \\ \text { Upper CL Dif } & 2.0363 \text { Prob }>|t| & 0.5012\end{array}$

$\begin{array}{lrll}\text { Upper CL Dif } & 2.0363 & \text { Prob }>|t| & 0.5012 \\ \text { Lower CL Dif } & -1.1806 & \text { Prob }>t & 0.2506\end{array}$

$\begin{array}{lrll}\text { Lower CL Dif } & -1.1806 & \text { Prob }>t & 0.2506 \\ \text { Confidence } & 0.95 & \text { Prob }<t & 0.7494\end{array}$

Means for Oneway Anova

\begin{tabular}{rrrrr} 
Level Number & Mean & Std Error & Lower 95\% & Upper 95\% \\
\hline 3 & 46.8507 & 0.40964 & 45.713 & 47.988
\end{tabular}

$\begin{array}{llllll}1 & 3 & 46.8507 & 0.40964 & 45.713 & 47.988 \\ 2 & 3 & 47.2785 & 0.40964 & 46.141 & 48.416\end{array}$

Std Error uses a pooled estimate of error variance

Oneway Analysis of Measured By Block Prep Method=PF, Glass ID=U-std, Oxide $=\mathrm{SO} 4(\mathrm{wt} \%)$, Targeted $=0$

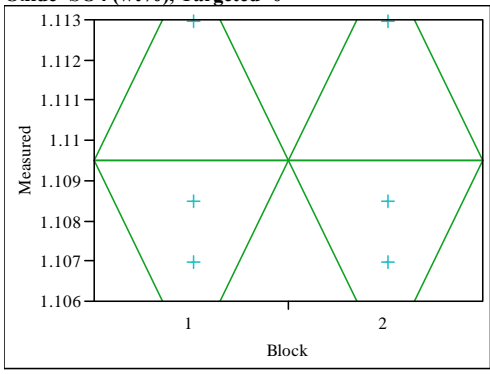

Oneway Anova

Summary of Fi

$\begin{array}{lr}\text { Rsquare } & 0 \\ \text { Adj Rsquare } & -0.25\end{array}$

Root Mean Square Error $\quad 0.003118$

Mean of Response

0.003118
1.109482

Observations (or Sum Wgts)

t Test

Assuming equal variances

Difference $\quad 0.00000$ t Ratio Std Err Dif $\quad 0.00255$ DF

Upper CL Dif 0.00707 Prob $>141.000$

Upper CL Dif 0.00707 Prob $>|t| 1.0000$

Lower CL Dif -0.00707 Prob $>$ t 0.5000

$\begin{array}{lll}\text { Confidence } & 0.95 \text { Prob }<\mathrm{t} \quad 0.5000\end{array}$ 


\section{Exhibit C3. Measurements by Block and Sub-Block for Samples of the ARG-1 and Ustd Standards with the Thorium Glasses by Oxide}

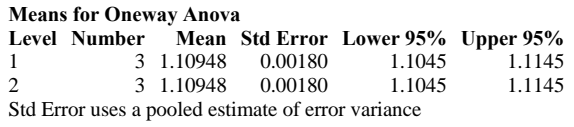

Oneway Analysis of Measured By Block Prep Method=PF, Glass ID=U-std, Oxide $=\mathrm{ThO} 2(\mathrm{wt} \%)$, Targeted $=0$

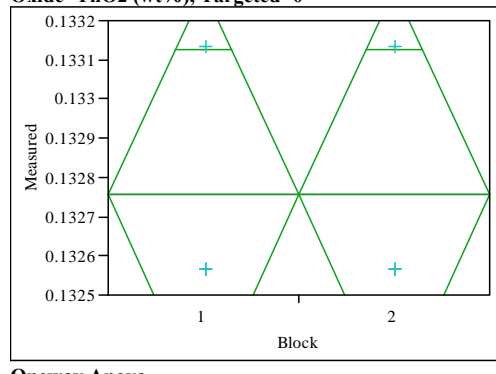

\section{Oneway Anova
Summary of Fit}

Rsquare

Adj Rsquare

Observations (or Sum Wgts) $\quad 6$

t Test

2-1

Assuming equal variances

Difference $\quad 0.00000$ t Ratio 0

Std Err Dif $\quad 0.00027$ DF

Upper CL Dif $0.00074 \quad$ Prob $>|t| 1.0000$

Lower CL D

Lower CL D
Confidence

Means for Oneway Anova

Level Number Mean Std Error Lower 95\% Upper 95\%

$\begin{array}{lrrrrr}1 & 3 & 0.132755 & 0.00019 & 0.13223 & 0.13328 \\ 2 & 3 & 0.132755 & 0.00019 & 0.13223 & 0.13328\end{array}$

Std Error uses a pooled estimate of error variance

Oneway Analysis of Measured By Block Prep Method=PF, Glass ID=U-std, Oxide $=$ TiO2 $(w t \%)$, Targeted $=1.049$

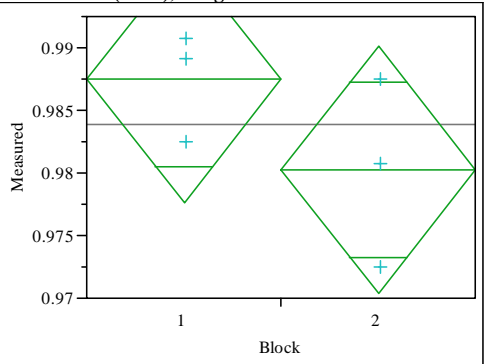

Oneway Anova

Summary of Fit

Rsquare

Adj Rsquare

0.006166 0.983842

\section{t Test}

2-1

Assuming equal variances

Difference $\quad-0.00723$ t Ratio $\quad-1.4356$ Std Err Dif 0.00503 DF

Upper CL Dif 0.00675 Prob $>|t| \quad 0.2244$

Lower CL Dif -0.02121 Prob $>t \quad 0.8878$

0.95 Prob $<t$

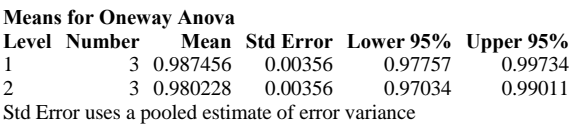

Oneway Analysis of Measured By Block Prep Method=PF, Glass ID=U-std, Oxide $=\mathbf{U} 308(\mathbf{w t} \%)$, Targeted $=\mathbf{2 . 4 0 6}$

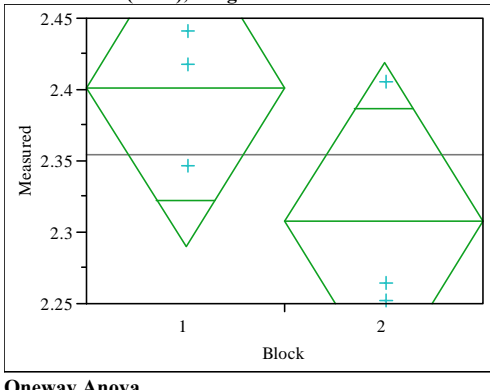

Summary of Fit

$\begin{array}{lr}\text { Rsquare } & 0.407932 \\ \text { Adj Rsquare } & 0.259915 \\ \text { Root Mean Square Error } & 0.069596 \\ \text { Mean of Response } & 2.354469 \\ \text { Observations (or Sum Wgts) } & 6\end{array}$

t Test

2-1

Assuming equal variances

Difference $\quad-0.09434$ t Ratio -1.66011

$\begin{array}{lrr}\text { Std Err Dif } & 0.05682 \text { DF } & 4 \\ \text { Upper CL Dif } & 0.06344 \text { Prob }>|t| & 0.1722\end{array}$

$\begin{array}{lrll}\text { Upper CL Dif } & 0.06344 & \text { Prob }>|t| & 0.1722 \\ \text { Lower CL Dif } & -0.25211 & \text { Prob }>t & 0.9139\end{array}$

$\begin{array}{lrl}\text { Confidence } & 0.95 \text { Prob }<\mathrm{t} & 0.0861\end{array}$

Means for Oneway Anova

Level Number Mean Std Error Lower 95\% Upper 95\%

$\begin{array}{lrrrrr}1 & 3 & 2.40164 & 0.04018 & 2.2901 & 2.5132 \\ 2 & 3 & 2.30730 & 0.04018 & 2.1957 & 2.4189\end{array}$

Std Error uses a pooled estimate of error variance

Oneway Analysis of Measured By Block Prep Method=PF, Glass ID=U-std, Oxide $=\mathrm{ZnO}($ wt $\%)$, Targeted $=0$

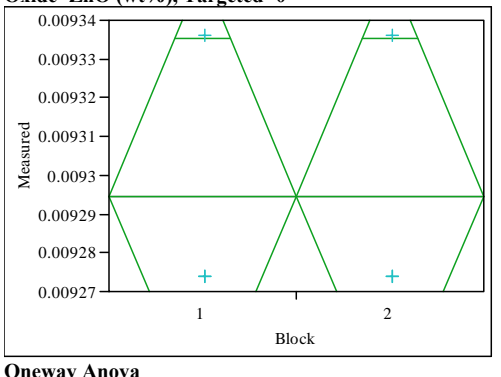

Oneway Anova
Summary of Fit

Rsquare

Adj Rsquare

$-0.25$

$\quad 0.000036$

Mean of

0.009295

Observations (or Sum Wgts)

t Test

2-1

Assuming equal variances

Difference $\quad 0.00000$ t Ratio $\quad 0$

Std Err Dif 2.934e-5 DF

Upper CL Dif 8.146e-5 Prob >|t| 1.0000

Lower CL Dif -8.15e-5 Prob $>$ t 0.5000

Confidence $\quad 0.95$ Prob $<\mathrm{t} \quad 0.5000$ 
Exhibit C3. Measurements by Block and Sub-Block for Samples of the ARG-1 and Ustd Standards with the Thorium Glasses by Oxide

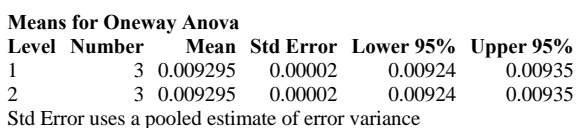




\section{Exhibit C4. Average Measured Versus Targeted Compositions by Glass ID by Oxide for the Thorium SB6 VS Glasses}

Overlay Plot Oxide=A12O3 (wt\%)

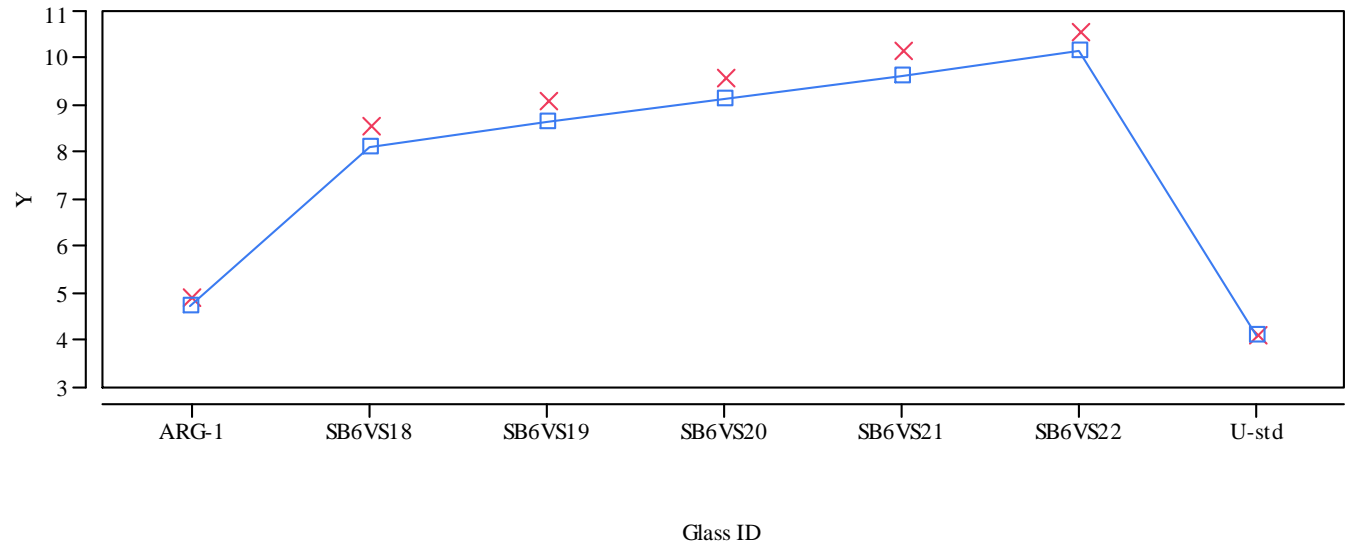

Overlay Plot Oxide=B2O3 (wt\%)

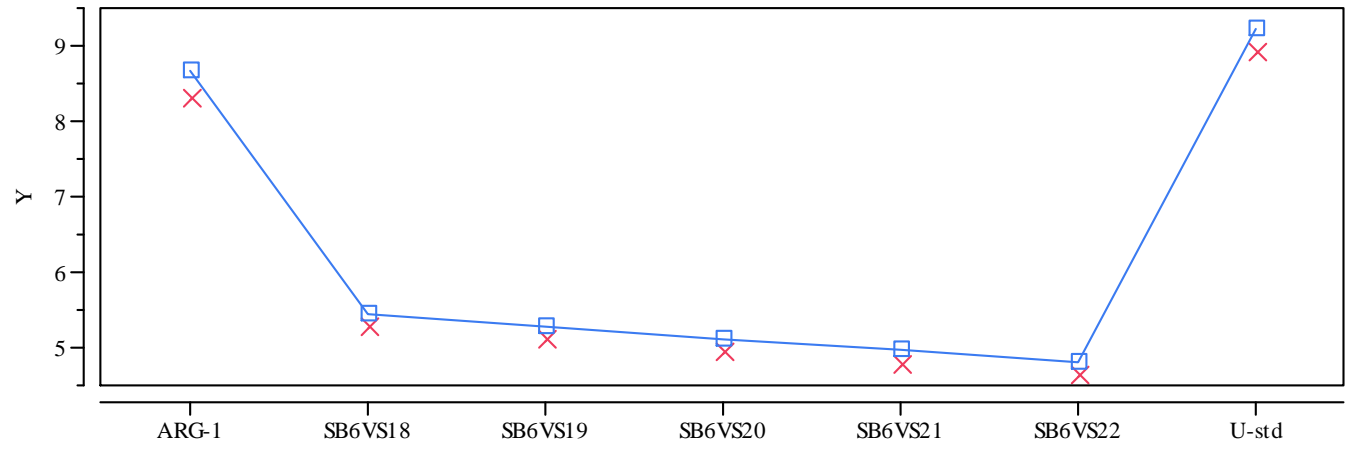

Glass ID

Overlay Plot Oxide $=\mathrm{BaO}(\mathbf{w t} \%)$

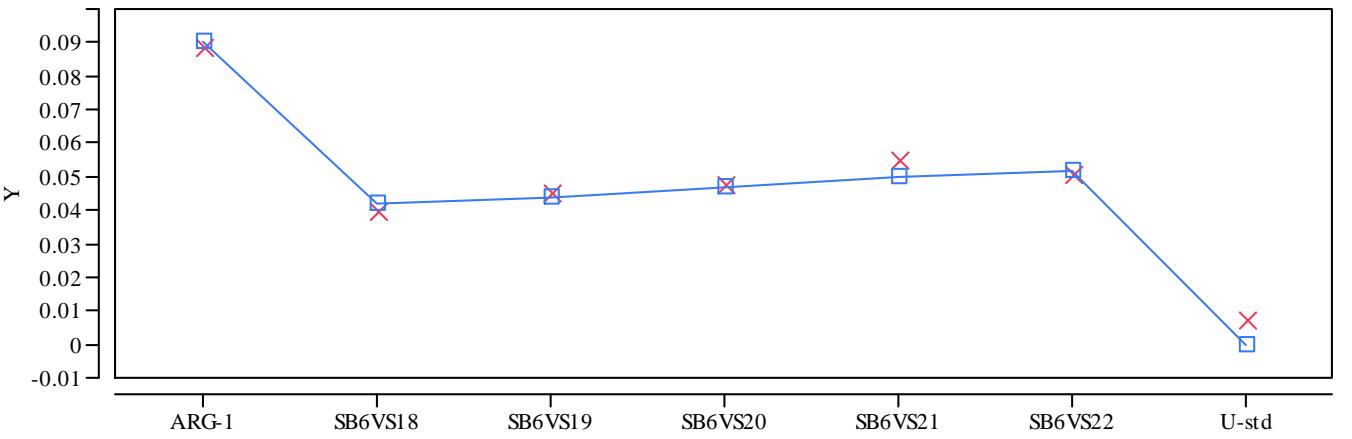

Glass ID

Y $\times$ Measured $\square-$ Targeted 


\section{Exhibit C4. Average Measured Versus Targeted Compositions by Glass ID by Oxide for the Thorium SB6 VS Glasses}

Overlay Plot Oxide $=\mathrm{CaO}\left(\mathrm{wt}_{\mathrm{t}} \%\right)$

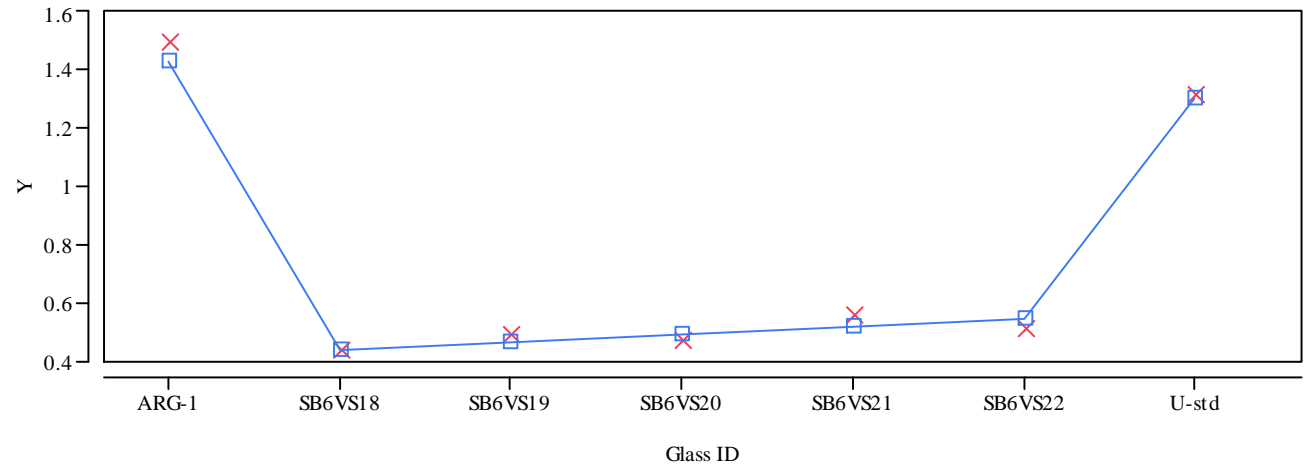

Overlay Plot Oxide $=\mathrm{CdO}(\mathrm{wt} \%)$

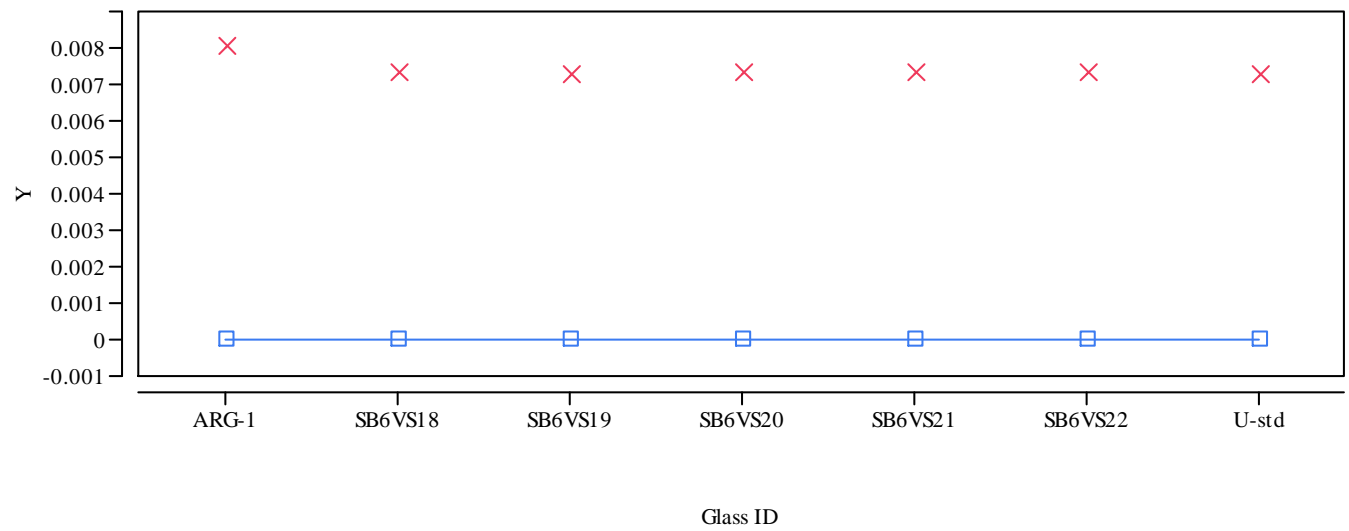

Overlay Plot Oxide $=\mathrm{Ce} 2 \mathrm{O3}(\mathrm{wt} \%)$

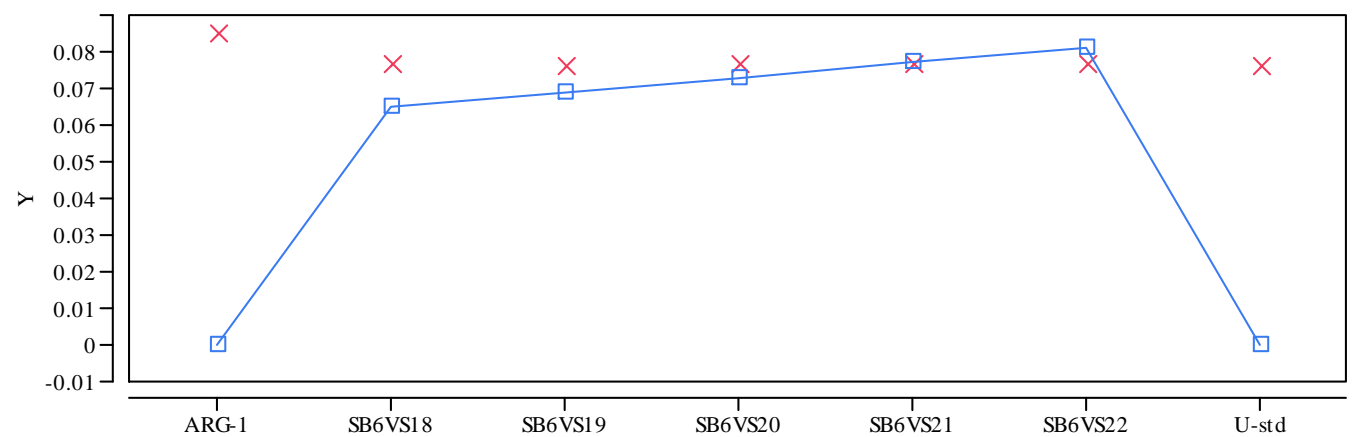

Glass ID

Y $\times$ Measured $\square$ Targeted 
Exhibit C4. Average Measured Versus Targeted Compositions by Glass ID by Oxide for the Thorium SB6 VS Glasses

Overlay Plot Oxide $=\mathrm{Cr2O3}(\mathrm{wt} \%)$

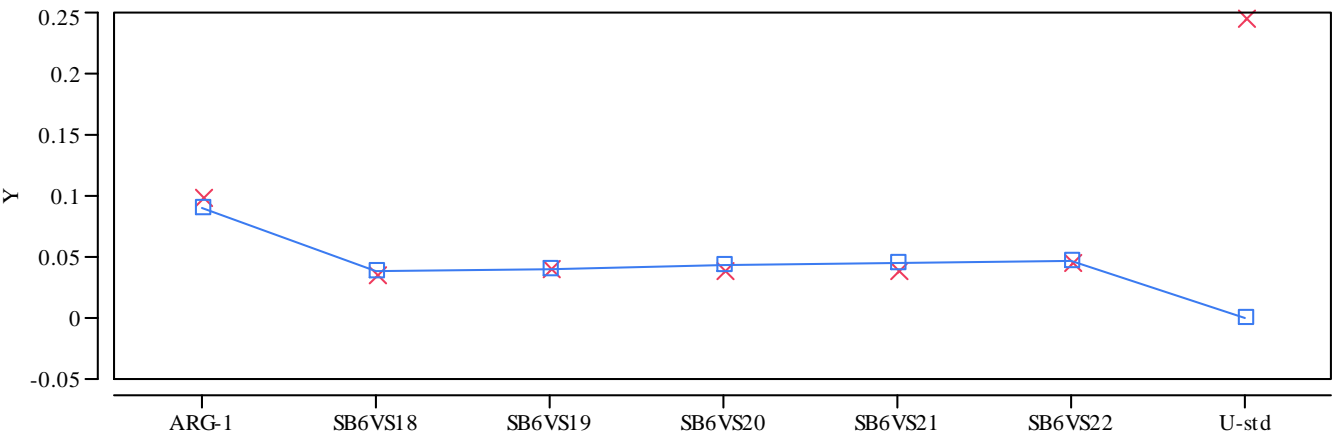

Glass ID

Overlay Plot Oxide=CuO $(w t \%)$

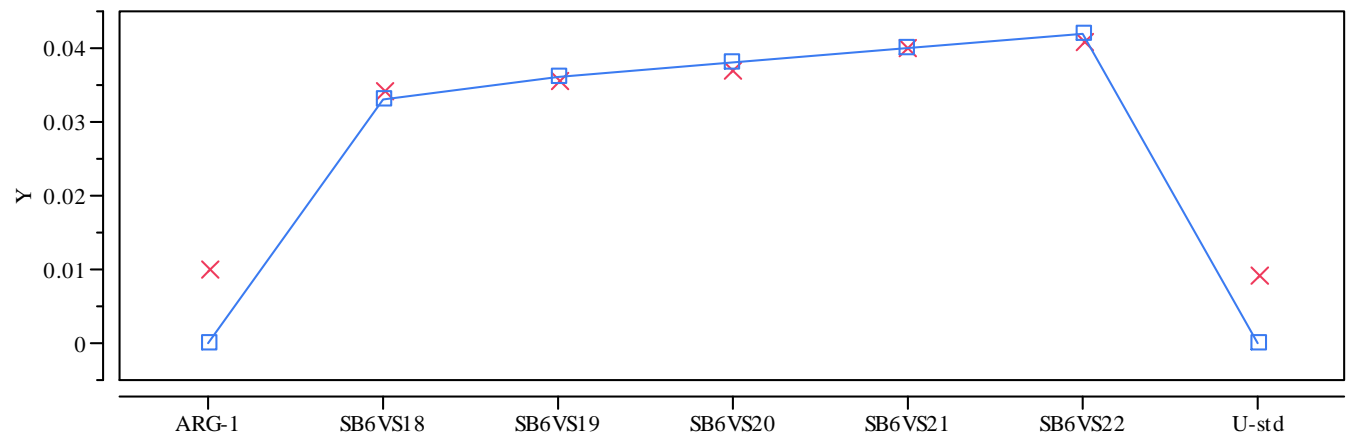

Glass ID

Overlay Plot Oxide $=$ Fe2O3 $(w t \%)$

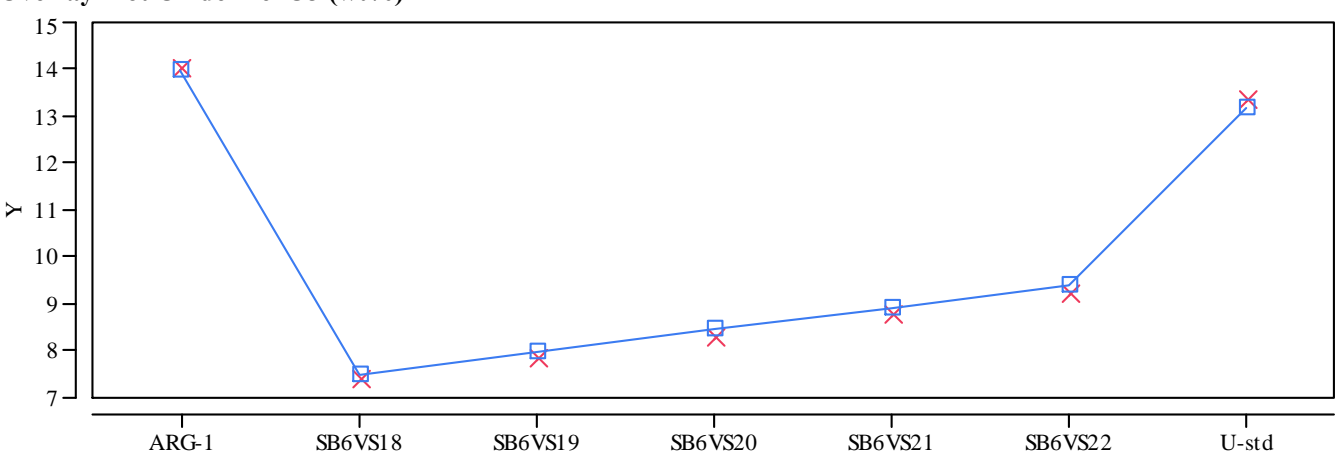

Glass ID

Y $\times$ Measured $\square$ Targeted 


\section{Exhibit C4. Average Measured Versus Targeted Compositions by Glass ID by Oxide for the Thorium SB6 VS Glasses}

Overlay Plot Oxide $=$ K2O (wt $\%)$

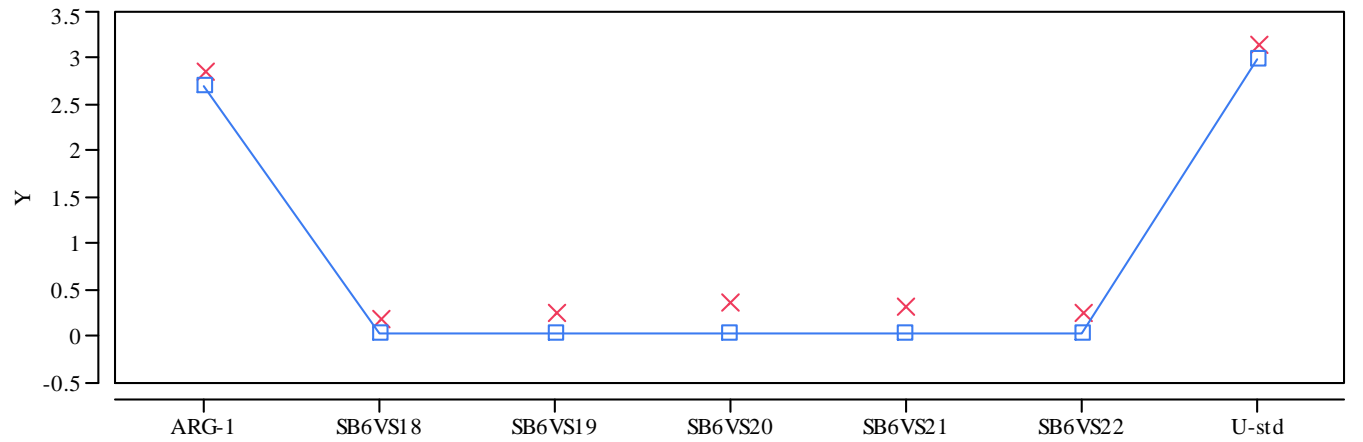

Glass ID

Overlay Plot Oxide $=$ La2O3 (wt\%)

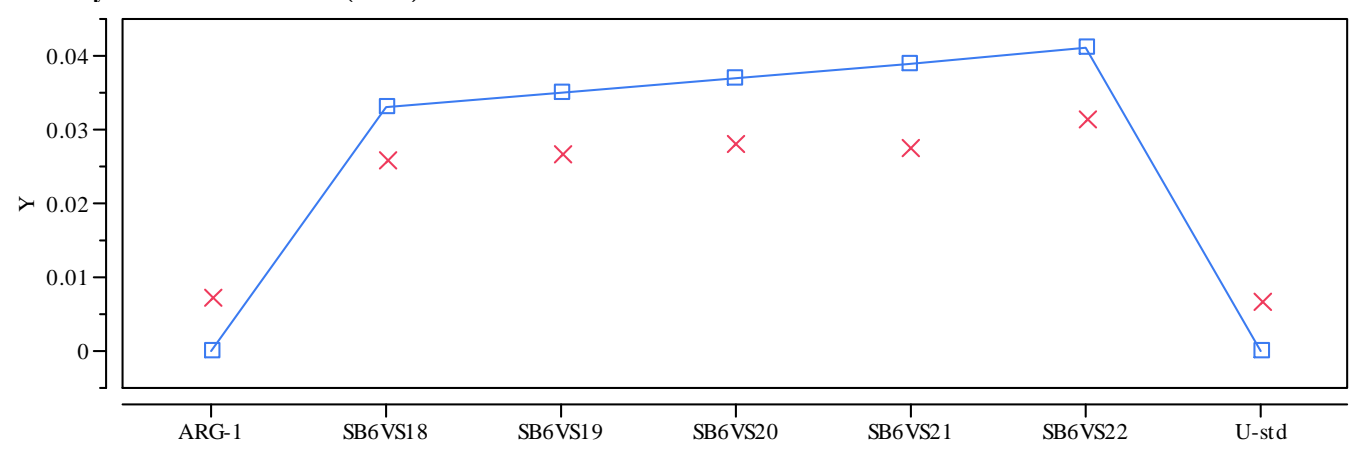

Glass ID

Overlay Plot Oxide $=$ Li2O $(w t \%)$

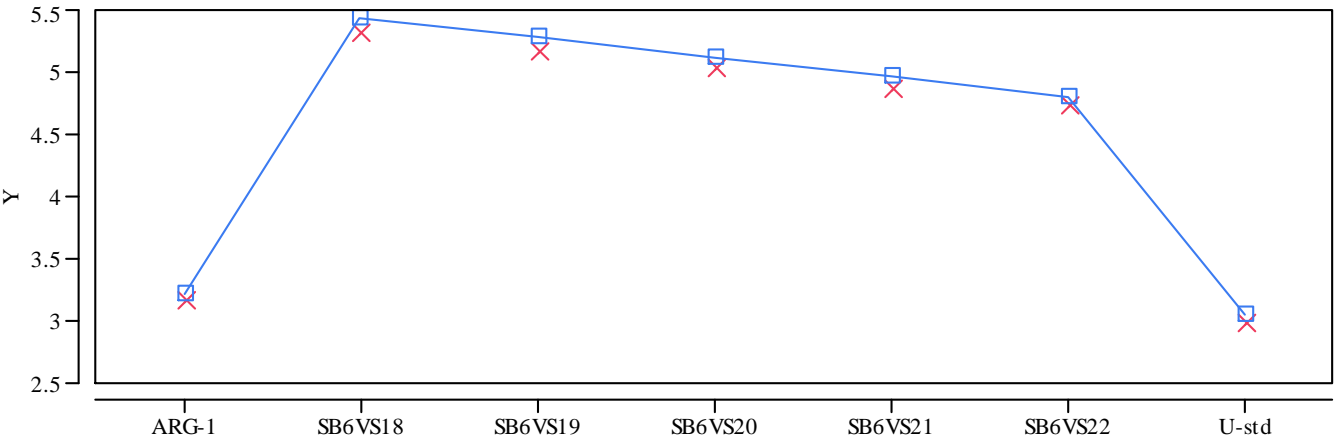

Glass ID

Y $\times$ Measured $\square$ Targeted 


\section{Exhibit C4. Average Measured Versus Targeted Compositions by Glass ID by Oxide for the Thorium SB6 VS Glasses}

Overlay Plot Oxide $=$ MgO $\left(w_{t} \%\right)$

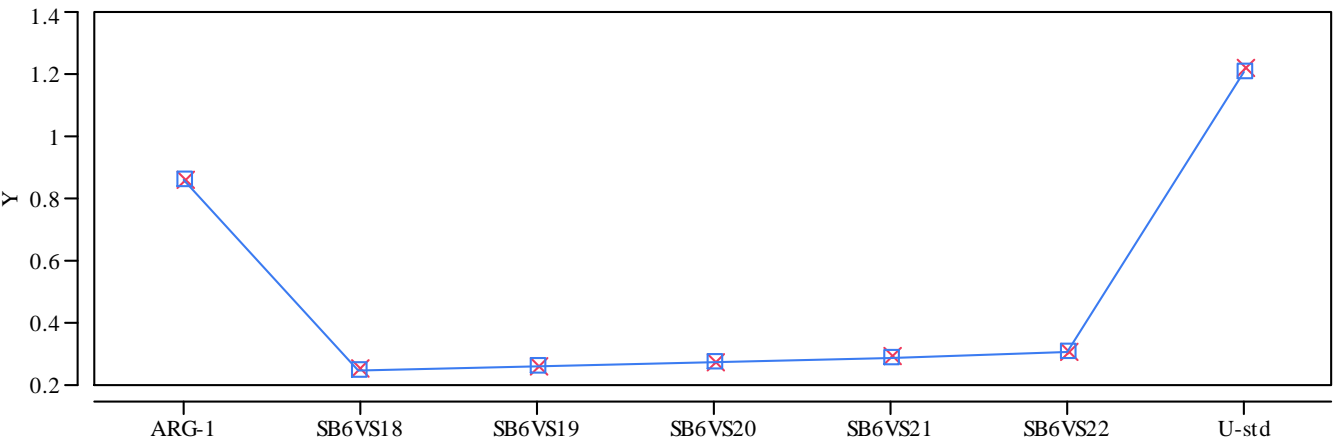

Glass ID

Overlay Plot Oxide=MnO (wt\%)

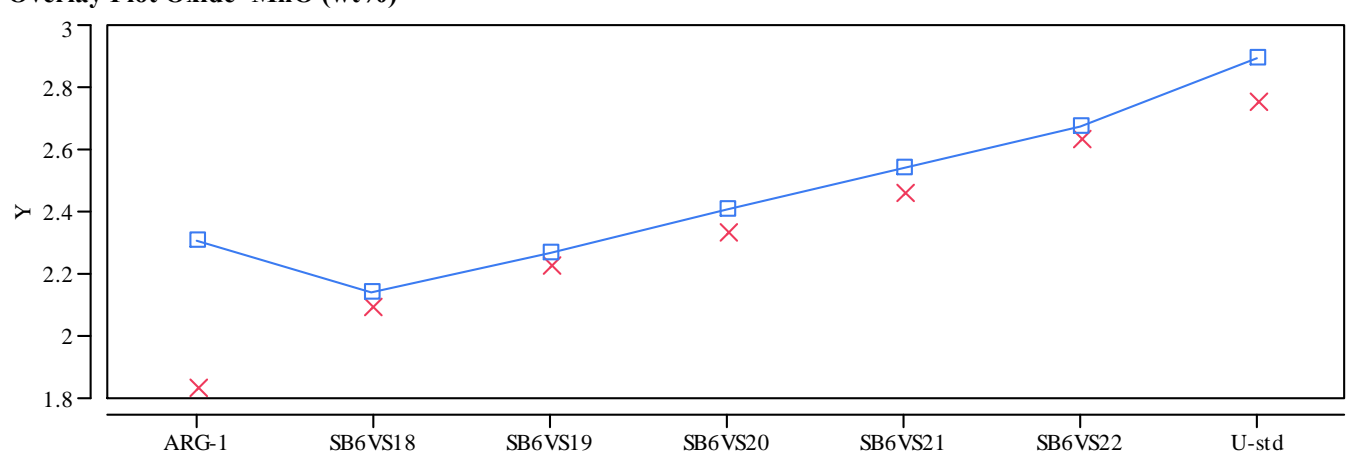

Glass ID

Overlay Plot Oxide $=\mathrm{Na} 2 \mathrm{O}(\mathrm{wt} \%)$

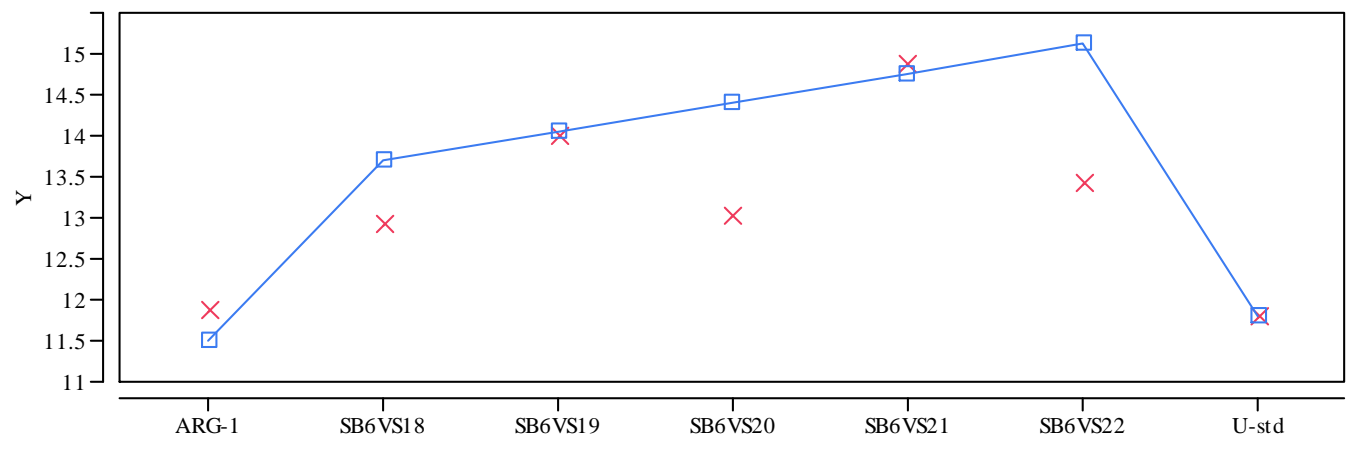

Glass ID

Y $\times$ Measured $\square-$ Targeted 


\section{Exhibit C4. Average Measured Versus Targeted Compositions by Glass ID by Oxide for the Thorium SB6 VS Glasses}

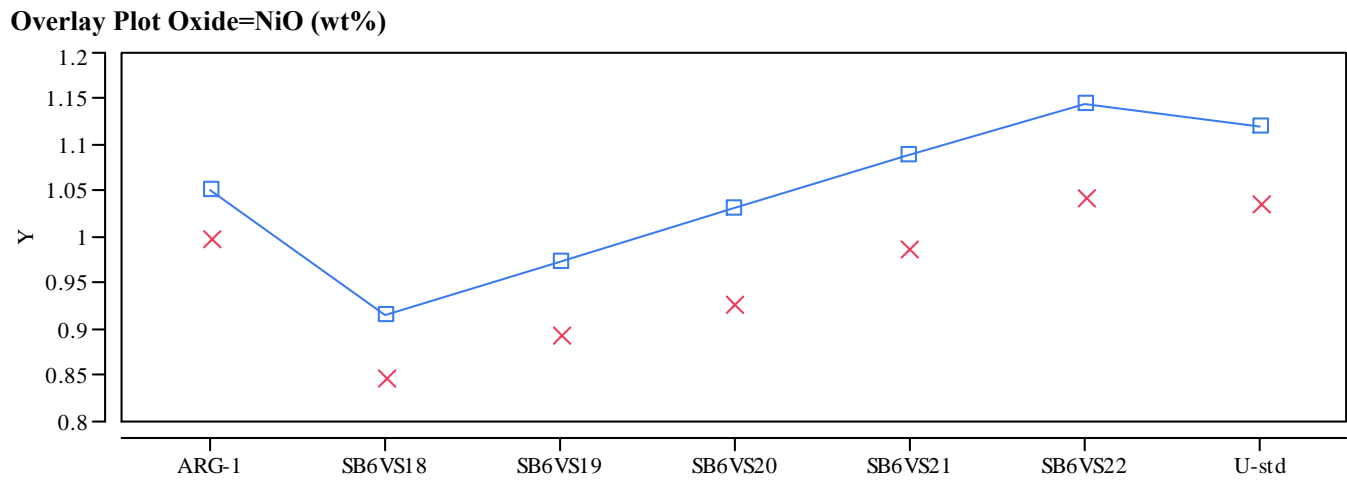

Glass ID

Overlay Plot Oxide $=$ P2O5 (wt\%)

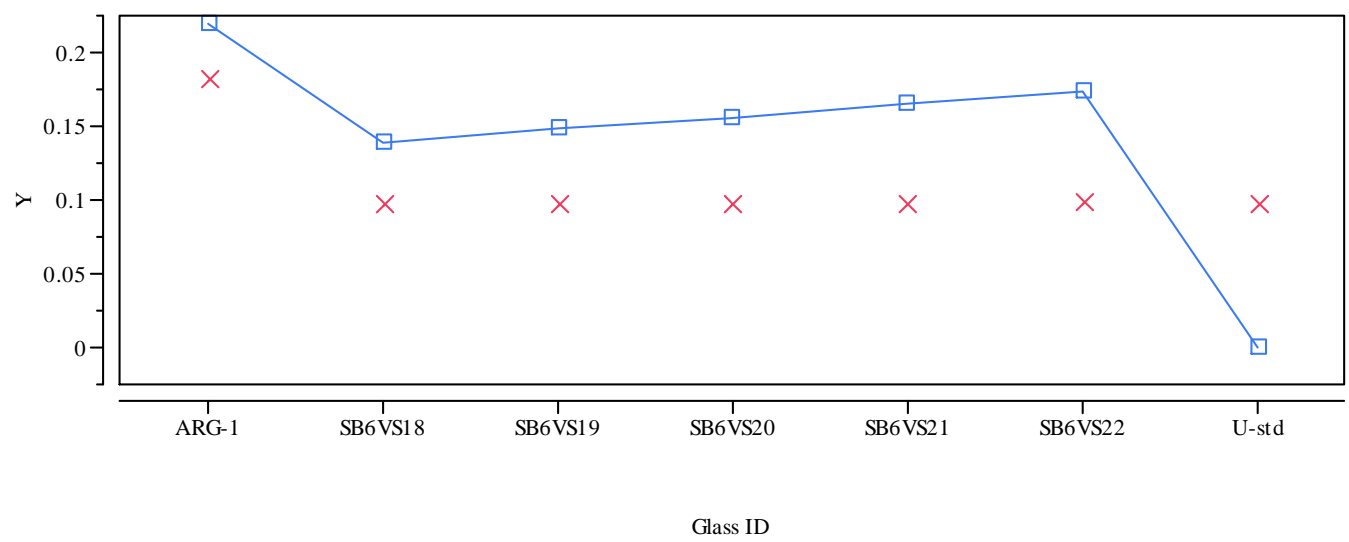

Overlay Plot Oxide $=$ PbO (wt\%)

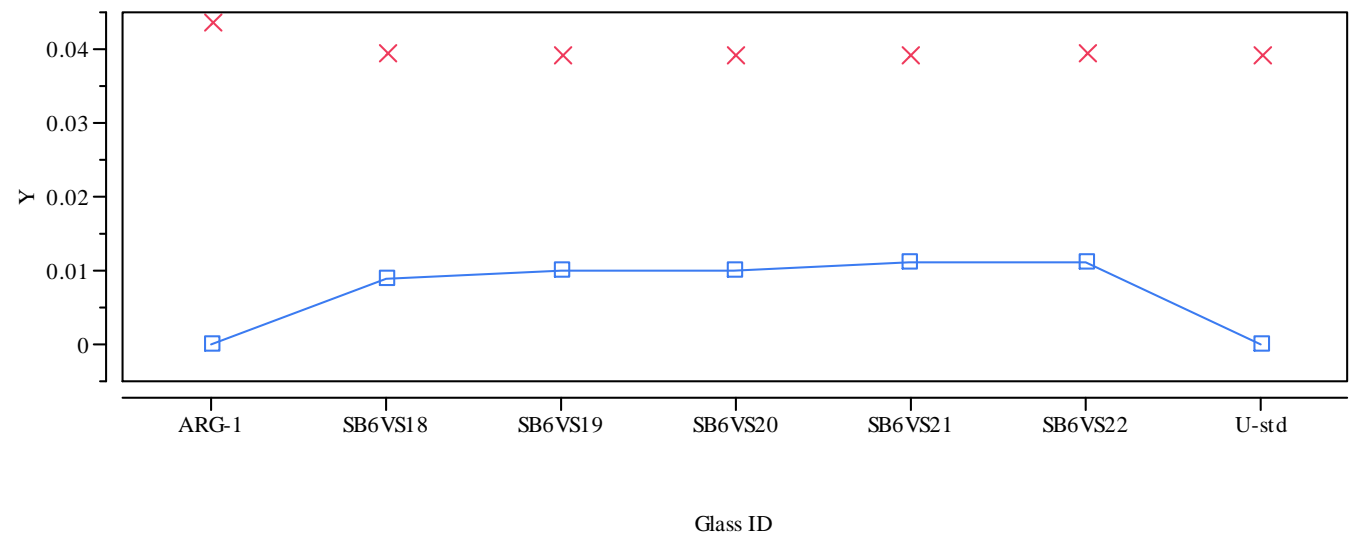

Y $\times$ Measured $\quad$ - Targeted 


\section{Exhibit C4. Average Measured Versus Targeted Compositions by Glass ID by Oxide for the Thorium SB6 VS Glasses}

Overlay Plot Oxide=SiO2 (wt\%)

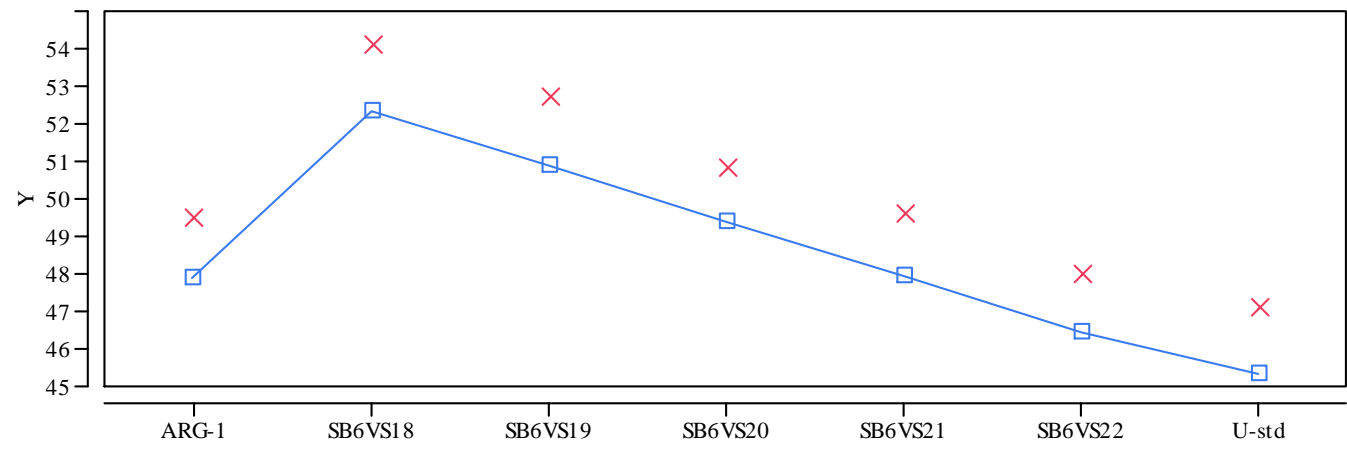

Glass ID

Overlay Plot Oxide=SO4 (wt\%)

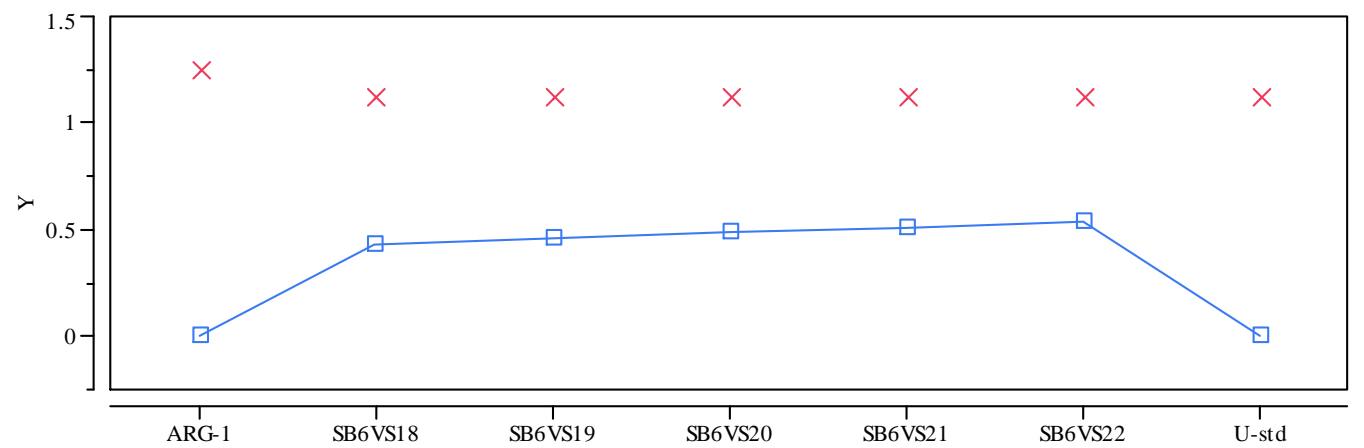

Glass ID

Overlay Plot Oxide $=$ ThO2 $(w t \%)$

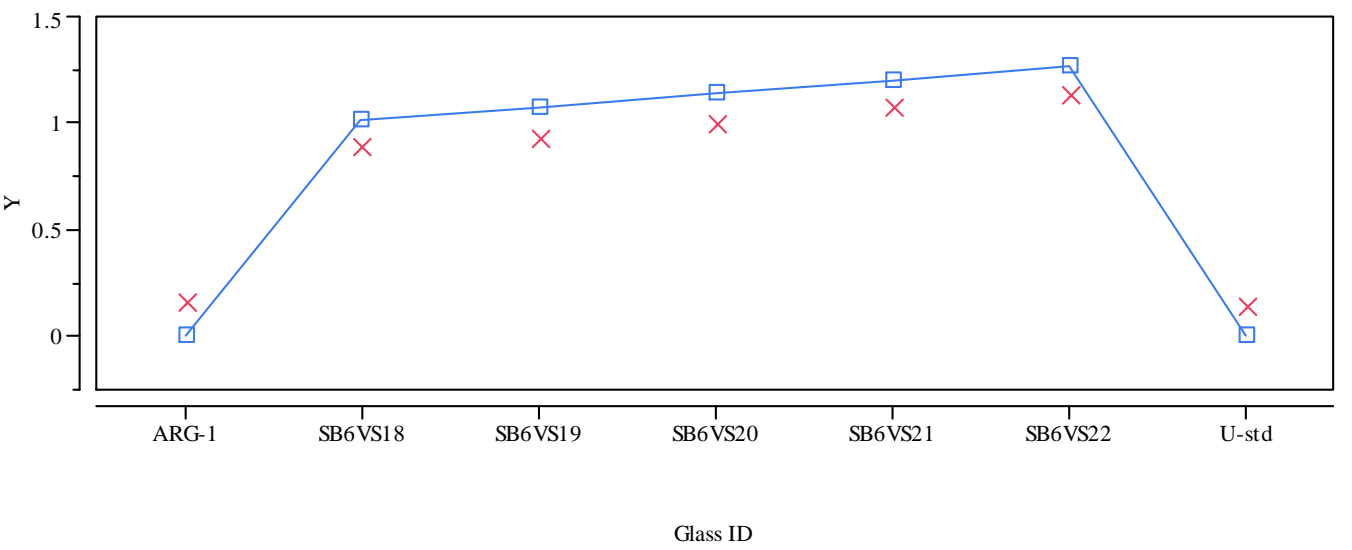

Y $\times$ Measured $\quad \square \longrightarrow$ Targeted 


\section{Exhibit C4. Average Measured Versus Targeted Compositions by Glass ID by Oxide for the Thorium SB6 VS Glasses}

Overlay Plot Oxide $=\mathrm{TiO} 2(\mathrm{wt} \%)$

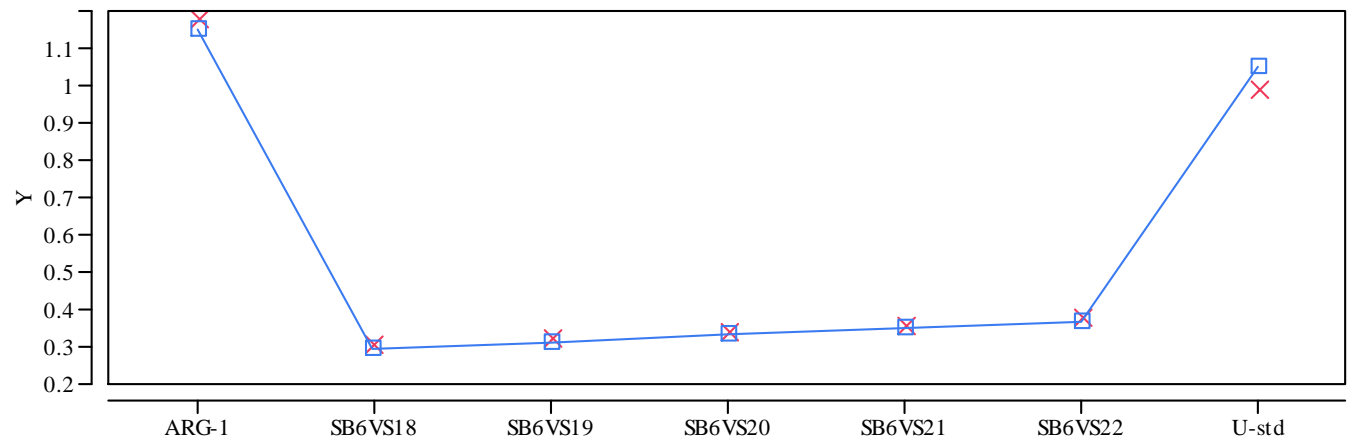

Glass ID

Overlay Plot Oxide $=\mathrm{U} 308(\mathrm{wt} \%)$

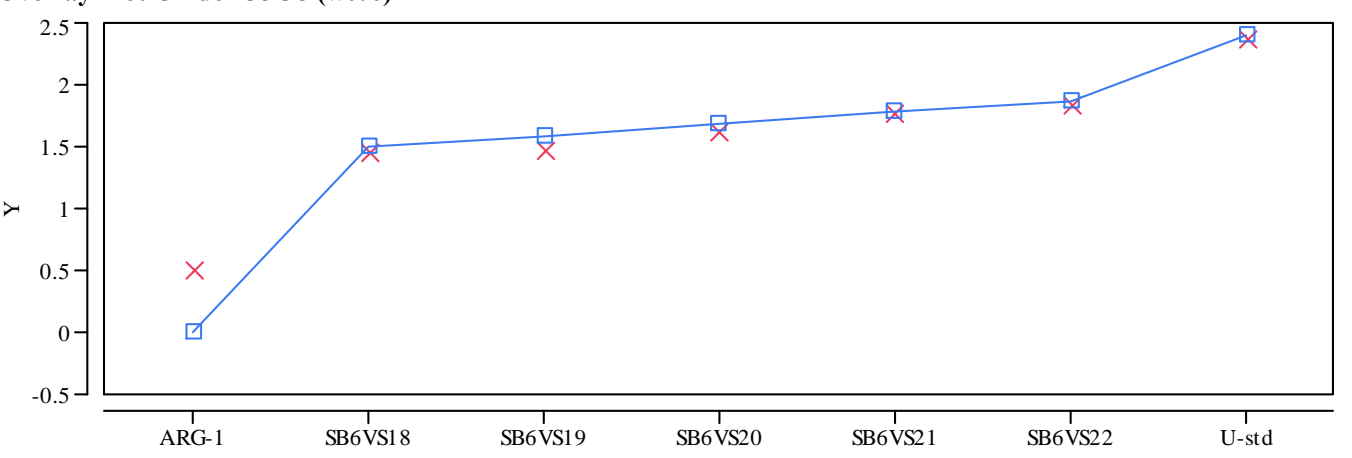

Glass ID

Overlay Plot Oxide $=\mathrm{ZnO}(\mathbf{w t} \%)$

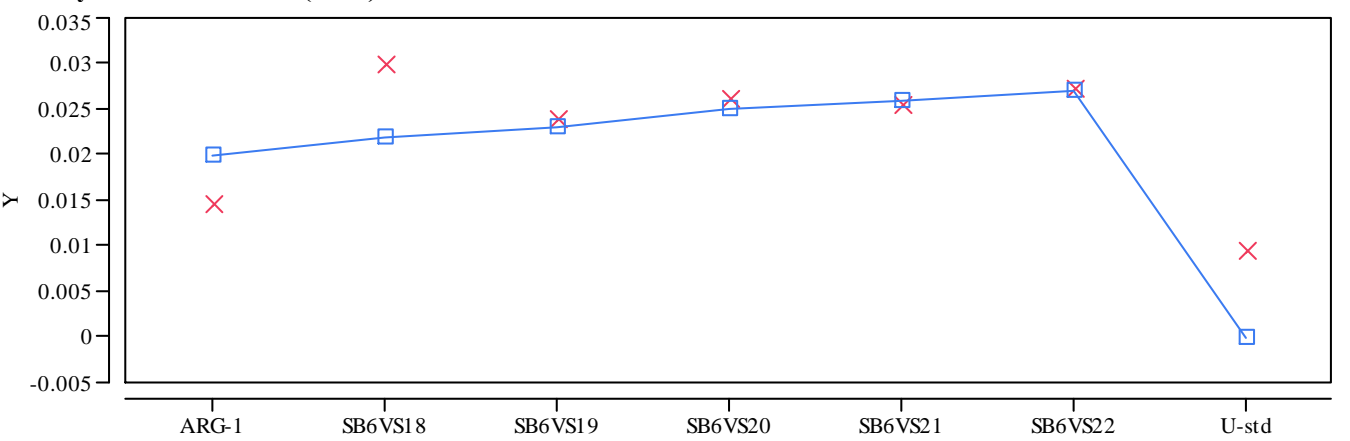

Glass ID

Y $\times$ Measured $\square-$ Targeted 


\section{Exhibit C4. Average Measured Versus Targeted Compositions by Glass ID by Oxide for the Thorium SB6 VS Glasses}

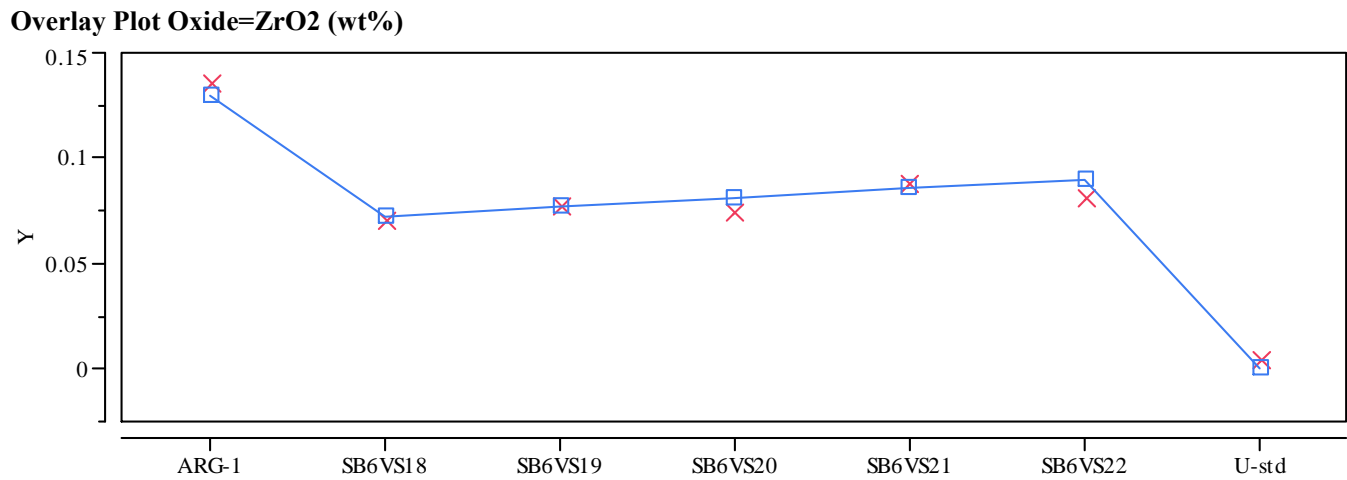

Glass ID

Overlay Plot Oxide $=$ Sum of Oxides

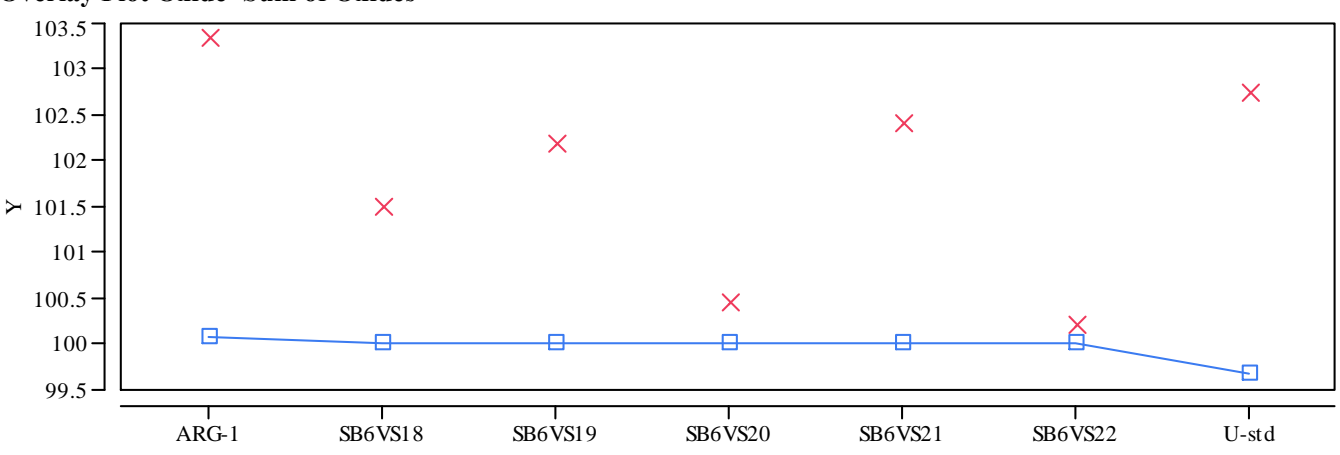

Glass ID

Y $\times$ Measured $\square$ - Targeted 


\section{Appendix D:}

\section{Tables and Exhibits Supporting the Analysis of the PCT Results for the Thorium Study \\ Glasses}


SRNL-STI-2010-00242

Revision 0

This page intentionally left blank. 
Table D1. Analytical Development's Measurements of the PCT Solutions AsReceived (ar) and After Appropriate Adjustments (in ppm) for the Thorium Glasses

\begin{tabular}{|c|c|c|c|c|c|c|c|c|c|c|c|c|}
\hline Glass ID & Heat Treatment & Block & Seq & Lab ID & B ar & Li ar & Na ar & Si ar & B (ppm) & $\mathbf{L i}(\mathbf{p p m})$ & $\mathrm{Na}(\mathrm{ppm})$ & Si (ppm) \\
\hline Soln Std & \begin{tabular}{|l|} 
ref \\
\end{tabular} & 1 & 1 & std-11 & 19.7 & 9.77 & 81.7 & 51.8 & \begin{tabular}{|l|}
19.700 \\
\end{tabular} & 9.770 & 81.700 & 51.800 \\
\hline SB6VS-21 & quenched & 1 & 2 & Y15 & 6.4 & 10.7 & 60.4 & 70 & 10.667 & 17.834 & 100.669 & 116.669 \\
\hline SB6VS-21 & CCC & 1 & 3 & Y18 & 6.31 & 11 & 55.7 & 69.4 & 10.517 & 18.334 & 92.835 & 115.669 \\
\hline SB6VS-20 & quenched & 1 & 4 & Y32 & 6.48 & 11.3 & 58.5 & 72.4 & 10.800 & 18.834 & 97.502 & 120.669 \\
\hline SB6VS-18 & $\mathrm{CCC}$ & 1 & 5 & Y06 & 7.03 & 12.7 & 50.7 & 79.3 & 11.717 & 21.167 & 84.502 & 132.169 \\
\hline blank & ref & 1 & 6 & $\mathrm{Y} 24$ & $<0.375$ & $<0.756$ & $<0.863$ & $<0.954$ & 0.313 & 0.630 & 0.719 & 0.795 \\
\hline SB6VS-19 & $\mathrm{CCC}$ & 1 & 7 & $\mathrm{Y} 20$ & 6.58 & 11.8 & 51.2 & \begin{tabular}{|l|}
73.6 \\
\end{tabular} & 10.967 & 19.667 & 85.335 & 122.669 \\
\hline SB6VS-20 & CCC & 1 & 8 & Y09 & 6.36 & 11.4 & 53.2 & 70.9 & 10.600 & 19.000 & 88.668 & 118.169 \\
\hline Soln Std & ref & 1 & 9 & std-12 & 19.8 & 9.89 & 82.7 & 52.2 & 19.800 & 9.890 & 82.700 & 52.200 \\
\hline SB6VS-22 & $\mathrm{CCC}$ & 1 & 10 & $\mathrm{Y} 01$ & 6.14 & 10.3 & 57.1 & 66.2 & 10.234 & 17.167 & 95.169 & 110.336 \\
\hline EA & ref & 1 & 11 & Y34 & 30.7 & 10.2 & 86.1 & 51.6 & 511.668 & 170.000 & 1435.003 & 860.002 \\
\hline SB6VS-22 & quenched & 1 & 12 & Y35 & 6.36 & 10.2 & 63.5 & 67.6 & 10.600 & 17.000 & 105.835 & 112.669 \\
\hline SB6VS-18 & quenched & 1 & 13 & Y30 & 6.74 & 12 & 52.6 & 75.9 & 11.234 & 20.000 & 87.668 & 126.503 \\
\hline ARM-1 & ref & 1 & 14 & Y31 & 10.3 & 8.37 & 22.1 & 37.5 & 17.167 & 13.950 & 36.834 & 62.501 \\
\hline SB6VS-19 & quenched & 1 & 15 & Y28 & 6.6 & 11.5 & 54.9 & 73.5 & 11.000 & 19.167 & 91.502 & 122.502 \\
\hline Soln Std & ref & 1 & 16 & std-13 & 19.8 & 9.87 & 82.6 & 51.9 & 19.800 & 9.870 & 82.600 & 51.900 \\
\hline Soln Std & ref & 2 & 1 & std-21 & 19.9 & 9.75 & 82.7 & 52.2 & 19.900 & 9.750 & 82.700 & 52.200 \\
\hline SB6VS-22 & quenched & 2 & 2 & Y10 & 6.04 & 9.36 & 61.3 & 65.2 & 10.067 & 15.600 & 102.169 & 108.669 \\
\hline SB6VS-21 & quenched & 2 & 3 & Y12 & 6.02 & 9.77 & 57.5 & 66 & 10.034 & 16.284 & 95.835 & 110.002 \\
\hline ARM-1 & ref & 2 & 4 & Y08 & 10.4 & 8.25 & 22.4 & 38.3 & 17.334 & 13.750 & 37.334 & 63.835 \\
\hline SB6VS-22 & quenched & 2 & 5 & Y37 & 6.22 & 9.73 & 62.9 & 67.1 & 10.367 & 16.217 & 104.835 & 111.836 \\
\hline SB6VS-20 & quenched & 2 & 6 & Y26 & 6.35 & 11 & 57.3 & 71.2 & 10.584 & 18.334 & 95.502 & 118.669 \\
\hline SB6VS-20 & CCC & 2 & 7 & Y16 & 6.42 & 11.3 & 53.3 & 72.4 & 10.700 & 18.834 & 88.835 & 120.669 \\
\hline SB6VS-19 & CCC & 2 & 8 & $\mathrm{Y} 04$ & 6.19 & 11 & 49.5 & 69.4 & 10.317 & 18.334 & 82.502 & 115.669 \\
\hline Soln Std & ref & 2 & 9 & std-22 & 19.7 & 9.84 & 82.2 & 51.7 & 19.700 & 9.840 & 82.200 & 51.700 \\
\hline SB6VS-19 & quenched & 2 & 10 & Y02 & 6.55 & 11.5 & 54.9 & 72.8 & 10.917 & 19.167 & 91.502 & 121.336 \\
\hline EA & ref & 2 & 11 & Y22 & 34.4 & 10.6 & 96.5 & 54.9 & 573.334 & 176.667 & 1608.337 & 915.002 \\
\hline SB6VS-18 & $\mathrm{CCC}$ & 2 & 12 & Y27 & 6.98 & 12.3 & 49.5 & 76.5 & 11.634 & 20.500 & 82.502 & 127.503 \\
\hline SB6VS-21 & CCC & 2 & 13 & Y23 & 6.21 & 10.5 & 55.6 & 69 & 10.350 & 17.500 & 92.669 & 115.002 \\
\hline SB6VS-18 & quenched & 2 & 14 & Y33 & 6.62 & 11.7 & 51.6 & 75.8 & 11.034 & 19.500 & 86.002 & 126.336 \\
\hline Soln Std & ref & 2 & 15 & std-23 & 19.8 & 9.67 & 82.3 & 51.6 & 19.800 & 9.670 & 82.300 & 51.600 \\
\hline Soln Std & ref & 3 & 1 & std-31 & 19.9 & 9.79 & 82.1 & 52 & 19.900 & 9.790 & 82.100 & 52.000 \\
\hline SB6VS-21 & CCC & 3 & 2 & Y05 & 6.47 & 11.1 & 56.6 & 70.9 & 10.784 & 18.500 & 94.335 & 118.169 \\
\hline SB6VS-19 & quenched & 3 & 3 & Y38 & 6.74 & 11.7 & 55.7 & 74.8 & 11.234 & 19.500 & 92.835 & 124.669 \\
\hline ARM-1 & ref & 3 & 4 & Y14 & 10.7 & 8.35 & 22.2 & 38 & 17.834 & 13.917 & 37.001 & 63.335 \\
\hline EA & ref & 3 & 5 & Y29 & 33.5 & 10.6 & 93.1 & 54.4 & 558.334 & 176.667 & 1551.670 & 906.668 \\
\hline SB6VS-22 & $\mathrm{CCC}$ & 3 & 6 & Y36 & 6.24 & 10.5 & 57.2 & 67.9 & 10.400 & 17.500 & 95.335 & 113.169 \\
\hline SB6VS-18 & $\mathrm{CCC}$ & 3 & 7 & Y21 & 7 & 12.5 & 50 & 78.5 & 11.667 & 20.834 & 83.335 & 130.836 \\
\hline SB6VS-22 & CCC & 3 & 8 & Y17 & 6.2 & 10.5 & 56.9 & 67.6 & 10.334 & 17.500 & 94.835 & 112.669 \\
\hline Soln Std & ref & 3 & 9 & std-32 & 19.9 & 9.86 & 81.8 & 52 & 19.900 & 9.860 & 81.800 & 52.000 \\
\hline SB6VS-19 & CCC & 3 & 10 & Y07 & 6.67 & 11.8 & 51 & 74.4 & 11.117 & 19.667 & 85.002 & 124.002 \\
\hline SB6VS-21 & quenched & 3 & 11 & Y03 & \begin{tabular}{|l|}
6 \\
\end{tabular} & \begin{tabular}{|l|}
9.77 \\
\end{tabular} & \begin{tabular}{|l|}
56.4 \\
\end{tabular} & \begin{tabular}{|l|}
66.3 \\
\end{tabular} & 10.000 & 16.284 & 94.002 & 110.502 \\
\hline blank & ref & 3 & 12 & Y19 & $<0.375$ & $<0.756$ & $<0.863$ & $<0.954$ & 0.313 & 0.630 & 0.719 & 0.795 \\
\hline SB6VS-20 & quenched & 3 & 13 & $\mathrm{Y} 25$ & \begin{tabular}{|l|}
6.38 \\
\end{tabular} & \begin{tabular}{|l|}
11 \\
\end{tabular} & \begin{tabular}{|l|}
56.8 \\
\end{tabular} & \begin{tabular}{|l|}
71.6 \\
\end{tabular} & 10.634 & 18.334 & 94.669 & 119.336 \\
\hline SB6VS-20 & CCC & 3 & 14 & Y11 & 6.39 & 11.4 & 52.6 & 71.4 & 10.650 & 19.000 & 87.668 & 119.002 \\
\hline SB6VS-18 & quenched & 3 & 15 & Y13 & 6.74 & 12.1 & 52 & 77.4 & 11.234 & 20.167 & 86.668 & 129.003 \\
\hline Soln Std & ref & 3 & 16 & std-33 & 19.8 & 9.9 & 82.7 & 51.9 & 19.800 & 9.900 & 82.700 & 51.900 \\
\hline
\end{tabular}


Exhibit D1. PCT Measurements (as Common Logarithms) in Analytical Sequence by Analytical Set for Thorium Glasses

Variability Chart for $\log [\mathrm{B}$ ppm]

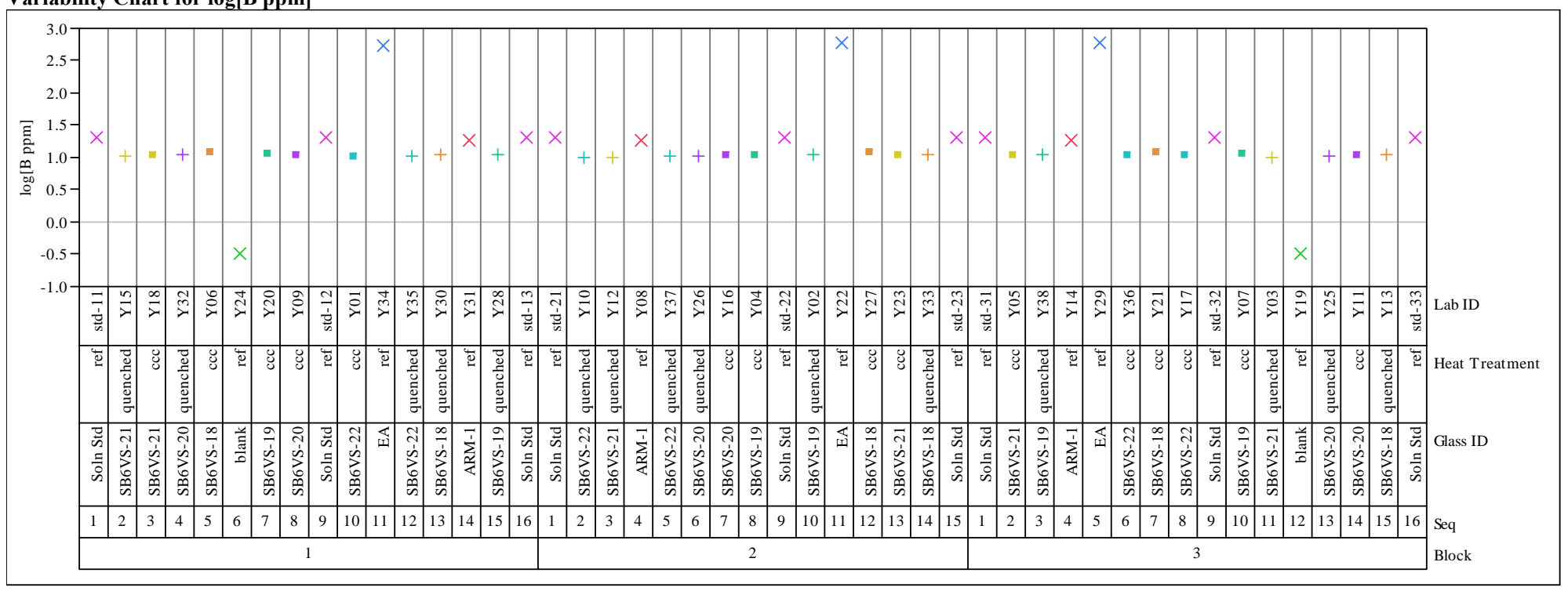

Variability Chart for $\log [\mathrm{Li}$ ppm]

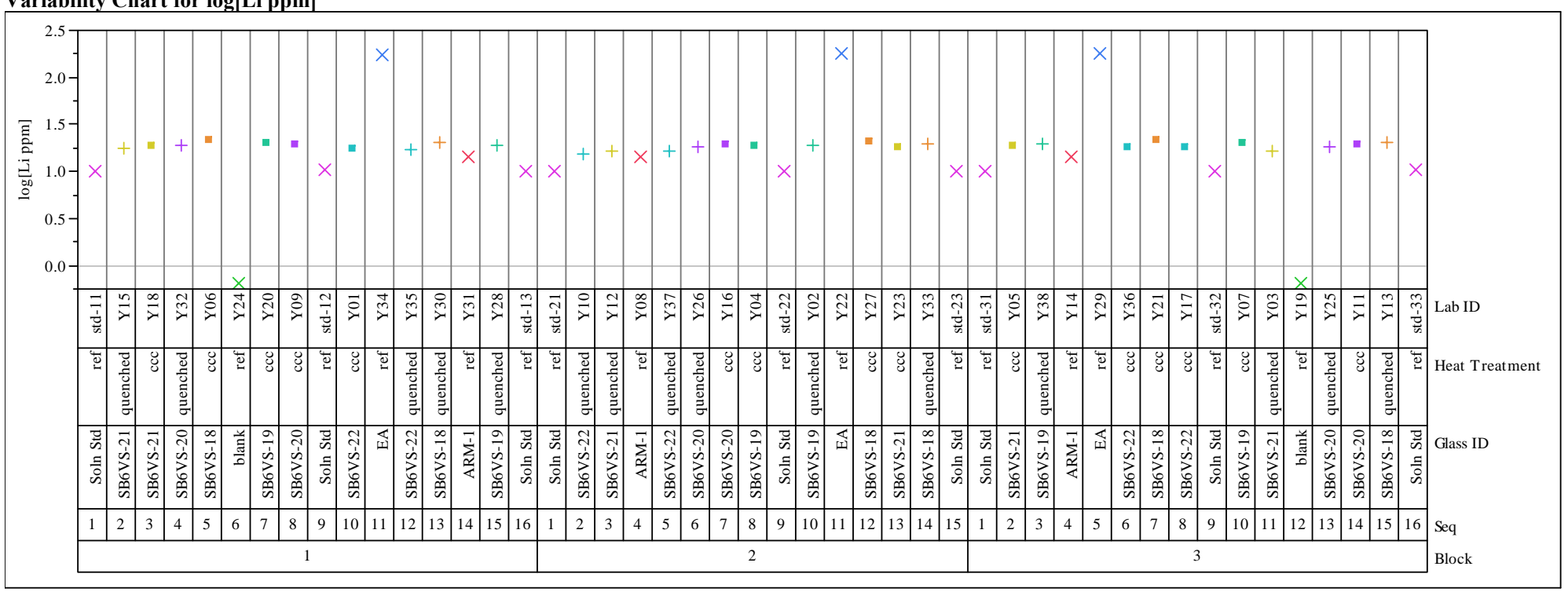


Exhibit D1. PCT Measurements (as Common Logarithms) in Analytical Sequence by Analytical Set for Thorium Glasses

Variability Chart for log[Na ppm]

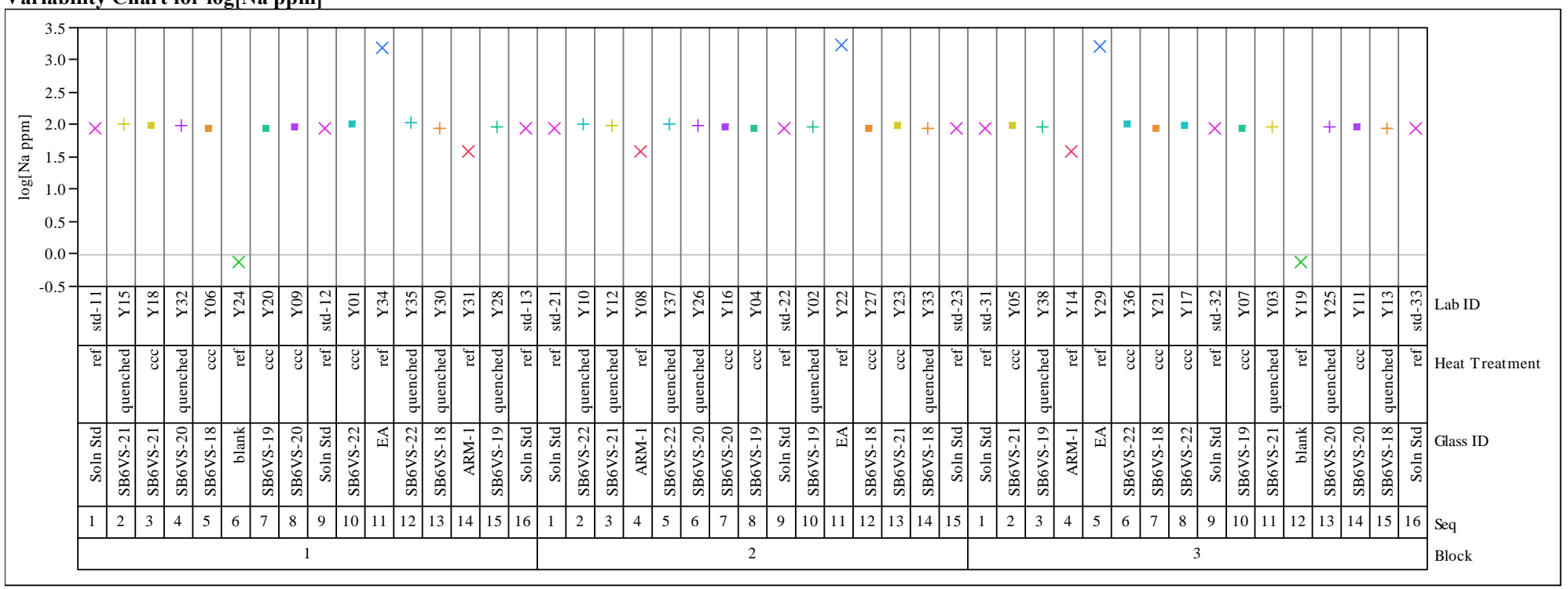


Exhibit D1. PCT Measurements (as Common Logarithms) in Analytical Sequence by Analytical Set for Thorium Glasses

Variability Chart for $\log [\mathrm{Si}$ ppm]

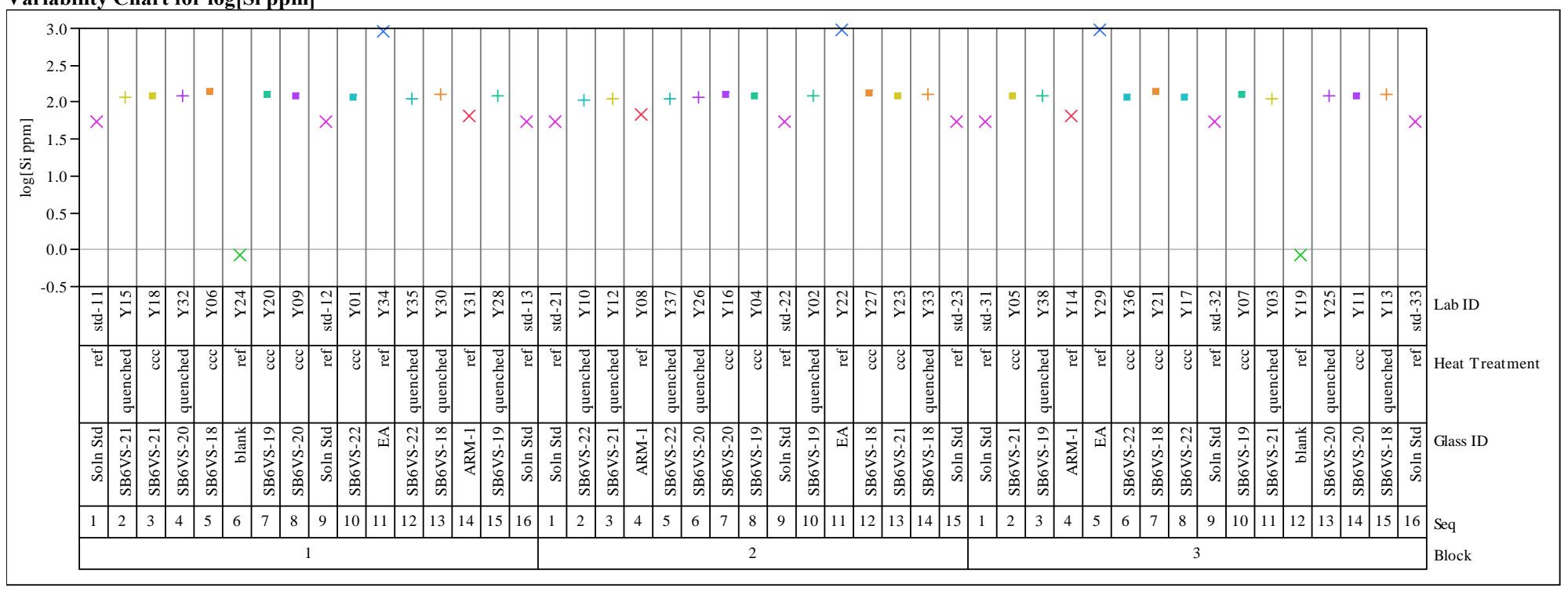




\section{Exhibit D2. Measurements of the Multi-Element Solution Standard by ICP Block for Thorium Glasses}

Oneway Analysis of B (ppm) By Block

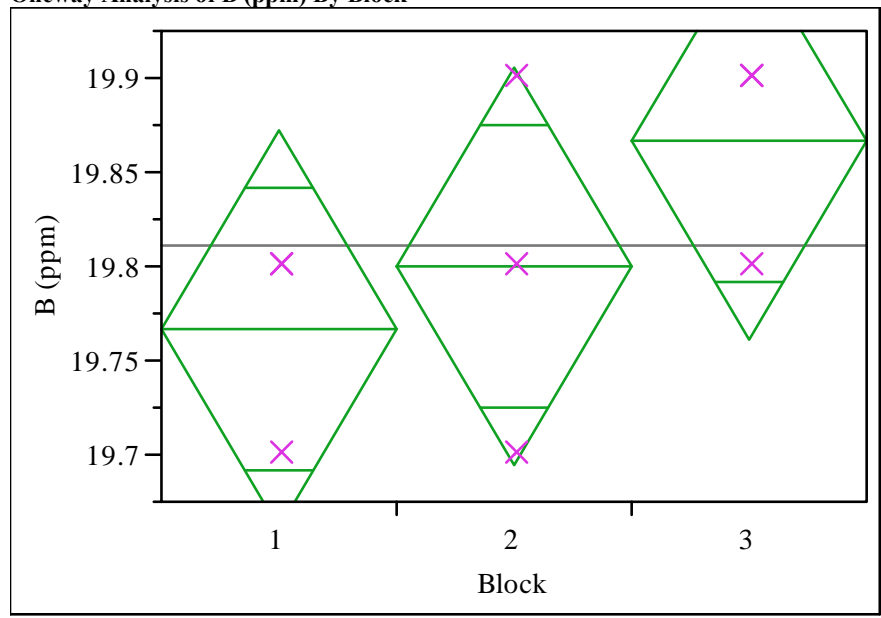

Summary of Fit

$\begin{array}{lr}\text { Rsquare } & 0.318182 \\ \text { Adj Rsquare } & 0.090909 \\ \text { Root Mean Square Error } & 0.074536 \\ \text { Mean of Response } & 19.81111 \\ \text { Observations (or Sum Wgts) } & 9\end{array}$

Analysis of Variance

Source DF Sum of Squares Mean Square F Ratio Prob $>$ F

$\begin{array}{llllll}\text { Block } & 2 & 0.01555556 & 0.007778 & 1.4000 & 0.3170\end{array}$

Error $\quad 6 \quad 0.03333333$

0.005556

C. Total $8-0.04888889$

\begin{tabular}{lrrrrr} 
Level Number & Mean & Std Error & Lower 95\% & Upper 95\% \\
1 & 3 & 19.7667 & 0.04303 & 19.661 & 19.872 \\
\hline & 3 & 19.8000 & 0.04303 & 19.695 & 19.905
\end{tabular}

$\begin{array}{llllll}1 & 3 & 19.7667 & 0.04303 & 19.661 & 19.872 \\ 2 & 3 & 19.8000 & 0.04303 & 19.695 & 19.905 \\ 3 & 3 & 19.8667 & 0.04303 & 19.761 & 19.972\end{array}$

Std Error uses a pooled estimate of error variance
Oneway Analysis of Li (ppm) By Block

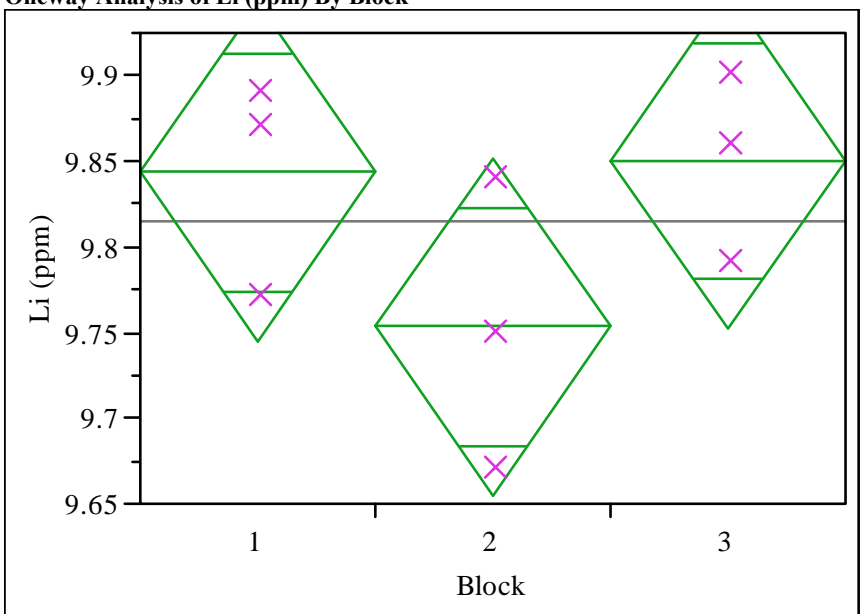

Oneway Anova

Summary of Fit

Rsquare

Adj Rsquare $\quad 0.16898$

Root Mean Square Error $\quad 0.069442$

Mean of Response $\quad 9.815556$

Observations (or Sum Wgts)

Analysis of Variance

Source DF Sum of Squares Mean Square F Ratio Prob $>$ F

$\begin{array}{llllll}\text { Block } & 2 & 0.01748889 & 0.008744 & 1.8134 & 0.2421\end{array}$

Error $\quad \begin{array}{lll}6 & 0.02893333\end{array}$ 0.004822

C. Total $8 \quad 0.0464222$

Level Number Mean Std Error Lower 95\% Upper 95\%

$\begin{array}{lrrrrr}1 & 3 & 9.84333 & 0.04009 & 9.7452 & 9.9414 \\ 2 & 3 & 9.75333 & 0.04009 & 9.6552 & 9.8514\end{array}$

$\begin{array}{llllll}1 & 3 & 9.75333 & 0.04009 & 9.6552 & 9.8514 \\ 3 & 3 & 9.85000 & 0.04009 & 9.7519 & 9.9481\end{array}$

Std Error uses a pooled estimate of error variance 


\section{Exhibit D2. Measurements of the Multi-Element Solution Standard by ICP Block for Thorium Glasses}

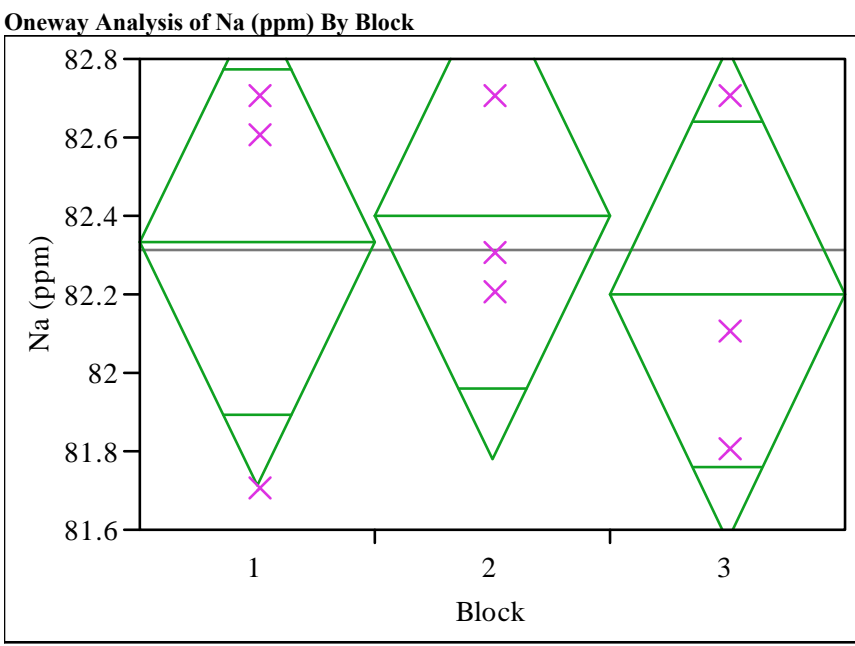

\section{Oneway Anova}

Summary of Fit

Rsquare

0.050633

Adj Rsquare $\quad-0.26582$

Mean of Response 82.31111

Observations (or Sum Wgts)

Analysis of Variance

Source DF Sum of Squares Mean Square F Ratio Prob $>$ F

$\begin{array}{llllll}\text { Block } & 2 & 0.0622222 & 0.031111 & 0.1600 & 0.8557\end{array}$

Error $\quad 6 \quad 1.1666667$

0.19444

C. Total $8-1.2288889$

Means for Oneway Anova

$\begin{array}{rrrrrr}\text { Level Number } & \text { Mean } & \text { Std Error } & \text { Lower 95\% } & \text { Upper 95\% } \\ 1 & 3 & 82.3333 & 0.25459 & 81.710 & 82.956 \\ 2 & 3 & 82.4000 & 0.25459 & 81.777 & 83.023\end{array}$

$\begin{array}{llllll}2 & 3 & 82.4000 & 0.25459 & 81.777 & 83.023 \\ 3 & 3 & 82.2000 & 0.25459 & 81.577 & 82.823\end{array}$

Std Error uses a pooled estimate of error variance

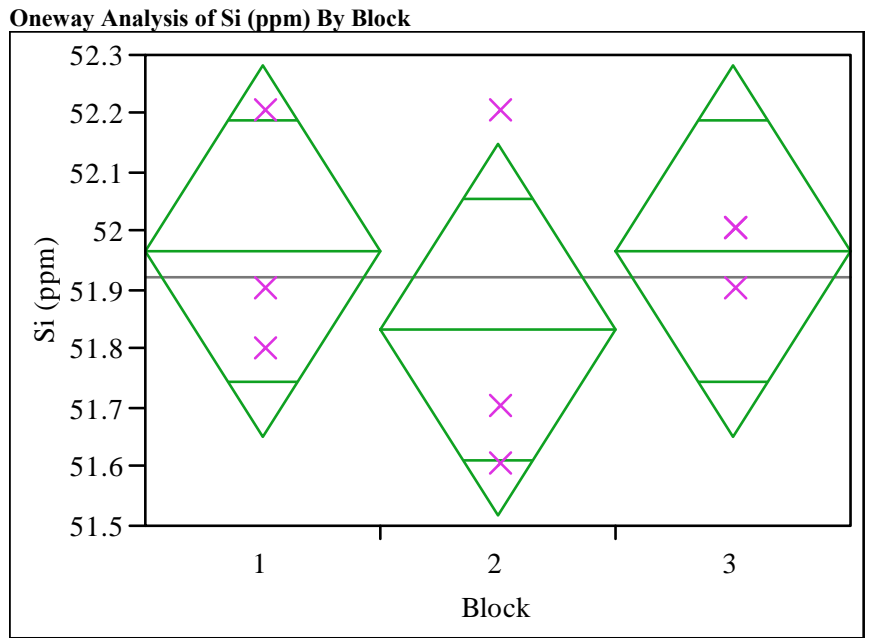

Oneway Anova

Summary of Fit

Rsquare

Mean of Response 51.92222

Observations (or Sum Wgts)

Analysis of Variance

Source DF Sum of Squares Mean Square F Ratio Prob $>$ F

$\begin{array}{llllll}\text { Block } & 2 & 0.03555556 & 0.017778 & 0.3556 & 0.7146\end{array}$

Error $\quad \begin{array}{lll}6 & 0.30000000\end{array}$

$\begin{array}{lll}\text { C. Total } & 8 & 0.33555556\end{array}$

\section{Means for Oneway Anova}

Level Number Mean Std Error Lower 95\% Upper 95\%

$\begin{array}{lllllr}1 & 3 & 51.9667 & 0.12910 & 51.651 & 52.283 \\ 2 & 3 & 51.8333 & 0.12910 & 51.517 & 52.149\end{array}$

$\begin{array}{llllll}1 & 3 & 51.8333 & 0.12910 & 51.517 & 52.149 \\ 3 & 3 & 51.9667 & 0.12910 & 51.651 & 52.283\end{array}$

Std Error uses a pooled estimate of error variance 
Exhibit D3. Laboratory PCT Measurements by Glass Identifier for Thorium Study Glasses and Standards
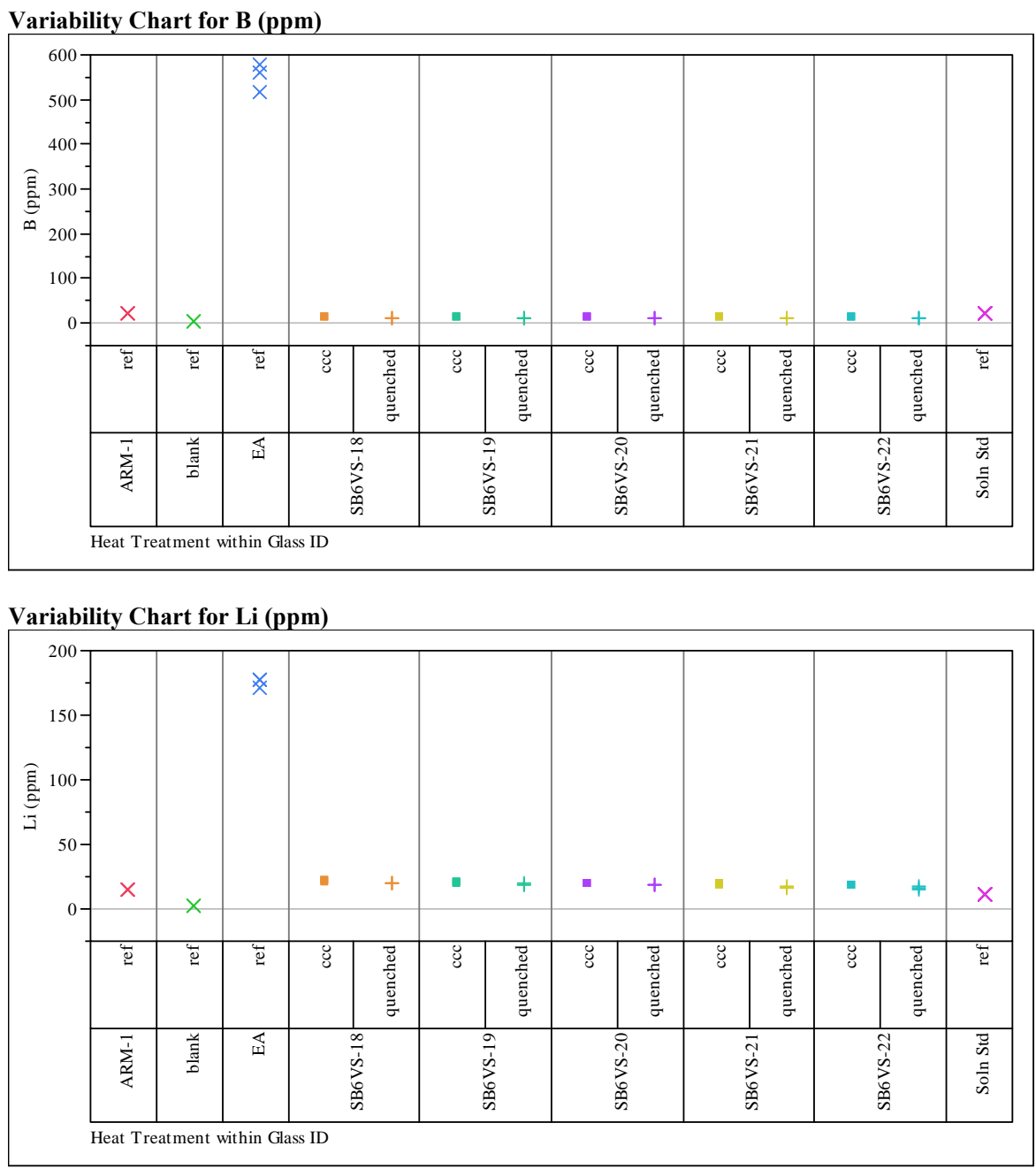

Variability Chart for Na (ppm)

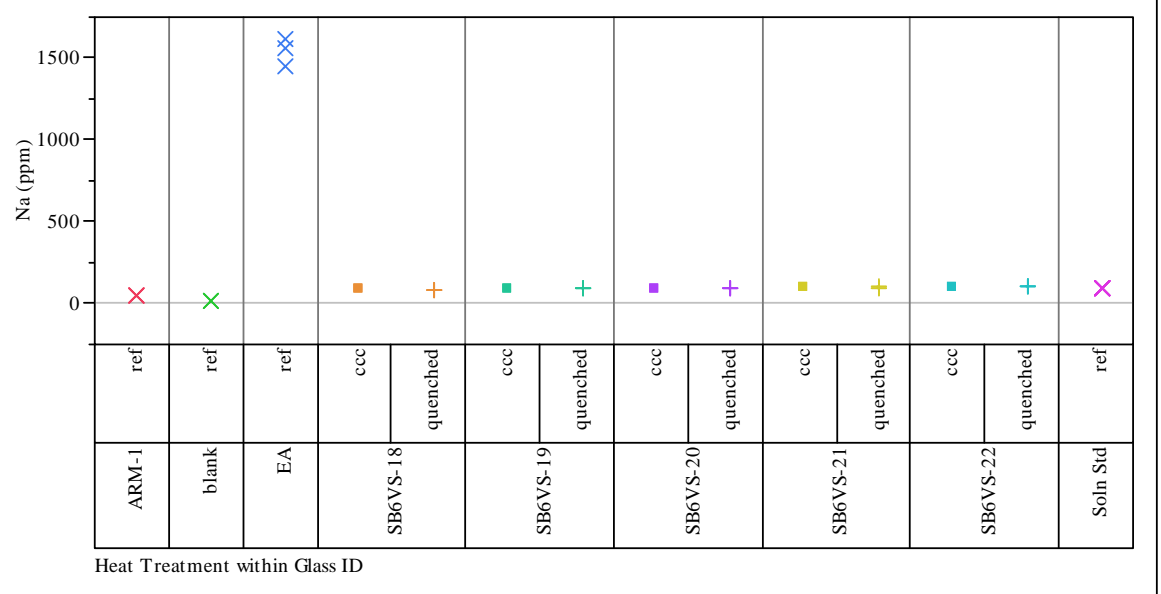


Exhibit D3. Laboratory PCT Measurements by Glass Identifier for Thorium Study Glasses and Standards
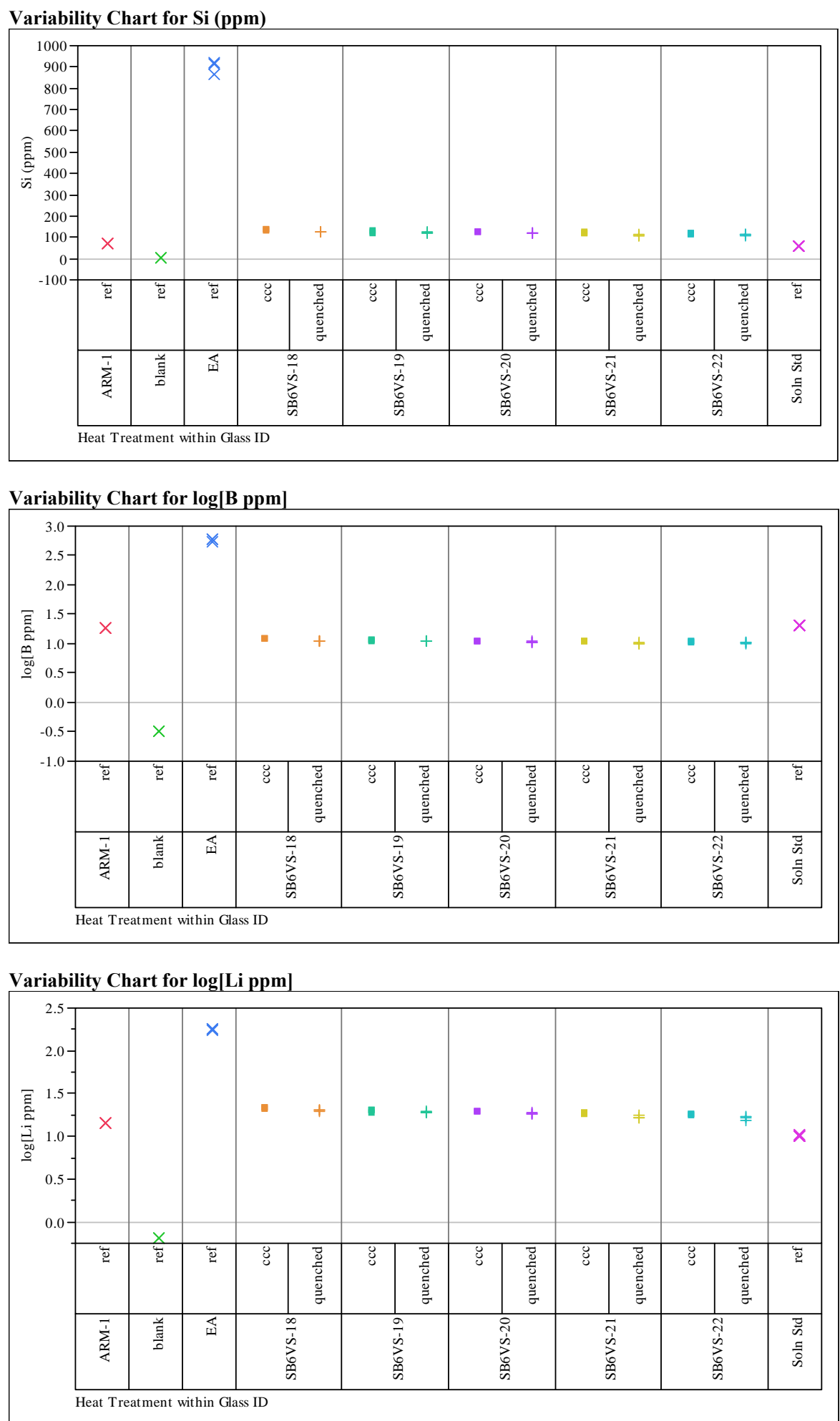
Exhibit D3. Laboratory PCT Measurements by Glass Identifier for Thorium Study Glasses and Standards
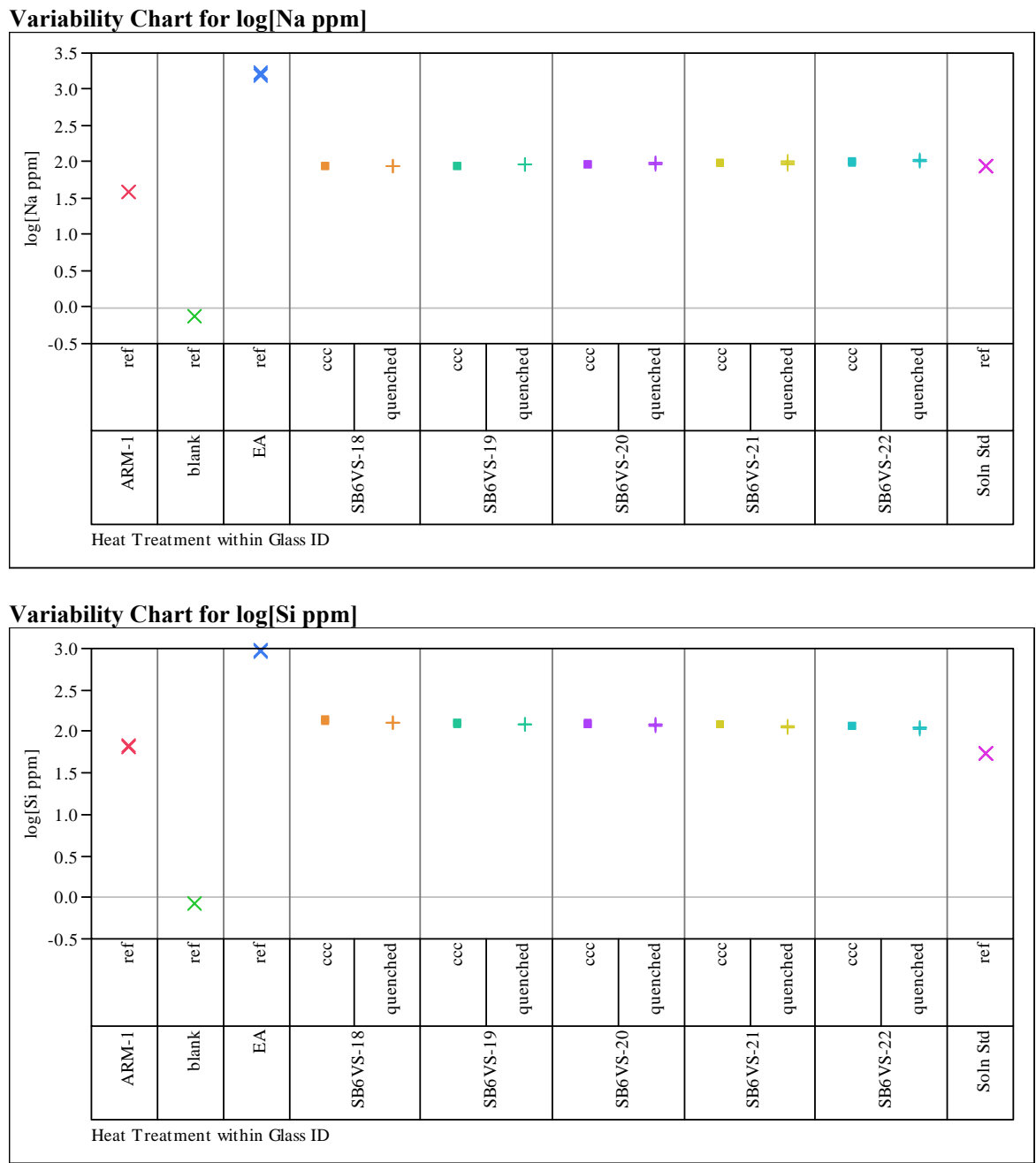


\section{Exhibit D4. Correlations and Scatter Plots of Normalized PCTs Over All Compositional Views and Heat Treatments for the Thorium Glasses}

\section{Multivariate \\ Correlations}

$\log \mathrm{NL}[\mathrm{B}(\mathrm{g} / \mathrm{L})]$

$\log \operatorname{NL}[\operatorname{Li}(g / \mathrm{L})]$

$\log \mathrm{NL}[\mathrm{Na}(\mathrm{g} / \mathrm{L})]$

$\log \mathrm{NL}[\mathrm{Si}(\mathrm{g} / \mathrm{L})]$
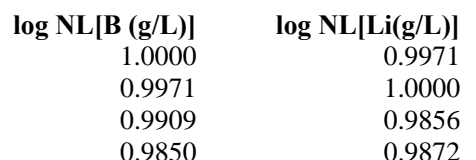
$\log \mathrm{NL}[\mathrm{Na}(\mathrm{g} / \mathrm{L})]$
0.9909
0.9856
1.0000

$\log \mathbf{N L}[\mathbf{S i}(\mathbf{g} / \mathbf{L})]$
0.9850
0.9872
0.9892
1.0000

\section{Scatterplot Matrix}

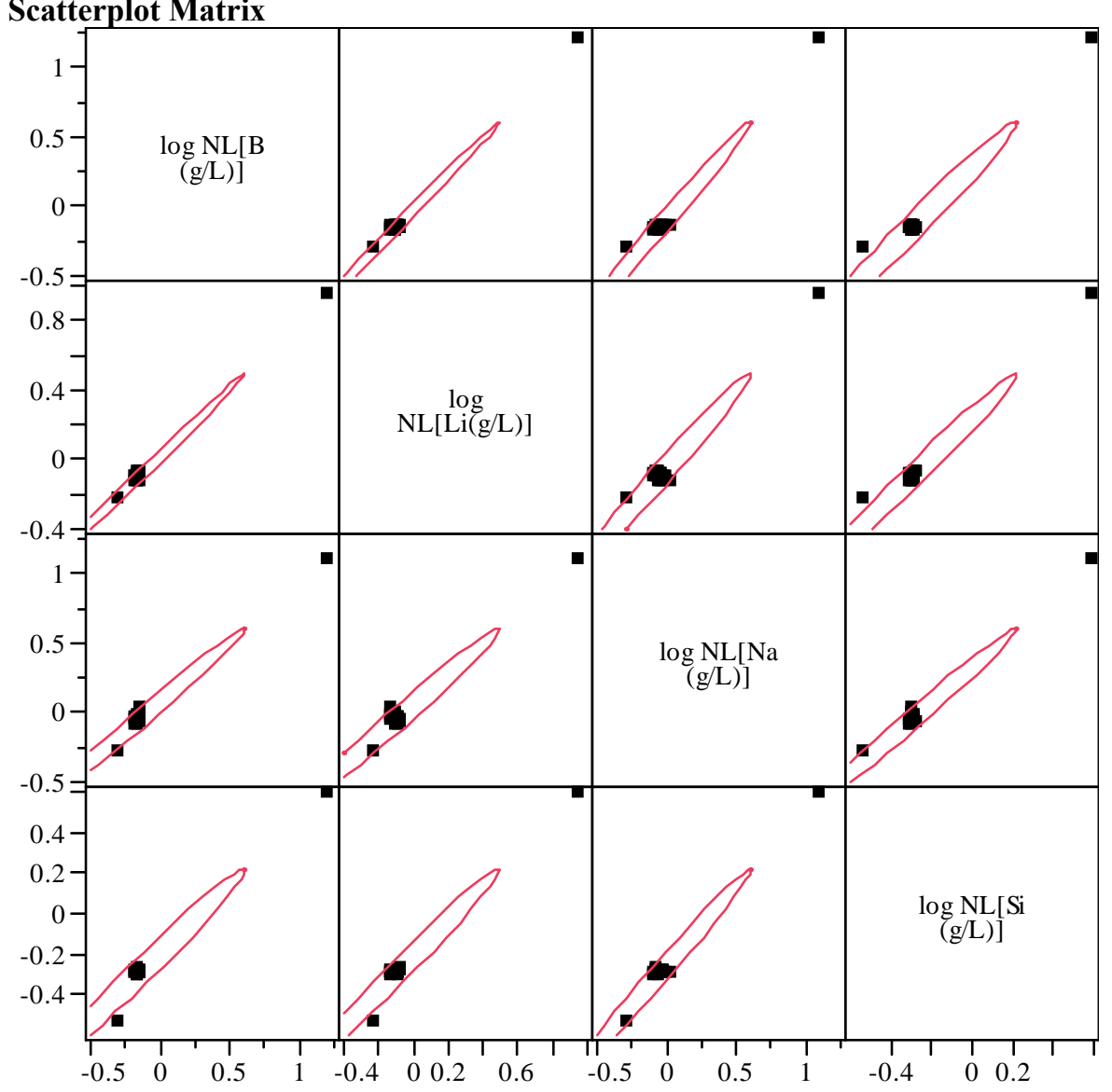




\section{Exhibit D5. Effects of Heat Treatment for Thorium Study Glasses by Compositional View}
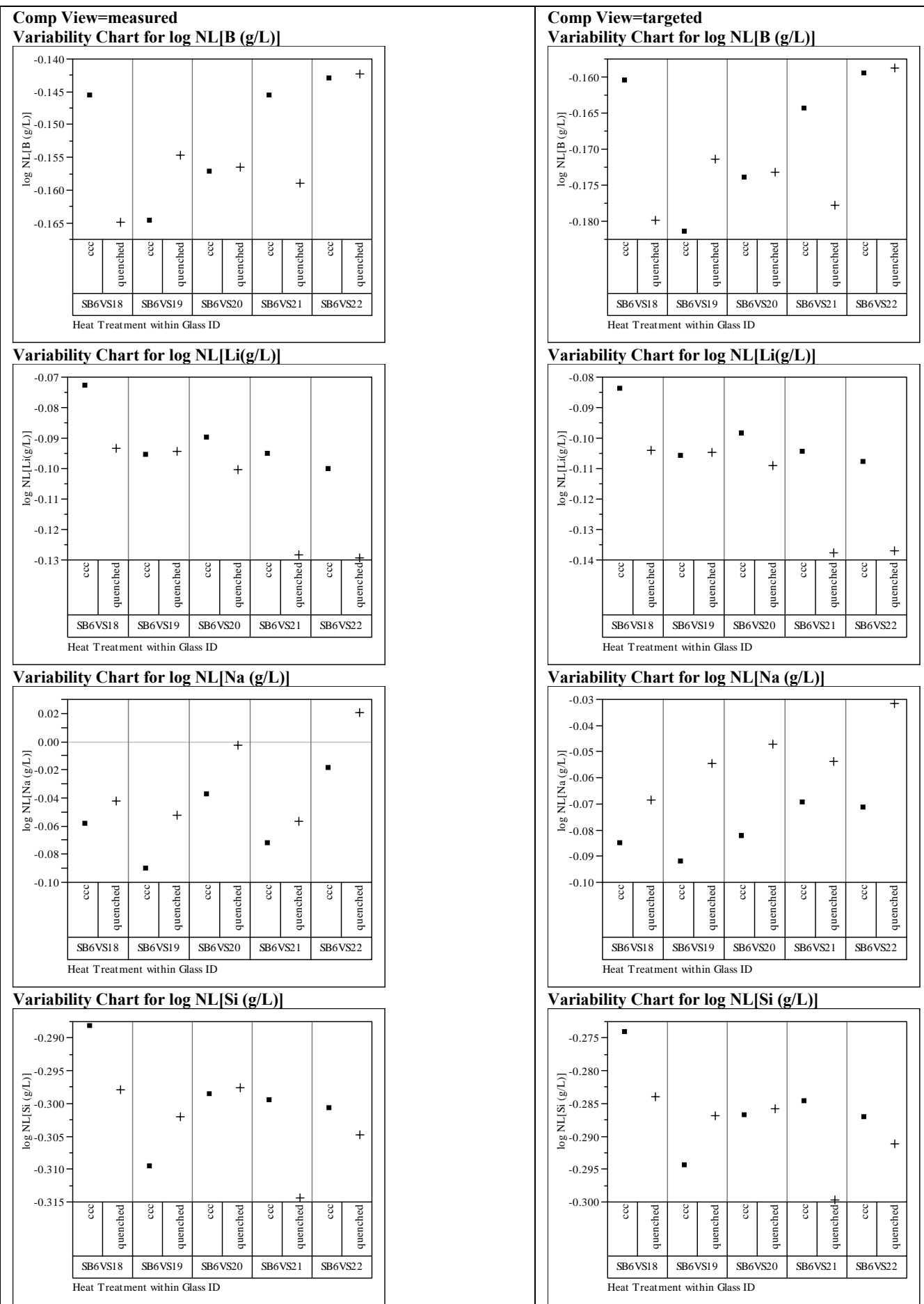

Variability Chart for $\log \mathrm{NL}[\mathrm{Si}(\mathrm{g} / \mathrm{L})]$

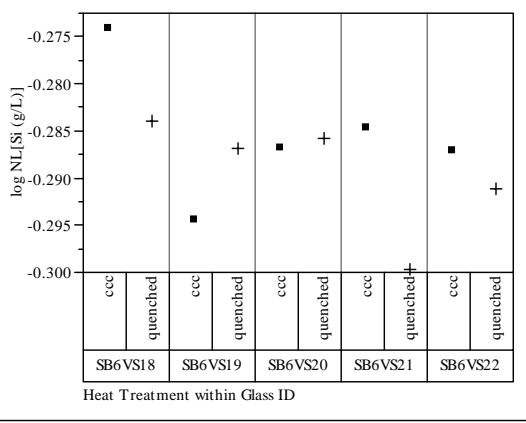


Exhibit D6. del Gp $\left(\Delta G_{p}\right)$ Predictions versus the Common Logarithm of the Normalized Leachate (log NL[.]) for B, Li, Na and Si for Targeted Compositions for the Thorium Glasses for Both Heat Treatments

\begin{tabular}{|c|c|}
\multicolumn{2}{c}{ Legend } \\
Symbol & $\begin{array}{c}\text { Standard/ } \\
\text { Comp View-Heat Treatment }\end{array}$ \\
\hline $\mathbf{Z}$ & EA \\
\hline$\diamond$ & ARM \\
\hline$\times$ & Targeted-ccc \\
\hline$\bullet$ & Targeted-quenched \\
\hline
\end{tabular}

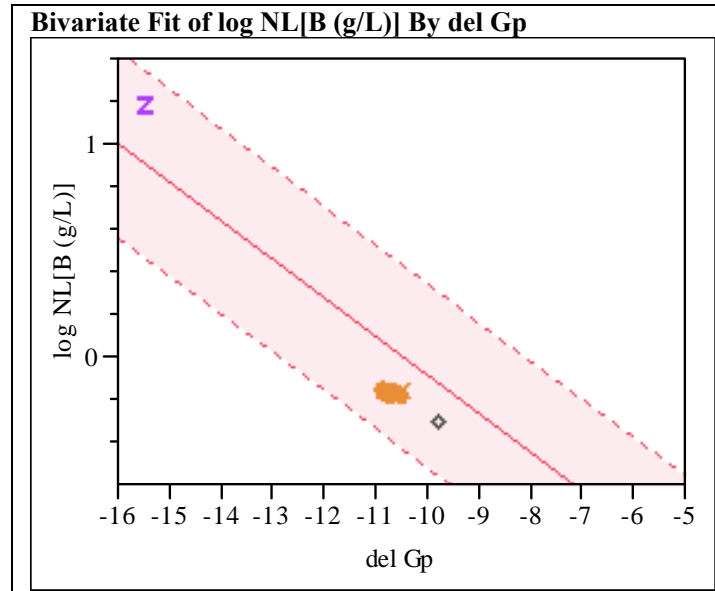

Bivariate Fit of $\log$ NL[Li (g/L)] By del Gp

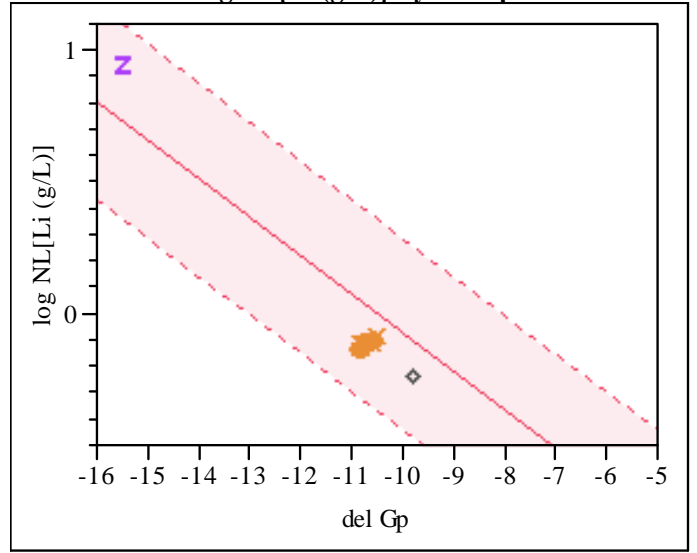

Bivariate Fit of $\log$ NL[Na (g/L)] By del Gp

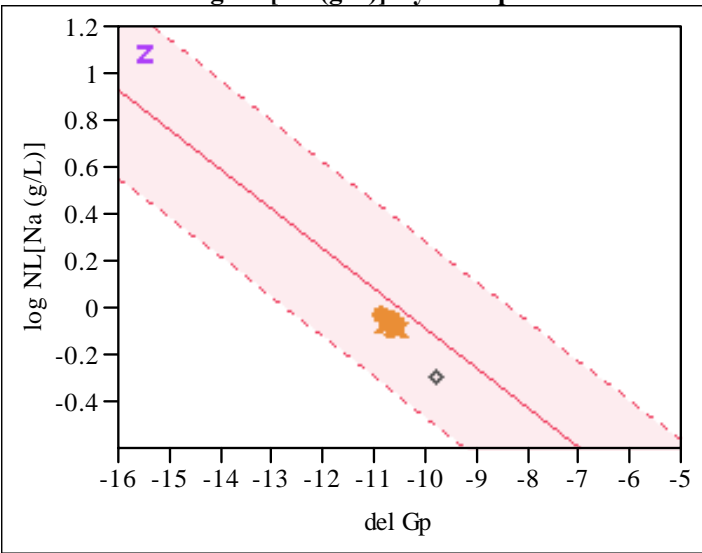

Bivariate Fit of $\log$ NL[Si (g/L)] By del Gp

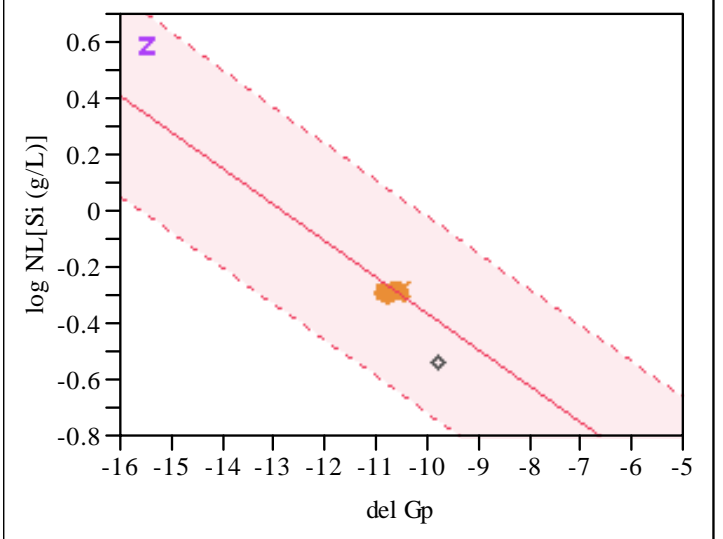


Exhibit D7. del Gp $\left(\Delta G_{p}\right)$ Predictions versus the Common Logarithm of the Normalized Leachate $(\log \mathrm{NL}[\cdot])$ for B, Li, Na and Si for Measured Compositions of the Thorium Glasses for Both Heat Treatments

\begin{tabular}{|c|c|}
\multicolumn{2}{c}{ Legend } \\
\hline Symbol & $\begin{array}{c}\text { Standard/ } \\
\text { Comp View-Heat Treatment }\end{array}$ \\
\hline$z$ & EA \\
\hline$\diamond$ & ARM \\
\hline$\times$ & Measured-ccc \\
\hline$\bullet$ & Measured-quenched \\
\hline
\end{tabular}

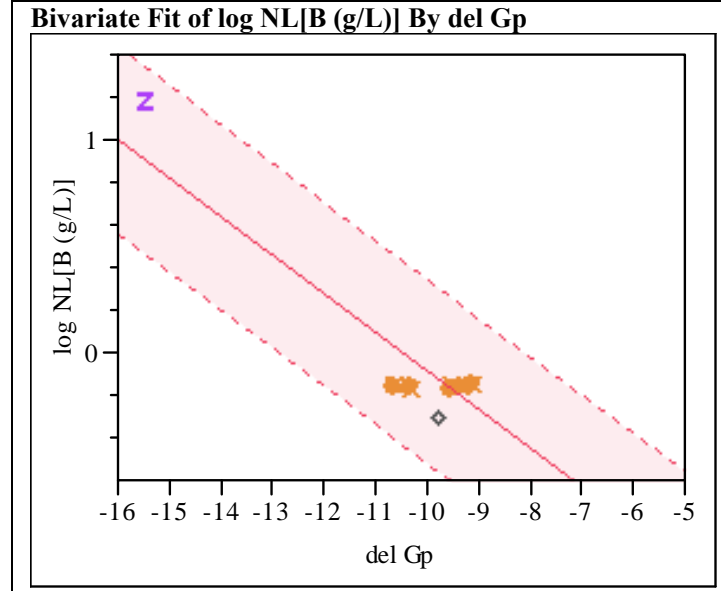

Bivariate Fit of $\log$ NL[Li (g/L)] By del Gp

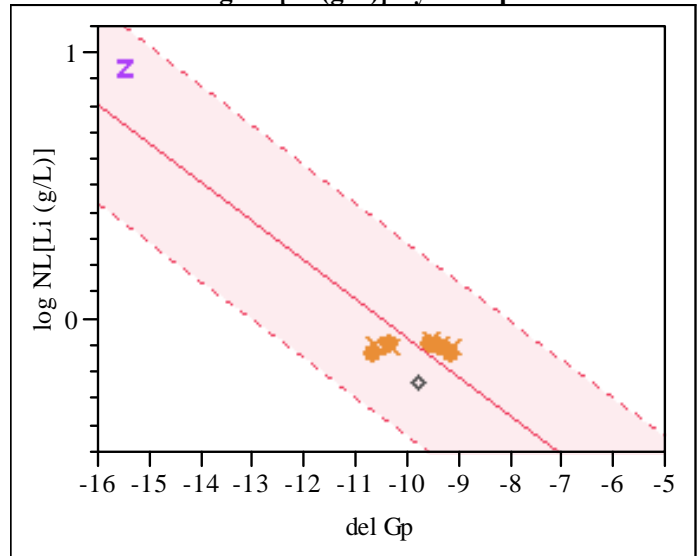

Bivariate Fit of $\log \mathrm{NL}[\mathrm{Na}(\mathrm{g} / \mathrm{L})]$ By del Gp

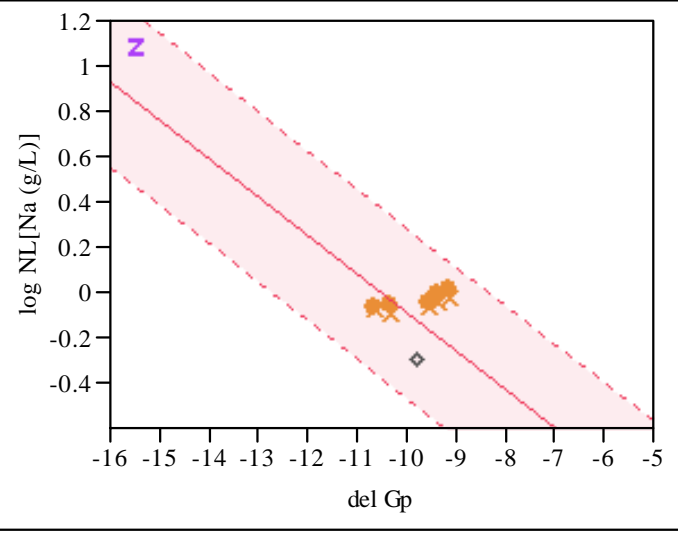

Bivariate Fit of log NL[Si (g/L)] By del Gp

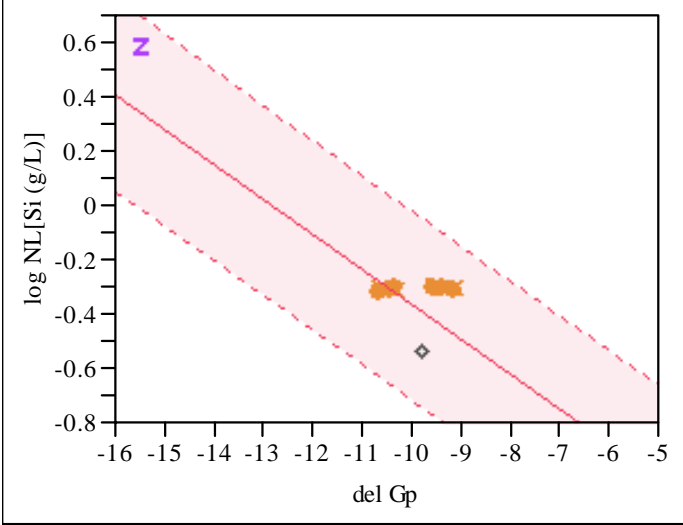




\section{Distribution:}

J.W. Amoroso, 999-W

C. J. Bannochie, 773-42A

A. B. Barnes, 999-W

A.L. Billings, 999-W

J. M. Bricker, 704-27S

M. A. Broome, 704-29S

D. A. Crowley, 773-43A

T.B. Edwards, 999-W

T. L. Fellinger, 704-26S

S. D. Fink, 773-A

K.M. Fox, 999-W

B. J. Giddings, 786-5A

J. M. Gillam, 766-H

B. A. Hamm, 766-H

C. C. Herman, 999-W

R. N. Hinds, 704-S

E. W. Holtzscheiter, 704-15S

J. F. Iaukea, 704-30S

P.R. Jackson, 703-46A

F.C. Johnson, 999-W

M. T. Keefer, 766-H

S. L. Marra, 773-A

D.H. Miller, 999-W

F. M. Pennebaker, 773-42A

J. E. Occhipinti, 704-S

D. K. Peeler, 999-W

J. W. Ray, 704-S

H. B. Shah, 766-H

D. C. Sherburne, 704-S

A.V. Staub, 704-27S

M. E. Stone, 999-W

J. P. Vaughan, 773-41 A 